\title{
Simulation of the effects of movement patterns and resource density on the egg distributions of Pieris rapae (Lepidoptera) at multiple spatial scales.
}

by

James Barritt

A thesis

submitted to the Victoria University of Wellington in fulfilment of the requirements for the degree of

Master of Science in Ecology and Biodiversity.

Victoria University of Wellington 



\begin{abstract}
This thesis presents a spatially explicit, agent based simulation, used to explore the ovipositing behaviour of the Small Cabbage White butterfly, Pieris rapae (Lepidoptera). The study concerns the effects of host-plant (Cabbage, Brassica oleracae) density upon $P$. rapae egg distribution patterns, at multiple scales.

A general review of the literature is provided which covers the ecology of animal movement, methods of quantifying movement, models of movement, ecological theory of herbivore responses to plant density (Resource Concentration Hypothesis) and the biology of Prapae

The construction of the simulation is described in detail and the source code plus an executable version of the software are available as a companion CD.

A number of simulation experiments are reported which demonstrate the basic behaviour of the simulation over a simplified resource layout. The framework is then used to explore more complex layouts which are compared to field experiments conducted as part of a separate PhD thesis (Hasenbank, in prep).

A Correlated Random Walk simulated a negative relationship between forager egg distributions and resource densities, observed at all scales. Including a diffuse attraction to resources (e.g. olfaction), simulated a negative relationship between egg distributions and resource densities at smaller scales, and a positive relationship at larger scales.

This work builds on a large body of previous simulation studies and attempts to produce a useful framework for subsequent researchers to explore the effects of animal movement through the use of random walks. It demonstrates the use of the framework with a specific example concerning the egg distributions of $P$. rapae and the effect of scale. It provides some useful insights into both the analysis of results from a complex spatial experimental layout, and potential responses which may be observed. It demonstrates that a simple model can, in the case of $P$ rapae be used to obtain relatively realistic egg distributions.
\end{abstract}

\title{
Keywords
}

Animal Movement, Brassicae, Correlated Random Walk, Ecology, Host-Plant Interactions, Java, Individual-Based, Landscape, Object-oriented, Pieris rapae L., R statistical language, Resource Concentration Hypothesis, Simulation, Scale, Spatial Ecology. 


\section{Acknowledgements}

I would like to thank my Supervisors Dr. Stephen Hartley and Dr. Marcus Frean of Victoria University Of Wellington. Without their shared interest in modelling and biology I would not have found a topic so interesting to myself for my masters degree and then travelled to New Zealand and had such a wonderful adventure. Marc Hasenbank (soon to be Dr. Hasenbank) has been a fantastic colleague and friend during the time I have spent in Wellington. We have had numerous discussions about the project and it has been great to have someone else who thinks about cabbages and butterflies more than I. Dr. Shirley Pledger provided some key advice throughout the project and was the inspiration to both Marc and myself to begin working with R, without which I don't think I could have completed the work. Thanks to all the members of "Bug Club" at Victoria University; especially Dr. Phil Lester, Rudi (soon to be Dr) Schnitzler, Dr. Kirsti Abbot, Alice McNatty, Alex Dixson and many more for welcoming me to Victoria and providing cakes once a week. Dr. K.C. Burns for taking an interest at my talks and for some interesting suggestions.

I would like to thank Prof. R.E. Jones for taking the time to respond to some of my questions about her work and taking an interest in the project, which was a great inspiration during writing up!

I would also like to thank the administrative staff in the School Of Biological Sciences Mary Murray, Patricia Stein, Sandra Taylor, Delwyn Carter-Jarratt and Paul Marsden who are always very welcoming and keep the place running. The department of Maths and Computer Science have also provided great support in use of server processing resources for running the experiments, without which it would not have been possible to conduct as many simulation experiments.

The Marsden Fund provided funding towards both the cost of fees and living expenses which were gratefully received.

I would most importantly like to thank my parents Wendy and Michael Barritt and the rest of my family, Marie (Nanny), Paul and Steve for their constant support (both emotionally and financially). Last but most definitely not least, Ms Kira McKernan for her beautiful artwork. 


\section{Flying Crooked}

The butterfly, a cabbage-white,

(His honest idiocy of flight)

Will never now, it is too late,

Master the art of flying straight,

Yet has -who knows so well as I?-

A just sense of how not to fly:

He lurches here and here by guess

And God and hope and hopelessness.

Even the acrobatic swift

Has not his flying-crooked gift.

\section{- Robert Graves (1895 - 1985)}

"Essentially, all models are wrong, but some are useful" - George E. Box. 


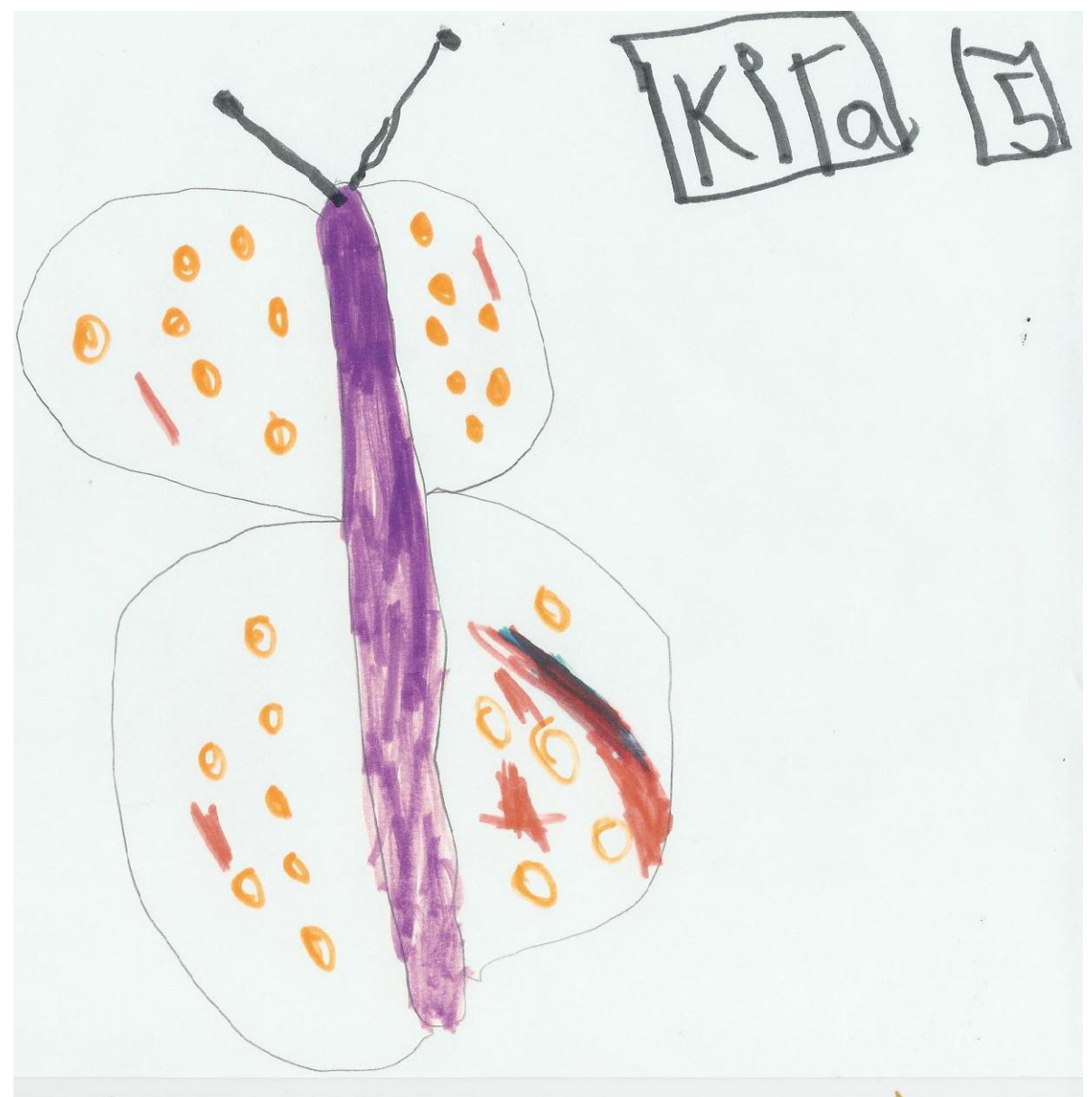

Figure 1: "Butterfly" By Kira McKernan, aged 5 


\section{Contents}

1 General Introduction 1

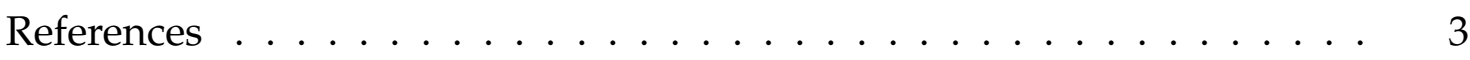

2 Review 5

2.1 Abstract .............................. 5

2.2 The ecology of animal movement . . . . . . . . . . . . 6

2.2.1 Ecological consequences of movement . . . . . . . . . . . 6

2.2.2 Searching for resources . . . . . . . . . . . . 7

2.3 Scale and the ecological landscape . . . . . . . . . . . . . . 11

2.4 The Resource Concentration Hypothesis . . . . . . . . . . . . . . . . . 13

2.5 Quantifying animal movement . . . . . . . . . . . . . 15

2.5.1 The geometry of directional data . . . . . . . . . . . . 15

2.5 .2 Measuring a path . . . . . . . . . . . . 17

2.6 Modelling movement . . . . . . . . . . . . . . . . 27

2.6.1 Random walks . . . . . . . . . . . . . . . . . . 29

2.6 .2 Sensory perception . . . . . . . . . . . . . . 31

2.7 Study species: Pieris rapae . . . . . . . . . . . . . . . . . 37

2.7 .1 Oviposition behaviour . . . . . . . . . . . 38

2.7.2 Trophic interactions . . . . . . . . . . . . . 46

2.8 Case study: Jones $(1977) \ldots \ldots \ldots$. . . . . . . . . . . . 46

2.8.1 Experimental resource layout . . . . . . . . . . . . . 47

2.8 .2 Plant types . . . . . . . . . . . . . . 47

2.8 .3 Experiments . . . . . . . . . . . . . . 47

2.8 .4 Field observations . . . . . . . . . . . . . . 48

2.8.5 Results from field data . . . . . . . . . . . . . . 51

2.8.6 Simulation construction and parameters . . . . . . . . 54

2.8 .7 Simulation results . . . . . . . . . . . . . . 58

2.8 .8 Case study summary . . . . . . . . . . . . . . . 60

2.9 Review summary . . . . . . . . . . . . . . . . . . 61

References ......................... 74 
3 Simulation framework $\quad 75$

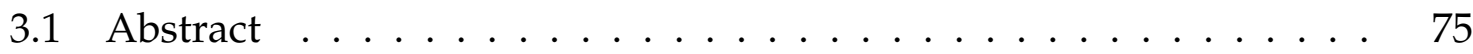

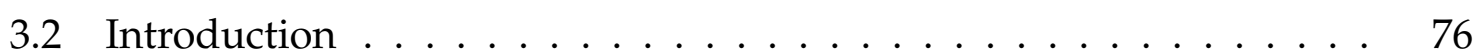

3.3 Methods: Conceptual model . . . . . . . . . . . . . . . . . . . 77

3.3 .1 Resources . . . . . . . . . . . . . . . 78

3.3.2 The correlated random walk $(\mathrm{CRW}) \ldots \ldots$. . . . . . 82

3.3.3 Olfactory perception . . . . . . . . . . . . . . 92

3.3.4 Visual perception . . . . . . . . . . . . . . . . . . . 101

3.3.5 A Signal-based Random Walk . . . . . . . . . . . . . . . . . 106

3.4 Methods: Simulation features . . . . . . . . . . . . . . . . . . . 111

3.4 .1 Landscape . . . . . . . . . . . . . . . . . 112

3.4 .2 Agents ......................... 113

3.4.3 Parameters (experiment editor) . . . . . . . . . . . . . . . 119

3.4.4 Execution (experiment controller) . . . . . . . . . . . . 121

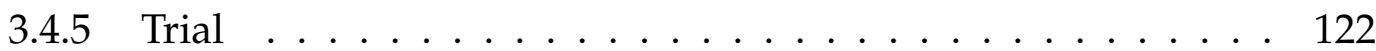

3.4.6 Results analysis ('R' reports $) \ldots \ldots \ldots 123$

3.4.7 Validation . . . . . . . . . . . . . . . . . . 125

3.5 Methods: Exploratory simulation experiments . . . . . . . . . . . . 128

3.5.1 Boundary effect simulation experiment . . . . . . . . . 133

3.5.2 Edge Effect simulation experiment . . . . . . . . . . . . . . 134

3.5.3 Analytical methods . . . . . . . . . . . . . . . . . 136

3.6 Results . . . . . . . . . . . . . . . . . 137

3.6.1 Boundary effect results . . . . . . . . . . . . . 137

3.6 .2 Edge effect results . . . . . . . . . . . . . . . . 142

3.7 Discussion . . . . . . . . . . . . . . . . . 156

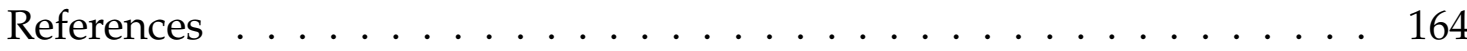

4 Sense and scale $\quad 165$

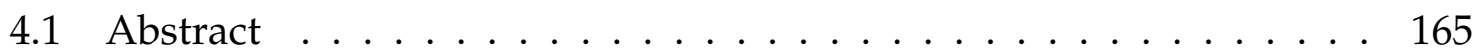

4.2 Introduction . . . . . . . . . . . . . . . . . 166

4.3 Methods ............................ 168

4.3.1 Experimental resource layout . . . . . . . . . . . . . . 168

4.3.2 Analysis of results . . . . . . . . . . . . . . . 175

4.3 .3 Experiments . . . . . . . . . . . . . . . 177

4.4 Results . . . . . . . . . . . . . . . . . . 182

4.4 .1 Field experiments . . . . . . . . . . . . . . . 182

4.4 .2 Calibration . . . . . . . . . . . . . . . . . . 184

4.4.3 Correlated Random Walk . . . . . . . . . . . . . . . . . . 185

4.4 .4 Vision . . . . . . . . . . . . . . . . . 187

4.4 .5 Olfaction . . . . . . . . . . . . . . 188 
4.4.6 Olfaction and vision . . . . . . . . . . . . . . . . . . . . . . 189

4.4 .7 Parameter estimation . . . . . . . . . . . . . . . . . . 190

4.5 Discussion . . . . . . . . . . . . . . . . . . . . . . . . . . . 208

References . . . . . . . . . . . . . . . . . . . 212

5 General discussion 213

References . . . . . . . . . . . . . . . . . . . . . . . 218

A Edge Effect detailed simulation results 219

A.1 Trial B (CRW) Single Egg results . . . . . . . . . . . . . . . . . 221

A.2 Trial B (CRW) Multiple Eggs results _ . . . . . . . . . . . . . 227

A.3 Trial C (Olfaction) Single Egg results . . . . . . . . . . . . . . . 233

A.4 Trial C (Olfaction) Multiple Eggs results . . . . . . . . . . . . . . 239

A.5 Trial D (Vision) Single Egg results . . . . . . . . . . . . . . 245

A.6 Trial D (Vision) Multiple Eggs results . . . . . . . . . . . . . . . . 251

A.7 Trial E (Olfaction and Vision) Single Egg results _ . . . . . . . . 257

A.8 Trial E (Olfaction and Vision) Multiple Eggs results . . . . . . . . 263

B Sense and scale detailed simulation results 269

B.1 Bray-Curtis Optimisation Statistics . . . . . . . . . . . . . . . . 270

B.2 Egg Distributions by $\mathrm{L}$ and $\mathrm{k} \ldots \ldots \ldots \ldots \ldots \ldots$

B.3 Regression Statistics . . . . . . . . . . . . . . . . 306

B.4 Field Breakdowns . . . . . . . . . . . . . . . . . . . . . . . . . 309

C Generation of Lévy dust 313

References . . . . . . . . . . . . . . . . . . . . . 318 


\section{List of Figures}

"Butterfly" By Kira McKernan . . . . . . . . . . . . . . . . . . v v

$2.1 \quad$ Theoretical responses to density $\ldots \ldots \ldots \ldots \ldots \ldots \ldots \ldots$

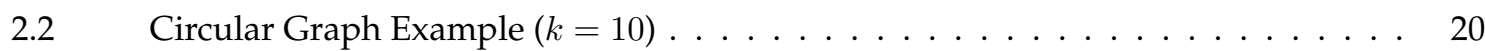

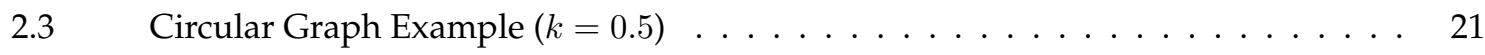

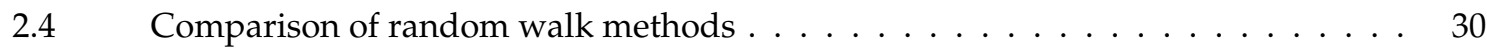

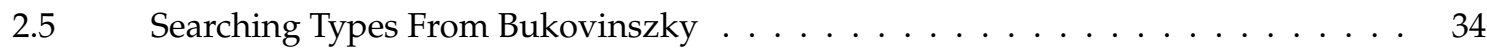

2.6 Jones (1977): Experimental Layouts _ . . . . . . . . . . . . . . . . . 48

$2.7 \quad$ Jones (1977): Flow Diagram . . . . . . . . . . . . . . . . . . . 54

2.8 Jones (1977): MOVE parameter $\ldots \ldots \ldots \ldots \ldots \ldots \ldots \ldots$

$3.1 \quad$ Radius of attraction $\ldots \ldots \ldots \ldots \ldots \ldots \ldots \ldots \ldots \ldots$

$3.2 \quad$ CRW Parameters . . . . . . . . . . . . . . . . . . . . . 83

3.3 Example paths, varying $k \ldots \ldots \ldots \ldots \ldots \ldots \ldots \ldots$

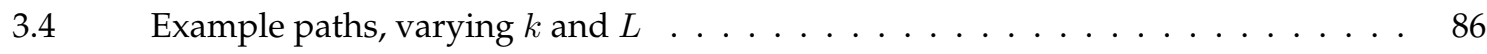

3.5 Example path $(k=10, L=5)$, varying $P$ and $R \ldots \ldots \ldots \ldots \ldots \ldots$

3.6 Example path $k=3, L=5$, varying $P$ and $R \ldots \ldots \ldots \ldots \ldots$

$3.7 \quad$ Example Path $(k=0.5, L=5)$, varying $P$ and $R \ldots \ldots \ldots \ldots \ldots$

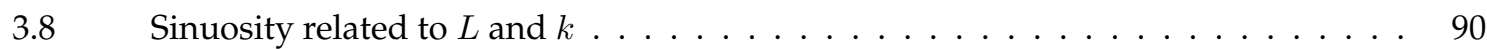

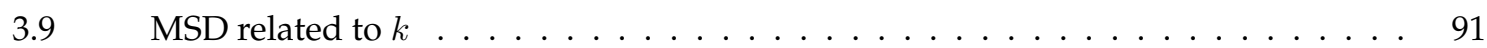

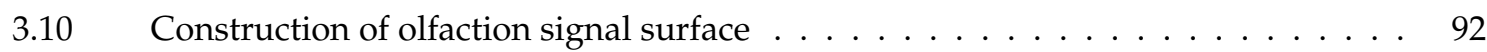

3.11 Olfaction signal surface $(\mathrm{SW}=20) \ldots \ldots \ldots \ldots \ldots \ldots \ldots$

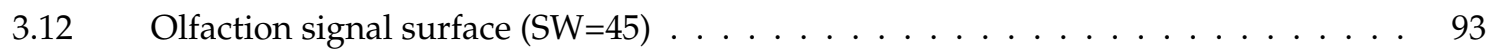

3.13 Olfaction signal surface $(\mathrm{SW}=90) \ldots \ldots \ldots \ldots \ldots \ldots \ldots$

3.14 Olfaction signal surface $(\mathrm{SW}=180) \ldots \ldots \ldots \ldots \ldots \ldots$

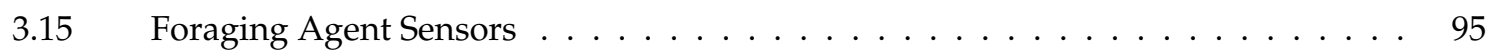

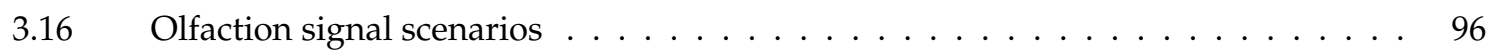

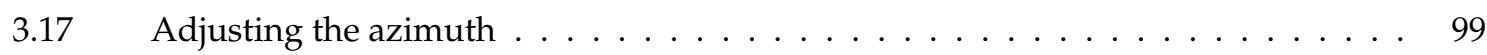

3.18 Difference in signal related to difference in direction . . . . . . . . . . . . . . 100

3.19 Visual Field of View . . . . . . . . . . . . . . . . . . . . . . . . . 102

3.20 Visibility as a function of distance . . . . . . . . . . . . . . . . . . 104

3.21 Visual Input Signal . . . . . . . . . . . . . . . . . . . . . . . . . . . . . 104

3.22 Probability of action from visual input . . . . . . . . . . . . . . . 105

3.23 Visual Noise . . . . . . . . . . . . . . . . . . . . . . . . . . . . . 108

3.24 Structure of Signal-Based CRW . . . . . . . . . . . . . . . . . . . . . . . . . 109

3.25 Calculated layout parameters . . . . . . . . . . . . . . . . . . . . . 115

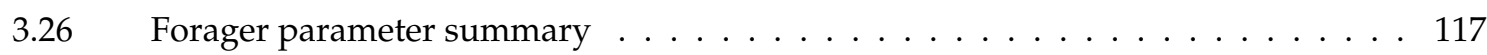

3.27 Release Boundary Parameters . . . . . . . . . . . . . . . . . . . 118 
3.28 Experiment Controller Flow Diagram . . . . . . . . . . . . . . . . . . . . . . . 124

3.29 Validation of Azimuth Generator histogram . . . . . . . . . . . . . . . . . . . 126

$3.30 \quad$ Validation of MSD . . . . . . . . . . . . . . . . . . . . . . . . . . 127

3.31 Corner-Centre-Edge Resource Categorisation . . . . . . . . . . . . . . . . . . . . . . 129

3.32 Resource Layout - Corner, Centre and Edge . . . . . . . . . . . . . . . . . . 130

3.33 Resource Layout - Corner and Centre only . . . . . . . . . . . . . . . . . . 131

3.34 Olfaction signal surface, $P=100, S W=360 \ldots \ldots \ldots \ldots \ldots \ldots \ldots \ldots$

3.35 Olfaction signal surface, $P=300, S W=360 \ldots \ldots \ldots \ldots \ldots \ldots \ldots$

3.36 Olfaction signal surface, $P=600, S W=360 \ldots \ldots \ldots \ldots \ldots \ldots$

3.37 Boundary effect - Trial $\mathrm{F}$ - centre:corner ratio vs release boundary . . . . . . . . . 138

3.38 Boundary effect - Trial B - k=0.5 . . . . . . . . . . . . . . . . . . . . . . 139

3.39 Boundary effect - Trial B - $\mathrm{k}=10 \ldots \ldots \ldots \ldots \ldots$

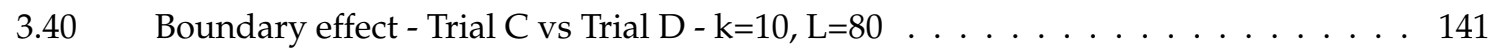

3.41 Centre ratio analysis structure . . . . . . . . . . . . . . . . . . . . 143

3.42 Search efficiency analysis structure . . . . . . . . . . . . . . . . . . . . . . 143

3.43 Edge effect - Trial B - Ratio summary - Single egg . . . . . . . . . . . . . . . . 144

3.44 Edge effect - Trial B - Ratio summary - Multiple eggs . . . . . . . . . . . . 144

3.45 Edge effect - Trial B (CRW) Single Egg - Centre ratio summary . . . . . . . . . 147

3.46 Edge effect - Trial B (CRW) Single Egg - Centre ratio vs. L and k . . . . . . . . . 147

3.47 Edge effect - Trial B (CRW) Single Egg - Centre ratio vs. layout . . . . . . . . . . 148

3.48 Edge Effect - Trial C (Olfaction) Multiple Eggs - Centre ratio vs. R and P . . . . . . 149

3.49 Edge effect - Trial D (Vision) Single Egg - Centre ratio vs. L and k . . . . . . . . . . 149

3.50 Edge Effect - Trial B (CRW) Single Egg - Search Efficiency Summary . . . . . . . . 154

3.51 Edge effect - Trial C (Olfaction) Multiple Eggs - Search efficiency vs. R and P . . 154

4.1 Field Layout - KAITOKE-04 . . . . . . . . . . . . . . . . . . . . . . . . . 170

$4.2 \quad$ Field Layout - KAITOKE-04 - Block K1 . . . . . . . . . . . . . . . . . . . 171

4.3 Field Layout - KAITOKE-04 - Blocks K2 to K4 . . . . . . . . . . . . . . . . . . . 172

4.4 Field Layout - LEVIN-06-II . . . . . . . . . . . . . . . . . . . . . . 173

4.5 Field Layout - LEVIN-06-II - Blocks _ . . . . . . . . . . . . . . . . . . . 174

4.6 Potential theoretical responses to Plant Density . . . . . . . . . . . . . . 176

4.7 Calibration - LEVIN-06-II Trial B3 . . . . . . . . . . . . . . . . . . . . . 185

$4.8 \quad$ Parameter Estimation Summary ～. . . . . . . . . . . . . . . . . . . . . . . . 190

4.9 Plant Density - KAITOKE-04 - Field Data . . . . . . . . . . . . . . . . . . . . 193

4.10 Plant Density - KAITOKE-04 - Trial C2 (CRW, Single Egg) . . . . . . . . . . . . . . . 193

$4.11 \quad$ Log-Log Regression - KAITOKE-04 - Field Data . . . . . . . . . . . . . . . . . . . . . 194

4.12 Log-Log Regression - KAITOKE-04 - Trial C2 (CRW, Single Egg) . . . . . . . . . . . 194

4.13 Scale Code - KAITOKE-04 - Field Data . . . . . . . . . . . . . . . . . . . . . . . . 195

4.14 Plant Density - LEVIN-06-II - Field Data . . . . . . . . . . . . . . . . . . . . . . 196

4.15 Plant Density - LEVIN-06-II - Trial D6 (Olfaction and Vision, Multiple Eggs) . . . . 196

4.16 Plant Density - LEVIN-06-II - Trial C1 (CRW, Single Egg) . . . . . . . . . . . . . . 196

$4.17 \quad$ Log-Log Regression - LEVIN-06-II - Field Data . . . . . . . . . . . . . . . . . . . . 197

4.18 Log-Log Regression - LEVIN-06-II - Trial D6 (Olfaction and Vision, Multiple Eggs) 197

4.19 Log-Log Regression - LEVIN-06-II - Trial C1 (CRW, Single Egg) . . . . . . . . . . 197

4.20 Scale Code - LEVIN-06-II - Field Data . . . . . . . . . . . . . . . . . . . . . . . . . . 198

$4.21 \quad$ Field Breakdown - LEVIN-06-II - Field Data . . . . . . . . . . . . . . . . . . . . . . . 199

4.22 Field Breakdown - LEVIN-06-II - Trial C1 (CRW) . . . . . . . . . . . . . . . . 200

4.23 Olfaction Signal Surface - LEVIN-06-II (Field E) . . . . . . . . . . . . . . . . . . . 201 
4.24 Olfaction Signal Surface - LEVIN-06-II (Field F) . . . . . . . . . . . . . . . . . . . . 201

4.25 Olfaction Signal Surface - LEVIN-06-II (Field G) . . . . . . . . . . . . . . . . . . . . 202

4.26 Olfaction Signal Surface - LEVIN-06-II (Field H) . . . . . . . . . . . . . . . . . . 202

4.27 Plant Density - LEVIN-06-II Trial D1 (Olfaction, Single Egg) . . . . . . . . . . . 203

4.28 Plant Density - LEVIN-06-II Trial D6 (Olfaction and Vision, Multiple Eggs) . . . . . 203

4.29 Log-Log Regression - LEVIN-06-II Trial D1 (Olfaction, Single Egg) . . . . . . . . . . 204

4.30 Log-Log Regression - LEVIN-06-II Trial D6 (Olfaction and Vision, Multiple Eggs) . 204

4.31 BC Response Surface - KAITOKE-04 - Trial C2 (CRW, Single Egg) . . . . . . . . . . 205

4.32 BC Response Surface - LEVIN-06-II - Trial C1 (CRW, Single Egg) . . . . . . . . . . . 205

4.33 BC Response Surface - LEVIN-06-II - Trial C3 (CRW, Multiple Eggs) . . . . . . . . . 205

4.34 BC Response Surface - LEVIN-06-II - Trial D1 (Olfaction, Single Egg) . . . . . . . . 206

4.35 BC Response Surface - LEVIN-06-II - Trial D3 (Vision, Single Egg) . . . . . . . . . . 206

4.36 BC Response Surface - LEVIN-06-II - Trial D2 (Olfaction and Vision, Single Egg) . 206

4.37 BC Response Surface - LEVIN-06-II - Trial D4 (Olfaction, Multiple Eggs) . . . . . 207

4.38 BC Response Surface - LEVIN-06-II - Trial D5 (Vision, Multiple Eggs) . . . . . . . . 207

4.39 BC Response Surface - LEVIN-06-II - Trial D6 (Olfaction and Vision, Multiple Eggs) 207

\section{Edge effect simulation results - Trial B (CRW), Single Egg}

A.1-1 Centre ratio summary . . . . . . . . . . . . . . . . . . . . . . . 221

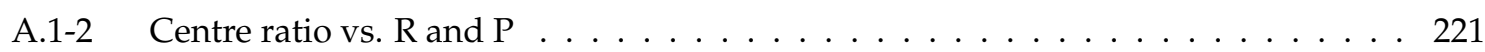

A.1-3 Search Efficiency Summary . . . . . . . . . . . . . . . . . . . . . 222

A.1-4 Search efficiency vs. $R$ and $P \ldots \ldots \ldots 22$

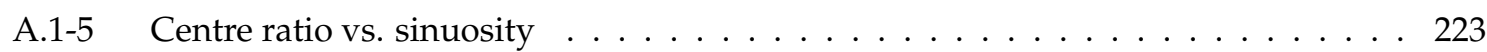

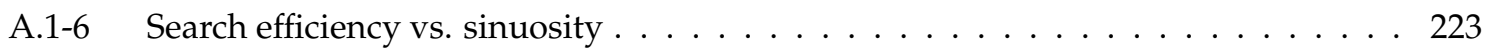

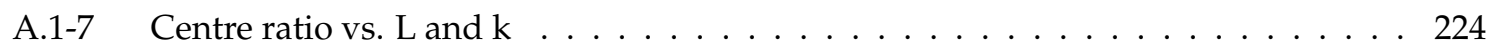

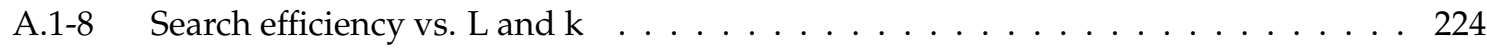

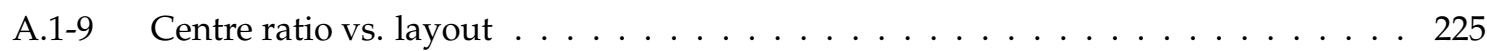

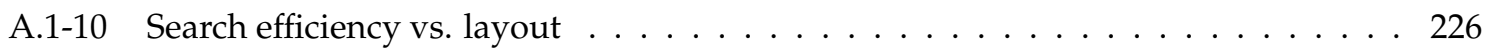

Edge effect simulation results - Trial B (CRW), Multiple Eggs

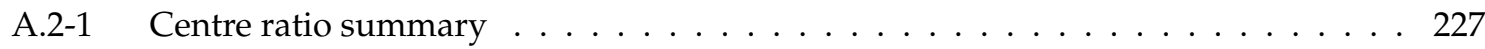

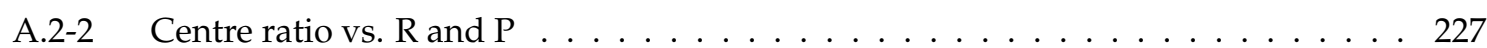

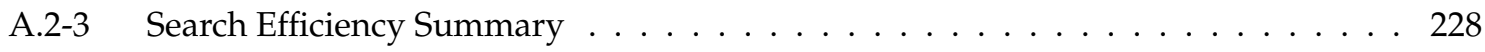

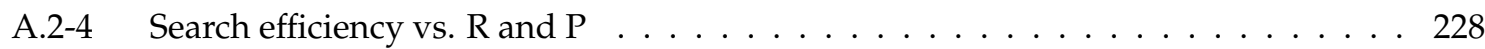

A.2-5 Centre ratio vs. sinuosity . . . . . . . . . . . . . . . . . . . . . . . . 229

A.2-6 Search efficiency vs. sinuosity . . . . . . . . . . . . . . . . . . . . . . . . . . 229

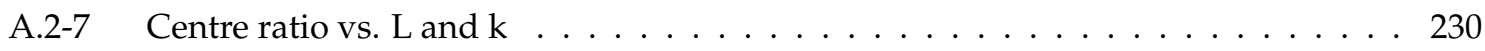

A.2-8 Search efficiency vs. $\mathrm{L}$ and $\mathrm{k} \ldots \ldots \ldots \ldots \ldots \ldots \ldots$

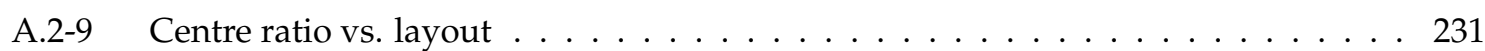

A.2-10 Search efficiency vs. layout . . . . . . . . . . . . . . . . . . . 232

Edge effect simulation results - Trial C (Olfaction), Single Egg

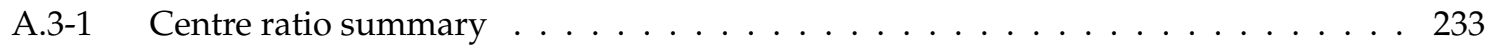

A.3-2 Centre ratio vs. $\mathrm{R}$ and $\mathrm{P} \ldots \ldots \ldots \ldots \ldots \ldots \ldots \ldots$

A.3-3 Search Efficiency Summary . . . . . . . . . . . . . . . . . . . . . 234

A.3-4 Search efficiency vs. $\mathrm{R}$ and $\mathrm{P} \ldots \ldots \ldots \ldots \ldots \ldots \ldots$

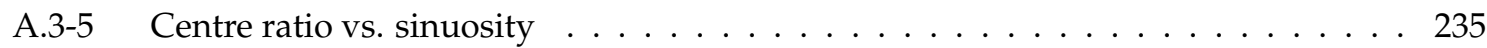

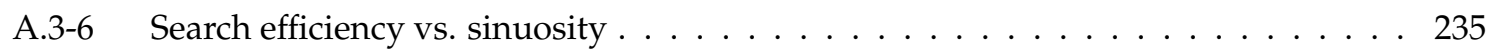

A.3-7 Centre ratio vs. $\mathrm{L}$ and $\mathrm{k} \ldots \ldots \ldots \ldots \ldots \ldots \ldots \ldots$ 


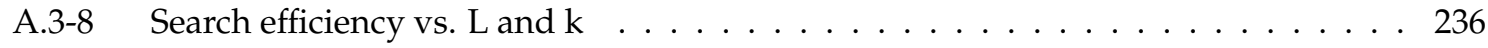

A.3-9 Centre ratio vs. layout . . . . . . . . . . . . . . . . . . . . . . 237

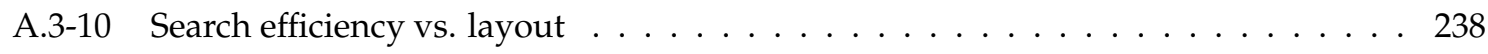

Edge effect simulation results - Trial C (Olfaction), Multiple Eggs

A.4-1 Centre ratio summary . . . . . . . . . . . . . . . . . . . . . . . . 239

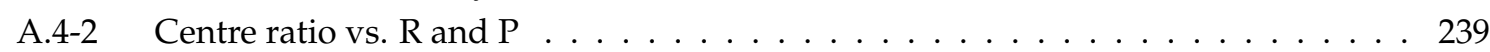

A.4-3 Search Efficiency Summary . . . . . . . . . . . . . . . . . . . 240

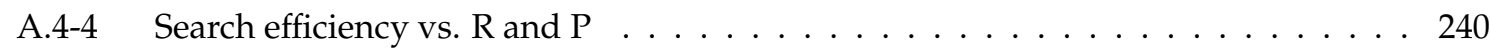

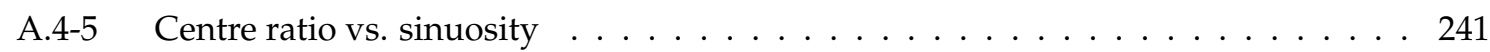

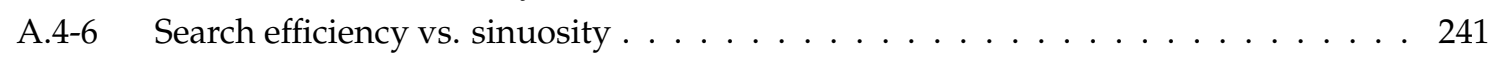

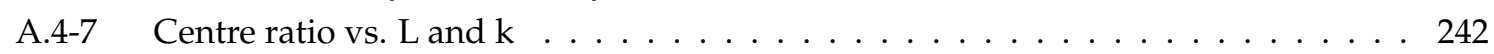

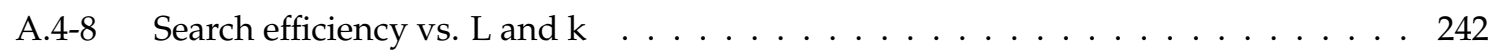

A.4-9 Centre ratio vs. layout . . . . . . . . . . . . . . . . . . . . . . 243

A.4-10 Search efficiency vs. layout . . . . . . . . . . . . . . . . . . . 244

Edge effect simulation results - Trial D (Vision), Single Egg

A.5-1 Centre ratio summary . . . . . . . . . . . . . . . . . . . . . . 245

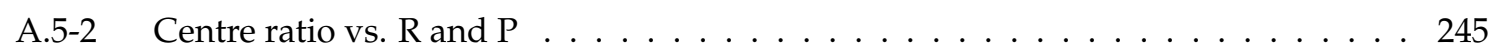

A.5-3 Search Efficiency Summary . . . . . . . . . . . . . . . . . . . . 246

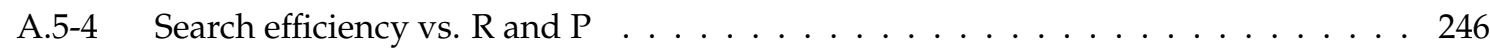

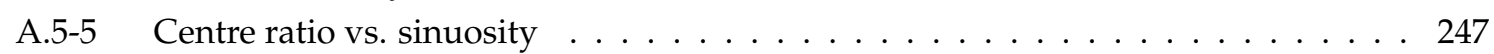

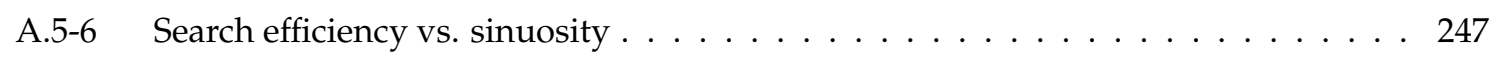

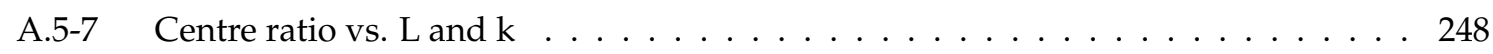

A.5-8 Search efficiency vs. $\mathrm{L}$ and $\mathrm{k} \ldots \ldots \ldots \ldots \ldots \ldots \ldots \ldots \ldots \ldots$

A.5-9 Centre ratio vs. layout . . . . . . . . . . . . . . . . . . . . . . 249

A.5-10 Search efficiency vs. layout . . . . . . . . . . . . . . . . . . 250

Edge effect simulation results - Trial D (Vision), Multiple Eggs

A.6-1 Centre ratio summary . . . . . . . . . . . . . . . . . . . . . . . . . . 251

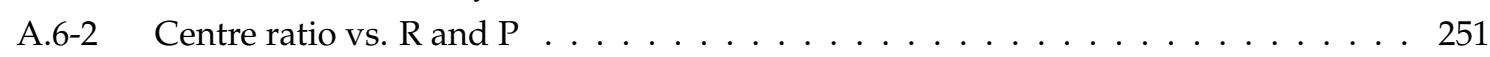

A.6-3 Search Efficiency Summary . . . . . . . . . . . . . . . . . . . . . . . 252

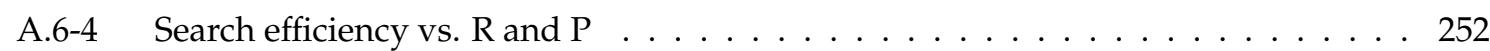

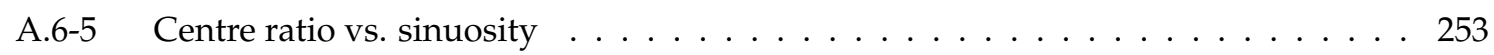

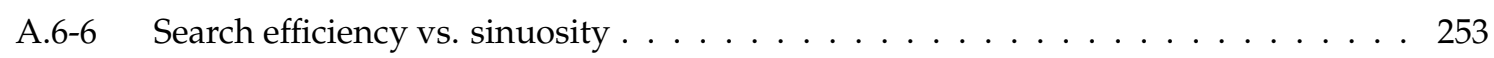

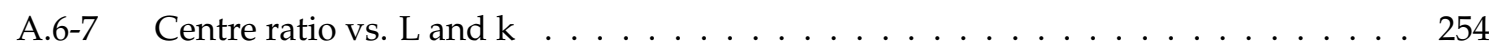

A.6-8 Search efficiency vs. $\mathrm{L}$ and $\mathrm{k} \ldots \ldots \ldots \ldots \ldots$. . . . . . . . . . . . . . 254

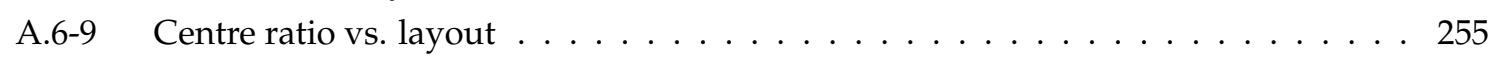

A.6-10 Search efficiency vs. layout . . . . . . . . . . . . . . . . . . . . 256

Edge effect simulation results - Trial E (Olfaction and Vision), Single Egg

A.7-1 Centre ratio summary . . . . . . . . . . . . . . . . . . . . . . 257

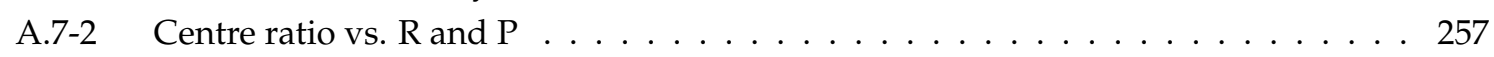

A.7-3 Search Efficiency Summary . . . . . . . . . . . . . . . . . . . . . 258

A.7-4 Search efficiency vs. $\mathrm{R}$ and $\mathrm{P} \ldots \ldots \ldots \ldots \ldots \ldots \ldots \ldots \ldots$

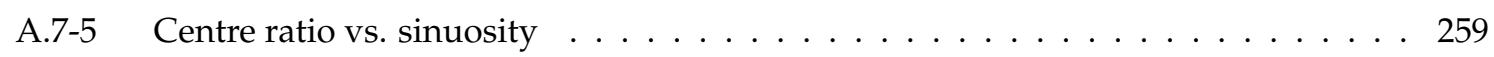

A.7-6 Search efficiency vs. sinuosity . . . . . . . . . . . . . . . . . . . . . 259

A.7-7 Centre ratio vs. $\mathrm{L}$ and $\mathrm{k} \ldots \ldots \ldots \ldots \ldots$

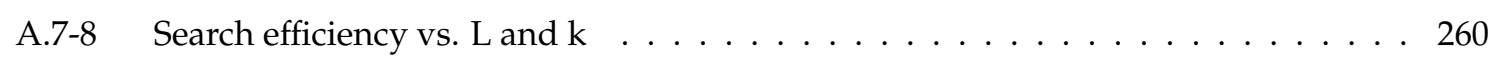

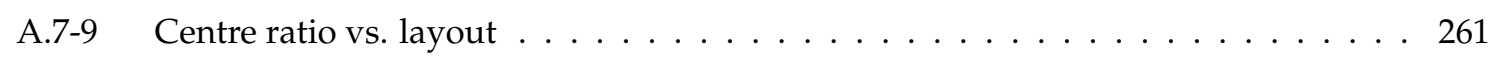


A.7-10 Search efficiency vs. layout . . . . . . . . . . . . . . . . . . . . . . . . 262

Edge effect simulation results - Trial E (Olfaction and Vision), Multiple Eggs

A.8-1 Centre ratio summary . . . . . . . . . . . . . . . . . . . . . . . 263

A.8-2 Centre ratio vs. $\mathrm{R}$ and $\mathrm{P} \ldots \ldots \ldots \ldots \ldots \ldots \ldots$

A.8-3 Search Efficiency Summary . . . . . . . . . . . . . . . . . . . . . . . . . . 264

A.8-4 Search efficiency vs. $\mathrm{R}$ and $\mathrm{P} \ldots \ldots \ldots \ldots$. . . . . . . . . . . . . 264

A.8-5 Centre ratio vs. sinuosity $\ldots \ldots \ldots \ldots \ldots \ldots \ldots$

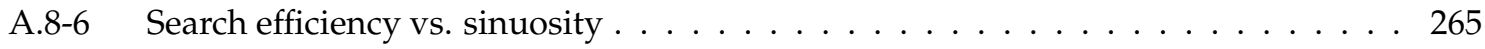

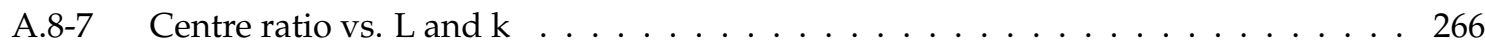

A.8-8 Search efficiency vs. $\mathrm{L}$ and $\mathrm{k} \ldots \ldots \ldots \ldots \ldots$

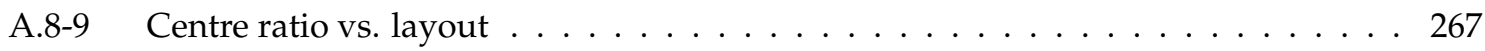

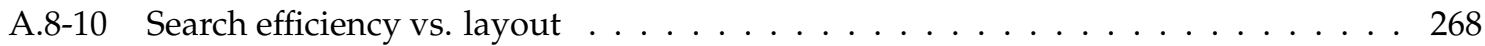

B.1 Response Summary - Trial C1 (CRW - LEVIN-06-II) - Single Egg - R=5 . . . . . . 279

B.2 Response Summary - Trial C1 (CRW - LEVIN-06-II) - Single Egg - R=10 . . . . . . 280

B.3 Response Summary - Trial C1 (CRW - LEVIN-06-II) - Single Egg - R=20 . . . . . . 281

B.4 Response Summary - Trial C2 (CRW - KAITOKE-04) - Single Egg - R=5 . . . . . . 282

B.5 Response Summary - Trial C2 (CRW - KAITOKE-04) - Single Egg - R=10 . . . . . 283

B.6 Response Summary - Trial C2 (CRW - KAITOKE-04) - Single Egg - R=20 . . . . . 284

B.7 Response Summary - Trial C3 (CRW - LEVIN-06-II) - Multiple Eggs - R=5 . . . . . . 285

B.8 Response Summary - Trial C3 (CRW - LEVIN-06-II) - Multiple Eggs - R=10 . . . . 286

B.9 Response Summary - Trial C3 (CRW - LEVIN-06-II) - Multiple Eggs - R=20 . . . . 287

B.10 Response Summary - Trial D1 (Olfaction - LEVIN-06-II) - Single Egg - R=5 . . . . 288

B.11 Response Summary - Trial D1 (Olfaction - LEVIN-06-II) - Single Egg - R=10 . . . . . 289

B.12 Response Summary - Trial D1 (Olfaction - LEVIN-06-II) - Single Egg - R=20 . . . . 290

B.13 Response Summary - Trial D2 (Olfaction and Vision - LEVIN-06-II) - Single Egg -

$\mathrm{FD}=100 \ldots \ldots \ldots$. . . . . . . . . . . . . . . . . . . . . . . . . 291

B.14 Response Summary - Trial D2 (Olfaction and Vision - LEVIN-06-II) - Single Egg -

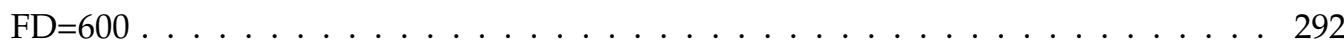

B.15 Response Summary - Trial D2 (Olfaction and Vision - LEVIN-06-II) - Single Egg -

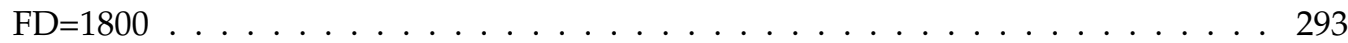

B.16 Response Summary - Trial D3 (Vision - LEVIN-06-II) - Single Egg - FD=100 . . . . . 294

B.17 Response Summary - Trial D3 (Vision - LEVIN-06-II) - Single Egg - FD=600 . . . . . 295

B.18 Response Summary - Trial D3 (Vision - LEVIN-06-II) - Single Egg - FD=1800 . . . . 296

B.19 Response Summary - Trial D4 (Olfaction - LEVIN-06-II) - Multiple Eggs - R=5 ․ . 297

B.20 Response Summary - Trial D4 (Olfaction - LEVIN-06-II) - Multiple Eggs - R=10 _ . 298

B.21 Response Summary - Trial D4 (Olfaction - LEVIN-06-II) - Multiple Eggs - R=20 . . . 299

B.22 Response Summary - Trial D5 (Vision - LEVIN-06-II) - Multiple Eggs - FD=100 . . . 300

B.23 Response Summary - Trial D5 (Vision - LEVIN-06-II) - Multiple Eggs - FD=600 . . . 301

B.24 Response Summary - Trial D5 (Vision - LEVIN-06-II) - Multiple Eggs - FD=1800 . . 302

B.25 Response Summary - Trial D6 (Olfaction and Vision - LEVIN-06-II) - Multiple Eggs -

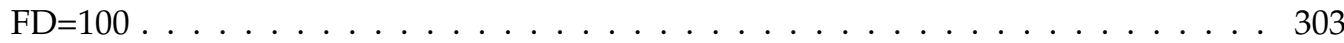

B.26 Response Summary - Trial D6 (Olfaction and Vision - LEVIN-06-II) - Multiple Eggs -

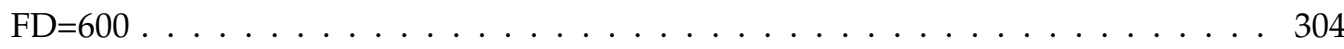

B.27 Response Summary - Trial D6 (Olfaction and Vision - LEVIN-06-II) - Multiple Eggs -

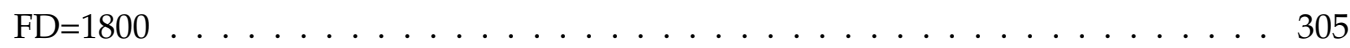

B.28 Levin 2006 Field breakdown of Egg Distribution . . . . . . . . . . . . . . . . . . . 309

B.29 Trial D6 - LEVIN-06-II - Olfaction and Vision - Multiple Egg - Field Breakdown . . 310 
xvi

B.30 Trial C1 - LEVIN-06-II - CRW - Multiple Egg - Field Breakdown . . . . . . . . . . 311

C.1 Probability Inferences . . . . . . . . . . . . . . . . . . . . . . . 314

C.2 Upper vs Lower Tailed Distributions . . . . . . . . . . . . . . . . . . . . . . 315

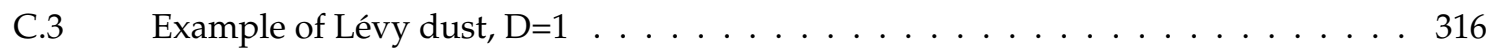

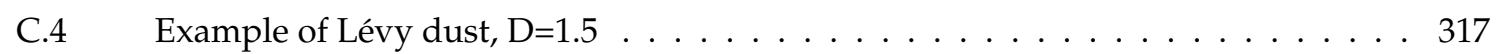

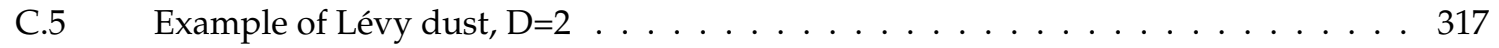




\section{List of Tables}

$2.1 \quad$ Foraging principles and constraints $\ldots \ldots \ldots \ldots \ldots$

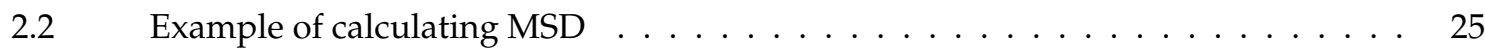

2.3 Bukovinszky's species and search type $\ldots \ldots \ldots \ldots \ldots \ldots \ldots \ldots \ldots$

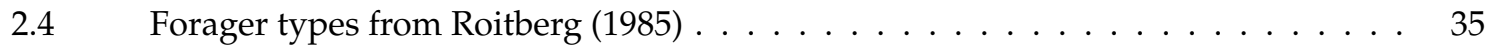

2.5 Summary of Pieris rapae behavioural categories . . . . . . . . . . . . . . 38

$2.6 \quad$ Jones (1977): Kale Types . . . . . . . . . . . . . . . . . . . . . . . 49

2.7 Jones (1977): Experimental observation summary . . . . . . . . . . . . . . 50

2.8 Jones (1977): Directionality Summary ～. . . . . . . . . . . . . . . . . . . 53

$2.9 \quad$ Jones (1977): Eggs per plant data . . . . . . . . . . . . . . . . . . 53

2.10 Jones (1977): Simulation Parameters . . . . . . . . . . . . . . . . . . . . 56

2.11 Jones (1977): Host Preferences . . . . . . . . . . . . . . . . . . . . . . . 59

2.12 Jones (1977): Plant Spacings . . . . . . . . . . . . . . . . . . . . . . . . 60

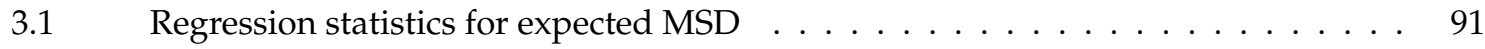

3.2 Signal differences and strengths $\ldots \ldots \ldots \ldots \ldots \ldots \ldots$

3.3 Commonly manipulated parameters . . . . . . . . . . . . . . . 120

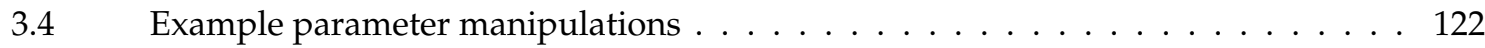

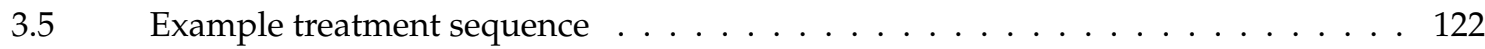

$3.6 \quad$ Boundary effect experimental trials $\ldots \ldots \ldots \ldots \ldots$

$3.7 \quad$ Boundary Effect Experiment Manipulations . . . . . . . . . . . . . . . . 134

$3.8 \quad$ Edge effect experimental trials . . . . . . . . . . . . . . . . . . . 134

$3.9 \quad$ Edge effect experiment: Common parameter manipulations . . . . . . . . . . 135

3.10 Example Egg Count . . . . . . . . . . . . . . . . . . . . . 136

3.11 Table of the Relative spacing for $\mathrm{R}$ and $\mathrm{P}$ parameters $\ldots \ldots \ldots$. . . . . . . . 145

3.12 Table of the Sinuosity of CRW parameters . . . . . . . . . . . . . . . . . . 150

4.1 Scales and Plant Densities . . . . . . . . . . . . . . . . . . . . . . . . . . . 169

4.2 Scale codes - KAITOKE-04 . . . . . . . . . . . . . . . . . . . . . . . . . . . . 169

4.3 Scale codes - LEVIN-06-II . . . . . . . . . . . . . . . . . . . . . . . . . . . . . . 169

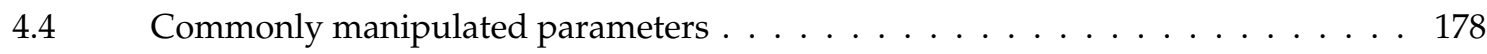

4.5 Egg counts from Field observations $\ldots \ldots \ldots \ldots \ldots \ldots \ldots$

4.6 Summary of flight parameters From Literature . . . . . . . . . . . . . . . . 179

$4.7 \quad$ Experimental Trials . . . . . . . . . . . . . . . . . . . . . . . . . . 181

$4.8 \quad$ Parameter value Manipulations . . . . . . . . . . . . . . . . . . . . . 182

$4.9 \quad$ Result Plot Types . . . . . . . . . . . . . . . . . . . . . . . . . . . 183

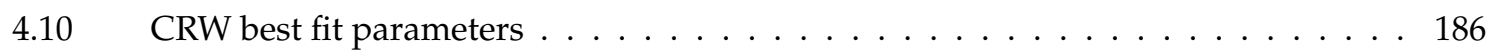

4.11 Vision best fit parameters - LEVIN-06-II . . . . . . . . . . . . . . . . . . . . 187

4.12 Olfaction and Vision best fit parameters - LEVIN-06-II . . . . . . . . . . . . . . 189 
4.13 Parameter estimation summary . . . . . . . . . . . . . . . . . . . 191

A.1 Structure of edge effect results sections $\ldots \ldots \ldots \ldots \ldots \ldots \ldots$

B.1 Optimisation - Trial C1 (CRW) - LEVIN-06-II . . . . . . . . . . . . . . . . . . 270

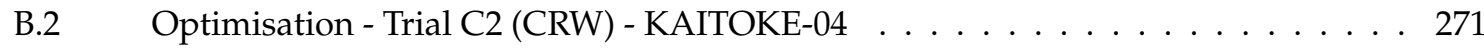

B.3 Optimisation - Trial C3 (CRW) - LEVIN-06-II . . . . . . . . . . . . . . . . . . 272

B.4 Optimisation - Trial D1 (Olfaction) - LEVIN-06-II . . . . . . . . . . . . . . . . 273

B.5 Optimisation - Trial D2 (Olfaction and Vision) - LEVIN-06-II . . . . . . . . . . . . 274

B.6 Optimisation - Trial D3 (Vision) - LEVIN-06-II . . . . . . . . . . . . . . . . . . 275

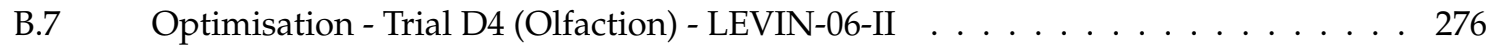

B.8 Optimisation - Trial D5 (Vision) - LEVIN-06-II . . . . . . . . . . . . . . . . . . 277

B.9 Optimisation - Trial D6 (Olfaction and Vision) - LEVIN-06-II . . . . . . . . . . . 278

B.10 Regression Statistics - KAITOKE-04 Field . . . . . . . . . . . . . . . . . . 306

B.11 Regression Statistics - LEVIN-06-II Field . . . . . . . . . . . . . . . . . . . . . 306

B.12 Regression Statistics - Trial C1 - LEVIN-06-II, CRW, Single Egg . . . . . . . . . . . . 306

B.13 Regression Statistics - Trial C2 - KAITOKE-04, CRW, Single Egg . . . . . . . . . . 306

B.14 Regression Statistics - Trial C3 - LEVIN-06-II, CRW, Multiple Eggs . . . . . . . . . 306

B.15 Regression Statistics - Trial D1 - LEVIN-06-II, Olfaction, Single Egg . . . . . . . . . 306

B.16 Regression Statistics - Trial D1 - LEVIN-06-II, Olfaction, Single Egg . . . . . . . . 307

B.17 Regression Statistics - Trial D2 - LEVIN-06-II, Vision and Olfaction, Single Egg . 307

B.18 Regression Statistics - Trial D3 - LEVIN-06-II, Vision, Single Egg . . . . . . . . . . . 307

B.19 Regression Statistics - Trial D4 - LEVIN-06-II, Olfaction, Multiple Eggs . . . . . . . 307

B.20 Regression Statistics - Trial D5 - LEVIN-06-II, Vision, Multiple Eggs . . . . . . . . 307

B.21 Regression Statistics - Trial D6 - LEVIN-06-II, Vision and Olfaction, Multiple Eggs 307

B.22 Regression Statistics - Trial D6 - LEVIN-06-II, Vision and Olfaction, Multiple Eggs 308 


\section{Chapter 1}

\section{General Introduction}

Insects are a vital component of the world's ecosystems. Their interactions with other species (in particular plants), can have dramatic effects upon the surrounding ecosystem (Levin, 1992; Swingland and Greenwood, 1984; Turchin, 1991). Herbivorous insects are in turn influenced by populations of parasitoids preying upon them. Movement is both crucial to their survival and strongly influences their effects upon the ecosystem. Thus, there is great interest in studying and quantifying both how insects (and animals in general) move and locate their prey or food resources, and the patterns of distribution that arise from these interactions.

An individual animal's movement behaviour determines the dispersal patterns of the species at the population level. Dispersal patterns affects a species' ability to exploit new niches in a changing environment, or to establish a population in an existing environment as an invasive species (Vos et al., 2001; Hastings et al., 2005).

At the largest scale we find animals migrating thousands of miles (Brower et al., 2006). At smaller scales many insects are constantly in motion, seeking potential hosts or sources of food. Anyone who has seen the Cabbage White butterfly (Pieris rapae) in flight will observe how they cover a large area rapidly in their apparently random and jerky flight, as the poem "Flying Crooked" relates, which is in the frontmatter of this thesis (Graves, 1965).

This thesis is concerned with the effect of spatial scale when analysing insect responses to host plants. The study species is Pieris rapae, chosen because it is well documented with a wealth of material pertaining to its life history and oviposition behaviour. Although many studies are concerned with manipulating host plant density, few were found that deliberately manipulate the scale at which density is measured.

The work reported here is a simulation study conducted in combination with a set of field experiments, which are described elsewhere (Hasenbank, in prep). The re- 
sults of the field experiments are compared to those of the simulation, in order to determine which parameter combinations produce the closest "fit" to the observed egg distributions.

A secondary objective of the thesis is to introduce a conceptual model, building on that of the Correlated Random Walk and taking into account potential attraction of the "walkers" towards resources. This allows for density dependent effects and exploration of responses to patterns of resources at different scales.

Finally, the thesis presents a simulation framework that was developed to facilitate these goals. It is hoped that this framework will in itself be useful to future researchers wishing to explore similar topics and is made available on a companion $\mathrm{CD}$ and the Internet at http://jimbarritt.com/academic/bugsim/download.htmI

The structure of the thesis is first to provide a review of relevant literature. The field is wide ranging and it would be impossible to provide a complete description, it is hoped that the review provides a reasonable summary and contains references at least to the key papers concerned.

The third chapter introduces the simulation framework itself and the details of the conceptual model it implements. This chapter provides the background necessary for the fourth chapter, which describes a set of simulation experiments that were conducted to explore the oviposition behaviour of $P$. rapae.

The final chapter provides a general discussion of the work and an analysis of the project and its usefulness both to this specific topic, and as a research tool. 


\section{References}

Brower, L. P., L. S. Fink, and P. Walford, 2006: Fueling the fall migration of the monarch butterfly. Integrative and Comparative Biology, 46(6), 1123-1142.

Graves, R., 1965: Flying crooked. In Collected Poems. Cassell, London, England.

Hasenbank, M., in preparation: Phd thesis. Victoria University (Wellington, New Zealand).

Hastings, A., K. Cuddington, K. F. Davies, C. J. Dugaw, S. Elmendorf, A. Freestone, S. Harrison, M. Holland, J. Lambrinos, U. Malvadkar, B. A. Melbourne, K. Moore, C. Taylor, and D. Thomson, 2005: The spatial spread of invasions: new developments in theory and evidence. Ecology Letters, 8(1), 91-101.

Levin, S., 1992: The problem of pattern and scale in ecology. Ecology, 73 (6), 1943-1967.

Swingland, I. and Greenwood, P., editors, 1984: The Ecology Of Animal Movement. Clarendon Press, Oxford.

Turchin, P., 1991: Translating foraging movements in heterogeneous environments into the spatialdistribution of foragers. Ecology, 72(4), 1253-1266.

Vos, M., S. M. Berrocal, F. Karamaouna, L. Hemerik, and L. E. M. Vet, 2001: Plant-mediated indirect effects and the persistence of parasitoid-herbivore communities. Ecology Letters, 4, 38-45. 


\section{Chapter 2}

\section{Pieris rapae, Random Walks, scale and the Resource Concentration Hypothesis: A review}

\subsection{Abstract}

This review brings together literature from a number of areas, centering around simulating the foraging behaviour of Pieris rapae. Animal movement and its relationship to general ecology sets the scene. The topic of scale and the landscape patch structure is introduced along with the "Resource Concentration Hypothesis" which is used to describe relationships between consumers and the density of their resources. Some general background to animal foraging strategies is provided. Movement is discussed in more detail, specifically in terms of methods of quantifying, and modelling, animal movement. A review of various simulations is provided together with a detailed case study of a particularly relevant simulation. Finally a summary of information known about the study species, P.rapae is given including oviposition behaviour and sensory perception.

\section{Keywords}

Agent-based, Brassica oleracae, Correlated Random Walk, Individual-based, Java, Pieris rapae, Simulation, Spatial Ecology 


\subsection{The ecology of animal movement}

"No animal lives alone" (Dethier and Stellar, 1964). Even the albatross, the epitome of solitary existence, must return from roaming the seas to find its own kind to reproduce. Animals which have no need of contact to reproduce such as the amoeba still interact with both food organisms and predators and parasites. Animals also react to their environment to aid their survival. For example a sparrow "fluffs" its feathers when cold, locusts orientate to the sun when hot and swallows fly south for the winter. Each of these examples represent active actions (behaviour) on the part of the individual animals to enable their survival and reproductive success (Dethier and Stellar, 1964)

Movement is a characteristic of animals (Swingland and Greenwood, 1984; Grove et al., 1966). Within this review, movement is defined as behaviours or actions which involve physical displacement of the animal in space. Movement may occur at different spatial and temporal scales from large scale migration to small scale foraging movements (Baker, 1978; Brower et al., 2006; Swingland and Greenwood, 1984). As a consequence of movement, distribution patterns may be observed across temporal scales, where displacement occurs over longer time periods due to population expansion, and is a result of cumulative small scale movements. This review is primarily concerned with smaller scale foraging movements as opposed to large (planetary) spatial scale migrations or temporal population displacement.

Much work is concerned with animals being dispersed in space according to their resources, and the effect that the spatial patterns of these resources have on the movements and distribution of the animals. Insects may use resources for food (nectar feeding for example) or hosts for larvae (Bach, 1988; Cromartie, 1975; Cronin, 2003; Fahrig and Paloheimo, 1988; King and With, 2002; Roitberg and Mangel, 1997; Levin and Segel, 1985). Conversely, animals may influence the spatial arrangement of resources, through seed dispersal, pollination and herbivorous damage for example (Tilman and Kareiva, 1997; Bolker and Pacala, 1997; Baker, 1978).

\subsubsection{Ecological consequences of movement}

Animal movement is a highly significant process when studying the structure of an ecosystem and the web of relations between its members (Turchin, 1998; Skellam, 1951). It can play a part in the interactions between species at different trophic levels e.g. Plant - Herbivores - Parasitoids (Vos et al., 2001; Vet and Dicke, 1992), provide an adaptive advantage to a particular species in competing for resources, or allow coexistence (Potthoff et al., 2006; Levin, 1974). Species with good dispersal abili- 
ties may be able to respond to fluctuating environments more efficiently (CastilloChavez et al., 1988; Levin and Paine, 1974) or take advantage of new resources made available more rapidly than species with poor dispersal abilities, leading to "invasions" (Hastings et al., 2005; Hong and Mladenoff, 1999; Cuddington and Hastings, 2004).

At the individual level, movement is essential to everyday survival (Baker, 1978). Even sedentary animals such as the barnacle have moving appendages in order to capture prey items, or for reproduction. The strategies available to an animal in searching for food will depend on their physical attributes (Ahmad, 1983; Bell, 1991).

At the population level, migration may allow a species to survive despite a highly fluctuating environment where local extinction events are common. These metapopulations are dependant on a level of inter-patch movement for persistence (Hanski and Gilpin, 1996; Conradt et al., 2001).

\subsubsection{Searching for resources}

Bell (1991) and Ahmad (1983) provide excellent coverage of the topic of searching strategies and mechanisms that animals and insects have available to them when foraging for food. Bell (1991) lists a set of principals or constraints that will affect a forager (Table 2.1). The assumption here is that food resources are distributed in the environment patchily. Aggregation tends to occur in plants due to their reproductive strategies (Bell, 1991). The definition of a patch is in itself a complex topic (Wiens, 1976). Hassell and Southwood (1978) define it in terms of an aggregation as perceived by the forager. In this thesis, a patch may also be an arbitrary area defined by the experiment.

Resource detection abilities can be divided into three categories (Bell, 1991):

- Olfactory

- Visual

- Contact

This is a categorisation shared by Bukovinszky et al. (2005), and is adopted in this thesis (Chapter 2). Contact searchers rely upon sensory appendages attached to themselves to "feel" prey items. This review is mostly concerned with searching within a patch, and so rules about when to leave a patch are not discussed, this topic however is closely related with that of animal movement and the concept of "Optimal Foraging" (Krebs, 1978; Krebs and Kacelnik, 1991; Stephens and Krebs, 1986), in terms of the energy costs and mortality risks associated with movement. 
Searching for resource patch Animals attempt to localise an assemblage of resources (patch)

Searching within a patch

No information available

Information available

Patch specific cues

Animal produced cues
Avoid leaving until becomes unprofitable to remain

Move so as to optimise the chances of finding resources and reduce chances of revisiting

Efficiency increases (See Chapter 3)

Information about potential but distant resource patches can be found

Resources associated with other community members may be located by interactions producing sensory signals

Table 2.1: Foraging principals and constraints from Bell (1991).

\section{Searching Patterns}

In the absence of information, animals may adopt a systematic search pattern. Spiralling patterns are observed for example in homing birds (Griffin, 1944) and desert Isopods attempting to find their burrows (Hoffmann, 1983b). The latter is an example of a contact searcher employing a systematic (spiral) pattern to locate their burrows (which can be only detected by coming into contact with them). It may be straightforward for an insect to adopt a spiral pattern simply by moving less on one side (Bell, 1991). Theoretically, the space between arms of the spiral should be twice the detection distance of the searcher to allow for left and right looking (Bell, 1991). This introduces the concept of the scale of perception of the searcher; for birds, the ideal spacing for the spiral might be the same size as its familiar territory (or "patch"), perhaps a small copse. It is this unit of pattern which it might recognise, although Bell (1991) notes that this behaviour had not actually been observed at the time of writing.

Conradt et al. (2000) report that the butterfly Maniola jurtina, when released, flew in "loops" like the petals of a flower, centered on the release point and suggests that this is a systematic searching mechanism.

As an accompaniment to the systematic search of the desert Isopods, Hoffmann (1983a) also discusses the function of a random element in the search process, which can improve the efficiency of the search. Bell (1991) notes that an entirely random walk has no examples, even in micro-organisms. Most are directed at least in some 
manner, or Correlated (Section 2.6.1). He gives an adaptive reason for this to be that the random walk has the effect of making it very likely to visit an area already covered. Even that apparently most random searcher, Gyrinus picipes (The Whirligig beetle) adopts a correlated (more directional) random walk when initially searching for food. However, upon contact with a prey item it shortens its step length and increases the randomness of its turning, approximating more closely a purely random path. This behaviour is adaptive in that it will increase the search efficiency in the vicinity of prey items (Winkelman and Vinyard, 1991). G. picipes is further example of a contact searcher.

An interesting observation of Jander (1975) is that, if the resources are patchy and easy to find, a straight line is the most efficient search. The probability of a target being detected is given by (2.1).

$$
T_{d}=\frac{\arcsin \left(\alpha / r_{0}\right)}{180}
$$

Where $\quad \alpha=$ detection radius

$r_{0}=$ distance between starting point and resource goal

The straight line is therefore a poor strategy for locating relatively small resource units. If the path is sufficiently long, search effort first increases with increasing "straightness" then declines. Bell (1991) writes:

"If an animal walks in straight lines, but where the distribution of directions taken is random, the probability of success is maximal when the length of the line spans a distance corresponding roughly to that from which the animal can detect a target. Hoffmann (1983b) explains it this way: if the directional constancy of a random search is too low, the animal searches only in the immediate vicinity of the starting point and therefore does not detect the target. If the directional constancy is too high the animal may walk a long way in an inappropriate direction. Hence, most species supplement straight moves by periodic turns to avoid the problem of becoming locked into an unprofitable path direction."

Two other theoretical search patterns are the expanding square and parallel sweep, used in human search and rescue but not undiscovered as animal search strategies (Bell, 1991).

\section{Environmental Cues}

The environment contains many cues by which animals may navigate. Even if they do not lead directly to food, they may be effective in covering a large area, reducing time spent revisiting patches (Table 2.1). For example males and females of the butterfly species Melitae trivia prefer to fly towards the top of local hills when seeking 
a mate (Pe'er et al., 2004). It has been noted that Pieris rapae fly in the shelter of a "leading line" such as a railroad. Although they have a preferred direction based upon the sun, if a leading line happens to be in the same direction they will delegate to this until it diverges too far (Baker, 1978).

Wolves (Canis lupis) foraging in groups tend to follow contours and topographical features until they detect the scent of prey in the wind (Bell, 1991). The shore crab leaves a trail of sand which it follows back to its lair, creating its own topographical landmarks (Bell, 1991).

\section{Area Restricted Searching}

This is a common term in movement ecology. When a forager encounters a patch or a food item, turning angle concentration and step lengths (Section 2.5) may decrease (path become less directional), causing less displacement but a more thorough coverage of the area. This response of altering movement behaviour in relation to some environmental factor is termed klinokinesis. Heinrich (1979b) conducted an elegant experiment using bumble bees foraging over clover where patches of clover were covered, allowing neighbouring patches to become depleted of nectar. The paths of bumble bees were then measured over the two patches and a significant increase in turning and a decrease in step length in the nectar-rich patch was observed. As mentioned, a similar effect is reported with G. picipes after encountering a prey item (Winkelman and Vinyard, 1991). An interesting paper by Kareiva and Odell (1987) suggests that if individuals in a swarm each adopt an area-restricted search, the overall swarm will exhibit prey taxis.

\section{Sensory perception (Olfaction and Vision)}

Most animals and insects have sophisticated sensory apparatus. These abilities are usually in the form of detection of volatile info-chemicals in the air or visual stimuli. In some cases a response may be triggered by a combination of sensory stimuli, e.g. the apple maggot fly (Rhagoletis pomonella) flies towards red spheres after searching behaviour is stimulated by apple odour (Prokopy, 1968a,b; Roitberg, 1985). Male sweat bees (Lasioglossum zephyrum) and house flies Musca domestica are attracted to small dark objects when they perceive female sex pheromone (Barrows et al., 1975). They do not respond or at least respond to a lesser degree when the olfactory cue is absent. This mechanism could increase the efficiency of resource finding within a patch of resources, and decrease the amount of time spent in detailed searching behaviour when no resources are nearby. 
Those using odours may find it easier to locate clumped rather than isolated plants because more odour is produced. The Milkweed bug (Oncopeltus fasciatus) seldom colonises small or sparse Milkweed patches (Ralph, 1977b).

A summary of the sensory abilities of the subject of this thesis, P. rapae is given in Section 2.7.

Parasitoids in particular are highly adapted to using information from the senses to locate prey, and can be very selective in their response. Leptopilina clavipes, an endoparasite of the Drosophilidae responds to the smell of decaying mushrooms, specifically at the time in which they are likely to contain host larvae (Vet and Dicke, 1992). Cardiochiles nigriceps, a parasitoid of the Tobacco bud-worm (Heliothis virescens) is attracted by the odour of tobacco plant leaf tissue when leaves are damaged by the host (Vinson et al., 1975). A similar response is reported for the parasitoid wasp Cotesia glomerata (a parasitoid of the larvae of $P$. rapae), where volatiles released from the cabbage leaves as they are eaten by the caterpillars are thought to signal areas of prey abundance (Horikoshi et al., 1997; Sato et al., 1999).

\subsection{Scale and the ecological landscape}

The scale of observation and the pattern of arrangement of biotic organisms and abiotic structures (the ecological landscape), has become a key feature of modern ecological studies (Levin, 1992; Wiens, 1989; Levin, 1988; Ricketts, 2001). Combined with studies and methods seeking to quantify spatial arrangements (Kareiva, 1994), a powerful theoretical framework is being created, within which the responses of individuals and populations can be explored. How animals respond to and move about in their environment, and the effects they have upon it are intrinsically linked to their physical and perceptual scales (With, 1994; Roitberg and Mangel, 1997). A given distribution of resources or individuals may appear to be more or less aggregated at different scales (Hartley et al., 2004; Tenhumberg et al., 2001).

When considering quantification of a spatial pattern, the terms "Isolation", "Density" and "Aggregation" are often encountered. These are all dependant on the scale of observation (Hartley and Kunin, 2003; Matter et al., 2005; Summerville et al., 2002). For example, a measure of plant density may be "Number of Plants per $10 \mathrm{~m}^{2 \prime \prime}$. The scale is set at $10 \mathrm{~m}^{2}$.

The scale of measurement is defined by the extent of the area measured and the grain of the measurements taken within that area (Wiens, 1989). The extent sets the scale at which the number of observations may be made, and hence the stability of those measurements, an upper limit to what may be encompassed. The grain sets the scale 
of resolution that may be observed, the minimum size of observation. Wiens (1989) describes an analogy to a sieve; the extent is the size of the sieve and the grain is the size of the holes in the mesh.

At whichever scale measurement is made, spatial patterns will be observed. These often relate to aggregation, or "clumped" distributions, resulting in "patchy" environments (Cronin, 2003). This "heterogeneous" distribution of resources can significantly affect the way in which the dynamics of populations both fluctuate in the real world and are modelled theoretically. For example, a non-spatial Lotka-Volterra model has fundamentally different dynamics to a spatially explicit metapopulation model defining groups (demes) of individuals interacting locally, with migration amongst demes (Hanski and Gilpin, 1996; Kareiva, 1990; Law and Dieckmann, 2000).

Spatial arrangement can affect the results of field experiments intrinsically, particularly in terms of spatial auto-correlation. This is essentially the premise that two points sampled closer together are more likely to share the same value than two distant points if there is a gradient in the feature being observed. If the feature is patchily distributed, correlation will depend on the relative spacing of the samples to the size and spatial distribution of the patches. Originally deriving from the geophysical sciences, statistical methods to both test for and adjust for spatial auto-correlation are being increasingly incorporated into ecological studies (Legendre et al., 2004, 2002).

The spatial arrangement of plants within a heterogeneous background of other species can impact both their own development and the behaviour of herbivorous insects feeding upon them (Johnson et al., 1992; Matter et al., 2005). Bach (1988) manipulated densities and patterns of Squash plants (Cucurbita maxima) and observed the response of the beetles Diabrotica undecimpunctata and Diabrotica virgifera. The "patches" of Squash plants were either surrounded or not by non-host Tomato plants. The tomato plants were either planted in the ground or in pots to isolate their competitive effect on the host (Squash) plants. The results showed that the growth and reproduction rates of the Squash plants were negatively affected by the surrounding Tomatoes, leading to generally lower densities of beetles. The beetles' movement was also affected by the surrounding Tomatoes. Beetles tended to leave patches without surrounding non-hosts more than those with a border, suggesting a "reflective" barrier.

This last example highlights the interplay of various effects that might be in operation on any given system. The measurement both of the observed variables (density, patch size) and the response (number of beetles, patch occupancy time) are all tied to the scale at which they are measured, and the underlying mechanisms to the scale 
of the organism (Holland et al., 2004; Banks, 1998). With (1994) applied fractal analysis to large and small species of grasshopper and reported that the smaller species showed more tortuous paths (See section 2.5) than the larger species, suggesting that they are interacting with the environment at a smaller scale.

Movement strategies which may be efficient for searching within patches (e.g. Area Restricted Searching) may be less efficient for larger scale, inter-patch movement and searching. Here, a combination of behaviours may be seen. Zollner and Lima (1999b) produced a model incorporating both systematic searching and Random Walking (Sections 2.2.2 and 2.6.1). Search patterns at larger scales suggested that a combination of the two was the most effective, a result echoed in Hoffmann (1983a). Jones et al. (1980) suggest an opposite response for P. rapae, which has a directional movement during a days' foraging, but that the preferred direction changes daily, resulting in a pure Random Walk when measured at a scale greater than a single day's foraging (Section 2.7).

Risk factors such as predation or starvation can also be at work shaping an individual's foraging actions if the landscape is heterogeneous and the forager's resources are dispersed patchily (Russell et al. 2003; Roitberg and Mangel 1997; Section 2.7). In these cases dispersal abilities become important to the viability of the population and thus the effects of small scale, individual behavioural decisions is seen at the larger population scale and is important for such models (Zollner and Lima, 2005).

\subsection{The Resource Concentration Hypothesis}

The study of ecology is the study of complex interacting systems (Begon et al., 1990). The search for pattern and abstraction in this complexity leads to the (often difficult) attempt to formulate general rules and hypotheses about the function of those systems (Levins, 1968).

The Resource Concentration Hypothesis ( $\mathrm{RCH}$ ) describes a general relationship between the density of resources and the density and diversity of the animals which utilise them. Introduced by Root (1973), it is defined thus:

"... herbivores are more likely to find and remain on hosts that are growing in dense or nearly pure stands; that the most specialised species frequently attain higher relative densities in simple environments; and that, as a result, biomass tends to become concentrated in a few species, causing a decrease in the diversity of herbivores in pure stands" (Root, 1973).

In its original form, this proposition was concerned with the effects of pure and mixed stand vegetation. In a more general sense this can be interpreted as "concentration" of the resources, pure stands being "dense" or "concentrated" and mixed 


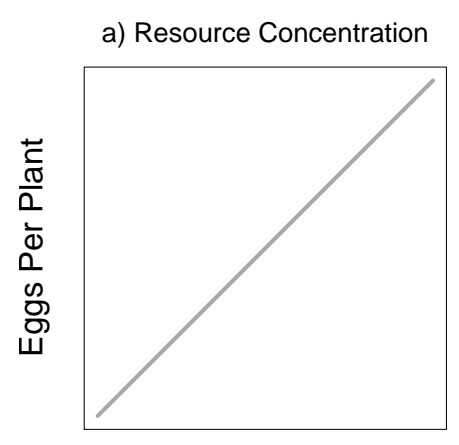

b) Ideal Free Distribution

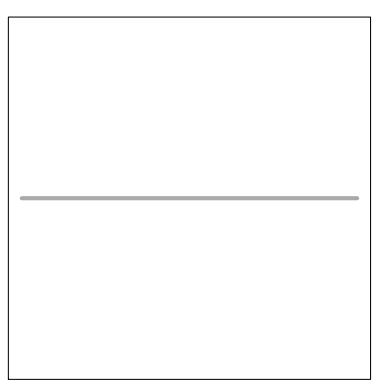

Resource Density c) Resource Dilution

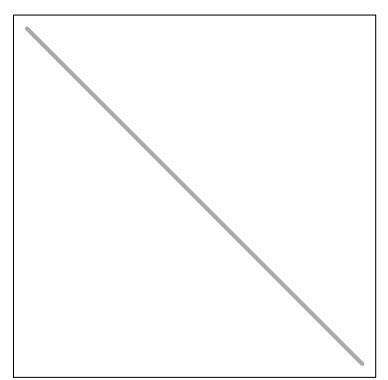

Figure 2.1: A simplified view of three theoretical responses to resource density.

stands containing lower densities, and more "Isolated" hosts. This effect was demonstrated in the original paper and followed up by several others (Cromartie, 1975; Ralph, 1977a; Bach, 1980; Tahvanainen and Root, 1972; Kunin, 1999). Resource Concentration can be envisaged as a simple, positive correlation between host plant density and herbivore densities (Figure 2.1). Hamback and Englund (2005) have recently proposed a theoretical framework that provides for a more complex response to resource density, which may provide a future direction of the theory.

In a subsequent paper, Root and Kareiva (1984) experimented with P. rapae and discovered that this species did not respond to the inter-cropped density of their hosts when ovipositing. The result was that areas of high host plant density experienced a collective "advantage" from their density, because the overall eggs per plant laid was lower than for more isolated plants. This effect is termed "Resource Dilution" and has been shown in several other papers, especially for P. rapae (Root and Kareiva, 1984; Yamamura, 1999; Jones, 1977; Otway et al., 2005). A visualisation of these responses is provided in Figure 2.1 . A "null" response is shown, assuming an Ideal Free Distribution (Cressman et al., 2004) where all plants receive an equal number of eggs. In reality, this response is likely to require the most complex interactions as the foragers would need to adjust their behaviour to exactly match the densities of resources, neither favouring highly dense areas, nor simply ignoring density.

As has been mentioned, density is a measure which depends upon the scale at which it is measured. It is possible then that the response of the $\mathrm{RCH}$ might also vary with scale (Chapter 4).

Root and Kareiva (1984) suggested that a potential evolutionary mechanism driving the "dilution" response might be one of "Spreading The Risk" in terms of predation risk to the Pieris larvae. Courtney (1986) argued in response to this that the effects of density dependant mortality (Jones and Ives, 1979) were strong enough in their own right to be the selective pressure driving the egg spreading behaviour. However, Root and Kareiva (1986) responded in the same publication, questioning the details 
of Courtney's assumptions, and in which they state "We never claimed to prove that risk-spreading is the basis for the ovipositional behaviour of $P$. rapae. We simply offered it as the hypothesis that best fit the available data". The conclusion has yet to be resolved ${ }^{1}$.

A related topic is that of the "Natural Enemies Hypothesis" which relates species abundance and diversity to the fact that heterogeneous environments provide a greater diversity of predators and thus maintain a balance preventing any one species becoming super-abundant. Mono-cultures should tend toward highly variable population explosions and extinctions (Letourneau, 1987; Gratton and Welter, 1999; Vet and Dicke, 1992).

\subsection{Quantifying animal movement}

Quantifying animal movement is an area which is relatively well documented. With the increased availability of technology such as radio tracking devices, this field is growing in popularity. Turchin (1998) provides good coverage although his focus is more the dispersal dynamics of populations and does not deal with statistical methods for analysing individual paths. To that end, Batschelet (1981) and Mardia (1972) provide excellent references; these texts are inevitably cited in any papers on the subject. The following section describes a selection of concepts and methods which were used during this thesis, in summary form.

Before discussing the specifics of measuring an animal's path, a recap of some geometrical principals which will be useful is provided.

\subsubsection{The geometry of directional data}

This topic is also covered in Batschelet (1981), Part 2. It was felt that an overview would be beneficial to this thesis for both understanding and as a statement of assumptions and terminology.

\section{Rectangular vs. Polar Coordinates}

A point in rectangular, 2D, continuous space can be defined by its $x$ and $y$ distance from the origin (bottom left corner), called the Rectangular Coordinate and symbolised by $P(x, y)$. An alternative representation is the straight line distance from the origin and the angle formed by the intersection of that line with the x-axis, measured

\footnotetext{
${ }^{1}$ As far as the author is aware
} 
anticlockwise and symbolised $P(\phi, r)$ where $r$ is the distance to the point ${ }^{2}$. By applying Pythagoras' theory to these measures it is possible to easily translate between the two representations. The conversion from rectangular to polar is summarised in (2.2). In these equations, $r$ is always the hypotenuse of the triangle, $x$ is the adjacent side and $y$ the opposite side.

$$
\begin{aligned}
P(\phi, r) & =P(x, y) & & \\
r & =\sqrt{x^{2}+y^{2}} & & \text { hypotenuse } \left.^{2}=\text { adjacent }^{2}+\text { opposite }^{2}\right) \\
\phi & =\arctan \left(\frac{y}{x}\right) & & \left(\tan (\phi)=\frac{\text { opposite }}{\text { adjacent }}\right)
\end{aligned}
$$

To obtain the angle however there are some exceptional circumstances to Equation 2.2c. Firstly, it only functions if $x \neq 0$. Further, the values of $\arctan (y / x)$ range between $-90^{\circ}$ and $+90^{\circ}$. This means that it only produces angles when $x>0$. When $x$ is less than zero, $180^{\circ}$ must be added to $\operatorname{arc} \tan (y / x)$, as $\tan \phi$ has a period of $180^{\circ}$. This results in (2.3).

$$
\phi= \begin{cases}\arctan (y / x) & \text { if } x 0 \\ 180^{\circ}+\arctan (y / x) & \text { if } x<0\end{cases}
$$

To this must be added exceptional cases when $x=0$ (Batschelet, 1981 p. 239).

$$
\phi= \begin{cases}90^{\circ} & \text { if } x=0 \text { and } y>0 \\ 270^{\circ} & \text { if } x=0 \text { and } y<0 \\ \text { undetermined } & \text { if } x=0 \text { and } y=0\end{cases}
$$

These rules are particularly important for computer software performing the calculation and so are repeated here. To convert from polar to rectangular is simpler (2.5).

$$
\begin{array}{rlrl}
P(x, y) & =P(\phi, r) & & \\
x & =r \times \cos (\phi) & & \left(\cos (\phi)=\frac{\text { adjacent }}{\text { hypotenuse }}\right) \\
y & =r \times \sin (\phi) & \left(\sin (\phi)=\frac{\text { opposite }}{\text { hypotenuse }}\right)
\end{array}
$$

The geometric angle $\phi$ is often measured in radians when passed to the trigonometric

\footnotetext{
${ }^{2}$ Derived from "radius" as it is the radius of the circle centred on the origin upon whose perimeter the point lies
} 
functions. Converting to and from degrees is possible using (2.6).

$$
\begin{aligned}
360^{\circ} & =2 . \pi \text { radians, } 180^{\circ}=\pi \text { radians } \\
\phi_{\text {deg }} & =\frac{180}{\pi \cdot \phi_{\text {rad }}} \\
\phi_{\text {rad }} & =\phi_{\text {deg }}\left(\frac{\pi}{180}\right)
\end{aligned}
$$

The geometric angle is always measured anti-clockwise from the x-axis.

\section{Azimuth vs Geometric Angle}

Directional data is generally recorded in terms of degrees from North, measured clockwise, as a compass bearing. This measurement is termed in navigation, the Azimuth and denoted here by $\theta$ to distinguish it from the geometric angle, $\phi$. The compass bearing can never be greater than $360^{\circ}$. Care should be taken when comparing the Azimuth and the geometric angle. When using them as input to statistical calculations there is no difference (Batschelet, 1981), but when converting between Polar and Rectangular Coordinates it is more consistent to first convert the Azimuth into the equivalent geometrical angle (2.7 and 2.8).

$$
\begin{aligned}
& \theta_{d e g}=\left(\left(360-\phi_{d e g}\right)+90\right) \bmod 360 \\
& \phi_{d e g}=\left((360+90)-\theta_{d e g}\right) \bmod 360
\end{aligned}
$$

Or in radians:

$$
\begin{array}{r}
\theta=((2 \pi-\phi)+0.5 \pi) \bmod 2 \pi \\
\phi=((2.5 \pi)-\theta) \bmod 2 \pi
\end{array}
$$

\subsubsection{Measuring a path}

Animals move in a continuous manner through continuous space, which is rather intractable to measurement. The common method for analysing a path is to sample points along that path in some way (e.g. by following and leaving markers at time intervals). These can then be joined together by straight line "steps" or "moves" (for consistency "step(s)" will be used hereafter), providing an approximation of the true path (Cain, 1989; Wiens et al., 1993; Zalucki and Kitching, 1982; Zalucki et al., 1980). 
The path can be sampled in three ways; a) at specified time intervals (e.g. every 3 seconds), b) at a specified step length (e.g. step lengths of $1 \mathrm{~m}$ ) and c) by "Point to Point" sampling. Method c) involves recording known positions at which an animal was observed, and using these as the points of the path, e.g. Plant-To-Plant movements of butterflies, recording whenever a butterfly alights upon a plant (Jones, 1977). Figure 3.2 in Chapter 3 demonstrates the sampling of a continuous path at specified intervals.

The path is thus recorded as a vector of Rectangular Cartesian Coordinates in space, which can be written as $P\left(x_{i}, y_{i}\right)$. The origin is the bottom left of the sampling area. In the field it would be usual to mark out a rectangular observation area and record positions relative to the bottom left of this.

\section{Step Length}

The first metric of the path is simply the distance between each point, or the length of each "step". Depending on the method of sampling, this may be constant. A common technique is to record the plant-to-plant steps e.g. (Jones, 1977) and so the step lengths can vary. A mean can be taken of these to give a single, characteristic value.

If $P\left(x_{1}, y_{1}\right)$ represents the rectangular coordinate of the start of a step, and $P\left(x_{2}, y_{2}\right)$ the end of the step, the start point can be considered the origin and a relative coordinate can be calculated for the end point, $P\left(x^{\prime}, y^{\prime}\right)(2.9)$. The calculations outlined in the previous section can be applied to calculate length of the step $(r)$.

$$
\begin{array}{r}
x^{\prime}=x_{2}-x_{1} \\
y^{\prime}=y_{2}-y_{1}
\end{array}
$$

\section{Turning Angle}

The second metric of the the path is the difference in direction between successive steps, or the Turning Angle. This assumes that the animal has a direction, so that each step represents a "turn" either to the left or the right, in this thesis represented as $\Delta \theta$, with limits (2.10).

$$
-180 \leqslant \Delta \theta_{\text {deg }} \leqslant+180
$$

Or in radians (2.11).

$$
-\pi \leqslant \Delta \theta \leqslant+\pi
$$




\section{Directionality}

Given a distribution of directions (azimuths or angles) it is possible to characterise them further in terms of a "mean direction" and a "mean vector length", which is a measure of the concentration of directions about the mean (Batschelet, 1981; Mardia, 1972). If the distribution contains angles of turn which are evenly distributed to the left and right, the mean direction will always be zero (straight ahead). The distribution can be plotted on a circular graph, where points are added at their respective azimuths and are stacked up when they are for the same azimuth (Figures 2.2 and 2.3). These two measures in combination are known as the "Mean Vector" (Batschelet, 1981).

To calculate the mean vector, the concept of a "unit circle" (a circle with radius $=1$ ) is used. Each observation (angle) is placed around this circle and given an equal weight. It is then possible to calculate the "Centre of Mass" of the distribution. If all the points are distributed evenly around the circle the centre of mass will be at the centre of the circle, the zero vector. The centre of mass is the mean vector, the polar coordinates of which are used to represent it, composed of the mean direction $(\bar{\phi})$ and a vector length $(r)$. This is represented by the small grey circle in Figures 2.2 and 2.3 and is notated by $m(\bar{\phi}, r)$.

Without repeating the complete description to be found in (Batschelet, 1981); to calculate the mean vector, first calculate the $x$ and $y$ components by taking the mean of the $x$ and $y$ components of each unit vector (Equation 2.14). These are denoted $\bar{x}$ and $\bar{y}$. The unit vector is from the centre of the unit circle to the point on its perimeter described by the observation angle.

$$
\begin{aligned}
& \bar{x}=\frac{1}{n}\left(\cos \phi_{1}+\cos \phi_{2}+\cdots+\cos \phi_{n}\right) \\
& \bar{y}=\frac{1}{n}\left(\sin \phi_{1}+\sin \phi_{2}+\cdots+\sin \phi_{n}\right)
\end{aligned}
$$

These could be written as (2.13)

$$
\begin{gathered}
\bar{x}=\frac{\sum_{i=1}^{i=n} \cos \phi_{i}}{n} \\
\bar{y}=\frac{\sum_{i=1}^{i=n} \sin \phi_{i}}{n}
\end{gathered}
$$

The mean vector length $(r)$ can then be calculated (2.14).

$$
r=\sqrt{\bar{x}^{2}+\bar{y}^{2}}
$$




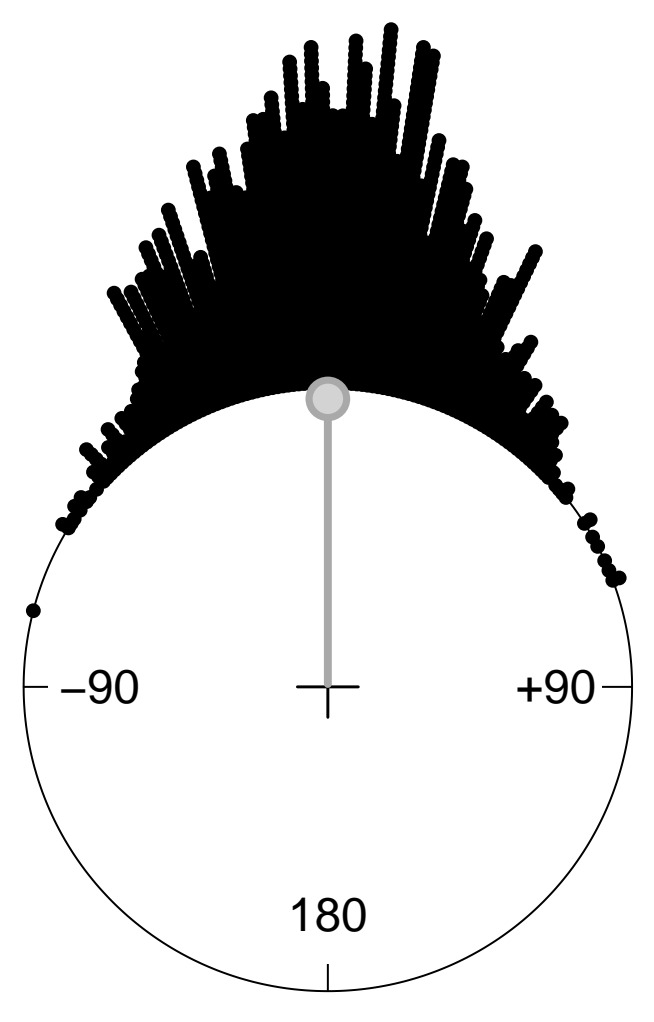

Figure 2.2: Example of a distribution of turning angles plotted as a circular graph, generated from Von Mises distribution ( $k=10,2000$ samples shown). The small grey circle represents the centre of mass and the grey line the mean vector length $(r=0.946)$ 


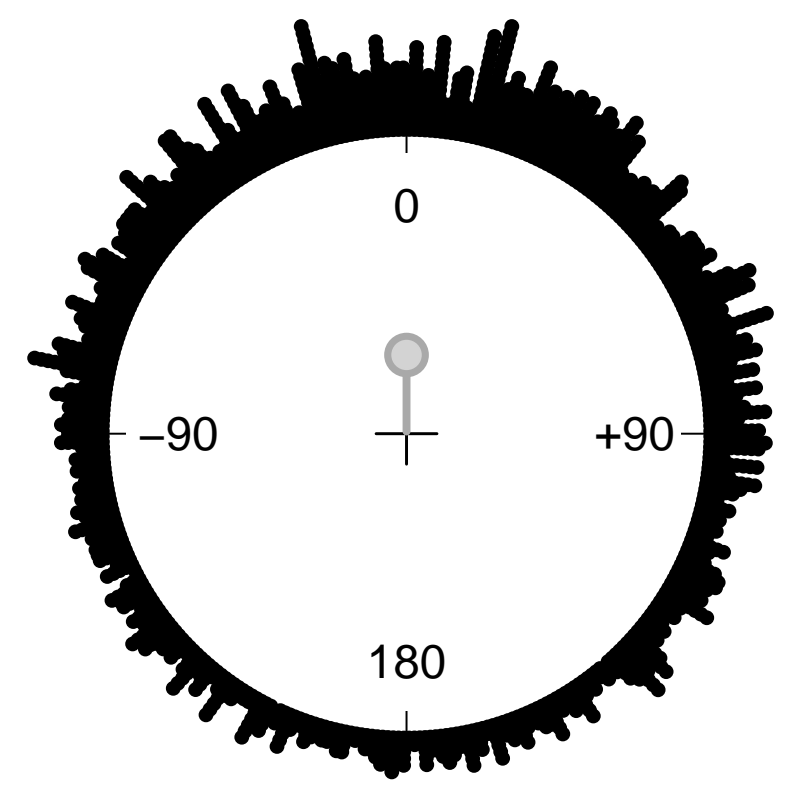

Figure 2.3: Example of a distribution of turning angles plotted as a circular graph, generated from Von Mises distribution ( $k=0.5,2000$ samples shown). The small grey circle represents the centre of mass and the grey line the mean vector length $(r=0.259)$. Notice that for this concentration the centre of mass is much closer to the centre of the unit circle because the observations are distributed more evenly around the perimeter 
The mean angle can similarly be calculated from $\bar{x}$ and $\bar{y}$ following 2.2 (2.15 and 2.16).

$$
\bar{\phi}= \begin{cases}\arctan (\bar{y} / \bar{x}) & \text { if } x 0 \\ 180^{\circ}+\arctan (\bar{y} / \bar{x}) & \text { if } x<0\end{cases}
$$

Again, account must be taken of the exceptional cases when $x=0$ (As with Equation 2.4).

$$
\bar{\phi}= \begin{cases}90^{\circ} & \text { if } \bar{x}=0 \text { and } \bar{y}>0 \\ 270^{\circ} & \text { if } \bar{x}=0 \text { and } \bar{y}<0 \\ \text { undetermined } & \text { if } \bar{x}=0 \text { and } \bar{y}=0\end{cases}
$$

When referring to the moments of circular directional distributions, Batschelet (1981) notates the Mean Vector as $\mu(\rho, \theta)$ where $\rho$ (rho) is the mean vector length and $\theta$ the mean angle. The calculations are the same as above. Other authors, such as (Jones, 1977) denote the mean vector length as $r$.

If turning angles, rather than absolute directions, are being measured, and if the distribution is symmetrical about the mean direction (i.e. same number of turns left as right), then the mean of the sines of the angles will be approximately equal to zero $(\bar{y} \approx 0)$ and so the mean vector length will simply be $\bar{x}$ (Equation 2.14). This is particularly useful when measuring paths which are known to fit a symmetrical distribution (See "Von Mises" Distribution below), or paths generated from a Von Mises distribution (Section 2.6.1).

The mean vector length can also be seen as a measure of the correlation between directions of successive steps. When there is no correlation $(r=0)$ a pure random walk (Section 2.6.1) is observed, when it is perfect $(r=1)$ the path will be a straight line.

There are deeper characterisations and higher order moments which are possible, such as Angular Deviation (equivalent to standard deviation of the Normal Distribution) which will not be explored here as they are not directly relevant. Batschelet (1981); Mardia (1972); Cain (1989); Turchin (1998) and Wiens et al. (1993) provide an excellent starting point, as well as covering the topics presented here in more depth.

\section{The Von Mises Distribution}

Once the distribution of turning angles has been quantified in this manner, it is also possible to compare it to theoretical probability distributions in the same way that a set of linear observations may be compared to the Normal Distribution. There are a class of Circular probability distributions which have similar features to the normal distribution. Whilst this is a detailed topic (Batschelet, 1981; Mardia, 1972), the most 
well used distribution is the "Von Mises Distribution", also known as the "Circular Normal Distribution". One of the properties of Circular probability distributions is that they incorporate trigonometric terms so that they "wrap" around 360 such that the value of the density function for 370 will be the same as that for 10 . The Von Mises density function is defined as (2.17)

$$
f(\phi)=\frac{1}{2 \pi I_{0}(k)} \exp [k \cos (\phi-\bar{\theta})]
$$

Where $k(k \geqslant 0)$ is a concentration parameter, similar to $r$ (and can be inter-converted from the tables in Batschelet), $\phi$ is the angle over which the density is calculated, and $\bar{\theta}$ is the mean direction. The parameters $k$ and $\bar{\theta}$ map closely to those of the mean vector (mean angle and direction). The function $I_{0}(x)$ is a Second family (sometimes refereed to as modified) Bessel Function of the order 0. The general definition for the Bessel function is given by (2.18).

$$
I_{n}(z)=\frac{1}{2 \pi} \int_{0}^{2 \pi} \exp (z, \cos \phi) \cos n \phi d \phi
$$

Conveniently, for the Zero Order the term $\cos n \phi d \phi$ reduces to $\cos 0=1$ and so the equation for $I_{0}(z)$ is reduced to (2.19).

$$
I_{0}(z)=\frac{1}{2 \pi} \int_{0}^{2 \pi} \exp (z, \cos \phi)
$$

This integral is not analytically solvable, but can be approximated by numerical integration (Press, 2002), where an algorithm (usually performed by a computer) calculates the area under the curve by sampling intervals from the distribution function.

It is possible to test a given distribution to determine the probability that it derives from a Von Mises distribution. One such test is Watson's goodness of fit test. Implementation of various circular distributions and tests can be found in the CircStats package (Agostinelli and Lund, 2006) for the R statistical programming language ( $R$ Development Core Team, 2005).

Other circular distributions are the Wrapped Normal and the Wrapped Cauchy distributions Batschelet (1981). These have similar properties to the Von Mises Distribution, but the Von Mises Distribution can be parameterised to match any of the other distributions. It is a popular choice because the $k$ parameter has a close association to the mean vector length $r$, and it has other convenient statistical properties similar to the linear Normal Distribution (Section 2.6.1). 


\section{Dispersal (Mean Squared Displacement)}

A useful measure of animal movement is how far an individual has dispersed over time, or its displacement from its original location. The path of an animal can be recorded as a vector of steps $(x, y)$. Each element represents the change in $x$ and $y$ for that move. The total displacement is given by Equation 2.20 (Kareiva and Shigesada, 1983).

$$
R_{n}=\sum_{m=1}^{n}\left(x_{m}, y_{m}\right)
$$

An example is given in Table 2.2. The displacement vector can then be calculated in terms of the mean vector, so $m(\phi, r)$ where $r$ is given by (2.21).

$$
r=\sqrt{\sum_{m=1}^{n}\left(x_{m}\right)^{2}+\sum_{m=1}^{n}\left(y_{m}\right)^{2}}
$$

If the squared displacement is considered, an expectation can be calculated of it's size over time, given a mean vector for the movement path, in other words the mean turning angle and mean direction (Kareiva and Shigesada, 1983; Jones, 1977; Johnson et al., 1992; Skellam, 1951). This expectation represents what the Mean Squared Displacement (MSD) for a set of individuals would be. The squared displacement is $r^{2}$ which reduces the equation (2.21) to (2.22).

$$
r^{2}=\sum_{m=1}^{n}\left(x_{m}\right)^{2}+\sum_{m=1}^{n}\left(y_{m}\right)^{2}
$$

To calculate the Mean Squared Displacement from a set of observed path, $r^{2}$ must be calculated for each individual, and then the mean taken. This measure relates to the area covered rather than the length of each path (linear displacement).

Calculation of the Expected MSD is discussed in both Kareiva and Shigesada (1983) and by N.E. Gilbert in the appendix of Jones (1977), the formula from Jones is more straightforward and is derived from original work by (Skellam, 1951), which underpins both derivations, so it is this that is repeated here. The Expected MSD, $E\left(R_{n}^{2}\right)$ is given by (2.23) where $n$ is the number of steps, $s$ is the fixed length of the steps, and $z$ is the mean value of $\cos (\phi)$ (as for Equation 2.13.a). $\phi$ is the angle drawn from a Circular Normal (Von Mises) distribution of turning angles with a mean direction of 0 . This formula is also reported with slightly different variable names in Johnson et al. (1992).

$$
E\left(R_{n}^{2}\right)=s^{2} \frac{1+z}{1-z} n-\frac{2 z\left(1-z^{n}\right)}{\left(1-z^{2}\right)}
$$

Equation 2.23 can easily be used to estimate the expected MSD for a population of 
paths which are generated using a Von Mises probability distribution and a fixed step length because when the turns are symmetrical about the mean, the $r$ is given only by $\bar{x}$ because the sum of $\sin (\phi)$ will be approximately zero (Section 2.5.2).

\begin{tabular}{rrr}
\hline & $\mathbf{x}$ & $\mathbf{y}$ \\
\hline 2 & 3 \\
-5 & 1 \\
3 & -3 \\
& -1 & 1 \\
\hline Total & -1 & 2 \\
\hline
\end{tabular}

Table 2.2: Example of a calculation of the Mean Squared Displacement

Benhamou (2004) provides a summary of refinements to these calculations which allow an approximation of the net linear displacement. He presents Equation 2.24 for this purpose.

$$
E(D)_{a p p}=\left[\frac{\pi E\left(D^{2}\right)}{4}\right]^{0.5}
$$

\section{Sinuosity}

The combination of step length and the distribution of turning angles defines the qualitative types of the path observed (Chapter 3). Shorter step lengths and widely ranging turning angles lead to a more tortuous path. Longer step lengths and less variability in turning angle leads to more directional paths, ultimately to a straight line. Bovet and Benhamou (1988) developed a measure which combines these two metrics into a single, relative measure of tortuosity which they term "Sinuosity".

The calculation of their measure was based around a Random Walk simulation (Section 2.6.1). They generated paths using a linear normal distribution of turning angles, "wrapped around" so that it appears to be circular, which is a method for approximating the Von Mises distribution. The turning angle concentration (equivalent to the $k$ parameter of the Von Mises distribution, or the $r$ of the mean vector) is controlled by the standard deviation $(\sigma)$ of the normal distribution. The $\sigma$ is related to the mean vector length by (2.25).

$$
r=e^{-\sigma^{2} / 2}
$$

Using the Random Walk simulation, 500 paths were generated with a fixed step 
length $(P)$, for 12 values of $\sigma$, ranging from 0.1 to 1.2 radians $^{3}$. In degrees the range is approximately $6^{\circ}$ to $70^{\circ}$. Each path was then re-discretised (re-sampled) with different step lengths $(R)$ varying from $0.6 P$ to $10 P$. The standard deviation of the rediscretised turning angle distribution $\left(\sigma_{R}^{*}\right)$ was calculated for each re-sampled path. From this a quadratic function was fitted to parameterise the relationship between the re-sampled $\sigma_{R}^{*}$ and that of the original $\sigma$. The result was (2.26), where $R / P$ is the ratio of the re-sampled length to the step length used to generate the path.

$$
\sigma_{R}^{*}=0.85 \sigma(R / P)^{1 / 2}
$$

They then separated the terms that belong to the model ( $P$ and $\sigma$ ) from the observations $\left(R\right.$ and $\left.\sigma_{R}^{*}\right)$ to produce their definition of sinuosity (2.27).

$$
S_{*}=\frac{\sigma}{\sqrt{P}}=\frac{1.18 \sigma_{R}^{*}}{\sqrt{R}}
$$

This derivation has two purposes; a) the first expression (2.28) can be said to be a theoretical definition of sinuosity and is useful as an abstract measure of the two parameters "Turning Angle Concentration" $(\sigma)$ and "Step Length" $(P), \mathrm{b})$ the second expression (2.29) can be applied to observed animal paths sampled in the field to provide an estimate of the true sinuosity, simply by knowing the re-discretisation step length $(R)$. They go on to show that you can calculate the s.d. of the $S^{*}$ values and that this can be used to test their model, which will not be covered here.

$$
\begin{gathered}
S^{*}=\frac{\sigma}{\sqrt{P}} \\
S^{*}=\frac{1.18 \sigma_{R}^{*}}{\sqrt{R}}
\end{gathered}
$$

The units of their measurement are $\mathrm{rad} . u^{-1 / 2}$ where $u$ is the unit of step length (e.g. rad.cm ${ }^{-1 / 2}$ ) although it is more useful as a relative measure than an absolute one. This measure is particularly relevant to this thesis because it allows the characterisation of a path generated with two parameters, by a single parameter. Equation (2.28) is used in Chapter 3 to aid interpretation of the parameter space of a Random Walk simulation, where both turning angle concentration and step length are varied simultaneously.

When applying this method to field observations, Bovet and Benhamou (1988) note that even if the step lengths are not all equal, the mean step length can still be used. Whether this would remain true for distributions such as the Levy Walk (Section

\footnotetext{
${ }^{3}$ The absolute value of $P$ is arbitrary because it is the relationship between the Step length and turning angle concentration (in this case, $\sigma$ ) which is important. For illustration it could be assumed that $P=1$
} 
2.6.1) is not clear.

The relationship between the standard deviation of a linear wrapped normal $(\sigma)$ and the mean vector length $(r)$ defined by Equation 2.25 can be re-arranged to make $\sigma$ the subject and thus afford two-way conversion by using the fact that (2.30) is true (Eason and Coles, 1989).

$$
\begin{aligned}
y & =e^{x} \\
\ln (y) & =x
\end{aligned}
$$

With $\sigma$ as the subject, Equation (2.25) becomes (2.31).

$$
\begin{aligned}
\ln (r) & =\frac{-\sigma^{2}}{2} \\
\sigma & =\sqrt{-2 \ln (r)}
\end{aligned}
$$

Using this transformation it is possible to calculate the sinuosity for any distribution for which $r$ is known, such as a set of observations or the Von Mises Distribution, using tables in Batschelet (1981) to convert between $k$ and $r$. This method is applied in Chapter 3.

\section{Fractal dimension}

One other measure of the tortuosity of a path is provided by calculating the fractal dimension (usually symbolised by $D$ ) of the path (Dicke and Burrough, 1988; Kenkel and Walker, 1996; Mandelbrot, 1983; Mark, 1984). The measure has the limits $1 \leqslant$ $D \leqslant 2$ where 1 is completely straight and 2 is so tortuous that it fills the entire $2 \mathrm{D}$ space. Dicke and Burrough (1988) use the "dividers method" for mite trails. Mark (1984) presents a similar method. As fractal geometry was not applied in this thesis the topic is left for the reader to indulge, it is an interesting extension to the field of path analysis. Some notes about generating fractal paths can be found in Appendix C.

\subsection{Modelling movement}

\footnotetext{
"A good model does not attempt to reproduce every detail of the biological system; the system itself suffices for that purpose as the most detailed model of itself. Rather, the objective of a model should be to ask how much detail can be ignored without producing results that contradict specific sets of observations, on particular scales of interest" (Levin, 1988).
}

"Simple ecological models typically are designed from the outset either primarily to expose ideas or primarily to describe particular systems" and tend "to include assumptions that 
enhance mathematical tractability but make the models difficult to test". More complex models, "intended to capture particular field systems are easier to estimate but tend to be considerably less tractable than are simple models of ideas". (Tilman and Kareiva, 1997).

"In general, adding more parameters to a model may be expected to give a better fit to observed data, but may result in a less reliable model for prediction" (Kareiva and Shigesada, 1983)

"Essentially, all models are wrong, but some are useful", Box and Draper (1987).

These quotations set the scene and the principals of modelling for this thesis, which is primarily presenting an ecological model of animal movement. It is important to remember that no model is ever a true representation of the real world. Sometimes as Tilman and Kareiva (1997) and Kareiva and Shigesada (1983) note, they can be used to aid conceptual thought processes and analysis of complex behaviour observed in the field, and thus become "useful".

The terms "Modelling" and "Simulation" are easy to interchange and are often used to describe the same process or artifact. Here, "Modelling" is defined as an abstract, logical process which generates a conceptual "Model" for a particular system. "Simulation" is defined as the concrete implementation of a particular model, specifically implemented as a computer program, although it would also be possible to run a "Pen and Paper" simulation of the same model which should produce the same result.

\section{Individual Based models}

This thesis is concerned with developing an individual-based model and so some definition of the term is warranted. Individual-based models may take two forms; a) those that recognise that individuals are a part of the system but aggregate their responses, and b) those that explicitly model and track the attributes of each individual within the system (Judson, 1994; Mamedov and Udalov, 2002; Uchmanski and Grimm, 1996; West and Cunningham, 2002). This thesis is concerned with the latter.

While it is possible to describe to some extent the result of the diffusion of many individuals following a particular movement pattern (Turchin, 1989, 1998; Holmes et al., 1994; Skellam, 1973), these models are necessarily based on simplistic movement behaviours (such as the Random Walk) and tend not to take into account adaptive behaviours such as being able to perceive resources. This can leave their results general and somewhat abstract. Turchin (1989) suggests that there is a need to incorporate such features into aggregated diffusion models and that this is mathemat- 
ically complex.

The converse is true of behaviourally complex and detailed individual models; it is difficult to produce analytically tractable behaviours from them, because of the number of variables and behaviours. As the opening quotes of this section suggest, adding more parameters and detail to a model may produce results that mimic the field observations more accurately but potentially at the cost of making it difficult to elucidate general principles.

\subsubsection{Random walks}

"Although organisms do not move randomly, the collective behaviour of large numbers of such individuals may be indistinguishable (at the scale of the population) from what would result if they did (Levin, 1992).

Once a logical abstraction of the way animals move has been constructed, it is natural (and very popular) to use this as a basis for a simulation model. The method of representing an animal's path as a series of straight line "steps" is derived from a mathematical model known as the "Random Walk", studied as early as Pearson (1905) and Einstein (1905). The Random Walk is applied in many other areas, for example Boccignone and Ferraro (2004) model gaze shift in the human eye using the Random Walk.

A "pure" Random Walk is often analogised with the classic story of a drunken man wandering home from the local pub staggering in every direction at random and taking a long time to get anywhere because his path has very little direction. The definition of this process is that each step is of a uniform random length and that the direction each step is a uniform random direction Pearson (1905). This model is the same as "Brownian Motion" (Einstein, 1905) . Diffusion of particles (or animals) as a result of this process is known as "Fickian Diffusion" (Johnson et al., 1992).

Animals tend to move forwards because of cephalo-caudal polarisation and bilateral symmetry (Bovet and Benhamou, 1988), thus their movement patterns rarely approximate truly Brownian Motion. By selecting the direction of each step from a unimodal probability distribution rather than the Uniform, a degree of directionality is introduced into the path. It becomes more likely that the animal will continue in the same direction as the previous step and less likely that it will turn completely around. This is known as the "Correlated Random Walk", because the direction of each successive step is "correlated" to the previous step's direction (Skellam, 1951; Siniff and Jessen, 1969; Cain, 1985; Byers, 2001; Berg, 1993; Marsh and Jones, 1988; Hoffmann, 1983a). 

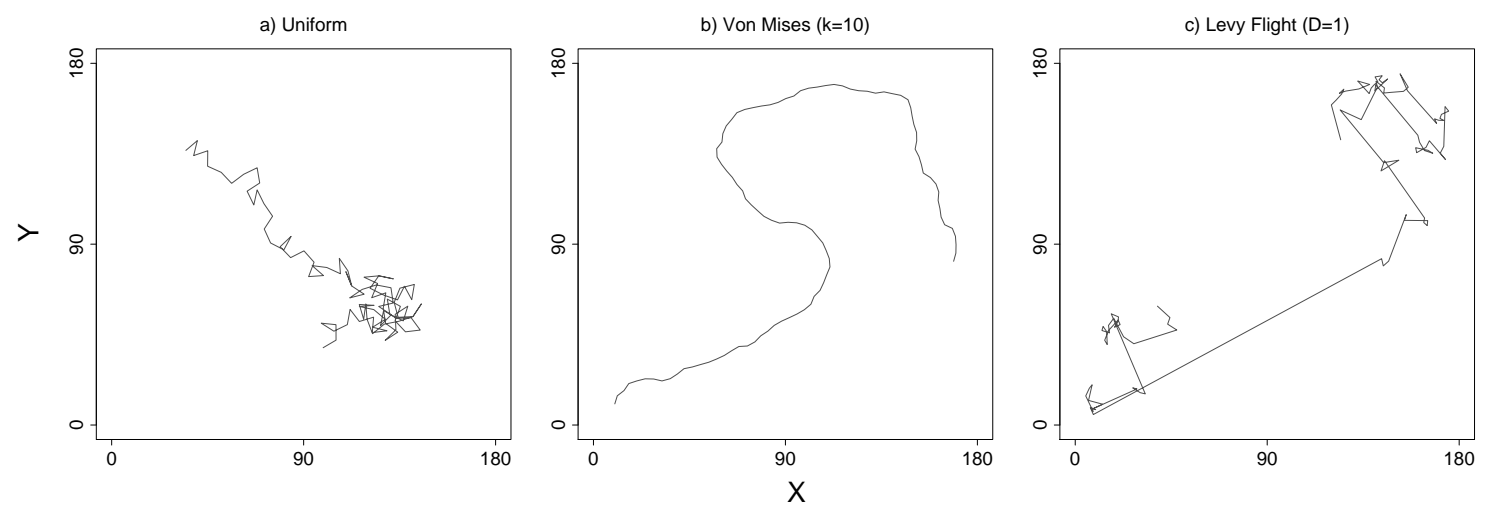

Figure 2.4: Comparison of Random Walk generation methods, each for 100 steps with a step length of 2. a) "pure" Random Walk, b) highly directional Correlated Random Walk and c) Léry Walk with a low fractal dimension $(D=1)$, the minimum step length is 2 and the maximum 1000.

Johnson et al. (1992) demonstrate an interesting property of diffusion by Correlated Random Walks as opposed to pure, "Fickian" diffusion. Over time, the log-log relationship between timesteps and MSD is linear, with a slope of close to 1 , as first perceived by Einstein (1905). In the short term (small number of timesteps) a Correlated Random walk will diverge from this and provide a greater displacement than expected (slope $>1$, because it is more directional). Over a longer time period however, the effect of the directionality is reduced; eventually, all directions have been sampled and the displacement reduces to that of the uncorrelated Random Walk.

Siniff and Jessen (1969) produced what is probably the original Random-Walk model applied to animal movement. Cain (1985) however presents a seminal paper concerning the relationship between a Random Walker and resource spatial arrangement. Cain (1985) measured the efficiency of searchers when moving over resources which were either spaced uniformly, or in clumps. In order to represent some ability for the searcher to "see" the resource (i.e. detect it), he introduced the concept of a "Radius of Attraction" surrounding the resource, which if intersected by the searcher led to it being discovered. A higher percentage of foragers discovered a resource over a uniform layout than a clumped layout, when resources were at higher densities. This provided an "escape" for the clumped plants. This was not simply related to overlapping radii of detection. When resources were hard to find (low radius of attraction, short step length), increased directionality in the search path made no difference to efficiency, but when resources were easy to find (high radius of attraction, long step lengths) increased directionality decreased success. Further exploration of this model is found in Chapter 3. 


\section{Lévy Walks (or Lévy Flights)}

The "Lévy Walk" (or Flight) model is an extension of the random walk that generates clusters of area restricted searching with occasional long "jumps" into a new area, and has been applied to various studies (Chechkin and Gonchar, 1999; Hastings, 1993; Kaye, 1989; Kenkel and Walker, 1993; Mandelbrot, 1999; Ogata and Katsura, 1991; Sugihara and May, 1990; Turchin, 1996).

Figure 2.4.c shows a Lévy Walk with a fractal dimension $(D)$ of $D=2$. . The basic principle is similar to a Correlated Random walk except that the length of the steps is not fixed, but is drawn from an inverse power law distribution so that most steps are quite short, but occasionally extremely large steps may be taken. In its pure form the inverse power law will have an infinite limit, so that there is no limit to the maximum step length.

Ogata and Katsura (1991) and Reynolds (2006) provide equations for the simulation of a Lévy path, however some interpretation is required! A method for calculating a Lévy "dust" (which is the point pattern generated from the "footprints" of a levy walk) is found in Appendix $C$ along with an example of a point pattern generated in this manner. The point pattern is interesting because it demonstrates a second use for the Lévy Walk which is to generate a spatial pattern with a particular fractal dimension. This could be used to simulate naturalistic patterns of resource distribution.

\subsubsection{Sensory perception}

"The utility of including details of search in foraging studies is evident. Evaluating perceptive and information-processing abilities is particularly important because it tells the researcher what the insect perceives to be available to it. It is, however, often much easier in theory than in practise to quantify many aspects of search behaviour" (Roitberg, 1985).

Roitberg's statement underlies all modelling of perceptual abilities. Most perceptual abilities are incorporated in an abstract way such as Cain (1985)'s "Radius of attraction". Bukovinszky et al. (2005) also created a model which took account of the three searching "categories" mentioned previously; Visual, Olfactory and Contact searchers (Figure 2.5). One of the problems with incorporating sensory perception into the model is, as Roitberg (1985) states, that it is difficult to quantify, particularly at the mechanistic level. A good deal of work has been done with the bee, Apis mellifera (Keasar et al., 1996; Spaethe et al., 2001; Barron and Sirnivasan, 2006) and there is a mounting volume of general work, e.g. Ahmad (1983); Hern et al. (1996); Courtney (1983); Bell (1985); Courtney et al. (1989); Finch and Collier (2000); Hogan (2006); 
Horikoshi et al. (1997); Prokopy and Owens (1983); Prokopy (1968a); Thorsteinson (1960); Visser (1986, 1988); Zollner and Lima (1999a); West and Cunningham (2002).

Thorsteinson (1960) is perhaps one of the original papers on the subject, in which he suggests that insects, by the nature of their neurophysiology cannot engage in both dispersal and feeding simultaneously, and that this is the fundamental pattern which all insect-plant interactions are based upon. Bernays and Chapman (1994) suggest that olfactory and visual cues operate in combination to find a suitable host. Visser (1988) presents an interesting analysis of the mechanisms by which insects process input and how this leads to search patterns, in an attempt to classify the various mechanisms and responses.

Miller and Strickler (1984) have a model of behaviour described in Hern et al. (1996) in which acceptance of a host plant is a relative balance between the inputs from the sensory apparatus of the insect, and that this is modulated by an internal "motivational state".

The Correlated Random walk represents a standardised and tractable model for movement. There is no general equivalent for perceptual responses although attempts are being made, e.g. Farnsworth and Beecham (1999); Grunbaum (2000). As mentioned, it seems reasonable to categorise such mechanisms under the three kinds of senses employed, but a standard method for representing these in simulations is lacking. One of the reasons for this is that although insects tend to share common features such as the compound eye and basic olfaction ability (Ahmad, 1983), the extent to which each species uses each and how they behave in combination is difficult to test experimentally. Section 2.7 includes a selection of work relating to $P$. rapae.

Bukovinszky et al. (2005) used a simulation framework (described in Potting et al., 2005) to compare the foraging strategies of three species which corresponded to the three categories of search; Visual, Olfactory and Contact. This framework represents the landscape as a grid of squares where a cell may either contain a resource or not. The contact searcher detects a resource if it lands on square which contains a plant. The Visual searcher has a "window" within which it can detect plants, and the olfactory searcher detects plants within larger patches with a probability that is proportional to the size of the patch. Figure 2.5 is repeated from the paper for convenience and describes the basic setup. The species that were modelled are listed in Table 2.3. The model also contained a parameter called "arrestment" which was the probability of leaving a plant and was dependant on plant type. Host plants had a higher arrestment value than non-hosts or locations with no plant.

The results from Bukovinszky et al. (2005) suggest that the distribution of insects observed in the field could be attributed to the different search strategies of three 
species of insect. The density of contact searchers was independent of the patch size, because aphids tend to travel by being"sucked up" into in air columns and thus do not move actively between hosts. For visual searchers they observed a negative response to plant density and patch size. This was because the smaller patches had a greater perimeter-area ratio and so were encountered more often. This effect was enhanced with a greater movement speed (equivalent to longer step length). Fastmoving insects with good perceptual abilities should discover the smaller patches more often, demonstrating a resource dilution effect. For olfactory searchers, those with a high displacement speed and strong arrestment responded positively to the patch density and size, demonstrating a resource concentration effect. These results are similar to those obtained in this thesis (Chapter 3 ).

\section{Search Type Species}

$\begin{array}{ll}\text { Contact } & \text { Cabbage aphid (Brevicoryne brassicae) } \\ \text { Visual } & \text { Cabbage White (P.rapae L.) } \\ \text { Olfactory } & \text { Diamond-back moth (Plutella xylostella L.) }\end{array}$

Table 2.3: Species and search Type from Bukovinszky et al. (2005).

There is a wealth of disparate knowledge concerning sensory the modelling of sensory perception in insects. In following sub-sections, two examples of studies involving the senses are selected for closer inspection; one visual, the other olfactory.

\section{Visual search}

Roitberg (1985) measured the visual response distance of a female tetraphid fruit fly (Rhagoletis pomonella) in some detail. He notes that when the flies are searching within trees, their search mechanism is primarily visual, as evidenced by the success of visual fruit mimic traps and the observation that they expend an equal amount of searching effort in trees with no fruits as those with fruits. The response of flies to clusters of fruit placed at various distances was measured. A positive response was recorded if the fly oriented toward the fruit and flew straight towards it. From this they were able to determine the "reactive envelope" surrounding the fly. This was an elliptical area which was plotted on polar co-ordinate paper. They note four principles required to complete a model of insect foraging behaviour:

1. Host Distribution

2. Forager Movement 
(a)

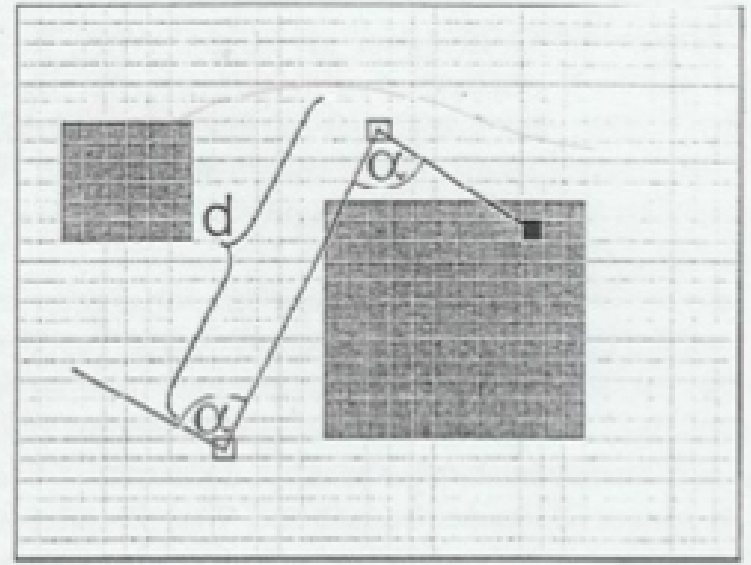

(b)

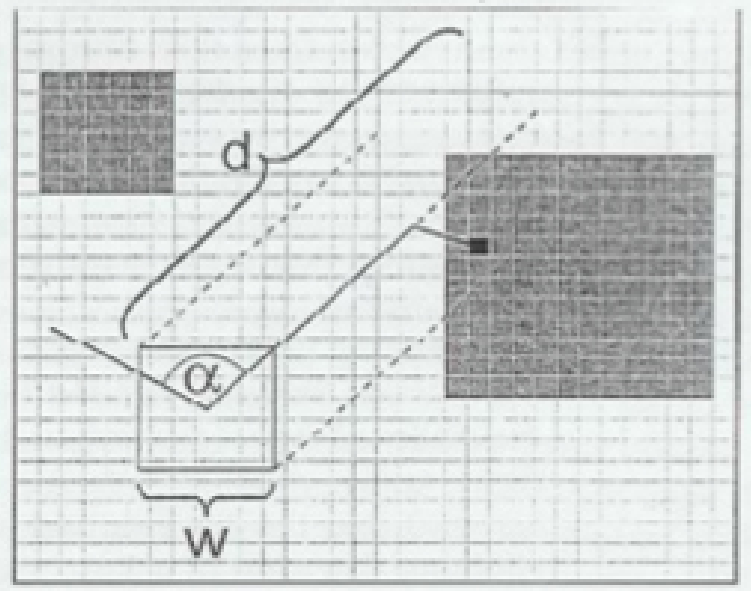

(c)

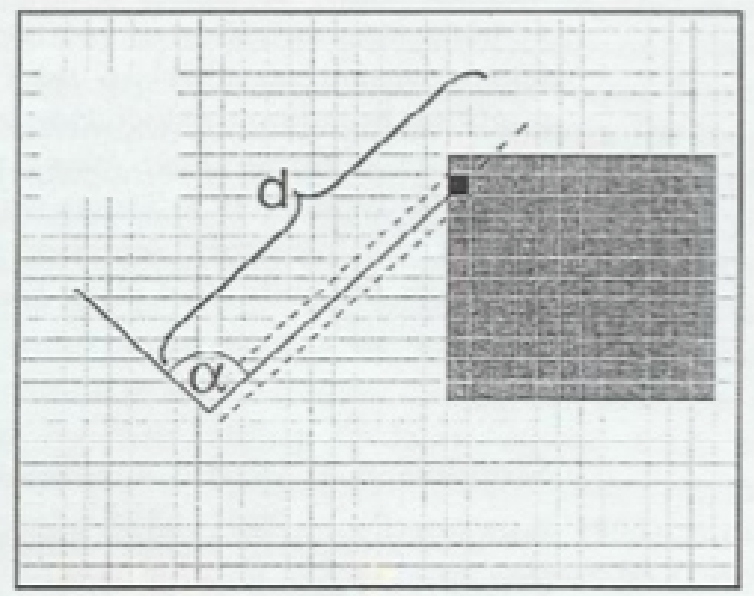

Fig. 3. Examples of the three foraging strategies used in the simulations. Grey patches indicate host plants. a-is the direction of the flight path between two successive random take-offs. $\mathrm{d}$-is the maximum distance covered in one hout chocen randomly between 0 and $\mathrm{d}$. (a) A "contact searcher" identifies its host plant upon landing. (b) A "visual seareher" is able to detect host plants in its flight path, where w is the width of scarch window. Cells in patclies of different sices were detected with the same probability. (c) An "olfactory searcher" that detects large patches with greater probability (dark grey) than small (light grey) ones proportional to their area.

Figure 2.5: From Bukovinszky et al. (2005) Figure 3 
3. Distance and angles at which resources are recognised

4. Information processing and retention abilities of the foragers

More information is becoming available about the learning abilities of insects (Bleeker et al., 2006; Kandori and Ohsaki, 1996; Lewis, 1986) and incorporating this into simulation studies would be interesting. Roitberg (1985) proceeded to incorporate the observations of reactive distance into a spatial foraging simulation. There were three "types" of forager in the simulation, shown in table 2.4. These were allowed to forage on two resource layouts with different levels of "clumping" or aggregation, "Low" and "High"

\begin{tabular}{llll}
\hline Type & Movement & Reactive Envelope & Encounter Behaviour \\
\hline Type I & Random Walk & Small ( $\mathrm{r}=1$ cell, symmetric $)$ & $\begin{array}{l}\text { Restricted to areas } \\
\text { surrounding prey for } 2 \\
\text { moves }\end{array}$ \\
\hline Type II & Random Walk & Large $(\mathrm{r}=2.25$ cells, symmetric $)$ & No response \\
\hline Type III & $\begin{array}{l}\text { Straight until } \\
\text { choose prey } \\
\text { containing cells } \\
\text { within search } \\
\text { radius }\end{array}$ & Large $(\mathrm{r}=2.25$ cells, symmetric $)$ & $\begin{array}{l}\text { Increase turning angle when } \\
\text { encounter prey } \\
\text { (klinokinetic) }\end{array}$ \\
\hline
\end{tabular}

Table 2.4: The forager types from Roitberg (1985)

The results showed that Type 3 foragers performed the best (across several measures) with Type 1 foragers the worst across both clumping levels. When Type 1 foragers were run with an increased reactive envelope size (1.5 times) they significantly increased their foraging performance. A similar response in efficiency is noted in Chapter 2. The variation in resource encounter rate was higher in the more clumped resource patches. This supports the concept that spatial distribution of resources influences forager search success, as well as prey density and forager movements.

Roitberg (1985) also concurs with Kareiva and Shigesada (1983) in suggesting that the foraging model might be represented as a first order Markovian process where previous events (i.e. position in a patch) determines probability of encountering prey in the future. For example, if on the edge of the patch and moving away from the centre, there is less probability of encountering a prey item in the next step than if on the edge and heading toward the centre.

Type II foragers did not respond to encountering a prey item (klinokinetic response). This could reduce the probability of re-encounter with prey items in the patch, which might be adaptive in the case of oviposition, preventing eggs being laid on the same 
plant twice and thus reducing competition between siblings. Roitberg (1985) notes that this would be particularly true if the prey are spread out and not in clumps. Root and Kareiva (1984) are cited as a demonstration of this kind of behaviour for P.rapae and the subject is discussed in more detail in Section 2.7.

Type III foragers are consistent with the idea introduced in Section 2.2.2 that in fact a straight line may be the most effective strategy for locating dispersed resources. In this case, the straight line moves are at the scale of the patch, helping to locate new patches and move between them, combined with an area restricted search once within the patch to maximise the potential of locating all resources in that patch. The resulting path might look similar to a Lévy flight and therefore be analysed as such, even though it is generated with a behavioural model that includes a response to the resources.

\section{Olfactory search}

Ralph $(1977 \mathrm{a}, \mathrm{b})$ investigated the search behaviour of the Large Milkweed Bug, Oncopeltus fasciatus (Hemiptera: Lygaeidae), noting that, in general, only a few species have been proven to use olfaction to locate their hosts. In the case of the Milkweed bug, the host plants are patchily distributed and hard to find in amongst non-host plants. In these papers it is demonstrated that the bugs can find the Milkweed plant even when hidden from view. Further, those released in larger patches remain there longer than when released in small patches, possibly due to the detection of an edge to the patch. This is an example of a Resource Concentration effect (Section 2.4). It is shown by the fact that the number of egg clutches in an area of observation correlated positively with Milkweed pod density.

In terms of the mechanics of odour dispersal in the atmosphere, Dusenbery (1989) created a mathematical model to find the optimum direction for searching for the source of odour in theoretical plumes of various shapes. He concludes that the optimum direction is to be downwind of the source for a spherical plume. However, as the active space elongates, the optimal course becomes that of a crosswind direction, although still somewhat downwind. A longer plume of a given volume has a greater apparency but increased wind speed does not necessarily increase the length of the plume. This is because the wind creates a turbulence which actually reduces the volume of the "active" area of the plume (the space in which the signal can be detected by the searcher).

In terms of modelling the odour plume itself, Hogan (2006) provides some background. The most simplistic model would assume that there is no wind and therefore the odour signal decays uniformly around the source as it disperses into the 
atmosphere.

\subsection{Study species: Pieris rapae, (Lepidoptera), the Small Cabbage White butterfly}

This review is primarily concerned with the consequences of movements associated with herbivorous insects. The insects in in question are the caterpillars of the small Cabbage White butterfly P. rapae (Lepidoptera) ${ }^{4}$. They are distributed by the female adult butterfly when she oviposits on cabbages (Muggeridge, 1942). The caterpillars themselves are unable to locate new hosts and so are reliant on the detection abilities and oviposition strategies of their mothers (Hern et al., 1996). Sensory cues eliciting or suppressing oviposition undoubtedly play a role in this process (Hern et al., 1996; Renwick and Chew, 1994).

Pieris rapae is well documented in the literature, an excellent summary of what is known is reported in detail in Hern et al. (1996). There is little point in repeating every detail here. However, a precis is provided below which highlights some interesting details relevant to the thesis, with information from other sources added when appropriate.

Pieris rapae is widely distributed throughout the northern hemisphere and Australasia (Ohsaki, 1979, 1980, 1982). Local populations may have between three (UK) and six (Missouri, Columbia) full generations a year. Adult females survive for approximately 19.8 days and males 23 days. The females may develop 450-500 eggs, 350 of which are laid (Richards, 1940). The caterpillars feed for five instars and overwinter as diapausing pupae (Jones, 1977). Pieris rapae flies only in moderate weather conditions. Nectar feeding and oviposition are restricted to sunny, warm $\left(18^{\circ}-20^{\circ}\right)$ periods with light to moderate winds. Most egg-laying occurs in the late morning or early afternoon (Root and Kareiva, 1984). Eggs are laid singly (Jones, 1977).

Pieris rapae is listed as a pest species more because the plants become contaminated with caterpillar frass (excretion) than because of actual damage to the leaves. They are an agriculturally significant pest which has driven much research into their biology (Hern et al., 1996).

\footnotetext{
${ }^{4}$ More correctly but less frequently referred to as Artogeia rapae $L$.
} 


\subsubsection{Oviposition behaviour}

Patterns of egg, and hence larval distribution are created by the oviposition behaviour of the females. This can be divided into several categories, shown in Table 2.5 (after Hern et al. 1996). These categories are grouped into behaviours which occur before the female lands on a plant, those that occur once landed on a suspected host, or at any point in the sequence.

\begin{tabular}{ll}
\hline Searching Phase & Behavioural Category \\
\hline Pre-Alighting & $\begin{array}{l}\text { Motivational State } \\
\text { Directionality } \\
\end{array}$ \\
& Visual Cues \\
& Olfactory Cues \\
\hline Post-Alighting & Contact Chemoreception \\
\hline Any & $\begin{array}{l}\text { Plant Condition } \\
\text { Any }\end{array}$ \\
Any & Oviposition deterrents \\
Any & Response to plant density \\
Any & Bearning From Prior Experience \\
\hline
\end{tabular}

Table 2.5: Pieris rapae behavioural categories from Hern et al. (1996).

Searching for resources is time consuming and prone to risk of predation (Bell, 1990). Host plant selection is therefore expected to be a complex process and subject to selection pressures (Hern et al., 1996).

Singer (1986) notes that observed larval distributions are a result of various effects, including:

- Initial adult distribution and density

- Oviposition preference and plant acceptability

- Egg survival patterns

- Larval movements, preference, performance and host plant suitability

- Differential larval predation

"Preference" as defined by Painter (1951) "is used to denote the group of plant characters and insect response that lead to or from the use of a particular plant or variety, for ovipo- 
sition, for food or shelter, or for a combination of the three. Anthropomorphic connotations should not be read into his terminology". Singer (1986) notes that "Preference can be used to refer to the behaviour of an insect towards parts of an individual plant, particular sets of plants of the same species, or towards a specified set of plant species".

\section{Motivational state}

The ovipositing behaviour of $P$. rapae may be influenced by the female's "Motivational State". For instance, if females are prevented from flying due to bad weather, the next sunny day available, they lay up to $78 \%$ more eggs (Gossard and Jones, 1977) and tend to lay these on the first avaialable plants, becoming less discriminating. As eggs are laid, the females return to being more discriminating and tend to move considerably more between laying bouts (Gossard and Jones, 1977; Jones, 1977; Root and Kareiva, 1984; Courtney et al., 1989; Finch and Collier, 2000). After a period prevented from flying, females passed over fewer available oviposition sites. In "normal" oviposition flight more than 40 collards were passed over with only 10 eggs laid. This was reduced to approximately 20 plants passed over and $15-20$ eggs laid after two days of bad weather ${ }^{5}$ (Root and Kareiva, 1984).

Tabashnik (1987) reports that as the time between oviposition increases, the female may accept less suitable or even non-host plants, the discrimination time in this setup seemed to be between 5 and 15 minutes. Hopkins and Van Loon (2001) showed by dissection that the number of mature eggs within a female's ovaries increased when females were kept on non-host plants.

\section{Directionality}

Root and Kareiva (1986) and (Jones, 1977) report that P. rapae tends to have a linear flight path. Jones et al. (1980) extends a small scale, detailed movement model, to measure and predict movement over multiple days. Individuals flew with sufficient directionality to remain within a $45^{\circ}$ angle over $1 \mathrm{~km}$. Each day, butterflies assumed a different direction, with the result that over many days, the pattern of movement was that of a Random Walk with a step length of approximately $450 \mathrm{~m}$. This was suggested to be a mechanism by which more ground could be covered. Fahrig and Paloheimo (1987) found that the residence time of a butterfly in a patch was 1.3 days and out of 102 butterflies marked in a patch containing 450 plants $^{6}, 74 \%$ of

\footnotetext{
${ }^{5}$ Values were estimated by sight from Figure 8 in Root and Kareiva (1984)

${ }^{6}$ They do not reference the exact dimensions of the patch, but a table is given for number of eggs laid where the patch size for 450 plants was $15 \times 15 \mathrm{~m}$, so it is likely that this is the size
} 
those butterflies were not found in the patch after 17 days. The dispersal rate of $P$. rapae is therefore high.

\section{Visual cues}

As described in Section 2.6.2, vision has been shown to be an important mechanism in host plant selection. Myers (1985) conducted experiments concerning the choice of plants and their physiological condition. The preferred plants had higher nitrogen and phosphorous content and higher transpiration rate and were greener in colour. "Greenness" was measured "using a Tectronix photometer fitted with filters to compare the reflectance of light in the red $(6750 \mathrm{~A})$ and infrared $(8000 \mathrm{~A})$ frequencies. The greener plants absorb more of the red wavelengths which increases the ratio of infrared to red reflectance" (Myers, 1985). An interesting note is that the linear flight patterns of $P$. rapae were modified if a suitable number of highly attractive plants are provided; butterflies remained to lay several eggs on these plants (Myers, 1985). The "motivational state" of the butterflies might also be influencing this observation.

Renwick and Radke (1988) have shown also that butterflies have a preference for "green". Hern et al. (1996) reports that while specific wavelengths of light have not been tested for $P$. rapae, $P$. brassicae have been tested for the effects on drumming (see "Contact chemoreception") and oviposition. These responses are elicited in the range $497 \mathrm{~nm}$ to $578 \mathrm{~nm}$, with the greatest stimulation at the wavelength of $548 \mathrm{~nm}$ (Kolb and Scherer, 1982). Higher light intensities increased oviposition response, but not for white light. Unpublished results are referred to in Renwick and Radke (1988) suggesting a preference in $P$. rapae for leaves having reflectance at $550 \mathrm{~nm}$.

Visual cues certainly play an important role in detection and acceptance of host plants, however it is likely that a combination of other cues, olfactory and particularly chemoreception are combined to locate the host plant (Bernays and Chapman, 1994).

\section{Olfactory cues}

"The volatile compounds emanating from plants are thought to play a major role in he orientation of insects to their host plants and in the avoidance of unsuitable plants. However, very few reports of the specific chemicals responsible for the attraction gravid female insects exist" (Hern et al., 1996). Renwick and Radke (1988) note that there is no evidence to suggest that volatile chemicals are involved in the orientation of P. rapae to the host plants. Hern et al. (1996) mentions that there is circumstantial evidence for a role in Pierids. Renwick and Radke (1988) have shown, that macerated cabbages may 
deter oviposition, the theory being that it is an inhibitory response to an already occupied plant. Takabayashi et al. (1998) suggest that the parasitoid Cotesia glomerata responds to artificially damaged plants. So there may be two adaptive reasons for avoiding host plant already infested with larvae, firstly overcrowding resulting in lack of food, and secondly parasitoid avoidance. Non-host volatiles can also deter females from cabbage plants according to Tabashnik (1987).

Fahrig and Paloheimo (1987) conclude that it is "unlikely that females can use olfactory detection to find host plants", but "Although (Traynier, 1979) concluded that vision alone could account for host plant location, he warned against concluding that olfaction plays no part in host location by P. rapae". Hern et al. (1996) conclude that evidence exists only for olfaction acting as an inhibitor and not as an attractor, but that $P$. rapae is able to perceive odours and that they are sensitive to Crucifer-specific compounds, and therefore it is likely to play some role. They state that this is the "...major knowledge gap in the ovipositional behaviour of P. rapae" (Hern et al., 1996). A search for articles citing Hern et al. (1996) reveals few subsequent investigations for P. rapae. Langan et al. $(2004,2001)$ conducted some experiments concerning gas exchange and transpiration rates and found no evidence for a direct increase in oviposition response but a general observation that plants with higher physiological activity received more eggs. Bruinsma et al. (2007) show that a hormone treatment (Jasmonic Acid) inhibits oviposition. They note that it is again an indirect effect and likely to be the result of changes to the plant after treatment rather than the hormone itself. No references citing Hern et al. (1996) were found specifically relating to olfaction.

\section{Contact chemoreception}

Once a potential host plant has been located, and the female has landed on a leaf, "drumming" (rapid moment of forelegs across the surface of the leaf) occurs. As most Brassica plant leaves are covered with a waxy cuticle, it is possible that the drumming dislodges wax crystals and allows the polar (chemically active for olfaction) stimulants necessary for oviposition to be released. The drumming may also provide physical information about the leaf (Renwick and Radke, 1988). The ovipositor is not used for assessing chemical cues on the leaf, rather tarsal chemoreception is the main source of information about the suitability of the host plant (Traynier, 1979). The detailed chemistry is covered in both Hern et al. (1996) and Traynier (1979), but the involvement of glucinosinolates, particularly glucobrassicin and sinigrin is noted. Renwick and Radke (1988) also report a population difference in response between American and Australian butterflies, with Australian butterflies often laying eggs on substrates which simply had water on them. 
Finch and Collier (2000) propose an interesting model for search behaviour, which they call the theory of "Appropriate / Inappropriate landings". It is exemplified by the cabbage root fly (Delia radicum), although seven test species are discussed. In their theory, the insects fly about searching for hosts and may be arrested by odour in the air, but the main attraction is visual, notably a preference for green objects, and a distaste for landing on brown areas. Thus they will land on host or non-hosts if they are green at which point contact chemoreception is used to discern the suitability of the host for oviposition. They note that $P$. rapae has been observed by Jones (1977) to make contact with several leaves, interspersed with short flights before laying an egg. These observations concur with the observations of (Hasenbank, in prep) during the field work associated with this thesis. In some cases, the orientation of the leaf also appeared to play a part, some leaves providing a convenient perch as the butterflies hang from their fore limbs and oviposit.

\section{Plant condition}

Myers (1985) observed that plants with high nitrogen and phosphorous content were preferred. Similar observations by Ives (1978) and Jones (1977) showed some discrimination based on leaf and plant size and age. Medium sized leaves are preferred, with younger leaves chosen if they are the same size. Scriber (1984) conducted a survey of the literature to find that $70 \%$ of studies indicated that increased plant nitrogen led to increased insect damage, growth, fecundity or abundance. Wolfson (1980) also reports a clear preference for fertilised (high nitrogen) plants. On plants given low nitrogen fertiliser dose, the choice of leaves changed from fully expanded middle aged leaves to the younger leaves being peferred, and recieving over $25 \%$ of the eggs. Letourneau and Fox (1989) report that leaf nitrogen concentration accounted for $90 \%$ of the variation in oviposition rate. As Myers (1985) notes plants with higher transipiration rates were also attractive, consistent with the findings of Langan et al. $(2004,2001)$ concerning gas exchange rates previously noted.

There does not seem to be a definitive causal explanation available for these observations although the association between plant condition and greenness is noted (Hern et al., 1996). Possibly the infochemicals released are also related to the plant condition in terms of processes of production of these being affected by available nitrogen and healthy transpiration.

\section{Oviposition deterrents}

Chemical inhibitors of oviposition certainly seem to play a role in the selection of host plants (Hern et al., 1996; Renwick, 2002). Tabashnik (1987) studied the effects of 
the non-host phyto-chemicals coumarin and rutin. Both these chemicals affected the pre-alighting rather than post-alighting response. Coumarin is thought to be mostly olfactory, whereas rutin is a non-volatile pigment and is thought to alter the visual stimuli emanating from the plant making it less attractive. Hern et al. (1996) note that the effect of the deterrent does not completely mask the general attractiveness of host plants, simply making them less acceptable, which in turn could be overridden by a high motivation to oviposit.

Renwick and Radke $(1985,1983)$ showed that macerated cabbage, extract of cabbage leaves and non-polar extracts (hexane) applied to acceptable cruciferous plants deterred oviposition by $P$. rapae. Hern et al. (1996) treat this topic in more depth, but conclude that ultimately the host acceptability is a balance of positive and negative stimuli from the plants. They also report that P. rapae is able to detect its own oviposition deterring pheromone, but that this is of minor importance in the overall host acceptance decision.

\section{Response to resource density}

The egg (and hence larval) distributions of P. rapae are observed to follow the general Resource Dilution pattern (Section 2.4), with eggs "spread" over a large area, laid singly (Jones, 1977; Root and Kareiva, 1984; Yamamura, 1999; Cromartie, 1975). The behaviour which leads to this distribution is one of high visual acuity (Hern et al., 1996), directional movements, tending to lay a single egg and showing no klinokinetic response (i.e. increased turning) to plant density. (Jones, 1977; Root and Kareiva, 1984). Root and Kareiva (1984) did observe a shortening of step length in response to host density, but this was not sufficient to counteract the dilution effect of many more plants (increased density of $400 \%$ resulted in move length decreasing by $20 \%$ ). A contrast is given with the nectar-feeding of the females, where increase turning was observed (Area Restricted Searching), females visiting on average $73 \%$ of inflorescences per patch as compared to $33 \%$ of host plants whilst ovipositing (Root and Kareiva, 1984). A key factor in Jones (1977)'s model was that the butterflies return to isolated plants if no others are found in the vicinity (from Jones, pers. comm.). Combined with the fact that the butterflies appear to be good at "spotting" the resources as they fly past, this leads to a natural increase in the number of eggs on isolated plants, and a sheltering effect (Cain, 1985) recieved by the cabbages in denser groups. This response is a significant departure from the behaviour of most other butterflies (Hern et al., 1996).

Hern et al. (1996) suggests a simple "rule of thumb" which might produce the negative response to plant density, based on the fact that $P$. rapae has only a limited number of eggs per day and that a patch is likely to contain more hosts than the 
female has eggs available. It involves a "motivational threshold" which determines whether the butterfly will lay an egg. If the acceptance level of the plants in the current patch are below this threshold, an egg will be laid. Once an egg is laid, the butterfly may become more discriminating and thus if she has few eggs left to lay, will move on from this patch (unless a particularly juicy cabbage is spotted). Presumably, the motivational threshold drops over time as more eggs are produced which leads to a return to ovipositing.

Mangel (1987) reports that egg clutch size may also vary with host distribution. When a host was more likely to be found, clutch sizes fell compared to when there were fewer hosts around, which is similar to the idea of "Motivational state" mentioned previously. This would also lead to more eggs on isolated plants in the same way that the parameter "MISS" from the model of Jones (1977) causes returns to plants (Section 2.8).

Shapiro (1970) Showed that females being harassed by males become more dispersive and directional, although this is not mentioned elsewhere as a potential influence on the patterns of movement it might be a factor in highly dense population such as intensive agricultural plots.

The evolutionary costs to of this searching behaviour are an increase in energy used for flying as well as increased amount of time spent flying (which could be spent on other activities) and an increased risk of predation (Hern et al., 1996; Heinrich, 1979a; Ohsaki and Sato, 1990; Ohsaki, 1986; Yamamura and Yano, 1999). Dempster (1983) shows that both predation and failing to lay a full complement of eggs are the two most important factors limiting butterfly populations.

There are two main suggestions for the evolutionary development of this strategy to "spread" eggs. The first, the "risk spreading" concept has been introduced in Section 2.4. Ohsaki and Sato (1994) also suggest that this strategy may be used to avoid parasitoids.

Secondly, Jones and Ives (1979) suggest that the differences observed between populations of Australian and Canadian butterflies in terms of clustering may be due to population-level selection. Individuals may in fact be behaving sub-optimally (i.e. increased cost in flying time and predation). Less aggregated egg clusters may be selected for because any increase in aggregation of larvae can lead to heavy infestations and the decimation of the host population. The high dispersal ability of the Cabbage Whites, laying of single eggs and less clustering may all be selected for in this manner (Jones, 1987b; Jones et al., 1982, 1987; Jones, 1987a; Jones and Ives, 1979). 


\section{Learning from past experience}

Hern et al. (1996) complete their review with mention of the learning capacities of insects, which are noted as another under-developed area with regards to $P$ rapae. Traynier $(1986,1984)$ and Kandori and Ohsaki (1998) have shown learning abilities in P. rapae. Traynier and Truscott (1991) showed that $P$. rapae associated the colour of a disc with the presence of host phytochemicals (sinigrin or glucobrassicin), but that they did not associate leaf shape characteristics with the presence of sinigrin, and were unable to learn to associate a negative stimulus with colour (Traynier, 1987). Pieris rapae has been shown to use learning to increase efficiency when foraging for nectar (Lewis, 1989, 1986) and so, as Hern et al. (1996) conclude, it is entirely possible that the mechanism is used for host plant location, and that it is likely to be used to "...improve future searching efficiency leading to a higher rate of encounter with host plants" Hern et al. (1996).

\section{Behavioural Plasticity}

Jones (1977) notes the variation in movement parameters and aggregation of eggs between Australian, UK and Canadian butterfly populations. It would appear that there is therefore a fair degree of plasticity in the behavioural traits of $P$. rapae which can be acted upon by selection and allow it to adapt to different environments. As mentioned, Jones' subsequent work (Jones et al., 1980) involved a study of the kinds of adaptation and behavioural selection which might occur in P. rapae, the summary of which is that larval density-dependant mortality has a strong impact on the population dynamics and is therefore likely to influence the selection of butterflies which exhibit more or less turning in their movements. Factors such as the number of flying hours and days were also important, for example the weather was more consistent in Australia, allowing more flying time and thus decreasing the associated cost, potentially allowing more directionality and less aggregation of eggs (Jones, 1987b; Jones et al., 1982, 1987; Jones, 1987a; Jones and Ives, 1979)

Recently $P$. rapae has been studied in the context of global climate change. One consequence of global climate warming is an increase in nocturnal temperatures. Whitney-Johnson et al. (2005) studied the effect of these on pupal development and found that although there were significant variations. On average, warmer nocturnal temperatures elicited a faster development rate associated with a decreased pupal mass. The conclusion is that the populations would multiply more quickly under this regime. This could have consequences in terms of selection pressure acting on the populations. Jones (1987b) tested the effects of pupal development on flight behaviour and found that increased development rate of the pupa or of its 
mother led to more eggs being laid per alighting. Different populations responded oppositely in terms of flight time, with Australians spending more time in flight than UK females with increased development rate. This demonstrates the wide potential for the effects of warming events, with the population distribution dependant entirely on the variable and adaptive oviposition behaviour.

\subsubsection{Trophic interactions}

This thesis focuses on the foraging behaviour of Pieris rapae. As Hern et al. (1996) note, the interactions between the larva and their predators and parasitoids may have a selection impact upon the searching behaviour of the females and so the ultimate distribution of individuals. The two main parasitoids of $P$. rapae are the wasps Cotesia glomerata and Cotesia rubelica. These parasitoids are known to use olfactory cues to orient toward the prey, and may respond to volatiles released by the larvae of $P$. rapae as they eat the foliage of the cabbages. (Ohsaki and Sato, 1999, 1990; Sato and Ohsaki, 2004; Takabayashi et al., 1998; Tanaka and Ohsaki, 2006; Jones, 1987a; Horikoshi et al., 1997; Tenhumberg et al., 2001; Yamamura, 2002). It is also possible that they drive the adaptiveness of the egg spreading behaviour observed in P. rapae.

\subsection{Case study: Jones (1977)}

Jones (1977) authored what is perhaps the seminal modelling paper concerning Pieris rapae, which has been cited many times (e.g.Turchin, 1989). Jones (1977) made several measurements of flight behaviour and then created a detailed behavioural model based on these parameters. Due to various observational biases, for some parameters, values were systematically adjusted until the best fit to the observed data was produced. These "trained" parameter set-ups were then tested against separate observations that were not included in the "training".

The paper contains a wealth of information and the experimental setup is reasonably complex, comprising two locations and various field layouts and plant types. It reports a series experiments which measure not only flight behaviour but also host preference in terms of species and life history. The information below has been extracted and arranged so as to provide an overview of the relevant measurements made. 


\subsubsection{Experimental resource layout}

Experiments were carried out in two locations, Canada (Vancouver) and Australia (Canberra). Here the two locations are referred to simply as either Canada (CAN) and Australia (AUS). The patch layouts consisted of 4 structures:

J1. Uniform Grid

J2. Clumped Grid

J3. Linear Array

J4. Commercial Plot

A visual representation of the experimental layouts is given in figure 2.6. The uniform grid consisted of plants aligned on the nodes of a grid layout with various spacings between plants $(1 m, 2 m, 4 m$ and $10 m)$. The clumped grid had groups of 4 plants with $1 \mathrm{~m}$ between each plant and $3 \mathrm{~m}$ between groups. The linear array had plants arranged in a line, with groups of either 3 (Canada) or 4 (Australia) plants with $20 \mathrm{~m}$ between groups. The spacing between plants in each group is not recorded. The commercial plot is detailed in terms of overall size, and the plants are described as "closely spaced". There were three such plots.

\subsubsection{Plant types}

In addition to the various plant layouts, different species of Crucifer were used; Cabbage, Kale, Raddish and Cauliflower, and various plant ages and sizes within these. The kale plants used in the Canadian experiments were categorised into 5 categories of size and age (Table 2.6). No description of how these plants were distributed in the layout is given. The Cauliflowers were categorised as large or small and the cabbages were all categorised as large.

In the Canadian experiments the Kale plants had cut flowers placed in their pots to provide feeding sources. Although no comment is made about this, it may be have influenced the results as Root and Kareiva (1984) have observed that the patterns of movement differ when females are ovipositing compared to when foraging for nectar.

\subsubsection{Experiments}

Table 2.7 contains a summary of experiments which were conducted at each location and which layouts and host species were used. Experiments (a), (c) and (e) were 
a) Uniform Grid Layout

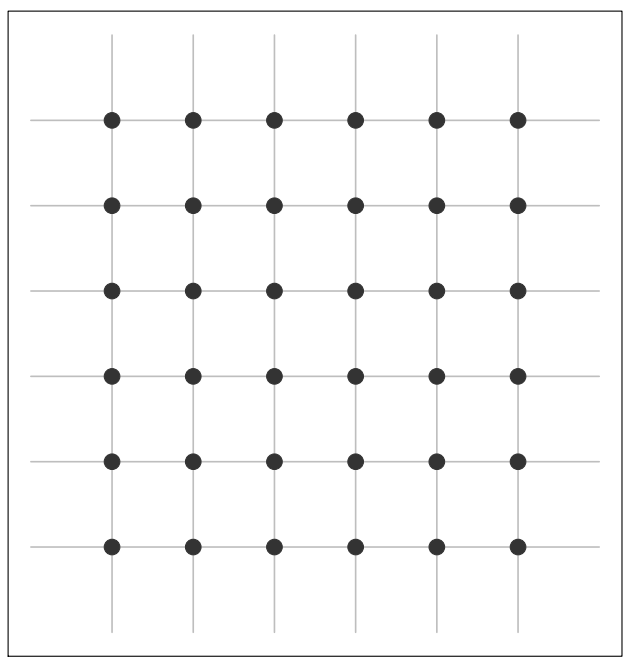

$1,2,4$ or $10 m$ b) Clumped Grid Layout

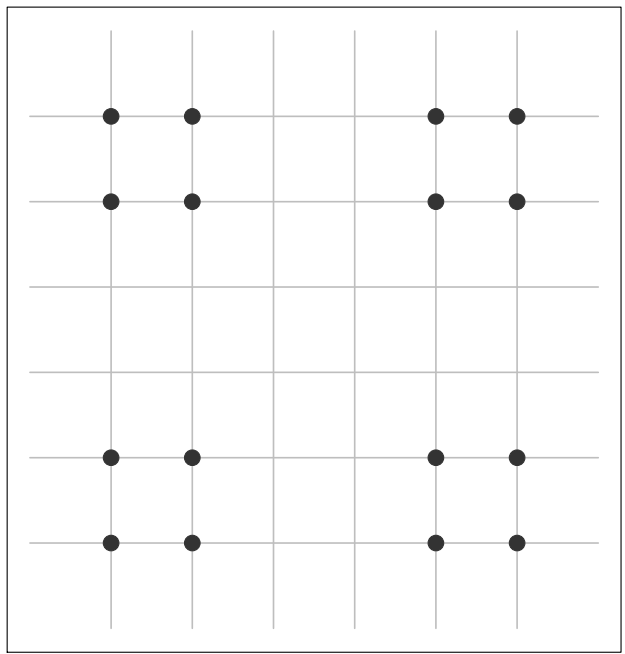

c) Linear Layout

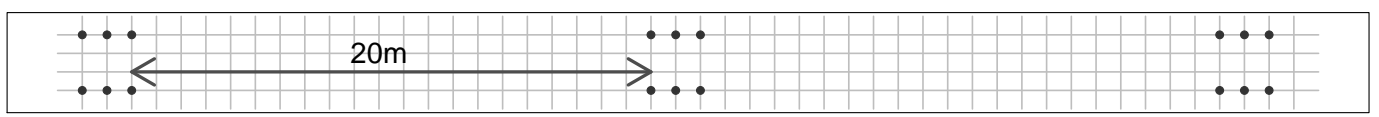

Figure 2.6: Graphical representation of Jones' experimental layout types. The Commercial layout type is not shown as there is no information about how the plants were arranged. Layout (a) is shown with the $1 \mathrm{~m}$ spacing. The same arrangement but with larger spacings between plants was also used at $1 \mathrm{~m}, 2 \mathrm{~m} 4 \mathrm{~m}$ and $10 \mathrm{~m}$. For layout (c) there are not enough details for an exact representation, however this is my interpretation of the description in the text. These are provided to give a sense of the spatial arrangements, the exact dimension and number of plants are not recorded

used to parameterise the model. Experiments (b) and (f) were used to compare the results of the model experiments. Experiments (g), (h) and (i) were used for plant preferences. The $10 \mathrm{~m}$ spacings were used to estimate the effect of fecundity on flight parameters.

\subsubsection{Field observations}

The butterflies were in the main wild butterflies flying over the experimental areas. However, in the Canadian experiments, reared butterflies were used but the results merged with those of the wild butterflies as they were "indistinguishable from each other" (Jones, 1977).

Three types of move were recorded: 


\section{Unit move}

2. Multiple move

3. Zero move

The plant to plant movements of the butterflies were recorded as Cartesian Coordinates. A Unit move is where the flight distance is less than $1.5 \mathrm{~m}$ (diagonal distance between plants at the $1 \mathrm{~m}$ spacing). A Multiple move is where the flight distance was greater than this. A Zero move was when the butterfly left a plant, flew around and then returned to the same plant. The length of this move was not recorded. The observations resulted in two response variables:

1. Mean move length

2. Directionality $r$ as from (Batschelet, 1981) (see Section 2.5)

Two measures of directionality are given, $r($ dir $)$ and $r($ turn $)$. Both are measures of the concentration of angles about a mean, as described in Batschelet (1981) and Section 2.5 .

$\mathbf{r}(\operatorname{dir})$

$r($ dir $)$ relates to the "preferred direction" of a population of butterflies. For example, if all butterflies observed flew in straight lines across the observation area toward the North East, $r$ (dir) would be close to 1 . If each butterfly flew in a different direction then $r(\operatorname{dir})$ would be close to 0 . This directionality is measured by taking the Azimuth (Section 2.5, e.g. N.E., N.N.W., S., etc.) of each step in the recorded path aggregated across all butterflies in the population. As noted in Jones et al. (1980) in Australia, where the butterflies were more directional, each butterfly had a different

\begin{tabular}{lrrr}
\hline Type & $\begin{array}{r}\text { Mean no. } \\
\text { leaves per } \\
\text { plant }\end{array}$ & $\begin{array}{r}\text { Mean leaf } \\
\text { length (cm) }\end{array}$ & $\begin{array}{r}\text { Weeks since } \\
\text { seedling } \\
\text { transplanted }\end{array}$ \\
\hline (1) Large old & 15.4 & 17.6 & $9-14$ \\
(2) Medium old & 10.4 & 8.4 & $9-14$ \\
(3) Medium medium & 9.3 & 9.2 & $6-10$ \\
(4) Medium young & 9.5 & 10.0 & $4-8$ \\
(5) Small very young & 5.1 & 4.3 & less than 4 \\
\hline
\end{tabular}

Table 2.6: Categorisation of kale plant types from Jones (1977) 


\begin{tabular}{lccccc}
\hline Layout & Spacing AUS CAN $\begin{array}{c}\text { Number Of } \\
\text { Plants }\end{array}$ & Patch Size & Plant Species \\
\hline (a) Uniform Grid & $1 \mathrm{~m}$ & $\checkmark$ & $\checkmark$ & - & $-\begin{array}{l}\text { Cabbage } \\
\text { (AUS),Kale (CAN) }\end{array}$ \\
(b) Clumped Grid & $3(1) \mathrm{m}$ & $\checkmark$ & $\checkmark$ & - & - Cabbage (AUS), \\
Kale (CAN)
\end{tabular}

Table 2.7: Summary of experimental observations carried out by (Jones, 1977). AUS = Australia, $C A N=$ Canada. For $(a)$ and $(b)$ no sizes are given but the total plot size in AUS was $20 \times 40 \mathrm{~m}$ so the patches could not be larger than this, it might be assumed that 3 patches of $20 \times 20$ plants were planted. For both $(b)$ and $(e)$ there are groups of plants and so the spacing values indicate the spacing between groups with the spacing between plants in brackets. For (e) the spacing between plants was not specified but for the purposes of the graphical illustration it has been taken to be $1 \mathrm{~m}$ as would appear consistent with (b). For experiment (d) it is not mentioned how the different species were distributed. For ( $f$ ) the description of spacing is "closely spaced" which has been taken here to mean less than $1 \mathrm{~m}$. Experiment $(g)$ contained 54 plants at $1 \mathrm{~m}$ spacing, no information is given as to the exact layout. For experiments $(h, i)$, the overall size of each patch is not given, so the exact layout is unknown. In a later table (Jones 1977 Table 7), the numbers of these cabbages are reported as 9 for cabbage and 36 each for Kale and Raddish this is unexplained but is possibly due to having 2 replicates for the latter at both spacings. 
preferred direction which would suggest the low values for $r($ dir $)$ which are seen in Table 2.8. The preferred direction was more variable for the Australian butterflies and less variable for Canadians.

The $r$ (dir) shown is for the population as a whole; if the values were calculated for individual tracks they were generally above 0.4 and sometimes close to 1 for the Australian butterflies, highlighting the directionality. For the Canadians there was little difference between individual tracks and the population as a whole (Jones, pers. comm).

r(turn)

$r($ turn) is estimated from the angle of turn at each step (Section 2.5). This is then the relative change in Azimuth between successive steps $(\Delta \theta)$. It will produce a distribution centered about a mean of $0^{\circ}$ (which represents straight line movement, no turning to left or right). As for $r($ dir $)$, the values shown are aggregated across all the individual paths observed. It is noticeable that $r($ turn $)$ is generally higher than $r($ dir $)$, particularly for the Australian butterflies.

$r($ turn $)$ is also transformed by multiplying by the cosine of the mean angle turned. As the mean angle turned varies from $0^{\circ}$ to $180^{\circ}$, so the cosine of this will vary from 1 to -1 . This was implemented because sometimes females flew in a "zigzag" pattern backwards and forwards across the path. This movement is averaged out with the standard calculation but adjusted with this transformation so that those "zig-zagging" will have a value of -1 and those truly moving in a highly directional manner will be 1 .

In terms of possible values for $r(d i r)$ and $r($ turn $)$ and their relationship, if all butterflies flew in straight lines but in different, random directions, $r($ turn $)$ would be 1 but $r$ (dir) would be 0 . There is a relationship between the two measures in that as $r$ (turn) decreases, the butterflies become less directional and therefore it becomes impossible to have a high $r($ dir $)$. In this way, $r($ turn $)$ is a limiting constraint upon $r($ dir $)$.

Several other measurements are used to parameterise the simulation and these are discussed with each parameter below.

\subsubsection{Results from field data}

Jones (1977) paper contains many gems regarding the observations that were made on both the flight behaviour and egg distributions in the experimental setups, sum- 
marised below.

\section{Flight patterns}

Table 2.8 summarises the general parameters observed in the flight observations. This table provides a summary of more general movement parameters which may be applicable to other modelling techniques such as a Random Walk (Section 2.6.1).

\section{Directionality}

Australian butterflies are observed to be considerably more directional using both measurements of directionality (Table 2.8). The $r$ (dir) directionality is a mean across each butterfly flight observed. The fact that the average $r(\operatorname{dir})$ is higher than 0 implies that there was some tendency to have a preferred direction. This is later backed up in (Jones et al., 1980) where again measurements were taken in a commercial patch and this time a graph (Jones' Figure 7) is shown with the distribution of preferred directions on each day, indicating that they do not maintain a preferred direction over the day. This directionality was only observed in the Australian population. This is the population studied in Jones et al. (1980), in which butterflies were followed for more than one day and the observations demonstrate that if their dispersal is greater than would be expected without directionality.

\section{Plant preferences}

Experiments (g), (h), and (i) (Table 2.7) were run over two seasons enabling an overall count of eggs to be made. These are then reported in Jones' table 7 (Summarised here in Table 2.9). It is seen that for kale, younger plants are preferred to older plants, and that stunted (transplanted later) older plants are least preferred. Between species, the preferred order of species (descending) is Cabbage, Kale, Raddish. No statistical comparison is reported, although standard errors are noted and the differences lie well beyond these.

\section{Plant spacing}

Here Jones reports that at higher densities (closer spacing) there are fewer eggs per plant than at lower densities (wider spacing). This is consistent with other results (Cain, 1985; Yamamura, 1999). 


\begin{tabular}{lccccccr}
\hline & \multicolumn{3}{c}{ Australian } & & \multicolumn{3}{c}{ Canadian } \\
\cline { 2 - 3 } \cline { 5 - 7 } Layout & Move & r(dir) & r(turn) & & Move & r(dir) & r(turn) \\
\hline Uniform 1m spacing & 1.39 & 0.28 & 0.36 & 1.76 & 0.15 & 0.11 \\
Uniform 4m spacing & 4.80 & 0.26 & 0.53 & - & - & - \\
Clumped 3(1)m & 2.45 & 0.19 & 0.33 & 2.25 & 0.08 & 0.22 \\
Commercial Large Plant & 2.52 & 0.16 & 0.49 & - & - & - \\
Commercial Small Plant & 4.08 & 0.21 & 0.66 & - & - & - \\
Linear 20(1)m & 7.70 & 0.13 & 0.10 & 5.04 & 0.10 & 0.05 \\
\hline
\end{tabular}

Table 2.8: Table of results from Jones (1977). The results are aggregated for ease of comparison, showing the directionality and mean, non-zero (i.e. did not return to same plant) move length. Move lengths are in metres.

\begin{tabular}{lrrrrr}
\hline & \multicolumn{5}{c}{ Experimental Layout } \\
\cline { 2 - 6 } Plant Species & $\begin{array}{r}\text { Uniform } \\
\text { 1m }\end{array}$ & $\begin{array}{r}\text { Clumped } \\
\mathbf{3 ( 1 ) \mathrm { m }}\end{array}$ & $\begin{array}{r}\text { Commercial } \\
\text { Cauliflower 20m }\end{array}$ & $\begin{array}{r}\text { Linear 20m } \\
\text { (CAU) }\end{array}$ \\
Cauliflower small & - & - & 0.28 & - & - \\
Kale Large old (1) & - & - & 0.42 & - & - \\
Kale Medium old (2) & 0.16 & 0.18 & - & - & - \\
Kale Medium medium (3) & 0.14 & 0.13 & - & - & - \\
Kale Medium young(4) & 0.25 & 0.15 & - & - & - \\
Kale Small v.young (5) & 0.44 & 0.41 & - & - & - \\
Cabbage & 0.58 & 0.64 & - & - & 0.68 \\
Kale & - & - & - & 1.00 & 0.49 \\
Raddish & - & - & - & 0.50 & 0.39 \\
\hline
\end{tabular}

Table 2.9: Mean number of eggs according to plant species and experimental layout (summarised from Jones, 1977). Although it is mentioned that Brussel sprouts were also used in the Australian $20 \mathrm{~m}$ linear layout, the eggs per visit for these are not recorded. No indication of size is given for the Cabbage, Radish and second Kale results. 


\subsubsection{Simulation construction and parameters}

Figure 2.7 reproduces the flow diagram from Jones (1977). The parameters are highlighted. Table 2.10 summarises the values for the parameters and their purpose which is described below.

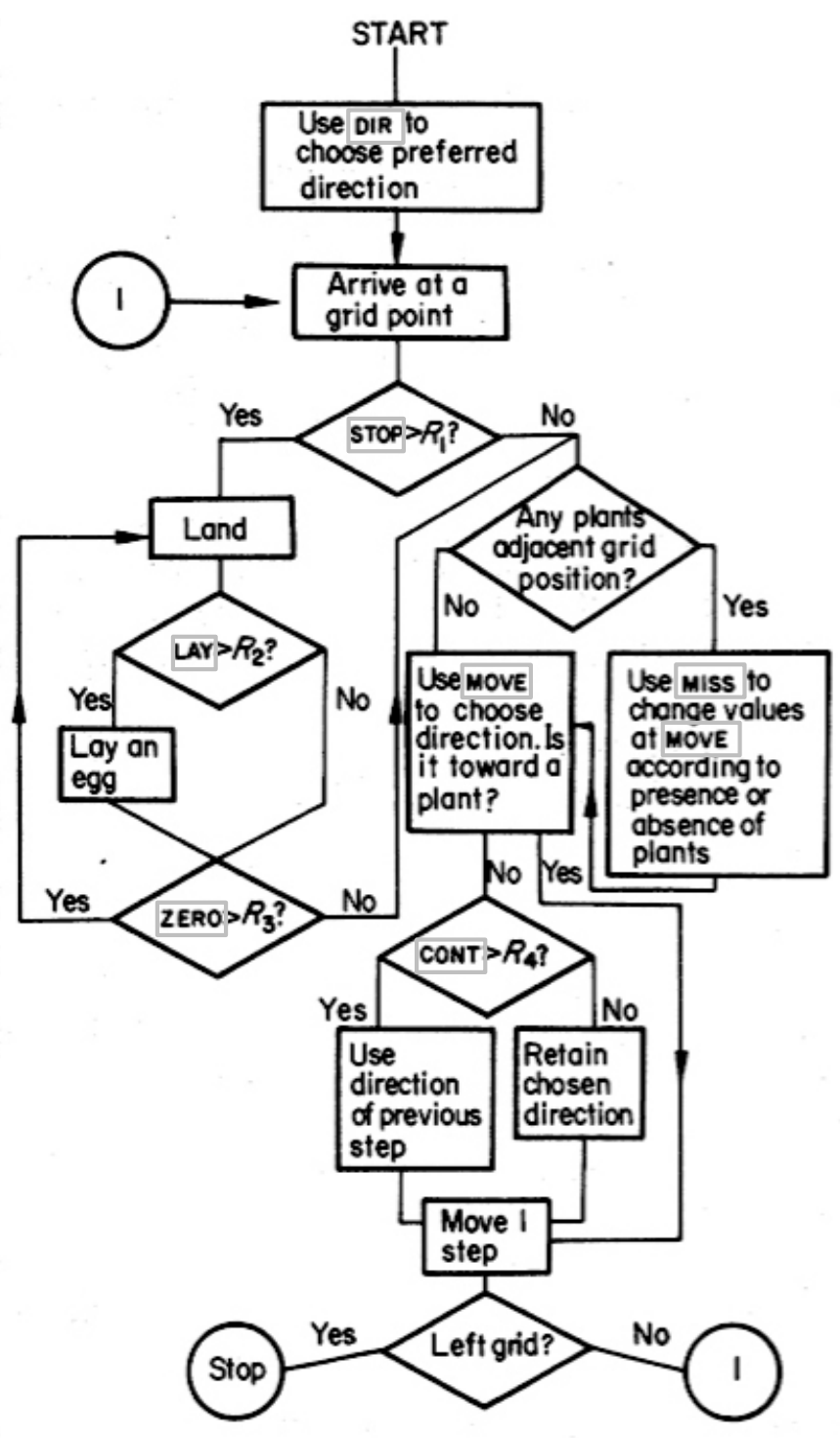

Figure 2.7: From Jones (1977, Figure 3). Flow diagram of the simulation model. Original caption reads "Flow chart of flight and egg-laying simulation model. $R_{1-4}$ are random numbers selected from a uniform distribution between 0 and 1 . Other symbols are described in the text."

\section{DIR}

DIR was used to chose a preferred direction for the butterfly. It has 4 possible values, corresponding to the closest of the four directions it is possible to take between the nodes on the grid. DIR has only four possible directions whereas MOVE may have 


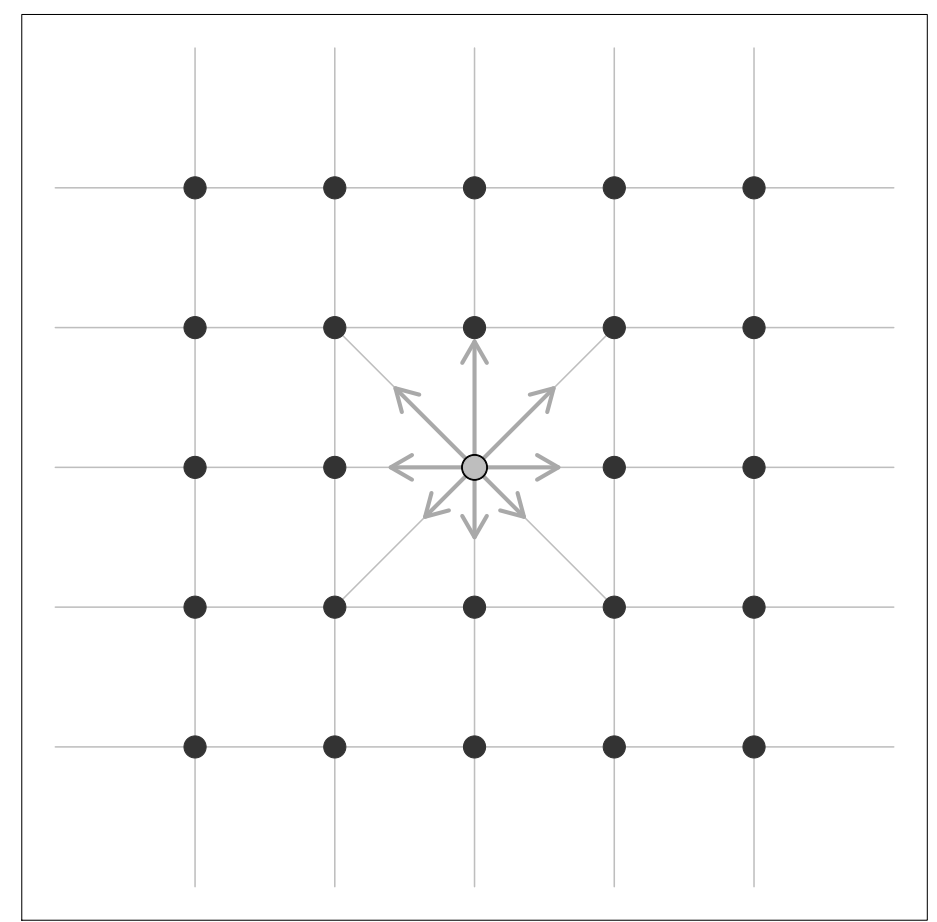

Figure 2.8: Graphical representation of Jones' MOVE parameter. The arrows represent the probability of moving in a particular direction. The longer the arrow the higher the probability. The most probable direction of movement in this case is "North" towards the top of the page. The calculation of the probabilities is described in the text.

8. DIR is used at the start of each butterfly's path to chose that butterfly's preferred direction. It is fixed for each butterfly and is decided by the frequency of mean directions observed in the $1 \mathrm{~m}$ spacing observations.

DIR represents the variation in preferred direction amongst the population of butterflies.

\section{MOVE}

Once the preferred direction is chosen, a probability distribution for all of the 8 possible moves (Figure 2.8) is created, $\operatorname{MOVE}(1)$ to $\operatorname{MOVE}(8) . \operatorname{MOVE}(1)$ is set to the the preferred direction. The frequency of moves in each direction for the $1 \mathrm{~m}$ data was used to estimate this. From the field observations it appears that Australian butterflies had a variable preferred direction but tended to stick to that direction when moving, whereas the Canadian butterflies seemed to go more in the same direction but move around more randomly.

MOVE represents the variation in angle of turn for an individual path. 


\begin{tabular}{|c|c|c|c|c|c|}
\hline Parameter & Definition & Dependent On & Australian & \multicolumn{2}{|c|}{ Canadian } \\
\hline \multicolumn{6}{|l|}{ Movement } \\
\hline DIR & Preferred Direction & & $\begin{array}{l}\text { Variable: } \\
\operatorname{DIR}(1)<0.45\end{array}$ & \multicolumn{2}{|c|}{$\begin{array}{l}\text { Less Variable: } \\
\operatorname{DIR}(1)>0.7\end{array}$} \\
\hline MOVE & Directionality & & $\begin{array}{l}\text { High: } \\
\text { MOVE(1)=0.6 }\end{array}$ & \multicolumn{2}{|c|}{ Low $\operatorname{MOVE}(1)=0.3$} \\
\hline MISS* & $\begin{array}{l}\text { Responsiveness to } \\
\text { host plants }\end{array}$ & Butterfly fecundity & $\begin{array}{l}\text { Less: } \\
1-0.03 x\end{array}$ & \multicolumn{2}{|l|}{$\begin{array}{l}\text { More: } \\
1-0.07 x\end{array}$} \\
\hline CONT $*$ & $\begin{array}{l}\text { Probability of } \\
\text { maintaining a } \\
\text { flight direction in } \\
\text { the absence of } \\
\text { hosts }\end{array}$ & & \multicolumn{3}{|c|}{ Similar $(0.6-0.8)$} \\
\hline STOP $*$ & $\begin{array}{l}\text { Probability of } \\
\text { landing }\end{array}$ & $\begin{array}{l}\text { Plant species, size, } \\
\text { age, butterfly } \\
\text { fecundity }\end{array}$ & 0.015 & \multicolumn{2}{|c|}{$0.012+0.003 x$} \\
\hline \multicolumn{6}{|l|}{ Egg Laying } \\
\hline LAY & $\begin{array}{l}\text { Probability of } \\
\text { laying an egg } \\
\text { during a visit }\end{array}$ & Plant species, age & \multicolumn{3}{|c|}{ Similar ( $0.7-0.9$ cabbage, 0.5 young kale) } \\
\hline ZERO $*$ & $\begin{array}{l}\text { Probability of } \\
\text { returning to host } \\
\text { without } \\
\text { intervening flight }\end{array}$ & $\begin{array}{l}\text { Plant species, size, } \\
\text { age }\end{array}$ & $\begin{array}{l}\text { Low: } \\
0.2-0.3\end{array}$ & Higher: & 0.5 \\
\hline
\end{tabular}

Table 2.10: Table of simulation parameters from (Jones, 1977). $x$ is the number of eggs the butterfly has available to lay. STOP values were the same for large host plants (0.65- 0.75 for cabbage) and then were modified to those shown after contact with a host. ZERO values are show for cabbage plants. Parameters in dark grey and marked with a * were "tuned" to the field data from the calibration experiments. 


\section{MISS}

MISS was a parameter which allows for attraction towards host plants. If some of the potential moves are towards a host, and some are not, the values of MOVE are altered to represent an attraction. This is achieved by examining each of the directions in turn. If this direction does NOT contain a plant, the probability of MOVE in that direction is multiplied by the value of MISS $(0 \leqslant$ MISS $\leqslant 1)$ and then they are all rescaled so that they sum to 1 . In this way the probability of moving into an empty space is reduced dependant on the value of MISS.

MISS can be interpreted as a representation of the "Motivational State" of the butterfly (Section 2.7.1), or as Jones' describes it, the "Fecundity" of the butterflies. Initially this parameter was set at a constant value but was later estimated by trying a sequence of values until a close fit to the data was obtained. This was then tested against a separate set of field observations which had not been used in the parameter estimation.

\section{CONT}

CONT was a representation of the "Correlation" of the random walk, or the probability of maintaining the current direction, in the absence of hosts. It is implemented in the model as the probability that the butterfly will continue in the current direction rather than select a new direction at random, based on the constraints of MOVE and MISS. Thus if CONT is high, the agent will hardly ever select a new direction at random and will move almost in straight lines. If it is low, it is more likely to move around towards host plants and then return to its preferred direction in their absence.

The step length in the model is always $1 \mathrm{~m}$, the CONT parameter was estimated using a series of values until it produced results that matched the $4 \mathrm{~m}$ plant spacing field observations.

\section{STOP}

STOP was the probability of landing at a given node in the grid. Different points on the grid may have different probabilities of "arrestment" depending on which plant types are there. This parameter allowed for the variations observed in plant species and condition. It was parameterised from the $1 \mathrm{~m}$ host plant patterns by calculating the ratio of unit moves/(unit moves + multiple moves), which is estimating the proportion of moves which resulted in a landing (the plant spacing is $1 \mathrm{~m}$ and a unit move is a move from one plant to another). 
The observation of this ratio is biased by the observations, in that if a butterfly flies $1 m$ west and then $2 m$ east, it would be recorded as a unit move, when in fact it had moved $3 \mathrm{~m}$. The parameter was adjusted in subsequent runs of the simulation until a good fit to the data was obtained.

\section{LAY}

LAY was the probability that a butterfly, having stopped at a node will lay an egg. It was estimated by taking total eggs laid/total visits for each plant type. It represents the "acceptability" of the host in terms of the characteristics discussed in section 2.7.1. It was highest for Cabbage, lower for Kale and lowest for radish. It was not affected by plant size, but decreased linearly with plant age.

\section{ZERO}

ZERO was the probability that a female will spend the next move on the same cabbage. This represents the situation where a female hops from one leaf to another, allowing more probability to lay an egg on that plant, potentially multiple eggs. This was estimated by the ratio of zero moves/total moves. The estimate is biased, because it could also represent the "zero moves" that were observed when females flew away from a plant and then back again to the same plant without stopping in between. So this parameter too was adjusted to fit the training data.

\subsubsection{Simulation results}

Here the results that were obtained by simulation are compared with those discovered from the field.

\section{Flight patterns}

The $1 \mathrm{~m}$ and $4 \mathrm{~m}$ uniform grid observations were used to parameterise the model and the $4 m$ grouped setup to check the results. The $20 m$ linear model was used for some of the parameterisation. No data is presented from the $10 \mathrm{~m}$ uniform grid observations.

The Canadian observations match closely those that were used to parameterise them (i.e. $1 \mathrm{~m}$ and grouped $3(1) \mathrm{m}$ ). For the Australian measurements in the commercial patch, the simulation appears to overestimate the directionality of the butterflies as the plants are more closely spaced and denser. Perhaps the simulation is not as 
responsive to the plant density as the real butterflies. The Australian simulation trials which most closely resemble the field structure $(1 \mathrm{~m}, 3(1) \mathrm{m}$ clumped and $4 \mathrm{~m})$, the simulation results closely mimics the field observations.

\section{Plant preferences}

In respect of the host species preference, the model produces a consistent pattern to the observed preferences, i.e. Cabbage, Kale, Radish in descending order. The model seems to exaggerate the preference for cabbage, with a greater (0.63) proportion than the observed (0.49). With the kale, the model seems to prefer older kale than younger which is the opposite effect to the field observations. A summary of the relevant observations is found in Table 2.11

\begin{tabular}{lcr}
\hline & \multicolumn{2}{c}{ Proportion eggs on each type } \\
\cline { 2 - 3 } Plant Species & Observed & Simulated \\
\hline May-June 1973 & & \\
Young Kale & 0.48 & 0.40 \\
Old Kale & 0.37 & 0.46 \\
Stunted Kale & 0.15 & 0.14 \\
\hline June-August 1975 & & 0.63 \\
Cabbage & 0.49 & 0.28 \\
Kale & 0.40 & 0.09 \\
Radish & 0.11 & \\
\hline
\end{tabular}

Table 2.11: Summary of observed and simulated host preferences from (Jones, 1977) table 7. Young Kale was 4-8 weeks old. Old Kale was 9-14 weeks old. Both were transplanted at 4 weeks, the stunted kale was 9-14 weeks old and transplanted at 8 weeks.

\section{Plant spacing}

The simulation showed a similar pattern to the observed ratios in that the $2 m$ spacing (lower density) showed fewer eggs per plant (Table2.12). This is consistent with (Yamamura, 1999) and others (see Sections 2.4 and 2.6.1). 


\begin{tabular}{lcccr}
\hline & \multicolumn{2}{c}{ Observed } & \multicolumn{2}{c}{ Simulated } \\
\cline { 2 - 3 } Spacing & Kale & Radish & & \\
\hline $\mathbf{2 m}$ & 0.68 & 0.57 & 0.61 \\
$\mathbf{1 m}$ & 0.32 & 0.43 & 0.39 \\
\hline Total Eggs & 171 & 63 & - \\
\hline
\end{tabular}

Table 2.12: Summary of observed and simulated ratios of eggs per plant for different plant spacings (Jones, 1977)

\subsubsection{Case study summary}

This is a pioneering paper. The process of breaking down the behaviour of the insects and attempting to measure so many aspects of their flight behaviour has to my knowledge, not been repeated for an other insect. The work also provides a wealth of information about the ovipositing behaviour of Prapae which, in its own right is valuable. Perhaps the greatest contribution then is that this simulation goes where few have been in terms of detail. It provides a centre-piece around which much of the other literature on the subject revolves.

It would be an interesting project to replicate the model and compare its output with more general, Random Walk models, as well as providing it with novel resource layouts which have been used for field observations.

Jones (1977) concludes with several points:

- Parameters concerned with landing and laying eggs depended on host plant quality (age and species of plant).

- The simulations correctly predict observed behaviour patterns.

- Canadian butterflies disperse less, and produce a more aggregated egg distribution and have a more efficient search at lower prey densities than their Australian Cousins.

- "More eggs are laid on isolated plants than on plants in small groups, and more on plants in small groups than on plants in large groups" Jones (1977).

- "butterfly density tends to vary inversely with host density" Jones (1977), even though the butterflies increase their response to plants as they become more "fecund", i.e. have more eggs to lay. 
The final two points are particularly relevant to this thesis in that they re-iterate the theme of less dense, more isolated plants receiving relatively more eggs per plant. The explanation proposed is that butterflies are able to visit the same plant again (i.e. the plant does not die after being "parasitised") and thus in the absence of more plants in the vicinity, the butterfly will tend to return to the single plant to lay more eggs, as opposed to continuing onward. It is also noted that this revisiting behaviour might lead to an "edge effect" at the edge of larger patches and that the model demonstrated this. This is a crucial finding of the paper and is interesting because it suggests that the parameter "ZERO" or chance of returning to a plant would be the most significant in producing the observed responses.

Other parameters such as directionality (CONT) vary the distribution as a whole, with the more directional Australian butterflies producing less aggregated egg distributions "at the cost of longer flight paths" Jones (1977). Canadian butterflies cover less distance but lay more aggregated distributions.

\subsection{Review summary}

The field of animal movement ecology is wide ranging. This review has touched on many papers involved, and has hopefully provided a starting point for further investigation as well as a reasonable background for the subsequent chapters of this thesis.

Animal movement and its ecological consequences are a key topic in the study of ecological systems. The scale at which individual animals operate and at which the consequences of their behaviour are measured can strongly influence the patterns that are observed.

The quantification of animal paths is a reasonably standardised process. The field of circular statistics allows for measurements and probability distributions that provide a complete description and analysis of directional data. Animal paths may be described in terms of a sequence of straight line moves, measured by the length of each "Step" and the Angle of turn between successive steps which relates usefully to the mathematical model of a Random Walk. These methods have been used in a number of situations (Wiens et al., 1993; Schtickzelle et al., 2007; Mardia, 1972; Turchin et al., 1991; Turchin, 1998).

Simulation of animal movement is strongly based around the theoretical concept of the Random walk. This conceptual model has been used successfully to describe at least aggregated distributions of individuals and may be extended into more formal population dynamics models. It has been applied to a number of study species 
(Bartumeus et al., 2005; Byers, 1996; Cain, 1985; Kareiva and Shigesada, 1983; Marsh and Jones, 1988; Turchin, 1989; Winkelman and Vinyard, 1991; Zalucki and Kitching, 1982).

While the real world animal may not move at random, the population level effects of the movements of many individuals may often be approximated well by that model (Turchin, 1998). This is particularly the case when resources in which the animal is interested are themselves distributed at random. The patterns created by an animal, although behaving deterministically will be strongly influenced by the structure of the landscape in which it moves and the scale at which it perceives those structures.

Detailed behavioural models are less common. One with particular relevance to this thesis was explored in detail in the form of Jones (1977)'s model of Pieris rapae oviposition behaviour. This model is relevant because it highlights areas where parameters above and beyond the simple model of the Random Walk are required in order to produce detail in egg distribution patterns at the small scale.

Animals no doubt use their senses to locate food, prey or reproductive sites and avoid danger. To what extent these senses operate at a detailed level to produce observed patterns of behaviour is difficult to quantify. It may be possible to incorporate a general model of "attraction" into the basic model of the Random Walk to allow for the influence of senses and adaptive behaviour, and thus allow for these responses in more general population models.

Pieris rapae is primarily a visual searcher (Hern et al., 1996). Two simulation methods for representing vision have been reviewed; that of Cain (1985)'s "radius of attraction" and that of Bukovinszky et al. (2005) and Roitberg (1985), in which a "field of view" or "reactive envelope" is used. The difference in representation is that the "field of view" is centered on the individual rather than the resource. This may influence the results of a simulation, particularly concerning the effects of resources at the edge of a patch, where the radius of attraction can act as a barrier, preventing foragers from passing through to resources behind (See Chapter 3).

The Cabbage White butterfly (Pieris rapae) is well studied and there is a wealth of material detailing its biology. It provides a fantastic research species for investigating interactions between its movements and one of its host species, the Cabbage (Brassicae oleracae). 


\section{References}

Agostinelli, C. and U. Lund, 2006: CircStats R package version 0.2-1. Internet.

Ahmad, S., editor, 1983: Herbivorous Insects: Host Seeking Behaviour and mechanisms. Academic Press, New York.

Bach, C. E., 1980: Effects of plant density and diversity on the population dynamics of a specialist herbivore, the striped cucumber beetle, Acalymma vittata (Fab.). Ecology, 61(6), 1515-1530.

Bach, C. E., 1988: Effects of host plant patch size on herbivore density: Underlying mechanisms. Ecology, 69(4), 1103-1117.

Baker, R., 1978: The Evolutionary Ecology of Animal Migration. Hodder and Stoughton, London.

Banks, J. E., 1998: The scale of landscape fragmentation affects herbivore response to vegetation heterogeneity. Oecologia, 117(1-2), 239-246.

Barron, A. and M. Sirnivasan, 2006: Visual regulation of ground speed and headwind compensation in freely flying honey bees (Apis melifera L.). Journal of Experimental Biology, 209, 978-984.

Barrows, E. M., W. J. Bell, and C. D. Michener, 1975: Individual odor differences and their social functions in insects. Proceedings Of The National Academy Of Sciences Of The United States Of America, 72(7), 2824-2828.

Bartumeus, F., M. G. E. Da Luz, G. M. Viswanathan, and J. Catalan, 2005: Animal search strategies: A quantitative. random-walk analysis. Ecology, 86(11), 3078-3087.

Batschelet, E., 1981: Circular Statistics In Biology. Mathematics in Biology. Academic Press, San Fransisco.

Begon, M., J. Harper, and C. Townsend, 1990: Ecology: Individuals, Populations and Communities. Blackwell Scientific Publishing.

Bell, W., 1991: Searching Behaviour: The behavioural ecology of finding resources. Chapman and Hall, London.

Bell, W. J., 1985: Sources of information controlling motor patterns in arthropod local search orientation. Journal Of Insect Physiology, 31(11), 837-847.

Bell, W. J., 1990: Searching behavior patterns in insects. Annual Review Of Entomology, 35, 447-467.

Benhamou, S., 2004: On the expected net displacement of animals' random movements. Ecological Modelling, 171(1-2), 207-208.

Berg, H., 1993: Random Walks in Biology. Princeton University Press.

Bernays, E. and R. Chapman, 1994: Host plant selection by phytophagous insects. Chapman and Hall, London.

Bleeker, M. A. K., H. M. Smid, J. L. M. Steidle, H. M. Kruidhof, J. J. A. Van Loon, and L. E. M. Vet, 2006: Differences in memory dynamics between two closely related parasitoid wasp species. Animal Behaviour, 71, 1343-1350.

Boccignone, G. and M. Ferraro, 2004: Modelling gaze shift as a constrained random walk. Physica A-Statistical Mechanics And Its Applications, 331(1-2), 207-218. 
Bolker, B. and S. W. Pacala, 1997: Using moment equations to understand stochastically driven spatial pattern formation in ecological systems. Theoretical Population Biology, 52(3), 179-197.

Bovet, P. and S. Benhamou, 1988: Spatial-analysis of animals movements using a correlated randomwalk model. Journal Of Theoretical Biology, 131(4), 419-433.

Box, G. and N. Draper, 1987: "Essentially, all models are wrong, but some are useful.". In Empirical Model-Building and Response Surfaces. Wiley and Sons, New York, 424.

Brower, L. P., L. S. Fink, and P. Walford, 2006: Fueling the fall migration of the monarch butterfly. Integrative and Comparative Biology, 46(6), 1123-1142.

Bruinsma, M., N. M. Van Dam, J. J. A. Van Loon, and M. Dicke, 2007: Jasmonic acid-induced changes in Brassica oleracea affect oviposition preference of two specialist herbivores. Journal Of Chemical Ecology, 33(4), 655-668.

Bukovinszky, T., R. P. J. Potting, Y. Clough, J. C. van Lenteren, and L. E. M. Vet, 2005: The role of preand post-alighting detection mechanisms in the responses to patch size by specialist herbivores. Journal of Chemical Ecology, 109(3), 435-446.

Byers, J. A., 1996: An encounter rate model of bark beetle populations searching at random for susceptible host trees. Ecological Modelling, 91(1-3), 57-66.

Byers, J. A., 2001: Correlated random walk equations of animal dispersal resolved by simulation. Ecology, 82(6), 1680-1690.

Cain, M. L., 1985: Random search by herbivorous insects - a simulation-model. Ecology, 66(3), 876888.

Cain, M. L., 1989: The analysis of angular data in ecological field studies. Ecology, 70(5), 1540-1543.

Castillo-Chavez, C., S. Levin, and F. Gould, 1988: Physiological and behavioural adaptations to varying environments: A mathematical model. Evolution, 42(5), 986-994.

Chechkin, A. and V. Gonchar, 1999: A model for ordinary levy motion. From the internet somewhere.

Conradt, L., E. J. Bodsworth, T. J. Roper, and C. D. Thomas, 2000: Non-random dispersal in the butterfly Maniola jurtina: implications for metapopulation models. Proceedings Of The Royal Society Of London Series B-Biological Sciences, 267(1452), 1505-1510.

Conradt, L., T. J. Roper, and C. D. Thomas, 2001: Dispersal behaviour of individuals in metapopulations of two british butterflies. Oikos, 95(3), 416-424.

Courtney, S. P., 1983: Models of hostplant location by butterflies - the effect of search images and search efficiency. Oecologia, 59(2-3), 317-321.

Courtney, S. P., 1986: Why insects move between host patches - some comments on risk-spreading. Oikos, 47(1), 112-114.

Courtney, S. P., G. K. Chen, and A. Gardner, 1989: A general-model for individual host selection. Oikos, 55(1), 55-65.

Cressman, R., V. Krivan, and J. Garay, 2004: Ideal free distributions, evolutionary games, and population dynamics in multiple-species environments. American Naturalist, 164(4), 473-489.

Cromartie, W. J., 1975: The effect of stand size and vegetational background on the colonization of cruciferous plants by herbivorous insects. The Journal of Applied Ecology, 12(2), 517-533. 
Cronin, J. T., 2003: Matrix heterogeneity and host-parasitoid interactions in space. Ecology, 84(6), 1506-1516.

Cuddington, K. and A. Hastings, 2004: Invasive engineers. Ecological Modelling, 178(3-4), 335-347.

Dempster, J., 1983: The natural control of populations of butterflies and moths. Boilogical Review, 58, 461-481.

Dethier, V. and E. Stellar, 1964: Animal Behaviour. Prentic-Hall Ltd, London.

Dicke, M. and P. Burrough, 1988: Using fractal dimensions for characterizing tortuosity of animal trails. Physiological Entomology, 13, 393-398.

Dusenbery, D. B., 1989: Optimal search direction for an animal flying or swimming in a wind or current. Journal Of Chemical Ecology, 15(11), 2511-2519.

Eason, G. and C. Coles, 1989: Mathematics and Statistics for the Bio-Sciences. Ellis Horwood Ltd, Chichester.

Einstein, A., 1905: The motion of elements suspended in static liquids as claimed in the molecular kinetic theory of heat. Annalen Der Physik, 17(8), 549-560.

Fahrig, L. and J. Paloheimo, 1988: Effect of spatial arrangement of habitat pathces on local population size. Ecology, 69(2), 468-475.

Fahrig, L. and J. E. Paloheimo, 1987: Interpatch dispersal of the cabbage butterfly. Canadian Journal Of Zoology-Reoue Canadienne De Zoologie, 65(3), 616-622.

Farnsworth, K. D. and J. A. Beecham, 1999: How do grazers achieve their distribution? a continuum of models from random diffusion to the ideal free distribution using biased random walks. American Naturalist, 153(5), 509-526.

Finch, S. and R. H. Collier, 2000: Host-plant selection by insects - a theory based on 'appropriate/inappropriate landings' by pest insects of cruciferous plants. Entomologia Experimentalis Et Applicata, 96(2), 91-102.

Gossard, T. W. and R. E. Jones, 1977: Effects of age and weather on egg-laying in Pieris rapae (L.). Journal Of Applied Ecology, 14(1), 65-71.

Gratton, C. and S. C. Welter, 1999: Does "enemy-free space" exist? experimental host shifts of an herbivorous fly. Ecology, 80(3), 773-785.

Griffin, D. R., 1944: The sensory basis of bird navigation. Quarterly Review Of Biology, 19(1), 15-31.

Grove, A., G. Newell, J. Carthy, and E. Mercer, 1966: Animal Biology. University Tutorial Press, London.

Grunbaum, D., 2000: Advection-diffusion equations for internal state-mediated random walks. Siam Journal On Applied Mathematics, 61(1), 43-73.

Hamback, P. and G. Englund, 2005: Patch area, population density and the scaling of migration rates: the resource concentration hypothesis revisited. Ecology Letters, 8, 1057-1065.

Hanski, I. and Gilpin, M., editors, 1996: Metapopulation Biology: Ecology, Genetics, and Evolution. Academic Press, USA.

Hartley, S. and W. E. Kunin, 2003: Scale dependency of rarity, extinction risk, and conservation priority. Conservation Biology, 17(6), 1559-1570. 
Hartley, S., W. E. Kunin, J. J. Lennon, and M. J. O. Pocock, 2004: Coherence and discontinuity in the scaling of species' distribution patterns. Proceedings of the Royal Society of London Series B-Biological Sciences, 271(1534), 81-88.

Hasenbank, M., in preparation: Phd thesis. Victoria University (Wellington, New Zealand).

Hassell, M. P. and T. R. E. Southwood, 1978: Foraging strategies of insects. Annual Review Of Ecology And Systematics, 9, 75-98.

Hastings, A., K. Cuddington, K. F. Davies, C. J. Dugaw, S. Elmendorf, A. Freestone, S. Harrison, M. Holland, J. Lambrinos, U. Malvadkar, B. A. Melbourne, K. Moore, C. Taylor, and D. Thomson, 2005: The spatial spread of invasions: new developments in theory and evidence. Ecology Letters, 8(1), 91-101.

Hastings, H.M., S. G., 1993: Fractals: A Users Guide for the natural sciences. Oxford University Press, Oxford

Heinrich, B., 1979a: Foraging strategies of caterpillars - leaf damage and possible predator avoidance strategies. Oecologia, 42(3), 325-337.

Heinrich, B., 1979b: Resource heterogeneity and patterns of movement in foraging bumblebees. Oecologia, 40(3), 235-245.

Hern, A., G. EdwardsJones, and R. G. McKinlay, 1996: A review of the pre-oviposition behaviour of the small cabbage white butterfly, Pieris rapae (Lepidoptera: Pieridae). Annals of Applied Biology, 128(2), 349-371.

Hoffmann, G., 1983a: The random elements in the systematic search behavior of the desert Isopod Hemilepistus reaumuri. Behavioral Ecology And Sociobiology, 13(2), 81-92.

Hoffmann, G., 1983b: The search behavior of the desert Isopod Hemilepistus reaumuri as compared with a systematic search. Behavioral Ecology And Sociobiology, 13(2), 93-106.

Hogan, W., 2006: Atmospheric dispersion modelling. In Biosurveillance in Handbook of Biosurveillance, Wagner, M., editor. Elsevier Academic Press, 289-299.

Holland, J. D., D. G. Bert, and L. Fahrig, 2004: Determining the spatial scale of species' response to habitat. Bioscience, 54(3), 227-233.

Holmes, E. E., M. A. Lewis, J. E. Banks, and R. R. Veit, 1994: Partial-differential equations in ecology - spatial interactions and population-dynamics. Ecology, 75(1), 17-29.

Hong, S. and D. J. Mladenoff, 1999: Spatially explicit and stochastic simulation of forest landscape fire disturbance and succession. Ecology, 80(1), 81-99.

Hopkins, R. J. and J. J. A. Van Loon, 2001: The effect of host acceptability on oviposition and egg accumulation by the small white butterfly, Pieris rapae. Physiological Entomology, 26(2), 149-157.

Horikoshi, M., J. Takabayashi, S. Yano, R. Yamaoka, N. Ohsaki, and Y. Sato, 1997: Cotesia glomerata female wasps use fatty acids from plant-herbivore complex in host searching. Journal of Chemical Ecology, 23(6), 1505-1515.

Ives, P. M., 1978: How discriminating are cabbage butterflies? Austral Ecology, 3(3), 261-276.

Jander, R., 1975: Ecological aspects of spatial orientation. Annual Review Of Ecology And Systematics, 6, 171-188. 
Johnson, A. R., B. T. Milne, and J. A. Wiens, 1992: Diffusion in fractal landscapes - simulations and experimental studies of tenebrionid beetle movements. Ecology, 73(6), 1968-1983.

Johnson, A. R., J. A. Wiens, B. T. Milne, and T. O. Crist, 1992: Animal movements and populationdynamics in heterogeneous landscapes. Landscape Ecology, 7(1), 63-75.

Jones, R. and P. Ives, 1979: The adaptiveness of searching and host selection behaviour in Pieris rapae (L.). Australian Journal of Ecology, 4, 75-86.

Jones, R. E., 1977: Movement patterns and egg distribution in cabbage butterflies. The Journal of Animal Ecology, 46(1), 195-212.

Jones, R. E., 1987a: Ants, parasatoids, and the cabbage butterfly Pieris rapae. The Journal of Animal Ecology, 56(3), 739-749.

Jones, R. E., 1987b: Behavioral evolution in the cabbage butterfly (Pieris rapae). Oecologia, 72(1), 69-76.

Jones, R. E., N. Gilbert, M. Guppy, and V. Nealis, 1980: Long-distance movement of Pieris rapae. Journal Of Animal Ecology, 49(2), 629-642.

Jones, R. E., J. R. Hart, and G. D. Bull, 1982: Temperature, size and egg-production in the cabbage butterfly, Pieris rapae (L.). Australian Journal Of Zoology, 30(2), 223-232.

Jones, R. E., V. G. Nealis, P. M. Ives, and E. Scheermeyer, 1987: Seasonal and spatial variation in juvenile survival of the cabbage butterly Pieris rapae - evidence for patchy density-dependence. Journal Of Animal Ecology, 56(3), 723-737.

Judson, O. P., 1994: The rise of the individual-based model in ecology. Trends In Ecology \& Evolution, 9(1), 9-14.

Kandori, I. and N. Ohsaki, 1996: The learning abilities of the white cabbage butterfly, Pieris rapae, foraging for flowers. Researches on Population Ecology, 38(1), 111-117.

Kandori, I. and N. Ohsaki, 1998: Effect of experience on foraging behavior towards artificial nectar guide in the cabbage butterfly, Pieris rapae crucivora (Lepidoptera: Pieridae). Applied Entomology and Zoology, 33(1), 35-42.

Kareiva, P., 1990: Population dynamics in spatially complex environments: theory and data. Philosophical Transactions of the Royal Society of London Series B-Biological Sciences, 330, 175-190.

Kareiva, P., 1994: Space - the final frontier for ecological theory. Ecology, 75(1), 1-1.

Kareiva, P. and G. Odell, 1987: Swarms of predators exhibit preytaxis if individual predators use area-restricted search. American Naturalist, 130(2), 233-270.

Kareiva, P. M. and N. Shigesada, 1983: Analyzing insect movement as a correlated random-walk. Oecologia, 56(2-3), 234-238.

Kaye, B., 1989: A random walkthrough fractal dimensions. VCH Publishers, New York, VCH P ublishers Verlagsgesellschaft, Weinheim (Germany), Basel (Switzerland);Cambridge;New York.

Keasar, T., A. Shmida, and U. Motro, 1996: Innate movement rules in foraging bees: Flight distances are affected by recent rewards and are correlated with choice of flower type. Behavioral Ecology And Sociobiology, 39(6), 381-388.

Kenkel, N. and D. Walker, 1993: Fractals and ecology. Abstracta Botanica, 17 (1-2), 53-70.

Kenkel, N. and D. Walker, 1996: Fractals in the biological sciences. Coenoses, 11, 77-100. 
King, A. W. and K. A. With, 2002: Dispersal success on spatially structured landscapes: when do spatial pattern and dispersal behavior really matter? Ecological Modelling, 147(1), 23-39.

Kolb, G. and C. Scherer, 1982: Experiments on wavelength specific behaviour of Pieris brassicae L. during drumming and egg laying. Journal of comparative Physiology A, 149, 325-332.

Krebs, J., 1978: Optimal Foraging: decision rules for predators. Blackwell Scientific Publications, Oxford, 23-63.

Krebs, J. and A. Kacelnik, 1991: Decision-Making. Blackwell Scientific Publishing, Oxford, 105-136.

Kunin, W. E., 1999: Patterns of herbivore incidence on experimental arrays and field populations of ragwort, Senecio jacobaea. Oikos, 84(3), 515-525.

Langan, A. M., C. P. Wheater, and P. J. Dunleavy, 2004: Biogenic gradients of $\mathrm{CO} 2$ and $\mathrm{H} 2 \mathrm{O}$ and oviposition by the small white butterfly (Pieris rapae L.) in cages. Applied Entomology And Zoology, 39(1), 55-+.

Langan, A. M., C. P. Wheater, and P. L. Dunleavy, 2001: Does the small white butterfly (Pieris rapae L.) aggregate eggs on plants with greater gas exchange activity? Journal of Insect Behavior, 14(4), $459-468$.

Law, R. and U. Dieckmann, 2000: A dynamical system for neighborhoods in plant communities. Ecology, 81(8), 2137-2148.

Legendre, P., M. Dale, M. Fortin, P. Casgrain, and J. Gurevitch, 2004: Effects of spatial structures on the results of field experiments. Ecology, 85 (12), 3202-3214.

Legendre, P., M. R. T. Dale, M.-J. Fortin, J. Gurevitch, M. Hohn, and D. Myers, 2002: The consequences of spatial structure for the design and analysis of ecological field surveys. Ecography, 25(5), 601615.

Letourneau, D. and L. Fox, 1989: Effects of experimental design and nitrogen on cabbage butterfly oviposition. Oecolgia, 80, 211-214.

Letourneau, D. K., 1987: The enemies hypothesis - tritrophic interactions and vegetational diversity in tropical agroecosystems. Ecology, 68(6), 1616-1622.

Levin, S., 1974: Dispersion and population interactions. The American Naturalist, 108 (960), 207-228.

Levin, S., 1988: Pattern, scale and variability: An ecological perspective. In Community Ecology, Levin, S. and Hastings, A., editors, volume 77 of Lecture Notes in Biomathematics. Springer-verlag, Berlin, 1-12.

Levin, S., 1992: The problem of pattern and scale in ecology. Ecology, 73 (6), 1943-1967.

Levin, S. and R. Paine, 1974: Disturbance, patch formation and community structure. Proceedings of the National Academy of Sciences of the United States Of America, 71 (7), 2744-2747.

Levin, S. and L. Segel, 1985: Pattern generation in space and aspect. SIAM review, 27 (1), 45-67.

Levins, R., 1968: Evolution in Changing Environmnents. Princeton University Press, New Jersey.

Lewis, A. C., 1986: Memory constraints and flower choice in Pieris rapae. Science, 232(4752), 863.

Lewis, A. C., 1989: Flower visit consitency in Pieris rapae, the cabbage butterfly. The Journal of Animal Ecology, 58(1), 1-13. 
Mamedov, A. and S. Udalov, 2002: A computer tool to develop individual-based models for simulation of population interactions. Ecological Modelling, 147(1), 53-68.

Mandelbrot, B., 1983: The Fractal Geometry Of Nature. W.H. Freeman and Co, New York, USA.

Mandelbrot, B. B., 1999: A multifractal walk down wall street. Scientific American, 280(2), 70-73.

Mangel, M., 1987: Oviposition site selection and clutch size in insects. Journal Of Mathematical Biology, 25(1), 1-22.

Mardia, K., 1972: Statistics of directional data. Academic Press, London, England.

Mark, D., 1984: Fractal dimension of a coral reef at ecological scales: a discussion. Marine Ecology Prgress Series, 14, 2930294.

Marsh, L. M. and R. E. Jones, 1988: The form and consequences of random-walk movement models. Journal Of Theoretical Biology, 133(1), 113-131.

Matter, S. F., T. Roslin, and J. Roland, 2005: Predicting immigration of two species in contrasting landscapes: effects of scale, patch size and isolation. Oikos, 111(2), 359-367.

Miller, J. and K. Strickler, 1984: Finding and accepting host plants. In Chemical Ecology of Insects, Bell, W. and Carde, R., editors. Chapman and Hall, London, 127-157.

Muggeridge, J., 1942: The white butterfly (Pieris rapae L.) 1. its establishment, spread and control in new zealand. The New Zealand Journal Of Science and Technology, 24(3), 107A-129A.

Myers, J., 1985: Effect of physiological condition of the host plant on the ovipositional choice of the cabbage white butterfly, Pieris rapae. Journal of Animal Ecology, 54, 193-204.

Ogata, Y. and K. Katsura, 1991: Maximum-likelihood-estimates of the fractal dimension for random spatial patterns. Biometrika, 78(3), 463-474.

Ohsaki, N., 1979: Comparative population studies of 3 pieris butterflies, Pieris rapae, Pieris melete and P. Napi, living in the same area .1. ecological requirements for habitat resources in the adults. Researches On Population Ecology, 20(2), 278-296.

Ohsaki, N., 1980: Comparative population studies of 3 pieris butterflies, Pieris rapae, Pieris melete and P. napi, living in the same area .2. utilization of patchy habitats by adults through migratory and non-migratory movements. Researches On Population Ecology, 22(1), 163-183.

Ohsaki, N., 1982: Comparative population studies of 3 pieris butterflies, Pieris rapae, Pieris melete and Pieris napi, living in the same area .3. difference in the annual generation numbers in relation to habitat selection by adults. Researches On Population Ecology, 24(1), 193-210.

Ohsaki, N., 1986: Body temperatures and behavioral thermoregulation strategies of 3 Pieris butterflies in relation to solar-radiation. Journal Of Ethology, 4(1), 1-9.

Ohsaki, N. and Y. Sato, 1990: Avoidance mechanisms of 3 Pieris butterfly species against the parasitoid wasp Apanteles glomeratus. Ecological Entomology, 15(2), 169-176.

Ohsaki, N. and Y. Sato, 1994: Food plant choice of pieris butterflies as a trade-off between parasitoid avoidance and quality of plants. Ecology, 75(1), 59-68.

Ohsaki, N. and Y. Sato, 1999: The role of parasitoids in evolution of habitat and larval food plant preference by three pieris butterflies. Researches on Population Ecology, 41(1), 107-119. 
Otway, S. J., A. Hector, and J. H. Lawton, 2005: Resource dilution effects on specialist insect herbivores in a grassland biodiversity experiment. Journal of Animal Ecology, 74(2), 234-240.

Painter, R., 1951: Insect resistance in crop plants. The MacMillan Company, New York.

Pearson, K., 1905: The problem of the random walk. Nature, 72, 294-294.

Pe'er, G., D. Saltz, H. H. Thulke, and U. Motro, 2004: Response to topography in a hilltopping butterfly and implications for modelling nonrandom dispersal. Animal Behaviour, 68, 825-839.

Potthoff, M., K. Johst, J. Gutt, and C. Wissel, 2006: Clumped dispersal and species coexistence. Ecological Modelling, 198(1-2), 247-254.

Potting, R. P. J., J. N. Perry, and W. Powell, 2005: Insect behavioural ecology and other factors affecting the control efficacy of agro-ecosystern diversification strategies. Ecological Modelling, 182(2), 199-216.

Press, W., 2002: Numerical recipes in C++. Cambridge, USA.

Prokopy, R. J., 1968a: Sticky spheres for estimating apple maggot adult abundance. Journal Of Economic Entomology, 61(4), 1082-\&.

Prokopy, R. J., 1968b: Visual responses of apple maggot flies Rhagoletis pomonella (Diptera Tephritidae) - orchard studies. Entomologia Experimentalis Et Applicata, 11(4), 403-\&.

Prokopy, R. J. and E. D. Owens, 1983: Visual detection of plants by herbivorous insects. Annual Review Of Entomology, 28, 337-364.

R Development Core Team, 2005: R: A language and environment for statistical computing. ISBN 3-900051-07-0.

Ralph, C. P., 1977a: Effect of host plant density on populations of a specialized, seed-sucking bug, Oncopeltus fasciatus. Ecology, 58(4), 799-809.

Ralph, C. P., 1977b: Search behavior of large milkweed bug, Oncopeltus fasciatus (Hemiptera Lygaeidae). Annals Of The Entomological Society Of America, 70(3), 337-342.

Renwick, J. A. A., 2002: The chemical world of crucivores: lures, treats and traps. Entomologia Experimentalis Et Applicata, 104(1), 35-42.

Renwick, J. A. A. and F. S. Chew, 1994: Oviposition behavior in lepidoptera. Annual Review of Entomology, 39, 377-400.

Renwick, J. A. A. and C. D. Radke, 1983: Chemical recognition of host plants for oviposition by the cabbage butterfly, Pieris rapae (Lepidoptera, Pieridae). Environmental Entomology, 12(2), 446-450.

Renwick, J. A. A. and C. D. Radke, 1985: Constituents of host plants and non-host plants deterring oviposition by the cabbage butterfly, Pieris rapae. Entomologia Experimentalis Et Applicata, 39(1), 21-26.

Renwick, J. A. A. and C. D. Radke, 1988: Sensory cues in host selection for oviposition by the cabbage butterfly, Pieris rapae. Journal of Insect Physiology, 34(3), 251-257.

Reynolds, A. M., 2006: On the intermittent behaviour of foraging animals. Europhysics Letters, 75(4), $517-520$.

Richards, O., 1940: The biology of the small white butterfly (Pieris rapae), with special reference to the factors controlling its abundance. The Journal of Animal Ecology, 9(2), 243-288. 
Ricketts, T. H., 2001: The matrix matters: Effective isolation in fragmented landscapes. The American Naturalist, 158(1), 87-99.

Roitberg, B. D., 1985: Search dynamics in fruit-parasitic insects. Journal Of Insect Physiology, 31(11), 865-872.

Roitberg, B. D. and M. Mangel, 1997: Individuals on the landscape: behavior can mitigate landscape differences among habitats. Oikos, 80(2), 234-240.

Root, R. B., 1973: Organization of a plant-arthropod association in simple and diverse habitats: The fauna of collards (Brassica oleracea). Ecological Monographs, 43(1), 95-124.

Root, R. B. and P. Kareiva, 1986: Is risk-spreading so unrealistic? Oikos, 47(1), 114-116.

Root, R. B. and P. M. Kareiva, 1984: The search for resources by cabbage butterflies (Pieris rapae): ecological consequences and adaptive significance of markovian movements in a patchy environment. Ecology, 65(1), 147-165.

Russell, R. E., R. K. Swihart, and Z. L. Feng, 2003: Population consequences of movement decisions in a patchy landscape. Oikos, 103(1), 142-152.

Sato, Y. and N. Ohsaki, 2004: Response of the wasp (Cotesia glomerata) to larvae of the large white butterfly (Pieris brassicae). Ecological Research, 19(4), 445-449.

Sato, Y., J. Takabayashi, S. Yano, and N. Ohsaki, 1999: Effect of herbivore species on host searching of a parasitoid, Cotesia glomerata (Hymenoptera: Braconidae). Applied Entomology and Zoology, 34(4), 459-461.

Schtickzelle, N., A. Joiris, H. Van Dyck, and M. Baguette, 2007: Quantitative analysis of changes in movement behaviour within and outside habitat in a specialist butterfly. BMC Evolutionary Biology, $7: 4$.

Scriber, J., 1984: Host-plant suitability. In Chemical Ecology of Insects, Bell, W. and Carde, R., editors. Chapman and Hall, London.

Shapiro, A. M., 1970: Role of sexual behavior in density-related dispersal of pierid butterflies. American Naturalist, 104(938), 367-\&.

Singer, M., 1986: The definition and measurement of oviposition preference in plant-feeding insects. In Insect plant Interactions., Miller, J. and Miller, T., editors. Springer Verlag, Berlin, 65-94.

Siniff, J. and C. Jessen, 1969: A simulation model of animal movement patterns. Adv. Ecol. Res., 6, 185-217.

Skellam, J., 1973: The formulation and interpretation of mathematical models of diffusionary processes in population biology. In The Mathematical theory of the dynamics of biological populations., Bartlett, M. and Hiorns, R., editors. Academic Press, London UK, 63-85.

Skellam, J. G., 1951: Random dispersal in theoretical populations. Biometrika, 38(1-2), 196-218.

Spaethe, J., J. Tautz, and L. Chittka, 2001: Visual constraints in foraging bumblebees: Flower size and color affect search time and flight behaviour. PNAS, 98 (7), 3898-3903.

Stephens, D. and J. Krebs, 1986: Foraging Theory. Princeton University Press, Princeton, New Jersey.

Sugihara, G. and R. M. May, 1990: Applications of fractals in ecology. Trends In Ecology \& Evolution, 5(3), 79-86. 
Summerville, K. S., J. A. Veech, and T. O. Crist, 2002: Does variation in patch use among butterfly species contribute to nestedness at fine spatial scales? Oikos, 97(2), 195-204.

Swingland, I. and Greenwood, P., editors, 1984: The Ecology Of Animal Movement. Clarendon Press, Oxford.

Tabashnik, B. E., 1987: Plant secondary compounds as oviposition deterrents for cabbage butterfly, Pieris rapae (Lepidoptera: Pieridae). Journal of Chemical Ecology, 13(2), 309-16.

Tahvanainen, J. and R. Root, 1972: The influence of vegetational diversity on the population biology of a specialized herbivore, Phyllotreta cruciferae (Coleoptera: Chrysomelidae). Oecologia, 10, 321346.

Takabayashi, J., Y. Sato, M. Horikoshi, R. Yamaoka, S. Yano, N. Ohsaki, and M. Dicke, 1998: Plant effects on parasitoid foraging: Differences between two tritrophic systems. Biological Control, 11(2), 97-103.

Tanaka, S. and N. Ohsaki, 2006: Behavioral manipulation of host caterpillars by the primary parasitoid wasp Cotesia glomerata (L.) to construct defensive webs against hyperparasitism. Ecological Research, 21(4), 570-577.

Tenhumberg, B., M. A. Keller, A. J. Tyre, and H. P. Possingham, 2001: The effect of resource aggregation at different scales: Optimal foraging behavior of Cotesia rubecula. American Naturalist, 158(5), 505-518.

Thorsteinson, A. J., 1960: Host selection in phytophagous insects. Annual Review Of Entomology, 5, $193-218$.

Tilman, D. and P. M. Kareiva, 1997: Spatial ecology: The role of space in population dynamics and interspecific interactions. Monographs In Population Biology, 30.

Traynier, R., 1979: Long term changes in the oviposition behaviour of the cabbage butterfly, Pieris rapae, induced by contact with plants. Physiological Entomology, 4, 87-96.

Traynier, R., 1984: Associative learning in the ovipositional behaviour of the cabbage butterfly, Pieris rapae. Physiological Entomology, 9, 465-472.

Traynier, R., 1987: Learining without neurosis in host finding and oviposition by the cabbage white butterfly, Pieris rapae. In Insects Plants, Labeyrie, V., Fabres, V., and Lachaise, D., editors. Dr W Junk, Doedrecht.

Traynier, R. M. M., 1986: Visual learning in assays of sinigrin solution as an oviposition releaser for the cabbage butterfly, Pieris rapae. Entomologia Experimentalis et Applicata, 40(1), 25-33.

Traynier, R. M. M. and R. J. W. Truscott, 1991: Potent natural egg-laying stimulant for cabbage butterfly Pieris rapae. Journal of Chemical Ecology, 17(7), 1371-80.

Turchin, P., 1989: Beyond simple diffusion: Models of not-so-simple movement of animals and cells. Comments on Theoretical Biology, 1, 65-83.

Turchin, P., 1996: Fractal analyses of animal movement: A critique. Ecology, 77(7), 2086-2090.

Turchin, P., 1998: Quantitative Analysis of Movement. Sinauer Associates, USA.

Turchin, P., F. Odendaal, and M. D. Rausher, 1991: Quantifying insect movement in the field. Environmental Entomology, 20, 955-963. 
Uchmanski, J. and V. Grimm, 1996: Individual-based modelling in ecology: What makes the difference? Trends In Ecology \& Evolution, 11(10), 437-441.

Vet, L. E. M. and M. Dicke, 1992: Ecology of infochemical use by natural enemies in a tritrophic context. Annual Review Of Entomology, 37, 141-172.

Vinson, S. B., R. L. Jones, P. E. Sonnet, B. A. Bierl, and M. Beroza, 1975: Isolation, identification and synthesis of host-seeking stimulants for Cardiochiles-Nigriceps, a parasitoid of tobacco budworm. Entomologia Experimentalis Et Applicata, 18(4), 443-450.

Visser, J., 1988: Host-plant finding by insects: Orientation, sensory input and search patterns. Journal Of insect Physiology, 34 (3), 259-268.

Visser, J. H., 1986: Host odor perception in phytophagous insects. Annual Review Of Entomology, 31, 121-144.

Vos, M., S. M. Berrocal, F. Karamaouna, L. Hemerik, and L. E. M. Vet, 2001: Plant-mediated indirect effects and the persistence of parasitoid-herbivore communities. Ecology Letters, 4, 38-45.

West, S. A. and J. P. Cunningham, 2002: A general model for host plant selection in phytophagous insects. Journal of Theoretical Biology, 214(3), 499-513.

Whitney-Johnson, A., M. Thompson, and E. Hon, 2005: Responses to predicted global warming in Pieris rapae (Lepidoptera): Consequences of nocturnal versus diurnal temperature change on fitness components. Environmental Entomology, 34(3), 535-540.

Wiens, J. A., 1976: Population responses to patchy environments. Annual Review Of Ecology And Systematics, 7, 81-120.

Wiens, J. A., 1989: Spatial scaling in ecology. Functional Ecology, 3(4), 385-397.

Wiens, J. A., T. O. Crist, and B. T. Milne, 1993: On quantifying insect movements. Environmental Entomology, 22(4), 709-715.

Winkelman, D. L. and G. L. Vinyard, 1991: Gyrinid searching tactics - empirical observations and a tactical model. Behavioral Ecology And Sociobiology, 28(5), 345-351.

With, K. A., 1994: Using fractal analysis to assess how species perceive landscape structure. Landscape Ecology, 9(1), 25-36.

Wolfson, J. L., 1980: Oviposition response of Pieris rapae to environmentally induced variation in Brassica nigra. Entomologia Experimentalis Et Applicata, 27(3), 223-232.

Yamamura, K., 1999: Relation between plant density and arthropod density in cabbage. Researches on Population Ecology, 41(2), 177-182.

Yamamura, K., 2002: Biodiversity and stability of herbivore populations: influences of the spatial sparseness of food plants. Population Ecology, 44(1), 33-40.

Yamamura, K. and E. Yano, 1999: Effects of plant density on the survival rate of cabbage pests. Researches on Population Ecology, 41(2), 183-188.

Zalucki, M. P., R. Kitching, D. Abel, and J. Pearson, 1980: A novel device for tracking butterflies in the field. Annals of the Entomological Society of America, 73, 262-265.

Zalucki, M. P. and R. L. Kitching, 1982: The analysis and description of movement in adult Danaus plexippus 1 (Lepidoptera, Danainae). Behaviour, 80, 174-198. 
Zollner, P. A. and S. L. Lima, 1999a: Orientational data and perceptual range: real mice aren't blind. Oikos, 84(1), 164-166.

Zollner, P. A. and S. L. Lima, 1999b: Search strategies for landscape-level interpatch movements. Ecology, 80(3), 1019-1030.

Zollner, P. A. and S. L. Lima, 2005: Behavioral tradeoffs when dispersing across a patchy landscape. Oikos, 108(2), 219-230. 


\section{Chapter 3}

\section{A software framework for simulating spatially explicit, individual foraging behaviour}

\subsection{Abstract}

An agent-based, spatially-explicit simulation framework was developed to enable the exploration of individual oviposition behaviour. The intended application was to model interactions of Pieris rapae (L.) with resources aggregated at different specified spatial scales. The framework was built upon the concept of the Correlated Random Walk. This was extended by incorporating a response to sensory information available about resources. Two modes of information gathering were modelled, equating to "Visual" and "Olfactory" senses, commonly found in insects (Hern et al., 1996). The framework supports a high level of parameterisation and provides for systematically exploring parameter space in the form of definable parameter manipulations. An analysis framework was constructed within the statistical package $\mathrm{R}$ to provide automated reports of the simulation output. The behaviour of the simulation is explored with regard to a simplified resource layout to demonstrate the response produced by various parameter combinations. The framework is intended to provide a useful tool for researchers to model any animal movement as a Correlated Random Walk.

\section{Keywords}

Agent-based, Brassica oleracae, Correlated Random Walk, Individual-based, Java, Pieris rapae, Simulation, Spatial Ecology 


\subsection{Introduction}

The random walk has been at the centre of much research, from as early as Einstein (1905) and Pearson (1905). "correlated" random walks (CRW) have been applied in many cases as a model for animal movement (Cain, 1985; Turchin, 1998; Byers, 2001; Root and Kareiva, 1984; Chapter 2).

This chapter presents a simulation framework that enables the study of the interaction between the movement path of a CRW model and a specified layout of resources. Designed around the work of Cain (1985), turning angles are generated from a Von Mises distribution (Batschelet, 1981). Interaction with resources occurs through a "radius of attraction", representing the animal's ability to perceive individual resources (Cain, 1985). The framework additionally includes two conceptual models for sensory perception, representing "vision" and "olfaction". The goal was to keep these mechanisms as simple as possible, whilst allowing the generation of a range of responses and a reasonable level of "realism". Thus the CRW is the basic model of movement to which is added the potential for an individual step to be influenced by sensory input from the environment.

The purpose in developing the framework was two-fold; a) to investigate the ovipositional response of Pieris rapae (Lepidoptera) to resource density across multiple spatial scales, and b) to produce a general framework enabling subsequent researchers to explore the CRW without developing specific, time consuming software (over half the time of this project was involved in the construction of the software). Chapter 4 details the results of the $P$. rapae experiments.

Some clarification of the term "foraging" may be required. Within this work, it refers to the general process of an animal moving around seeking resources. In this way "ovipositional" behaviour (i.e. searching for sites to lay eggs) represents a sub-set of the general behaviour of "foraging". Foraging may also be specifically foraging for food resources (in the case of butterflies, nectar). Because the simulation framework presented here is a general framework for movement, the two terms are often used interchangeably. It is hoped that this does not cause undue confusion.

\section{Background}

Kareiva and Shigesada (1983) reported that the expected mean squared displacement (MSD) of a CRW was consistent with the observed MSD of P. rapae whilst ovipositing but not when foraging for nectar. Cain (1985) and Cain et al. (1985) showed that based on a CRW, clumped resources should receive a lower number of visits per plant, but only at high enough resource densities. Jones (1977a) developed 
a sophisticated behavioural model which included parameters representing a more direct response to the resources. For example, the parameters "MISS" and "ZERO" respectively represented the butterfly's likelihood of moving toward plants or returning to the same plant in the absence of nearby hosts. This work showed that isolated plants received more eggs per plant than those in dense areas (consistent to Cain's model). Jones notes that the model was particularly sensitive to the parameters controlling perceptual range and host responsiveness (Jones, pers. comm.). A simulation produced by Bukovinszky et al. (2005) incorporated a response to density based on 3 characteristic foraging strategies; random, visual and olfactory. They also observed a negative response to patch density in the field. In simulation experiments the "visual" strategy used by Bukovinszky et al. (2005) produced similar behaviour due to the fact that smaller patches had a larger perimeter-area ratio, i.e. they were more likely to be "noticed" by a good visual searcher, particularly one that moved at a higher speed. They equate this to the movements of $P$. rapae for which they gathered the observational data. For a more detailed review please refer to Chapter 2.

This previous work led to the creation of the current framework in order to incorporate aspects from each of the modelling strategies in a consistent manner.

The methods section has been sub-divided into three parts; Section 3.3 describes the concepts behind the simulation and where appropriate, the mathematical representations. Section 3.4 describes in detail the features of the simulation framework in terms of its functionality and the main parameters. Section 3.5 describes two experiments that were executed in order to explore and demonstrate the responses that the simulation is capable of producing. Box 3.1 details a selection of terms relating to the simulation. Box 3.2 lists a selection of geometrical terms which are used throughout the chapter.

\subsection{Methods: Conceptual model}

The conceptual model is based around the concept of discrete timesteps during which the agents (Box 3.1) such as a forager (butterfly) or a resource (cabbage), are allowed to "behave". A resource may increase in size over time. In the case of the foragers, "behaviour" means moving from the current location to a new one. How far to move and in which direction is influenced by a number of parameters. In the absence of any host resources, the foragers move with a Correlated Random Walk (CRW) having a fixed step length. If the "olfaction" and "visual" senses are enabled, the direction taken will be influenced by resources which are detected. 
The manner in which the CRW is derived, and the response to the resources can be described entirely in terms of an abstract, conceptual model which is independent of the implementation of the framework and should allow complete replication of the results. Figure 3.24 shows a summary of the various conceptual elements. These are described in the following sections, beginning with a description of the resources, moving from the CRW through each of the "senses" and ending with a description of how they interact to form the behaviour of the foraging agents.

\subsubsection{Resources}

The primary aim of the framework is to explore interactions between forager movement and resources (in our experiments, cabbages) which are represented simply as a point on the landscape surrounded by a "radius of attraction" (Cain, 1985). An alternative representation is included which works in combination with the visual perception (Section 3.3.4), and involves the concept of apparency. Both represent the ability of the forager to perceive the resource. It would be possible to vary the parameters associated with detection of a resource on a per resource basis, representing variance in plant age, size or nutritional state for example, this has not been explored here.

Resources have a second attribute relating to the purpose of the simulation which is an "egg count". This allows foragers to add eggs to a resource and is the basic response variable for the simulation. The egg count was the response variable that was measured in the field, and so the distributions of eggs across the layouts can be compared between field and simulation.

The following sub-sections detail the radius of attraction, apparency, behavioural responses of the foragers and layout of the resources.

\section{Radius of attraction ( $\mathrm{R})$}

The radius of attraction is a circular zone which has a radius (denoted by $R$ ), centred on the resource location. It represents a zone of detection within which a forager is certain to be able to detect the resource. A smaller $R$ represents resources which are harder to find. Biologically this may be due to the size of the plant, or to its distinction relative to the environment (a lone cabbage in a dense field of lettuce is hard to spot).

When describing foraging behaviour, $R$ is similar to a concept of a random "contact" searcher, discussed in Bukovinszky et al. (2005). Figure 3.1 demonstrates a simple layout which will be used throughout the chapter with several values of $R$. An 


\section{Agent-based and agent}

Agent-based simulation or software refers to a specific level of encapsulation within the software architecture. In this case, an insect is represented within the software, referred as an agent. This representation can have both attributes (e.g. size, number of eggs) and behaviour (e.g. movement behaviour). Object-Oriented (OO) programming (Meyer, 2000) provides a natural way to build such a system and is used here. "Agents" represent a somewhat higher level of aggregation than individual "Objects". An agent, for example may be composed of many "Objects". Adopting this strategy allows complex systems of interacting agents to be constructed whilst retaining a manageable high-level conceptual organisation.

\section{Landscape}

The total area available to the agents in the simulation. Agents moving outside this area are considered to be removed from the simulation. This is as opposed to a "wrap around" world commonly used where agents disappear from one side to appear on the opposite side of the landscape.

\section{Logical vs. scaled units}

The spatial model represents an abstract logical space, e.g. it might be that the landscape is $100 \times 100$ units. Within this everything is relative and so the scale is arbitrary. The qualitative effects will remain, such that a step length of 1 vs that of 50 will have the same relation to the overall size of the landscape. This arbitrary unit can be "scaled" into a "real world" representation by assigning a number of real world units to each logical unit. The default scale of the simulation is $1 \mathrm{~cm}$, so 1 logical unit represents $1 \mathrm{~cm}$ in reality.

\section{Patch}

A rectangle which contains a set of resources. The term patch is usually used to define a density of resources in a convenient manner. For example, there might be 3 patch sizes, $1 \mathrm{~m} \times$ $1 m, 3 m \times 3 m$ and $6 m \times 6 m$ each containing 16 resources evenly distributed. This would result in 3 "scales" of measurement.

\section{Parameter space}

This is the set of all possible values for all parameter combinations, which could be seen as a multi-dimensional "space". When exploring the behaviour of a simulation, parameters are generally changed systematically in order to observe the response. This is termed "exploring the parameter space". The more parameters and the wider the range of values possible for each parameter, the larger the parameter space. This can become computationally very expensive, even with only a few parameters. Here, the focus tends toward selecting a subset of parameters representing interesting scenarios. A more comprehensive exploration of the parameters space would be desirable but would require further processing time.

\section{Box 3.1: Common terms specific to the Simulation used throughout the chapter.}




\section{D continuous space}

A geometric plane which has 2 dimensions, commonly (and here) labelled $\mathbf{x}$ and $\mathbf{y}$. Continuous means infinite resolution, i.e. not subdivided into grid squares or discrete units of measurement.

\section{Rectangular coordinate}

A point in 2D continuous space may be defined by its rectangular distance in both $x$ and $y$ dimensions from the origin of the space. We state that the origin is the bottom left of a given area of space to correspond with a "map" view.

\section{Polar coordinate}

In geometry, angles are conventionally measured from the x-axis, i.e. a horizontal line across the 2D space passing through the origin. They are measured anti-clockwise from here. A polar coordinate consists of two measurements, the angle (usually denoted by $\theta$ ), and the distance from the origin, e.g. $P(\theta, d)$.

\section{Azimuth (direction)}

Polar coordinates are useful because they are the conventional form to deal with geometric calculations. When dealing with animal movement, it is more common to be concerned with "direction". Azimuth is a term traditionally used in navigation to represent the angle of bearing on a compass, with $0^{\circ}$ being North (Soanes and Stevenson, 2004). It is adopted here to distinguish between this specific interpretation and the geometrical concept of an angle used in the polar coordinate system. This may seem to be a minor point but becomes important when discussing calculations based upon an angle, such as calculating the Euclidean distance between two points.

Box 3.2: Geometrical terms used throughout the chapter. 
interesting aspect to be noted here is that as $R$ increases, the space between the resources through which an animal may pass. This can lead to an "edge effect" (Section 3.5.2) as a barrier is formed preventing animals from reaching the inner plants (effectively "trapping" them at the edge).

a) $\mathrm{R}=2$

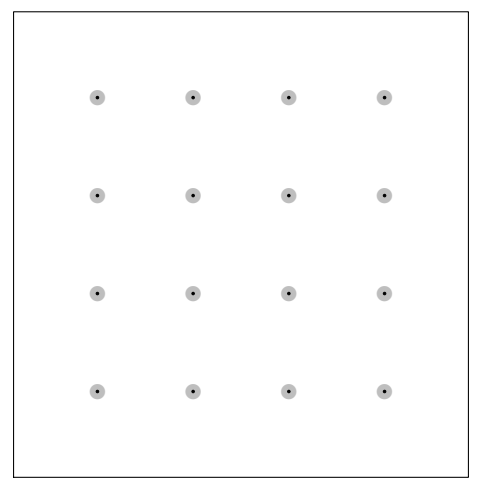

b) $R=5$

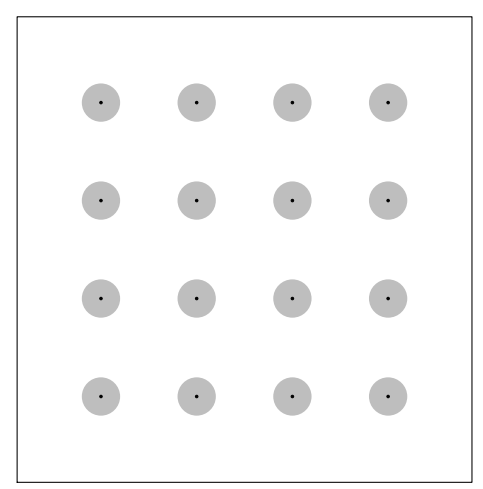

c) $R=15$

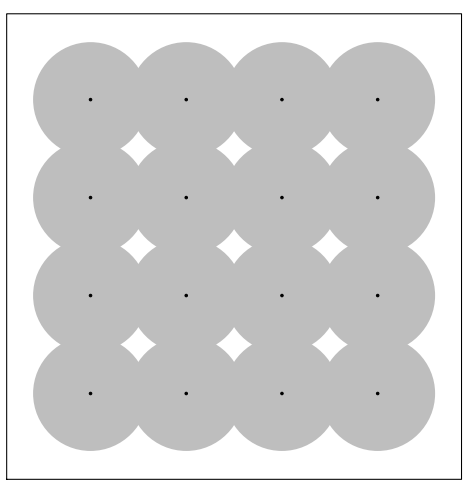

Figure 3.1: Demonstration of the radius Of attraction. Units are cm, the "patch" is $1 \mathrm{~m} \times$ $1 \mathrm{~m}, R$ varies from $2 \mathrm{~cm}$ to $15 \mathrm{~cm}$.

\section{Visual Apparency $(\alpha)$}

Apparency is denoted by $\alpha$ and represents attributes relating to the plant itself, to give some measure of visibility. The size of plant may be taken into account; age, amount of herbivory, or more interestingly, isolation index (Ricketts, 2001; Tischendorf et al., 2003). The latter would represent the case where isolated plants are either more or less visible. Due to the background vegetation, an isolated plant in a dense foliage may be less visible (Root, 1973). An isolated plant on bare ground may be more visible due to an increased perimeter-area ratio (Bukovinszky et al., 2005).

The model defines $\alpha$ to have a range between 0 and 1 (Equation 3.1), where 0 is invisible and 1 represents a maximal ability to perceive a resource (it can either be seen or not; once seen, it is not possible to see it more).

$$
0 \leqslant \alpha \leqslant 1
$$

The experiments described in this chapter assume constant apparency $(\alpha=1)$.

\section{Behavioural response (egg count)}

If the path of a forager intersects a circle defined by the radius of attraction, it has detected the plant. This could be seen in reality as similar to a pit-fall trap, and 
indeed the framework has been used to this effect in analysing a layout of pitfall traps designed to sample ant populations (Hartley, unpublished data).

The response of a forager upon intersecting a resource is to oviposit, which results in incrementing the egg count of the resource by one egg ${ }^{1}$. The movement path of the forager is not affected, the model simply assumes that any resources intersected are potential oviposition sites ${ }^{2}$. If more than one resource is intersected, the behaviour will depend on the number of eggs that the forager is parameterised to lay. Where possible, experiments were run with both a single egg and multiple eggs. If the forager has a single egg, the egg will be deposited on the closest resource to the starting location for that move. Once all eggs are laid, the forager is removed from the simulation.

\section{Resource layout $(\mathrm{P}, \mathrm{I})$}

When referring to a set of resources, they are referred to as a "patch". There are two parameters that can describe the layout, if the resources within the patch are evenly distributed. These are the dimensions of the patch $(P)$ and the spacing between the plants, or inter-edge spacing $(I)$. The spacing is the distance between the edge of the radius of attraction of each plant. If the patch is square, $P$ reduces to a single number, e.g. $P=100$ indicates a patch size of $100 \mathrm{~cm}$. The patch is taken to be the enclosing rectangle, with a border of $S / 2$ around the edge of the plants. Figure 3.25 provides a graphical representation of these parameters.

\subsubsection{The correlated random walk (CRW)}

Individual animal paths can be quantified in terms of a series of straight line moves (Turchin, 1998; Wiens et al., 1993), Figure 3.2 shows this graphically. A correlated random walk (Cain, 1985; Byers, 2001) is a random walk (Pearson, 1905) where there is a tendency to keep going in the same direction, i.e. there is a correlation between successive angles of turn. In terms of a sequence of events, an animal has a "current" direction, or azimuth (Box 3.2). It moves 1 step length in that direction. For the following step, an angle by which to turn is drawn from a Von Mises distribution (Batschelet, 1981), which means that there will be a change of direction centred around 0 , with equal probability of being negative ("left") or positive ("right"). Chapter 2 contains a detailed review of this process.

\footnotetext{
${ }^{1}$ Although other responses are theoretically possible, such as feeding from nectar. Similarly "clutches" of eggs could be deposited

${ }^{2}$ There is also a parameterisable behaviour whereby the forager will move to the centre of the resource, the effects of which have yet to be explored
} 
In terms of simulation, the CRW has two parameters; the step length $(L)$ and the turning angle concentration $(k)$.

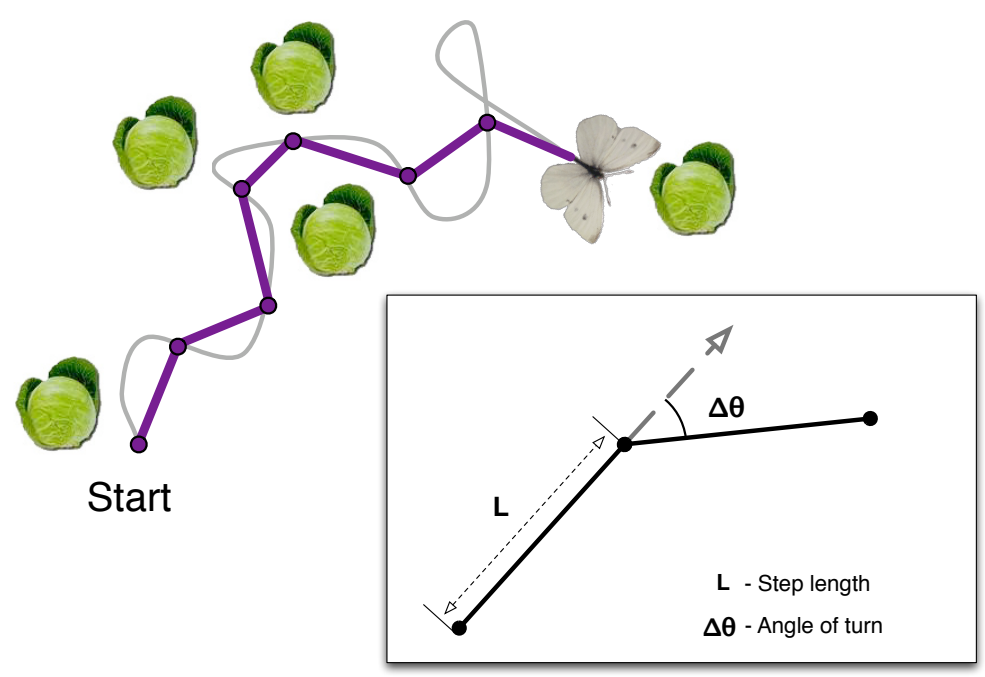

Figure 3.2: Quantifying the movement path of an animal. The butterfly moves along a continuous path which is then sampled (in this case) at discrete time intervals, producing a vector of "steps".

\section{The Von Mises distribution}

When analysing a path, it is common to refer to the "width" of the distribution of successive angles of turn as the turning angle concentration (Batschelet, 1981). This is also a parameter of the density function for the Von Mises probability distribution, usually denoted with $k$. Figure 3.3 shows the Von Mises distribution for three turning angle concentrations and below them an example of the path which is generated the step length in each case is constant. Figure 3.4 shows both changes in $k$ and in step length $(L)$.

Alternative circular distributions are available which achieve the same ends. These are discussed in Batschelet (1981) and are the wrapped Cauchy and wrapped normal distributions. Qualitatively they all produce the same result and indeed can all be represented by a particular parametrisation of the Von Mises distribution. It is also possible to use a linear Gaussian distribution, which again yields similar results. The Von Mises distribution was chosen as a default (and used throughout this chapter). Although computationally slightly more complex, it has parameters easily compared to those estimated from field data and is generally accepted as the "normal" circular distribution. The framework supports all of these distributions as a simple matter of configuration, should they be required (Section 3.4). 

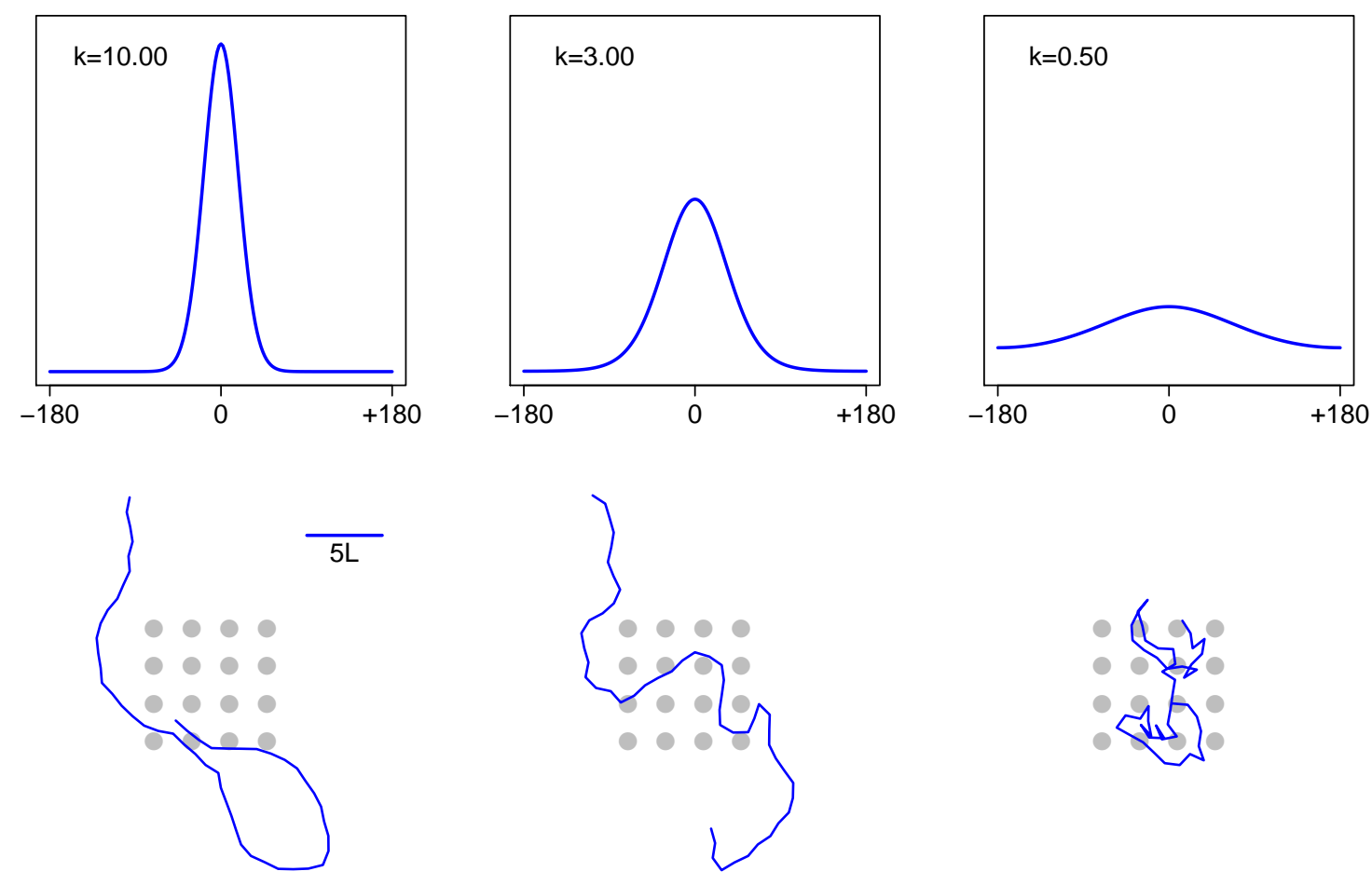

Figure 3.3: Example Paths for various turning angle concentrations. Grey circles represent potential resources and provide some indication of scale for comparison between paths.

\section{Sinuosity of path $\left(\mathrm{S}^{*}\right)$}

Sinuosity is a combination of both the step length and turning angle concentration (Bovet and Benhamou, 1988). Highly sinuous paths will have short step lengths and a large variation in turning angle concentration. Figure 3.8 shows the relationship between sinuosity and the CRW parameters $L$ and $k$.

Sinuosity can be interpreted biologically as a searching mechanism (Bovet and Benhamou, 1988; Bell, 1990, 1991). In situations where the animal "wishes" to search, it should increase the sinuosity of its path, covering a smaller area more thoroughly (Figure 3.3). This is often referred to as area-restricted searching (Bell, 1991). The scale at which it is searching may affect the step length. If searching a relatively large area, step length should be high, if searching a relatively small area, step length should be smaller. In situations where the animal would be better off somewhere else (e.g. low levels of resource) it should increase step length and decrease angle of turn, in other words become more directional. 


\section{Mean Squared Displacement (MSD)}

A useful property of the random walk is that over time, it is possible to analytically predict what the expected average squared displacement of a set of individuals would be (Turchin, 1998; Johnson et al., 1992; Kareiva and Shigesada, 1983). Johnson et al. (1992) notes that over short timescales (low number of steps) a CRW path will have a greater displacement than a pure random (or Fickian) walk. As the number of steps increases, the diffusion approximates that of the pure random walk and as Einstein (1905) resolved, is proportional with time. Figure 3.9 is based on these calculations, showing an analytical calculation of the expected MSD over a number of timesteps, and varying the turning angle concentration $(k)$. 

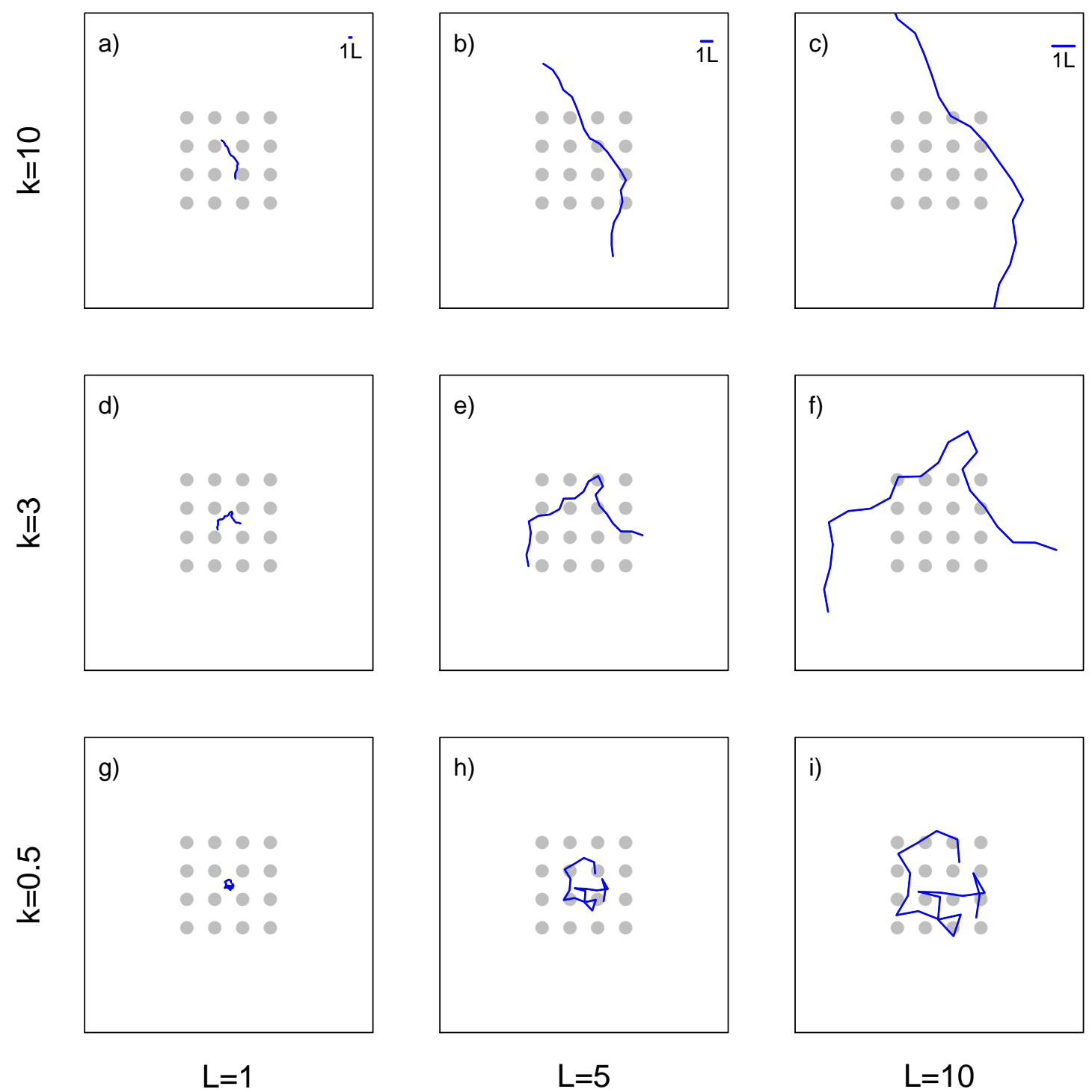

Figure 3.4: Example paths for various turning angle concentrations and step lengths. Grey circles represent potential resources and provide some idea of scale. Here we can see the scaling effect of increasing the step length. The turning angle concentration increases toward the top of the page, step length $(L)$ increases from left to right. A maximum of 20 steps is shown. 

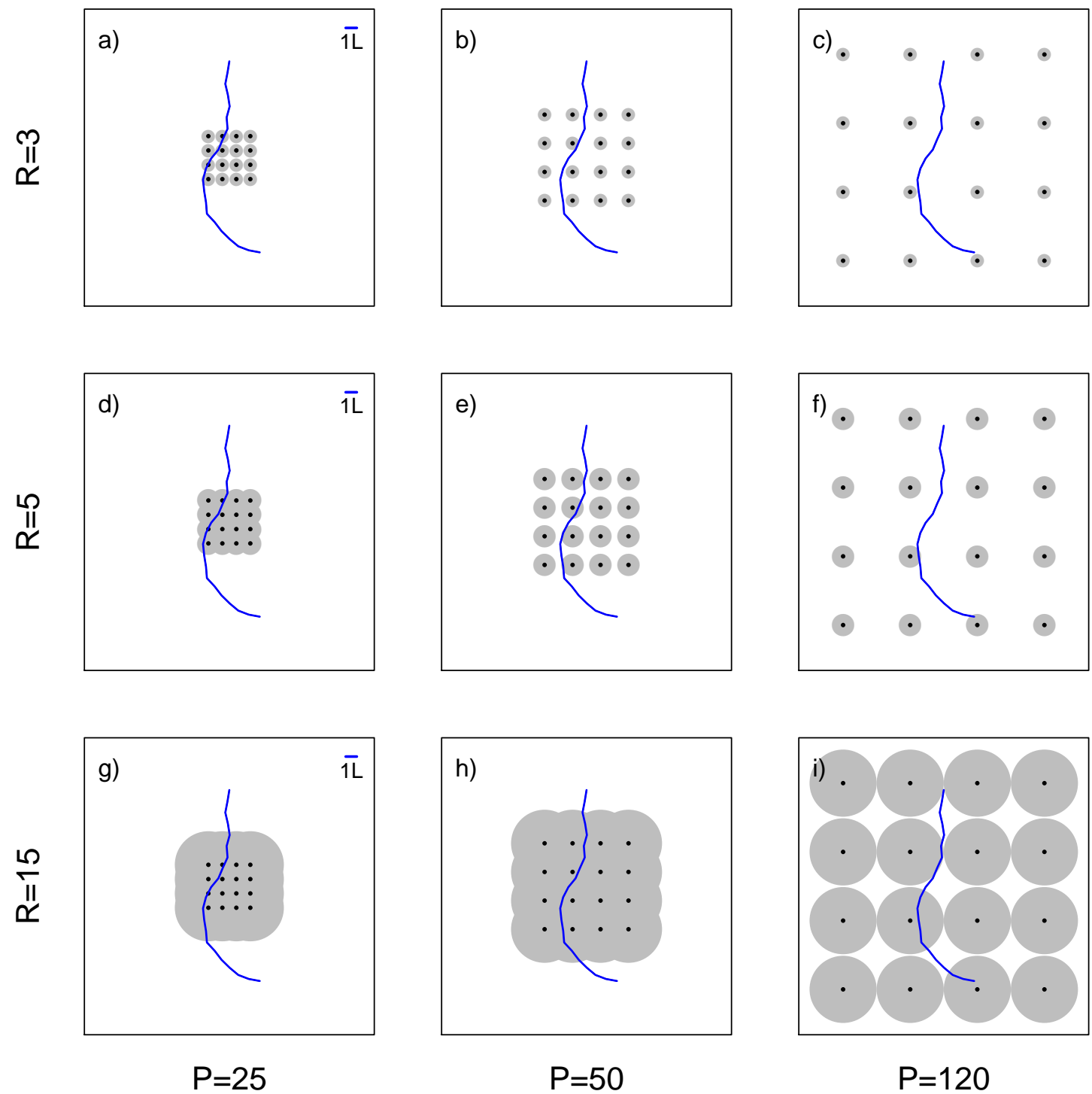

Figure 3.5: Example path ( $k=10, L=5$ ) overlaid onto resource patches of various sizes $(P)$ with different radii of attraction $(R)$. A maximum of 20 steps is shown. 

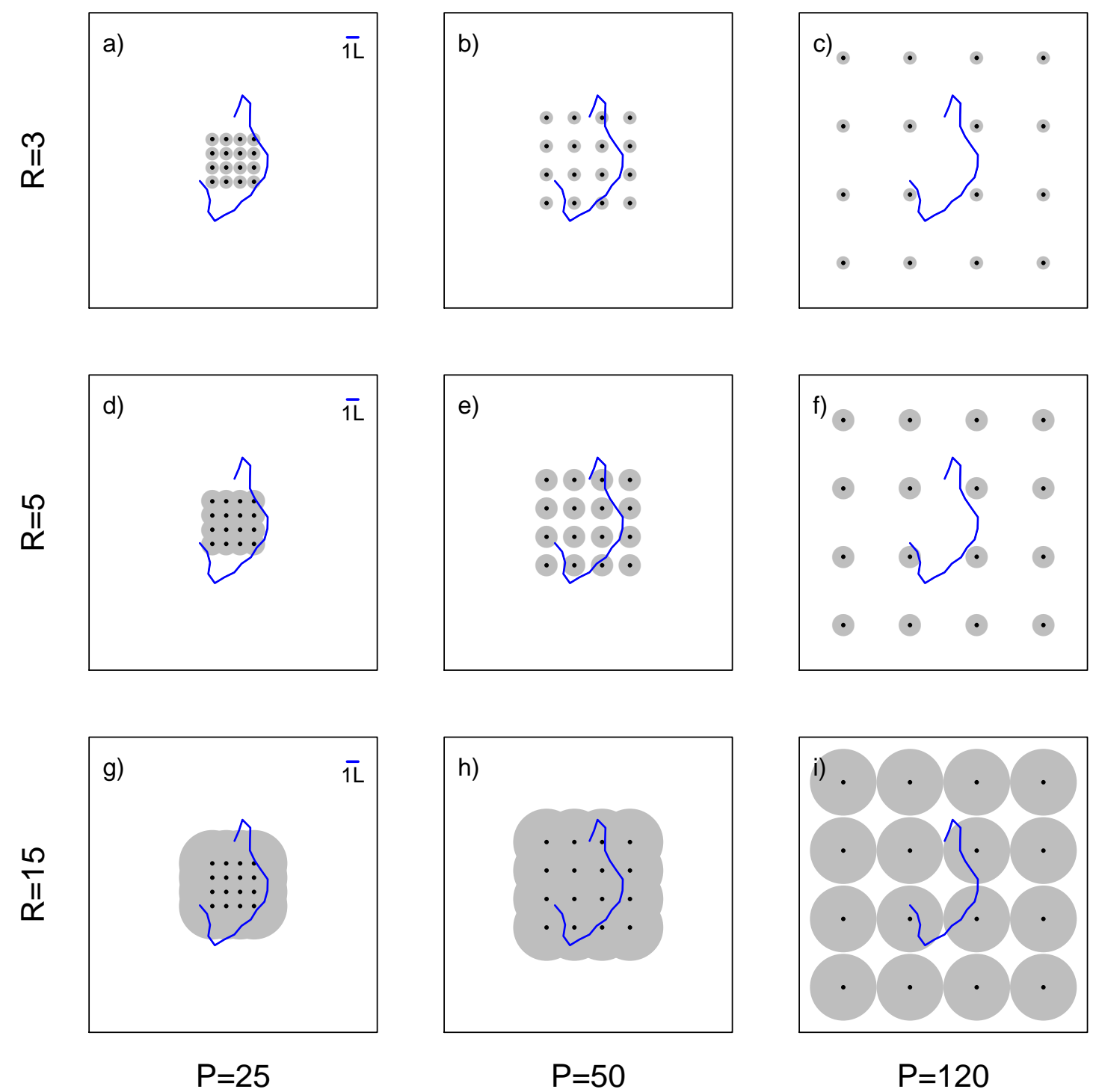

Figure 3.6: Example path ( $k=3, L=5$ ) overlaid onto resource patches of various sizes $(P)$ with different radii of attraction $(R)$. A maximum of 20 steps is shown. 

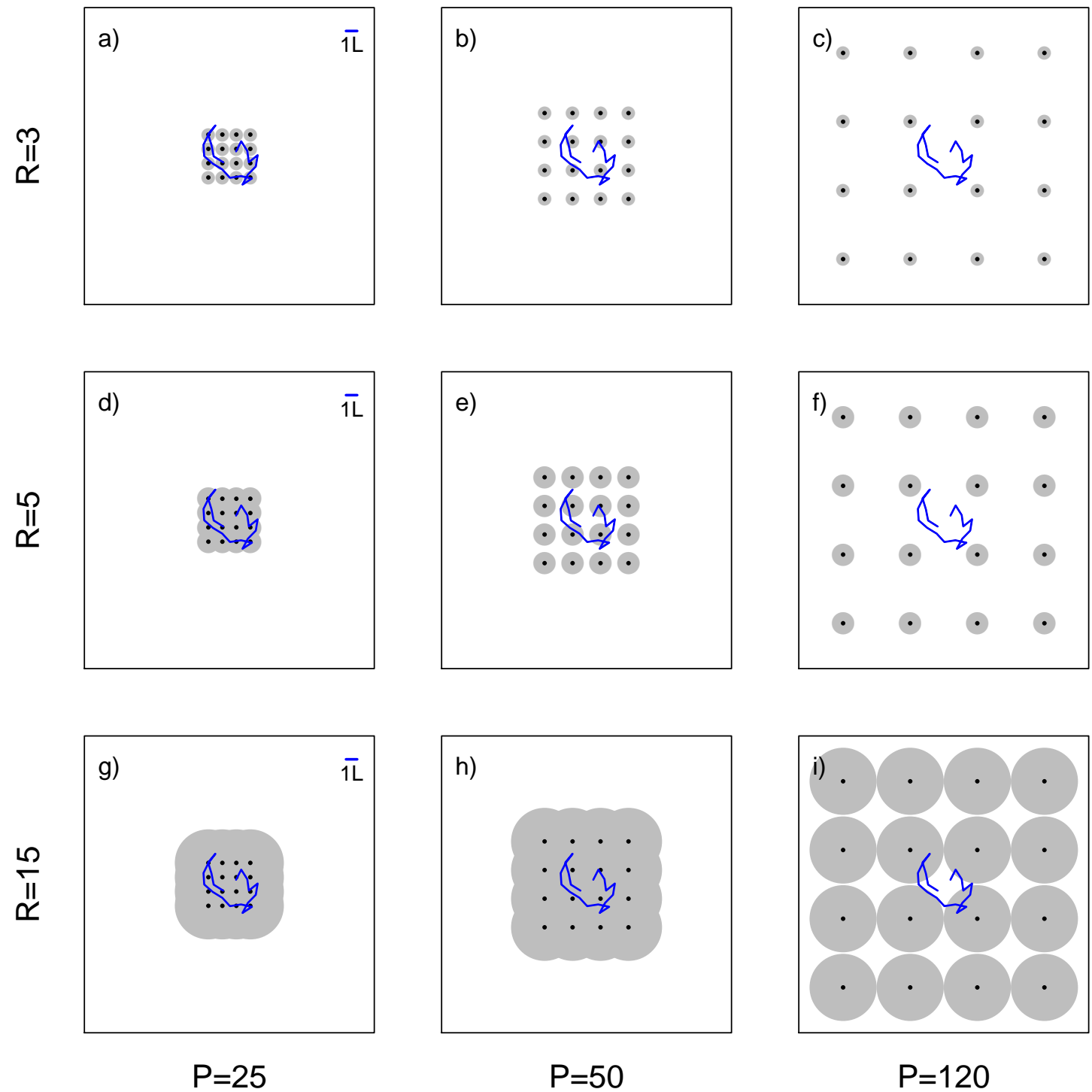

Figure 3.7: Example path ( $k=0.5, L=5$ ) overlaid onto resource patches of various sizes $(P)$ with different radii of attraction $(R)$. A maximum of 20 steps is shown. 


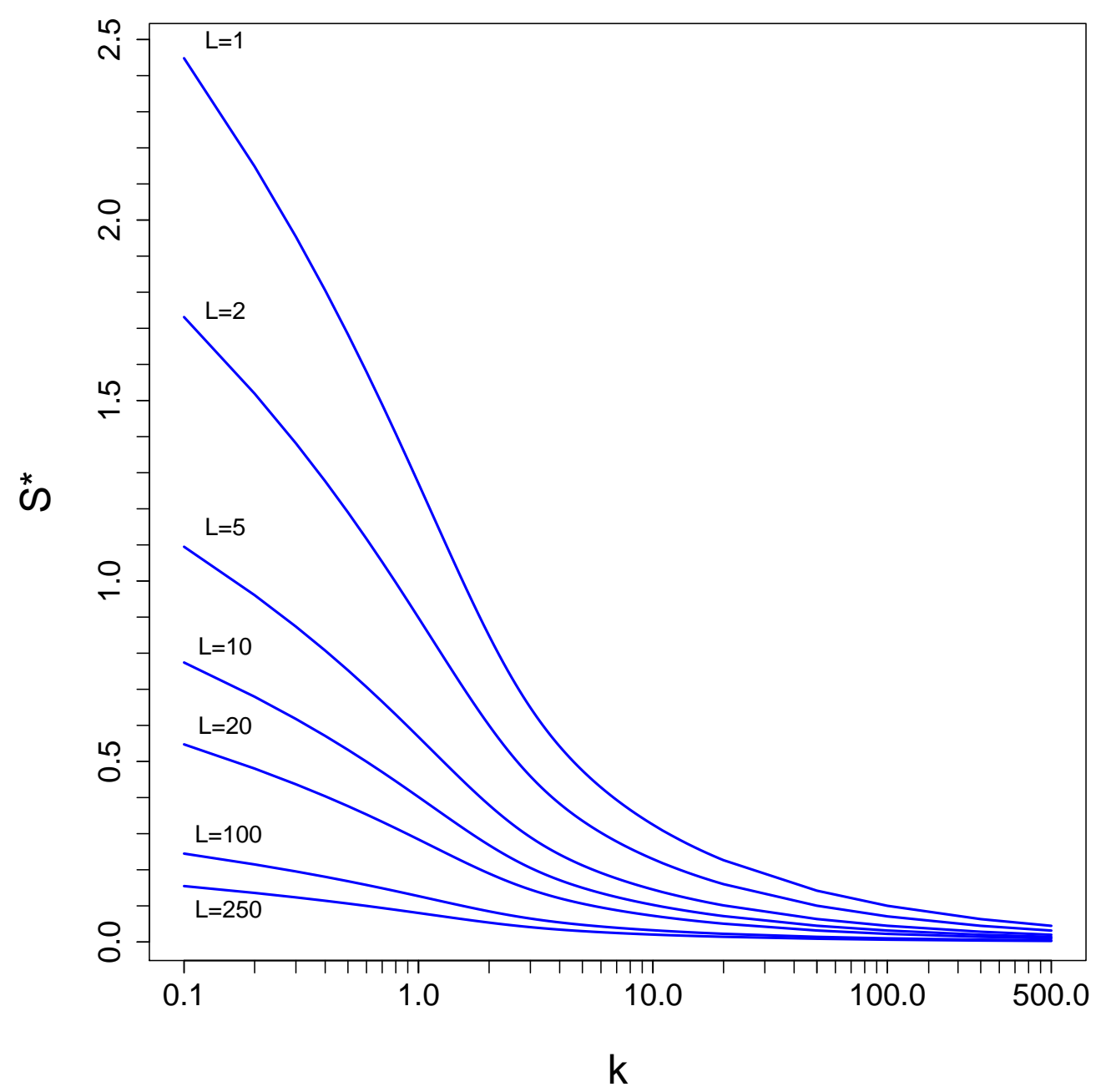

Figure 3.8: The relationship between sinuosity $\left(S^{*}\right), k$ and $L$. The $k$ axis is plotted on a $\log$ scale. 
a) $k=0.50, L=1$

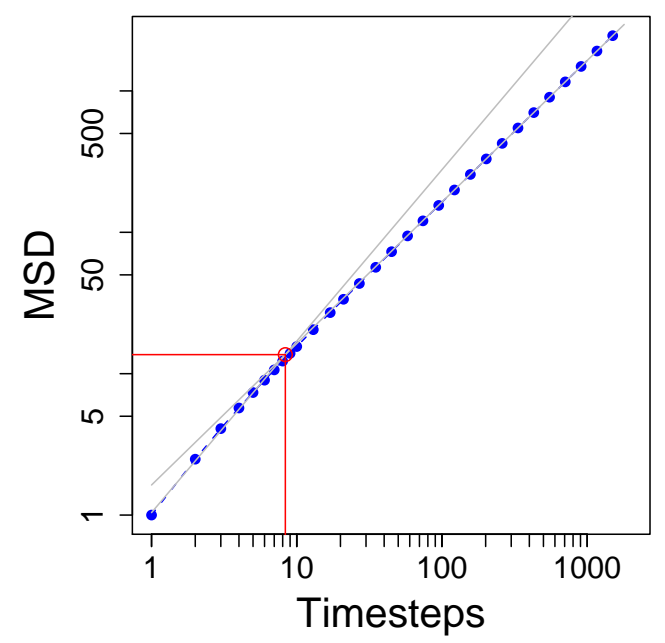

c) $k=10.00, L=1$

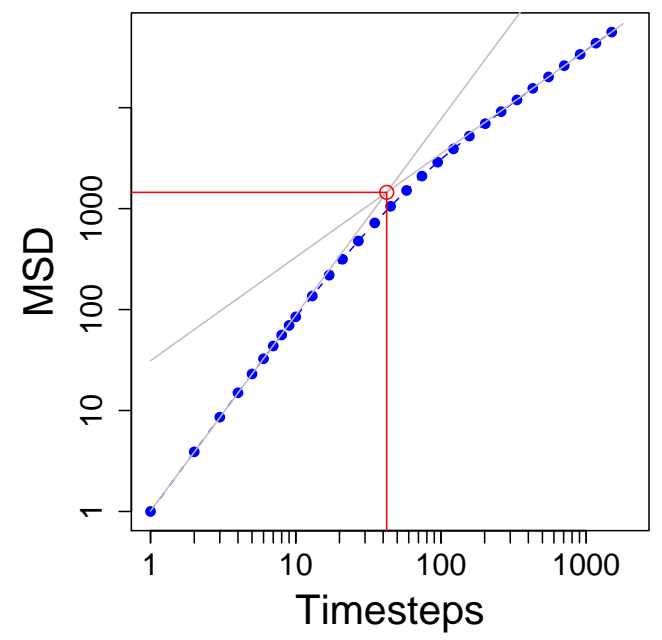

b) $k=3.00, L=1$

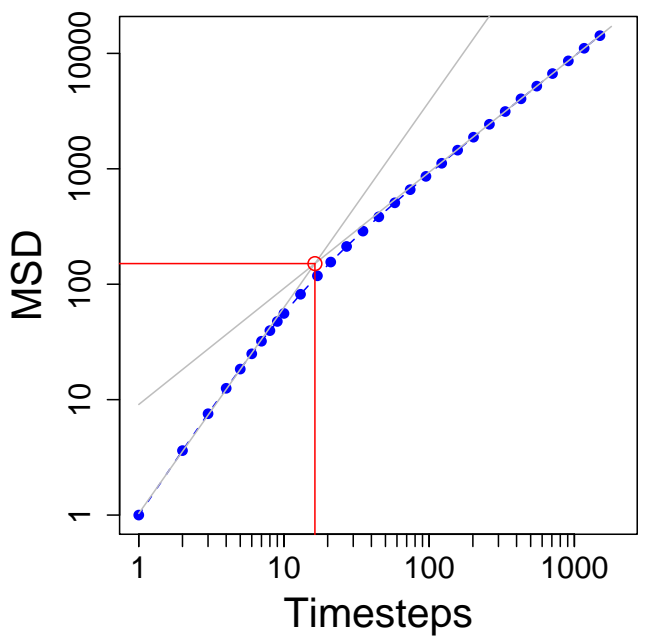

d) $k=20.00, L=1$

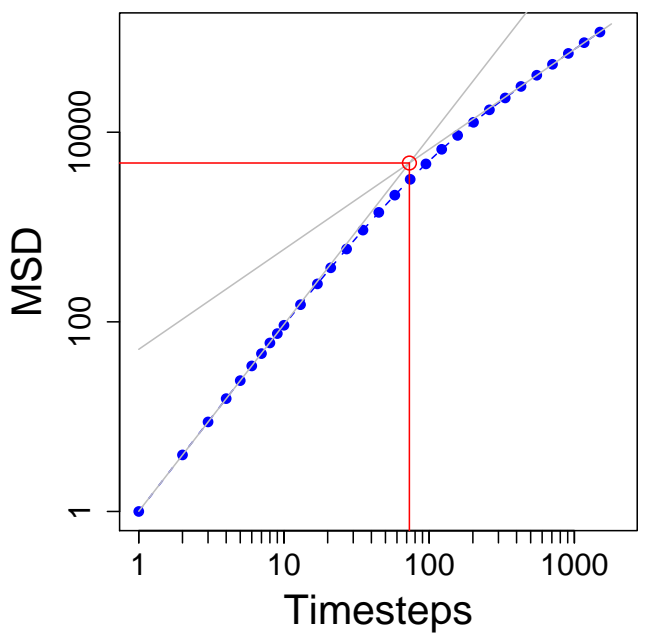

Figure 3.9: The relationship between expected mean squared displacement, $E\left(R_{n}^{2}\right)$ (here labelled "MSD"), $k$ and number of timesteps $(n)$. Both axes are log scales. The red lines indicate the point at which the deviation from a pure random walk occurs (after Johnson et al., 1992). As $k$ increases, the "directionality" of the walk produces a steeper slope than a pure random walk, for a greater number of timesteps. Details of the regressions are in Table 3.1 .

\begin{tabular}{rrrrrr}
\hline $\mathbf{k}$ & Slope A & Slope B & xTimesteps & xMSD & xRMSD \\
\hline $\mathbf{0 . 5}$ & 1.212 & 1.001 & 8 & 14 & 3.74 \\
$\mathbf{3}$ & 1.785 & 1.006 & 16 & 151 & 12.29 \\
$\mathbf{1 0}$ & 1.941 & 1.026 & 42 & 1450 & 38.08 \\
$\mathbf{2 0}$ & 1.971 & 1.054 & 73 & 4736 & 68.82 \\
\hline
\end{tabular}

Table 3.1: Regression statistics for expected MSD showing the intersections of the fitted regression lines (xTimesteps, $x M S D$ ) and the square root of the displacement intercept, $x$ RMSD. These relate are expressed graphically in Figure 3.9. Slope $A$ is the slope of the regression line for low timesteps $(<=7)$, and Slope $B$ for Timesteps $>=400$, after (after Johnson et al., 1992) 


\subsubsection{Olfactory perception}

Olfaction in the framework can be sub-divided into three components; the "odour signal" in the environment, the sensors available to the foraging agent (used to detect the odour) and the behavioural response of the agent to the information it is receiving.

\section{Odour "signal"}

Odour is assumed to be airborne and thus diffuses away from its source. In reality this will be odour plumes of from volatile chemicals released into the air by host plants (Hern et al., 1996; Hogan, 2006; Langan et al., 2004; Visser, 1986). In the current version of the framework no account is made for wind, so it is assumed that the odour degrades with distance uniformly in all directions, with the form of a Gaussian distribution. The main parameter for controlling the "width" of the signal is the standard deviation of the Gaussian distribution and is denoted by $S W$ (signal width). $S W$ is relative to the scale of the landscape. For larger landscapes, the $\mathrm{S} W$ needs to be increased to represent information available over greater distances. Foragers are able to "sample" the strength of the signal at any given point in the landscape.

a) $S W=20$

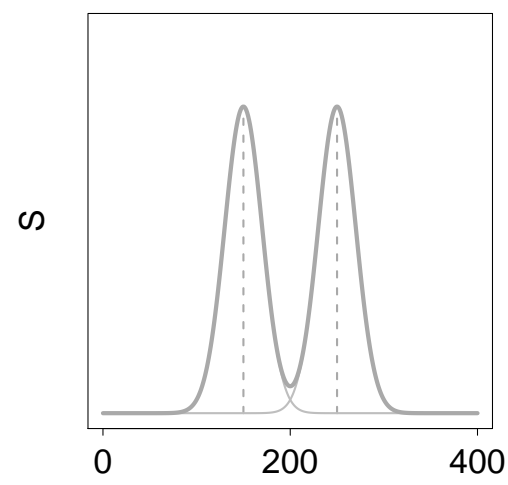

b) $\mathrm{SW}=35$

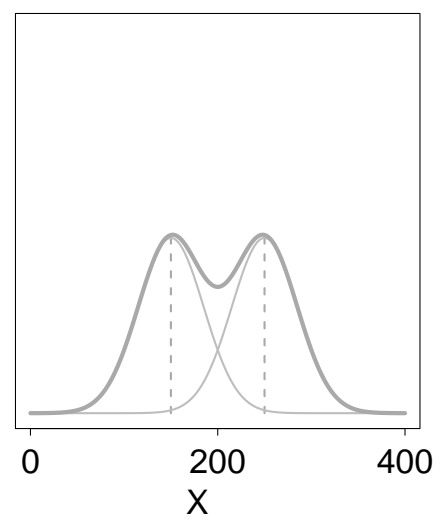

c) $\mathrm{SW}=70$

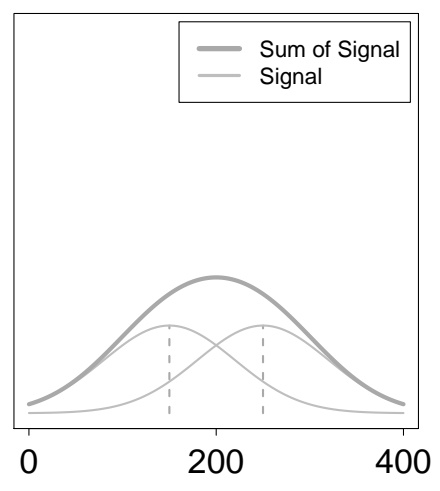

Figure 3.10: Construction of the olfaction signal surface. Shown are two resources (vertical dotted lines), each producing a Gaussian odour plume. The signal width $(S W)$ varies from narrow on the left to wide on the right.

Where more than one source is present, the odours from each source are summed to produce a signal "surface" (Figures 3.10, 3.11 to 3.14 ). An interesting result of this is that for wider signal widths, the signal peak occurs in-between the plants, and allowing a range of scenarios to be expressed. Figure 3.10.a represents a shorter range but highly directional olfactory signal (perhaps appropriate for parasitoids homing 

a) Image map
b) 3D Surface
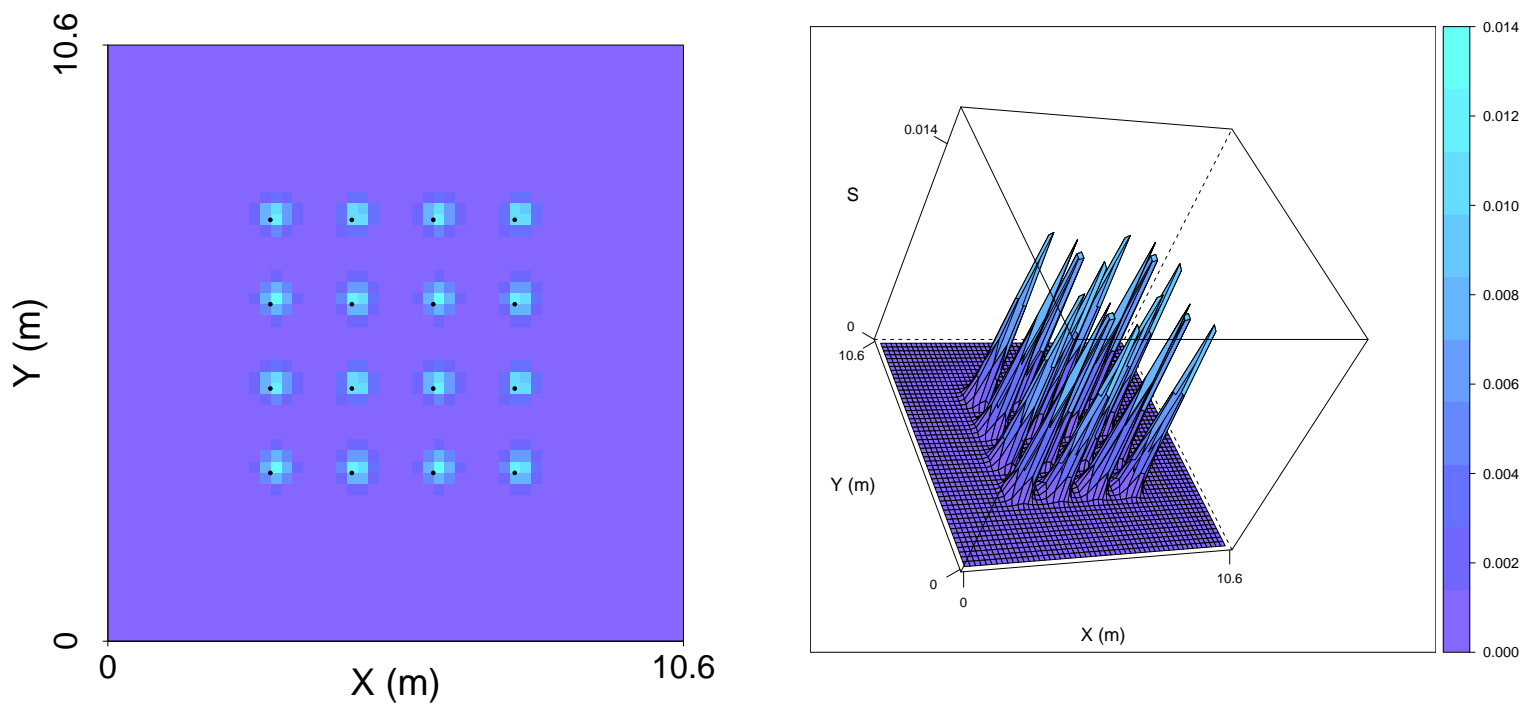

Figure 3.11: Olfaction signal surface, $S W=20$ )
a) Image map
b) 3D Surface
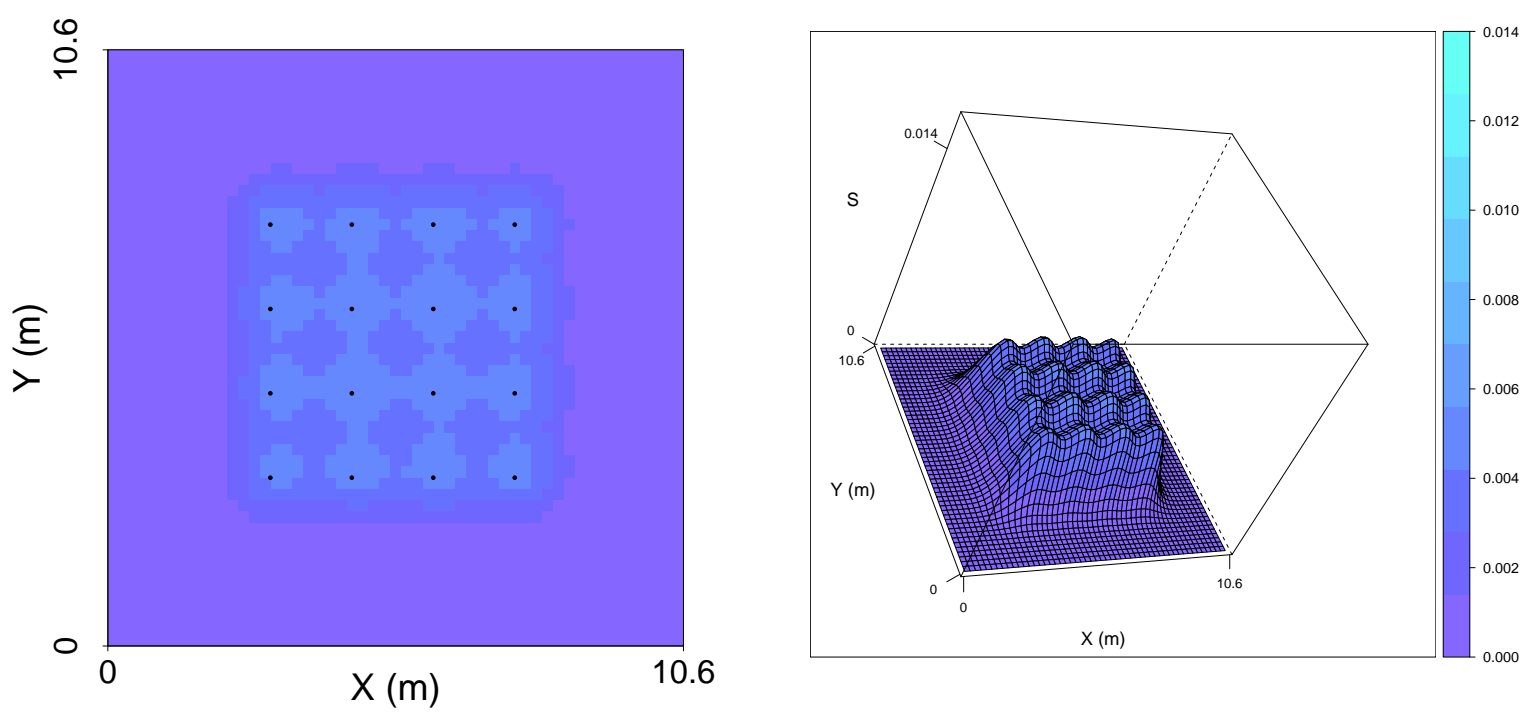

Figure 3.12: Olfaction signal surface, $S W=45$ ) 
a) Image map

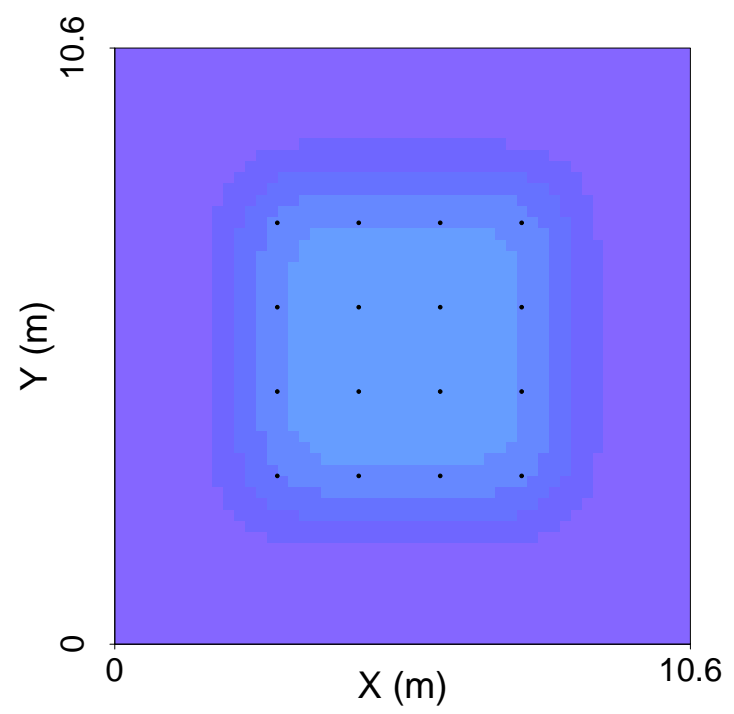

b) 3D Surface

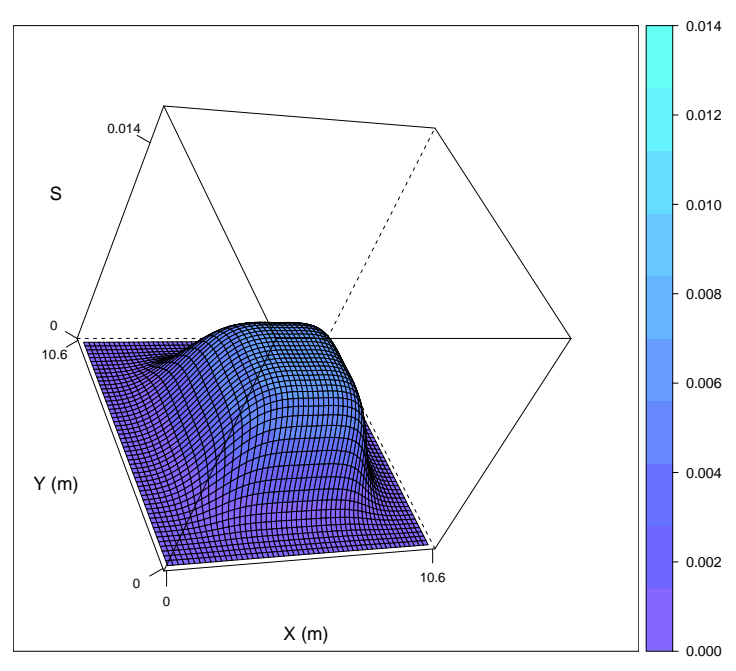

Figure 3.13: Olfaction signal surface, $S W=90$ )

a) Image map

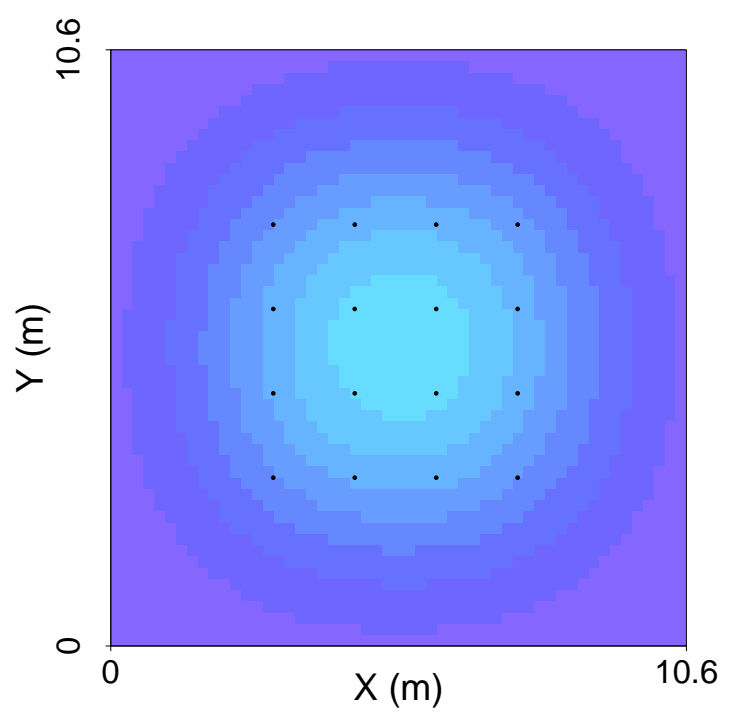

b) 3D Surface

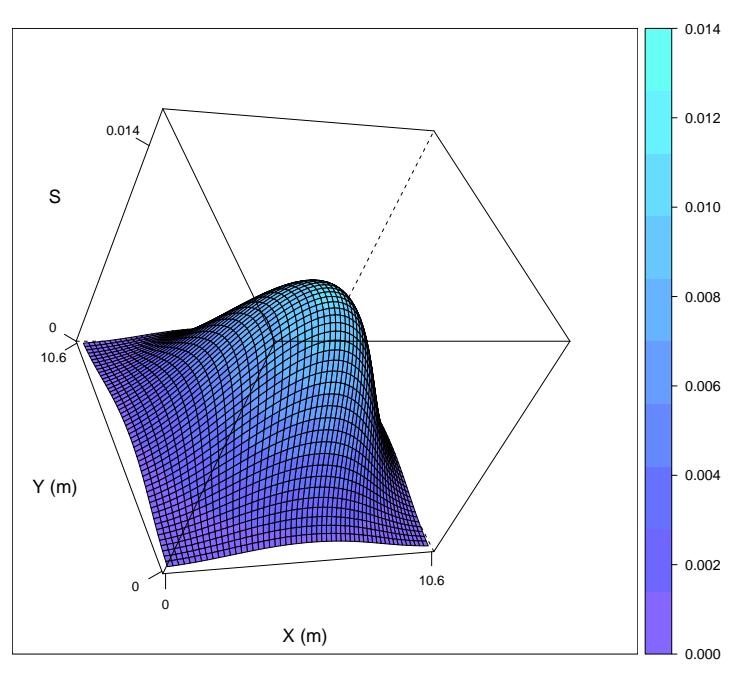

Figure 3.14: Olfaction signal surface, $S W=180$ ) 
in upon prey), whereas Figure 3.10.c represents a more diffuse but less accurate signal (perhaps representing the smell of cabbage in a field).

\section{Olfaction "Sensors"}

A foraging agent can be configured with any number of olfaction "sensors". Each sensor is capable of "sampling" the height of the signal surface at its location. The sensors are located at a specified distance and azimuth from the current location of the agent. Several configurations were experimented with but an effective (and realistic) setup involves two sensors located one on each side of the forager, at $40^{\circ}$. This configuration is shown in Figure 3.15, and is used throughout the thesis.

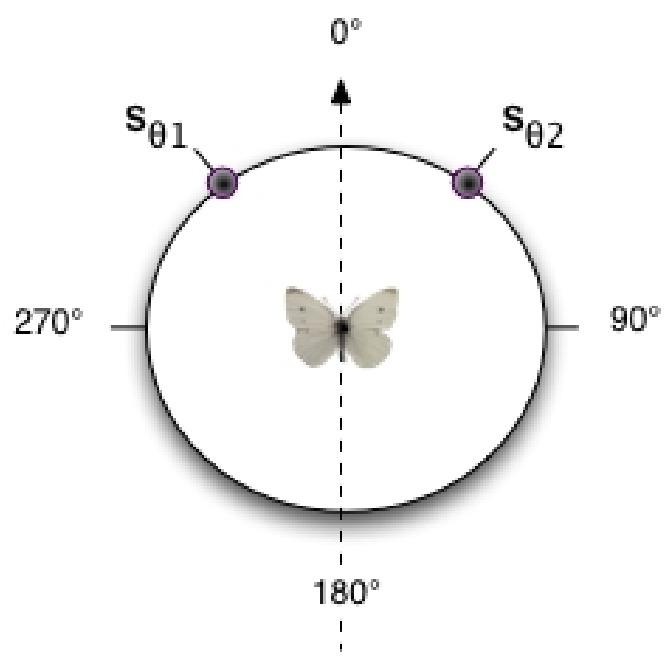

Figure 3.15: Foraging agent (Butterfly) with 2 olfaction sensors indicated by $S_{\theta 1}$ and $S_{\theta 2}$ at $\pm 40^{\circ}$

At any given timestep the forager has available to it a signal value for each of its sensors. This value is denoted by $S_{\theta i}$ where $\theta$ represents the fact that the signal is derived from a particular azimuth (relative to the forager's current direction), and $i$ is the index of the sensor. Two pieces of information are processed in order to affect the behaviour of the forager:

- The difference in signal between two or more sensors

- The total strength (sum) of the signal across all sensors

The difference in signal can provide information about the gradient of the signal, i.e. is there a larger signal to the left or to the right, and what is the difference? The total strength of the signal provides information about the surrounding environment, i.e. is the forager in an area of generally high or low signal?.

The difference in signal is calculated based on the assumption that the forager may only have two possible actions: turn left or turn right, which means that the actual 
a)

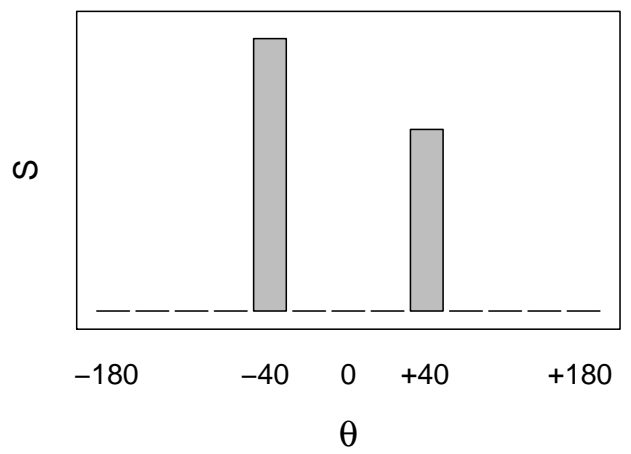

c)

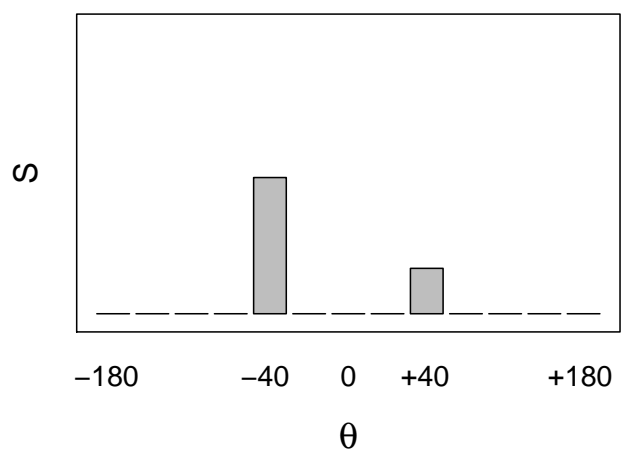

e)

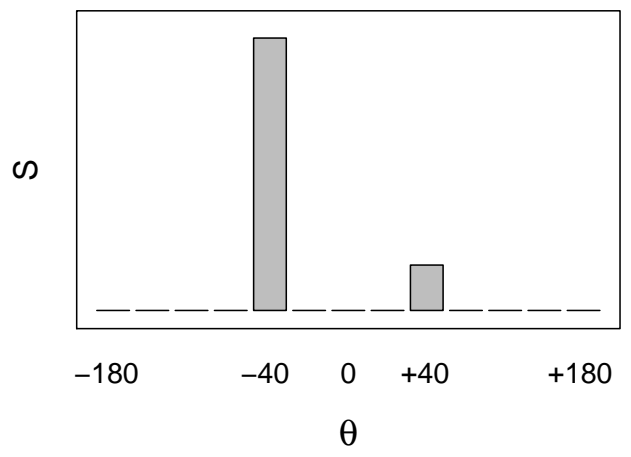

b)

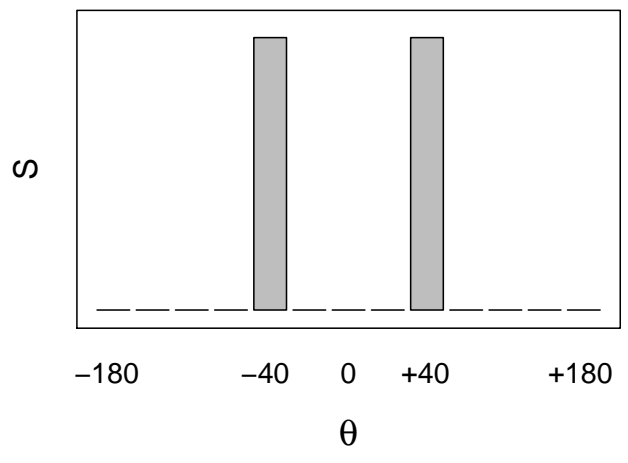

d)

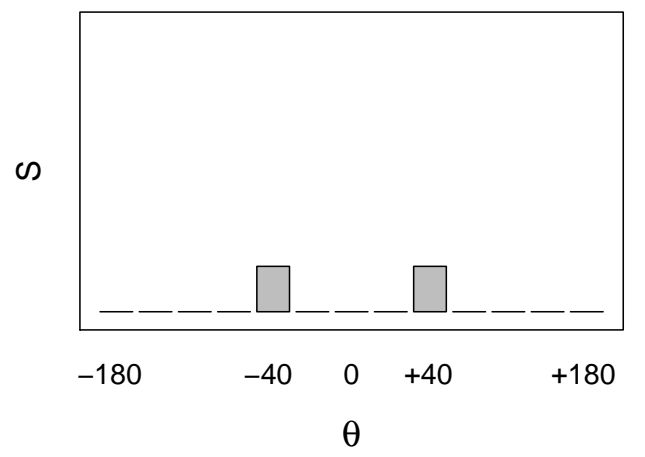

Figure 3.16: Possible signal values.

\begin{tabular}{lll}
\hline Figure & $\boldsymbol{\Delta} \mathbf{S}$ & $\sum\left(\mathbf{S}_{\theta \mathbf{i}}\right)$ \\
\hline 3.16.a & LOW & HIGH \\
3.16.b & ZERO & HIGH \\
3.16.c & LOW & LOW \\
3.16.d & ZERO & LOW \\
3.16.e & HIGH & MID \\
\hline
\end{tabular}

Table 3.2: Signal differences $(\Delta S)$ and total strengths $\left(\sum\left(S_{\theta i}\right)\right)$ 
azimuth of the sensors is not part of the calculation. The difference is defined as the sum of the signal from all the sensors on the right side minus the sum of the signal from all the sensors on the left side (3.2). The difference is denoted by $\triangle S_{\text {in }}$ and defined by Equation 3.3. If a signal is received at exactly $\theta=0$, it is ignored, therefore no sensor should be placed exactly at $0^{\circ}$.

$$
\begin{gathered}
\triangle S_{\text {left }}=\sum_{\theta>-180}^{<0}\left(S_{\theta i}\right) \quad \triangle S_{\text {right }}=\sum_{\theta>0}^{\leqslant 180}\left(S_{\theta i}\right) \\
\triangle S_{\text {in }}=S_{\text {right }}-S_{\text {left }} \\
\sum\left(S_{\text {in }}\right)=S_{\text {right }}+S_{\text {left }}
\end{gathered}
$$

The difference is normalised over the total sum of the signal (3.4) to create a proportional value in the range $-1 \leqslant \Delta S \leqslant 1$ (3.5).

$$
\triangle S=\frac{\triangle S_{i n}}{\sum\left(S_{i n}\right)}
$$

A larger signal on the left will result in a negative $\Delta S_{i n}$. Conversely, a larger signal on the right will result in a positive $\Delta S_{i n}$. Box 3.3 provides details of some subtleties with this calculation.

In practise, it was discovered that the absolute difference in the signal strength was usually too small to shift the azimuth significantly, so an amplification factor was added to Equation 3.5, producing (3.9), where $c$ represents a constant amplification of the signal. This was parameterisable but kept constant for all simulations reported, its value was derived by trial and error until the foragers responded sufficiently to the odour ${ }^{3}$.

$$
\triangle S=\frac{\triangle S_{\text {in }} \times c}{S_{\text {total }}}
$$

Figure 3.16 demonstrates five potential information scenarios, given a forager with two sensors at $\pm 40^{\circ}$ (figure 3.15), and the signals received represented by $S_{\theta 1}$ and $S_{\theta 2}$ respectively. They can be characterised in terms of difference in signal vs overall signal strength to produce Table 3.2.

\footnotetext{
${ }^{3}$ This could be explored more formally in a subsequent study
} 


\section{Behavioural Response}

The movement behaviour of the forager may be affected in one of three ways:

- Adjust the current azimuth (turn "left" or "right")

- Adjust the turning angle concentration of the CRW $(k)$

- Adjust the step length of the CRW $(L)$

This chapter is concerned with adjusting the current azimuth. The framework supports the other responses but they were not explored. It would be possible to allow the forager to respond to plant density for example, by decreasing step length and increasing angle of turn in areas of high signal strength, resulting in an "area restricted", klinokinetic search (Bell, 1990; Chapter 2).

The mechanism for adjusting the azimuth makes use of the information about the difference between signal strengths of the sensors. The conceptual model for this is that the forager is able to detect on which side the signal is stronger and will direct its movement "uphill" toward the source, i.e. it may chose to turn "right" or "left". If the difference is zero, there is no information to indicate whether to go left or right, even if the overall signal is strong, i.e. "There are many cabbages here somewhere, but where are they?!". Overall signal strength does provide some information in that if it is high, the forager must be closer to the source than if it is low, exemplified by 3.16.b.

The information given does not provide an exact location for the source of the sig$\mathrm{nal}^{4}$, so the forager adjusts it's current azimuth and then randomly selects the direction to move in as for the CRW. Figure 3.17 provides a visualisation of the adjustment. This could also be seen as "noise" in the system, i.e. either the information received is inaccurate, or the ability to accurately move in the desired direction is limited.

An additional response might be to "narrow" the turning angle concentration in response to the magnitude of the difference, so that when more reliable information is received (large difference between left and right) the forager becomes less random and more directed toward the source.

A limit (parameterisable) is placed on how far to the left or right the forager may turn. The idea behind this is that in reality a forager in flight may not be able to suddenly turn $160^{\circ}$ to the left. This might depend upon species. For the current investigations, this limit remained set to $\pm 90^{\circ}$. Jones $(1977 \mathrm{a})$ reports movements of $>90^{\circ}$ which is why a transformation of the measurement of directionality was used

\footnotetext{
${ }^{4}$ An interesting difference between the olfaction and vision models
} 


\section{Range for $\Delta \theta$}

A minor, but non-trivial point when implementing calculations as software, is the exact range of $\Delta \theta$ (3.6).

$$
-180>\Delta \theta \leqslant 180
$$

As -180 is congruent to +180 , both cannot be included at the same time. In terms of probability intervals, if regular intervals of $1^{\circ}$ are taken, the first interval is (3.7), and the last interval is (3.8).

$$
\begin{aligned}
& S_{\theta \min }=-180>\Delta \theta \leqslant-179 \\
& S_{\theta \max }=+179>\Delta \theta \leqslant+180
\end{aligned}
$$

A location of 180 is both +180 and -180 simultaneously. It is assumed that the animal is not able to direct itself completely backwards (by limiting the range over which the delta can be affected). Potentially this could become a special case in the calculations, but the solution is not limpid.

In practise, sensors are discreet, they have a definite location, and the simulation can be configured to not have sensors directly at either $180^{\circ}$ or $0^{\circ}$. In the context of this chapter there are only ever 2 sensors, at $\pm 40^{\circ}$

Box 3.3: Notes on the range of $\Delta \theta$ and the location of olfactory sensors.

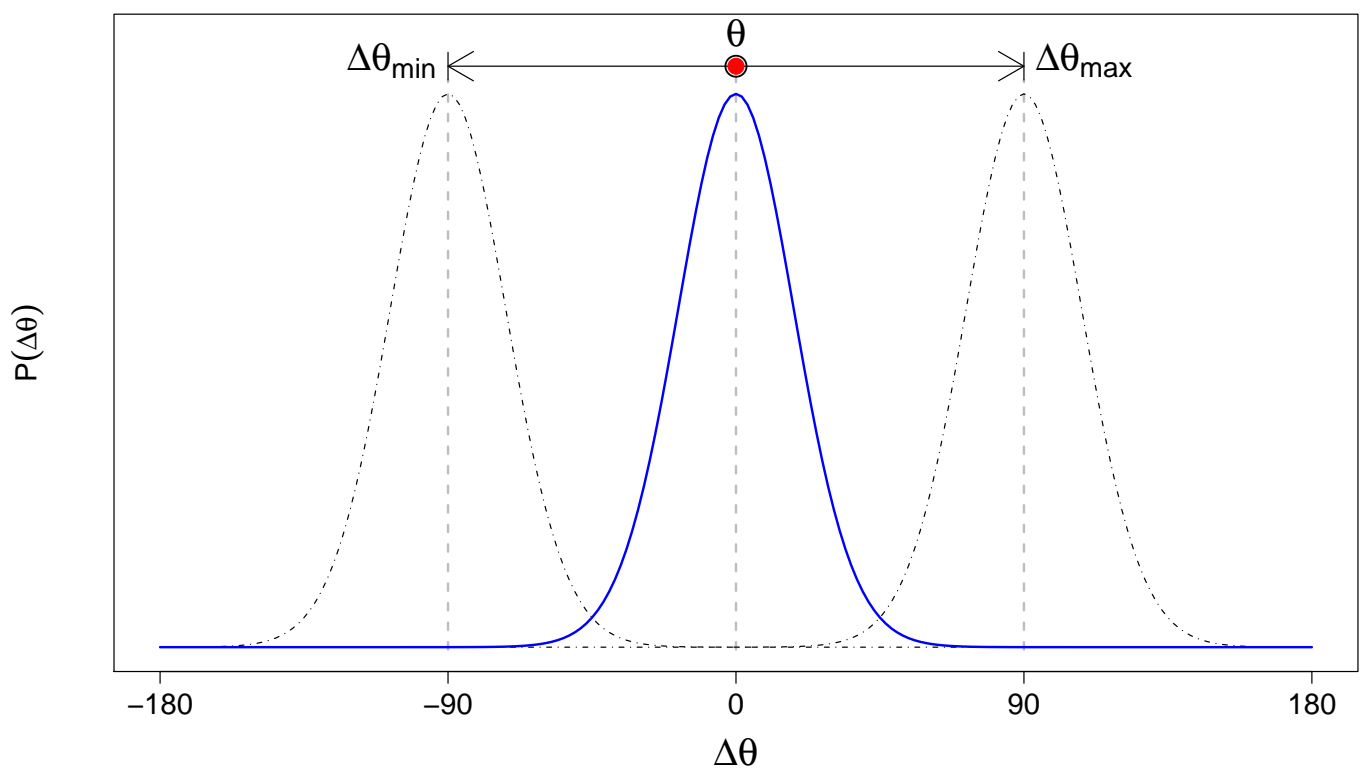

Figure 3.17: Adjusting the current azimuth based on the olfaction information, moving it to either the left or the right. $\theta$ is the current azimuth, $\Delta \theta$ is the change in azimuth to be applied and $-180 \leqslant \Delta \theta \leqslant+180 . \Delta \theta_{\min }$ and $\Delta \theta_{\max }$ represent a parameterisable limit to the movement, or the "range" over which the forager is able to turn left or right 
(Chapter 2), so this may not be completely realistic. However, it generally allows the foragers to orient toward patches producing olfactory signal. Further exploration of this parameter may be required.

In order to respond to the difference in signal, a change in azimuth $(\triangle \theta)$ is defined as a function of $\triangle S(3.10)$.

$$
\triangle \theta=f(\triangle S)
$$

This function should tend towards a minimum when $\triangle S \rightarrow-1$ and a maximum when $\triangle S \rightarrow+1$. The $\tan ()$ function provides a useful transformation. It is assumed that $\Delta \theta_{\max }=-\Delta \theta_{\min }$, i.e. the limit is symmetrical (the forager can turn to the same maximum on each side). In this case the tan of the signal difference is calculated as a proportion of $\tan (1)$ so that the result will vary between -1 and 1 . Multiplying this by the maximum delta, the change in azimuth is given by Equation 3.11 and is in the range (3.12).

$$
\begin{aligned}
& \triangle \theta=\Delta \theta_{\max } \times \frac{\tan (\triangle S)}{\tan (1)} \\
& -\Delta \theta_{\max } \leqslant \Delta \theta \leqslant+\Delta \theta_{\max }
\end{aligned}
$$

Figure 3.18 shows the function with $\triangle \theta_{\max }=90$. As the difference in signal tends toward each side, so the turn becomes sharper in that direction.

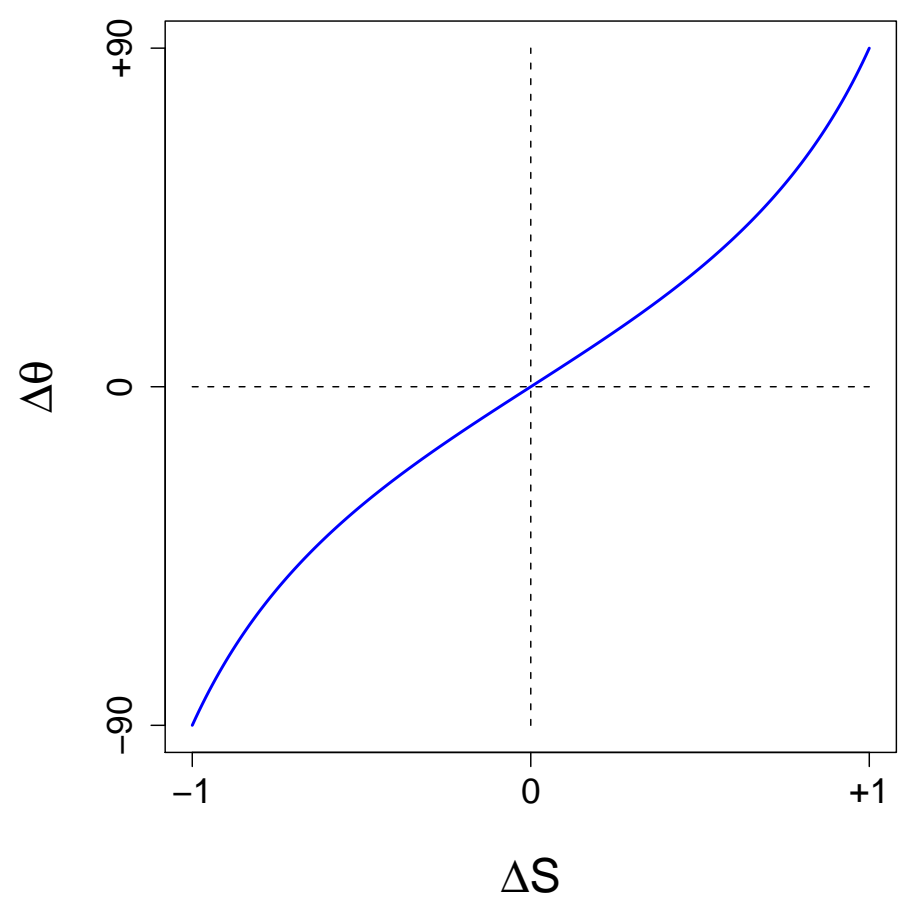

Figure 3.18: Difference in signal related to difference in direction. 


\section{Olfaction summary}

The difference between the left and right hand side signal inputs is calculated. A function based on the $\tan ()$ function transforms this into a relative adjustment of azimuth (negative to turn "left", positive to turn "right"). If the signal difference is high, this will be a tighter turn to the side with the highest signal. If the difference zero, no adjustment in azimuth will occur. In this case the model degrades to a simple CRW. In any case, once the azimuth has been adjusted, a random change remains, representing the imprecise nature of the information, or alternatively, "noise" in the system.

For this implementation, there is no response to overall signal strength, but it could be connected to the parameters of turning angle concentration and step length through an additional functional response.

This olfaction model should generally result in behaviour which leads the forager to high resource density areas.

\subsubsection{Visual perception}

For the purposes of the framework, Vision is assumed to be a shorter-range sense, but to be more informative, i.e. beyond a certain distance objects cannot be distinguished, but if an object is perceived, the forager has information about its exact location. As with olfaction, the visual model can be broken into three subcomponents; a mechanism to determine which objects are visible to the forager (the field of view), generation of a signal input, and a behavioural response to that information.

\section{The field of view}

A visual range is defined within which the forager has the potential to detect objects. The shape of the field of view is a segment of a circle centred on the forager and aligned so the the current azimuth bisects it. Figure 3.19 shows the field of view. Resources which are potentially seen by the organism are highlighted with darker circles in the centre. It's size is governed by two parameters, the radius of the circle segment (named Visual Field Depth, and denoted FD), and the width of the segment in angular degrees (named visual field width, and denoted $F W$ ). Resources are either inside the field of view or not. This is calculated by determining the azimuth from the forager to the centre of each resource $\left(\theta_{r}\right)$. If this lies within the range (3.13), and the Euclidean distance to the resource is less than the field depth 
$\left(d_{r} \leqslant F D\right)$, the resource is within the field of view. No account is taken of the radius of attraction of the resource ${ }^{5}$. The size of the forager is similarly not included, all calculations are from it's current location (assumed to be its centre).

$$
-(F W / 2) \leqslant \theta_{r} \leqslant+(F W / 2)
$$

A narrower field of view represents the concept of "conservation of motion", i.e. that although the forager may be right next to a plant on its left, it is not able to turn toward it due to physical limitations of its flight. By setting a field of view $<180^{\circ}$ the forager is also constrained to a "forward" perception, the forager is unaware of resources behind it. This differs crucially from the model of Jones (1977a) in which foragers were able to return to plants behind them if no further plants were encountered in the vicinity, the implications of which are discussed in section 3.7 .

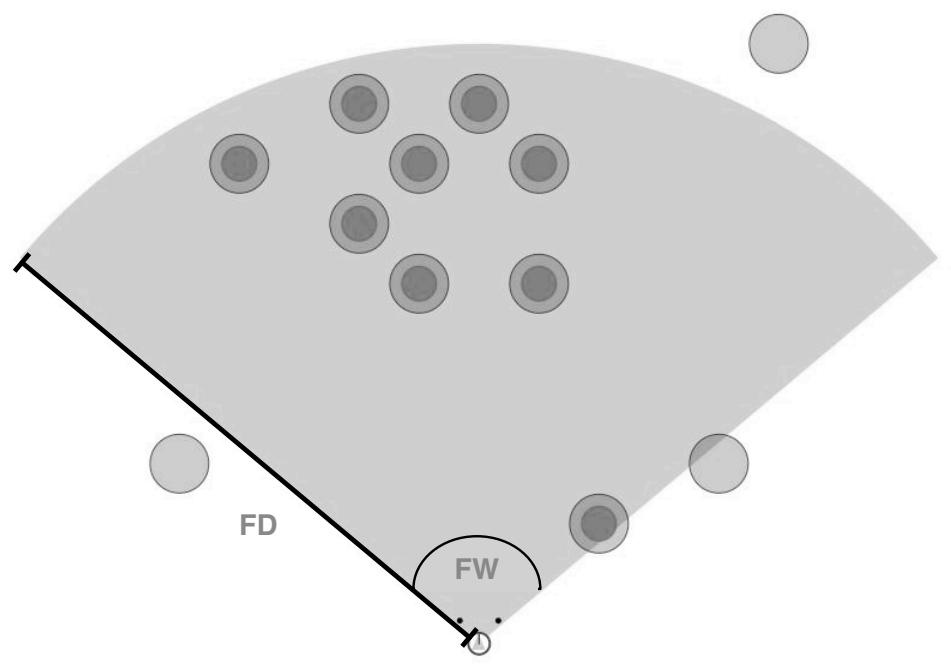

Figure 3.19: Example field of view $\left(F W=100^{\circ}, F D=100\right)$

To create the signal input, each resource within the field is assigned an apparency (Section 3.3.1), denoted by $\alpha$ and representing a "probability of detection".

\section{Signal input}

The information from the resources in view is represented as a set of signal inputs $\left(S_{\theta i}\right)$ corresponding to their direction from the forager relative to the current azimuth (negative is left, positive, right). The signal strength is a function of two parameters,

\footnotetext{
${ }^{5}$ Although this means resources right on the edge might not be included, the field width is a relatively arbitrary parameter and so this will not qualitatively affect the results (simply increase the field width to include more resources)
} 
apparency $(\alpha)$ and distance $(d)$ from the forager, defined as (3.14).

$$
S_{\theta i}=f\left(\alpha_{i}, d_{i}\right)
$$

The effect of distance is represented in the model by the factor $\lambda$, and has the range (3.15). Roitberg and Mangel (1997) calculated the exact angle subtended by a particular object in the field of view, based on its distance ${ }^{6}$, which is a similar model.

$$
0 \leqslant \lambda \leqslant 1
$$

The value of $\lambda$ decreases exponentially with distance. A scaling factor $\gamma$ provides control over the slope of the curve which is defined by (3.16) and plotted in Figure 3.20 .

$$
\lambda=e^{-\gamma d}
$$

The signal inputs are calculated as a discrete set of intervals of $1^{\circ}$, for example the first interval is defined by (3.17).

$$
-180<S_{\theta 1} \leqslant-179
$$

The signal input for a given interval $\left(S_{\theta i}\right)$ is defined by Equation 3.18 and has the range (3.19).

$$
\begin{gathered}
S_{\theta i}=\alpha_{i} \lambda_{i} \\
0 \leqslant S_{\theta i} \leqslant 1
\end{gathered}
$$

Signals from resources which occur within each interval are summed. Based on the layout in Figure 3.19, an example of the input signals is shown in Figure 3.21. Note that the resource which is closest has a much higher signal.

In the experiments described in this and subsequent chapters, $\alpha=1$ and $\gamma=0$ (so $\lambda=1$ irrespective of distance). This has the effect of removing the effect of both apparency and distance. The model is described here for completeness. Including manipulations of these parameters would have increased the parameter space beyond what was achievable within the time-frame of the thesis.

\footnotetext{
${ }^{6}$ Large objects further away will have the same "apparency" as smaller objects closer up
} 


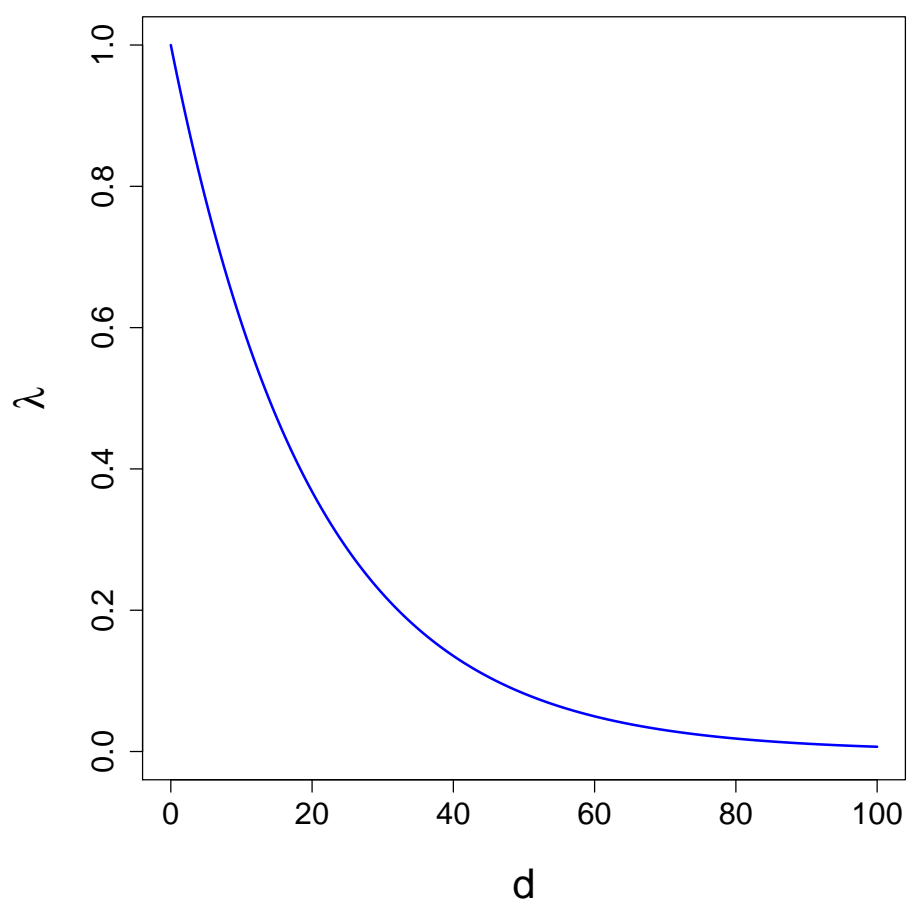

Figure 3.20: "Visibility" as a function of distance, $\gamma=0.05$

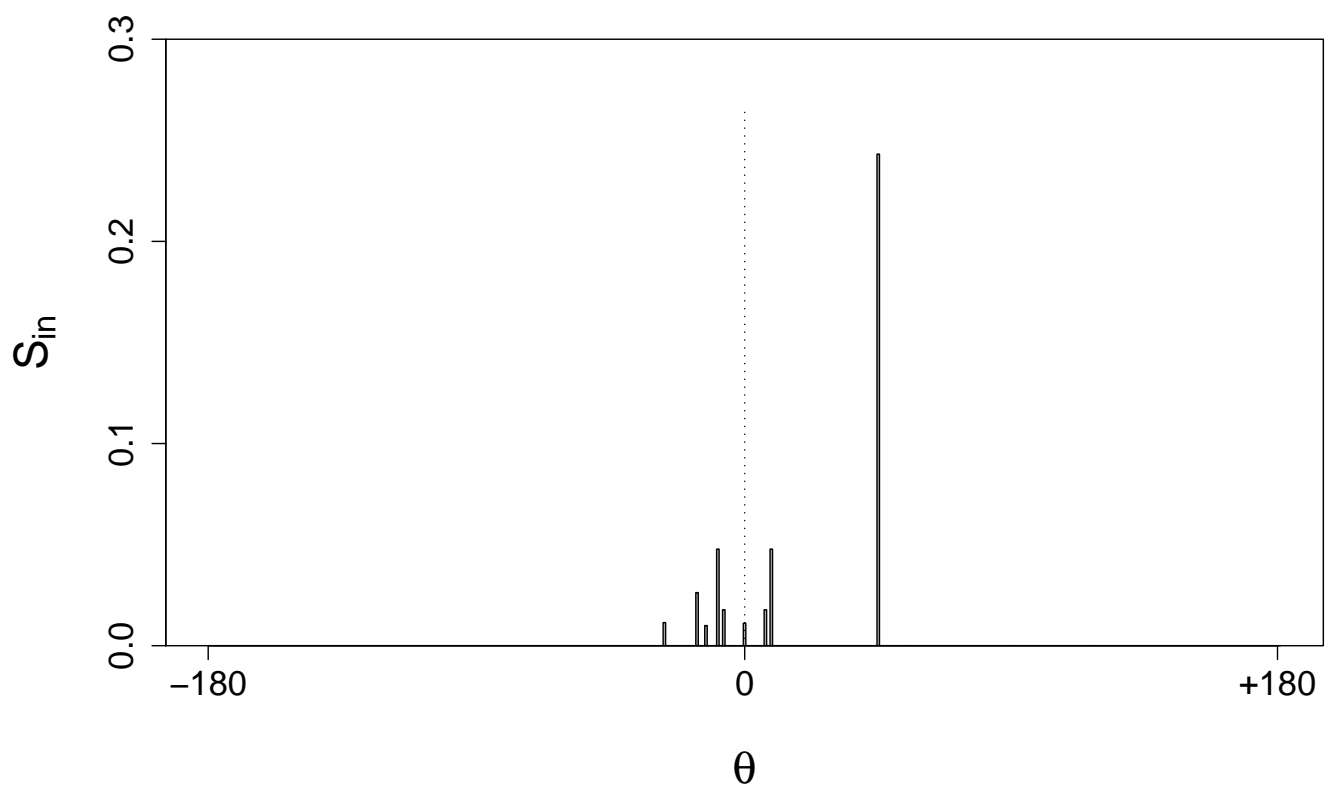

Figure 3.21: Visual Input Signal, $\gamma=0.05$, alpha $=1$ 


\section{Behavioural response}

The behavioural response of the foragers to visual information is more deterministic than for olfaction. Rather than adjusting the current azimuth and then choosing a random direction, the forager directly chooses its direction to be toward one of the resources. The assumption in terms of "real" behaviour is that the forager must make a choice and select a single plant to move towards.

A probability distribution is generated from the signal inputs (3.20) where $P\left(a_{\theta i}\right)$ represents the probability of choosing a particular azimuth (a represents "action" in this notation).

$$
P\left(a_{\theta i}\right)=\frac{S_{\theta i}}{\sum_{i=1}^{n}\left(S_{\theta i}\right)}
$$

The result based on Figure 3.21 is shown in Figure 3.22. This is used to select a new azimuth at random. As mentioned, the experiments that were run were parameterised to remove the effects of apparency and distance which meant that all resources in view had an equal chance of being chosen. If distance is allowed to affect the result, although close resources may generate more signal, a large number of resources in the near distance may actually out-weigh the close resource. In Figure 3.22 , the close resource actually only has a $P \approx 0.5$ just over half a chance of being selected, although it still has a relatively high chance on an individual basis. Once the agent moves away from it toward the group, it is unlikely to be found again.

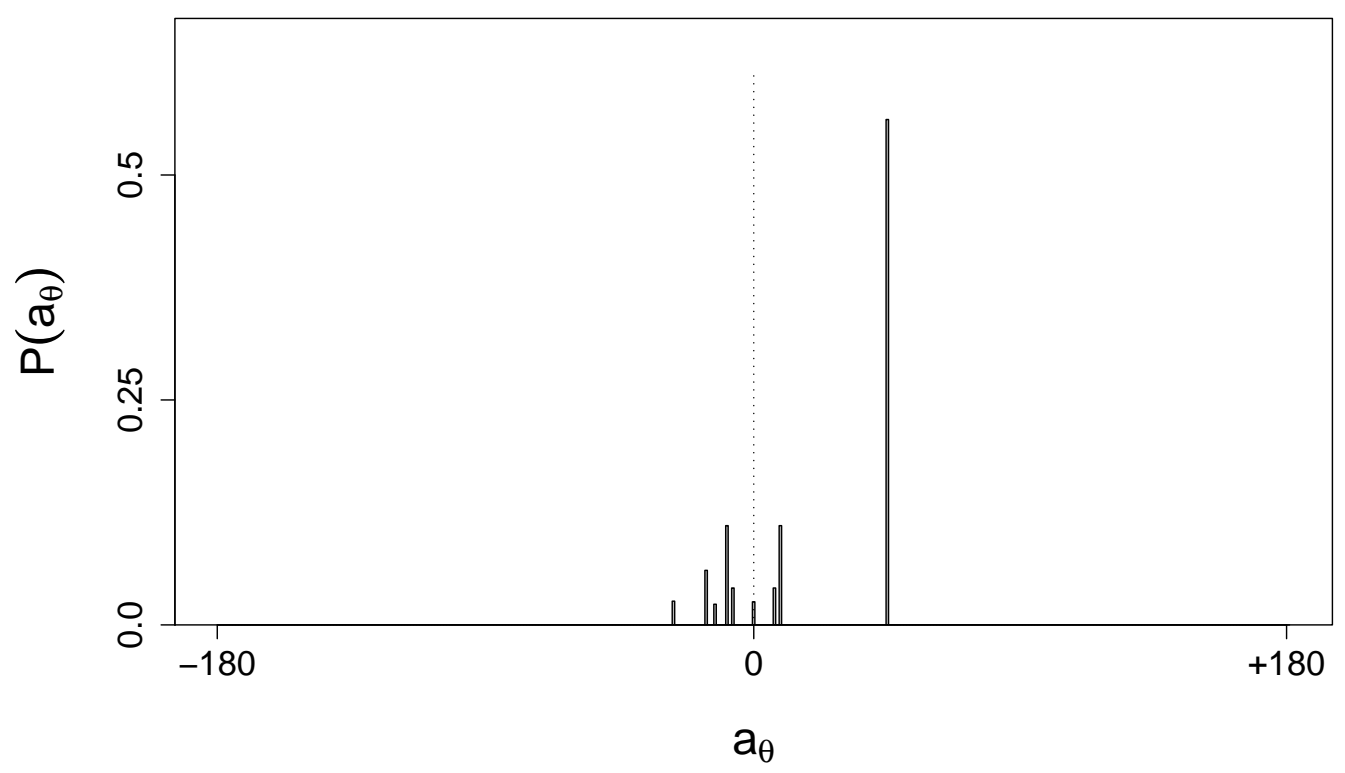

Figure 3.22: Probability of action, $\gamma=0.05$, alpha $=1$ 


\section{Vision summary}

A forager in the model has a field of view within which it can potentially detect all resources and know their direction. A signal strength is calculated for each resource in view based on a function of apparency (ease of detection) and distance from the forager. Apparency is a product of resource attributes, shape size age, isolation. Distance from the forager results in some decrease in the ability to detect it.

These input signals are used to derive a discreet probability vector and one of the resources is chosen from this at random. The forager then moves one step length directly towards this resource.

In a similar manner to the olfaction model, it would be possible for the parameters of the random walk (step length and turning angle concentration) to respond to the total signal value. For example, if the forager sees many resources it may take shorter step lengths and increase its turning, producing an area-restricted pattern.

There are two possible behaviour scenarios that could be modelled with this system:

- Prefer groups of plants to individual ones, even if they are close

- Always choose closest plant

The first is the setup which has been used throughout. If all plants irrespective of distance from the forager are given equal weight, the forager will more often than not head toward a group of plants than an isolated one, within the scale of the field of view. The second scenario could be generated by parameterising an decrease in signal strength with distance, setting this to the extreme would mean that plants at the edge of the visual field would rarely be moved towards. This could also be achieved by reducing the visual range which is another reason it was not explored in the current study.

One issue with the field of view model is that if there is a single plant in the field of view, it will dominate the view. Box 3.4 describes this issue in more detail.

\subsubsection{A Signal-based Random Walk}

Combining visual and olfactory signals does not have an immediately obvious solution. For example the forager may see many resources at close range to the left and yet "smell" a strong signal to the right, presenting a conflict. One method to represent this would be attempt to "weight" the incoming signals with and give them an overall probability distribution so that all alternatives could be selected from at random. Some models along these lines were constructed but they became rather complex. 


\section{Visual Signal Noise}

There is a potential issue with the visual perception model. If there is only a single plant in the field of view, it will be certain that the forager will move directly toward it, irrespective of distance, which may introduce a bias in the resulting egg distributions. This functionality was not actually put into practise, but is described here for information purposes.

Two solutions were considered; a) introduce a secondary random generation which determines whether the plant is "seen" or not, or b) instead of directly setting the azimuth, use a mechanism similar to the olfaction and in-fact only "suggest" a direction, following which the final azimuth is drawn from randomly. This would equate to a visual noise in the same way as with olfaction, and would depend on distance, so that the farther away the resource, the more random the movement. As the forager gets closer to the resource, it becomes more deterministic.

It was chosen to implement b) as it had a more "natural" behaviour and also a) would be computationally intensive. However, unless distance is a factor in the visual signal, it will be ignored and so is described here only for completeness as it was not in effect for any of the simulation experiments described.

\section{Calculation}

As $k$ increases, the angle of turn becomes more concentrated. It is assumed that $(k)$ can not fall below that currently parametrised $\left(k_{c u r r}\right)$. So In order to become more directional, a functional response to distance is required that adjusts the $k$ parameter of the Von Mises distribution from its current value $k_{c u r r}$ to some maximum $\left(k_{\max }\right)$. The distance to the resource $\left(d_{\theta i}\right)$ is first represented as a proportion of the total field depth $\left(d_{F D}\right)$, so that it will be in the range $0 \leqslant d_{F D} \leqslant 1$ (3.21) The resulting relative distance measure is denoted $D$.

$$
D=\frac{d_{\theta i}}{d_{F D}}
$$

Figure 3.3.4 graphs this response for $k_{\text {curr }}=2, k_{\max }=10$. This relationship is defined by (3.22) where we calculate a "noise" factor to add to our current $k$. This is represented by the function $\xi\left(k_{\max }, P_{S \theta}\right)$, where $P_{S \theta}$ is the Probability that this Signal direction was chosen.

$$
k=k_{\text {curr }}+\xi\left(k_{\text {curr }}, D, k_{\max }\right)
$$

The noise function is defined as (3.23).

$$
\xi\left(k_{\text {curr }}, D, k_{\max }\right)=\left(\psi \times k_{\max }\right)-\left(\psi \times D \times k_{\max }\right)
$$

Where $\psi$ is defined by (3.24) and ensures that the slope of the response fits the line between $k_{\text {curr }}$ and $k_{\text {max }}$.

$$
\psi=\frac{k_{\max }-k_{c u r r}}{k_{\max }}
$$

Box 3.4: Notes on visual signal noise 


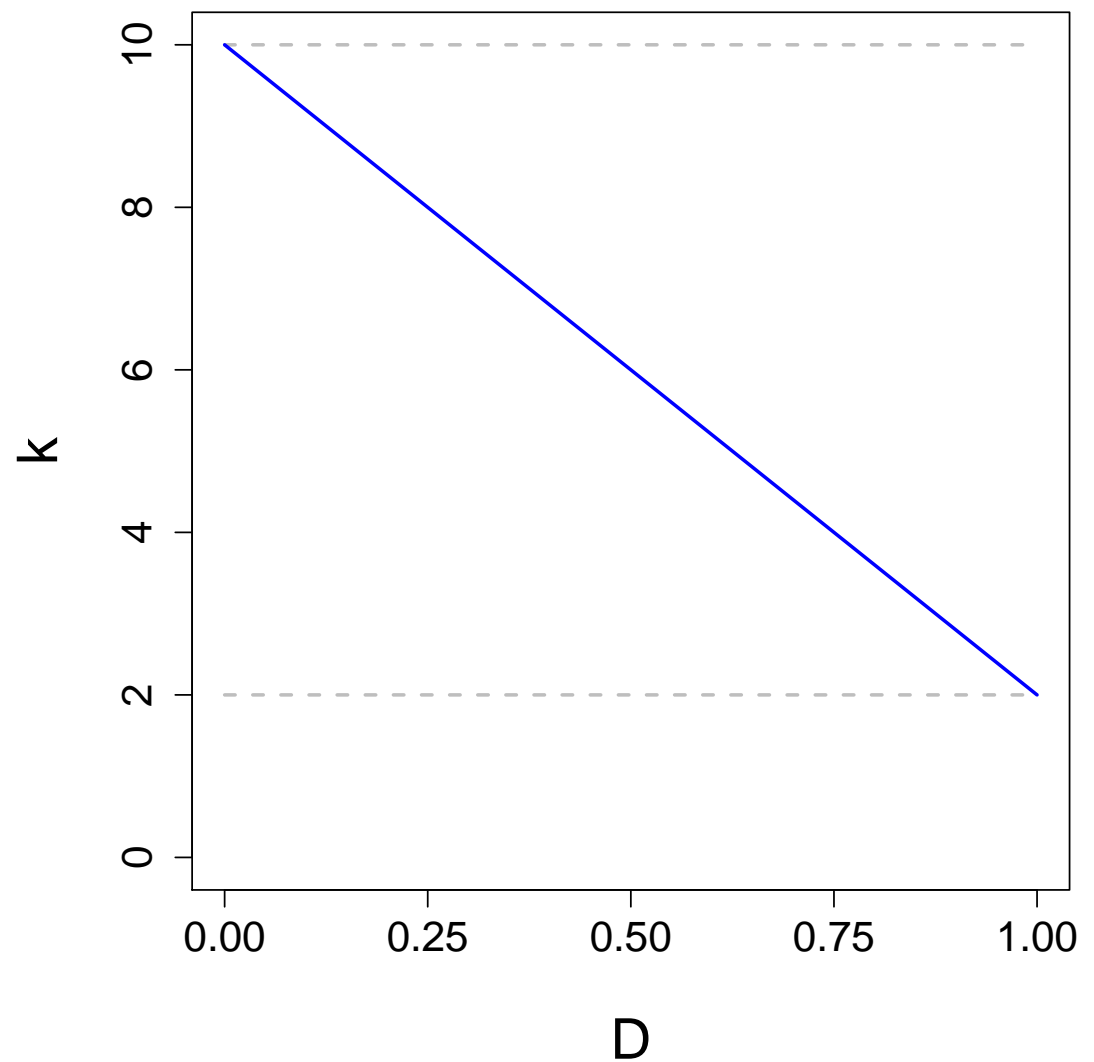

Figure 3.23: To accompany Box 3.3. The relationship between relative distance of the resource $(D)$ and $k$. 


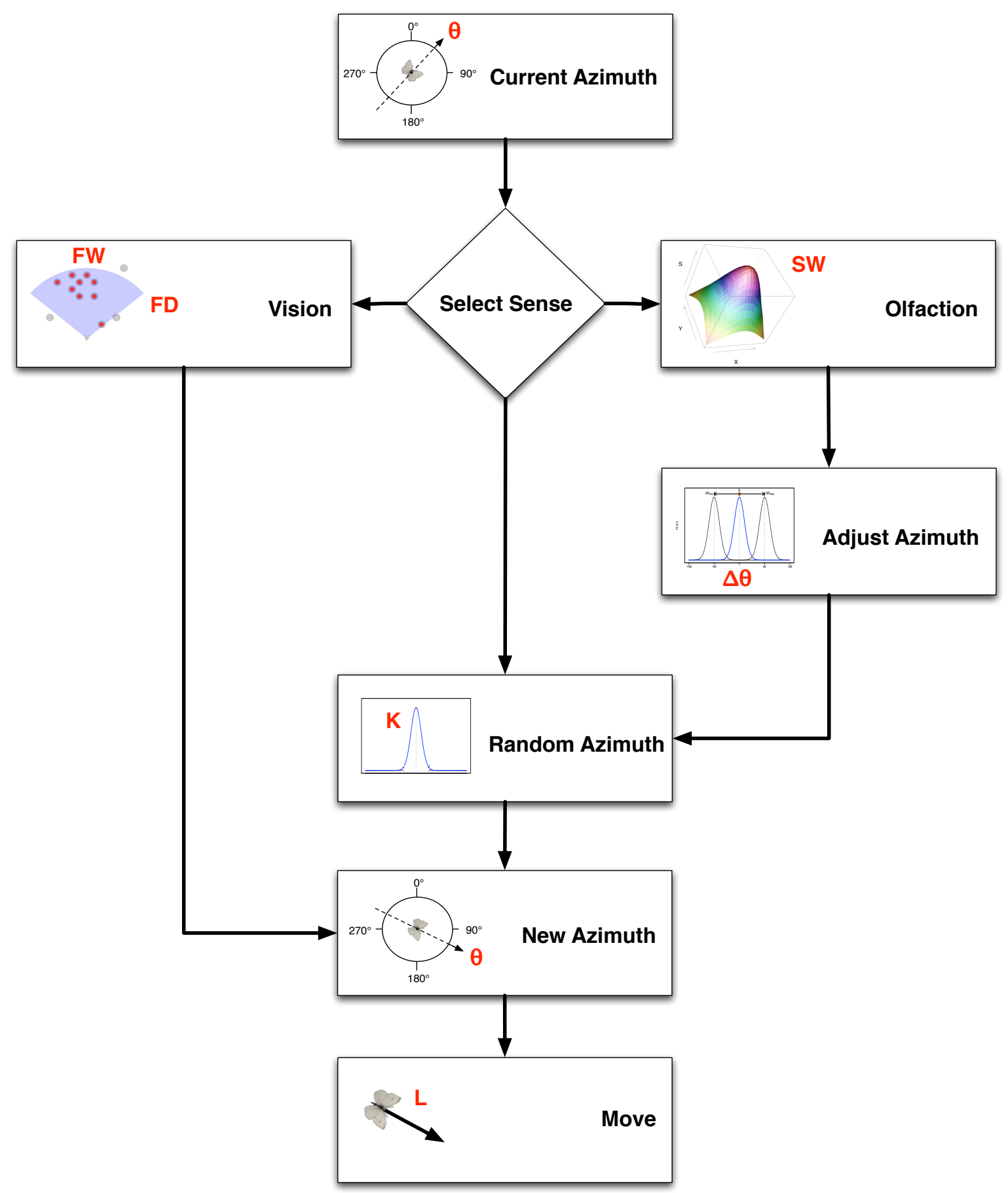

Figure 3.24: Summary of conceptual model for a signal-based Correlated Random Walk. The main parameters discussed in the text are shown in red. 
In order to keep the framework as simple as possible a simple rule applies that visual stimuli will always over-ride olfaction. The assumption here is that whilst olfaction may help a forager to locate a patch, once within the patch, visual recognition will be far more effective. In the absence of either signal, the movement is that of a CRW. It is possible to enable or disable both "senses", and therefore configure foragers which are entirely visual, entirely olfactory, purely random or any combination of the three. Figure 3.24 shows the overall structure of the behaviour of the model.

The movement behaviour of the forager begins with its current azimuth. At the first time step, this is usually selected at random (Section 3.4). A decision is then made about which sense to use to determine a new azimuth. This is based on whether there is a signal present and whether each sense is to be included. In the absence of any signal the flow moves directly to the generation of a random azimuth from a probability distribution which by default is the Von Mises distribution. A visual signal will over-ride the olfactory signal.

Once the new azimuth is chosen, the forager moves 1 step length $(L)$ in that direction. It is at this point that "foraging" behaviour will be executed. If the move has intersected with one or more resources, the forager will oviposit an egg on each resource (dependant on the number of eggs it has). Once all eggs are laid, the forager is removed from the simulation.

A refinement that is available in the framework is to limit the level of visual signal which "activates" the vision processing ${ }^{7}$. However, this will only be effective if the signal depends on distance and has yet to be tested experimentally.

There are two further concepts which although not included in the experiments are noted here for interest, a dynamic "Motivational State" of the forager (Chapter 2), and a possible extension concerning external factors affecting the foragers movement (Noise in the action).

\section{Motivational State}

One aspect of the oviposition behaviour not represented in this model is any kind of behavioural "state" of the forager. It would be possible to extend the behaviour of the foragers to include such a motivational state. For example, immediately after oviposition, the forager may no longer respond to the information it receives about host resource location, and return to a random movement. This could continue for a fixed number of timesteps at which point the "desire to oviposit" would be re-

\footnotetext{
${ }^{7}$ There is a visual signal "threshold" parameter. The simulation tests the maximum visual signal received to see if it exceeds the threshold and if so, the forager is said to be receiving a visual signal. Generally this threshold is set to zero so that all signals are responded to
} 
instated and the forager would respond once more to the resources. This is termed here a "refactory period" as it might represent a period of time when the butterfly is producing more eggs and thus be equivalent to the "motivational state" of the forager (Hern et al., 1996). An equivalent is found in Jones (1977a)'s model, where parameters were linked to the current fecundity of the forager, defined by how many eggs it had left to lay and estimated from observed oviposition rates and dissections.

\section{Noise in the action}

The cabbage white has a relatively erratic flight (Graves, 1965), the cause of which may not be certain but the effect of less than straight line movement might be important to a simulation model. It might be termed "noise" in the resulting action of the forager. Although it may perceive resources in a certain direction, and attempt to move in that direction, the actual result may be that it is displaced to another location, either because its actual movement mechanism is inherently erratic, or through movement of the substrate through which it is moving (wind in the case of butterflies, perhaps water in the case of aquatic animals).

The model does not include a concept of wind but a potential addition would be to translate the simulated move by some vector representing the wind, much as a sailor accounts for currents and wind when charting a course.

Random noise may already be represented at least in the olfaction model, as a random azimuth is always chosen. The architecture of the framework also supports randomness in the azimuth when using the visual mechanism (Box 3.4).

\subsection{Methods: Simulation features}

The implementation of the conceptual model (hereafter referred to a "the framework"), is described here in some detail, providing an overview of the features that the framework supports. Not all of these are directly related to the behaviour of model.

The framework was implemented in the Java (TM) programming language. This is an Object-Oriented (OO), language that was chosen because it has a fast development time and because software developed with it can be run easily on both Linux and Windows systems which makes it more available to future researchers. Faster execution times might be possible by re-implementing at least the execution engine in a lower level language such as $\mathrm{C}++$, however, other methods of optimisation are 
available as reported below, particularly the use of multiple processors or computers.

A key concept to making use of the framework is that of the "experiment plan" which represents a particular set of initial parameters combined with a "plan of action" concerning which parameters should be varied to explore the parameter space. When organising the experiments it was found helpful to create a general experiment grouping and within that, have a set of "Trials" which represent variations on a theme. Two examples of these are described in more detail in Section 3.5.

The framework itself is composed of three basic sub-systems:

- Parameter configuration (experiment editor)

- Execution (experiment controller)

- Results analysis ( $\mathrm{R}$ "reports")

Each sub-system is a stand-alone application. The experiment editor application allows the researcher to set the many parameters and produces an $\mathrm{XML}^{8}$ file which completely defines a particular run of the simulation. This "experiment plan" is then executed by the experiment controller. The output is in the form of a structured set of $\mathrm{CSV}^{9}$ files which contain the results of the experiment. These files are analysed with scripts written in the $\mathrm{R}$ statistical programming language ( $\mathrm{R}$ Development Core Team, 2005). The scripts are organised to represent various "reports" which are usually specific to an experiment but may have a general use. These make use of a library of routines that was created to generate PDF files from within $\mathrm{R}$ via an intermediate $\mathrm{ET}_{\mathrm{E}} \mathrm{X}$ file (Mittelbach, 2004).

This section begins by describing the features of the framework from the perspective of implementing the conceptual model described in Section 3.3. It then describes in some detail each of the sub-systems involved and concludes with some notes concerning the validation (testing) of the implementation.

\subsubsection{Landscape}

The "landscape" represents the "world" in which the agents live. It is an area of 2D continuous space and is responsible for containing the agents and allowing them to communicate. An example would be that a foraging agent is able to discover which other agents are in its vicinity from the landscape. The landscape can also be used to represent other environmental features, for example the odour signal is part of the landscape.

\footnotetext{
${ }^{8}$ eXtensible Markup Language

${ }^{9}$ Comma Separated, Variable-length field text file
} 
More complex behaviour would be possible, particularly wind or water movement. An interesting option would be to integrate a GIS (Geographic Information System) module to the landscape that would allow researchers to represent more complex environments with "real" features such as roads, fences, rivers, etc. These could be directly loaded from existing GIS maps. This however is not covered in the described implementation. In essence the landscape is a rather simple, clean place to exist.

\subsubsection{Agents}

There are two agents of interest that can "live" in the landscape; foragers and resources. All agents which have a representation in the landscape are derived from an abstract "physical agent", which means that they can be interacted with by other agents and always have a current "location". The location is a 2D point in the landscape space denoted by $P(x, y)$.

The framework provides a structured way to build both agents and strategies and allow them to be parametrised. With some work, it would be possible for third parties to develop new agents and register them with the framework dynamically without need to recompile the entire system. Another possibility would be to create a generic, "scriptable" agent whose behaviour is controlled by a scripting interface and therefore investigators would be able to create new agent behaviours based on the basic agent structure. These concepts could provide a powerful tool for researchers to explore agent-based, spatial simulation without requiring the detailed software development skills that were necessary to create the framework.

The experiments described here are limited to a single class of foraging agent, representing butterflies. It is also possible to parameterise a second "species" of forager and a "species" of parasitoid. The intention was to conduct some experiments involving population dynamics in terms of predator-prey and competition models. Unfortunately time constraints prevented this work, but it remains a future possibility.

\section{Resources}

The resources have three attributes; a location, a radius and an egg count. Further attributes such as age, colour, etc could be added. There are two methods for initialising resource layouts; a) generate a layout according to a predefined algorithm, and b) specify the layout in a file. The first option is used for the experiments in this chapter with a simple layout algorithm that creates a square "patch" of resources 
evenly spaced (Figure 3.1). The second option is used to specify layouts which were also used in field experiments (Chapter 4).

The simple layout algorithm can be parametrised In terms of patch size $(P)$ and radius of attraction ( $R$, spacing is calculated), and $\mathrm{b}$ ) in terms of radius of attraction $(R)$, or by how much spacing is desired (patch size, $P$ is calculated). It is straightforward to calculate between the two. The spacing is denoted by $I$ for "inter-edge spacing" (i.e. the spacing between the edges of the radii of attraction). Figure 3.25 summarises these parameters visually.

The input file for the resources is a simple CSV file containing a list of $(x, y)$ locations and a radius for each resource. It is possible to specify a radius that will be applied to all resources.

Further layout algorithms are possible. For example a fractal algorithm was constructed using a Lévy flight (Kenkel and Walker, 1996; Mandelbrot, 1983) to generate a spatial pattern with a particular fractal dimension (Appendix C). Cain (1985) had two kinds of layout, "clumped" and "even". It would be simple to implement a calculated layout of this type.

\section{Foragers}

Forager agents are composed of a number of behavioural "strategies". Each strategy is essentially an algorithm that defines an aspect of the agent's behaviour, and may be replaced by a different strategy if required. The behaviour is divided into three categories:

- Sensors (visual and olfactory)

- Behaviour (movement and oviposition)

- Population (immigration, reproduction, and mortality)

Figure 3.26 shows a summary of the structure of a forager agent, showing the main parameters which are in each category. The sensors connect the forager to the landscape and allow it to receive the signal input. The movement behaviour strategy (signal-based random walk) encapsulates the entire flow described in Figure 3.24. It can be parameterised to enable or disable the two sensory inputs. Movement itself is divided into two sub-strategies, the movement generator and the azimuth generator. This provides extra flexibility in terms of controlling the move length which would allow for example a Lévy flight (Kenkel and Walker, 1996; Mandelbrot, 1983). The azimuth generator can be swapped between various probability distributions; The Von Mises, a wrapped Cauchy, wrapped normal, and a linear Gaussian. 


\section{Calculated Layout Parameters}

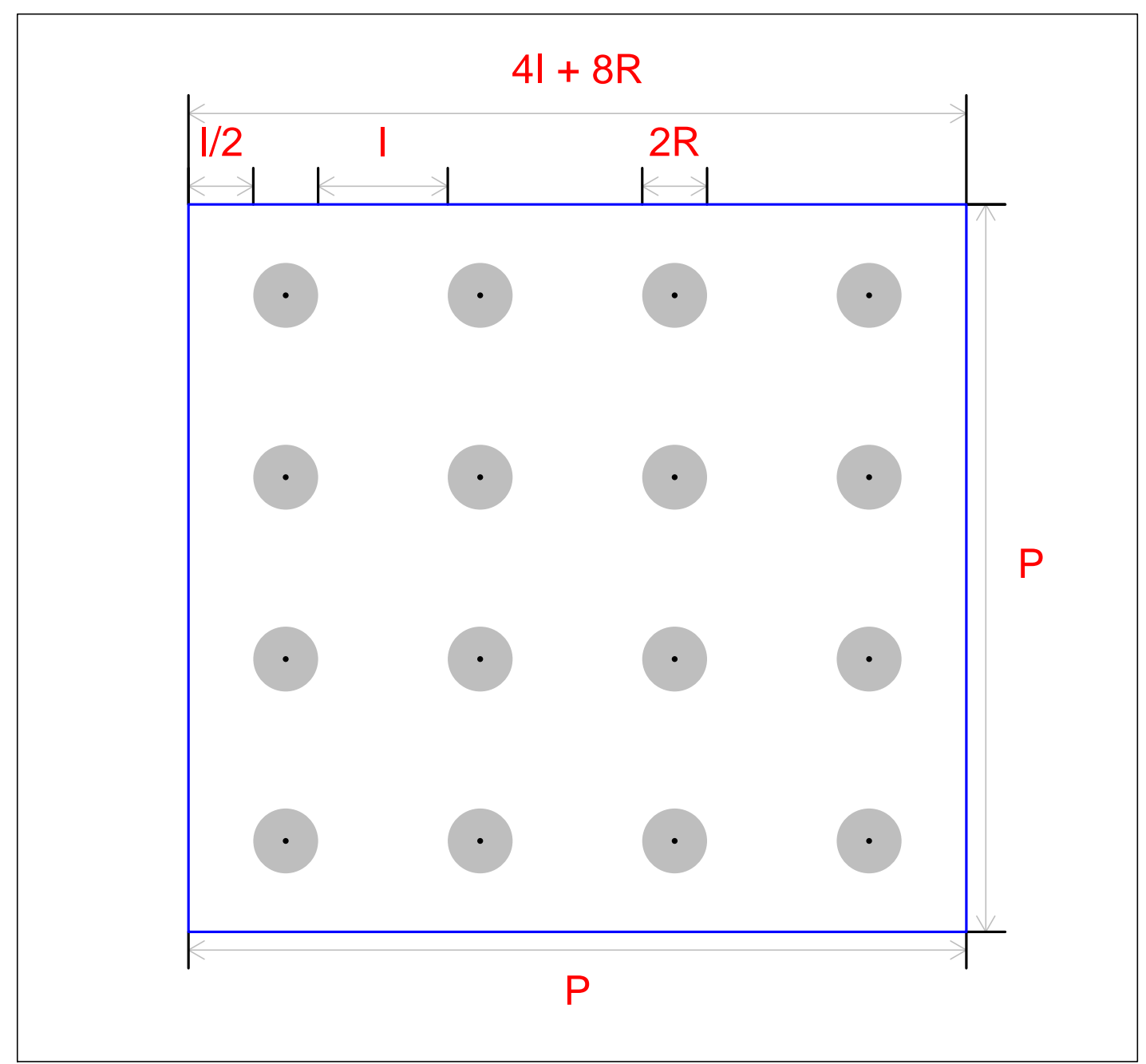

Figure 3.25: Representation of the parameters used to control the simple calculated layout. $I$ is the inter-spacing between radii of attraction, $R$ is the radius of the circle of attraction, and $P$ represents the dimensions of the patch. In a square patch (usual case) $P$ is simply the length of the side of the square. The default is to have a $4 \times 4$ resource layout but this can also be parameterised, in which case the size of $P$ becomes $P=(n \times I)+(n \times 2 R)$ where $n$ is the number of resources on one side (4 in this diagram). 
The population strategy controls how foragers come into existence in the simulation, and how they are removed, or "die". Initially the experiments were concerned with immigration into a patch, i.e. the population of butterflies passes over the experimental area. The addition of a new forager into the simulation is termed "release" if it is immigrating, and "birth" if it is created in the location of a plant which had an egg on it, although it is known that caterpillars disperse away from the host plant to pupate (Jones, 1977a,b). Foragers "die" either when they have no more eggs or have reached a specified age (number of timesteps).

After initial trials it was decided that releasing one forager at a time until a certain number eggs had been laid was the optimal configuration for generating comparable results. Releasing many foragers at once introduced a bias in the result because as they reached a resource they would "die". Resources that were closer to the release boundary therefore received a disproportionately greater number of eggs because execution would halt whilst other foragers were still alive and still "en route" to resources that were farther away. This bias increases as the number of simultaneous foragers released increased, but is only a problem if the treatment is completed when a specified egg limit is reached.

\section{Release Boundary}

In order to implement the "release" of foragers in a meaningful way, the framework has the concept of a "release boundary". This can be seen as equivalent to a "window" over a larger area, into which over time, foragers enter and leave, as if looking down on a large field. When outside the area of observation nothing is known about the foragers. It was decided to adopt a circular boundary centred on the experimental patch, distanced from the experimental area. It would be possible (and is supported by the framework) to use a square boundary, but this seemed less intuitive and potentially involves some bias at the corners.

Figure 3.27 shows the overall layout of the landscape in the simulation. The experimental area is in the centre. There are in fact three boundaries, two of which are parameterisable. The "Zero Boundary" represents the minimum release distance. It is the enclosing circle of the experimental layout. The release boundary is then circular and can be distanced from the zero boundary. The parameter $B$ controls this distance and is equivalent to the difference in radius between the two boundaries. The landscape itself has a boundary beyond which foragers are not observed, which is distanced from the release boundary by $L B$. If a forager moves outside this boundary it is removed from the simulation. This represents the "area of observation". In all experiments reported here, the landscape boundary and the release boundary are set to the same distance from the resources $(L B=0)$. 


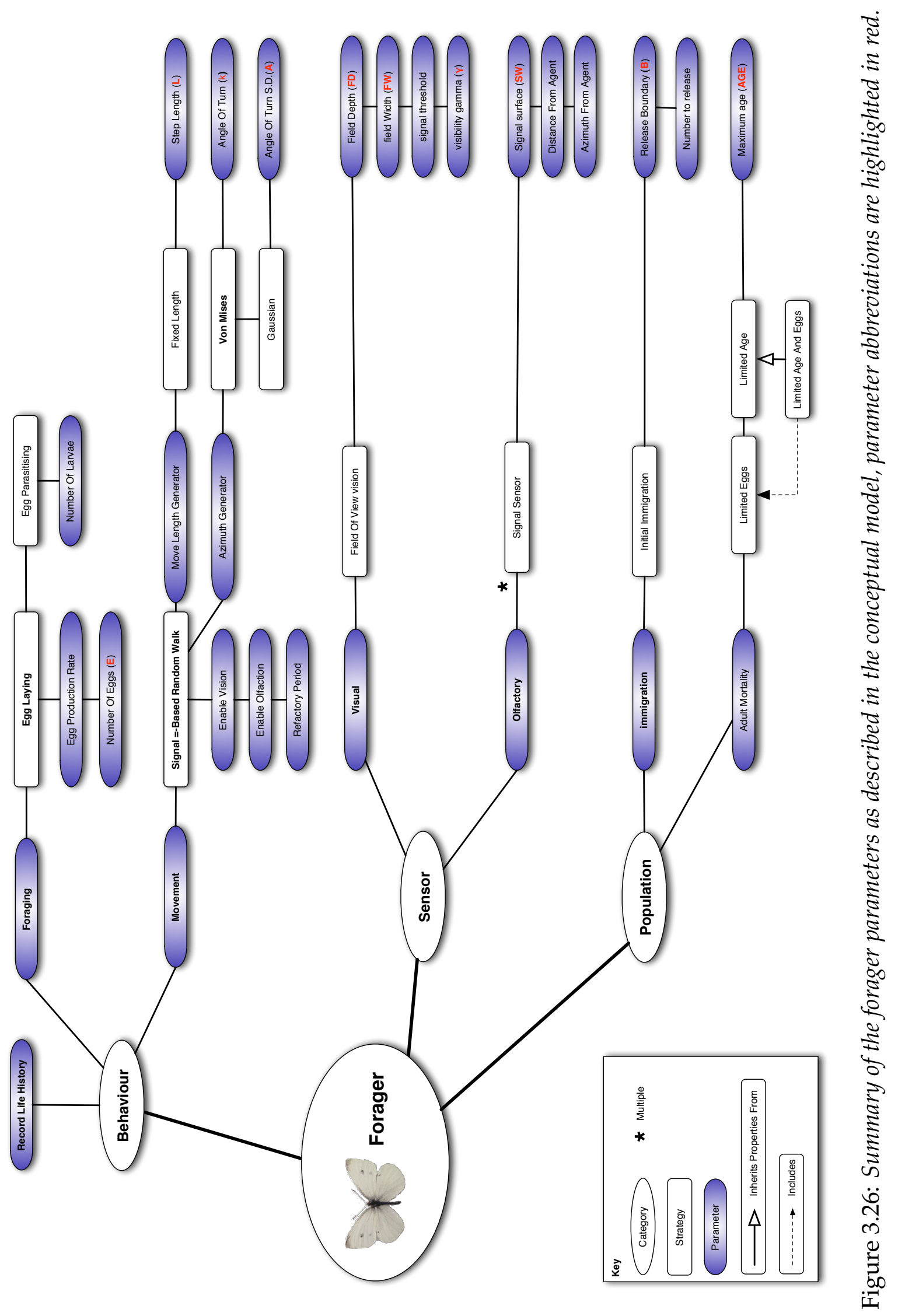




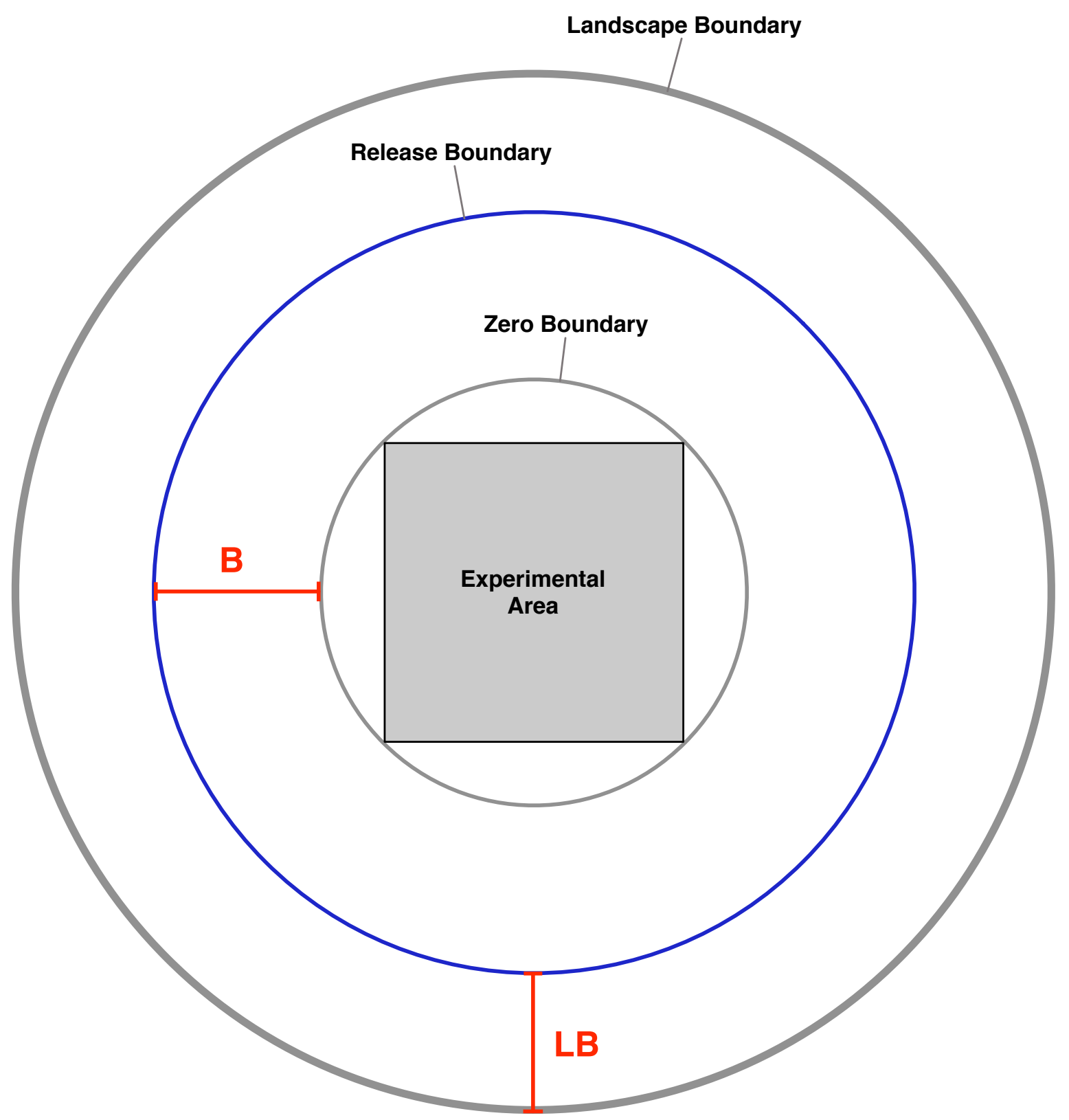

Figure 3.27: Representation of the parameters used to control the release boundary. A circular boundary is shown here, although the framework supports rectangular boundaries. In the centre is a square "Experimental Area" where the resources are placed. The foragers are "released" at a random point on the "Release Boundary". In general the landscape boundary is set at the same distance from the resources as the release boundary, it is shown here distanced by a further amount. The distance of the release boundary is parameterised by $B$ and the landscape boundary by LB. 
Foragers are initially created with a uniform random azimuth ${ }^{10}$, at a random point on the release boundary. Their first move will be in this initial direction unless sensory input diverts their attention. Thereafter they behave as described in (Figure 3.24) They are allowed to move through the landscape until they "die", or move outside the landscape, or "escape".

\subsubsection{Parameters (experiment editor)}

When building a behavioural model, it is often unknown at the outset which parameters will have the greatest effect on the response. One of the goals in the design of the framework was to allow any aspect of the behaviour to become part of the parameter space. Whilst this leads inevitably to a larger parameter space, it also means no behaviour is ignored. In order to make this feasible, a structured approach to managing the parameters was required. This applies to the configuration of the parameters for a particular experiment, to the definition of a set of manipulations desired, and to the analysis of the results (i.e. maintaining a link between which set of parameters generated a particular result).

The result was that a component of the framework was developed that specifically manages an arbitrary set of parameters, which may be organised hierarchically into groups (categories), and may contain behavioural "strategies"11. This is referred to as a "Parameter Map". A subset of the total parameter map has been shown in figure 3.26 .

A list of parameters which are most commonly manipulated is given in table 3.3. Strategies may be replaced and may themselves contain parameters. Some parameters are dependant on the values in other parameters; for example the size and location of the release boundary is dependant on the size and location of the experimental area. This is made possible through a specialisation of a parameter, called a "derived parameter" which may contain a reference to one or more source parameters and an algorithm for calculating its derived value.

By generalising the parameter framework a number of useful tasks may be accomplished:

- Consistent, structured, code-level organisation

\footnotetext{
${ }^{10}$ There is an optimisation which filters out foragers which would move outside the landscape within the first step to help processing speed

${ }^{11}$ Another way to view a strategy is as a parameterisable algorithm
} 


\begin{tabular}{lll}
\hline Code & Parameter & Values \\
\hline AGE & Age limit for forager & 1000 \\
E & Number of eggs per forager & 1,10 \\
G & Egg count limit (run until this many eggs laid) & $50 \rightarrow 2000$ \\
\hline L & Step length & $100 \rightarrow 10000$ \\
$\mathbf{k}$ & Turning angle concentration & $0.5 \rightarrow 10$ \\
VE & Vision enabled & true, false \\
OE & Olfaction enabled & true, false \\
FD & Visual field depth & $100 \rightarrow 1800$ \\
FW & Visual field width & 120 \\
\hline B & Release boundary distance - (Figure 3.27) & $0 \rightarrow 800$ \\
I & Inter edge separation (Figure 3.25) & $-75 \rightarrow 140$ \\
$\mathbf{P}$ & Patch size (Figure 3.25) & $100 \rightarrow 600$ \\
$\mathbf{R}$ & Resource radius (Figure 3.25) & $5 \rightarrow 50$ \\
SW & Signal width for odour "plume" & 1500 \\
\hline
\end{tabular}

Table 3.3: List of most commonly manipulated parameters. Example values are shown, these include simulations from Chapter 4. The values for step length will be relative to the size of the landscape. In all simulations based on field layouts, the scale was 1unit $=1 \mathrm{~cm}$. A length of $10 \mathrm{~m}$ (10,000units) was used as the landscape was larger than $200 \mathrm{~m}^{2}$.

- Generic Parameter Manipulation

- Simple representation as an XML file

- Generic Reporting

At the code level, it is useful to have a consistent and well defined way to access and manipulate the parameters. One task suited to this functionality was the method by which parameter values are systematically adjusted to explore the parameter space, termed "parameter manipulation". A set of manipulations are defined (e.g. vary $L$ using the values "1", "10", "100" and vary $k$ with " 0.5 ", "3", "10"). The framework constructs a matrix of each combination of these two manipulations which is executed as a set of treatments (see Section 3.4.4).

The combination of a set of initial parameter values and a set of parameter manipulations is termed an "experiment plan". The experiment plan can then be written as an XML file which can be passed to the experiment controller for execution. As each parameter combination is executed, the results are stored separately along with an 
XML representation of the specific set of parameters that were used to generate the results. This allows a generic reporting framework to be constructed which eases the analysis of the results (Section 3.4.6). More importantly it means a complete record of the parameters used is maintained directly with the results. It is then possible to not only read the parameter setup but to re-execute that particular experiment directly.

The parameter management component of the framework is independent of the core functionality and could be packaged as a separate framework, useful to many other applications, particularly simulations. It could also be implemented easily in a number of other programming languages ( $\mathrm{C \#}, \mathrm{C}++$ for example). This is also true of the experiment execution component.

\subsubsection{Execution (experiment controller)}

The Experiment Controller has the responsibility of executing a predefined Experiment Plan. The execution of an experiment is actually divided between two subcomponents, the Controller and the "Simulation". The controller is a generic component which could be used to control any simulation that was based on the concept of timesteps. The simulation component is a specific implementation for this framework which has knowledge about the landscape, foragers, resources, etc. Several important terms are defined in Box 3.5.

The simulation component provides the ability to define multiple "generations" within each "treatment", this provides for exploration of longer timescale patterns than purely immigration. No experiments had been conducted with this aspect of the framework at the time of writing.

The flow of execution is shown in Figure 3.28. It is reasonably straight-forward. The most important steps are "Update Agents" and "End Of Treatment?". The first is where the agents are called to execute whatever behaviour occurs for that timestep. The second decides, based on a number of criteria, whether the current treatment is complete. The treatment will be complete when either there are no more foragers to release, or the total egg count has reached a specified number. The latter allows the simulation to configured to run until the same number of eggs have been laid as were observed in the field data.

\section{Server processing}

The experiment controller can be used in one of two modes, "interactive" or "server". In interactive mode, a visual display of the landscape is available which allows the 


\section{Parameter manipulation}

The combination of a named parameter and a set of values which are to be systematically set for it, e.g. set the values "1", "10", and " 100 " into the parameter "step length $(L)$ ".

\subsubsection{Trial}

Experiments refer to general exploration of the parameter space, having a common set of parameter manipulations and similar resource layouts. For example, the "Edge Effect" experiment. In order to group the explorations in a meaningful manner, the term "Trial" is used. So a particular experiment may have several Trials, each exploring an aspect of the theme of that experiment.

\section{Treatment}

A treatment is often used to describe a particular setup for an experiment. Here it refers to the execution of a single combination of parameters as defined by the set of parameter manipulations. For example, if the manipulations were those in table 3.4, the resulting treatment plan would be that in table 3.5 .

\begin{tabular}{ll}
\hline Parameter & Values \\
\hline Step length (L) & $1,10,100$ \\
Turning angle concentration $(k)$ & $0.5,3.0,10.0$ \\
\hline
\end{tabular}

Table 3.4: Example parameter manipulations.

\begin{tabular}{lrr}
\hline Trial & $\mathbf{L}$ & $\mathbf{k}$ \\
\hline $\mathbf{0 0 1}$ & 1 & 0.5 \\
$\mathbf{0 0 2}$ & 1 & 3.0 \\
$\mathbf{0 0 3}$ & 1 & 10.0 \\
$\mathbf{0 0 4}$ & 10 & 0.5 \\
$\mathbf{0 0 5}$ & 10 & 3.0 \\
$\mathbf{0 0 6}$ & 10 & 10.0 \\
$\mathbf{0 0 7}$ & 100 & 0.5 \\
$\mathbf{0 0 8}$ & 100 & 3.0 \\
$\mathbf{0 0 9}$ & 100 & 10.0 \\
\hline
\end{tabular}

Table 3.5: Example treatment sequence.

\section{Replicate}

The term replicate is taken directly from the statistical concept of "replication" i.e. repeating an identical experimental configuration a number of times to improve the statistical "power" of the results. Here, each trial can be repeated a specified number of times. Each repeat is termed a "replicate". Thus the results of an experiment may contain several trials, each with several replicates. 
dynamic behaviour of the agents to be observed. This is particularly useful in ironing out problems with the software but also enables a more intuitive feel for the way the simulation is behaving. In "server" mode there is no visible user interface and so a communications protocol ${ }^{12}$ is available which allows a remote process to be queried across the internet for progress, and results to be easily downloaded for processing. This feature was particularly useful as the some of the final experiments were run on several available servers, physically placed in New Zealand, whilst being controlled from the UK.

The number of treatments can easily become large, and for certain parameter combinations (high degree of turning, short step lengths, large experimental area, no sensory perception), each treatment may take many hours to complete, the longest experiments took more than 40 hours of total processing time. The easiest way to optimise a process is to split the work across multiple processors or computers. This was built into the framework such that the treatment plan could be divided amongst servers. Specifying "1of4" as a parameter to the experiment controller is sufficient. In this way, larger parameter spaces can be explored.

\section{Reporting results}

Some of the experiments configured had over 100 treatments. Depending on the experiment, various response variables might be required, for example, the life history of each agent (i.e. its path and behaviours), the number of eggs on each cabbage, and a matrix of signal strengths for the olfaction surface which can be used to generate 3D images. Results reporting is itself a sub-component of the experiment controller, and as with parameters has a structured mechanism for creating consistent file structures and organising the files into sub-directories, so that it is possible to easily navigate the mass of information generated for each experiment.

\subsubsection{Results analysis (' $R$ ' reports)}

The statistical software package ' $R$ ' ( $R$ Development Core Team, 2005) provided an invaluable platform for the processing of the results from the simulation. The "S4 Object" language environment was used within $\mathrm{R}$ to allow something like objectoriented programming. In this way a structured representation of the output files was constructed. This was combined with a library that was developed which allows $\operatorname{LT}_{\mathrm{E}} \mathrm{X}$ (Mittelbach, 2004) files to be generated. The combination allowed specific "reports" to be built for each experiment, providing tables of the most important pa-

\footnotetext{
${ }^{12}$ Implemented using TCP/IP sockets
} 


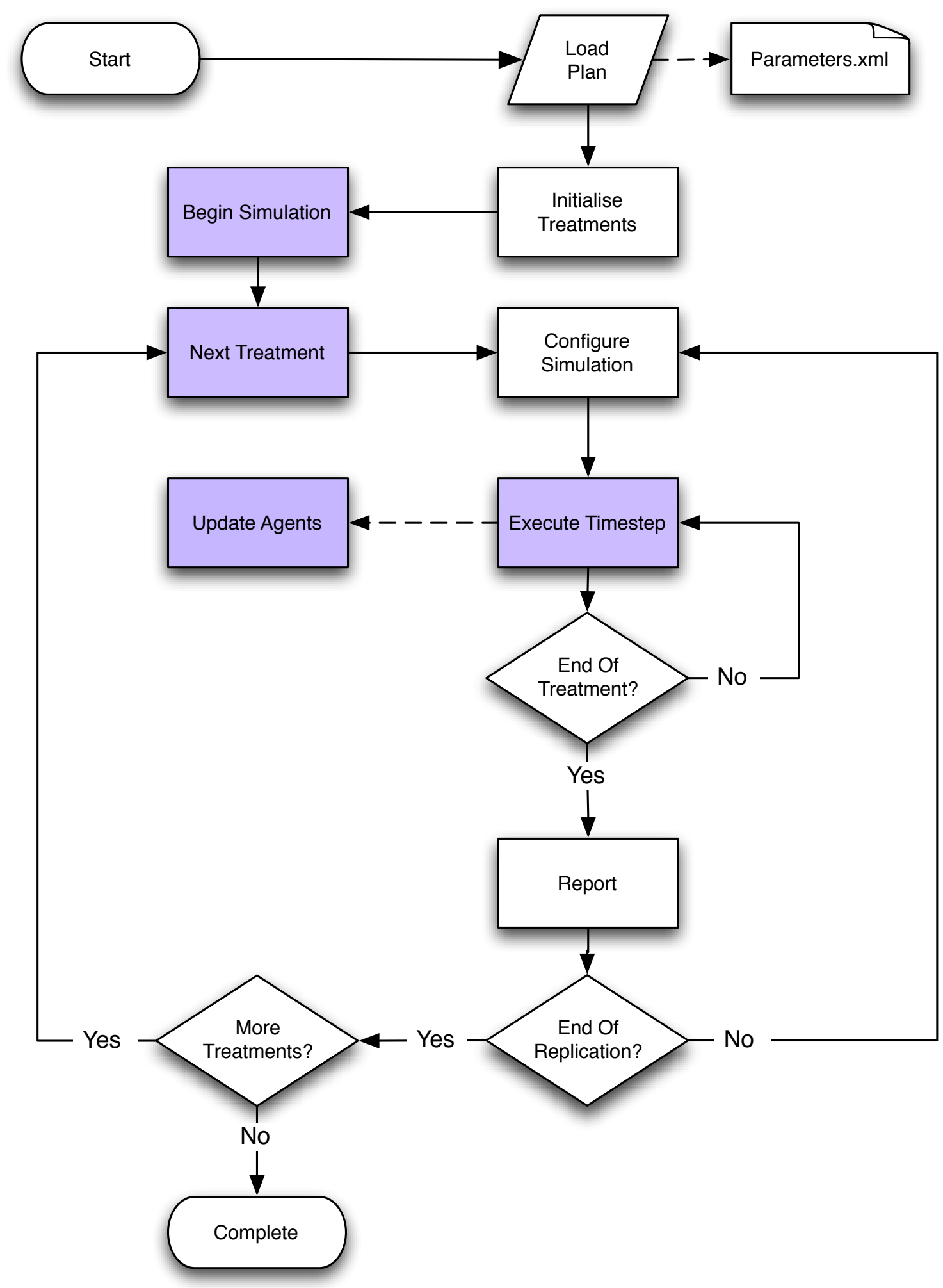

Figure 3.28: Summary flow diagram of simulation execution by the experiment controller. 
rameters, and incorporating graphs of the data. This was a vital element in managing the complexity of the data that was output as it enabled the researcher to simply execute a script and a PDF report was generated containing all of the results at a glance. Many experiments (see also Chapter 4) had different parameters, but identical reports. This separated the work of actually processing result data from the cognitive analysis of results and allows an overview of complex masses of data in a controlled manner.

A simple extension to the experiment controller would be to automatically execute a script in $\mathrm{R}$ at the end of an experiment and store the resulting PDF file with the results, potentially e-mailing the report to the researcher. A further advantage of the $\mathrm{HT}_{\mathrm{E}} \mathrm{X}$ files is that it is then possible to "cut and paste" sections of the reports which for example have built a table of results, or incorporated a graph, directly into some other $\mathrm{HT}_{\mathrm{E}} \mathrm{X}$ document (such as a paper or thesis), facilitating the process between parameterisation, execution and publishing of simulation data.

This functionality highlights one of the design goals of the framework; to remove as much as possible the researcher from the laborious result of automating such tasks. In the best case no knowledge of the underlying framework is required, in the worst case, it still enables a separation of work between the two tasks, a set of reports is developed during the design of the experiment, once complete, it is a simple matter to explore the parameter space at leisure which affords a much greater ability to process the results and direct the exploration.

\subsubsection{Validation}

The movement paths generated with the CRW were analysed and tested using the CircStats package in R (Agostinelli and Lund, 2006). The paths were recorded in a "life-history" CSV file which had a separate line for each timestep (and thus movement) of the forager. The angle of turn is recorded at each step in this file. These were then tested using the CircStats package to confirm that they conformed to a Von Mises distribution of the expected parameters. Graphs were also plotted of the actual vs expected distributions (Figure 3.29) and a circular distribution graph was plotted for visual analysis (e.g. Chapter 2, Figures 2.2 and 2.3). This was repeated for several values of $k$. A chi squared comparison was also performed as this was the method used by Cain (1985). The initial azimuth generation was tested using the Rayleigh test for uniformity. As a qualitative test, we reproduced the results of Johnson et al. (1992), shown in Figure 3.30. All statistical tests provided no significant difference to the expected distributions.

The importance of this validation was not only that the underlying random number 
generators were functioning, but also that the entire framework was performing as expected, from parameter configuration to results analysis. The random number generators used were those provided by the default Java programming language, which uses a 48-bit seed, modified using a linear congruential formula (See Knuth, 1998, Section 3.2.1.).

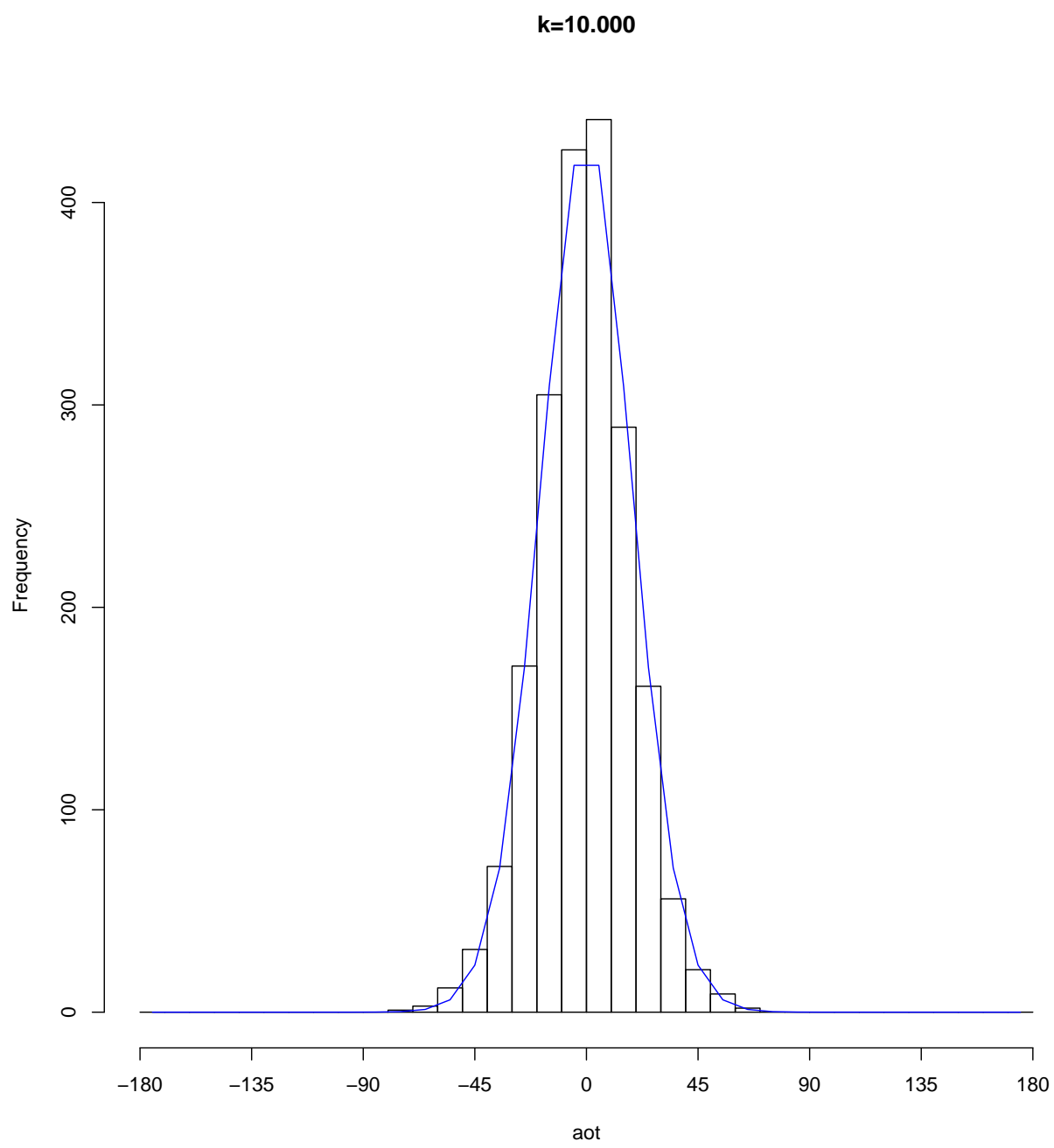

Figure 3.29: Validation of Azimuth Generator - Histogram $k=10$. Line shows expected density calculated from Von Mises density function. 


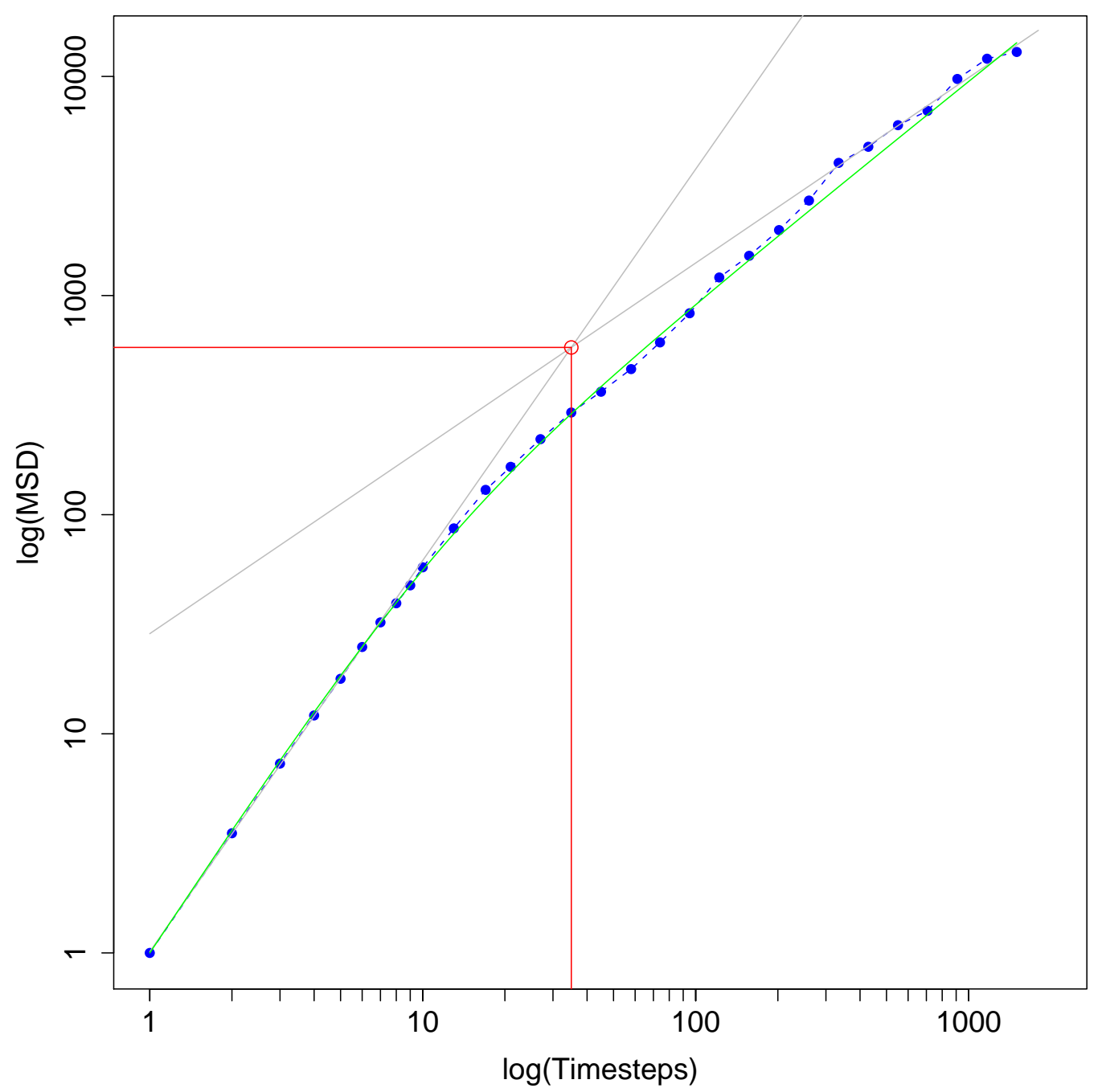

Figure 3.30: Validation of MSD. Following the method of Johnson et al. (1992), a number of random walkers were released and their squared displacement was recorded at various timestep intervals up to 1000 timesteps. The dotted blue line on the graph represents the values generated by simulation, the mean MSD for 1000 foragers. The green line is the theoretical expectation of the MSD. Regression lines were created by calculating the linear regression for the set of short step time intervals $(<=7$ timesteps)) and those of longer intervals ( $>=400$ timesteps). The slopes of these lines demonstrate that over the short timescale the CRW demonstrates a greater displacement and tends towards the slope of Fickian (purely random) diffusion over greater timescales, the slope of a CRW tends toward 1. The movement parameters in this instance were $L=1, k=3$, chosen because they matched they most closely matched the results presented in Johnson et al. (1992) 


\subsection{Methods: Exploratory simulation experiments}

Two experiments are reported here which were executed in order to gain some insight to the basic responses possible using a simple layout of resources, before moving on to explore more complex layouts that were compared to field observations (Chapter 4). The first concerns manipulating the distance between the release boundary and the resources, the "boundary effect" experiment. The second explored each of the behaviours of the foragers (CRW, vision and olfaction). The relative number of eggs on "edge" versus "central" resources was recorded, to explore the relationship between the various parameters and the observed response. This experiment is referred to as the "edge effect" experiment.

\section{Resource layout}

The layout used in both experiments was a simple, uniform spacing of 16 resources in a square (Section 3.4). With this design, the resources can be categorised in three groups; "Edge", "Corner" or "Centre" (Figure 3.31). A variation was used for the "edge effect" experiment in which the "Edge" resources were removed to simplify the layout further. The ratio of the numbers of eggs between each group was recorded. Experiments were run until a specified number of eggs had been laid, and the number of foragers that were released was recorded to provide a measure of "searching efficiency". Figures 3.32 and 3.33 demonstrate the difference between the two layouts, and the various combinations of patch size $(P)$ and radius of attraction $(R)$ that were used.

It is difficult to distinguish between the effects of plant spacing and radius of attraction when analysed independently as they can result in congruent layouts. For example, decreasing $R$ has the same effect as increasing $I$. If relative spacing is used however, it is possible to combine the two factors into single scale. Relative spacing $\left(I_{R}\right)$ was calculated from inter-edge spacing $(S)$ as a proportion of $R$ (Equation 3.25). In order to scale the index so that it is related to the relative spacing between patches, i.e. across all the layouts, this proportion is taken by taking spacing as a proportion of the size of the patch and radius as a proportion of the largest patch in the comparison.

$$
I_{R}=\frac{I / P}{R / P_{\max }}
$$

A statistic that can be used to compare layouts, is the relative area covered by the radii of attraction. A simple measure was devised and labelled $R_{P}$ and calculated as Equation 3.26. It is effectively a measure of the relative proportion of the patch area covered. It relates $R$ and $P$ in a meaningful way. This measure is useful for comparing 
forager success, as it is reliant on a combination of these factors.

$$
R_{P}=R_{\text {area }} \times \frac{P^{2}}{P_{\max }^{2}}
$$

Where $R_{\text {area }}$ is the area covered by all the resources, $P$ is the local patch size (i.e. the size used to parameterise the layout) and $P_{\max }^{2}$ is the size of the largest patch (in this case, $\left.P_{\max }^{2}=600 \times 600\right)$.

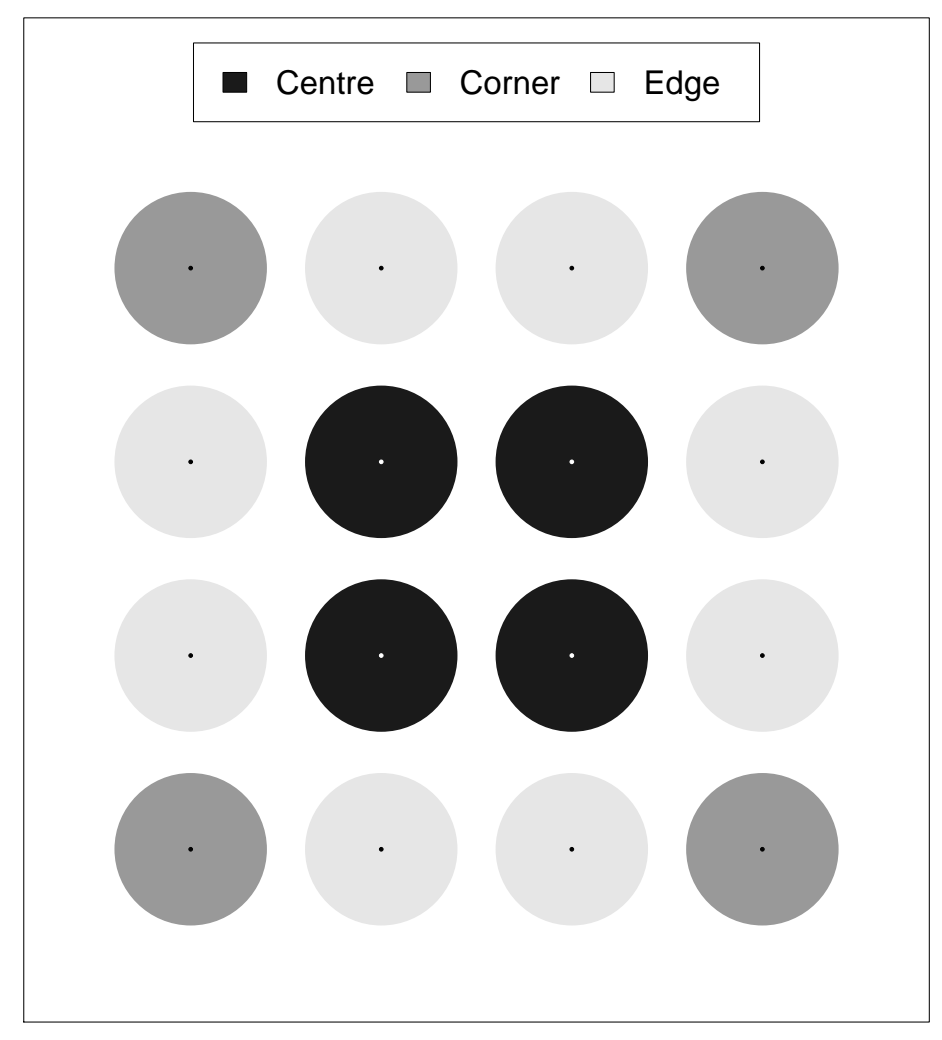

Figure 3.31: Demonstration of the categorisations of the resources in a simple $4 \times 4$ layout, showing the corner, centre and edge resources.

\section{Olfaction signal surface}

Figures 3.34 to 3.36 show the signal surfaces for each value of the patch size $(P)$ which is the parameter which will control the shape of the surface because $R$ is not relevant to the odour signal. The signal width is set to be relatively "wide" so there is a concentration of odour over the centre of the patch, as opposed to "spikes" over each resource (Figure 3.11). 
a) $I=15, I_{R}=18.0, R_{P}=34.9$

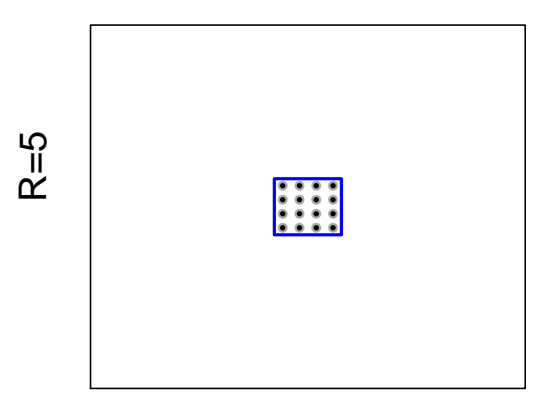

d) $I=-5, I_{R}=-2.0, R_{P}=176.0$

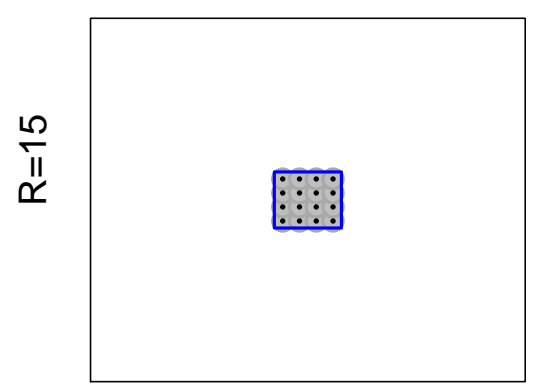

g) $I=-75, I_{R}=-9.0, R_{P}=765.6$

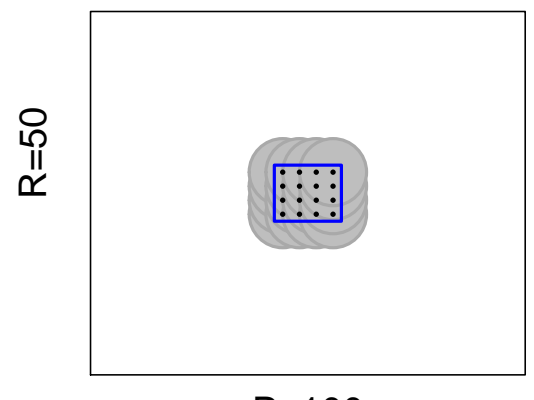

$\mathrm{P}=100$ b) $I=65, I_{R}=26.0, R_{P}=314.2$

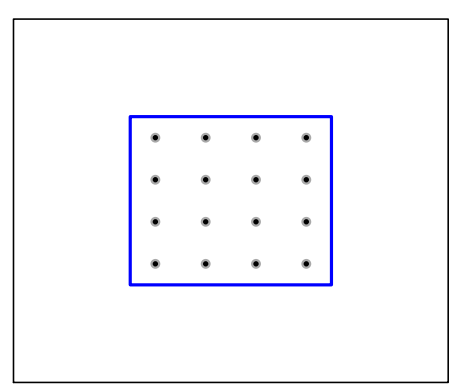

e) $I=45, I_{R}=6.0, R_{P}=2827.5$

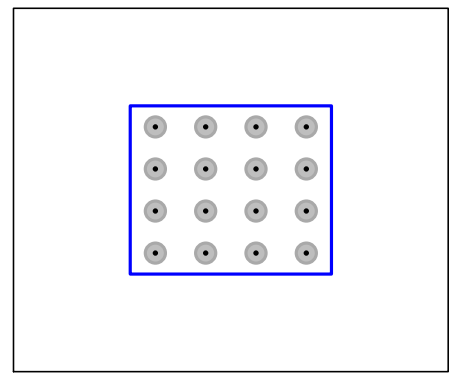

h) $I=-25, I_{R}=-1.0, R_{P}=16533.5$

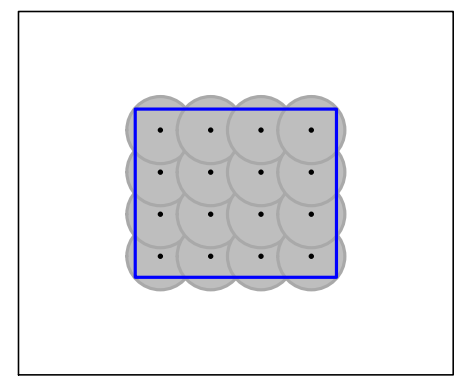

$P=300$ c) $I=140, I_{R}=28.0, R_{P}=1257.0$

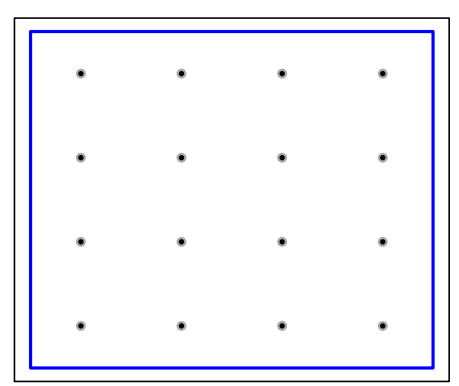

f) $I=120, I_{R}=8.0, R_{P}=11310.0$

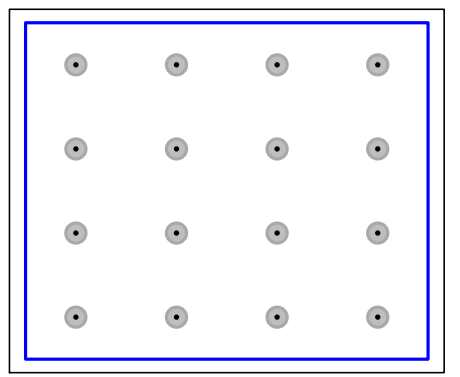

i) $I=50, I_{R}=1.0, R_{P}=125664.0$

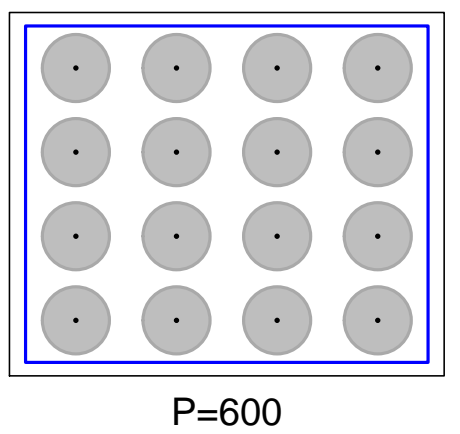

Figure 3.32: Resource layouts with different spacings (left to right) and different radii of attraction (top to bottom) of resources. 
a) $I=15, I_{R}=3.0, R_{P}=0.2$

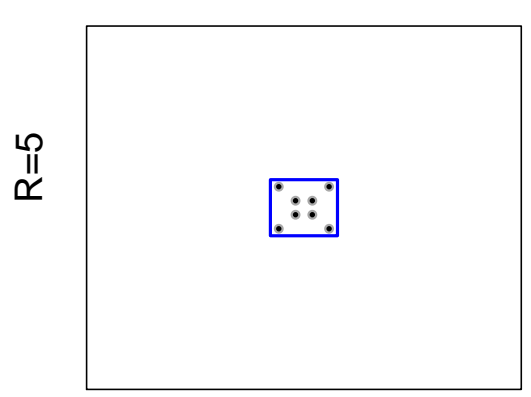

d) $I=-5, I_{R}=-0.3, R_{P}=1.3$

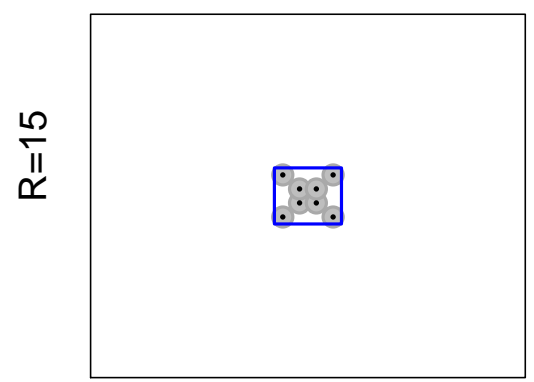

g) I=-75, I $I_{R}=-1.5, R_{P}=7.7$

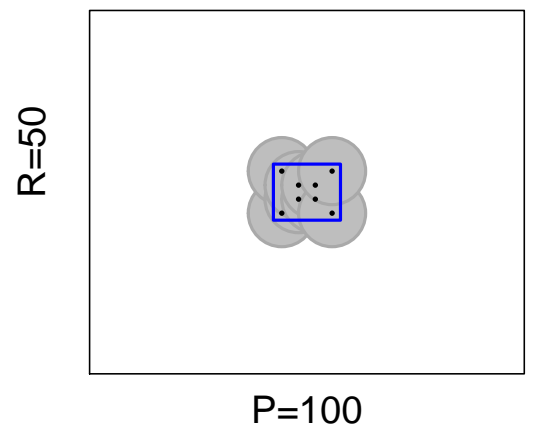

b) $I=65, I_{R}=13.0, R_{P}=0.5$

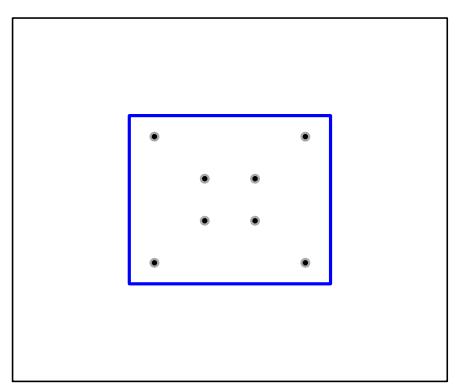

e) $I=45, I_{R}=3.0, R_{P}=4.7$

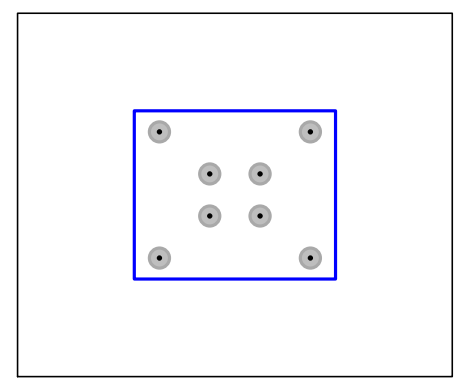

h) $I=-25, I_{R}=-0.5, R_{P}=44.1$

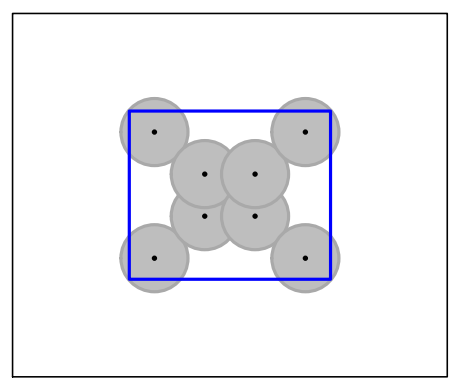

$P=300$ c) $I=140, I_{R}=28.0, R_{P}=1.0$

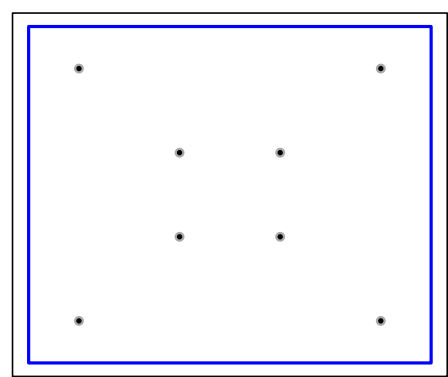

f) $I=120, I_{R}=8.0, R_{P}=9.4$

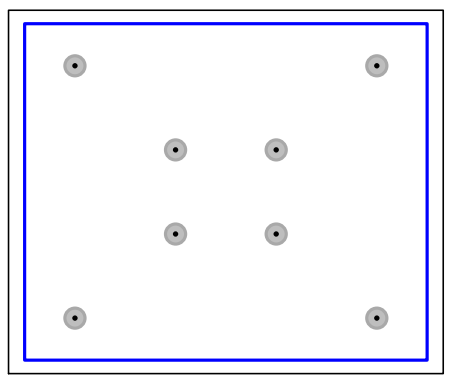

i) $I=50, I_{R}=1.0, R_{P}=104.7$

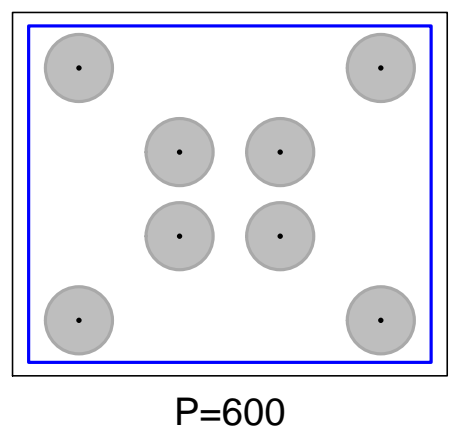

Figure 3.33: Resource layouts with corner and centre resources only. 

a) Image map
b) 3D Surface
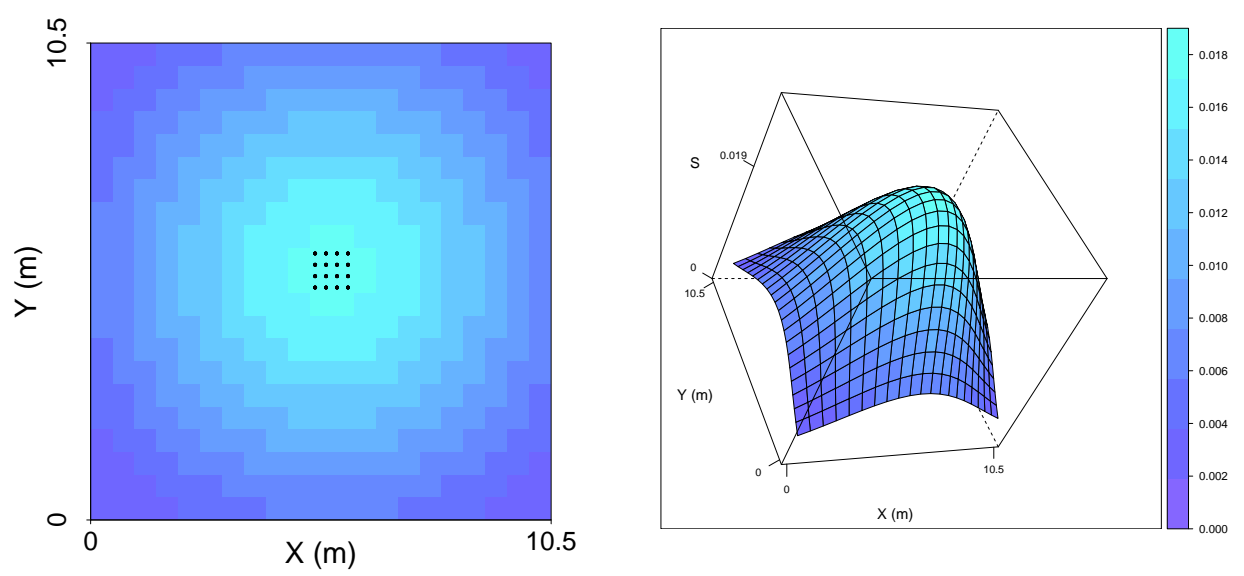

Figure 3.34: Olfaction signal surface, $P=100, S W=360$.
a) Image map
b) 3D Surface
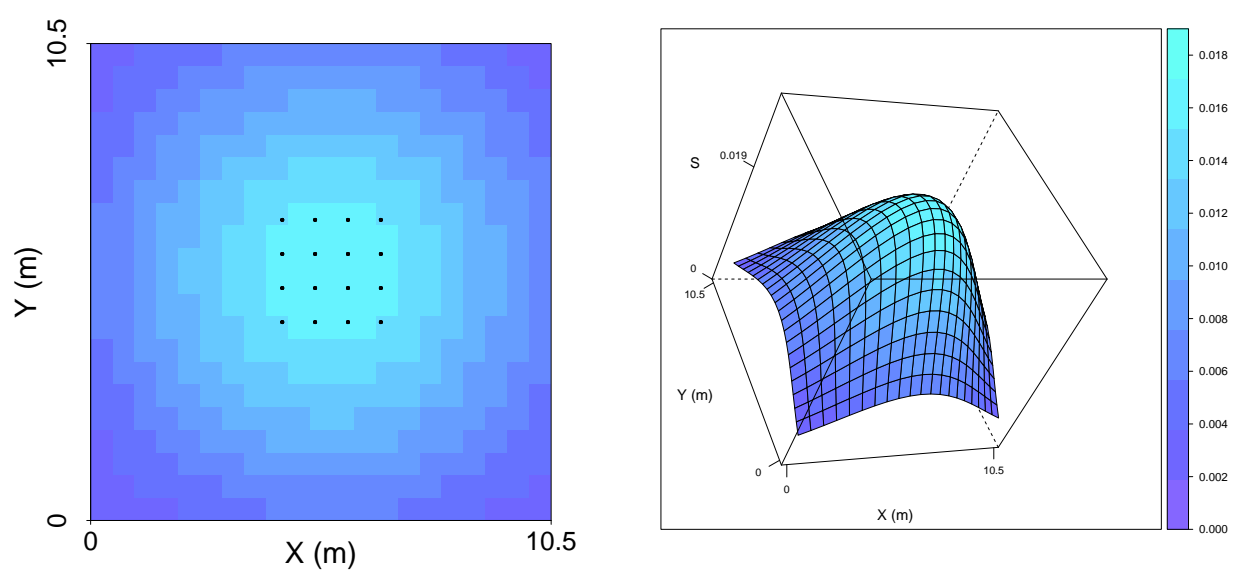

Figure 3.35: Olfaction signal surface, $P=300, S W=360$.
a) Image map
b) 3D Surface
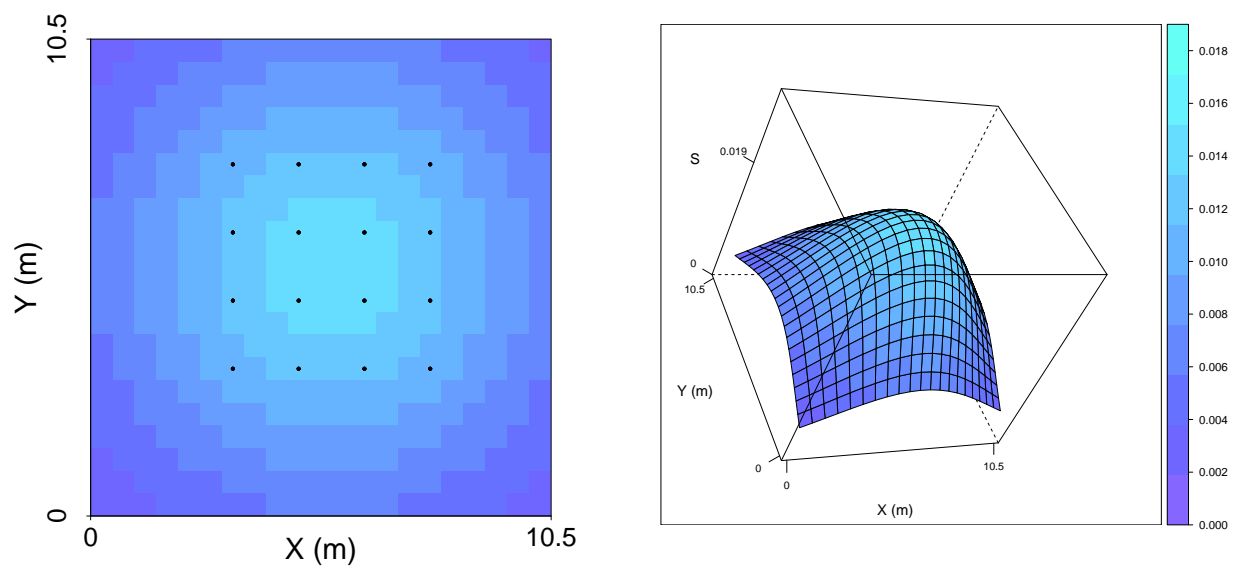

Figure 3.36: Olfaction signal surface, $P=600, S W=360$. 


\subsubsection{Boundary effect simulation experiment}

The "release boundary" representing a window on the landscape has been introduced previously. Agents are released at random around the perimeter, with a uniform random azimuth and so should simply represent a sample of the paths that have crossed in from outside, irrespective of distance from the central patch. It was hypothesised that, as the agents are initially placed at random around the perimeter and when moving at random (no vision or olfaction), the radius of the release boundary (i.e. its distance from the resources, $B$ ) should not qualitatively affect the results.

The consequence of releasing further away, particularly for high turning angle concentrations and shorter step lengths, is that the simulations take longer to run. This is because these highly sinuous paths tend to cover a smaller area more thoroughly and it takes many more timesteps for them to reach the central patch. Many foragers do not even arrive at the patch and at some point escape.

It was intended therefore to test the effect of release distance simply in order to show that foragers could be released closer to the patch in order to improve execution times of the simulation. The Trials conducted are listed in Table 3.6

\begin{tabular}{ll}
\hline Trial & Description \\
\hline A & Effect of egg limit $(G)$ \\
B & Effect of path sinuosity $(L, k)$ \\
C & Near vs far comparison (release on perimeter) \\
D & Near vs far comparison (release In "zone") \\
E & Near vs far comparison (less directional) \\
F & $\begin{array}{l}\text { Detailed exploration of release boundary (less } \\
\text { directional) }\end{array}$ \\
\hline
\end{tabular}

Table 3.6: Summary of trials run for the boundary effect experiment. Details are given in the text.

The experiment manipulated the release distance $(B)$, step length $(L)$, turning angle concentration $(k)$ and the total number of eggs to be laid $(G)$. The exact values used are shown in Table 3.7. The concept of "trials" is demonstrated here, with each trial representing a specific subset of manipulations within the experiment. Each trial was executed and analysed separately.

Trial A was designed to explore the effects of the total number of eggs laid. Trial B was concerned with the interaction between release boundary and the parameters of the CRW. Trials $C$ to $E$ were specific comparisons between a close release boundary and a distant one, $\mathrm{C}$ and $\mathrm{D}$ differ in that in $\mathrm{D}$ foragers were released at random 
locations within a "zone" (or annulus), rather than exactly on the perimeter. The width of the annulus was from the perimeter to the "Zero Boundary" (Figure 3.27). Trial $F$ provided a more detailed exploration of the effect of the release distance, at the expense of a single set of CRW parameters. Some of the parameterisations were decided upon after the results of previous trials had been analysed.

\begin{tabular}{llrrrr}
\hline Trial & Rep. & G & B & k & L \\
\hline A & 20 & $2,25,50,100,150,200,300$ & 100 & 10 & 10 \\
B & 5 & 100 & $0,5,10,20,100$ & $0.5,3,10$ & $1,5,10,40,80$ \\
C & 50 & 500 & $0,80,800$ & 10 & 80 \\
D & 50 & 500 & 80,800 & 10 & 80 \\
E & 50 & 500 & 0,800 & 8.8 & 80 \\
F & 50 & 500 & $0,5,10,20,100,400,800$ & 8.8 & 80 \\
\hline
\end{tabular}

Table 3.7: Manipulations for the boundary effect experiment. "Rep." is an abbreviation of "Number of Replicates. Other symbols are described in Table 3.3. In all cases $R=5$ and $P=80$.

\subsubsection{Edge Effect simulation experiment}

The second experiment explored the effect of movement patterns upon the ratio of eggs recieved by the outer versus inner resources. The sinuosity of the paths was varied and tested against several patch layouts, manipulating the spacing between resources $(I)$, radius of attraction $(R)$ and overall patch size $(P)$. The ratio of eggs received on the centre of the layout compared to the edge and corner resources was recorded for varying CRW parameters, as well as with vision and olfaction enabled. The simple layout represents a sub-component of more complex layouts which are explored in Chapter 4 , and therefore help to conceptualise the responses observed for these layouts. Table 3.8 lists the trials created for this experiment.

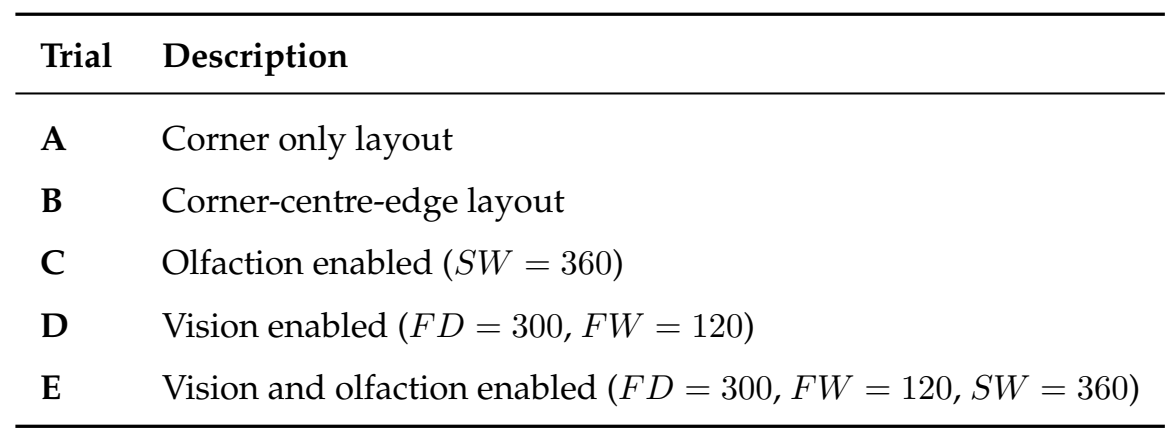

Table 3.8: Summary of trials run for the edge effect experiment. All trials varied parameters controlling the sinuosity of the path and the layout of the resources as defined in Table 3.9. 
The goal was to explore the "edge effect", i.e. where resources on the edge of a dense patch may "trap" approaching individuals and thus reduce the egg load on those in the centre. Jones (1977a) mentions such an effect, citing Harcourt $(1963,1961)$ and Kobayashi (1965) as sources, although in these cases the effect was suggested to be foragers leaving the patch and then returning to it.

A secondary objective was to record a measure of "forager efficiency" (defined as number of foragers released per egg laid). This is similar to Cains' work (1985) and was intended to demonstrate the effect of smaller, denser patches vs larger, more spaced patches. An alternative measure would be number of hosts encountered per distance flown (Jones, 1977a).

\begin{tabular}{lr}
\hline Parameter & Manipulations \\
\hline Replicates & 5 \\
B & 100 \\
G & 100 \\
E & 1,5 \\
k & $10,3,0.5$ \\
L & $50,150,250$ \\
R & $5,15,50$ \\
P & $100,300,600$ \\
I & $15,65,140,-5,45,120,-75,-25,50$ \\
\hline
\end{tabular}

Table 3.9: Common parameter manipulations for all trials as listed in Table 3.8. In total there were 162 combinations (treatments), replicated 5 times each with a constant Release Boundary of 100 and 100 eggs. For a description of the parameters refer to Table 3.3. The inter-edge separation (I) was not manipulated directly but was calculated based on the radius of attraction $(R)$ and the patch size $(P)$ as shown in Figure 3.25

Five trials were conducted (Table 3.8). Trial A used the layout without edges and no sensory perception. Trial B had the $4 \times 4$ layout for comparison. Trial C introduced a fixed configuration for olfaction, Trial $\mathrm{D}$ a fixed configuration of vision, and Trial $\mathrm{E}$ a combination of both vision and olfaction. Subsequent investigations could be configured to explore the parameter space of the senses more completely, however the general results obtained here were regarded as sufficient for the purposes of demonstrating potential responses with respect to the foragers.

Affecting the foragers, step length $(L)$, turning angle concentration $(k)$, number of eggs per forager $(E)$, visual and olfactory senses $(V E, O E)$ were manipulated. Affecting the resources, patch size $(P)$ and resource radius $(R)$ were manipulated which resulted in various measures of resource spacing $(I)$. Table 3.9 details the parameter manipulation values that were used. 


\subsubsection{Analytical methods}

Two response variables were measured for each experiment:

- Centre Ratio the ratio of the number of eggs per plant on centre resources relative to those laid on the other (edge or corner) resources, providing an indicator of the "edge effect".

- Number of eggs laid per forager, providing indicator of how well a movement pattern intersects the resource layout.

The first of these was the ratio of the eggs on corner resources to those in the centre and the edge. First the eggs per resource are calculated for each group. This is more commonly referred to as "eggs per plant" with field data and this metric will be adopted henceforth. The eggs per plant is simply the total eggs divided by the number of plants. With the calculated layout, the number of plants is fixed at 4 for both corner and centre, and 8 for the edges. Once the eggs per plant are calculated, the relative proportions for each group are calculated as a proportion of the total eggs per plant. Table 3.10 provides a sample egg count and follows the calculation through for an example.

\begin{tabular}{lrrrr}
\hline & Centre & Corner & Edge & Total \\
\hline Eggs & 10 & 20 & 50 & $\mathbf{8 0}$ \\
Plants & 4 & 4 & 8 & $\mathbf{1 6}$ \\
Eggs per Plant & 2.5 & 5 & 6.25 & $\mathbf{1 3 . 7 5}$ \\
\hline Ratio & $\mathbf{0 . 1 8}$ & 0.36 & 0.46 & $\mathbf{1}$ \\
\hline
\end{tabular}

Table 3.10: Example egg count for the calculated resource layout. If there are no "edge" plants this column is simply removed but the calculation remains the same

The Corner ratio allows comparison between the two layouts, in terms of relative proportions of eggs per plant on the different categories of resource (Figure 3.31). The "ideal free distribution" (Cressman et al., 2004) of the proportions of eggs per plant will be slightly different between the layouts. If If the same number of eggs were laid on each plant, for the corner-centre layout, the evenly divided proportion is simply $0.5: 0.5$. For the corner-centre-edge layout, it would be $0 . \overline{33}: 0 . \overline{33}: 0 . \overline{33}$. Either of these situations represents a balanced distribution of eggs per resource and so when contemplating the graphs in the results section, comparison to these ratios should be made (Figures 3.43 and 3.44). If the centre ratio is greater than 0.5 or 0.3 the centre resources are receiving disproportionately high numbers of eggs and vice-versa. 
The second response measured was forager success rate. The maximum value for this will be the value of the parameter "Number of eggs per forager $(E)$ ". There cannot be a greater efficiency than every forager released laying its complete set of eggs. The more foragers which escape from the landscape without locating a resource, or die of old age, the less efficient the searching behaviour.

\section{Statistical Tests}

Where mean values have been calculated, the results presented are shown with standard error bars. The error of the samples can be affected by both the number of eggs laid and the number of replications of each treatment, both decreasing the standard error. Increasing either of these parameters produces more consistent results and reduces the standard errors.

In general, statistical tests were not performed on the results since the response sought was of a qualitative nature, the trend of which might then also be observed in field observations. In a simulation environment, the p-values associated with any real difference between treatments can be made significantly small by increasing the number of replicates. As a "rule of thumb", if the standard error bars of two treatments do not overlap it was considered that there was a significant difference between them.

In the case of the boundary effect experiment, a test was used to compare the means across all treatments as additional evidence that a difference in response exists.

\subsection{Results}

\subsubsection{Boundary effect results}

Unexpectedly, the hypothesis that the distance of the release boundary from the resources should have no qualitative effect was not supported by the results. In fact, changing the release boundary distance did alter the proportion of eggs reaching the centre resources (Figures 3.37, 3.38). This was tested with Kruskall-Wallace test with a null hypothesis of equal means, which was significantly rejected $(H=106.05$, $d . f .=6, p<0.001)$. This result was obtained using the corner-centre only layout, so no edge resources.

Figure 3.37 shows the results for a relatively long step length ( $L=80$ relative to the patch size of $P=80)$, and medium-high directionality $(k=8.8)$. The release distance of $B=0$ has a higher number of eggs on the centre resources than $B=5$. 
Between $B=5$ and $B=800$, the number of eggs on the centre resources consistently increases. For a less directional path of the same step length $(L=80, k=0.5)$, this does not occur, and there is a more consistent decline towards the common ratio of approximately 0.45 (Figure 3.38). It is the very long steps ( $L=40$ and $L=80$ ) which show the steepest decline.

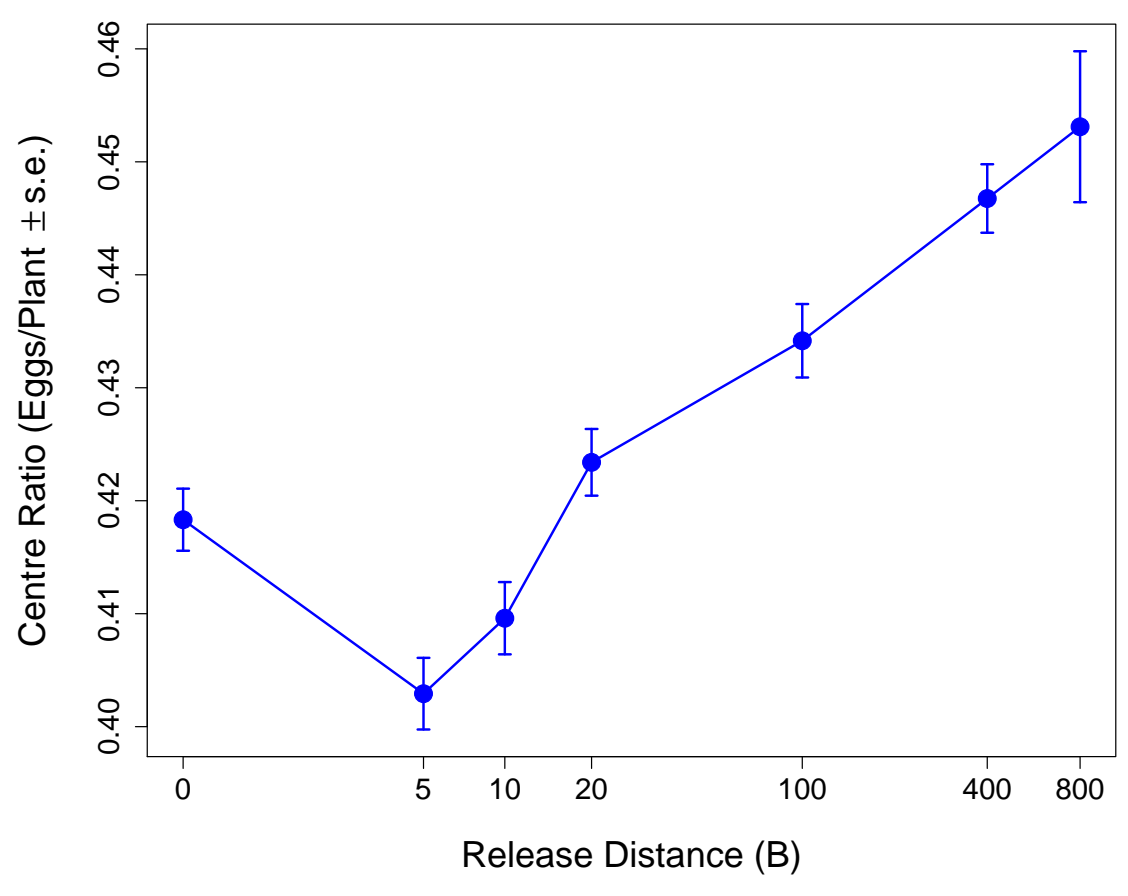

Figure 3.37: Trial F - centre:corner ratio plotted against $B(L=80, k=8.8, P=80$, $S=10, R=5, G=500$, Replicates $=50$, Layout $=$ corner - centre $)$.

Decreased turning angle concentrations (e.g. $k=0.5$ ) and shorter step lengths (e.g. $L=1$ to $L=10$ ) produce an "area restricted" search pattern, covering a smaller area, but more efficiently (Figure 3.3, also Chapter 2). With a low turning angle concentration $(k=0.5)$, more foragers are "caught" on the corner resources, and there is a strong effect of step length (Figure 3.38). Longer step lengths increase the number of eggs per plant in the centre.

As $k$ increases, the paths become less sinuous and more directed. A path with parameters of $L=1, k=10$ should tend toward the behaviour of a path with a higher step length but lower turning angle concentration, such as $L=80, k=3$.

In these experiments, when $k=10$, the responses are increasingly grouped around a common ratio near 0.4 (Figure 3.39). It would be reasonable to say that for highly directional paths, $L$ has a reduced effect on the centre ratio, except for very small step lengths which allow for more turning (increasing sinuosity and the number of foragers "caught" on the edge).

The patch size is $P=80$ which means that with relatively long step length (e.g. 


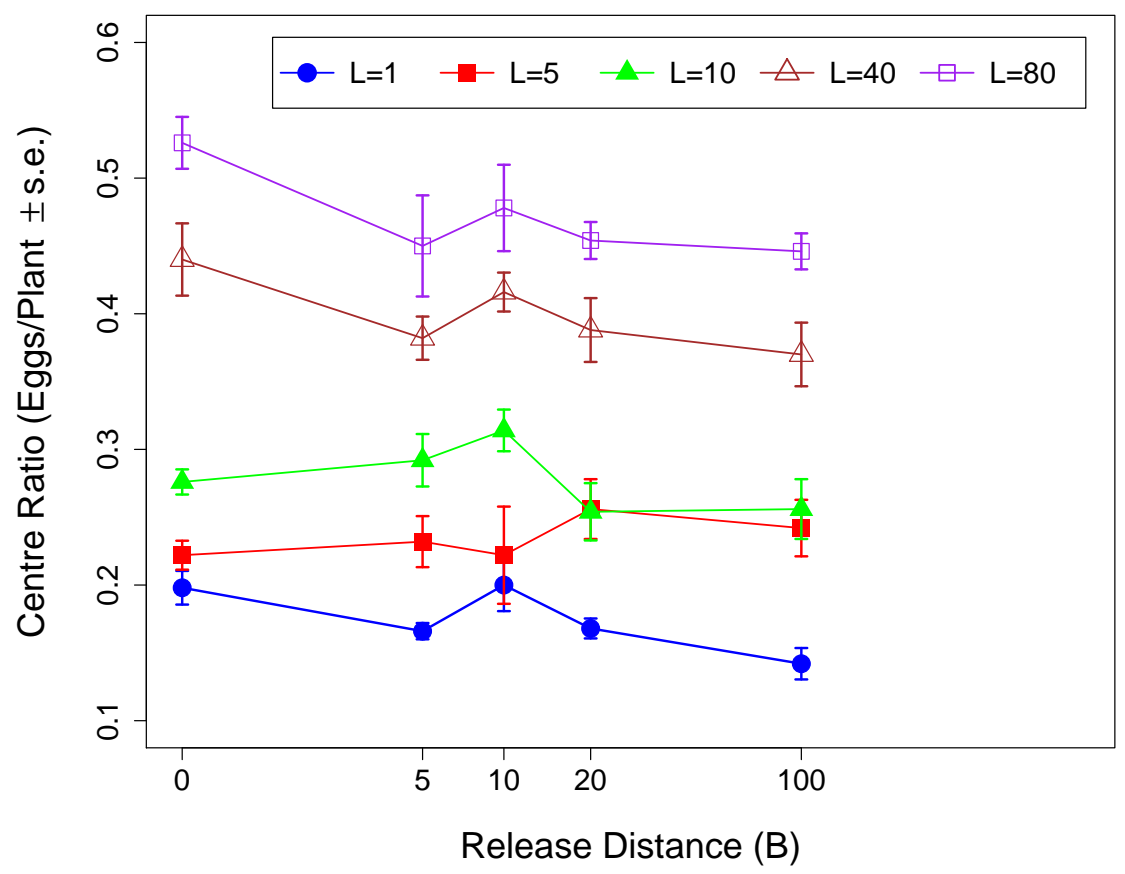

Figure 3.38: Trial $B$ results, $k=0.5$, varying $L$ and $B . \quad(G=100$, Replicates $=5$, $P=80, S=10, R=5$, Layout $=$ corner - centre)

$L=80$ ), a forager will potentially cross the patch and immediately escape. Even increasing the release boundary by a small amount allows for a greater number of steps to occur and thus more changes of direction. In other words, the parameter $k$ will have more influence as the release boundary $B$ is increased. As noted, a decrease in $k$ (more random turning) produces an "area restricted" search, resulting in more foragers being caught on the corners, which might account for the drop in ratio between $B=0$ and $B=5$ seen in Figure (3.37). $B=0$ is more directional and thus penetrates the centre more effectively than $B=5$. By this reasoning, as $B$ is increased, the number of eggs in the centre should decrease. This is observed for $k=0.5$ (Figure 3.38), but for $k=8.8$ (Figure 3.39), the centre ratio increases with $B$. This is a complex interaction and will require further study to understand completely.

An interesting point about these results (Figures 3.38 and 3.39) is that there is a peak at a release distance of $B=10$, consistent across all values of $L$, except $L=$ 5 where it is reversed (Figure 3.38). With increased directionality the disturbance is still observed although more random in nature (Figure 3.39). The fact that the peak is present for each treatment suggests a real effect. It is possible that for a given spacing between the resources, there might be release distance which allows optimum penetration to the centre, although this would be expected to vary with step length. To investigate further, patches of different spacings could be used. 


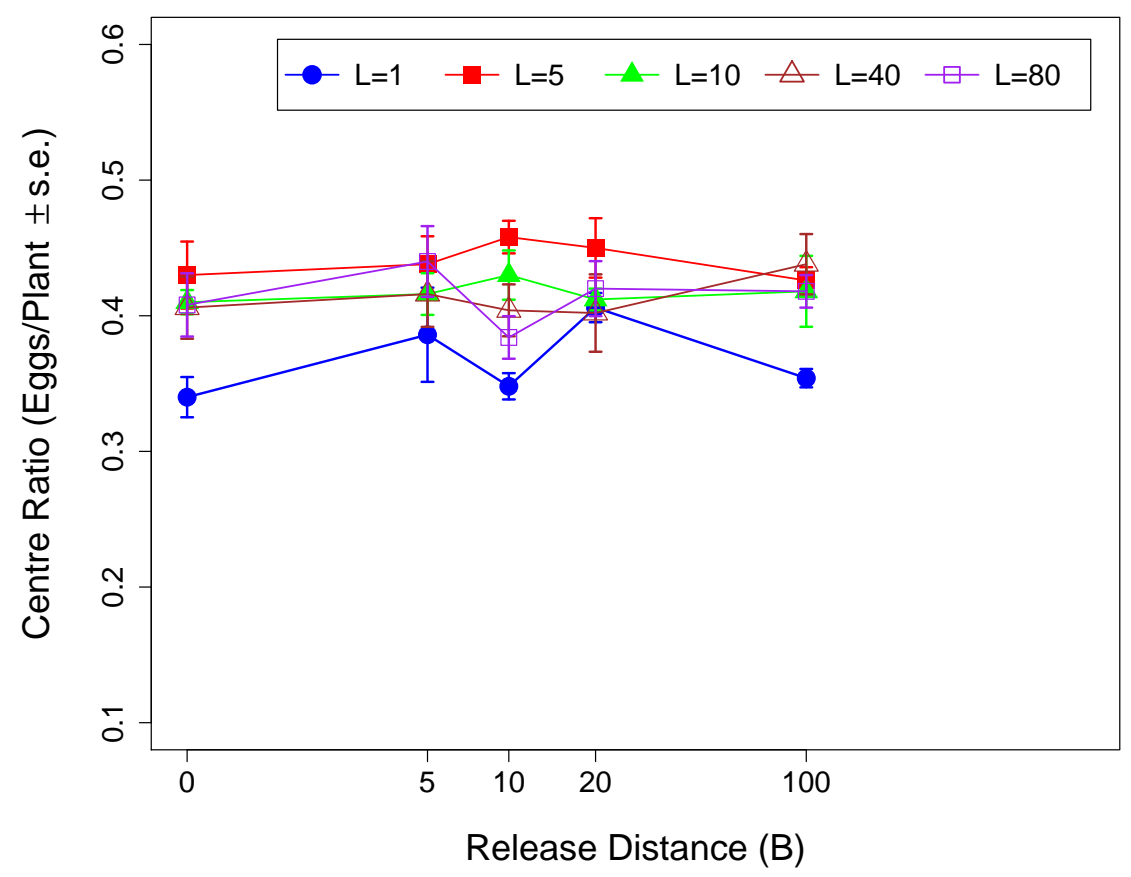

Figure 3.39: Trial $B: k=10$, varying $L$ and $B .(G=100$, Replicates $=5, P=80$, $S=10, R=5$, Layout $=$ corner - centre)

Increasing $B$ should have an analogous effect to reducing the step length in that it will increase the variation in moves across the patch. At the zero boundary with a step that always goes right across the patch (say $L=250$ ), the turning angle parameter should have no effect as the initial move is drawn from the same, uniform distribution.

The motivation for releasing foragers close to the resources without biasing the results was to improve processing speed. With this in mind, a potential "solution" was devised, which involved releasing foragers at a random point within a "zone" of release, rather than all from the same distance, exactly on the perimeter of the release boundary. This should be more representative of a sample of individuals arriving from further afield.

A simplified experiment was conducted (Trial D) where a comparison was made between releasing in a zone with an outer perimeter of $B=80$ (chosen because it is the step length) and a standard perimeter release at $B=800$. The null hypothesis is that releasing in a zone inside $B=80$ should produce the same results as releasing on the perimeter at $B=800$. Figure 3.40 shows these results, along with those of Trial $C$ which replicates a perimeter release at $B=0, B=80$ and $B=800$. The Kruskall-Wallis test for Trial D $(H=8.17$, d.f. $=1, p<0.004)$ shows a reduced statistic compared to Trial C $(H=30.32, d . f .=2, p<0.001)$, but the treatments of $B$ remained significantly different. From a visual inspection, the "zoned" release at 


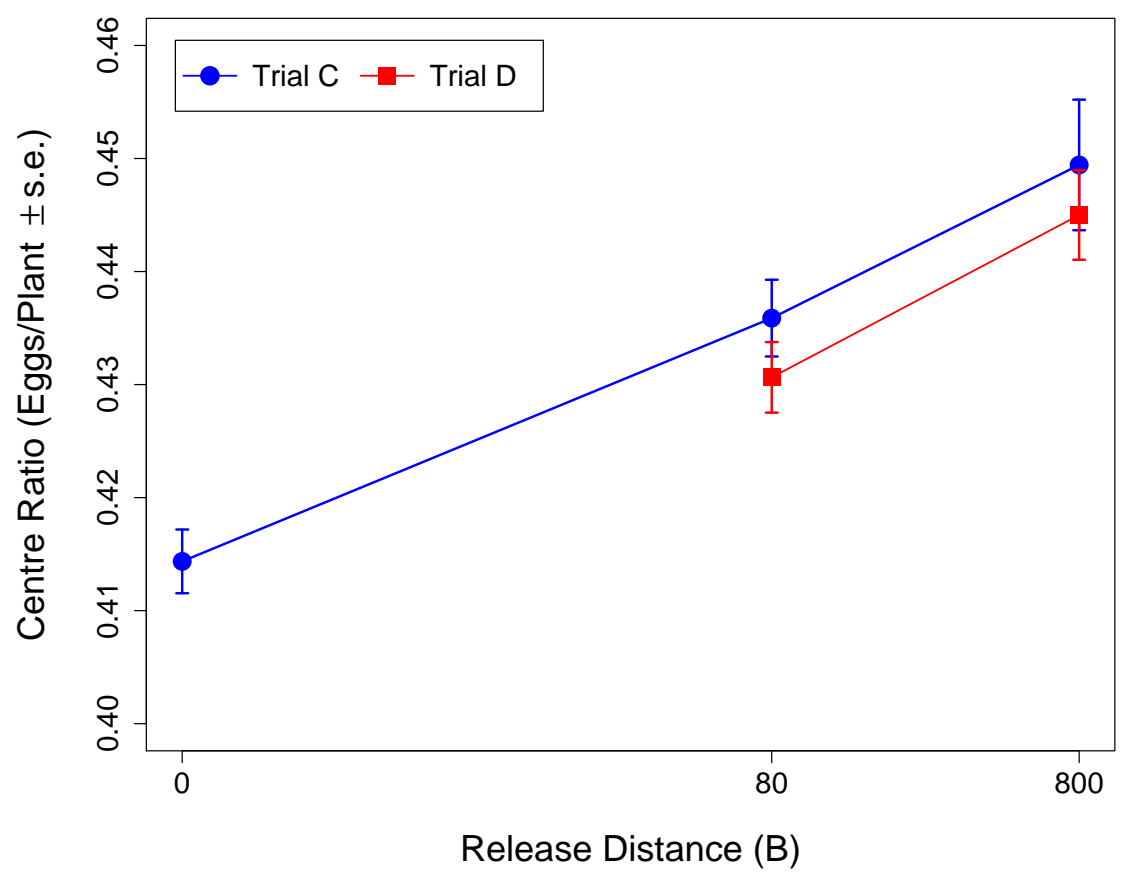

Figure 3.40: Foragers released within a "zone" around the zero boundary (Trial D) compared with a perimeter release (Trial C). $k=10, L=80 . \quad(G=500$, Replicates $=50$, $P=80, S=10, R=5$, Layout $=$ corner - centre $)$.

$B=80$ is not significantly different to a perimeter release at $B=80$. These results would need to be repeated for other movement parameters, but this might offer a solution as releasing in a zone is more efficient. A further option for future trials would be to release in a zone outside the perimeter of $B$, with a size equal to the current step length, $L$.

In summary, at a particular scale of resource patch, an increase in step length $(L)$ will reduce the effect of $k$. Conversely, as $k$ increases, the effect of $L$ is reduced. If the resources are "scaled up" (i.e. more widely spaced relative to $L$ ), the effect of $k$ will increase. Increasing $B$ increases the influence of $k$ as more steps (and thus random turns), will be executed.

Although these results are interesting, further investigation as to the causal factors was curtailed to allow progress on the rest of the thesis. A subsequent study could investigate the phenomenon more thoroughly. In practical terms, it can be seen that once a distance of $B=400$ is reached the effect is less pronounced. Subsequent trials in the edge effect experiment were released at $B=100$ as a compromise between efficiency and accuracy. It is also likely that as long as all trials are always released from the same distance, even if there is a bias, it will be common and so any interpretations which rely on relative comparisons will be sound. Finally, in larger, more complex layouts, the release boundary is much larger and relative to the size of the 
resources much further away, reducing the likelihood of an artefactual effect.

\subsubsection{Edge effect results}

The edge effect experiment was run with both layouts previously described (Figures 3.32 and 3.33). In analysing the results, the corner-centre-edge layout provides a more distinctive response because it has an increased "edge effect", or shielding of the central resources by those on the edge of the patch. It also has relevance in that it is the same layout which forms part of a larger layout in a subsequent chapter. This discussion will focus on the results from this layout only.

Each trial explored a different behavioural response to the resources:

- Correlated random walk (Trial B)

- CRW with olfaction (Trial C)

- CRW with vision (Trial D)

- CRW with vision and olfaction (Trial E)

For each of these, the same parameter space was manipulated:

- Movement parameters $(L, k)$

- Plant spacing and radius of attraction $(P, R)$

- Foragers laying single eggs vs multiple egg ( $M E, 5$ eggs per forager)

There are two response variables, the ratio of eggs per plant in the centre group of resources and the search efficiency of the foragers (calculated as eggs per forager released). Figures 3.41 and 3.42 graphically represent the breakdown of analysis for each response variable. The complete set of figures for each of these sections can be found in Appendix A. Selected figures have been repeated in this chapter for convenience. In the following sections, each of the areas of analysis are discussed with reference to the effect that the behavioural responses had on the results.

\section{Centre ratio}

Figures 3.43 and 3.44 show the overall ratios of eggs per resource group. For a single egg (Figure 3.43), the ratio is as would be expected from the boundary effect experiment, the centre resources are generally shielded by the edge resources. 


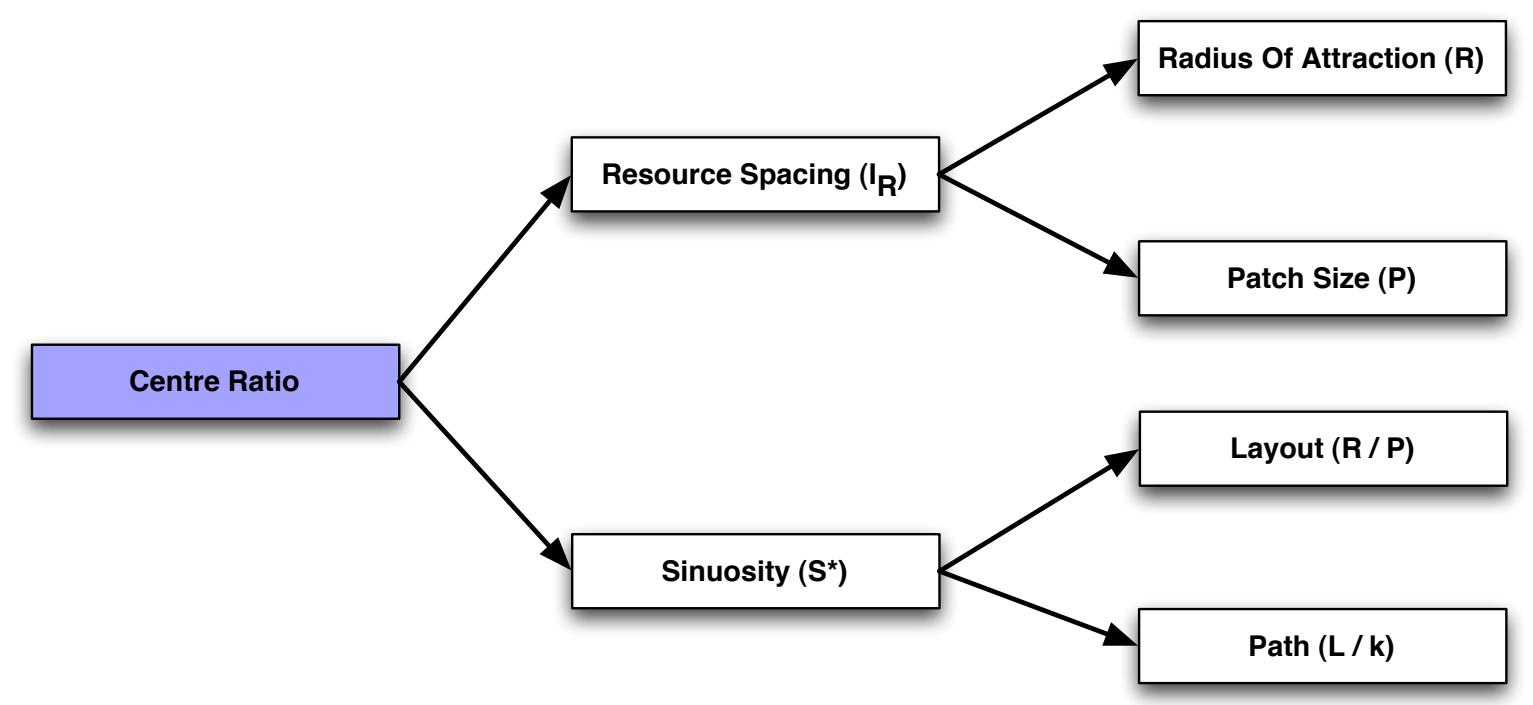

Figure 3.41: Structure of the analysis for centre ratio response.

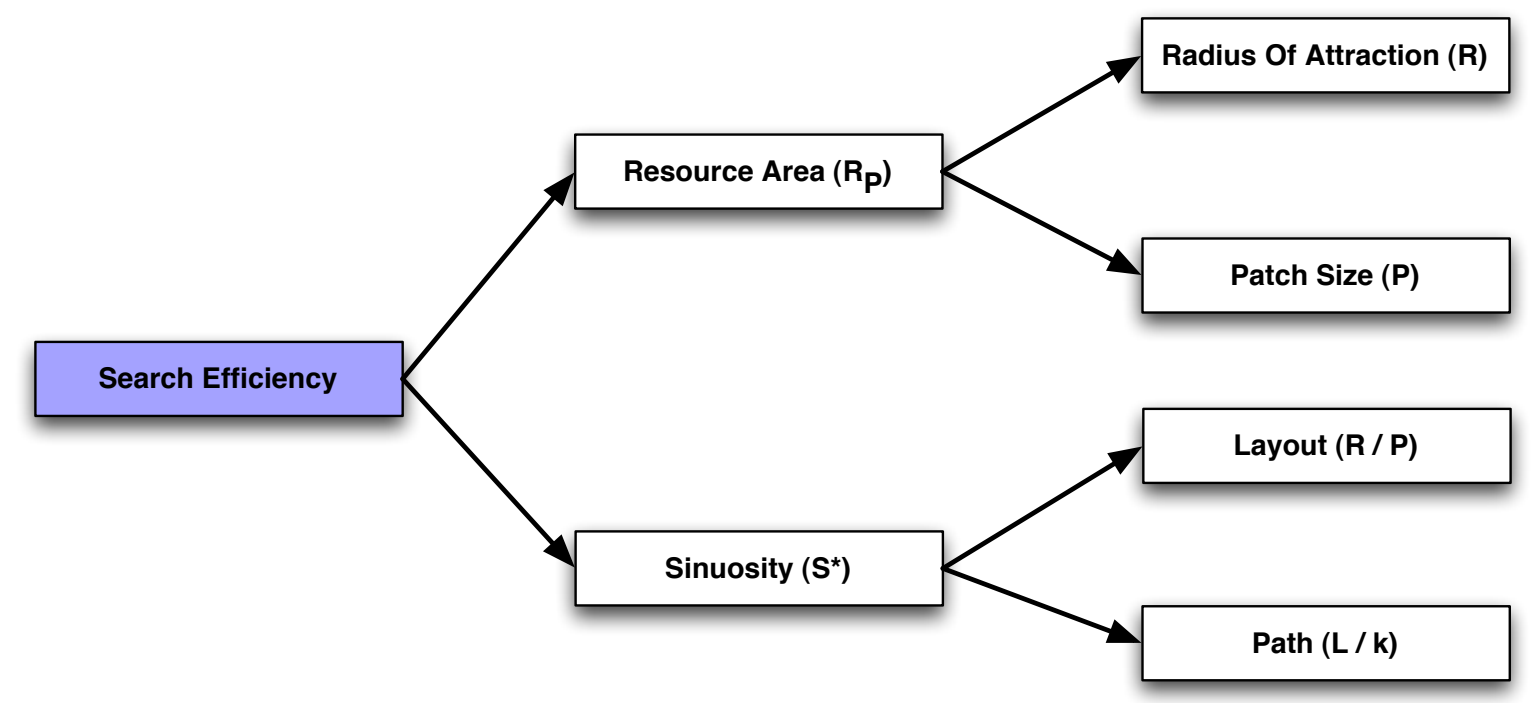

Figure 3.42: Structure of the analysis for search efficiency response. 
a) Total Eggs

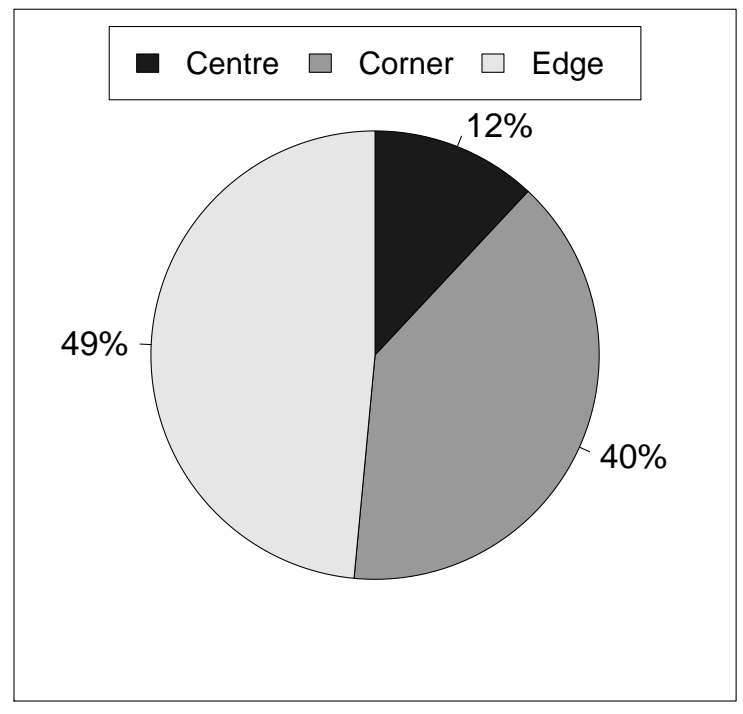

b) Eggs Per Plant

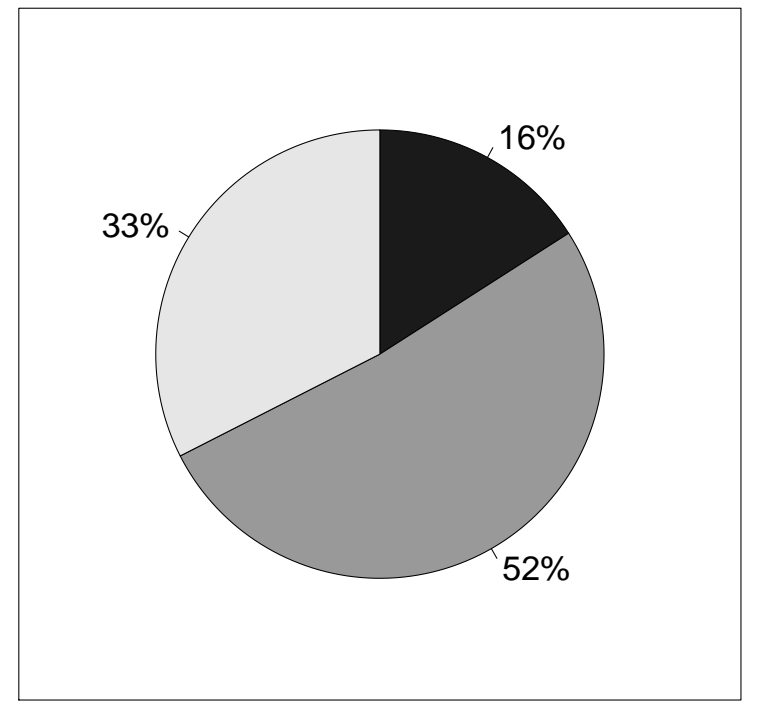

Figure 3.43: Ratio summary. Single egg.

a) Total Eggs

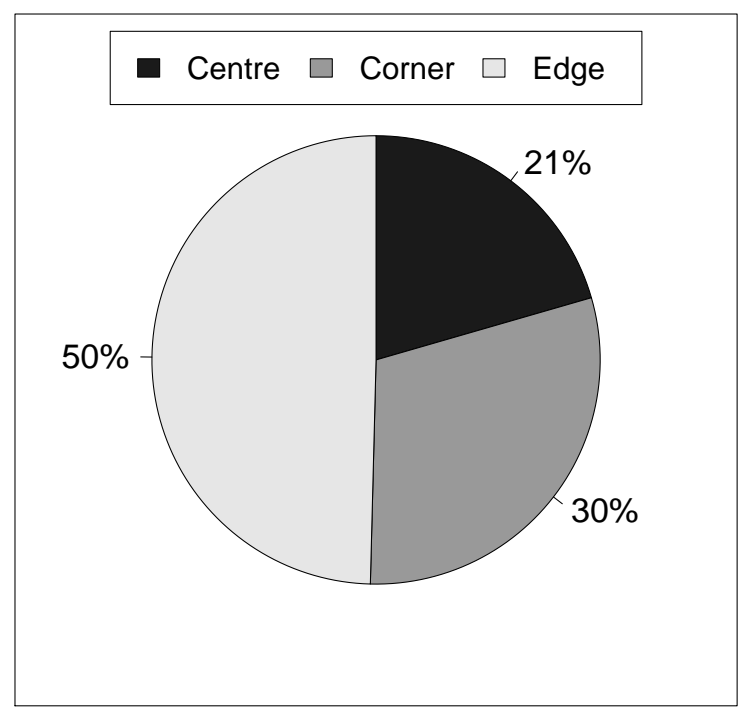

b) Eggs Per Plant

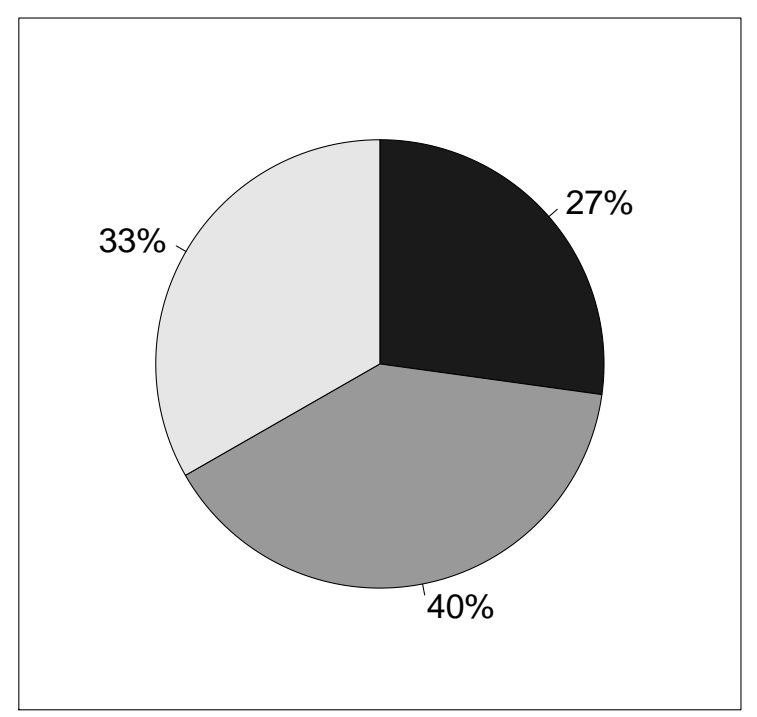

Figure 3.44: Ratio summary. Multiple eggs. 
When the foragers can lay more than one egg, this pattern changes, and we see something akin to the idealised ratio of $0 . \overline{33}: 0 . \overline{33}: 0 . \overline{33}$, although the order is still the same (Figure 3.44). These graphs also highlight the difference between measuring total eggs and adjusting for the number of plants by measuring eggs per plant.

\section{Centre ratio and resource spacing $\left(I_{R}\right)$}

The basic correlated random walk shows a straightforward response to the measure of relative spacing. As spacing increases, there is an increase in the ratio of eggs in the centre (Figure 3.45). This is not significantly affected by allowing multiple eggs except that the number of eggs per plant in the centre generally increases (Figure A.2-1). With larger gaps between resources, more foragers are able to penetrate to the centre of the patch.

\begin{tabular}{rrrr}
\hline $\mathbf{I}_{\mathbf{R}}$ & $\mathbf{R}$ & $\mathbf{P}$ & $\mathbf{I}$ \\
\hline-9 & 50 & 100 & -75 \\
-2 & 15 & 100 & -5 \\
-1 & 50 & 300 & -25 \\
1 & 50 & 600 & 50 \\
6 & 15 & 300 & 45 \\
8 & 15 & 600 & 120 \\
18 & 5 & 100 & 15 \\
26 & 5 & 300 & 65 \\
28 & 5 & 600 & 140 \\
\hline
\end{tabular}

Table 3.11: The relative spacing $\left(I_{R}\right)$ of the resource parameters ( $R$ and $P$ ) for each of the layouts shown in Figure (3.32).

With olfaction enabled, more eggs per plant are laid in the centre, particularly with multiple eggs, as can be seen from the scale of Figures A.3-1 and A.4-1. Generally the pattern is similar between olfaction and the CRW, as is more easily seen by examining the breakdowns by $R$ and $P$ (Figures A.1-2, A.2-2, A.3-2 and 3.48).

When multiple eggs and olfaction are enabled, a particularly strong relationship between patch size $(P)$ and centre ratio is seen (Figure 3.48). The foragers will tend to move toward the centre following the signal surface (Figures 3.34 to 3.36) and as the spacing increases they are less likely to become trapped by the outer resources. The influence of radius of attraction is reduced somewhat with $R=5$ and $R=15$ producing very similar responses, except in smaller patches $(P=100$, Figure 3.48). With a large $R$ an edge effect persists, reducing the eggs per plant in the centre.

When vision is enabled (Figure A.5-1), the overall picture is similar to both the CRW 
and olfaction trials. When the response is analysed by patch size $(P)$, a somewhat different picture emerges (Figure A.5-2), where for the lower radii of attraction ( $R=$ 5 and $R=15)$, increasing the patch size $(P)$ decreases the eggs per plant in the centre. Although the resources are closer together, once within visual range the foragers may make a "bee-line" for the centre resources and thus avoid becoming "trapped" in the radii of attraction of the edges. If however the spacing becomes very wide, the effect is the opposite and the number reaching the centre drops. This is perhaps because the central resources often fall outside the field of view whereas the edge resources are always likely to be detected, particularly for a tortuous path. This result could also be different if the probability of choosing a plant within the field of view was dependant upon distance from the resource (see discussion in Section 3.7). This effect can be seen as a "peak" in the ratio of eggs per plant in the centre in the mid range patch sizes (Figure A.5-2).

When multiple eggs and vision are enabled (Figures A.6-1 and A.6-2), a decrease in eggs in the centre is still noted with increasing patch size, but when patch size is small $(P=100)$, larger radii of attraction $(R=15, R=50)$ still have an influence, preventing foragers reaching the centre resources. It is also possible that foragers move away from the centre and then return to the edges, increasing the edge effect as patch size increases, similar to the effect noted by (Jones, 1977a).

A further point of interest with vision and multiple eggs occurs at the very low relative spacing ${ }^{13}$. The layout in Figure 3.32.g, receives more eggs in the centre than Figure 3.32.d, because the radii of attraction overlap so much as to actually reduce the edge effect (Figure A.6-9). As the spacing increases, a perfect barrier is created with less overlap and thus fewer get into the centre, even when there are multiple eggs laid.

In summary, as relative spacing increases, more resources in the centre receive eggs. This is consistent across all behavioural responses, although there is variation in the detail. The olfaction response generated exaggerated numbers in the centre at wider spacings, because the foragers are heading more directionally for the centre. With vision enabled, there may be an optimal spacing where more eggs are laid on the central resources, although this is influenced by the radius of attraction.

\section{Centre ratio and sinuosity}

The CRW has two main parameters, step length $(L)$ and turning angle concentration $(k)$. These can have similar effects, for instance increasing the step length is similar to having many shorter, highly directional steps. Sinuosity (here represented by $S^{*}$,

\footnotetext{
${ }^{13}$ Here in fact, the spacing is negative, the radii of attraction are overlapping
} 


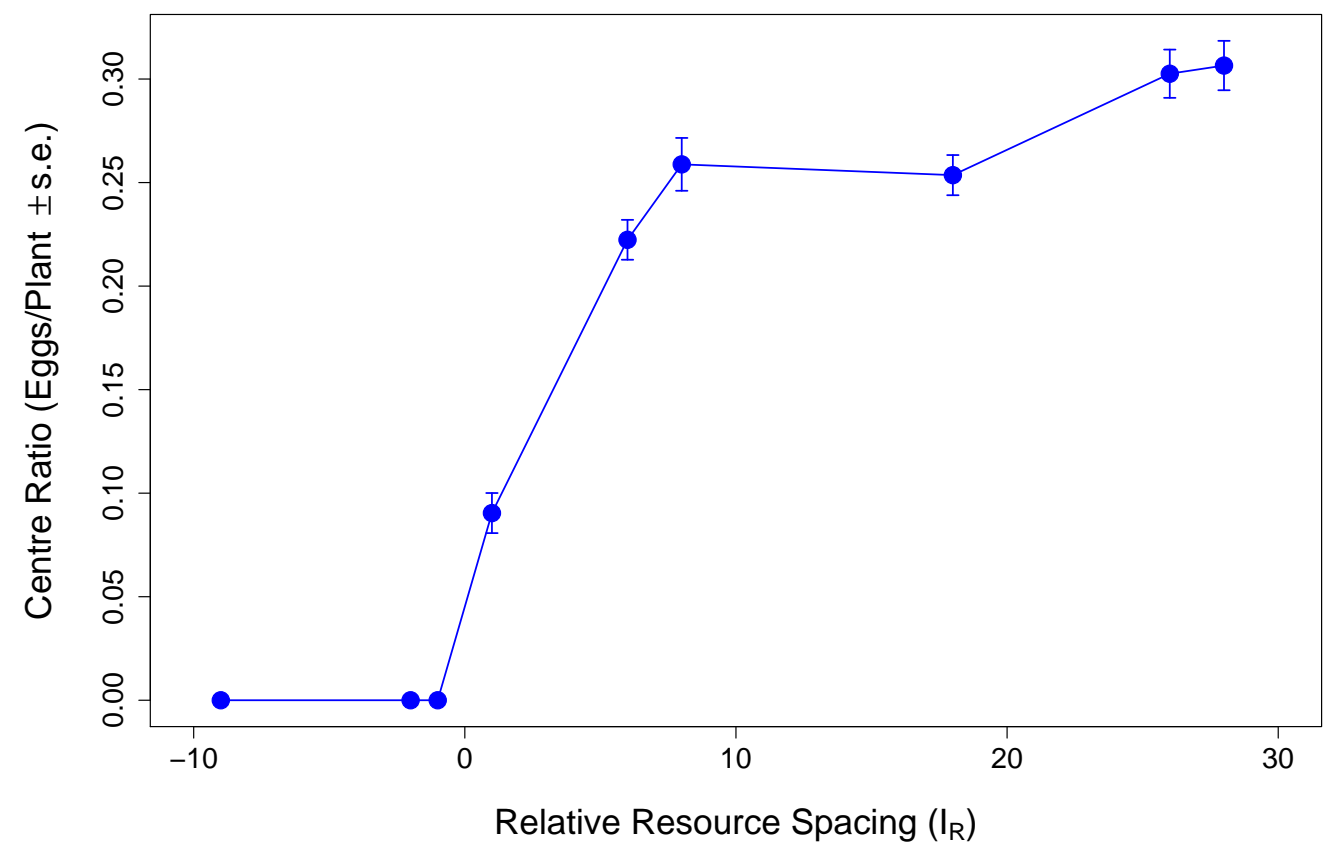

Figure 3.45: Centre ratio summary - Trial B (CRW), Single Egg. Proportion of eggs per plant on centre resources ( $y$-axis) vs. relative resource spacing, $I_{R}$ ( $x$-axis). Negative values of $I_{R}$ indicate that the radii of attraction $(R)$ are overlapping.

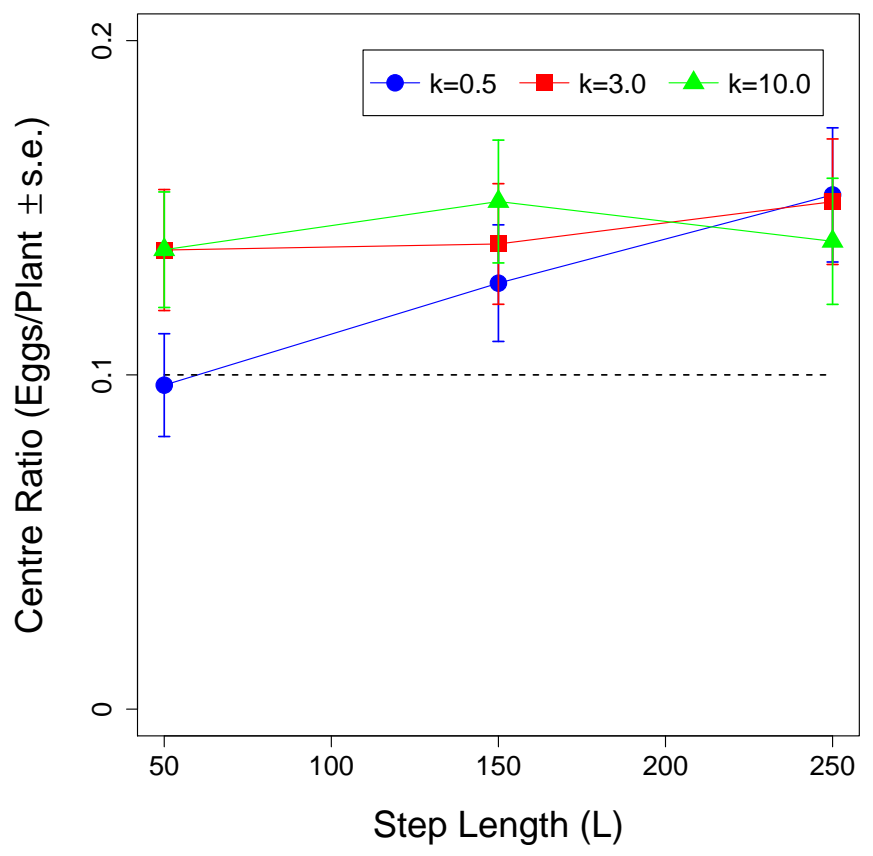

Figure 3.46: Centre ratio vs. L and $k$ - Trial B (CRW), Single Egg. Proportion of eggs per plant on centre resources ( $y$-axis) vs. step length $(L)$ for various turning angle concentrations $(k)$. Results are averaged over all combinations of $R$ and $P$. 
a)

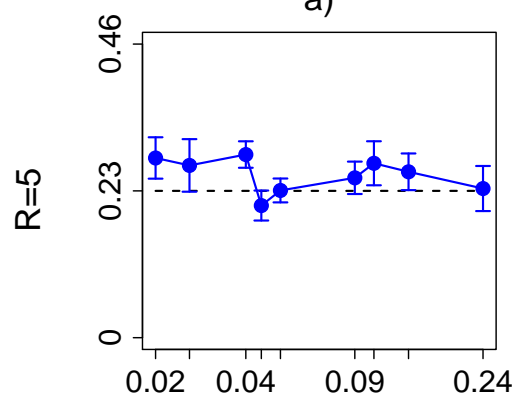

d)

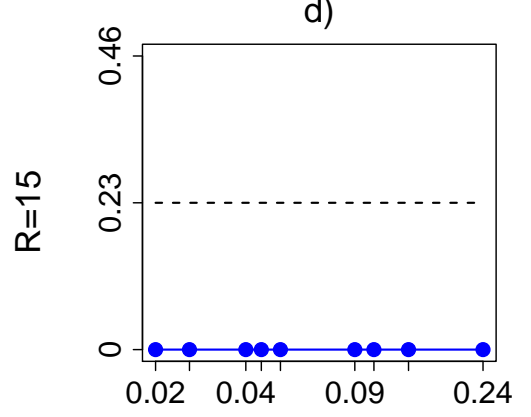

g)

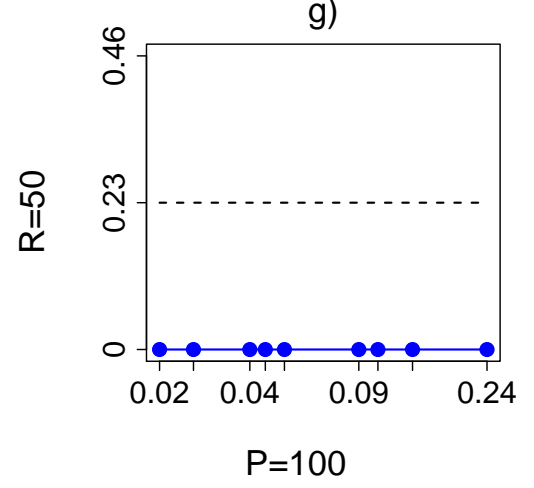

b)

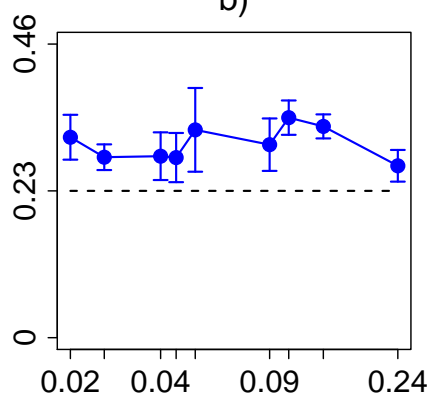

e)

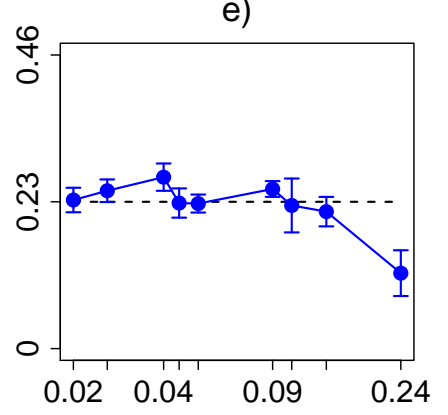

h)

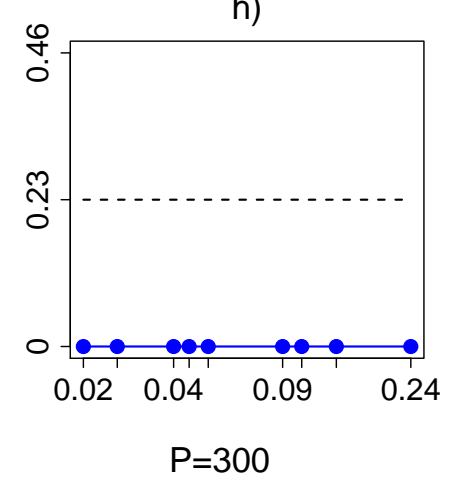

c)

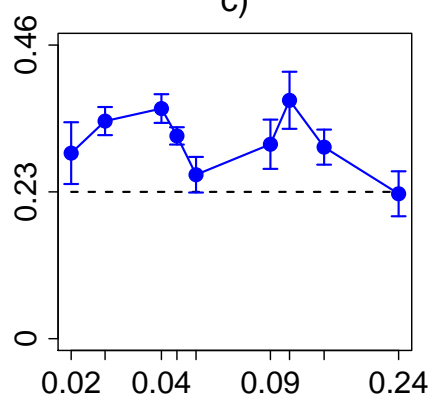

f)

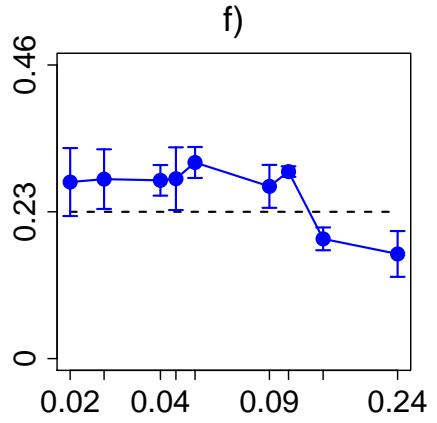

i)

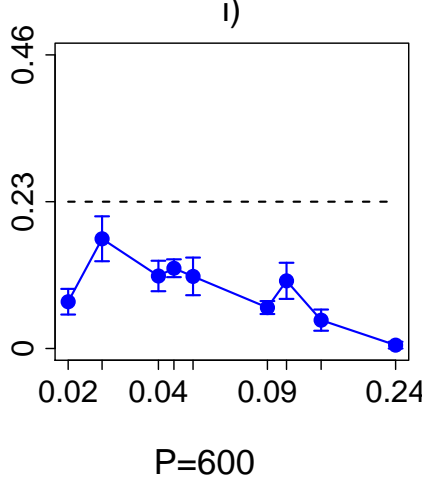

Figure 3.47: Centre ratio vs. layout - Trial B (CRW), Single Egg. Proportion of eggs per plant on centre resources (y-axis) vs. Sinuosity is plotted for combinations of radius of attraction $(R)$ and patch size $(P)$ in each sub-plot $(a-i)$. Radius of attraction increases from top to bottom and patch size increases from left to right. The layout is such that these results may be compared with Figure 3.32 to see the patch layouts. 
a)

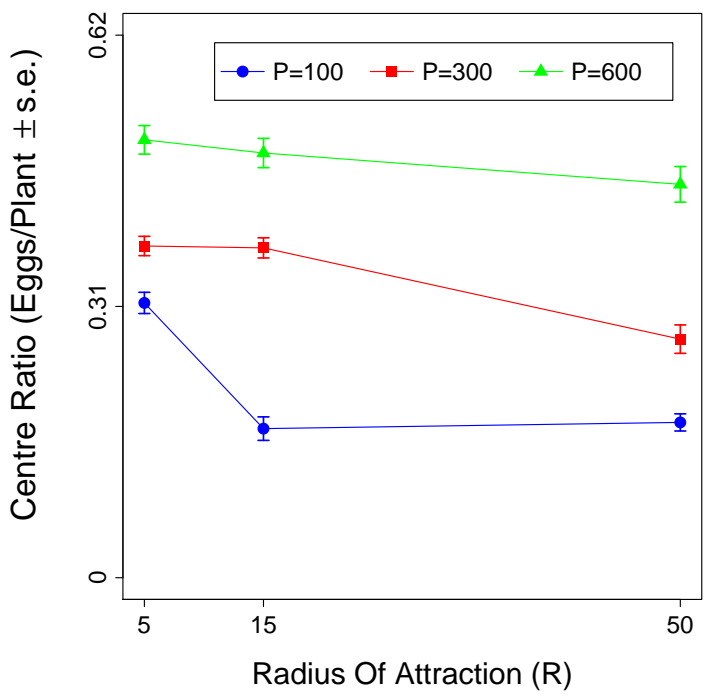

b)

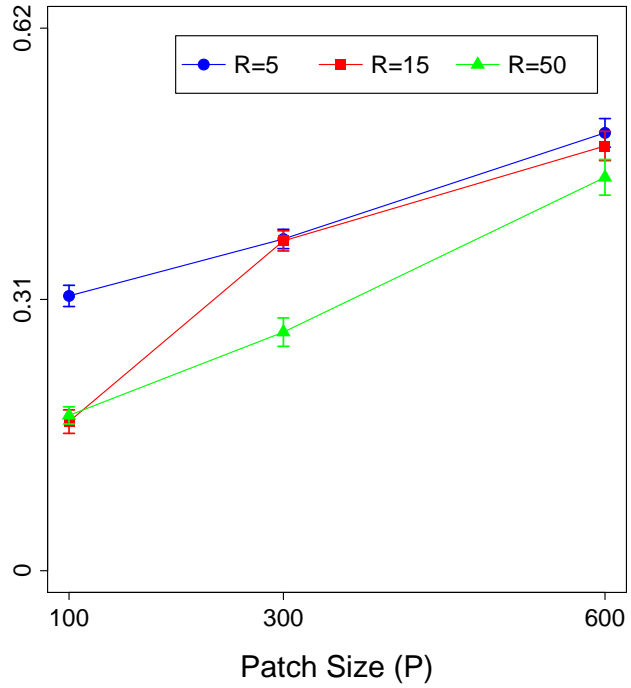

Figure 3.48: Centre ratio vs. $R$ and P - Trial C (Olfaction), Multiple Eggs. Proportion of eggs per plant on centre resources ( $y$-axis) vs. a) radius of attraction $(R)$ and $b$ ) patch size $(P)$. Results are averaged over all combinations of $L$ and $k$.

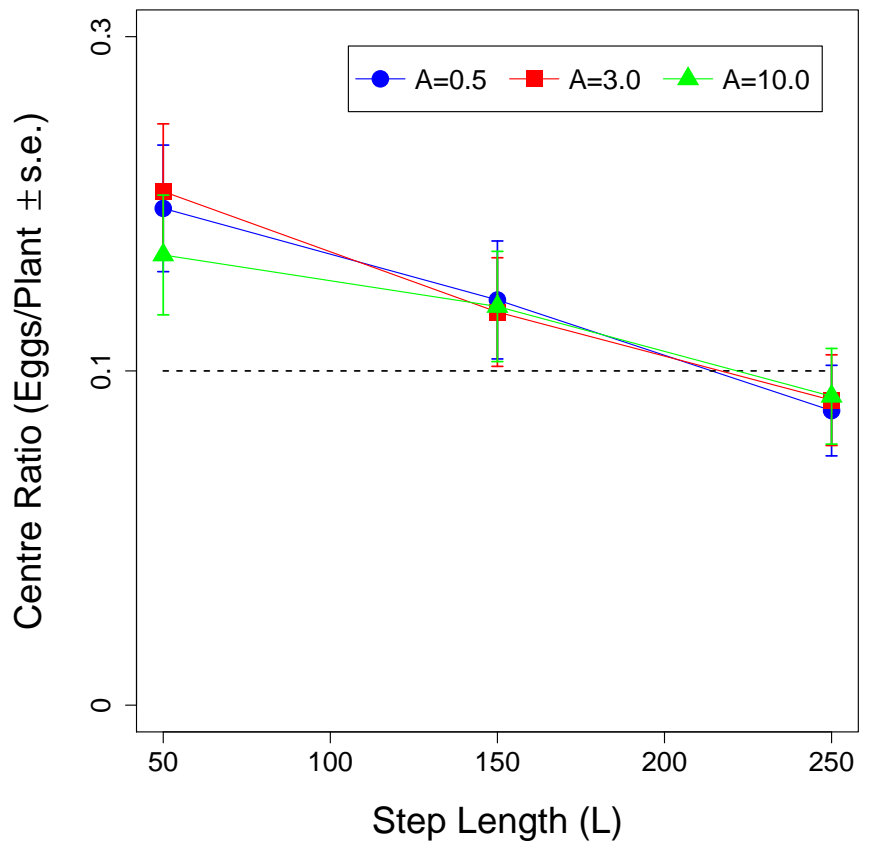

Figure 3.49: Centre ratio vs. L and $k$ - Trial D (Vision), Single Egg. Proportion of eggs per plant on centre resources ( $y$-axis) vs. step length $(L)$ for various turning angle concentrations $(k)$. Results are averaged over all combinations of $R$ and $P$. 
from Bovet and Benhamou 1988) is a useful metric which allows the combination of these parameters to be grouped along a gradient from straight line $\left(S^{*}=0\right)$ to so tortuous that all available space is occupied in a given area $\left(S^{*}=1\right)$. Table 3.12 shows the sinuosity calculated for the various parameter combinations that were used in the edge effect experiment. Generally this has the effect of ordering the combination as might be expected, the combination $L=250$ and $k=3$ is more directed (less sinuous) than a shorter step length with a much higher angle of turn. A step length of $L=1$ would require a $k>10$ before it approached the same sinuosity (Figure 3.8).

\begin{tabular}{rrr}
\hline Sinuosity & $\mathbf{L}$ & $\mathbf{k}$ \\
\hline 0.021 & 250 & 10.0 \\
0.027 & 150 & 10.0 \\
0.041 & 250 & 3.0 \\
0.046 & 50 & 10.0 \\
0.053 & 150 & 3.0 \\
0.092 & 50 & 3.0 \\
0.106 & 250 & 0.5 \\
0.137 & 150 & 0.5 \\
0.238 & 50 & 0.5 \\
\hline
\end{tabular}

Table 3.12: Sinuosity $\left(S^{*}\right)$ of the CRW parameter combinations used for the Edge Effect experiment.

A summary of the response of corner ratio to the sinuosity for the CRW is shown in Figure A.1-5. The range of movement parameters ( $L$ and $k$ ) and resource layout parameters ( $R$ and $P$ ) explored seem to have little effect on the number of eggs in the centre, except at the highest sinuosities. At high sinuosity, the number of eggs reaching the centre decreases, supporting the idea that more tortuous paths are more likely to encounter the outer resources first, as noted for the boundary effect experiment.

Olfaction exaggerates the effect of foragers becoming "trapped" on the outer resources, showing a reduction in the centre ratio (Figures A.3-5 and A.4-5). The foragers are moving toward the centre and yet still cover a large area around them because of their sinuous walk. With a very low turning angle concentration $(k=0.5)$, they may even turn back on themselves and move further away from the centre until they become attracted back in again by the odour. As noted in the section concerning spacing, this is comparable to the edge effect noted by Jones (1977a) where edge plants trap foragers that move away, and then back towards, a patch. 
Vision allows even tortuous paths to gain access to the centre (Figures A.5-5 and A.6-5). Once within the field of view, the forager is no longer moving randomly, but toward individual plants. This reduces the effect of becoming "trapped" by edge plants, except where $R$ is very high. This will occur as long as the field depth $(F D)$ of the foragers is deep enough that they can see past the edge resources into the centre when outside the patch.

Figure 3.46 shows the centre ratio broken down for each of the movement parameter values ( $L$ and $k$ ) for a CRW. All of the parameter combinations are reasonably close to each other, except for very low turning angles $(k=0.5)$. Low turning angle concentrations result in a positive relationship between step length and eggs per plant in the centre. It is likely that the effect of increased step length penetrating the centre is more apparent with higher turning angles. There is a slight "peak" for the more directional $(k=10)$ path, although the error bars suggest that this is not significant. This analysis suggests that for the CRW, the spatial arrangement and "arrestment" of the resources $(P$ and $R$ ) has a greater overall influence on the eggs reaching the central resources than the movement parameters ( $L$ and $k$ ) of the foragers.

With olfaction and more directional paths $(k=3, k=10)$, increasing step length decreases the number of eggs in the centre (Figure A.3-7). There is almost no effect for low directionality $(k=0.5)$, although a slight decrease in the centre ratio is seen for shorter step lengths $(L=50)$. Introducing multiple eggs accentuates the pattern (Figure A.4-7).

When vision is enabled, all turning angle concentrations show a similar decrease in centre ratio with increase in step length (Figures 3.49 and A.6-7). It is interesting to note that here, increased step length reduces the number in the centre, opposite to the effect observed with a CRW where increased step length is more effective in penetrating to the centre. Intuitively, a shorter step length combined with vision should result in more eggs on the edge resources. It is possible that with shorter step lengths there are more "opportunities" to move toward the edge cabbages and so the number "trapped" on the outer resources is increased, which could also explain the negative response found with olfaction (Figures A.3-7 and A.4-7).

Where vision and olfaction are both enabled the results match closely the visual search (Figures A.7-7 and 3.49, respectively). The vision component overwhelms the olfaction due of the structure of the model (vision is always preferred to olfaction) and the fact that the experimental area is not large enough relative to the field of view to allow for olfaction to have an effect. The foragers can usually see the cabbages very soon after reaching them by olfaction. Although efficiency might be increased (foragers remain in the patch because of olfaction), the responses are very 
like vision alone.

Figure 3.47 show the results for a CRW broken down by resource layout. Each plot shows the corner ratio by sinuosity. The arrangement on the page is the same as for Figure 3.32, for comparison. Overall, a similar pattern is seen across layouts, but the response to sinuosity is variable, producing "peaks", particularly for the combinations $L=250, k=0.5$ and $L=250, k=3$ (sinuosities of $S *=0.106$ and $S *=0.041$, respectively). For $R=5$, as the patch size increases, there is an increase in the number in the centre as would be expected. Where the radius of attraction is larger (easier to find resources) there is a decline in the number of eggs reaching the centre as the paths become more sinuous. The extreme is $R=50$, where there is a strong decline, again symptomatic of more directional paths penetrating to the centre more easily. Interestingly in the case of plot 3.47.i $(R=50, P=600)$, the most directional path also appears to be less penetrating. It is potentially too directional. In other words, when plants are widely spaced but easy to find, paths of intermediate sinuosity are most able to penetrate to the centre of the patch.

Introducing olfaction increases the number of eggs in the centre for all path combinations (Figures A.3-9 and A.4-9). The patterns appear similar to the CRW, but more pronounced. Allowing multiple eggs does not have a significant effect except at high radius $(R=50)$ where the more directional paths are achieving significantly higher centre ratio than with single eggs.

The visual results show no clear pattern other than that the centre ratio seems less dependant on sinuosity as measured here (Figures A.5-9 and A.6-9). There are peaks and troughs but it is difficult to elicit a clear pattern. Introducing multiple eggs most certainly increases the number of eggs in the centre. The decreased centre ratio observed with a CRW at high sinuosities and patch sizes (e.g. Figure A.1-9.i) is not observed for visual searchers. Enabling vision has the general effect of reducing the influence of $k$, because when resources are within the field of view, the foragers are no longer choosing their direction at random.

As with the other data, the combination of vision and olfaction is very similar to vision solely.

\section{Search efficiency and resource area $\left(R_{P}\right)$}

Search efficiency is related to the area covered by the radii of attraction rather than the spacing, as this should be the overriding factor that affects the success of the foragers. Indeed with a CRW, the response is again straightforward, and shows a curved trend when plotted on a $\log x$-axis (Figure 3.50). Introducing multiple eggs shows a very similar pattern but with much greater efficiency (Figure A.2-3). This 
results demonstrates that smaller, more dense patches result in lower forager search efficiency, especially when resources are hard to find (small radius of attraction), supporting the results from Cain (1985). Radius of attraction and patch size interact to produce this effect (Figure A.1-4).

With olfaction enabled, a mid-range patch size is found the most easily, except when $R$ is very high (Figure A.3-4). This is because the foragers are generally being attracted to the centre of the patch, and as the spacing increases, the edge plants are moved further from the centre, where the foragers spend most of their search time. The pattern remains consistent when multiple eggs are introduced (Figure 3.51), except that the overall values for efficiency are higher.

When vision is enabled, for foragers laying single eggs, a strong positive correlation between patch size $(P)$ and efficiency is observed, with no effect of radius of attraction (Figure A.5-4). The vision "sense" is in an alternative mechanism of detection to the radius of attraction and in this case completely negates any effects of $R$. When multiple eggs are introduced, the radius of attraction again has an influence, larger radii resulting in greater search efficiency. When the results are aggregated and plotted versus the relative area, the response is no longer so clear and peaks and troughs appear (Figure A.5-3). This pattern persists with multiple eggs (Figure A.63). The pattern is not well defined from these results and further experimentation and analysis would be required to determine the causes of this variability.

\section{Search efficiency and sinuosity $(\mathrm{S} *)$}

Search efficiency for the CRW when analysed by sinuosity shows a similar response to the centre ratio, in that it is fairly constant except for the higher sinuosities, where it declines (Figure A.1-6). Introducing multiple eggs does somewhat reduce the variation (Figure A.2-6). As the sinuosity of the path increases, a smaller area is covered, so fewer foragers make it to the centre, and more are required to populate the patch with the specified number of eggs $(G)$. These simulations also take longer to run (approximately 140 processing hours for $L=1, k=0.5$ ). As was noted in the boundary effect experiment moving the release boundary further away is analogous to reducing the step length, representing a kind of scaling.

Olfaction is most efficient when combined with mid-range sinuosities (Figure A.36). Highly sinuous paths (as previously noted) are less efficient in covering the area, but highly directed paths (particularly if step length is high) allow less orientation within the odour plume (the resolution of the steps is too large to allow navigation to the peak of odour concentration). Multiple eggs particularly exaggerates the response (Figure A.3-6), lower and higher range sinuosities having considerably 


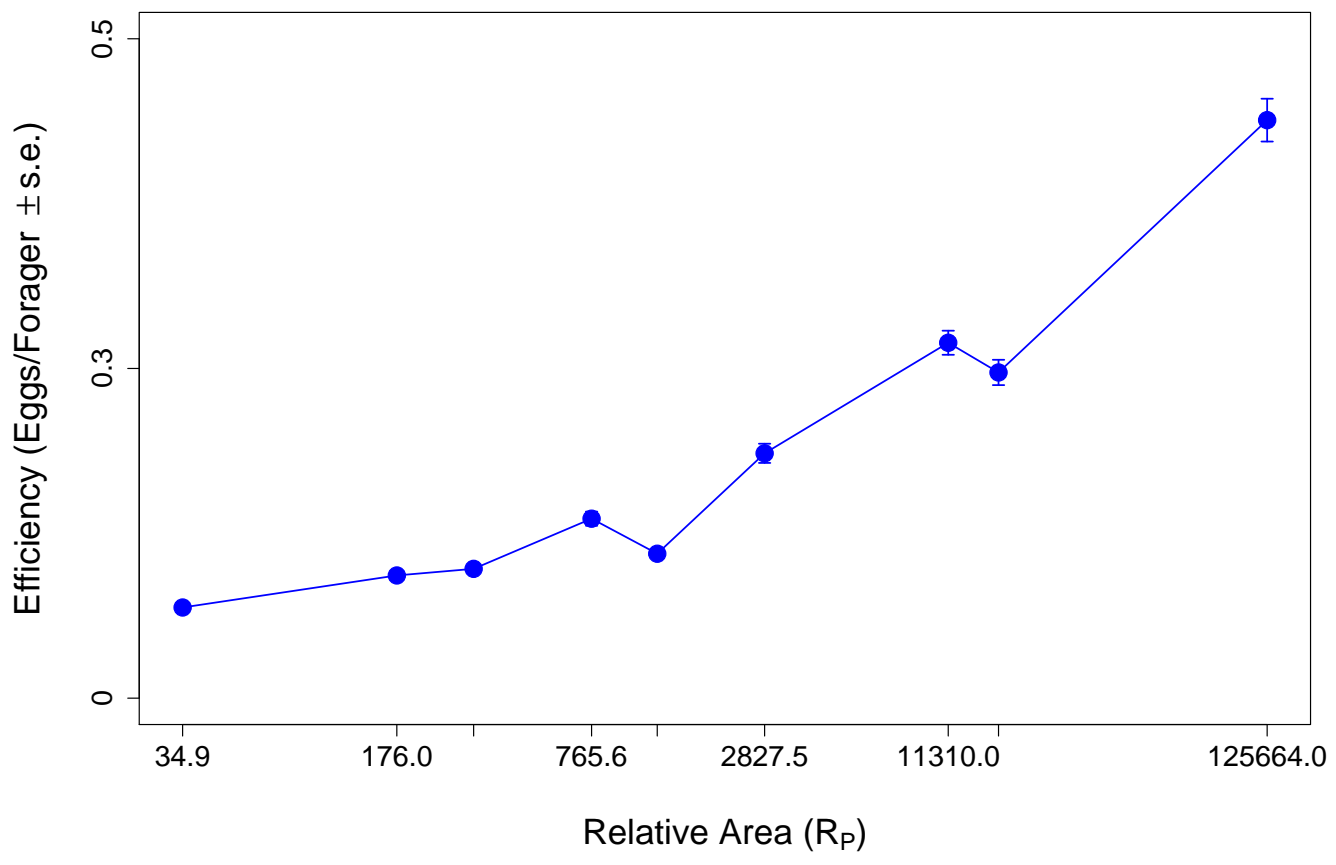

Figure 3.50: Search Efficiency Summary - Trial B (CRW), Single Egg. Proportion of eggs per Forager released (y-axis) vs. Relative Area $\left(R_{P}\right)$. See Chapter 3 details of this calculation.

a)

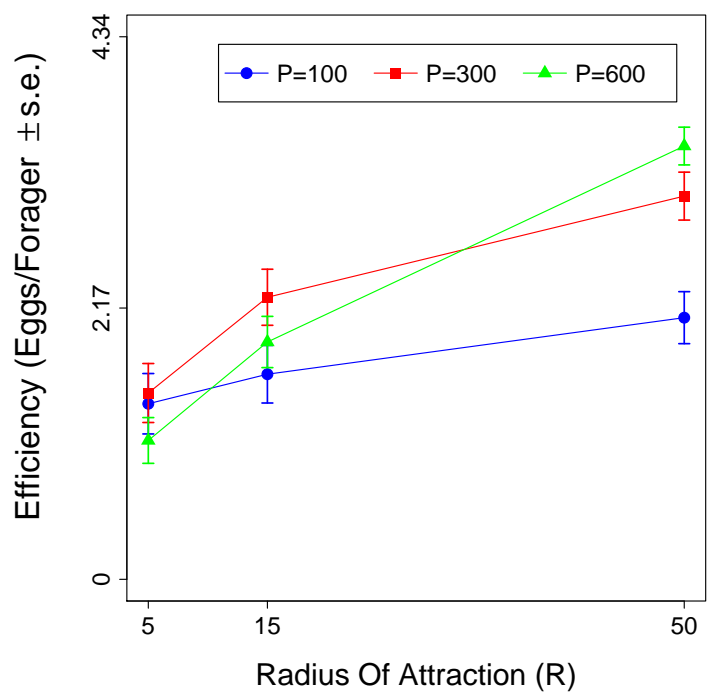

b)

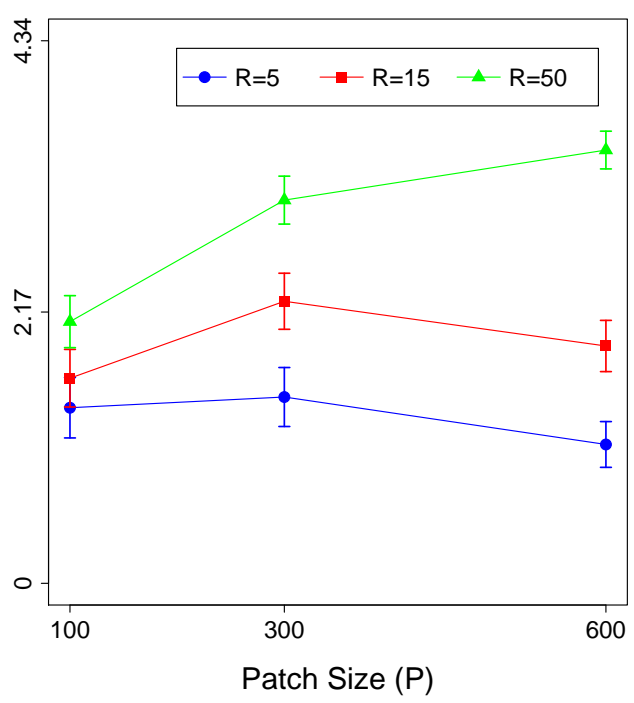

Figure 3.51: Search efficiency vs. $R$ and P - Trial C (Olfaction), Multiple Eggs. Proportion of eggs per forager released ( $y$-axis) vs. a) radius of attraction $(R)$ and $b$ ) patch size $(P)$. Results are averaged over all combinations of $L$ and $k$. 
lower efficiency than the mid-ranges. Here, the mid-range step lengths have more opportunity to roam over the patch and are prevented from escaping by being attracted back into the patch by the odour. This should also be a scale effect, the step lengths representing an optimal response being mid-range, relative to the size of the experimental patch, although further experiments with different patch sizes would be required to confirm this.

One of the behaviours noticed with this model of olfaction is that if a forager is moving directly away from the odour plume, it will maintain a straight line path because the signal is of equal strength on each side. In this case, introducing more random turning (decreasing $k$ ) can increase the efficiency of the search, as it allows for turning to the left and right, thus "exploring" the gradient of the odour surface and re-orienting back toward the peak. Hoffmann (1983) has also noted that a random element to systematic search can increase efficiency for desert Isopods searching for their burrows.

Vision, as before, tends to negate the effect of sinuosity, as mentioned due to the fact that the foragers are moving less randomly (Figure A.5-6). Introducing multiple eggs does elevate forager efficiency at higher sinuosities (Figure A.6-6).

As with the centre ratio, when the response of the CRW is analysed by step length and turning angle concentration, it is the low turning angle concentration $(k=0.5)$ which shows the mot definite response. With an increase in step length, there is an increase in efficiency. With the most directed path $(k=10)$ efficiency is reduced with increasing step length (Figure A.1-8).

When olfaction is enabled, efficiency generally declines with step length, especially when multiple eggs are laid (Figures A.3-8 and A.4-8). Longer step lengths make it harder for the forager to respond to the olfaction signal, especially over a relatively smaller patch size, they will continually step right across the patch and then back again, often escaping the landscape completely.

Similarly to the centre ratio, vision reduces the effect of the angle of turn upon search efficiency and brings the response of all parameter combinations closer together (Figure A.5-8). When multiple eggs are laid, the response is also negative (Figure A.6-8). Longer step lengths produce less efficient foragers, for similar reasons to the olfaction perception. If the step lengths are too long relative to the patch size and resource spacing, the foragers have less opportunity to orientate towards resources. The reduction in efficiency will be particularly apparent when multiple eggs are laid as this confers an advantage to the mid-range sinuosities, in terms of being able to move around the patch once it has been discovered. Again, this response is dependant upon the scale of the step lengths relative to the size and spatial arrangement of the resource patch. 
The results for search efficiency are consistent across the layouts, such that these detailed graphs provide little extra insight beyond the summary graph. A general trend for the CRW is that the efficiency increases with $R$ and $P$, and that multiple eggs exaggerates the patterns observed. The trend for mid-range sinuosities to be the most efficient is re-iterated (Figures A.1-10, A.2-10, A.3-10, A.4-10, A.5-10, A.610, A.7-10 and A.8-10).

\subsection{Discussion}

In this chapter a simulation framework was introduced for exploring correlated random walks with an option for including responses to perceptual information about the resources. The primary aim was to apply this framework to the movement behaviour and egg distributions of $P$. rapae which were observed in a separate set of field experiments (Hasenbank, unpublished data). The correlated random walk is a tried and tested method of modelling individual animal paths (Cain, 1985; Chapter 2). It is also known that sensory perception plays an important role in the searching behaviour of ovipositing insects, particularly visual perception for P. rapae (Hern et al., 1996; Chapter 2).

Previous simulations have been constructed which have many behavioural parameters (Jones, 1977a) and have produced a close match to observed egg distributions. Other models have incorporated concepts of visual and olfactory perception, for example the model of Cain (1985) introduced the concept of a "radius of attraction". Bukovinszky et al. (2005) incorporated a response to density representing olfaction and a "window of perception" representing vision, executed on a grid based landscape.

Here, a simulation framework is described which balances simplicity and realism. A model is suggested which based upon a correlated random walk with provision for both visual and olfactory senses, executed in continuous space.

A radius of attraction provides a representation of the "arrestment" of a passing forager to a host plant. The visual perception is modelled as a "field of view" within which the searcher will respond to objects, much like the "reactive envelope" of Roitberg (1985). The olfaction perception is based on the concept of an "odour plume" which is present in the air and which increases in concentration with an increase in number of resources emitting the odour (Hogan, 2006; Dusenbery, 1989). Foragers may turn left or right to orientate themselves towards the highest area of odour concentration (the "peak" of the odour signal surface).

The first aim of the framework was to provide an environment in which the ovipo- 
sition behaviour of Pieris rapae could be simulated, resulting in egg distributions which can be compared with those observed in the field.

Chapter 4 presents the results of simulation experiments conducted using resource layouts that were used in field experiments as part of the same project.

A second aim of the framework was to create an environment in which various simulation strategies could be applied to a specified spatial arrangement of resources in a consistent manner, providing common features for representing the resources, controlling and searching the parameter space and reporting results. There is a significant effort of work required to manage complexity of both input and output to such a simulation and so the framework attempts to provide a set of standardised mechanisms for this which are independent of the logic of the behavioural model.

The framework has been completed to a point where it could be of use to external researchers wishing to experiment with a random walk model over a particular resource layout. With some work required, there is provision within the framework to incorporate population level effects. The current thesis is concerned only with immigration to a patch, but the framework also supports "birth" within the patch, mortality and multi-species interactions. For example it would be possible to allow competing species to interact and have an effect on the mortality of each others larvae. Parasitoids could be allowed to parasitise the eggs of the foragers. Finally, it would be possible to extend the landscape component of the framework to incorporate more realistic features, possibly even soured from GIS information.

In order to thoroughly test the utility of the framework and explore the model's basic behaviours, two "simple" experiments were conducted with reduced layouts, the aims of which were to explore the effect of the "release boundary" and the shielding effect of edge resources in a patch. For each experiment a subset of the parameter space was explored.

\section{The "boundary effect"}

The "boundary effect" simulation experiment showed that releasing foragers closer to the resources produced a slight change in the ratio of those in the "centre" resources to the "corner" resources. This was an unexpected result, initially the experiment was designed as a simple calibration test. The most likely factor affecting the result is "shading" of the centre resources by the corners, combined with increased randomness and hence coverage as the boundary is increased, or the step length decreased.

The main reason for preferring a close release distance was processing time due to 
the area restricted nature of the correlated random walk (Chapter 2), particularly at low step lengths and low turning angle concentrations.

Subsequently, we performed informal experiments using the concept of simply creating random steps that cross a boundary. These demonstrate that, infact, the orientation of steps which cross the boundary are not uniformly distributed with respect to the centre of the patch. The actual distribution shows that more of the steps will be headed directly for the centre of the patch. This is an effect of the fact that as the start position moves further from the boundary, the range of possible azimuth's which would cause the step to cross the boundary is reduced, until at a distance equal to the step length, there is no chance of the step entering the boundary, even if it moves directly towards the centre. This is an underlying bias in the movement and thus it is not as surprising as initially thought that there is an effect on the distribution of "eggs".

Although interesting, it was not the main focus of research and so further study was not continued. Several attempts to quantify the effect were made and future work could continue this line of enquiry. In the end, the magnitude of the effect was relatively minor and the layouts used in Chapter 4 were much larger, so there is less likely to be a bias. If all experiments are executed with the same release distance, the same bias, if any, should apply.

It should be borne in mind that the potential for a bias exists, particularly if an interpretation of a particular result depends on a very slight quantitative difference. In this situation, the experiment could be re-run with a greater release distance to check for bias.

\section{The "edge effect"}

The edge effect simulation experiment was designed as a simple exploration of the interaction between forager movement, perception and the spatial arrangement of the resources. The edge plants act as a "trap" to incoming foragers. The closer these resources are to each other, or the wider their arrestment zone (radius of attraction), the more impenetrable this boundary is. In the real world, this effect might be particularly prominent if the resources were for example pitfall traps, in which case the situation would be as for the single egg experiments conducted where a forager once attracted to a resource has no further interactions (it is in the "pit"). Jones (1977a), amongst others, noted that there is the potential for an edge effect as foragers leave a patch because they reach an area with few resources and so turn back toward the patch, re-engaging and thus visiting more often the plants on the edge.

The protection of the centre plants was, unsurprisingly, strongly influenced by the 
radius of attraction and plant spacing. Generally, more tortuous paths increase the number of foragers "trapped" on the outer resources. This is because with an increase in tortuosity, the area of the search becomes increasingly restricted, and more thoroughly covered, as is indicated by the mean squared displacement (for the same number of steps, there will be a lower MSD when tortuosity is high). The costs of an area restricted search are particularly important as Cain (1985) noted, when mortality is high. More time spent covering an area more thoroughly may not be available with a limited life span or high predation risk. Longer step lengths penetrate the centre more effectively. When the foragers can lay multiple eggs, the "edge effect" is reduced, as the foragers are able to make subsequent moves from the edge into the centre of the patch.

When visual perception is enabled, at closer spacings, the centre plants receive more eggs and so the effect of the outer plants is reduced. The visual foragers can "see past" the edge plants. This demonstrates a difference between the representations of the radius of attraction vs the "field of view". With the radius of attraction, if it is high enough, the edge plants will always trap the forager. This might influence the results.. When the plant spacings become wider, visual searchers return to concentrating their eggs on the edge plants. This might be an effect similar to that noted in Jones (1977a) whereby as the foragers move to the outside of the patch, particularly if they are moving with a high degree of randomness, they may turn and be re-attracted to the edge plants. In this way, vision is comparable to the MISS parameter of Jones' model (Section 2.8), which served the similar purpose of representing moves back towards a plant in the absence of any others nearby.

One of the features of the framework that was implemented but unused in the experiments here, was the facility to have the the probability of choosing a resource within the visual field become a function of the distance to the resource (Section 3.3.4). This might change some of the patterns observed, particularly it was noticed that the edge effect was reduced with vision enabled for some layouts. Possibly this result would not be observed if the foragers always prefer resources which are closer to them, in this case the situation is more like that of the simple radius of attraction model.

It was observed when comparing the movement parameters against the patch layout (Figure 3.47), that for easy to find, widely spaced plants, there appeared to be an optimum sinuosity for penetrating the centre resources (Sub-figure 3.47.i). Such responses often indicate a stabilising process in terms of ecological systems and it is interesting to observe that such a relationship may exist between an animal's pattern of movement and the spatial arrangement of its resources.

In terms of foragers success, the foragers are generally more successful when the 
resources are more uniformly spaced, supporting the results from Cain (1985). With an increase in radius of attraction, longer, straighter paths are more optimal. When olfaction is introduced with a longer step length, the success is reduced because the foragers either escape or do not have enough steps to orientate as well to the centre of the patch. One interesting observation from the olfaction behaviour is that because the foragers are only able to turn left or right, if they begin to head directly away from the resource, they tend to keep going in that direction because the relative signals are equal on each side. In this case, a more "random" movement (i.e. lower turning angle concentration) increases the efficiency of the search pattern, allowing a greater sampling of the environment, as observed by (Hoffmann, 1983; Bell, 1991).

It was also found that an olfactory search was most efficient when combined with mid range sinuosities. This provides a similar message to that found in Vergassola et al. (2007) and suggested in Bell (1991) that having a random element to the movement can increase the efficiency of the search. In the case of Vergassola et al. (2007) the situation examined was one of locating a resource in a highly variable olfactory plume (relative to the scale of the organism's perception). In the case of this study, increasing the number of random moves allowed better orientation to the source which is a simple demonstration of the effect of random movement to aid orientation toward a resource.

\section{Comparative model analysis}

Although the current implementation contains a specific set of behaviours for the foragers, it would be entirely possible to replicate other models from the literature, such as Jones (1977a)'s within the framework in the future and thus explore comparatively the effect of the model structure itself on the results. An integrated approach would benefit the field in general, and particularly aid future researchers in assessing how these models function or field researchers wishing to apply these models to their own data, without the expense of detailed software programming. This framework is a step in that direction.

\section{Summary}

The simulation framework presented has been thoroughly tested and provides a novel mechanism for incorporating a sensory response into simulations of animal movements. Further it provides a platform within which any model of behaviour may be represented for the purposes of comparative study. For example, one avenue considered was to incorporate the "motivational state" of a butterfly as described by Hern et al. (1996) and reviewed in Chapter 2. The framework has been successfully 
used to explore the oviposition behaviour of $P$. rapae (Chapter 4 ) and it is hoped that it will provide a useful tool to future researchers. 


\section{References}

Agostinelli, C. and U. Lund, 2006: CircStats R package version 0.2-1. Internet.

Batschelet, E., 1981: Circular Statistics In Biology. Mathematics in Biology. Academic Press, San Fransisco.

Bell, W., 1991: Searching Behaviour: The behavioural ecology of finding resources. Chapman and Hall, London.

Bell, W. J., 1990: Searching behavior patterns in insects. Annual Review Of Entomology, 35, 447-467.

Bovet, P. and S. Benhamou, 1988: Spatial-analysis of animals movements using a correlated randomwalk model. Journal Of Theoretical Biology, 131(4), 419-433.

Bukovinszky, T., R. P. J. Potting, Y. Clough, J. C. van Lenteren, and L. E. M. Vet, 2005: The role of preand post-alighting detection mechanisms in the responses to patch size by specialist herbivores. Journal of Chemical Ecology, 109(3), 435-446.

Byers, J. A., 2001: Correlated random walk equations of animal dispersal resolved by simulation. Ecology, 82(6), 1680-1690.

Cain, M. L., 1985: Random search by herbivorous insects - a simulation-model. Ecology, 66(3), 876888.

Cain, M. L., J. Eccleston, and P. M. Kareiva, 1985: The influence of food plant dispersion on caterpillar searching success. Ecological Entomology, 10(1), 1-7.

Cressman, R., V. Krivan, and J. Garay, 2004: Ideal free distributions, evolutionary games, and population dynamics in multiple-species environments. American Naturalist, 164(4), 473-489.

Dusenbery, D. B., 1989: Optimal search direction for an animal flying or swimming in a wind or current. Journal Of Chemical Ecology, 15(11), 2511-2519.

Einstein, A., 1905: The motion of elements suspended in static liquids as claimed in the molecular kinetic theory of heat. Annalen Der Physik, 17(8), 549-560.

Graves, R., 1965: Flying crooked. In Collected Poems. Cassell, London, England.

Harcourt, D., 1961: Spatial pattern of the imported cabbage-worm, Pieris rapae L. on cultivated crucifers. Canadian Entomology, 93, 945-52.

Harcourt, D., 1963: Biology of cabbage caterpillars in eastern ontario. Proceedings of The Entomological Society of Ontario, 93, 61-75.

Hartley, S. Ant baiting regimes for a study at airports. unpublished data.

Hasenbank, M., in preparation: Phd thesis. Victoria University (Wellington, New Zealand).

Hern, A., G. EdwardsJones, and R. G. McKinlay, 1996: A review of the pre-oviposition behaviour of the small cabbage white butterfly, Pieris rapae (Lepidoptera: Pieridae). Annals of Applied Biology, 128(2), 349-371.

Hoffmann, G., 1983: The random elements in the systematic search behavior of the desert Isopod Hemilepistus reaumuri. Behavioral Ecology And Sociobiology, 13(2), 81-92.

Hogan, W., 2006: Atmospheric dispersion modelling. In Biosurveillance in Handbook of Biosurveillance, Wagner, M., editor. Elsevier Academic Press, 289-299. 
Johnson, A. R., B. T. Milne, and J. A. Wiens, 1992: Diffusion in fractal landscapes - simulations and experimental studies of tenebrionid beetle movements. Ecology, 73(6), 1968-1983.

Jones, R. E., 1977a: Movement patterns and egg distribution in cabbage butterflies. The Journal of Animal Ecology, 46(1), 195-212.

Jones, R. E., 1977b: Search behavior - study of 3 caterpillar species. Behaviour, 60, 236-259.

Kareiva, P. M. and N. Shigesada, 1983: Analyzing insect movement as a correlated random-walk. Oecologia, 56(2-3), 234-238.

Kenkel, N. and D. Walker, 1996: Fractals in the biological sciences. Coenoses, 11, 77-100.

Knuth, D., 1998: The Art Of Computer Programming: Volume 2 / Seminumerical Algorithms, volume 2. Addison-Wesley, Boston.

Kobayashi, S., 1965: Influence of parental density on the distribution pattern of eggs in the common cabbage butterfly, Pieris rapae crucivora. Researches on Population Ecology, 7(2), 109-117.

Langan, A. M., C. P. Wheater, and P. J. Dunleavy, 2004: Biogenic gradients of CO2 and $\mathrm{H} 2 \mathrm{O}$ and oviposition by the small white butterfly (Pieris rapae L.) in cages. Applied Entomology And Zoology, 39(1), 55-+.

Mandelbrot, B., 1983: The Fractal Geometry Of Nature. W.H. Freeman and Co, New York, USA.

Meyer, B., 2000: Object-Oriented Software Construction. Prentice-Hall Resource, New Jersey, 2nd edition.

Mittelbach, F., 2004: The $\mathrm{HT}_{E} \mathrm{X}$ Companion. Addison-Wesley, Boston, 2nd edition.

Pearson, K., 1905: The problem of the random walk. Nature, 72, 294-294.

R Development Core Team, 2005: R: A language and environment for statistical computing. ISBN 3-900051-07-0.

Ricketts, T. H., 2001: The matrix matters: Effective isolation in fragmented landscapes. The American Naturalist, 158(1), 87-99.

Roitberg, B. D., 1985: Search dynamics in fruit-parasitic insects. Journal Of Insect Physiology, 31(11), 865-872.

Roitberg, B. D. and M. Mangel, 1997: Individuals on the landscape: behavior can mitigate landscape differences among habitats. Oikos, 80(2), 234-240.

Root, R. B., 1973: Organization of a plant-arthropod association in simple and diverse habitats: The fauna of collards (Brassica oleracea). Ecological Monographs, 43(1), 95-124.

Root, R. B. and P. M. Kareiva, 1984: The search for resources by cabbage butterflies (Pieris rapae): ecological consequences and adaptive significance of markovian movements in a patchy environment. Ecology, 65(1), 147-165.

Soanes, C. and Stevenson, A., editors, 2004: Concise Oxford English Dictionary 11th Ed. Oxford University Press.

Tischendorf, L., D. J. Bender, and L. Fahrig, 2003: Evaluation of patch isolation metrics in mosaic landscapes for specialist vs. generalist dispersers. Landscape Ecology, 18(1), 41-50.

Turchin, P., 1998: Quantitative Analysis of Movement. Sinauer Associates, USA. 
Vergassola, M., E. Vilermaux, and S. B.I., 2007: Infotaxis as a strategy for searching without gradients. Nature, 445, 406-409.

Visser, J. H., 1986: Host odor perception in phytophagous insects. Annual Review Of Entomology, 31, 121-144.

Wiens, J. A., T. O. Crist, and B. T. Milne, 1993: On quantifying insect movements. Environmental Entomology, 22(4), 709-715. 


\section{Chapter 4}

\section{Sense and Scale: Simulating the effects of movement patterns on the egg distributions of Pieris rapae at multiple spatial scales, from random walks to information-based foraging}

\subsection{Abstract}

By simulation, the egg distributions of an ovipositing forager responding to plant density and spatial distribution were explored. Experimental layouts were used that had been used in field experiments and which contained a mixture of resource densities apparent at several scales of measurement. Using a correlated random walk simulation, a negative response to density was observed at all scales, which is consistent with both known literature for Pieris rapae (Lepidoptera) and the field observations. Further effects were explored by introducing a behavioural response to the resources in the form of both "visual" and "olfactory" perception. Including a reaction to information in the environment, a negative response to density at the smaller scale and yet a positive response at a larger scale was simulated. This work provides some interesting insights into both the behaviour of random walk models over complex resource layouts, and potential responses of foragers to resource aggregation at different scales. 


\section{Keywords}

Pieris rapae, Correlated Random Walk, Insect Sensory Perception, Resource Concentration Hypothesis, Agent-based Simulation

\subsection{Introduction}

A large body of research exists concerning the modelling of animal movements. It is generally accepted that individual movement is a key factor in the distribution of animals and hence their impact on the containing ecosystem (Swingland and Greenwood, 1984; Turchin et al., 1991). A great deal of work also exists on the effects of scale when considering and measuring ecosystem function (Banks, 1998; Levin, 1992; Wiens, 1989; With and Crist, 1996). This chapter explores the effects of scale when measuring the egg distributions of the small cabbage white butterfly, Pieris rapae. The species was chosen because there is an abundance of information available concerning its behaviour (Bukovinszky et al., 2005; Hern et al., 1996; Jones, 1987; Jones and Ives, 1979; Minnich, 1924; Renwick and Radke, 1988; Root and Kareiva, 1984). Field experiments were conducted in which the resource densities of its host plant, cabbages (Brassicae oleracea Capitata group; var "Summercross"), were manipulated at various scales (Hasenbank, in prep). In conjunction with these, simulation studies were conducted, which are reported here. Much work is available as a basis for the simulation, for both general insect movement and in particular applied to P.rapae (Cain, 1985; Jones, 1977; Kareiva and Shigesada, 1983; Root and Kareiva, 1984).

Root (1973) developed the "resource concentration hypothesis" (RCH) relating to the effect of host density on insect distributions. This states that animals are expected to seek out areas of high host density and therefore be found more abundant in high density stands. Applied to the oviposition behaviour of butterflies, a higher number of eggs per plant in areas of higher plant density would be expected. In fact, for $P$. rapae, the opposite response is observed, and isolated plants receive more eggs per plant, referred to as "Resource Dilution" (Root and Kareiva, 1984; Yamamura, 1999).

Previous simulations of $P$. rapae movement fall into two categories; Correlated random walks (CRW) (Kareiva and Shigesada, 1983; Cain, 1985) and detailed behavioural models, e.g. Jones (1977).

Kareiva and Shigesada (1983) demonstrated that the ovipositional behaviour of the cabbage white could be represented by a correlated random walk (CRW), by measuring the Mean Squared Displacement (MSD) of the observed flight path over time 
and comparing it with that expected from a CRW. When foraging for nectar however, the MSD deviated from the expected, as the foragers increased their turning angle and the pattern of movement represented an "area restricted search" (Bell, 1991).

Jones (1977)'s study has been reviewed many times, Turchin (1998) provides a good summary. Various movement parameters were recorded such as flight distance, turning angle and oviposition rate. A model was then derived from these parameters which both predicted the distributions of eggs at a small scale, and was later extended to operate at a larger scale, over several days (Jones et al., 1980). The environment of the model is a set of discrete nodes in a grid layout so the foragers may move in one of 8 directions to neighbouring nodes. The basic movement of this model in the absence of hosts is similar to a CRW, except that the movement has a consistent bias or "preferred" direction around which turns are made at random. This large scale "drift" could be a response to wind direction or an innate behaviour to encourage long-distance dispersal. Behavioural responses to the resources are represented by several parameters which might affect the move at any given timestep. These parameters are either estimated from the field observations or manipulated until they produce a close match to the observed egg distributions (Chapter 2). Jones (1977)'s model was not replicated here because it would have been a lengthy process to interpret and then parameterise the model. Furthermore, the correlated random walk is tractable in other modelling situations such as the context of reaction-diffusion equations (Turchin, 1989; Farnsworth and Beecham, 1999). Building a simulation using this model should provide a more general tool.

This chapter is based around several questions which might be asked about the movement behaviour of $P$. rapae.

The results presented are very much concerned with "immigration" into a patch. Given a patch exists in some landscape, and butterflies are moving around the landscape searching for oviposition sites; How do individuals discover and respond to the resource layout? ${ }^{1}$

The MSD may correlate well with that expected from a CRW, but as Turchin (1989) notes, the CRW does not account for an attraction toward resources and the MSD is a rather general measure which does not provide information about the detailed patterns of egg distributions. Is it possible to simulate a detailed egg distribution using a CRW which can be compared with that observed in the field?

If not, are there more generic models for responses to resources which can be used, based around a conceptual model of "visual" and "olfactory" senses which achieve a similar match

\footnotetext{
${ }^{1}$ The framework also provides the facility to study longer term population dynamics in terms of "Birth" into the experimental area, which is left to subsequent study.
} 
to a very detailed model and yet can easily be applied to other organisms?

In terms of scale, is it possible to observe a change in response with a change in scale? i.e. simulate at one scale a negative response to density (resource dilution) but at another scale, a positive response (resource concentration)? If so, what kinds of movement patterns are required?

\subsection{Methods}

\subsubsection{Experimental resource layout}

The simulation framework described in Chapter 3 allowed resource layouts to be created using the positions of cabbages recorded from the field experiments. Here, comparisons are made between simulations and two field experiments (Hasenbank, unpublished data). The field experiments were conducted at separate locations in New Zealand and are referred to here by the names of the locations and the years in which they were collected; "LEVIN-06-II" (it was the second run at this location) and "KAITOKE-04". The field layouts differ in complexity and the scales included, but follow a similar concept in terms of blocks of resources with different plant spacings. Within each set-up there are multiple densities at multiple scales.

KAITOKE-04 is composed of four "fields", one in each corner, which are themselves composed of four "blocks", labelled "K1" to "K4" (Figures 4.1 to 4.3). Each "field" is a replicate with the blocks arranged in a different sequence. Blocks K1 and K4 have the same structure but different spacings, so that $\mathrm{K} 1$ represents an expanded version of $\mathrm{K} 4$

LEVIN-06-II consisted of 4 replicate "fields" each of which was $36 \times 36 m$ and had a combination of 4 possible "blocks" within it, labelled here "L1 - L4" (Figures 4.4 and 4.5). Each field has the same set of blocks, in a different sequence.

In each layout, windows of different sizes or scales can be created, within which the number or density of plants is measured. The scales were $1 \times 1 \mathrm{~m}, 6 \times 6 \mathrm{~m}, 36 \times 36 \mathrm{~m}$ and $48 \times 48 \mathrm{~m}$. The resource densities were; $1,4,16$ or 40 plants (Table 4.1 ). The 40 plants were also laid out in a nested design, consisting of sub-patches of 1,4 and 16 plants. Other densities were spaced evenly within their respective scale areas. Tables 4.3 and 4.2 show the various combinations for each layout. The combinations were each given a "scale code" which consists of the densities of plants at each scale measured, in the form "small-medium-large" so for example if there is 1 plant at all scales (e.g. Figure 4.3.b), the scale code would be "1-1-1", if there was one plant at 


\begin{tabular}{lr}
\hline Scale & Plant density \\
\hline $1 \times 1 \mathrm{~m}$ & $1,4,16$ \\
$6 \times 6 \mathrm{~m}$ & $1,4,16,40$ \\
$36 \mathrm{~m} \times 36 \mathrm{~m}$ & 61 \\
$48 \mathrm{~m} \times 48 \mathrm{~m}$ & $4,16,40$ \\
\hline
\end{tabular}

Table 4.1: Possible densities at each scale.

\begin{tabular}{lrrr}
\hline & \multicolumn{2}{c}{ Scale of measurement } \\
\cline { 2 - 4 } Scale Code & 1x1m & 6x6m & $\mathbf{4 8 x 4 8 m}$ \\
\hline $1-1-4$ & 1 & 1 & 4 \\
$1-1-40$ & 1 & 1 & 40 \\
$1-40-40$ & 1 & 40 & 40 \\
$4-4-40$ & 4 & 4 & 40 \\
$4-40-40$ & 4 & 40 & 40 \\
$16-16-16$ & 16 & 16 & 16 \\
$16-16-40$ & 16 & 16 & 40 \\
$16-40-40$ & 16 & 40 & 40 \\
\hline
\end{tabular}

Table 4.2: Number of plants at each scale of measurement and the associated scale codes for KAITOKE-04 field layout.

\begin{tabular}{lrrr}
\hline & \multicolumn{3}{c}{ Scale of measurement } \\
\cline { 2 - 4 } Scale Code & 1x1m & 6x6m & 36x36m \\
\hline $1-1-61$ & 1 & 1 & 61 \\
$1-4-61$ & 1 & 4 & 61 \\
$1-16-61$ & 1 & 16 & 61 \\
$1-40-61$ & 1 & 40 & 61 \\
$4-40-61$ & 4 & 40 & 61 \\
$16-40-61$ & 16 & 40 & 61 \\
\hline
\end{tabular}

Table 4.3: Number of plants at each scale of measurement and the associated Scales Codes for LEVIN-06-II field layout. 


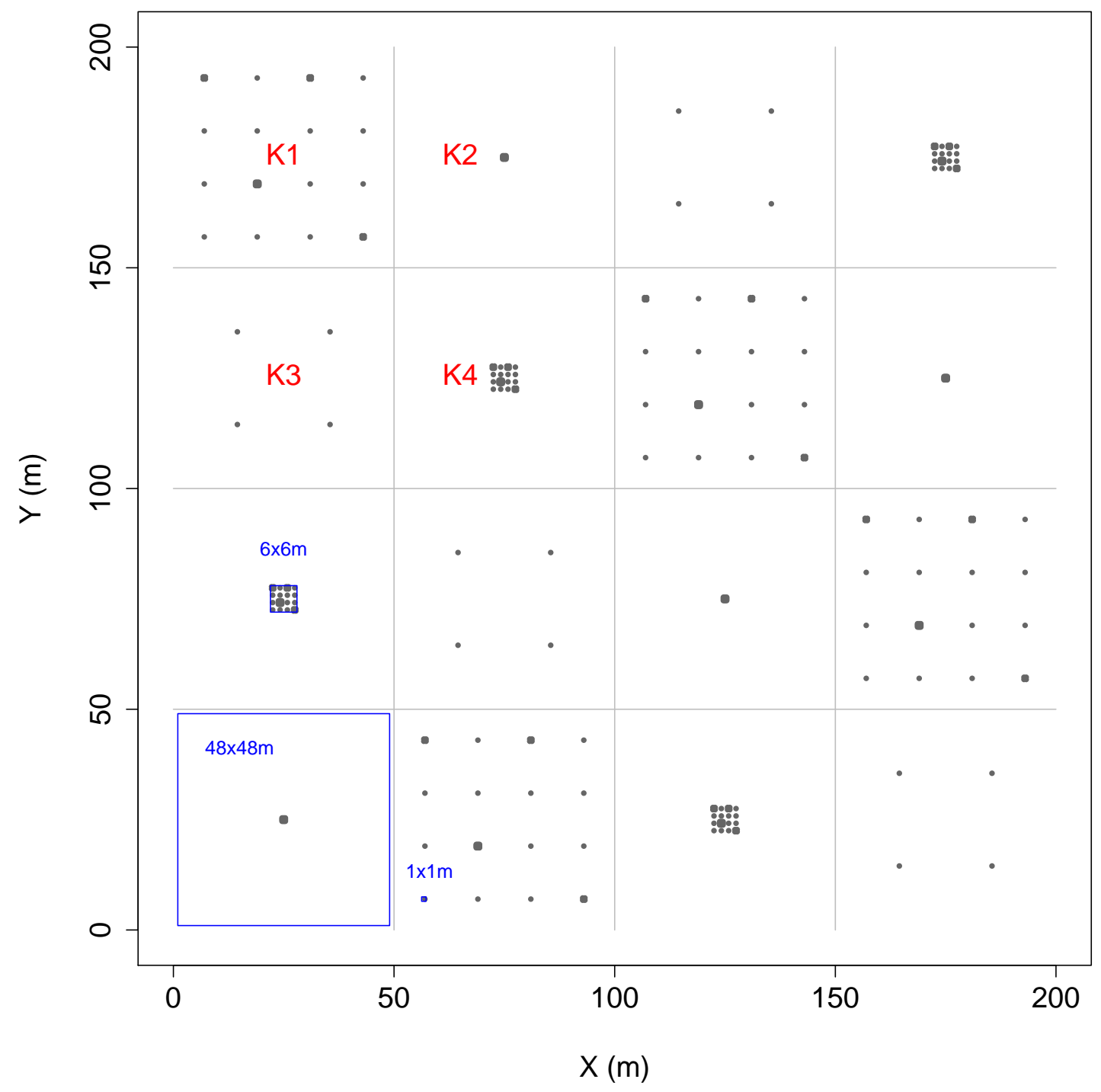

Figure 4.1: Field Layout - KAITOKE-04. Rectangles of each of the scales is shown. The four repeated 'blocks' are indicated by $\mathrm{K} 1$ - K4. 


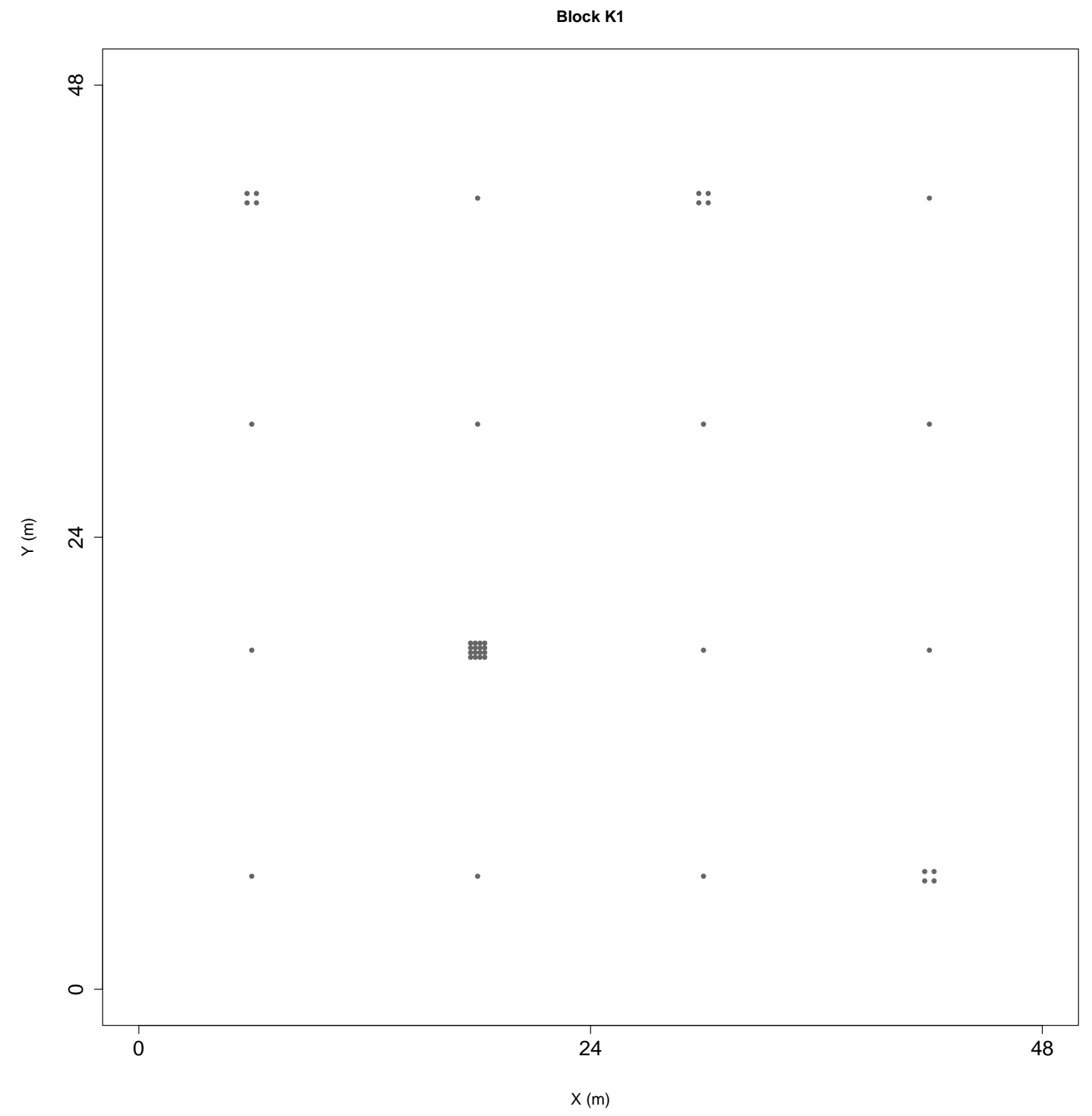

Figure 4.2: Field Layout - KAITOKE-04. Block Breakdown (Block K1). There are 12 single plants (1 plant $\left./ \mathrm{m}^{2}\right), 3$ clusters of 4 plants (4 plants $\left./ \mathrm{m}^{2}\right)$, and 1 cluster of 16 plants (16 plants $\left./ \mathrm{m}^{2}\right)$. 
a) Block K2

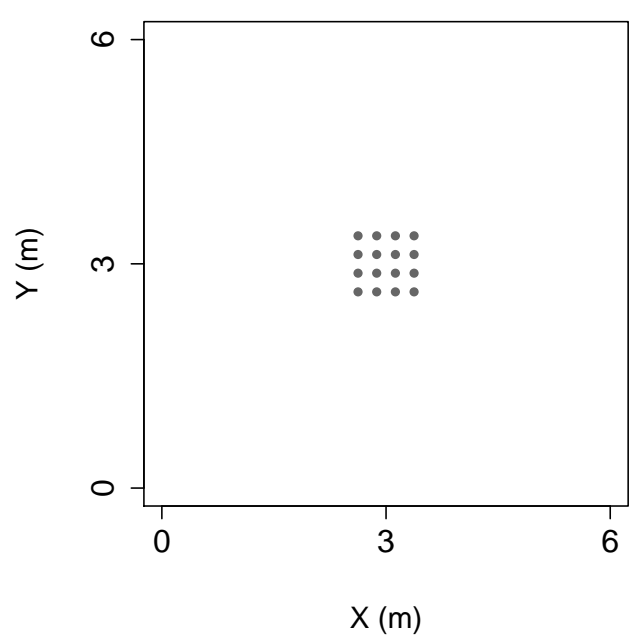

c) Block K4

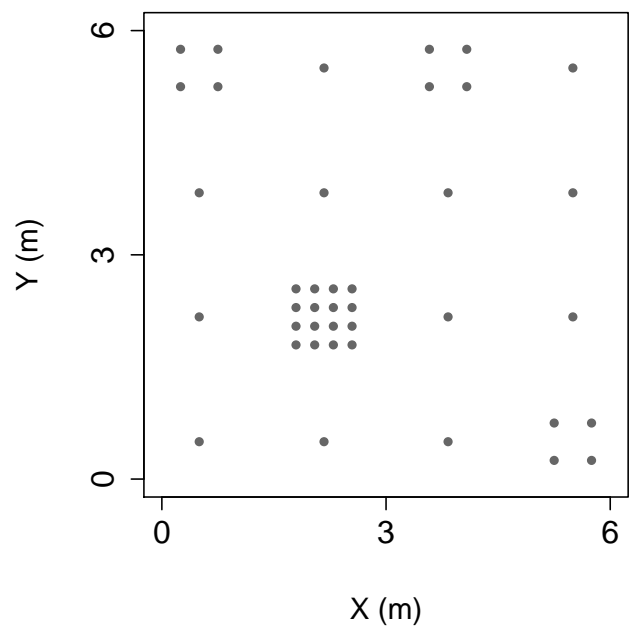

b) Block K3

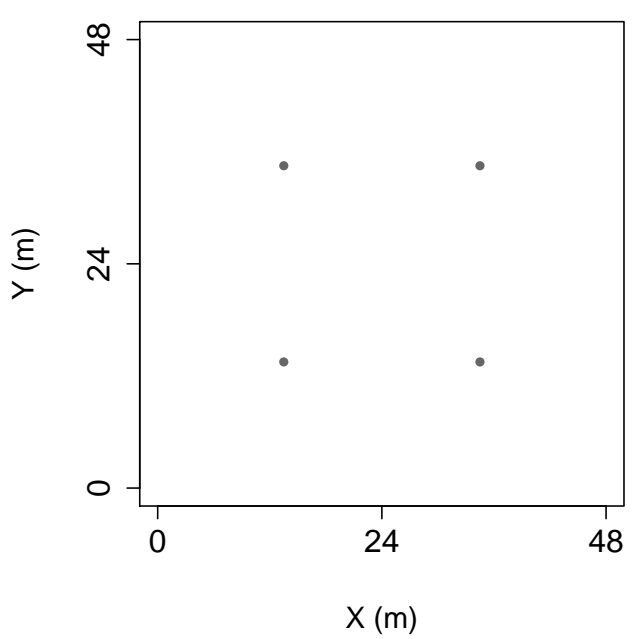

Figure 4.3: Field Layout - KAITOKE-04. Block breakdown for blocks K2-K4. 
a) Field E

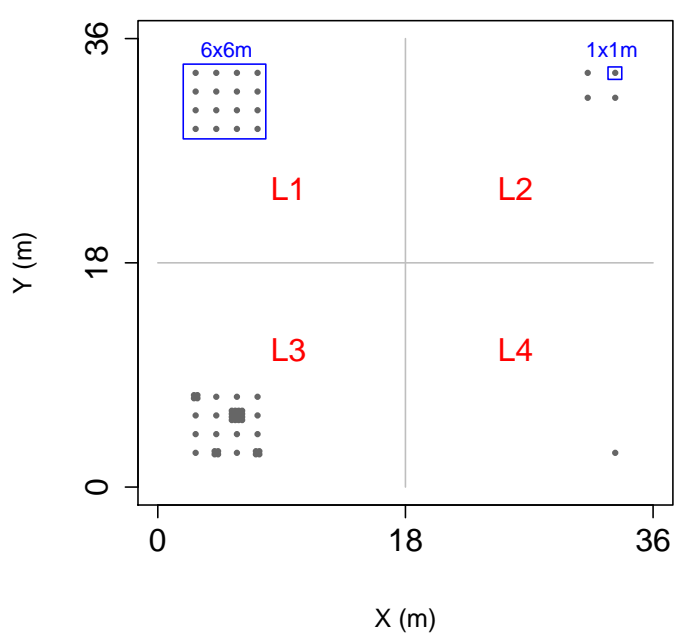

c) Field G

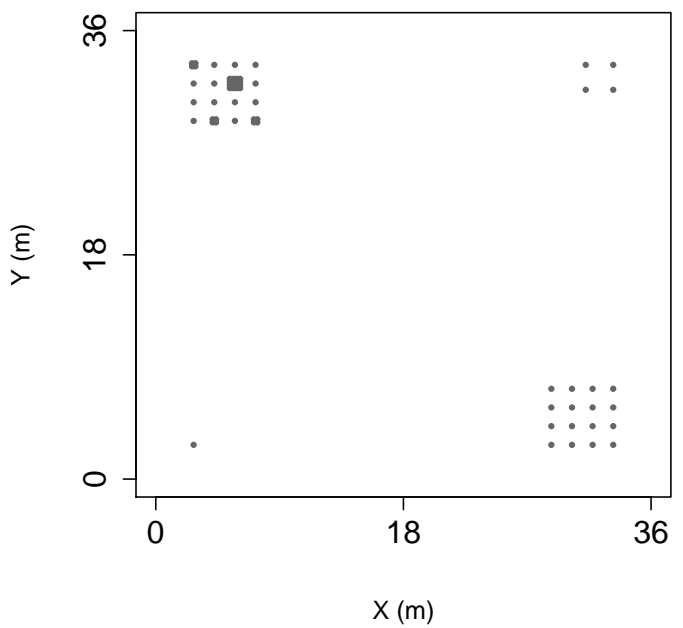

b) Field F

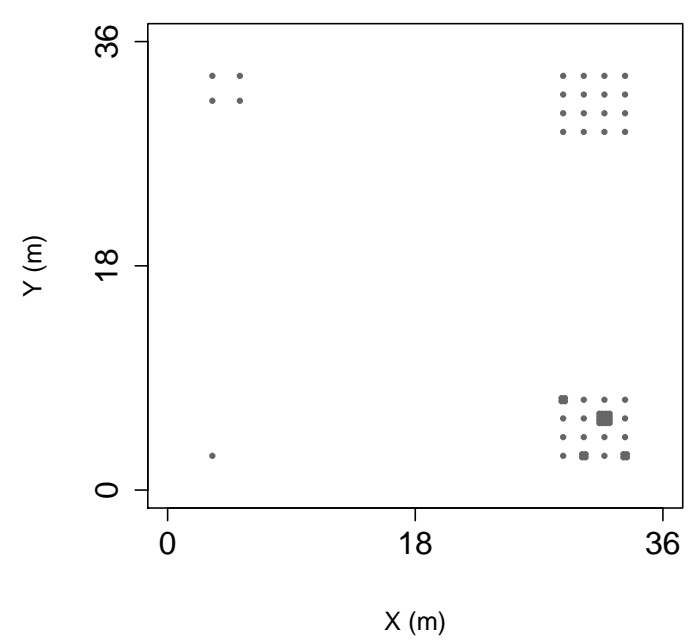

d) Field H

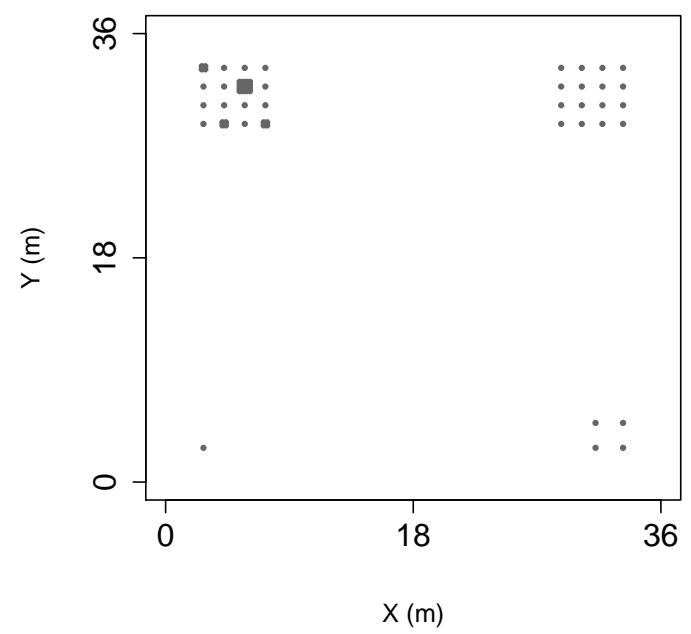

Figure 4.4: Field Layout - LEVIN-06-II. 
a) Block L1

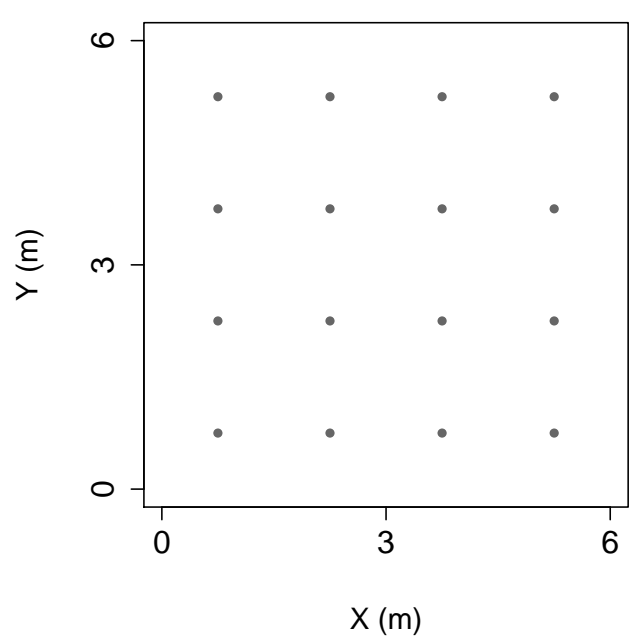

c) Block L3

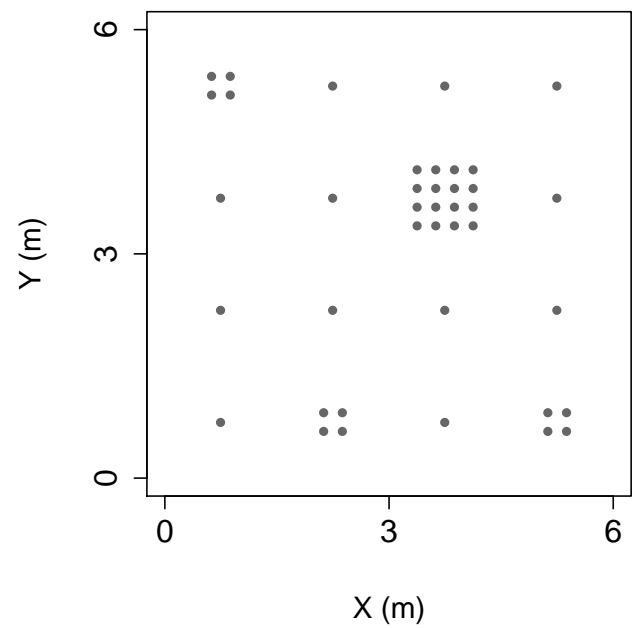

b) Block L2

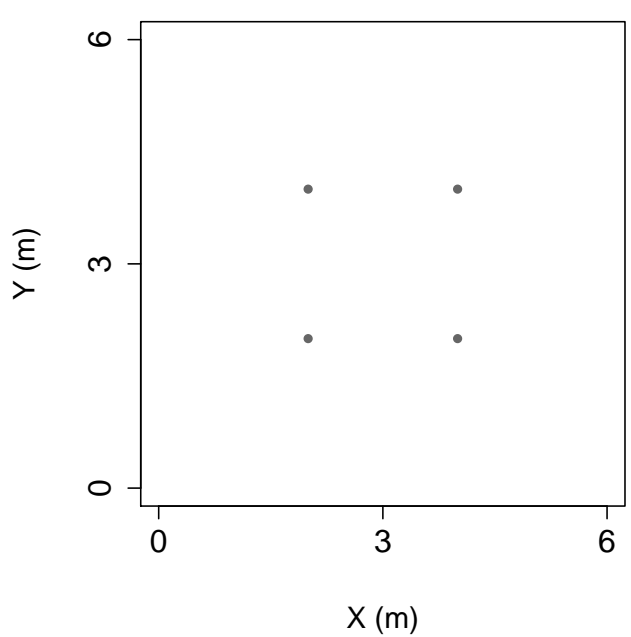

d) Block L4

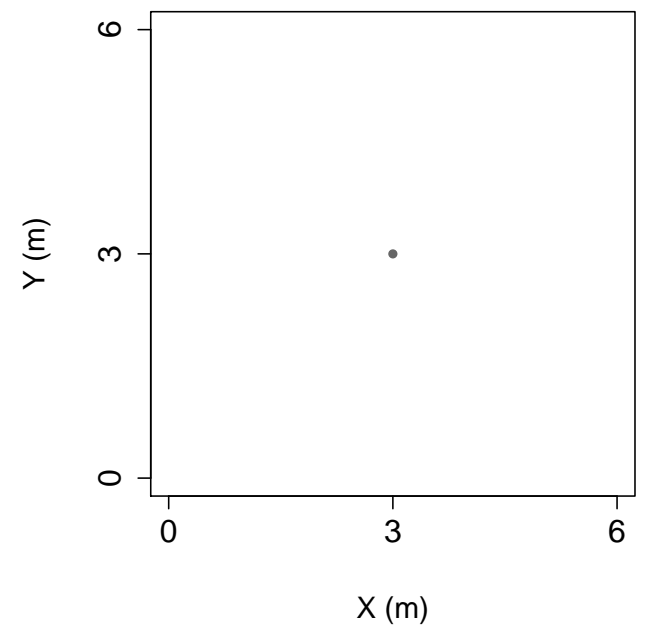

Figure 4.5: Field Layout - LEVIN-06-II. Block breakdown. Each of the blocks L1 to L4 is repeated in each field ( $E$ to $H$ ) but in different corners of the layout. 
the $1 \times 1 \mathrm{~m}$, sixteen plants at $6 \times 6 \mathrm{~m}$, and sixty-one plants at $36 \times 36 \mathrm{~m}$ or $48 \times 48 \mathrm{~m}$, the scale code would be "1-16-61".

The purpose of the nested nature of the layouts is to elicit responses at particular scales, in particular those which may depend on the surrounding density of plants. For example, a single plant at the $1 \times 1 \mathrm{~m}$ scale may receive more (or less) eggs depending on whether it is nested within a $6 \times 6 m$ area containing $1,4,16$ or 40 plants, which in turn may depend upon the number of plants in the containing $36 \times 36 m$ or $48 \times 48 m$ window. The resulting egg distribution may be influenced by these, surrounding plants.

\subsubsection{Analysis of results}

\section{Scale and resource density}

The goal of both the field and simulation experiments was to investigate the effect of resource density on the response variable, eggs per plant. There are two ways to characterise the resources in terms of density and scale. The first is to aggregate by plant density for each scale, as shown in Table 4.1. This provides a simple aggregated response which is useful for comparison between field and simulation egg distributions.

One problem with this grouping is that the sub-patches at each scale, particularly the $1 \times 1 m$ and $6 \times 6 m$ scales are themselves nested within larger scale patches. Therefore, for the KAITOKE-04 experiment there are actually 3 "subtypes" of patches which have a single plant at the $1 \times 1 \mathrm{~m}$ scale (Table 4.2), while for the LEVIN-06-II experiment there are four sub categories of single plants at the $1 \times 1 \mathrm{~m}$ scale (Table 4.3). Aggregating by scale and density, may obscure the potential influence of the surrounding patches by averaging the eggs per plant and so some detail is lost.

The second grouping is the "scale-code" for each of the groups of plants. This provides a more detailed picture of the results. Here, choosing a relative order depends on which scale is required for analysis. For example if the density is sorted for KAITOKE-04 based on the number of plants at the $6 \times 6 \mathrm{~m}$ scale, the sequence is different to that at the $6 \times 6 \mathrm{~m}$ scale and $48 \times 48 \mathrm{~m}$ scale. When these results are presented, a separate plot, ordered at each scale is shown. The KAITOKE-04 layout benefits particularly from this detail, the LEVIN-06-II layout is simpler. For this reason only the KAITOKE-04 results are discussed in the context of scale-code.

It would be possible to use some other index of density and scale, such as an isolation index (Bender et al., 2003; Matter et al., 2005; Tischendorf et al., 2003; Hasen- 
bank, in prep). However, this chapter is concerned with exploring the dynamics of the simulation, and whether or not it can produce similar results to the field. For this purpose, the choice of response measurement is almost arbitrary; if the simulation can accurately reproduce the observed egg distribution, any metric used for comparison should be consistent. This chapter deals with the high level, aggregated measures. Subsequent research could delve deeper into the detail and try to replicate the results at more complex levels of measurement. In particular the variation observed between results.

\section{Eggs per plant}

The response variable measured in all cases is eggs Per plant. This accounts for the fact that a larger number of plants will by default receive a larger number of eggs. In a null model of ideal free distribution, the foragers do not respond to the density of the plants in any way and it should be expected that the eggs per plant will be constant. In modelling terms this would be equivalent to removing the "spatial" element from the simulation, and simply randomly assigning a number of eggs to each plant. The three possible theoretical responses are shown in Figure 4.6. These can be interpreted in terms of resource concentration (Root, 1973) or dilution (Yamamura, 1999), or as a null model, an ideal free distribution (Cressman et al., 2004). Across all of the experiments, the eggs per plant varies considerably and so in order to present them all on the same scale of axis and to expand the lower range, the eggs per plant are presented with a square root transformation.

a) Resource Concentration

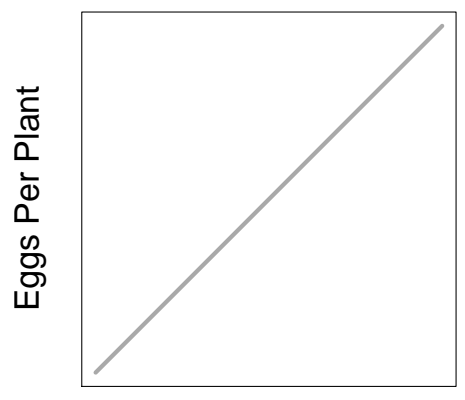

b) Ideal Free Distribution

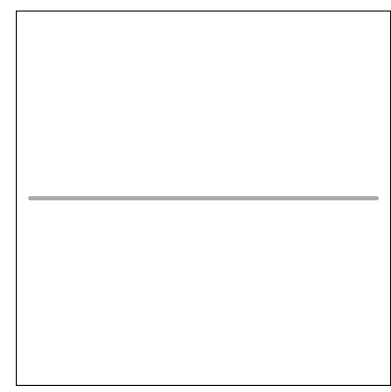

Resource Density c) Resource Dilution

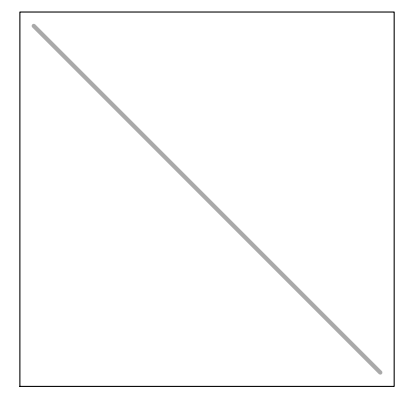

Figure 4.6: Potential theoretical responses to plant density.

\section{A measure of resource concentration}

Using the data aggregated at the density grouping, plots of eggs per plant vs resource density were plotted on log transformed axes, to which a linear regression 
line was fitted. The slope provided an indication of the strength of resource concentration or dilution, or at least the direction (positive or negative) of the response, and hence which of the theoretical situations from Figure 4.6 was observed.

\section{Comparison to field data}

In order to compare the output of the simulation with the observed egg distribution, the simulation was run until the same number of eggs had been laid in each field as had been observed (Table 4.5). Each experimental trial was run for 4 replicates. The eggs per plant were measured both by scale code and by plant density and then compared to the field data. A chi-squared test was used to test for an overall significant difference between field and simulation results. The Bray-Curtis (BC) dissimilarity distance (0 being identical and 1 maximally distant, Quinn and Keough, 2002) was then used to provide a sliding scale measure so that different parameter combinations could be compared in terms of "best fit" to the field data. The calculation for the Bray-Curtis measure is given in Equation 4.1, where $y_{1}$ and $y_{2}$ are two arrays of observations of equal length. The index $j$ refers to each set of plants for a particular density, or scale code.

$$
B C=1-\frac{2 \sum_{j=1}^{p} \min \left(y_{1 j}, y_{2 j}\right)}{\sum_{j=1}^{p} y_{1 j}+y_{2 j}}
$$

The BC value was calculated for both the density grouping and scale-code groupings of the egg distributions. Finally an average was calculated which allowed ranking of all trials on a consistent index of similarity.

\subsubsection{Experiments}

\section{Parameters}

A list of the parameters that are available in the simulation framework (Chapter 3) is presented in Table (4.4). For a complete description of the parameters and their function refer to Chapter 3 .

Several authors have reported information regarding the flight behaviour of P.rapae (Chapter 2). A summary of this information is shown in table 4.6. Where necessary, values have been converted into the $k$ parameter of the Von-Mises distribution so that the relation to the simulation parameters can be seen. 


\begin{tabular}{lll}
\hline Code & Parameter & Values \\
\hline AGE & Age limit for forager & 1000 \\
E & Number of eggs per forager & 1,10 \\
$\mathbf{G}$ & Egg count limit (run until this many eggs laid) & $50 \rightarrow 2000$ \\
\hline $\mathbf{L}$ & Step length & $100 \rightarrow 10000$ \\
$\mathbf{k}$ & Turning angle concentration & $0.5 \rightarrow 10$ \\
$\mathbf{V E}$ & Vision enabled & true, false \\
$\mathbf{O E}$ & Olfaction enabled & true, false \\
$\mathbf{F D}$ & Visual field depth & $100 \rightarrow 1800$ \\
$\mathbf{F W}$ & Visual field width & 120 \\
\hline $\mathbf{B}$ & Release boundary distance $-($ Figure 3.27$)$ & $0 \rightarrow 800$ \\
$\mathbf{I}$ & Inter edge separation (Figure 3.25) & $-75 \rightarrow 140$ \\
$\mathbf{P}$ & Patch size (Figure 3.25) & $100 \rightarrow 600$ \\
$\mathbf{R}$ & Resource radius (Figure 3.25) & $5 \rightarrow 50$ \\
$\mathbf{S W}$ & Signal width for odour "plume" & 1500 \\
\hline
\end{tabular}

Table 4.4: List of most commonly manipulated parameters and the ranges of values that were explored, including experiments from Chapter 3. The units for distance measurements such as $L$ are $\mathrm{cm}$.

\begin{tabular}{lr}
\hline Field & Egg Count \\
\hline LEVIN-06-II - Field E & 613 \\
LEVIN-06-II - Field F & 476 \\
LEVIN-06-II - Field G & 590 \\
LEVIN-06-II - Field H & 438 \\
\hline LEVIN-06-II - Total & 2117 \\
\hline KAITOKE-04 & 2061 \\
\hline
\end{tabular}

Table 4.5: Egg counts from the various field experiments. 


\begin{tabular}{|c|c|c|c|}
\hline Reference & $\begin{array}{l}\text { Step } \\
\text { Length } \\
(\mathrm{L} \mathrm{cm})\end{array}$ & $\begin{array}{l}\text { Turning } \\
\text { Angle } \\
\text { (k) }\end{array}$ & Notes \\
\hline Byers (2001) & 256 & 1.735 & $\begin{array}{l}\text { Converted using tables in } \\
\text { Batschelet (1981) from a s.d. of } \\
\text { 48.06. Byers extracted parame- } \\
\text { ters from Kareiva and Shigesada } \\
\text { (1983) }\end{array}$ \\
\hline Cain (1985) & & 1.2 & \\
\hline Jones (1977) Australia & 192 & 0.75 & $\begin{array}{l}\text { Averaged from } 1 \mathrm{~m} \text { even }(139 \mathrm{~cm} \text {, } \\
0.36 \text { rho }) \text { and } 3 \mathrm{~m} \text { clumped ob- } \\
\text { servations }(245 \mathrm{~cm}, 0.33 \text { rho }) \\
\text { (Chapter ??) }\end{array}$ \\
\hline Jones (1977) Canada & 201 & 0.5 & $\begin{array}{l}\text { Averaged from } 1 \mathrm{~m} \text { even }(176 \mathrm{~cm} \text {, } \\
0.11) \text { and } 3 \mathrm{~m} \text { clumped observa- } \\
\text { tions }(225 \mathrm{~cm}, 0.22 \mathrm{rho}) \text { (Chapter } \\
\text { ??) }\end{array}$ \\
\hline
\end{tabular}

Table 4.6: Summary of flight parameters obtained from literature.

The estimations vary somewhat, which highlights the difficulty in using such observations directly in a simulation environment. Each measure was obtained using different methodologies, with different populations and different resource types and layout. As Jones (1977) notes, significant differences were observed even over very similar layouts between Australian and Canadian populations, let alone such great differences in method. However, as a guide to the general scale of the observations, a step length of $2-2.5 \mathrm{~m}$ seems appropriate, with a turning angle concentration of $k>1.0$, although it is difficult to take a mean of the $k$ parameter as it does not have a linear scale.

Here we should like to note that "step length" is itself an arbitrary measure which depends upon the method of measurement. In some cases in the Jones (1977) paper, this is the result of measuring "plant to plant" moves. In terms of calibrating the simulation, the end goal is to represent the movement in an abstract manner, using parameters which can be estimated from the field. The simulated paths themseleves will never be representational of actual flight paths, and so it is assumed that the abstraction of "step length" is valid and useful in this circumstance.

Three experiments were constructed which tested very simple models of distributing eggs, as a "null" model against which to compare the more complex behavioural results. These were Trials B1, B2 and B3 (Table 4.7). 


\section{Calibration}

Before an extensive parameter exploration, several preliminary simulations were run to explore the general behaviour of the model. In these, various parameters were experimented with over a single field of the LEVIN-06-II field, field "E". This provided a basic understanding of the model and what potential responses could be obtained. These results are not reported here for brevity.

\section{Parameter estimation}

The greater number of experiments consisted of systematically manipulating the various behaviours and parameters, exploring the parameter space and comparing the output with that observed in the field, using the Bray-Curtis measure described above. A summary of the trials that will be referred to is given in Table 4.7. Due to time constraints, the KAITOKE-04 layout (which was much larger and required much longer to execute) was only compared to a set of random walk parameters. The LEVIN-06-II layout was compared against all combinations of CRW, olfactory and visual search behaviours. Each behaviour was also run with both a single egg per forager and 10 eggs per forager. For a single egg situation, once laid, the forager was removed from the simulation (Chapter 3). All trials were run until a specified number of eggs had been laid $(G)$. These numbers were set from the field observations and are recorded in Table 4.5 .

The KAITOKE-04 layout was a single, large layout. The LEVIN-06-II layout was divided into four "fields". Each "field" was executed separately in the simulation and then results were aggregated for analysis, in the same manner in which the field observations were processed.

For all simulation trials there were a consistent set of parameter manipulations (Table 4.8). The step length $(L)$ was different when running against the KAITOKE-04 layout because this layout was much larger and it was necessary to "scale up" the step length to ensure a reasonable processing time and coverage of the experimental area. It was not necessary to adjust the turning angle concentration $(k)$.

Notice that the release boundary distance $(B)$ was constant for these experiments. Although as discussed in Chapter 3 the release boundary distance may have an effect, it was felt that as long as a consistent boundary distance was set, any bias would be consistent accross results. In the event that results were shown to be particularly sensitive, it would have been possible to repeat experiments with different values for $B$, but this was not the case.

The step length parameter for LEVIN-06-II was chosen to be "realistic" (remember- 


\begin{tabular}{clll}
\hline Trial & Field Layout & Forager Eggs (G) & Description \\
\hline B1 & LEVIN-06-II & - & $\begin{array}{l}\text { Equal Eggs (15) On Each } \\
\text { Plant } \\
\text { Random Plant selection } \\
\text { (no space) } \\
\text { Random Spatial Location } \\
\text { (eggs from the sky) }\end{array}$ \\
B3 & LEVIN-06-II & - & Correlated Random Walk \\
\hline C1 & LEVIN-06-II & Single Egg & Correlated Random Walk \\
C2 & KAITOKE-04 & Single Egg & Correlated Random Walk \\
C3 & LEVIN-06-II & Multiple Eggs & Olfaction \\
\hline D1 & LEVIN-06-II & Single Egg & Olfaction and Vision \\
D2 & LEVIN-06-II & Single Egg & Vision \\
D3 & LEVIN-06-II & Single Egg & Olfaction \\
\hline D4 & LEVIN-06-II & Multiple Eggs & Vision \\
D5 & LEVIN-06-II & Multiple Eggs & Olfaction and Vision \\
D6 & LEVIN-06-II & Multiple Eggs & \\
\hline
\end{tabular}

Table 4.7: Experimental Trials, the preliminary trials, $A$ are not shown. Trial $B$ is a theoretical trial executed in the ' $R$ ' statistical package, to demonstrate a baseline of how the response would appear if either all the plants had the same number of eggs (B1), if the plants were simply selected at random (B2) or if a random point in the patch is chosen and if it falls within the radius of attraction an egg is laid on that plant. When running with multiple eggs, 10 eggs per forager were set.

ing that it is an arbitrary measure) based on the values reported in the literature (Table 4.6), values selected so as to "bracket" around the $250 \mathrm{~cm}$ mark. The turning angle concentration was chosen to represent a cross-section of directionality; high $(k=10)$, medium $(k=3)$ and low $(k=0.5)$. These values were also used in the experiments of Chapter 3 .

\section{Sensory perception}

Adding the extra parameters of the sensory perception behaviours multiplies the dimensions of the parameter space for exploration. With this in mind, when including olfaction, a single value for signal width $(S W)$ was chosen, representing a wide, diffuse olfactory signal (e.g. Figure 4.23). For vision, the field width $(F W)$ was kept constant at $F W=120$ which represents a wide field of view, but only forwardlooking. The field depth $(F D)$ was the most influential parameter and this was manipulated with three values, representing short $(F D=100)$, medium $(F D=600)$ and long $(F D=1800)$ range, relative to the scale of the layouts. 


\begin{tabular}{lr}
\hline Parameter & Values \\
\hline Replicates & 4 \\
B & 100 \\
L (LEVIN-06-II) & $150,250,350$ \\
L (KAITOKE-04) & $10000,1000,250$ \\
A & $0.5,3,10$ \\
R & $5,10,20$ \\
G & 1,10 \\
FD & 120 \\
FW & 1500 \\
SW & 600,1800 \\
\hline
\end{tabular}

Table 4.8: Parameter value Manipulations. These were used in each experimental trials from C1 to D6 (Table 4.7).

\subsection{Results}

The results are organised by the behavioural model used, which corresponds to the set of trials listed in table 4.7. A brief description of the field data is given, followed by results obtained with a basic CRW. From here, the effect of "visual", "olfactory" and a combination of both searching strategies is described. Finally a comparison of all strategies in terms of which produced the closest "fit" to the observed field egg distributions.

The figures are presented with the field observations alongside the equivalent simulation results, for easy comparison. Table 4.9 describes the various plot types found in the results section.

\subsubsection{Field experiments}

Figures 4.9, 4.11, and 4.13 show the egg distributions observed in the field for the KAITOKE-04 experiment. Figures $4.14,4.17$ and 4.20 show the results for the LEVIN06-II layout.

Both layouts demonstrate a "resource dilution" effect, i.e. a negative response of eggs per plant to plant density at all scales. This is supported by the log-log regression analysis statistics (Tables B.10 and B.11 in Appendix B). This is consistent with the literature (Jones, 1977; Kareiva and Shigesada, 1983; Yamamura, 1999). A 


\begin{tabular}{|c|c|}
\hline Plot Type & Description \\
\hline Plant density & $\begin{array}{l}\text { Shows the mean eggs per plant plotted against plant den- } \\
\text { sity, for each of the scales measured. This shows the over- } \\
\text { all relationship between plant density and egg load at each } \\
\text { scale. Eggs per plant are shown on a square root scale, to } \\
\text { expand the lower values, and provide a consistent scale } \\
\text { across all graphs. }\end{array}$ \\
\hline Log-log regression & $\begin{array}{l}\text { Plots a single point for each plant, showing the number of } \\
\text { eggs against the plant density group to which that plant } \\
\text { belongs. Values are "jittered" to produce a cloud of points. } \\
\text { Plots for the simulation data have four times the number of } \\
\text { points because there were } 4 \text { replicates. The plots are made } \\
\text { using a log transformation of both eggs per plant and den- } \\
\text { sity. In order to allow the log plot of eggs per plant, they } \\
\text { are first transformed by adding } 1 \text { to the count (to remove } \\
\text { zeros), the labels on the graph have been adjusted to rep- } \\
\text { resent the "real" count. A linear model is constructed for } \\
\text { each, the details of which can be found in Tables B.10 to } \\
\text { B.22 in Appendix B. These regression graphs and statistics } \\
\text { can be compared to the theoretical responses in Figure } 4.6 \\
\text { to give an indication of which response is observed. }\end{array}$ \\
\hline Scale-code & $\begin{array}{l}\text { Groups the resources by the "scale-code" (Tables } 4.2 \text { and } \\
\text { 4.3. This grouping aggregates across the scale measured, } \\
\text { and distinguishes between single plants at all scales and } \\
\text { those which are nested within a larger patch at larger } \\
\text { scales. They can be sorted in increasing density at each } \\
\text { scale, and a plot for each order is given. The points are } \\
\text { coded to show which group in the layout they come from } \\
\text { (Figures } 4.1 \text { and } 4.4 \text { ). }\end{array}$ \\
\hline Field breakdown & $\begin{array}{l}\text { Shows the Plant Density plot for each field within the } \\
\text { LEVIN-06-II layout, in order to see the variation between } \\
\text { fields. }\end{array}$ \\
\hline Signal surface & $\begin{array}{l}\text { Comprising two sub-plots, these show the "height" of the } \\
\text { signal surface over the entire simulation "landscape". Plot } \\
\text { a) is as if viewed from above with the locations of the } \\
\text { plants marked by black dots. Plot b) shows a 3D rendering } \\
\text { of the surface. The agents are motivated to try and move } \\
\text { "uphill" to areas of high odour concentration. }\end{array}$ \\
\hline Bray-Curtis surface & $\begin{array}{l}\text { Where many combinations of parameters were compared } \\
\text { using the Bray-Curtis index }(B C) \text {, these plots show a 3D } \\
\text { view of those parameters. The step length }(L) \text { and turning } \\
\text { angle concentration }(k) \text { are on the } x \text { and } y \text { axes and the } \\
B C \text { value is the height. In each case a third parameter is } \\
\text { varied which was either the radius of attraction }(R) \text { or the } \\
\text { visual field depth }(F D) \text {. A separate plot is shown for each } \\
\text { value of this third parameter. Lower values of } B C \text { indicate } \\
\text { a closer fit to the field data. }\end{array}$ \\
\hline
\end{tabular}

Table 4.9: Plot types shown in the results section. 
detailed discussion of these results and implications can be found in (Hasenbank, in prep).

The "Scale-code" graphs are shown to demonstrate the effect of aggregating the results at such a high level. It is noticeable that at the $48 \times 48 \mathrm{~m}$ scale for KAITOKE-04 and at the $6 \times 6 m$ scale, the 40 plant density grouping appears slightly elevated in relation to the 16 plant density. These groupings are actually aggregated over several sub-groups which becomes apparent when compared in Figures 4.13 and 4.20. Here, when they are ordered at the $48 \times 48 m$ scale for KAITOKE-04, the pattern of resource dilution is still seen but is broken into three sub groupings, which depend upon the density at the $6 \times 6 m$ scale. For LEVIN-06, the same pattern is seen, with two sub-groups, again dependant on the number of plants at the $6 \times 6 \mathrm{~m}$ scale $(1,4$ or 16 vs. 40$)$.

When calculating the Bray-Curtis dissimilarity, the results from these Scale-code groupings as well as from the plant density groupings were included, to allow for subtle variations. In theory, if the simulation mimics the results exactly, both responses should be the same, irrespective of how the plants are grouped. The graphs for the simulation data are not shown for clarity.

\subsubsection{Calibration}

Three simulation experiments were conducted as a control, or "null" experiment in order to analyse the response of the field layout without any animal movement whatsoever. These consisted of three simple strategies for creating an egg distribution; a) give each plant an equal number of eggs, b) choose plants at random and lay eggs with no regard to spatial arrangement and c) choose locations over the patch at random, and if that location falls within the radius of detection of a plant, lay an egg on that plant.

These results demonstrate the effective "ideal" distribution where the spatial arrangement and forager path have little, or indeed no effect on the response. Figure 4.7 shows these results. A flat response is seen in all cases, which is interesting to contrast to the responses observed in the field and from the other simulations, and highlights the fact that a response is occurring. Each of these experiments behaves in a similar way, as there is an equal chance for each individual cabbage to receive an egg, therefore the fact that eggs per plant is calculated, produces a flat line response. The absolute number of eggs in this case is unimportant; for the "equal eggs" scenario, 15 eggs were chosen, for the others, the same number of eggs as laid in the field were laid. The radius of attraction was fixed at $R=20$ and so none were overlapping. 
a) Equal Eggs

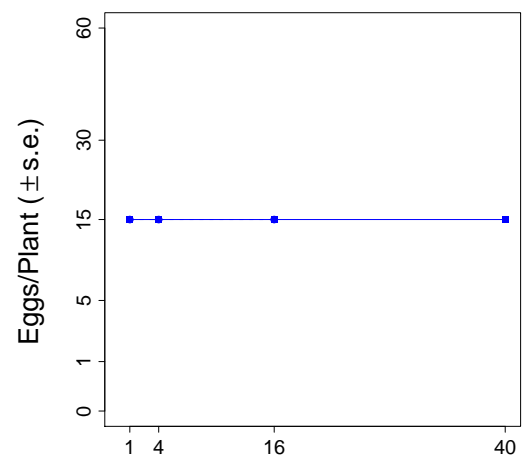

b) Random Plant

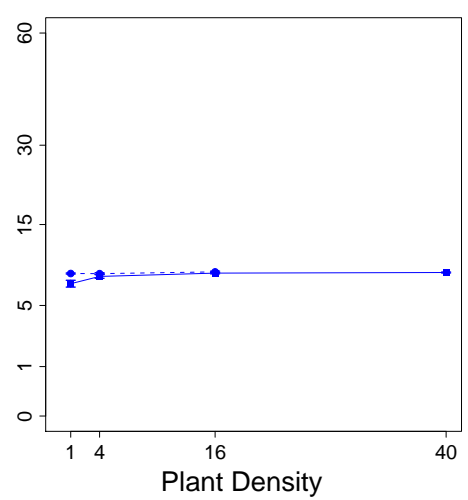

c) Random Location

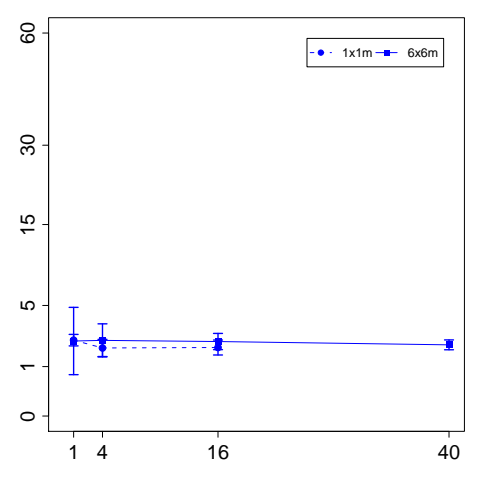

Figure 4.7: Calibration - LEVIN-06-II Trial B3. Distribution created by dropping eggs "from the sky" 4 replicates of each field with the same number of eggs laid as observed in field, $R=20$.

\subsubsection{Correlated Random Walk}

The results from the CRW experiments are striking in their overall similarity to the field data (Figures 4.10 and 4.16). Shown are the parameter combinations that most closely resembled the field data in terms of the Bray-Curtis measure. As with the field data, a general trend for "resource dilution" is simulated (Figures 4.12 and 4.19), and supported by the regression statistics (Tables B.13 and B.12). It should be noted that because the simulation experiments were replicated 4 times, there are 4 times the number of points on the regression graphs for these results, when compared to the field data.

Detailed breakdowns for each parameter combination can be found in Appendix B (Figures B.1 to B.27). From these, the effect of the radius of attraction $(R)$ can be seen; across all combinations of step length and turning angle, the "dilution effect" is magnified (i.e. isolated plants receive increasingly disproportionate eggs per plant). As $R$ increases, so the isolated plants become more "visible" and are more likely to be intercepted by the foragers. Because a single egg is laid, the result is that foragers searching over the area containing a single plant are more likely to intercept it (Block L4, Figure 4.5.d). The effect is reduced with a more directional path (longer step length, higher turning angle concentration) because the area containing the single plant is searched less thoroughly.

With the KAITOKE-04 layout, the "best fit" plot (Figure 4.10) shows a less pronounced response than the field, possibly the Bray-Curtis calculation has selected a result which is on average a closer match, but which actually represents the lowest common denominator. The plots shown in figures B.4 to B.6, particularly for $L=250, k=0.5$, appear to have a qualitatively better match. Perhaps there is another, more accurate measure? It could also be a problem with the fact that the 
results are aggregated at such a high level.

The negative response to plant density appears to be increased with an increase in $R$ (Figures B.1 to B.3). in other words, the "curve" of the graphs is steeper when $R=20$. The absolute measure of difference (the Bray-Curtis index), however, is higher for the larger $R$ values (Table B.1). Either the Bray-Curtis measure is not accurate in this situation, or the overall eggs per plant for the single plants becomes much greater in the simulation and therefore quantitatively the response is removed from that of the field.

\begin{tabular}{rlrrr}
\hline $\mathbf{R}$ & Eggs (G) & L & k & mean BC \\
\hline $\mathbf{5}$ & Single Egg & 150 & 0.5 & 0.1133 \\
$\mathbf{1 0}$ & Single Egg & 350 & 0.5 & 0.1361 \\
$\mathbf{2 0}$ & Single Egg & 250 & 0.5 & 0.1415 \\
\hline $\mathbf{5}$ & Multiple Egg & 350 & 10 & 0.1842 \\
$\mathbf{1 0}$ & Multiple Egg & 350 & 10 & 0.1590 \\
$\mathbf{2 0}$ & Multiple Egg & 250 & 10 & 0.1679 \\
\hline
\end{tabular}

Table 4.10: Table of the best fit for CRW behaviour LEVIN-06-II.

When multiple eggs are introduced (only run for LEVIN-06-II), an interesting response occurs in terms of fit to the field data. Rather than the shorter stepped, more tortuous paths being the closest match, longer steps and higher turning angle concentration are the best fit (Table 4.10). As noted in Chapter 3, more directional paths penetrate to the centre of resource patches more efficiently. Perhaps a similar effect is occurring here, allowing a greater area of the layout to be reached and thus providing more "realistic" results. With multiple eggs, the more sinuous paths may remain "trapped" at the edge of the layouts.

One feature of the field results is a high variability between fields (Figure 4.21). Each field has quite different plots and it is only when aggregated that a common trend is observed. The simulation produces generally more consistent results (Figure 4.22). These results demonstrate that although aggregating stochastic observations can produce a response which is tractable and may be modelled, it may be at the cost of loss of finer scale detail (Levin, 1992). A model which is able to replicate the variance as well as the aggregated response would be an interesting future project. However, we may never know what environmental factors, if any, may have caused the extra variability between fields. 


\subsubsection{Vision}

Vision is similar to the idea of the radius of attraction in terms of being a direct attractant to the forager (Chapter 3). The effects on the response are also similar in terms of stabilising the pattern, i.e. The visual attraction has no random element so the effect of the random walk parameters is reduced and so the response depends less upon the values of $L$ and $k$. Vision amplifies the trend of increased numbers of eggs on isolated cabbages (Figures B.16 to B.18 and B.22 to B.24).

\begin{tabular}{rlccc}
\hline FD & Eggs (G) & L & k & mean BC \\
\hline $\mathbf{1 0 0}$ & Single Egg & 350 & 10 & 0.1418 \\
$\mathbf{6 0 0}$ & Single Egg & 150 & 10 & 0.3338 \\
$\mathbf{1 8 0 0}$ & Single Egg & 150 & 10 & 0.3566 \\
\hline $\mathbf{1 0 0}$ & Multiple Eggs & 350 & 3.0 & 0.1651 \\
$\mathbf{6 0 0}$ & Multiple Eggs & 250 & 0.5 & 0.1370 \\
$\mathbf{1 8 0 0}$ & Multiple Eggs & 250 & 0.5 & 0.1430 \\
\hline
\end{tabular}

Table 4.11: Table of the best fit for Vision behaviour LEVIN-06-II.

Indeed, with a high field depth $(F D)$, the isolated plants received $>60$ eggs per plant, greater than the scale of the graph (it was decided to keep the graphs at the same scale for comparison across results). Introducing multiple eggs reduces the proportion which are laid on the single, isolated plants, allowing the foragers to penetrate into the more dense patches. When a high field depth is set, the isolated plants have a high probability of entering the field of vision if a forager is in that quadrant; once "fixated" the plant will dominate the field of view and thus always receive an egg, an issue discussed in Chapter 3. If $F D>L$, a forager having a high variance of turning angles $(k=0.5)$ with multiple eggs may even become "trapped" and lay all its eggs on the same plant.

With vision enabled, longer step lengths but higher turning angle concentrations (that is to say more directional paths) provide the best fit (Tables 4.11 and B.6). When multiple eggs are enabled, more turning provides a better fit (Table B.8). In general with vision the results were closer to the field with multiple eggs. Possibly more tortuous paths allow a greater chance for the foragers to "re-discover" isolated plants, an effect suggested by Jones (1977), and is consistent with the results from the CRW trial (Section 4.4.3) where increased radius of attraction $(R)$ increased the likelihood of discovering isolated plants.

A "short-range" depth of field $(F D=100)$ has the best fit (Table 4.11). As the field depth increases, it exaggerates the response to isolated plants so much that it ceases to re- 
semble the field data.

\subsubsection{Olfaction}

The signal surfaces for the four "fields" in the LEVIN-06-II layout are shown in Figures 4.23 to 4.26 . The Signal Width $(S W)$ was deliberately set to be 1500 which is relatively wide and causes a "hill" shaped surface to be created, with the signals from each plant merging and making it impossible to distinguish individual plants (Chapter 3).

One of the goals of the project of which this chapter is a part was to look for responses to plant density that might vary when measured at different scales. This was not observed in the field data, which may be because the observations were not made over a wide enough range of scales. The results when a long range, diffuse attractant (olfaction) was built into the model demonstrate that, at least in theory, this pattern is possible (Figures 4.27 and 4.29). Here, at the $1 \times 1 \mathrm{~m}$ scale, a dilution effect is still observed, while at the larger $6 \times 6 m$ scale the opposite response, "resource concentration" is shown, and the foragers at this scale lay more eggs in the higher density patches (regression statistics in Table B.16).

The reason that these two opposite responses are seen is a result of the wide signal width $(S W=1500)$. The foragers are generally attracted to the group at $6 \times 6 \mathrm{~m}$ scale which has 40 plants (it being the most dense and providing the greatest signal). Because the signal is diffuse, once within that area there is less information as to exactly where the plants are, and so the foragers are effectively moving at random again. As demonstrated in the previous sections, random movement results in a dilution pattern. Thus at the $1 \times 1 m$ scale within the $6 \times 6 m$ block (L3, Figure 4.5), resource dilution is still observed.

In terms of the parameter space, Figures B.10 to B.12 show that at higher turning angles and shorter step lengths, a dilution pattern still occurs at all scales. The olfaction behaviour is still influenced by the random walk and so the effect of intercepting the isolated plants still occurs.

This result was an exciting discovery because it helped the researchers involved conceptualise under what conditions this opposite response might occur. Although not formally observed in the field experiments, it was apparent that there were many more butterflies in the large, commercial cabbage field than adjacent, non-cabbage fields. The olfaction model in the simulation need not literally represent a sense of smell, rather it might represent any mechanism that results in an attraction toward a larger mass of resources. This might be in reality a completely different process, perhaps not even behavioural in nature, for example it may be demographic with 
greater levels of recruitment within a large patch at a much greater scale. If the scale of the field experiment were changed so that the layout covered a much larger area (say $5 \mathrm{~km}$ ), and at each location of a plant were a patch of 500 plants, perhaps this overall change in response could be observed. This field set-up would of course be extremely costly in effort and cabbage plants.

\subsubsection{Olfaction and vision}

When foragers lay a single egg, and when vision and olfaction are combined, the visual behaviour overwhelms the other behaviours due to the fact that it is the "preferred" sense (Figures B.13 to B.15). A similar effect is noted in Chapter 3. Again the eggs per plant on isolated plants is very large, exceeding 60 per plant and the scale of the graphs.

With multiple eggs, the response the isolated plants do not receive such disproportionate numbers of eggs (Figures B.25 to B.27). For multiple eggs, and when the field depth is in the mid-range $(F D=600)$, vision and olfaction combined produces the closest match to the field data of all the combinations according to the Bray-Curtis measure (Tables 4.8 and 4.12, Figures B.25 to B.27).

\begin{tabular}{rlrrr}
\hline FD & Eggs (G) & L & k & mean BC \\
\hline $\mathbf{1 0 0}$ & Multiple Eggs & 350 & 0.5 & 0.1993 \\
$\mathbf{6 0 0}$ & Multiple Eggs & 250 & 3.0 & 0.0534 \\
$\mathbf{1 8 0 0}$ & Multiple Eggs & 150 & 3.0 & 0.1281 \\
\hline
\end{tabular}

Table 4.12: Table of the best fit for Olfaction and Vision behaviour LEVIN-06-II.

For a shorter visual field depth $(F D=100)$, the opposing negative response to density at $1 \times 1 \mathrm{~m}$ and positive response at $6 \times 6 \mathrm{~m}$ is once more observed (Figure 4.28). This is similar to olfaction with no vision (Trial D1, Figure 4.27). Visually, the response appears more pronounced for Trial D6, i.e. There appears to be a greater curvature to the response at both scales. However, If the slope of the regression line is taken as an indicator of the strength of the response, Trial D1 has a steeper slope at both scales (Tables B.16 and B.22).

This leads to an interesting situation in which the combination of both vision and olfaction can produce a wide range of responses, from a very close fit to the field (Figures 4.8 and 4.15), to the most extreme deviation (opposite responses at different scales) from the field (Figure 4.28). This exemplifies the concept that as more parameters and possible behaviours are added to a model, a more flexible response 
is created, at the cost of an increasingly complex model (Jones, 1977; Kareiva and Shigesada, 1983; Levin, 1992).

\subsubsection{Parameter estimation}

Table 4.13 shows a summary of each of the behaviours tested along with the details of the measures of the Bray-Curtis index $(B C)$. This was calculated by comparing at each of the scales the eggs per plant for each density group i.e. The "plant density" plot, and also for each scale code i.e. the "scale code" plot (Table 4.9). The mean of these was then used for the comparison. Table 4.13 shows the lowest $B C$ value for each trial (trials are described in Table 4.7). Figure 4.8 Shows these results as a bar-plot, ordered with the lowest $B C$ value on the left, in ascending order.

For the random walk and olfaction experiments, the radius of attraction $(R)$ had a strong influence and so the results were grouped by $R$ for analysis. For the visual experiments, the field depth $(F D)$ played a similar role ( $R$ was kept constant at $R=5$ to minimise interference) these results are grouped accordingly, by $F D$. The detailed results are found in Tables B.1 to B.9 of Appendix B.

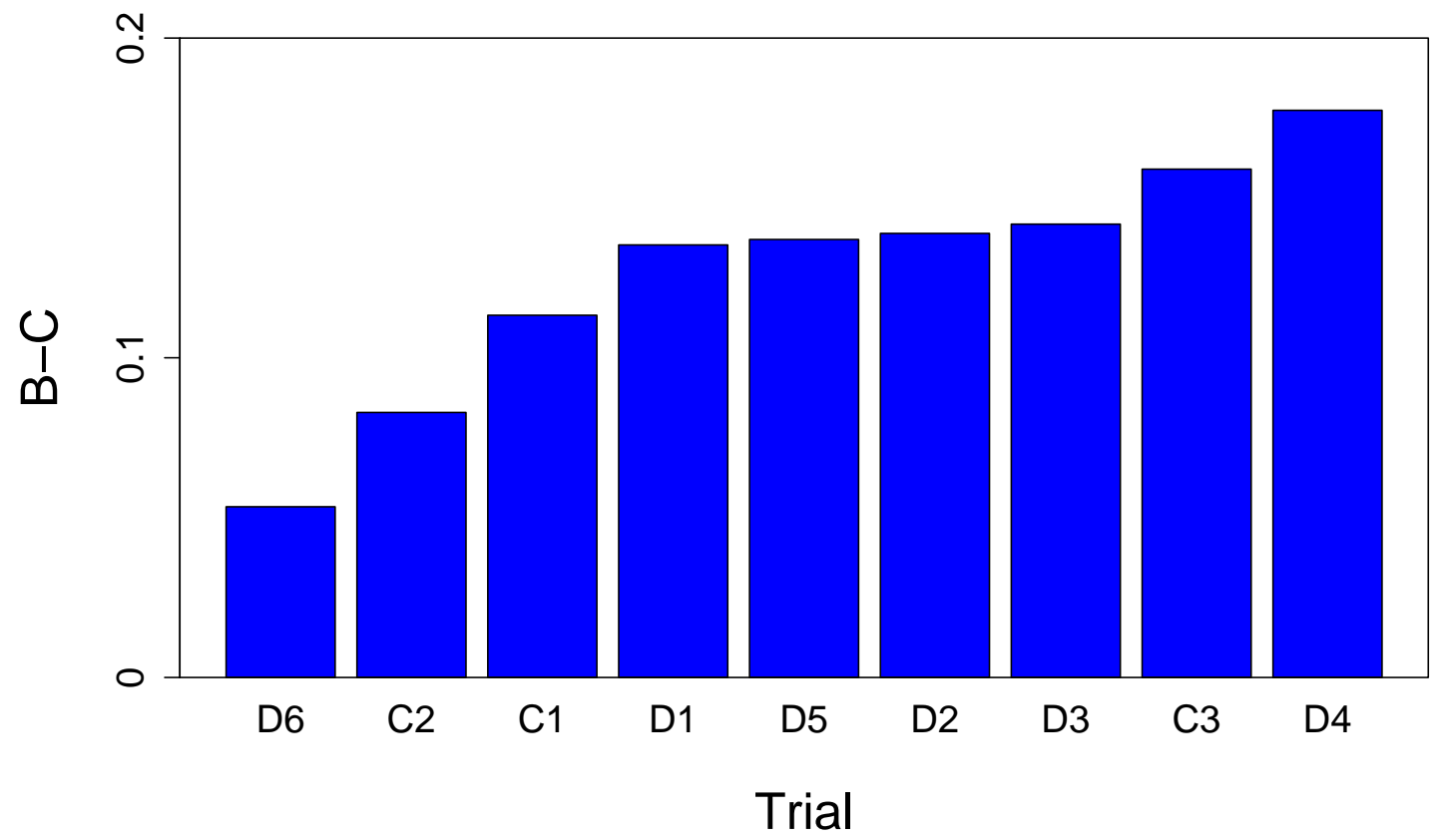

Figure 4.8: Bar-plot of the lowest average Bray-Curtis index (best fit) for each of the behaviour types (listed by trial code), sorted with lowest first. Lower values indicate a greater similarity to the field data. The trial codes are described in Table 4.7.

Figures 4.31 to 4.39 show a summary of the entire parameter space explored as 3D response surfaces. Some general points can be added from these to the previous results. 
The KAITOKE-04 CRW simulation (Figure 4.31) matched the field results overall more closely than the LEVIN-06-II CRW, although with a small radius of detection and sinuous path, the LEVIN-06-II results became closer to the field observations. There is an interesting "spike" in the KAITOKE-04 response when $R=20$ (Figure 4.31.c), at low step length $(L=250$ and low turning angle $(k=0.5)$, which is the opposite to the others. When compared visually with Figure B.6 the response looks very similar to the field observations. It is possible that the method of comparing the results is not so effective for this layout, because it is larger, more complex and has more scales.

\begin{tabular}{|c|c|c|c|c|c|c|c|c|c|c|c|c|c|c|c|c|}
\hline \multirow[b]{2}{*}{ Trial } & \multicolumn{3}{|c|}{ Parameters } & \multicolumn{3}{|c|}{ 1x1m (2 d.f.) } & \multicolumn{3}{|c|}{$6 \times 6 \mathrm{~m} 3$ d.f. } & \multicolumn{3}{|c|}{ 48x48m (2 d.f.) } & \multicolumn{3}{|c|}{ ScaleCode (5/6 d.f.) } & \multirow[b]{2}{*}{ mean $\mathrm{BC}$} \\
\hline & $\mathrm{R} / \mathrm{FD}$ & $\mathbf{L}$ & $\mathbf{k}$ & BC & $\chi^{2}$ & (p) & BC & $\chi^{2}$ & (p) & BC & $\chi^{2}$ & (p) & BC & $\chi^{2}$ & (p) & \\
\hline $\mathrm{C} 1$ & 5 & 150 & 0.5 & 0.040 & 5.72 & 0.057 & 0.157 & 74.89 & $<0.001$ & - & 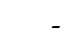 & - & 0.143 & 80.04 & $<0.001$ & 0.1133 \\
\hline $\mathrm{C} 2$ & 5 & 250 & 0.5 & 0.106 & 45.21 & $<0.001$ & 0.080 & 23.63 & $<0.001$ & 0.044 & 2.15 & 0.342 & 0.102 & 56.88 & $<0.001$ & 0.0829 \\
\hline $\mathrm{C} 3$ & 10 & 350 & 10.0 & 0.040 & 6.23 & 0.044 & 0.239 & 80.41 & $<0.001$ & - & - & - & 0.197 & 83.77 & $<0.001$ & 0.1590 \\
\hline D1 & 10 & 150 & 0.5 & 0.106 & 45.75 & $<0.001$ & 0.155 & 93.61 & $<0.001$ & - & - & - & 0.145 & 101.59 & $<0.001$ & 0.1353 \\
\hline D2 & 100 & 350 & 0.5 & 0.164 & 96.10 & $<0.001$ & 0.125 & 118.41 & $<0.001$ & - & - & - & 0.128 & 140.74 & $<0.001$ & 0.1389 \\
\hline D3 & 100 & 350 & 10.0 & 0.161 & 92.48 & $<0.001$ & 0.134 & 108.59 & $<0.001$ & - & - & - & 0.131 & 137.79 & $<0.001$ & 0.1418 \\
\hline D4 & 10 & 350 & 0.5 & 0.040 & 5.68 & 0.059 & 0.274 & 83.65 & $<0.001$ & - & - & - & 0.219 & 88.00 & $<0.001$ & 0.1774 \\
\hline D5 & 600 & 250 & 0.5 & 0.090 & 29.02 & $<0.001$ & 0.157 & 152.96 & $<0.001$ & - & - & - & 0.164 & 170.57 & $<0.001$ & 0.1370 \\
\hline D6 & 600 & 250 & 3.0 & 0.047 & 9.48 & 0.009 & 0.046 & 38.75 & $<0.001$ & - & - & - & 0.067 & 68.10 & $<0.001$ & 0.0534 \\
\hline
\end{tabular}

Table 4.13: Best fit across all trials for different behaviour combinations. For a graphical representation see Figure 4.8 .

Multiple eggs for the CRW experiment produces a flatter surface (Figure 4.33), and at low and high $\mathrm{R}$ are generally not as good a fit as single eggs. The mid range ( $R=10$, Figure 4.33.b) however, provides a fit comparable to the CRW with a single egg (Figure 4.32).

Olfaction produces a steeply sloping surface, with turning angle the dominating factor. Lower turning angle concentrations result in a better fit (Figure 4.34). A lower turning angle concentration produces a more tortuous, area restricted search and so isolated plants receive more eggs and the pattern is closer to the field observations.

Multiple eggs seems to have little effect on the olfaction response, except to generally move the Bray-Curtis surface higher, i.e. more distant from the field (Figure 4.37).

Where vision is enabled, results appear to be particularly sensitive to the vision parameter of field depth $(F D$, Figure 4.35). With a short range $(F D=100)$ the match is overall similar to the CRW, but at higher levels has the greatest difference to the field, even though as can be seen from figures B.16 to B.18 and B.22 to B.18, the qualitative shape of the response is similar to the field. 
With multiple eggs and vision, the surface is changed dramatically, sloping towards the lower turning angles (Figure 4.38). Perhaps with multiple eggs, foragers can move about within the patch, rather than always approaching from the outer edge of the landscape. In this way, the isolated plants that are found "en route" to the central patches receive relatively fewer eggs.

Finally we see the interesting range of behaviours possible when both vision and olfaction are enabled (Figure 4.36), particularly when multiple eggs are introduced (Figure 4.39). Here, both extremes (of a very good fit and a very poor fit to the field data) are represented, and it is clear from looking at all the plots together that Figure 4.39.b (Trial D6) has the closest overall match to the field (lowest $B C$ ) of all the behaviours, as confirmed by Figure 4.8 and Table 4.13 . 


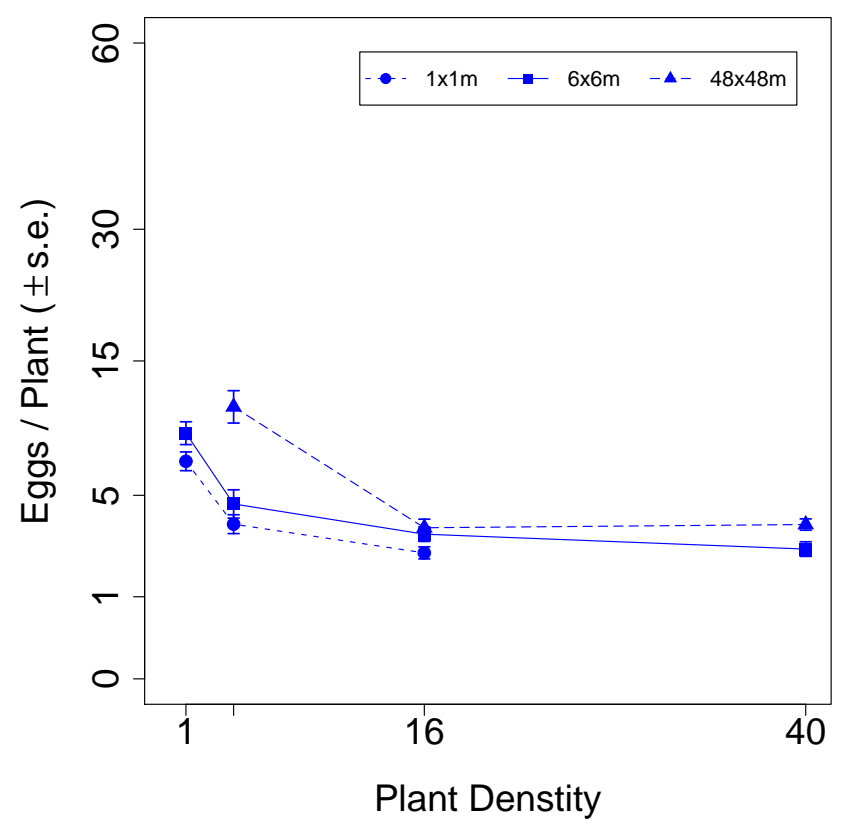

Figure 4.9: KAITOKE-04 Plant Density - Field Data.

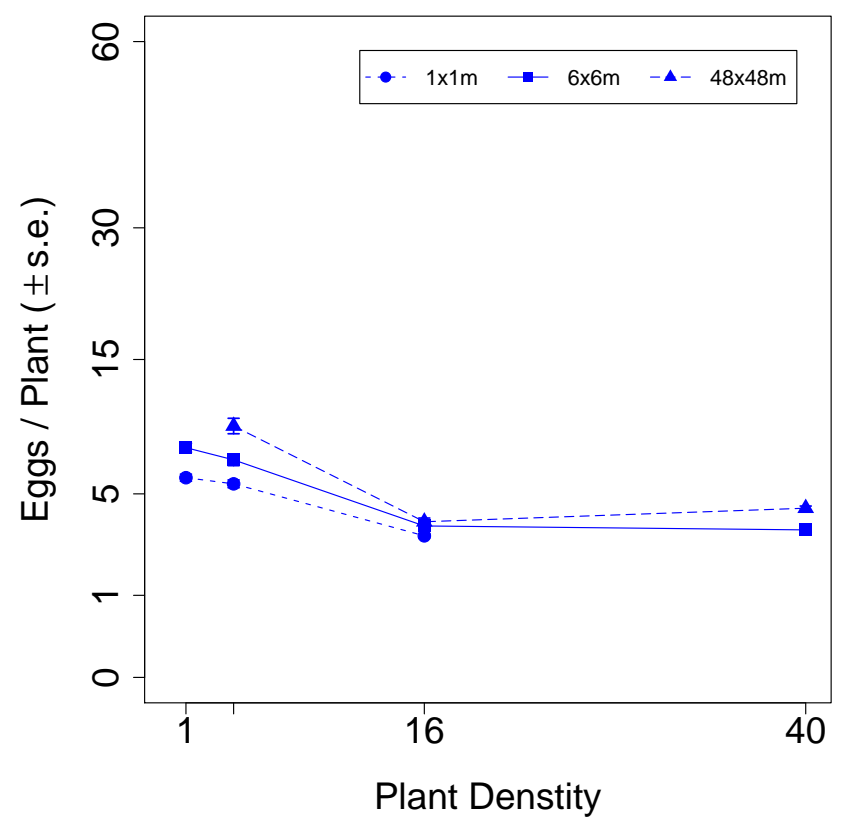

Figure 4.10: KAITOKE-04 Plant Density - Trial C2 (CRW, Single Egg). Best-fit $R=5$, $L=250, k=0.5$. 

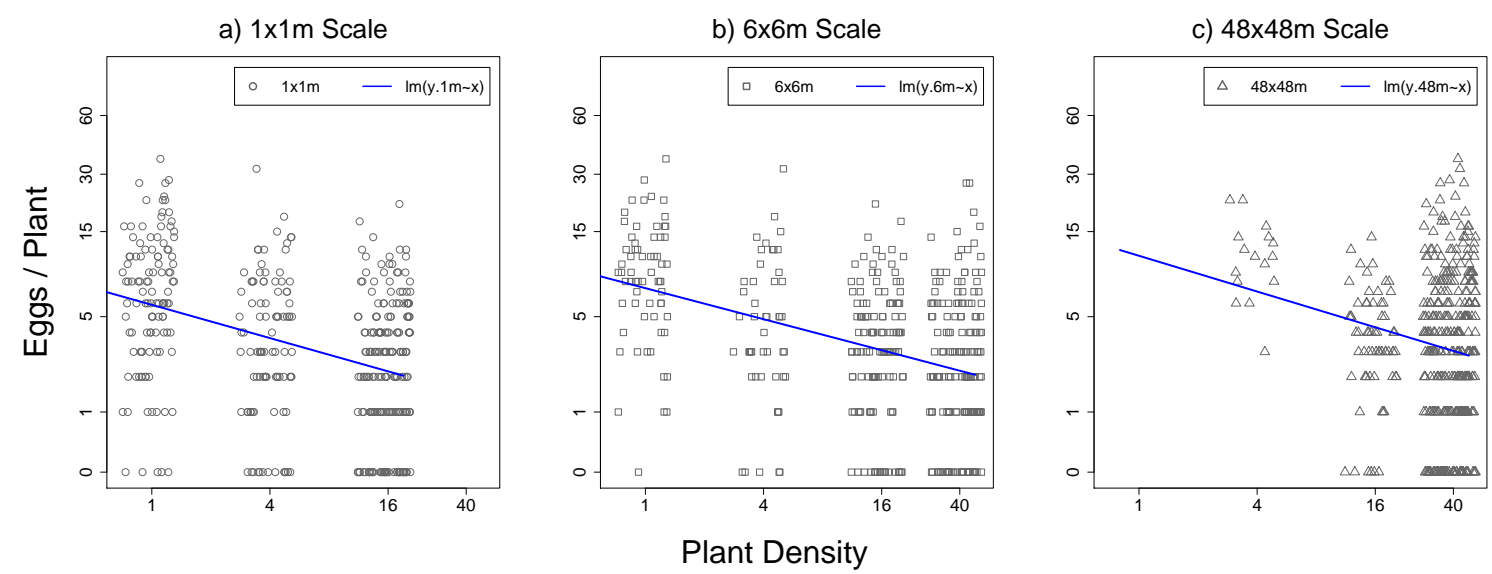

Figure 4.11: Log-Log Regression - KAITOKE-04 - Field Data. For statistics see Table B.10.

a) $1 \times 1 \mathrm{~m}$ Scale

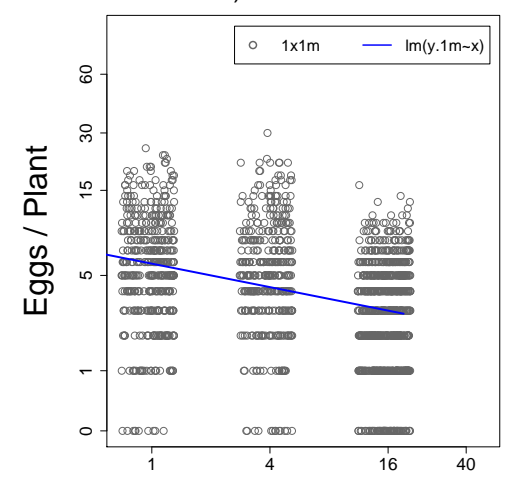

b) $6 \times 6 \mathrm{~m}$ Scale

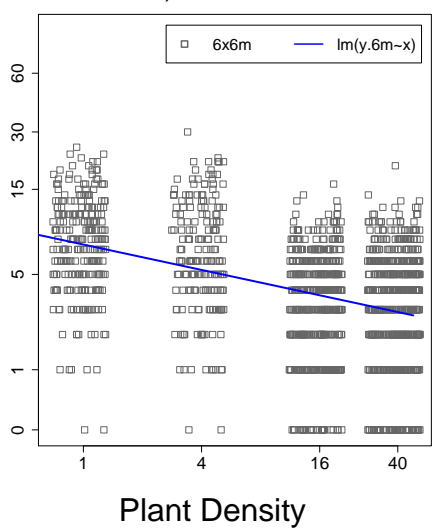

c) $48 \times 48 m$ Scale

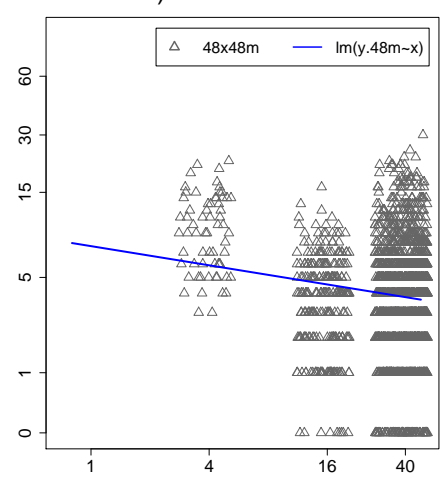

Figure 4.12: Log-Log Regression - KAITOKE-04 - Trial C2 (CRW, Single Egg). Best-fit $R=5, L=250, k=0.5$. For statistics see Table B.13. Note that there are more points for the simulation results because they were replicated 4 times. 
a) Sorted at $1 \mathrm{M}$ Scale

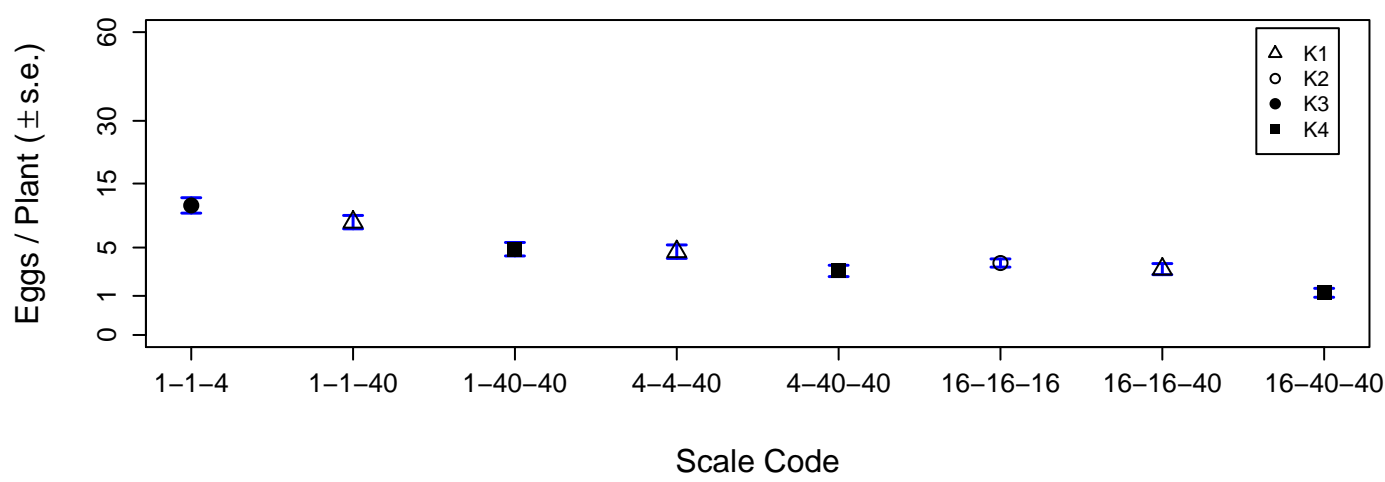

b) Sorted at $6 \mathrm{M}$ Scale

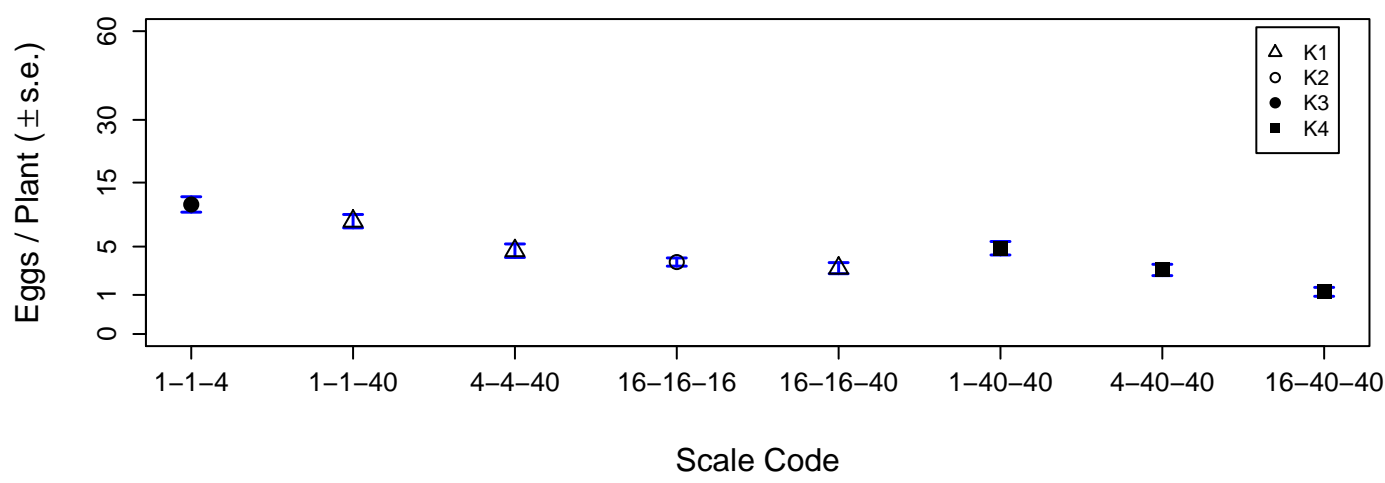

c) Sorted at $48 \mathrm{M}$ Scale

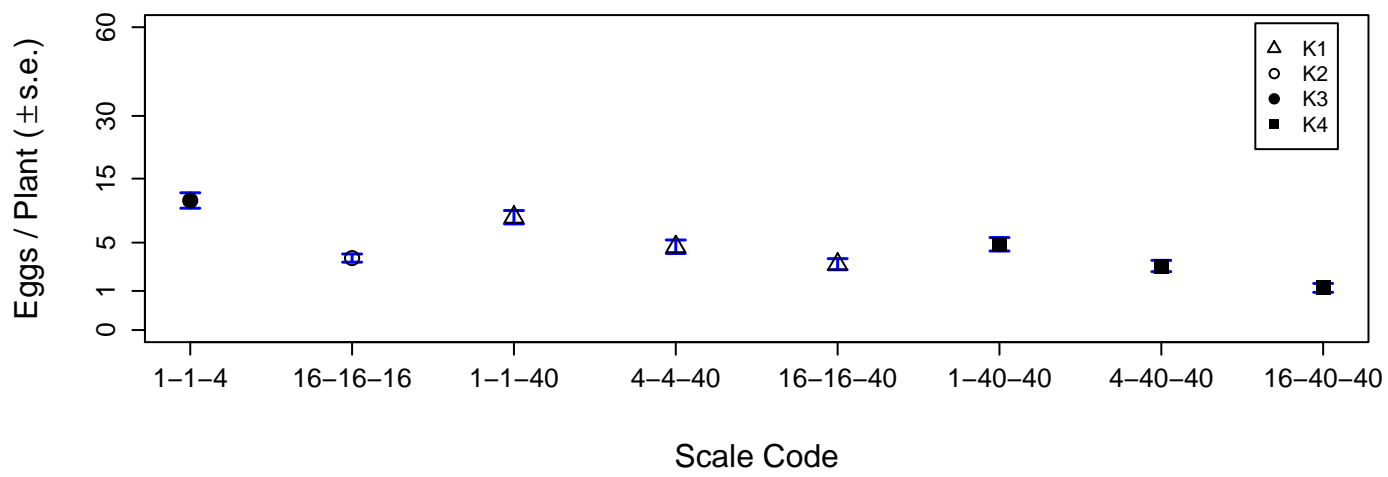

Figure 4.13: Scale Code - KAITOKE-04 - Field Data. The scale code is composed of the number of cabbages in a $1 \times 1 \mathrm{~m}, 6 \times 6 \mathrm{~m}, 48 \times 48 \mathrm{~m}$ square respectively. 


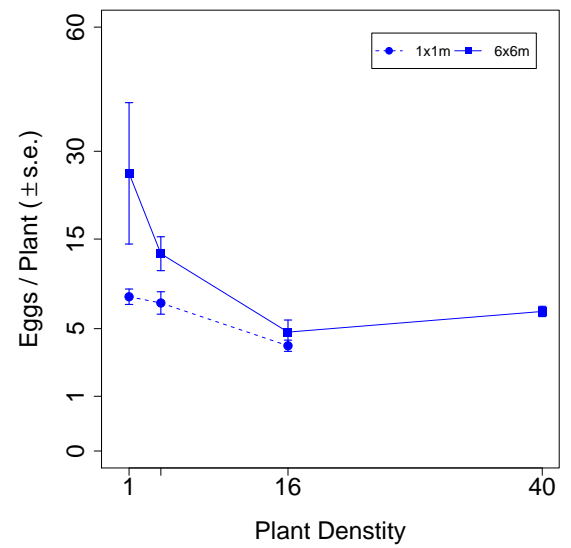

Figure 4.14: Plant Density - LEVIN-06-II - Field Data .

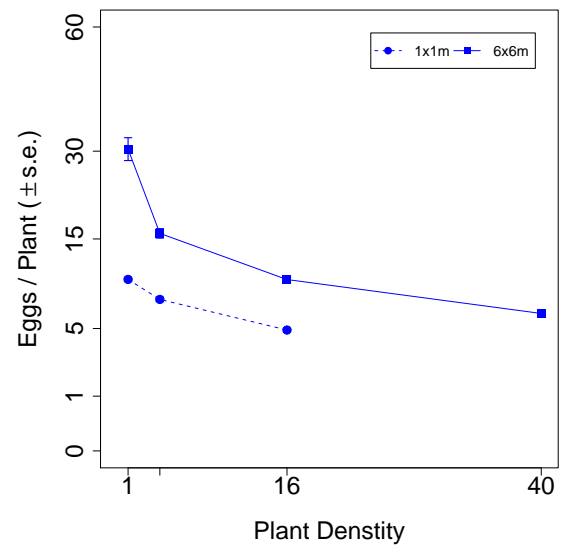

Figure 4.15: Plant Density - LEVIN-06-II - Trial D6 (Olfaction and Vision, Multiple Eggs). Best Fit $-F D=600, R=5, L=250, k=3.0$.

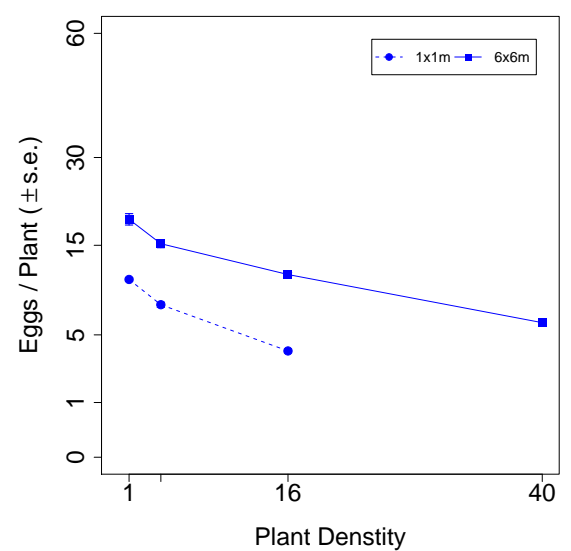

Figure 4.16: Plant Density - LEVIN-06-II - Trial C1 (CRW, Single Egg). Best-fit - R=5, $L=150, k=0.5$. 


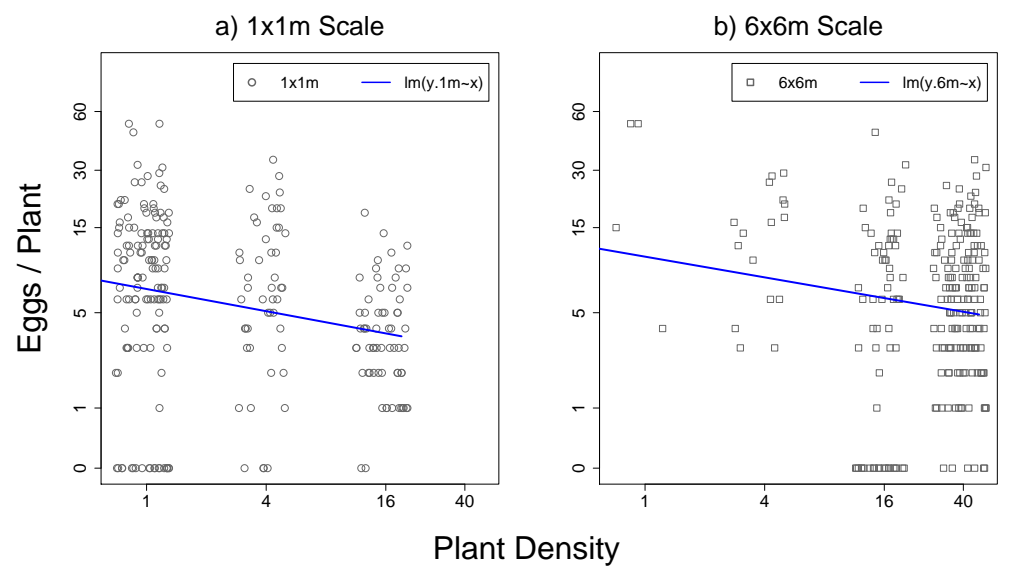

Figure 4.17: Log-Log Regression - LEVIN-06-II - Field Data. For statistics see Table B.11.

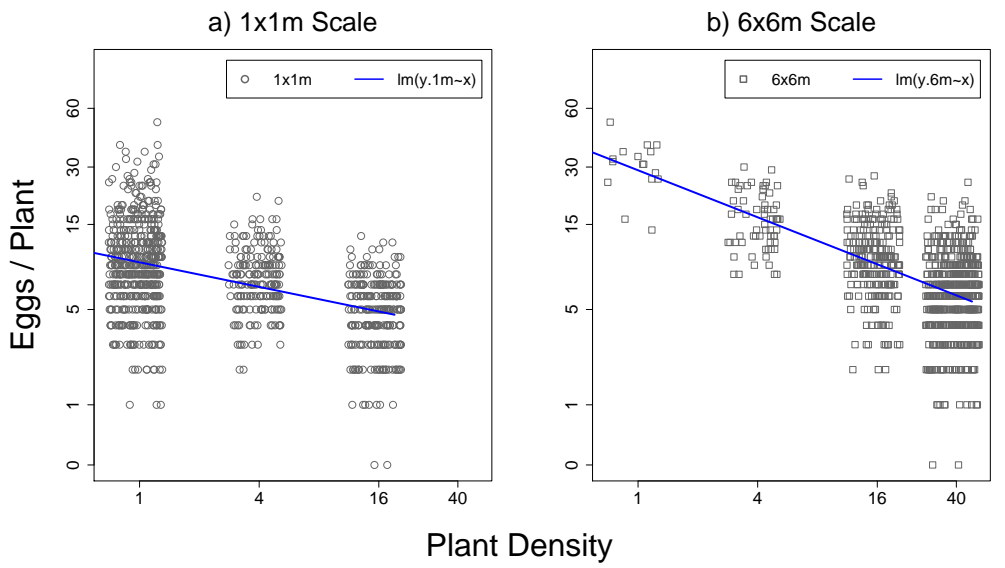

Figure 4.18: Log-Log Regression - LEVIN-06-II - Trial D6 (Olfaction and Vision, Multiple Eggs, $F D=600, L=250, k=3.0)$. For statistics see Table B.21. Note that there are more points for the simulation results because they were replicated 4 times.
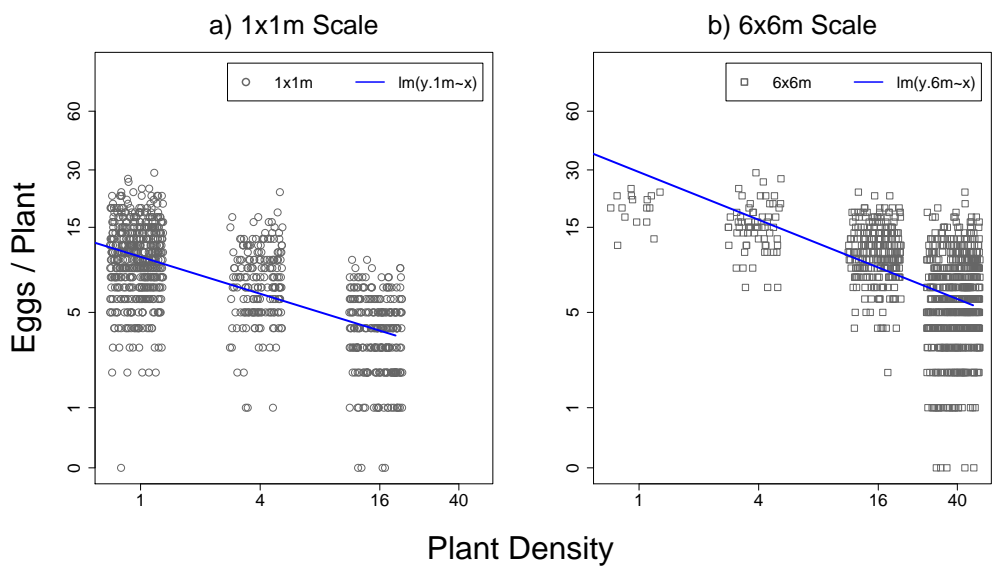

Figure 4.19: Log-Log Regression - LEVIN-06-II - Trial C1 (CRW, Single Egg, $R=5, L=150$, $k=0.5)$. For statistics see Table B.12. Note that there are more points for the simulation results because they were replicated 4 times. 
a) Sorted at $1 \mathrm{M}$ Scale

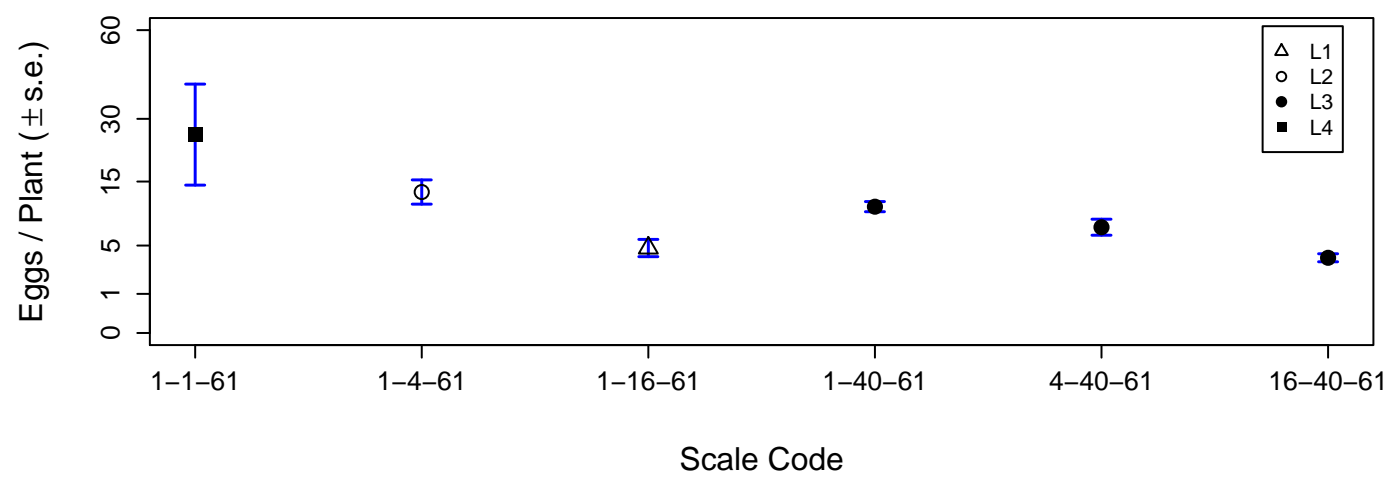

b) Sorted at $6 \mathrm{M}$ Scale

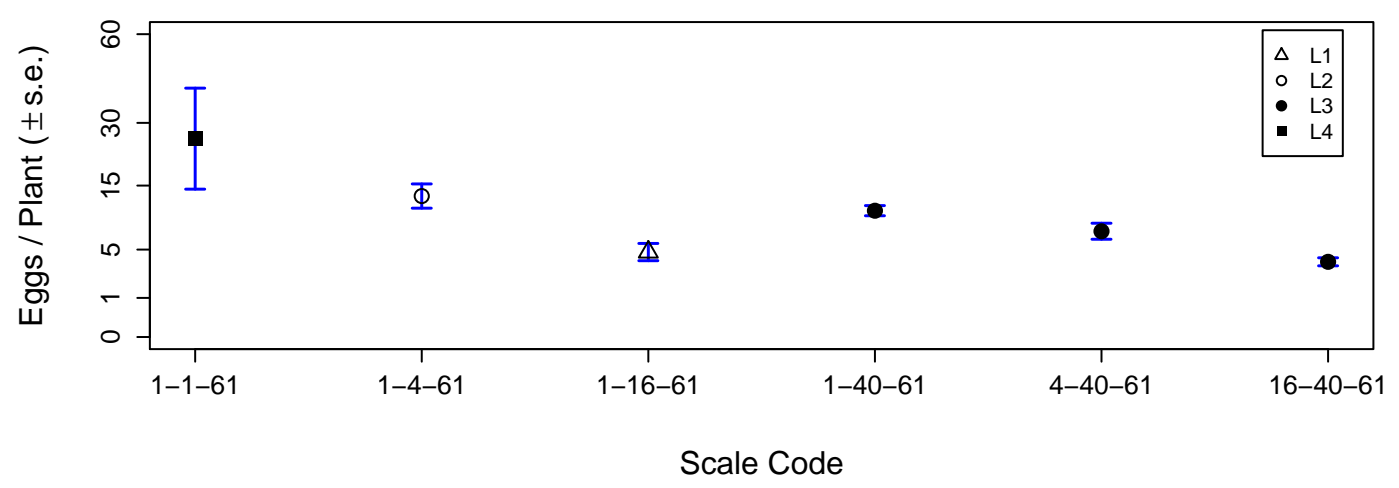

Figure 4.20: Scale Code - LEVIN-06-II - Field Data. The scale code is composed of the number of cabbages in a $1 \times 1 \mathrm{~m}, 6 \times 6 \mathrm{~m}, 36 \times 36 \mathrm{~m}$ square respectively. 

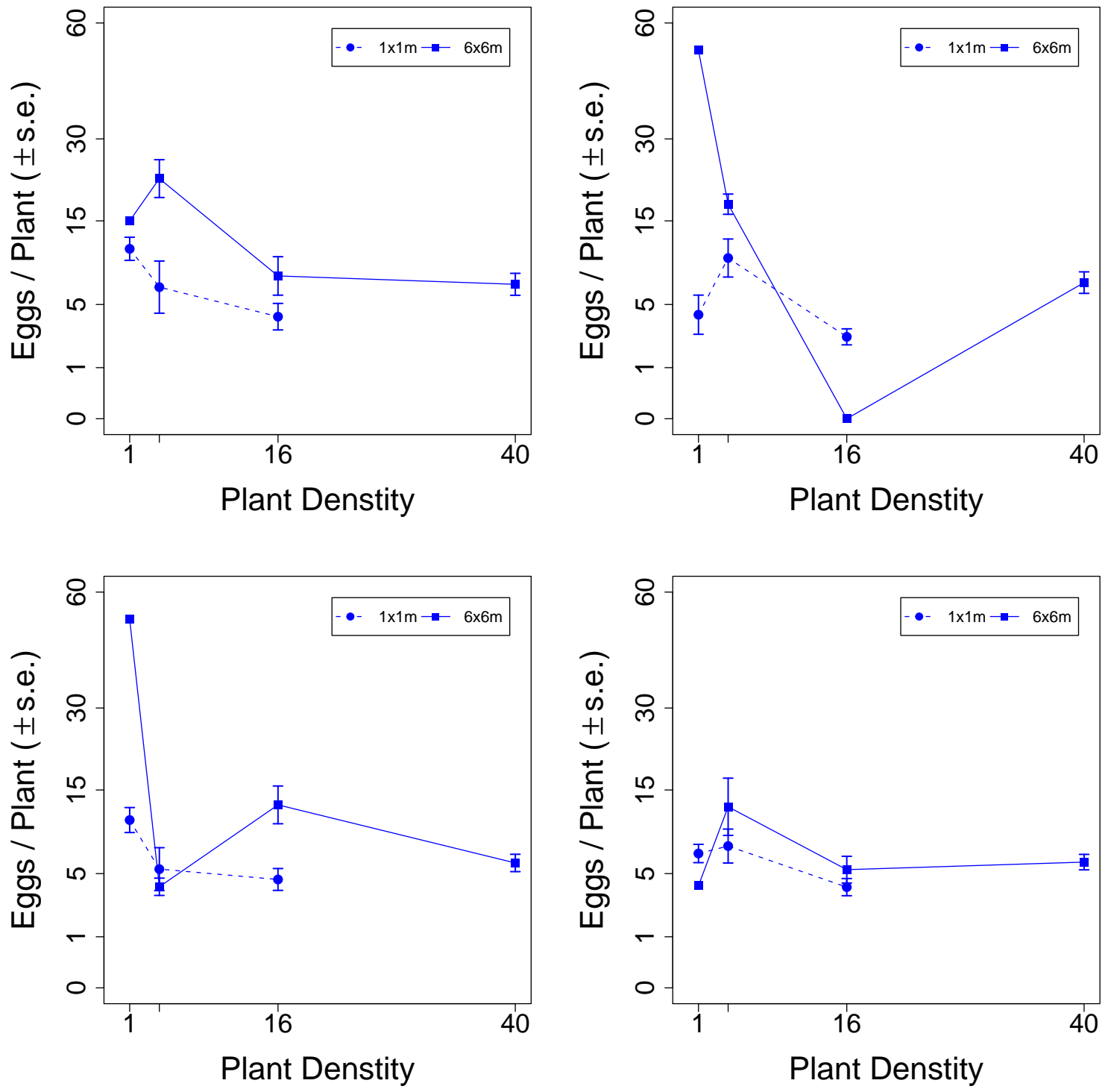

Figure 4.21: Field Breakdown - LEVIN-06-II - Field Data. Results from fields E, F, G and $H$ respectively, as in Figure 4.4. 

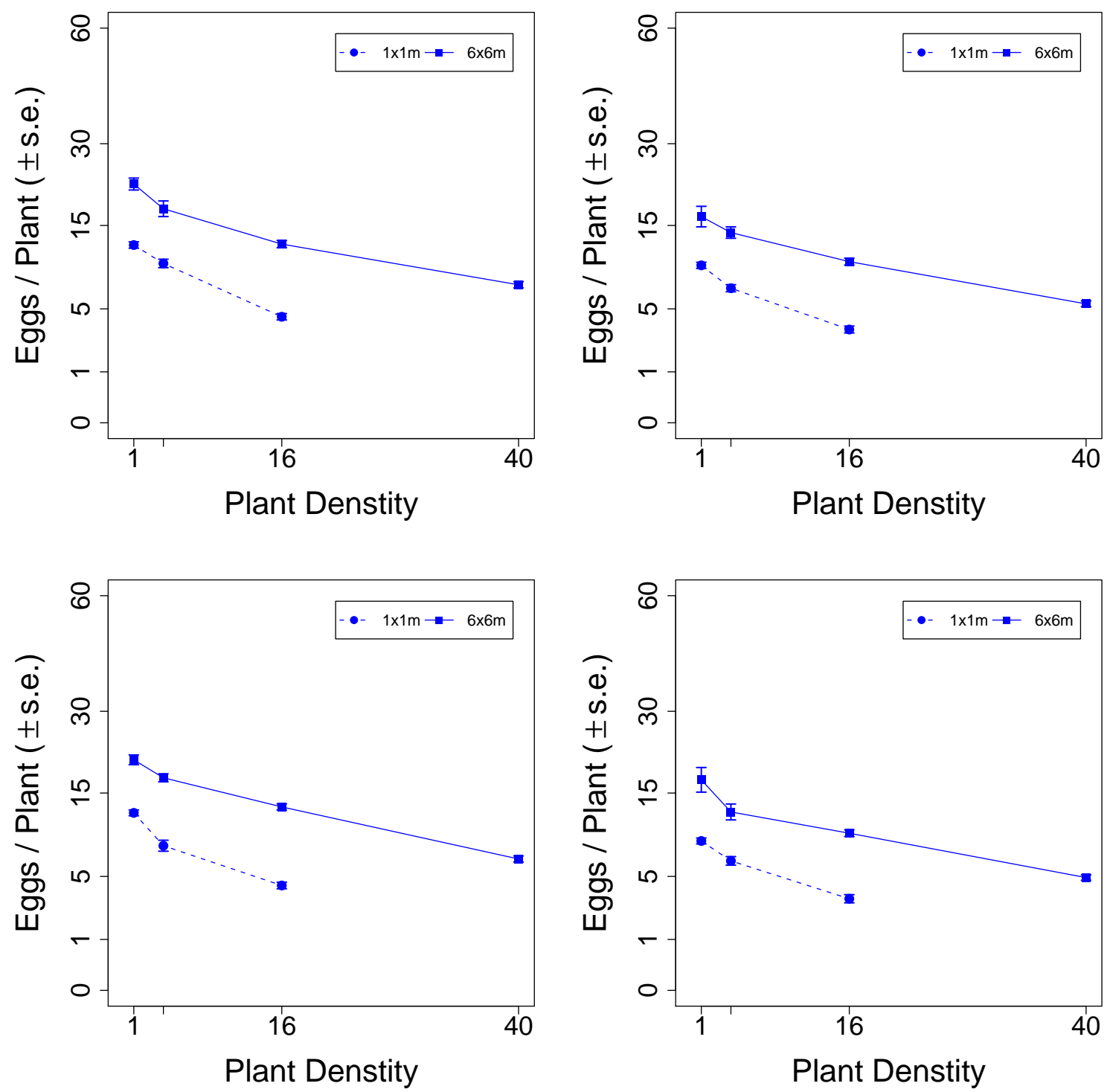

Figure 4.22: Field Breakdown - LEVIN-06-II - Trial C1 (CRW). Best fit $R=5, L=150$, $k=0.5$. Results from fields E, F, G and $H$ respectively, as in Figure 4.4. 
a) Image Map

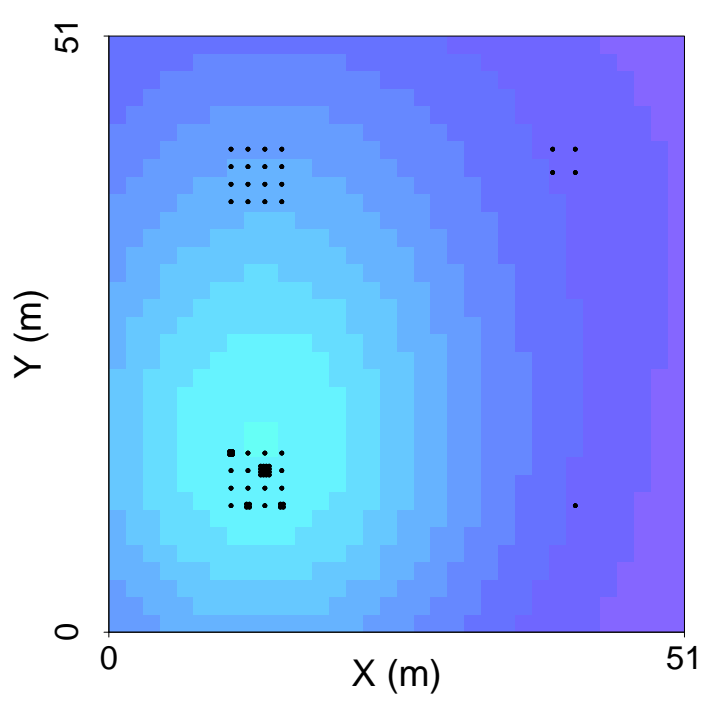

b) 3D Surface

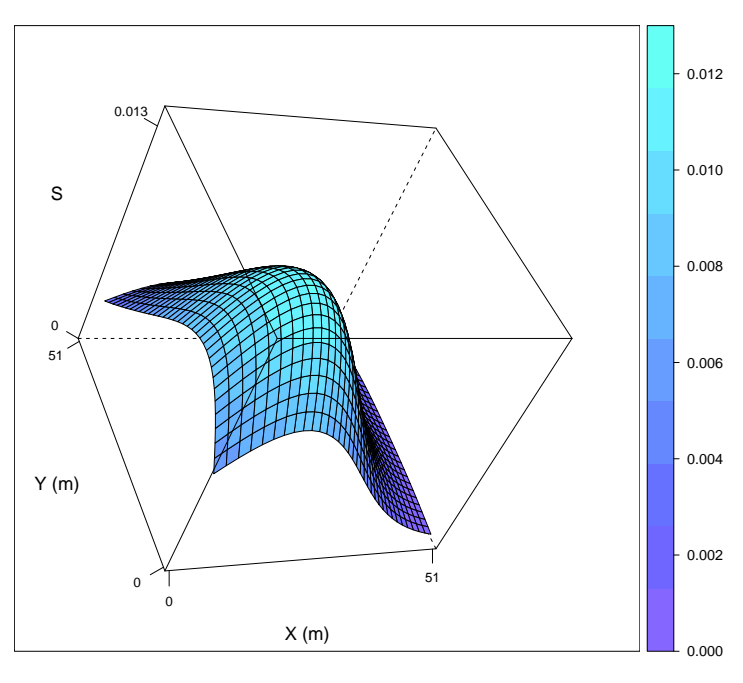

Figure 4.23: Olfaction Signal Surface - LEVIN-06-II (Field E), Treatment 1, SW=1500.

a) Image Map

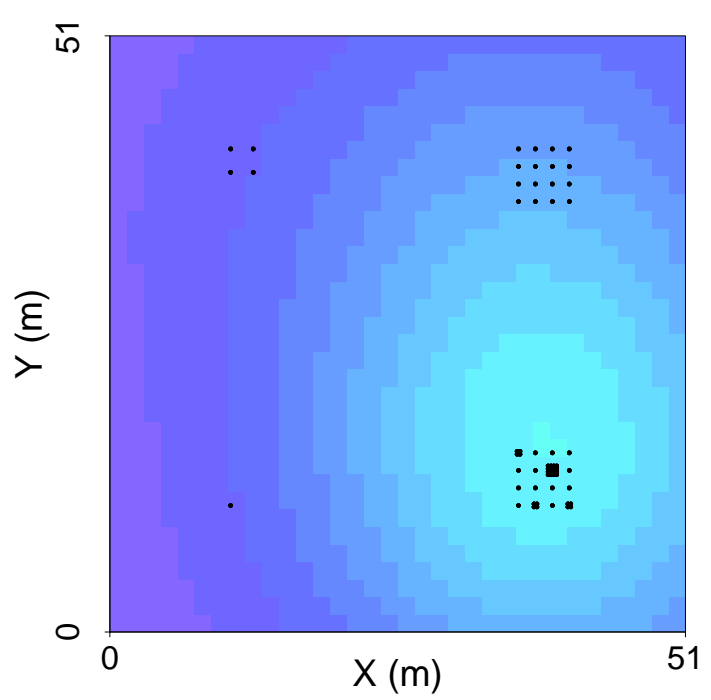

b) 3D Surface

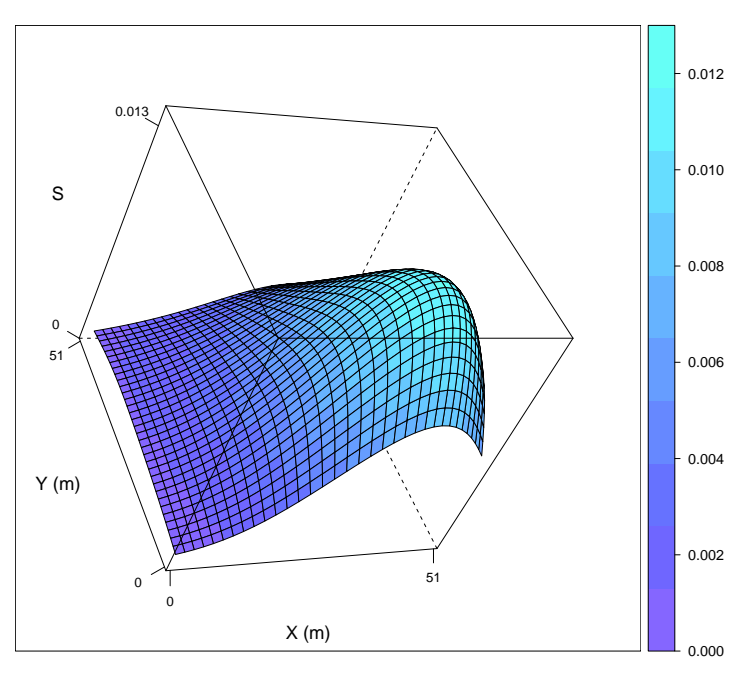

Figure 4.24: Olfaction Signal Surface - LEVIN-06-II (Field F). Treatment 40, SW=1500. 
a) Image Map

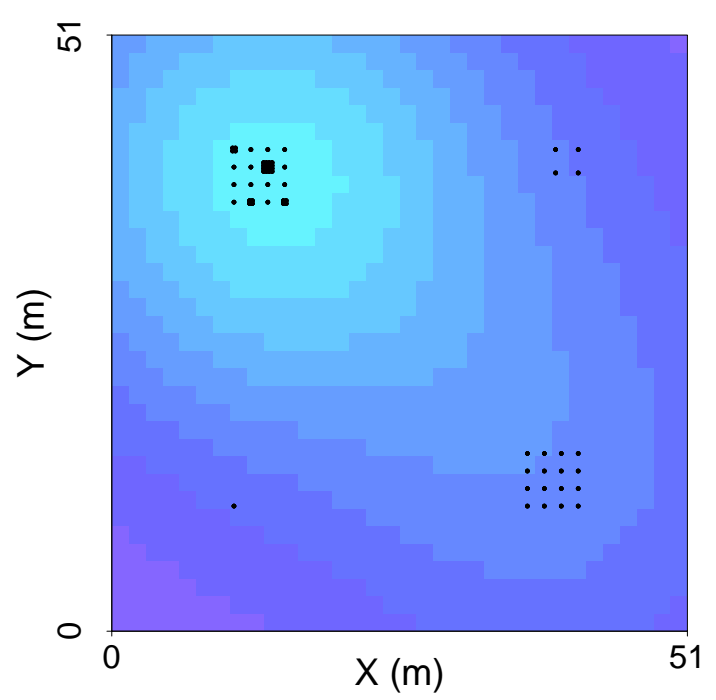

b) 3D Surface

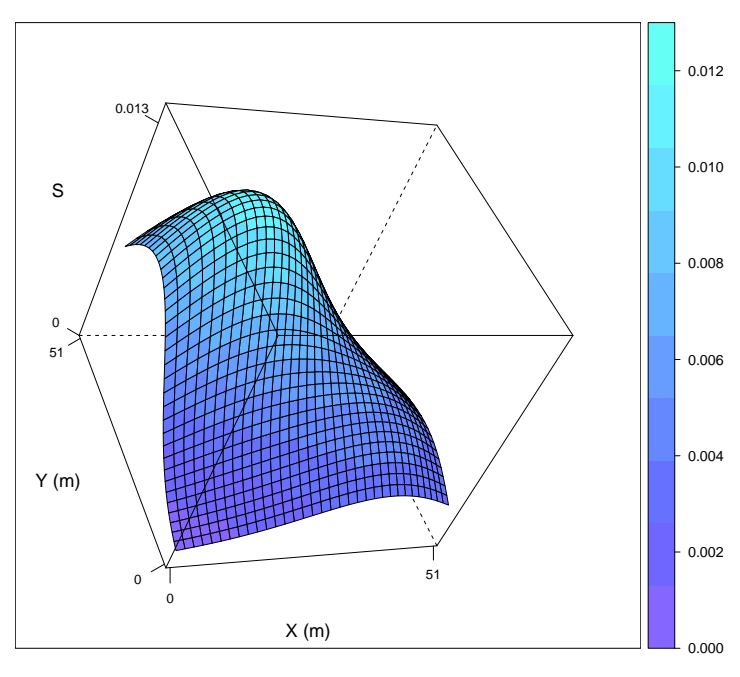

Figure 4.25: Olfaction Signal Surface - LEVIN-06-II (Field E). Treatment 60, SW=1500.

a) Image Map

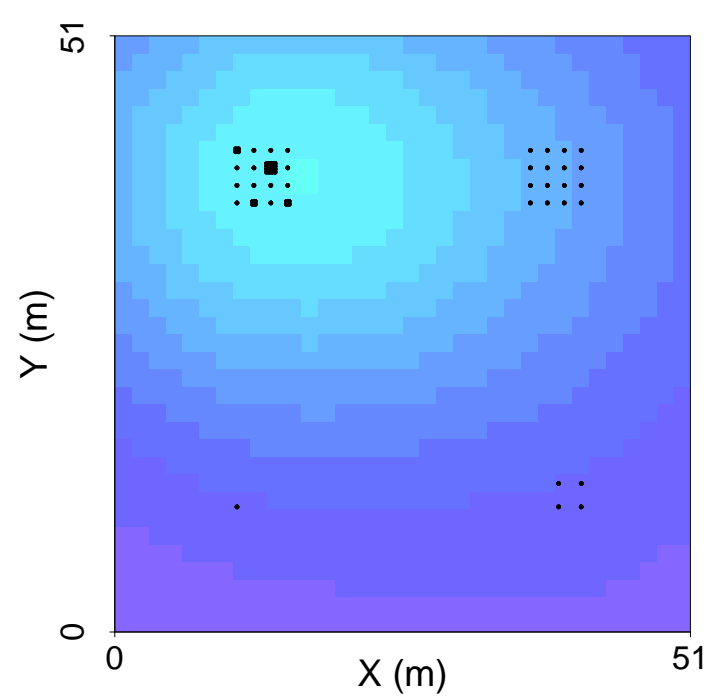

b) 3D Surface

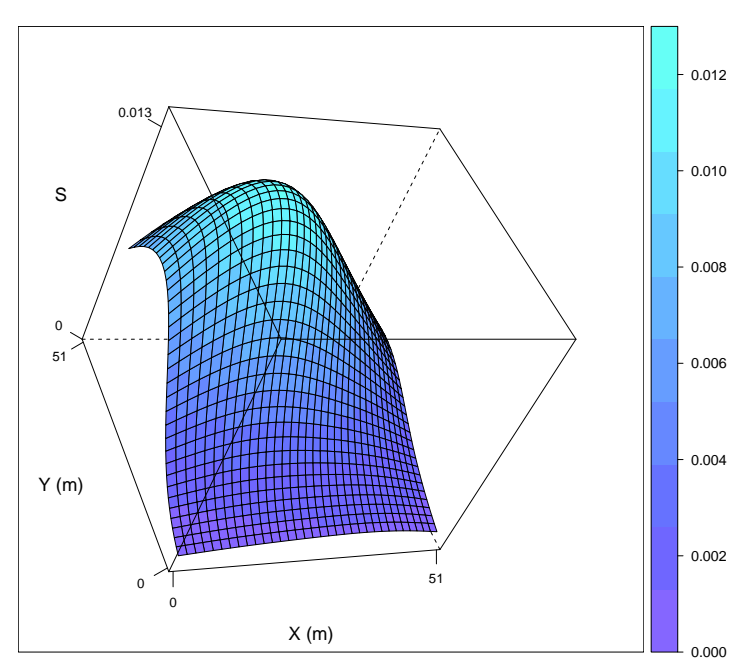

Figure 4.26: Olfaction Signal Surface - LEVIN-06-II (Field H). Treatment 100, SW=1500. 


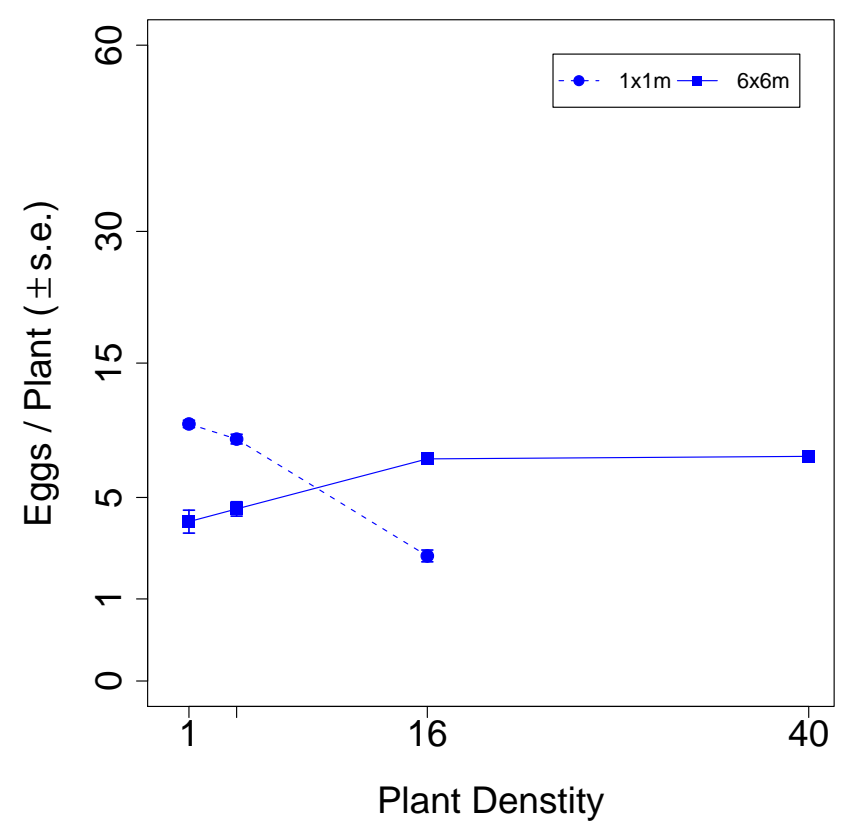

Figure 4.27: Plant Density - LEVIN-06-II Trial D1 (Olfaction, Single Egg). Opposite Response at Different Scales, $R=20, L=150, k=10$.

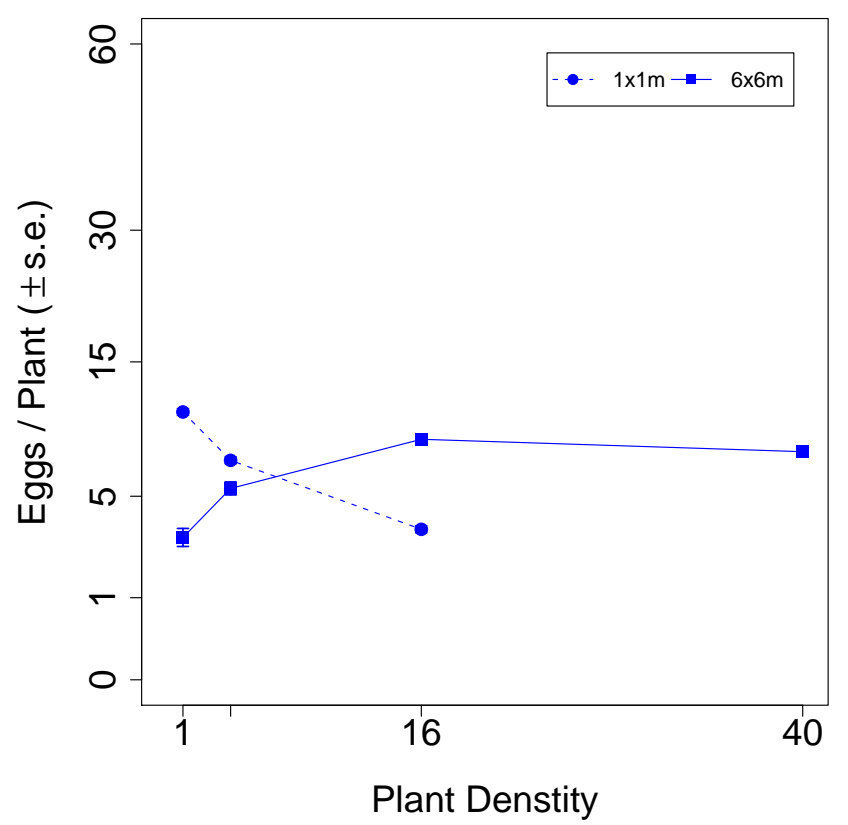

Figure 4.28: Plant Density - LEVIN-06-II Trial D6 (Olfaction and Vision, Multiple Eggs). Opposite Response at Different Scales, $F D=100, R=5, L=250, k=10$. 

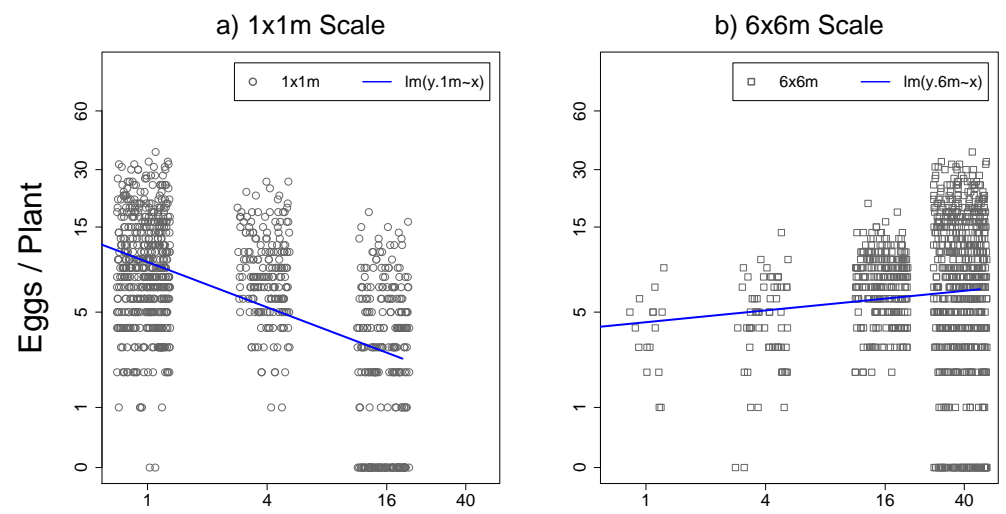

Plant Density

Figure 4.29: Log-Log Regression - LEVIN-06-II Trial D1 (Olfaction, Single Egg). $R=20$, $L=150, k=10$. For statistics see Table B.16.
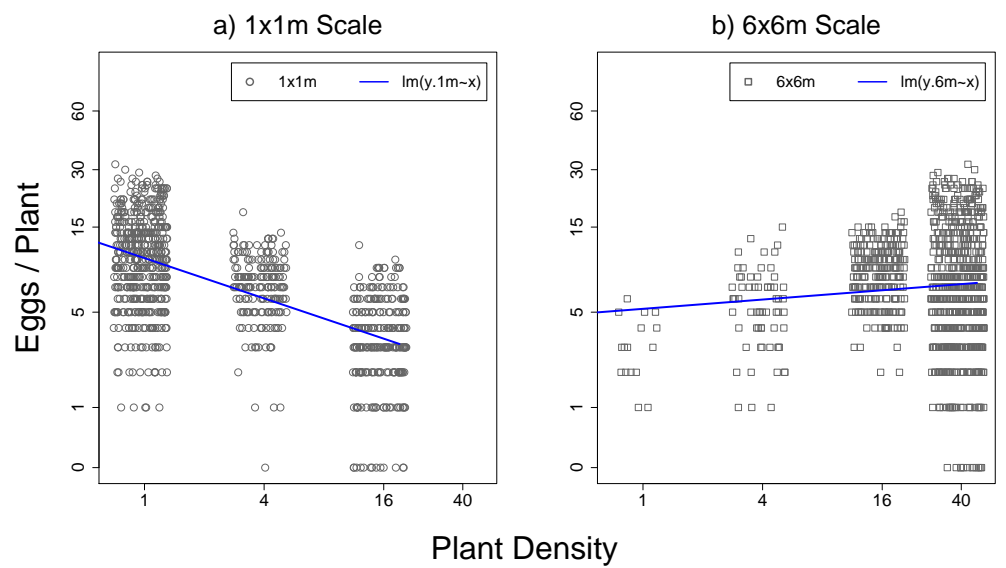

Figure 4.30: Log-Log Regression- LEVIN-06-II Trial D6 (Olfaction and Vision, Multiple Eggs). $F D=100, R=5, L=250, k=10$. For statistics see Table B.22. 


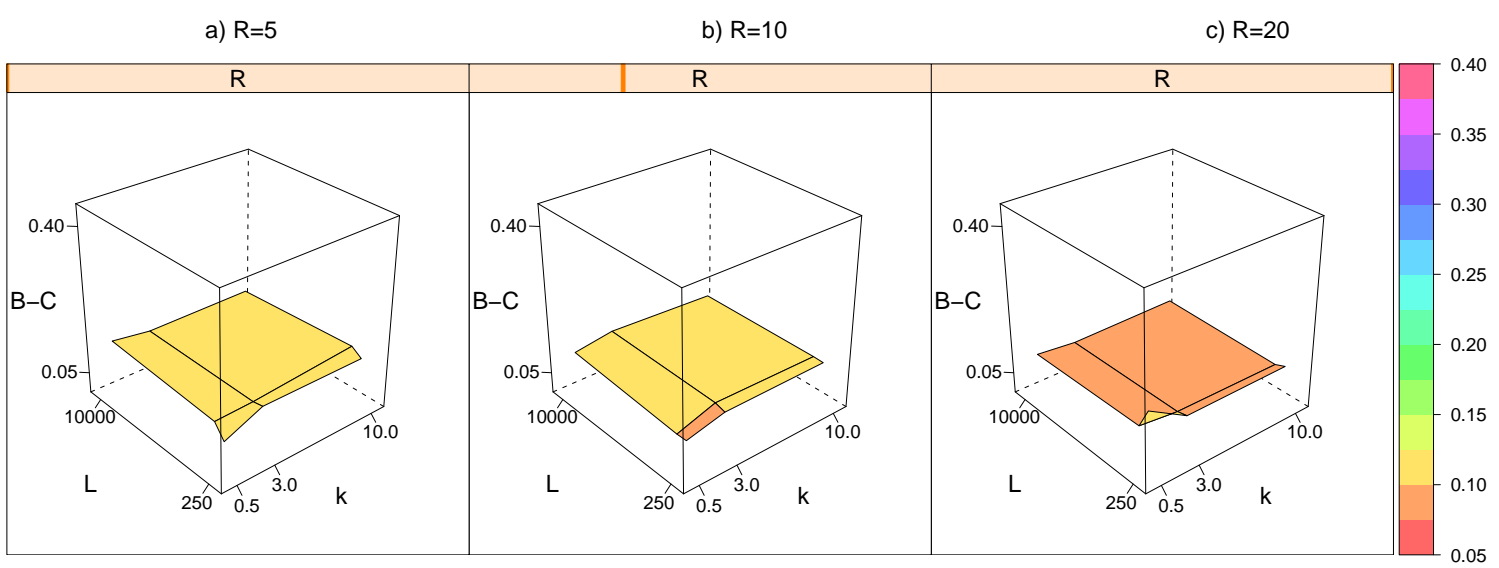

Figure 4.31: BC Response Surface - KAITOKE-04 - Trial C2 (CRW, Single Egg).

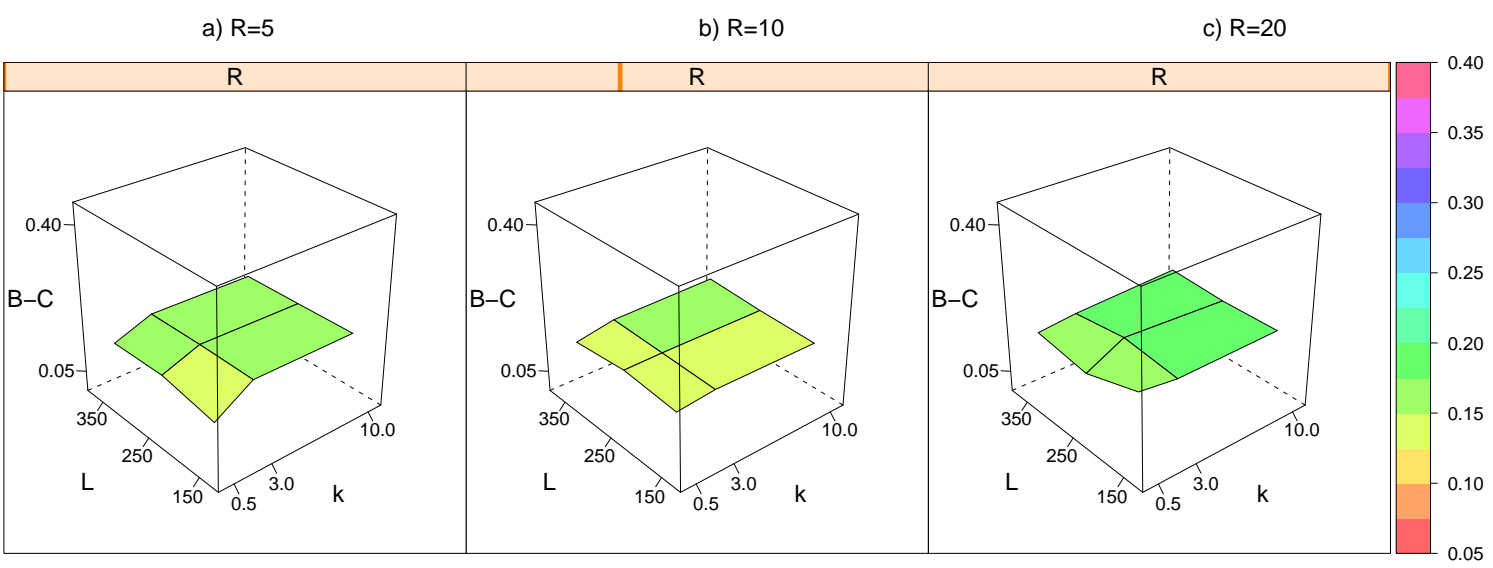

Figure 4.32: BC Response Surface - LEVIN-06-II - Trial C1 (CRW, Single Egg).

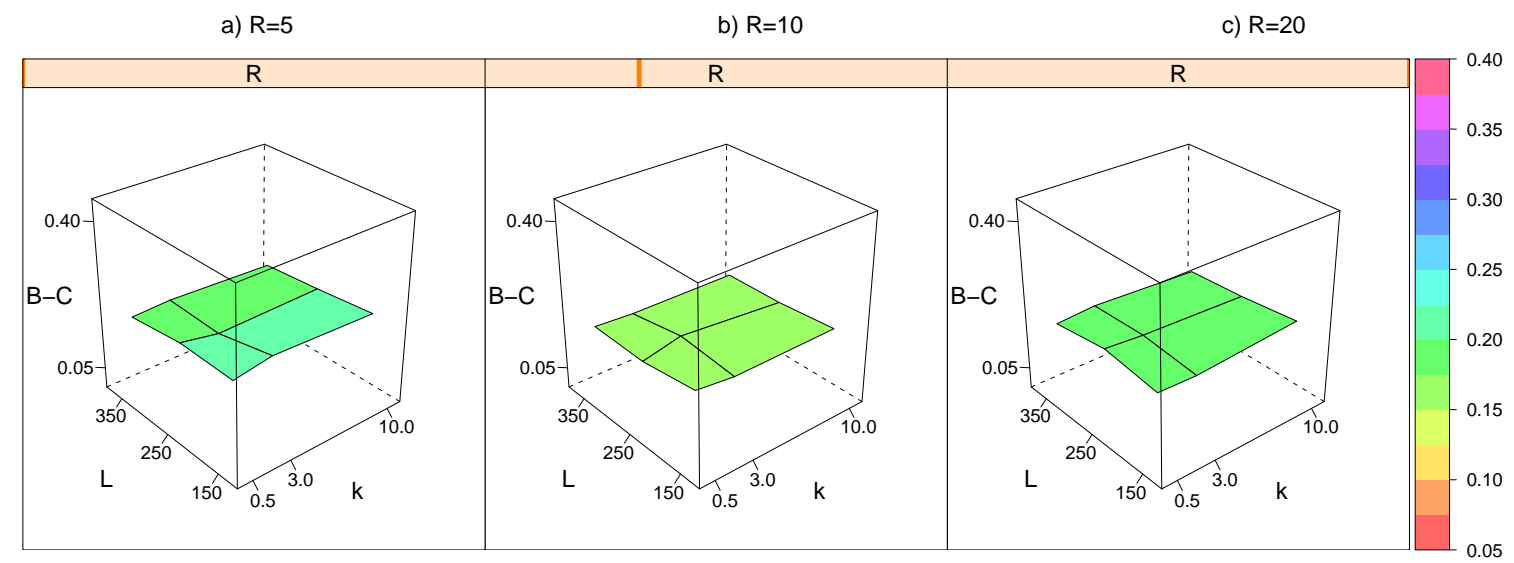

Figure 4.33: BC Response Surface - LEVIN-06-II - Trial C3 (CRW, Multiple Eggs). 


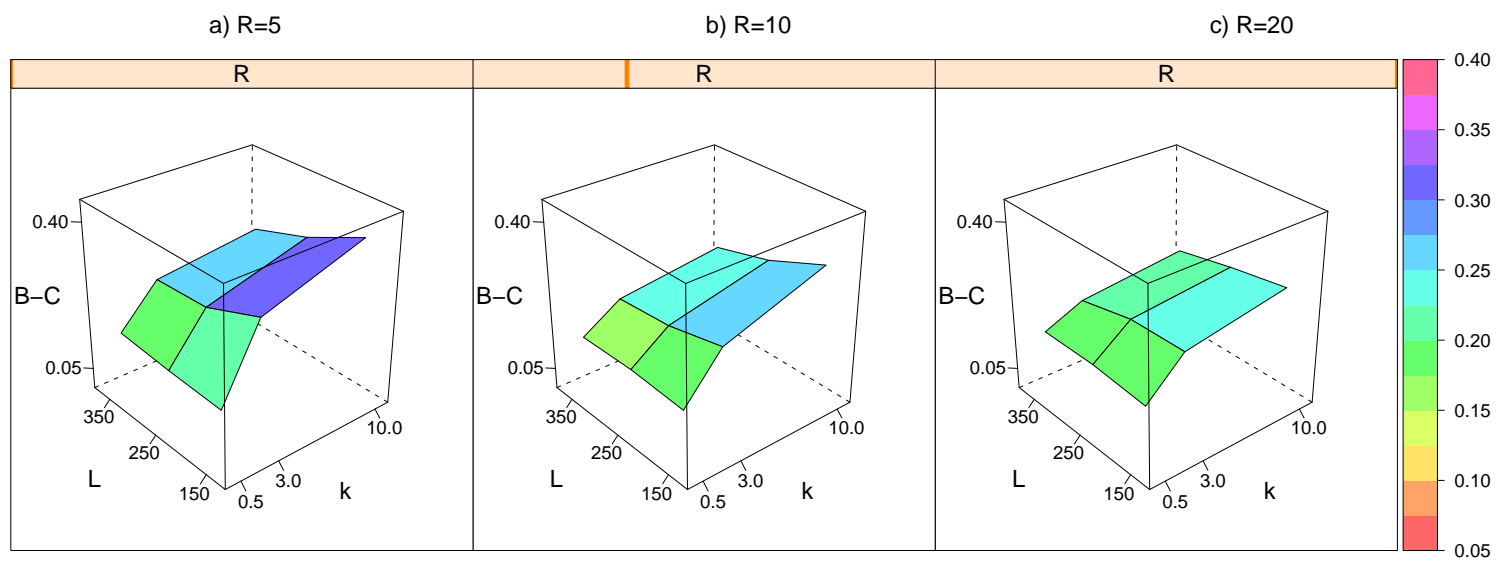

Figure 4.34: BC Response Surface - LEVIN-06-II - Trial D1 (Olfaction, Single Egg).

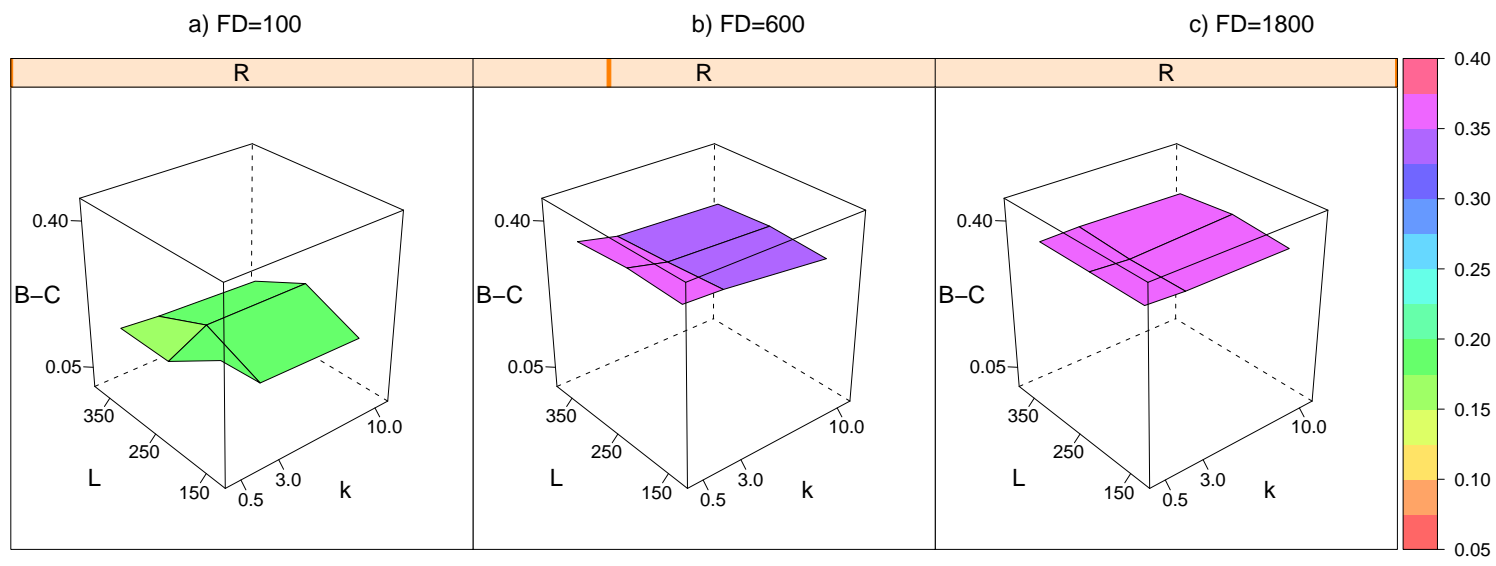

Figure 4.35: BC Response Surface - LEVIN-06-II - Trial D3 (Vision, Single Egg).

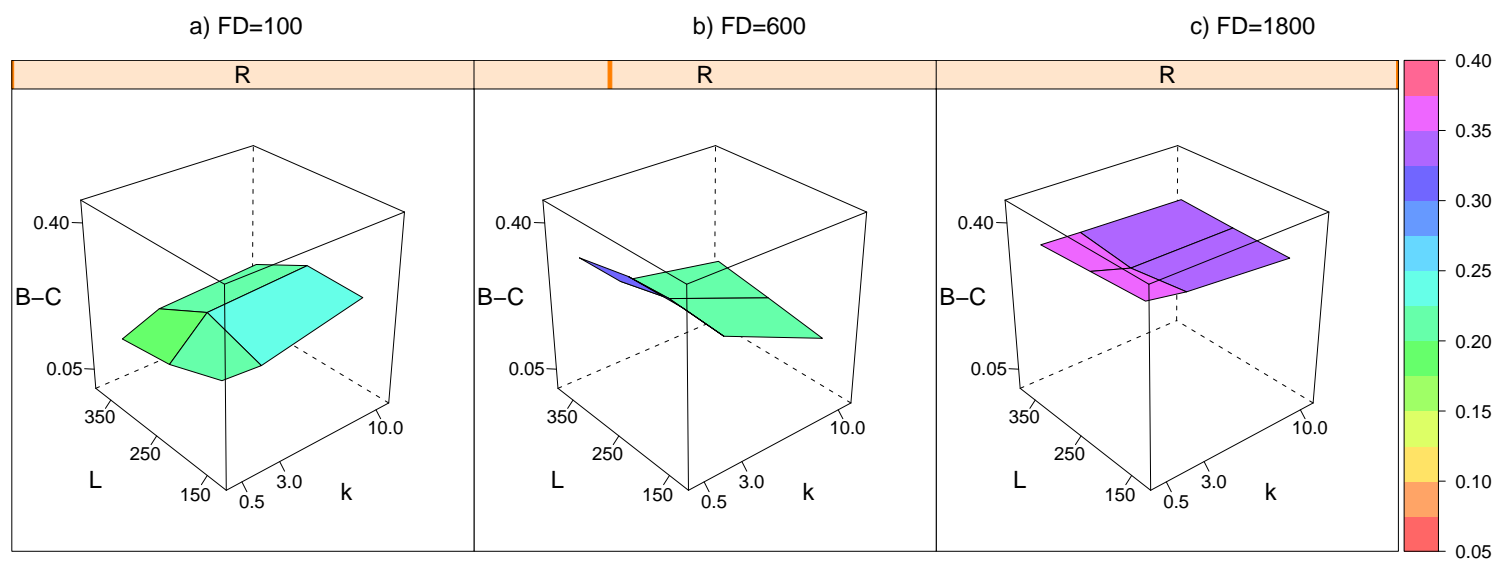

Figure 4.36: BC Response Surface - LEVIN-06-II - Trial D2 (Olfaction and Vision, Single Egg). 


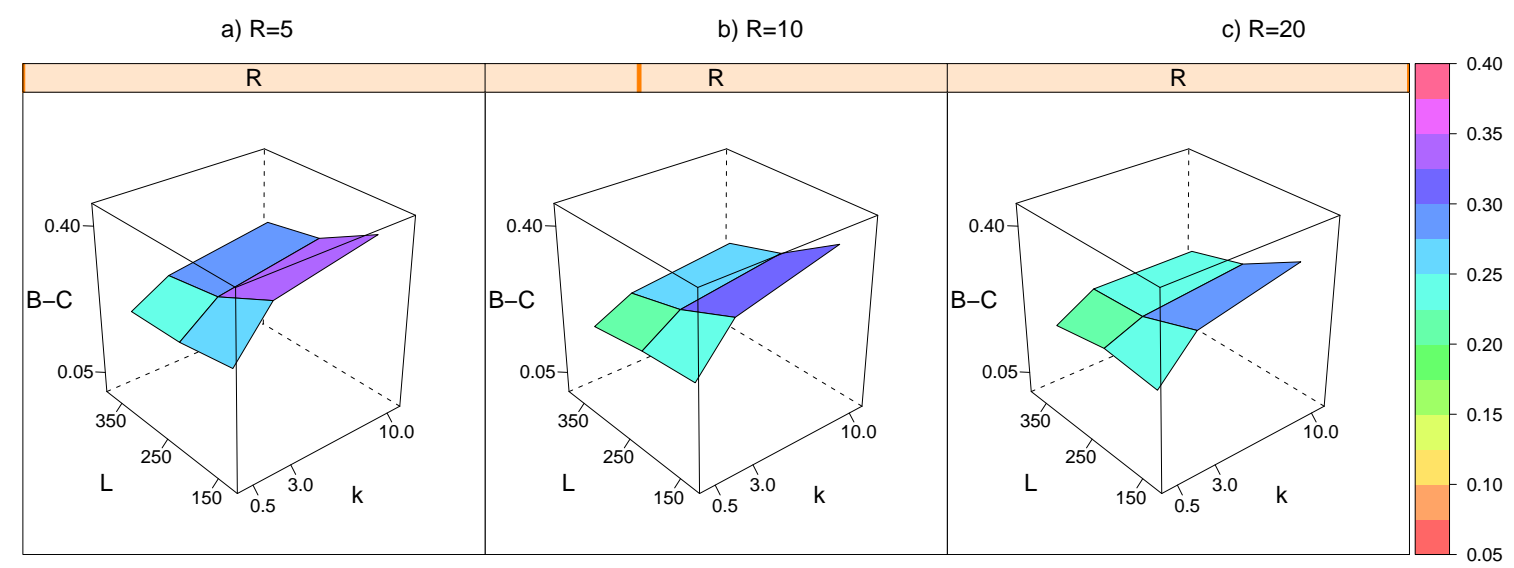

Figure 4.37: BC Response Surface - LEVIN-06-II - Trial D4 (Olfaction, Multiple Eggs).

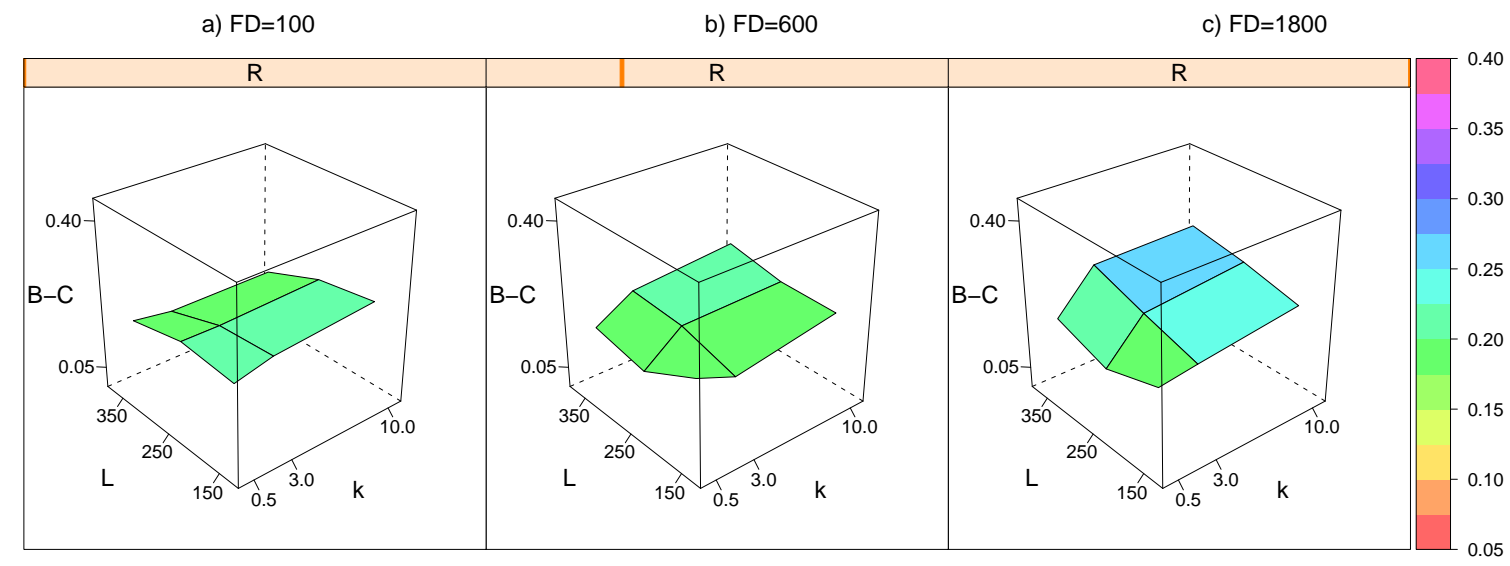

Figure 4.38: BC Response Surface - LEVIN-06-II - Trial D5 (Vision, Multiple Eggs).

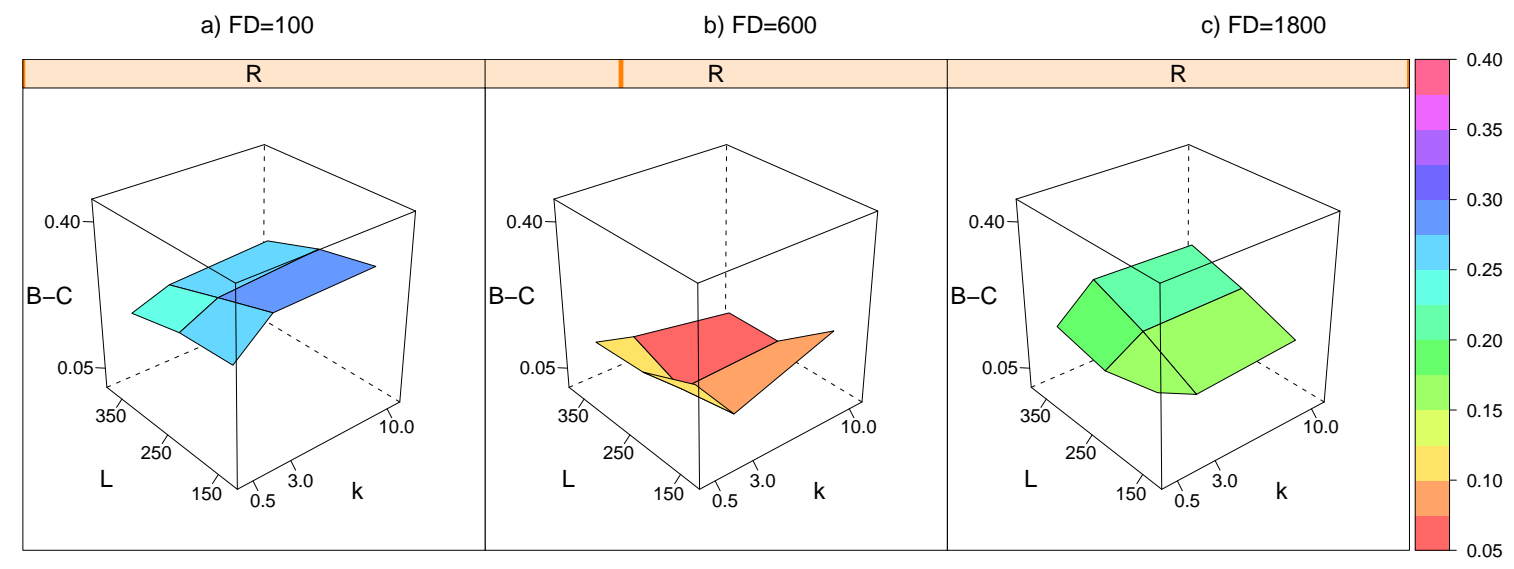

Figure 4.39: BC Response Surface - LEVIN-06-II - Trial D6 (Olfaction and Vision, Multiple Eggs). 


\subsection{Discussion}

This chapter utilised the simulation framework described in Chapter 3 to simulate egg distributions over resource layouts which were also used in field experiments involving P. rapae. These layouts were spatially complex, providing various aggregations of resources at different scales. The results were then compared to field data by use of the Bray-Curtis measure of similarity. The key parameters were systematically manipulated to explore the "parameter space" as effectively as computer processing time would allow. Pieris rapae butterflies are known to show a negative response to host plant density by virtue of their oviposition flight patterns. These involve directional movements, laying single eggs and being unresponsive to host plant density, i.e. they do not increase turning when in areas of high density, and pass over suitable hosts (Root and Kareiva, 1984; Hern et al., 1996). The result is that isolated plants receive more eggs per plant than denser patches. This behaviour is different to other butterflies and is potentially a means of "spreading the risk" or mediated by population factors such as density dependant larval mortality (Jones and Ives, 1979; Root and Kareiva, 1984).

The field experiments were designed to attempt to answer the question "Does the response to density vary with scale?" and so included different densities at various scales of measurement. The field data showed a negative response to density at all scales measured. The simulations were intended to explore this behaviour and seek potential parameter combinations which would alter this response. With a Correlated Random Walk, the response was invariably a negative "dilution" effect. With a visual search enabled, this was also the case, the visual search reducing the differences between the results if compared visually. The closest matches between field and simulation were found when the random walkers were following tortuous, area restricted search paths (shorter step lengths, low turning angle concentration), with one or multiple eggs per butterfly. This is because they are more effective in coming into contact with the isolated, low density plants. With vision enabled, more directional paths produced closer matches to the field data. In general the responses observed matched closely the field, at least when aggregate measures were compared.

With olfaction, an exciting result was produced where at the small scale $(1 \times 1 \mathrm{~m})$ a dilution effect was still observed, but at a larger scale $(6 \times 6 \mathrm{~m})$, a concentration of eggs was found on the denser patches. This occurred because the olfaction signal resulted in a bias toward the larger patch, but the signal was set to be widely dispersed and so within the larger patch there was little differentiation between areas, resulting in movement which returned to being random, and thus produced the dilution effect within the sub-patch. 
When both vision and olfaction are combined an interesting situation occurs, where both the best fit to the field data, and the result with the most extreme variance across scales was observed. This follows the observations of Kareiva and Shigesada (1983) that adding more parameters to a model will result in being able to create a better fit, but at a cost to analytical tractability.

Several observations from the literature were noted in Table 4.6. A reasonable approximation of known $P$. rapae flight would be a step length of $L=2-2.5 \mathrm{~m}$ and turning angle concentration of $k>1$. The closest simulation parameters to this that were explored are $L=250, k=3$. If the Bray Curtis metrics are compared for all behaviours at these particular values, it is found that Trial D6, the combination of vision and olfaction achieves the closest fit. Perhaps then this is the most "realistic" of the behavioural mechanisms implemented. Bearing in mind the observation that more parameters produce a more flexible model, it is difficult to determine whether this best fit is because a realistic mechanistic model has been achieved, or because it has the most flexible response.

These simulation results agree with other models (Kareiva and Shigesada, 1983; Bukovinszky et al., 2005) in general terms. The concept of scale within the resource layout is novel, and provided a useful insight into both the effects of the experimental layout and the possible theoretical responses. It is possible that the scale at which the field experiments were conducted was too small to be able to observe this reversal of response between dilution and concentration. If the layout is scaled up so that each "plant" represents a patch of 20 plants, say and the entire area becomes a size of $1 \mathrm{~km}$ instead of $40 \mathrm{~m}$, would it be possible to observe a similar concentration effect in the field? From personal observations, a field with many cabbages generally had more butterflies in it than neighbouring fields with no cabbages, although this was not quantified.

The model of olfaction does not need to be a literal, mechanistic representation of olfaction, it could represent a more abstract, general attraction to a dense group of resources, whatever the underlying mechanism. Turchin (1989) suggests that this inclusion of attraction would be useful in more general population modelling. Working with individual, stochastic models having simple behaviours may lead towards making more general descriptions possible as has been achieved with Correlated Random Walks and diffusion models (Turchin, 1989).

\section{Summary}

The observed results in the field could be approximated with a simple correlated random walk, although the parameters that gave the best fit suggested a greater 
tortuosity than previous published observations of the flight of P. rapae. However, introducing either a visual sensory element and or allowing foragers to lay multiple eggs, resulted in more directional paths creating a good match. The introduction of an olfactory response led to an opposite response to density at different scales, a novel result which is believed not to be reported elsewhere. The combination of both visual and olfactory senses produce a more flexible response and a closer fit to the data, as well as producing the most extreme variation across scales. "olfaction" in this model can be regarded as any general, density dependant attraction of the foragers to host plants. 


\section{References}

Banks, J. E., 1998: The scale of landscape fragmentation affects herbivore response to vegetation heterogeneity. Oecologia, 117(1-2), 239-246.

Batschelet, E., 1981: Circular Statistics In Biology. Mathematics in Biology. Academic Press, San Fransisco.

Bell, W., 1991: Searching Behaviour: The behavioural ecology of finding resources. Chapman and Hall, London.

Bender, D. J., L. Tischendorf, and L. Fahrig, 2003: Using patch isolation metrics to predict animal movement in binary landscapes. Landscape Ecology, 18(1), 17-39.

Bukovinszky, T., R. P. J. Potting, Y. Clough, J. C. van Lenteren, and L. E. M. Vet, 2005: The role of preand post-alighting detection mechanisms in the responses to patch size by specialist herbivores. Journal of Chemical Ecology, 109(3), 435-446.

Byers, J. A., 2001: Correlated random walk equations of animal dispersal resolved by simulation. Ecology, 82(6), 1680-1690.

Cain, M. L., 1985: Random search by herbivorous insects - a simulation-model. Ecology, 66(3), 876888.

Cressman, R., V. Krivan, and J. Garay, 2004: Ideal free distributions, evolutionary games, and population dynamics in multiple-species environments. American Naturalist, 164(4), 473-489.

Farnsworth, K. D. and J. A. Beecham, 1999: How do grazers achieve their distribution? a continuum of models from random diffusion to the ideal free distribution using biased random walks. American Naturalist, 153(5), 509-526.

Hasenbank, M., in preparation: Phd thesis. Victoria University (Wellington, New Zealand).

Hern, A., G. EdwardsJones, and R. G. McKinlay, 1996: A review of the pre-oviposition behaviour of the small cabbage white butterfly, Pieris rapae (Lepidoptera: Pieridae). Annals of Applied Biology, 128(2), 349-371.

Jones, R. and P. Ives, 1979: The adaptiveness of searching and host selection behaviour in Pieris rapae (L.). Australian Journal of Ecology, 4, 75-86.

Jones, R. E., 1977: Search behavior - study of 3 caterpillar species. Behaviour, 60, 236-259.

Jones, R. E., 1987: Behavioral evolution in the cabbage butterfly (Pieris rapae). Oecologia, 72(1), 69-76.

Jones, R. E., N. Gilbert, M. Guppy, and V. Nealis, 1980: Long-distance movement of Pieris rapae. Journal Of Animal Ecology, 49(2), 629-642.

Kareiva, P. M. and N. Shigesada, 1983: Analyzing insect movement as a correlated random-walk. Oecologia, 56(2-3), 234-238.

Levin, S., 1992: The problem of pattern and scale in ecology. Ecology, 73 (6), 1943-1967.

Matter, S. F., T. Roslin, and J. Roland, 2005: Predicting immigration of two species in contrasting landscapes: effects of scale, patch size and isolation. Oikos, 111(2), 359-367.

Minnich, D. E., 1924: The olfactory sense of the cabbage butterfly, Pieris rapae linn., an experimental study. Journal Of Experimental Zoology, 39(2), 339-356. 
Quinn, G. and M. Keough, 2002: Experimental Design and Data Analysis for biologists. Cambridge University Press, Cambirdge.

Renwick, J. A. A. and C. D. Radke, 1988: Sensory cues in host selection for oviposition by the cabbage butterfly, Pieris rapae. Journal of Insect Physiology, 34(3), 251-257.

Root, R. B., 1973: Organization of a plant-arthropod association in simple and diverse habitats: The fauna of collards (Brassica oleracea). Ecological Monographs, 43(1), 95-124.

Root, R. B. and P. M. Kareiva, 1984: The search for resources by cabbage butterflies (Pieris rapae): ecological consequences and adaptive significance of markovian movements in a patchy environment. Ecology, 65(1), 147-165.

Swingland, I. and Greenwood, P., editors, 1984: The Ecology Of Animal Movement. Clarendon Press, Oxford.

Tischendorf, L., D. J. Bender, and L. Fahrig, 2003: Evaluation of patch isolation metrics in mosaic landscapes for specialist vs. generalist dispersers. Landscape Ecology, 18(1), 41-50.

Turchin, P., 1989: Beyond simple diffusion: Models of not-so-simple movement of animals and cells. Comments on Theoretical Biology, 1, 65-83.

Turchin, P., 1998: Quantitative Analysis of Movement. Sinauer Associates, USA.

Turchin, P., F. Odendaal, and M. D. Rausher, 1991: Quantifying insect movement in the field. Environmental Entomology, 20, 955-963.

Wiens, J. A., 1989: Spatial scaling in ecology. Functional Ecology, 3(4), 385-397.

With, K. A. and T. O. Crist, 1996: Translating across scales: Simulating species distributions as the aggregate response of individuals to heterogeneity. Ecological Modelling, 93(1-3), 125-137.

Yamamura, K., 1999: Relation between plant density and arthropod density in cabbage. Researches on Population Ecology, 41(2), 177-182. 


\section{Chapter 5}

\section{A Simulation Framework For Information Based Random Walks: General Discussion}

This thesis has brought together techniques of analysing and modelling insect movement to create a simulation framework, that lays the foundations of what is a general tool for implementing movement simulations. Some elements of the framework are more general still and could be applied to any simulation which has a set of parameters which must be explored. It has been applied to a specific case of simulating the egg distributions of $P$. rapae over multiple scales.

\footnotetext{
"Essentially, all models are wrong, but some are useful." (Box and Draper, 1987) is an oft cited quote by George E. Box, commenting on goodness of fit to statistical models. It is equally applicable in this case. It is known that neither the random walk, nor this particular implementation of the sensory response is mechanistically accurate in representing the real world. It can, though, be useful to provide insights as to what kinds of pattern may be observed in the field, helping to improve intuition for understanding observed data and for testing experimental layouts against various "null" hypotheses.
}

The intention of introducing some response to information was therefore not to mechanistically re-create the function of vision and olfaction but rather to create a model which with as few parameters as possible could represent the kind of interactions thought to occur. Hern et al. (1996) provides a good review of knowledge about the sensory abilities of $P$. rapae. 


\section{Main Findings}

In Chapter 2, a selection of the literature concerning simulation of Pieris rapae oviposition was reviewed. Pieris rapae is a mainly visual forager, tending to lay more of its eggs on isolated plants. This pattern is essentially a product of the fact that it does not appear to alter its behaviour significantly in response to resource density and thus a "resource dilution" effect is observed in areas of high host plant density. Its displacement over time has been shown to be approximated by the predictions of a correlated random walk (CRW) Model (Kareiva and Shigesada, 1983).

In Chapter 3, a simulation framework was introduced which allows the exploration of this behaviour for any specified resource layout. The framework incorporates a model of the CRW and two representations of sensory mechanisms, "visual" and "olfactory". The framework also provides a set of general tools for manipulation of parameters and reporting of results in a controlled manner.

It was discovered in some situations that the release distance of foraging agents from the resources can influence the response. More research into this effect would be interesting.

A demonstration of the interaction between spatial arrangement of resources and movement patterns was presented, in terms of a shielding, or "edge effect". Outer resources tend to intercept incoming foragers and thus receive higher relative egg loads than those in the centre. The strength of the "edge effect" depended upon the details of the resource layout and the parameters of the movement behaviour. For example, an intermediate sinuosity was found to be the most successful in reaching centre plants for a particular resource layout. When intermediate strategies are "optimal", this can lead to a stabilising selection pressure on oviposition behaviour, adapted to particular environments of resource density and distribution (Jones and Ives, 1979).

Chapter 4 reports results from several simulation experiments which were conducted using the simulation framework. The subject of these experiments were resource layouts which were used in companion field studies conducted by (Hasenbank, unpublished data).

A reasonable approximation to the field results was possible with a correlated random walk model, simulating a negative response of eggs per plant to resource density. This supports evidence published in the literature concerning the lack of klinokinetic response of $P$. rapae to host plant density (Kareiva and Shigesada, 1983; Yamamura, 1999). The parameter space of the simulation was large and a more thorough exploration remains for future work. More detailed knowledge of the mechanistic perceptual abilities of $P$. rapae, particularly in terms of its visual range 
would be beneficial in determining a "realistic" range of parameters to explore.

An exciting discovery was that when a model of olfaction was introduced, both a negative and positive response to plant density can be observed simultaneously, but at different scales of measurement. This supports the growing body of work highlighting the importance of scale in the interpretation of ecological phenomena (Levin, 1992). It also provided an interesting conceptual demonstration of the factors which may lead to such a response, even though it was not observed in the field at the scales measured. This demonstrates the utility of such modelling exercises as conceptual tools to aid researchers attempting to understand complex ecological processes.

\section{Alternative Applications}

The simulation framework, at the end of this project, with no further refinements provides a useful tool for subsequent research. In fact, it has already been utilised in a different context, to aid interpretation of the effectiveness of a baiting regime for sampling ant populations (Hartley, unpublished data).

Subsequent researchers conducting similar experiments involving spatial patterns of resources could use the framework to explore the design of their particular experimental layouts in relation to both random walks and to incorporating a concept of a response to resource density.

Theoretical experimentation would be possible, exploring the consequences of isolated fragments of resources in a heterogeneous landscape. The importance of these isolated fragments as "stepping-stones" or "honeypot traps" for foragers moving across a landscape could be explored.

The concept of random walkers interacting with resources may have novel applications beyond the system of insect foragers. For example it was suggested by a colleague (Dr. K.C. Burns) that the "resources" could also be used as a model representing sessile feeders, and that the agents moving at random would be the food particles moving around them. The question in this case would be: What spatial arrangements of the sessile feeders would be optimum for given movement parameters of the food?

\section{Future Directions}

Time constraints meant that several areas of interest had to be abandoned in order to complete the main themes. Possibly the main thread would be a comparative analysis with other models available, particularly that of Jones (1977). It would be 
entirely possible to represent the structure of these models within the framework, and thus compare the output over the same resource layouts.

One future "direction" would be to introduce a concept of "drift" into the random walk model, choosing a direction for each step based upon an absolute azimuth, rather than left and right turning from the previous direction. This would lead to a more directional flight path than that produced by the CRW, and would accord with results from the observations and model of Jones (1977) and Jones et al. (1980), reporting highly directional paths for Australian butterfly populations.

Pieris rapae butterflies are observed to sometimes return to a plant after flying away from it (pers observation and Jones, 1977). This kind of behaviour can be approximated by setting a low turning angle concentration to the random walk in the current model $(k=0.5)$. This has the side affect of decreasing the overall directionality and area covered, which may not be as realistic given that a high degree of directionality has been recorded. A behaviour which allows foragers to return to a plant once visited might produce more accurate results. Possibly, as discussed by Hern et al. (1996), this could even involve a "memory" mechanism. This behaviour has an equivalent in the ZERO parameter of Jones (1977)'s model.

The topic of fractals, specifically the Lévy flight was briefly covered in Chapter 2and Appendix $C$. This area may provide some interesting results and it would have been interesting to build the mechanism into the framework. Both animal movement paths and resource distributions can be generated using fractal methods and the interaction of the two might be fruitful in terms of identifying scales of interaction. Roitberg (1985) presented a type of simulated foraging strategy involving straight lines followed by area restricted searching. Schtickzelle et al. (2007) have published observations of the bog fritillary butterfly Proclossiana eunomia, exhibiting exactly this kind of movement pattern. The resulting path might look similar to a Lévy flight and therefore be analysed as such, even though it is generated with a behavioural model that includes a response to the resources.

Hern et al. (1996) suggests a simple "rule of thumb" for modelling P. rapae movement involving the "motivational state" of a butterfly controlling it's discrimination. This concept was implemented and discussed at various points during the work of the thesis but was never formally tested. In relation to this, as discussed in Chapter 2, Mangel (1987) suggested that $P$. rapae might vary their clutch size in response to plant acceptability or density, or current physiological state (heavy egg load results in greater egg laying rate). This behaviour would be straightforward to include in the model. As a point of caution, it is worth remembering that adding more parameters might produce a more flexible response (and therefore more accurate match to the field), at the cost of obfuscating the general principals at work (Levin, 1992). 
Whilst the simulation produced reasonably accurate comparisons to the field observations when compared at the aggregated level, there was much less variation between fields (and replicates) in the simulation data. It suggests that, as Levin (1992) notes, the model is good at representing the pattern generated from the aggregation of many individuals but, as it is not a completely accurate mechanistic representation of the individuals, the variation is not described well.

Finally, it would be wonderful to extend the entire framework into three dimensional space, and thus afford modelling of air-borne or water-borne movement, and even perhaps more complex modelling of the true flight of $P$. rapae taking into account the fact that being farther from the ground may allow a greater range of visual perception.

\section{Conclusion}

This work has been useful in conceptualising and understanding the field observations discussed here. It is hoped that it will contribute to the body of work surrounding random walks and animal movement simulation. The results support existing studies suggesting that the oviposition behaviour of Pieris rapae can be represented to some degree by a correlated random walk model. A method for incorporating responses to information into random walk models has been constructed and explored, demonstrating a novel, opposite response to host plant density simultaneously observed at multiple spatial scales. Finally, the idea that a consistent framework allows a comparative execution of a variety of different model structures from previous workers is introduced. For example, the simulation experiments constructed were similar to those of Cain (1985), a more exact reproduction would be straightforward.

The ecological consequences of animal movement are undoubtedly significant and, as remote monitoring technology becomes increasingly available more field ecologists are likely to become interested in the mathematical tools available. With this in mind, any advances toward simplifying and standardising these modelling tools is an advantage. The process of synthesising the wide ranging literature on the subject of animal and insect foraging has proven an excellent introduction to the field. Once everything has been brought together and consolidated as a thesis, it forms a first step into the world of modelling animal movement. It will hopefully be of interest and practical use to those following the "path". Of course many more questions have arisen than have been answered, but hopefully the reader was kept interested throughout. Thank you for reading! 


\section{References}

Box, G. and N. Draper, 1987: "Essentially, all models are wrong, but some are useful.". In Empirical Model-Building and Response Surfaces. Wiley and Sons, New York, 424.

Cain, M. L., 1985: Random search by herbivorous insects - a simulation-model. Ecology, 66(3), 876888.

Hartley, S. Ant baiting regimes for a study at airports. unpublished data.

Hasenbank, M., in preparation: Phd thesis. Victoria University (Wellington, New Zealand).

Hern, A., G. EdwardsJones, and R. G. McKinlay, 1996: A review of the pre-oviposition behaviour of the small cabbage white butterfly, Pieris rapae (Lepidoptera: Pieridae). Annals of Applied Biology, 128(2), 349-371.

Jones, R. and P. Ives, 1979: The adaptiveness of searching and host selection behaviour in Pieris rapae (L.). Australian Journal of Ecology, 4, 75-86.

Jones, R. E., 1977: Movement patterns and egg distribution in cabbage butterflies. The Journal of Animal Ecology, 46(1), 195-212.

Jones, R. E., N. Gilbert, M. Guppy, and V. Nealis, 1980: Long-distance movement of Pieris rapae. Journal Of Animal Ecology, 49(2), 629-642.

Kareiva, P. M. and N. Shigesada, 1983: Analyzing insect movement as a correlated random-walk. Oecologia, 56(2-3), 234-238.

Levin, S., 1992: The problem of pattern and scale in ecology. Ecology, 73 (6), 1943-1967.

Mangel, M., 1987: Oviposition site selection and clutch size in insects. Journal Of Mathematical Biology, 25(1), 1-22.

Roitberg, B. D., 1985: Search dynamics in fruit-parasitic insects. Journal Of Insect Physiology, 31(11), 865-872.

Schtickzelle, N., A. Joiris, H. Van Dyck, and M. Baguette, 2007: Quantitative analysis of changes in movement behaviour within and outside habitat in a specialist butterfly. BMC Evolutionary Biology, $7: 4$.

Yamamura, K., 1999: Relation between plant density and arthropod density in cabbage. Researches on Population Ecology, 41(2), 177-182. 


\section{Appendix A}

\section{Edge Effect detailed simulation results}

This appendix presents detailed results from the edge effect experiment of Chapter 3. A consistent set of graphs was produced for each trial which combine to form the "response" measurement of the simulation experiment. Table A.1 lists the graphs found in each section. Each figure is labelled with the section of the appendix followed by the identifying number from this table, e.g. Figure A.1-1 is the first figure for the first trial. There are 10 figures for each trial.

Each trial conducted was run once with a single egg and once with multiple eggs $(E=10)$ and so there are sections for both for each trial in the following appendix. By presenting all the results here in a consistent format it is hoped that it will be easier to refer to and make comparison between the various behaviours such as olfaction vs vision, etc.

The two response variables are generally the centre ratio (proportion of eggs per plant laid in the centre resources) and the search efficiency (proportion of eggs laid to number of foragers). See Chapter 3 for details of these response variables. 


\begin{tabular}{lll}
\hline$\#$ & Graph title & Description \\
\hline 1 & Centre ratio summary & $\begin{array}{l}\text { Shows the response of the centre ratio plotted against the } \\
\text { relative resource spacing }\left(\mathbf{I}_{\mathbf{R}}\right) \text { which is a measure of the } \\
\text { spacing between resources relative to the patch size and } \\
\text { radius of attraction (Chapter 3) }\end{array}$
\end{tabular}

2 Centre ratio vs. $\mathbf{R}$ and $\mathbf{P}$ The centre ratio plotted against both the radius of attraction $(R)$ in graph a) and the patch size $(P)$ in graph b). These two parameters are the factors which determine and are therefore summarised by the relative resource spacing $\left(\mathbf{I}_{\mathbf{R}}\right)$.

3 Efficiency summary

Shows the response of the search efficiency of the foragers against the relative area $\left(\mathbf{R}_{\mathbf{P}}\right)$ which is a measure of the area of the radii of attraction $(R)$ relative to the patch size $(P)$.

$4 \quad$ Efficiency vs. $R$ and $P$

As with the centre ratio graphs, these graphs show search efficiency plotted against the component factors of $R$ and $P$ separately as opposed to the summary measure of relative area $\left(\mathbf{R}_{\mathbf{P}}\right)$

5 Centre ratio vs. sinuosity The centre ratio is plotted against the sinuosity $(S *)$ of the paths which is an aggregated measure including both step length $(L)$ and turning angle concentration $(k)$.

6 Efficiency vs. sinuosity

The search efficiency plotted against sinuosity $(S *)$.

7 Centre ratio vs. $\mathrm{L}$ and $\mathrm{k}$

Sinuosity represents a combination of both step length $(L)$ and turning angle concentration $(k)$. These plots show the centre ratio plotted against each factor.

8 Efficiency vs. L and $\mathrm{k}$

As with the centre ratio, these graphs show the search efficiency plotted against both $L$ and $k$

9 Centre ratio vs. layout

These figures contain 9 sub-figures which represent the different patch size and radius of attraction parameters of the experimental layouts (Figure 3.32). Each sub graph plots centre ratio against sinuosity $(S *)$ for each combination of $R$ and $P$.

10 Efficiency vs. layout

These figures contain 9 sub-figures, each plotting search efficiency against sinuosity $(S *)$ for each combination of $R$ and $P$.

Table A.1: Details of the graphs found for each trial in the results appendix. 


\section{A.1 Trial B (CRW) Single Egg results}

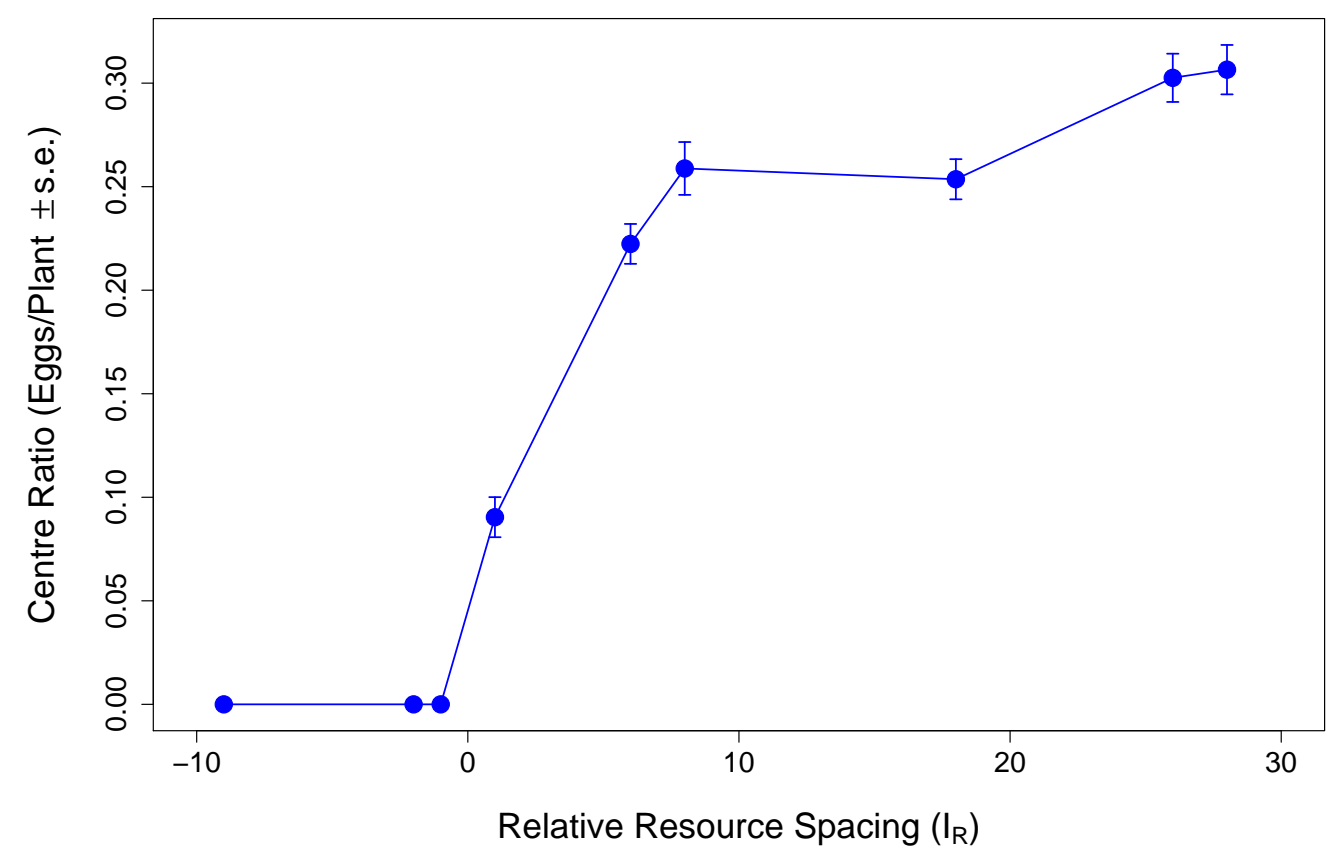

Figure A.1-1: Centre ratio summary - Trial B (CRW), Single Egg. Proportion of eggs per plant on centre resources ( $y$-axis) vs. relative resource spacing, $I_{R}$ ( $x$-axis). Negative values of $I_{R}$ indicate that the radii of attraction $(R)$ are overlapping.

a)

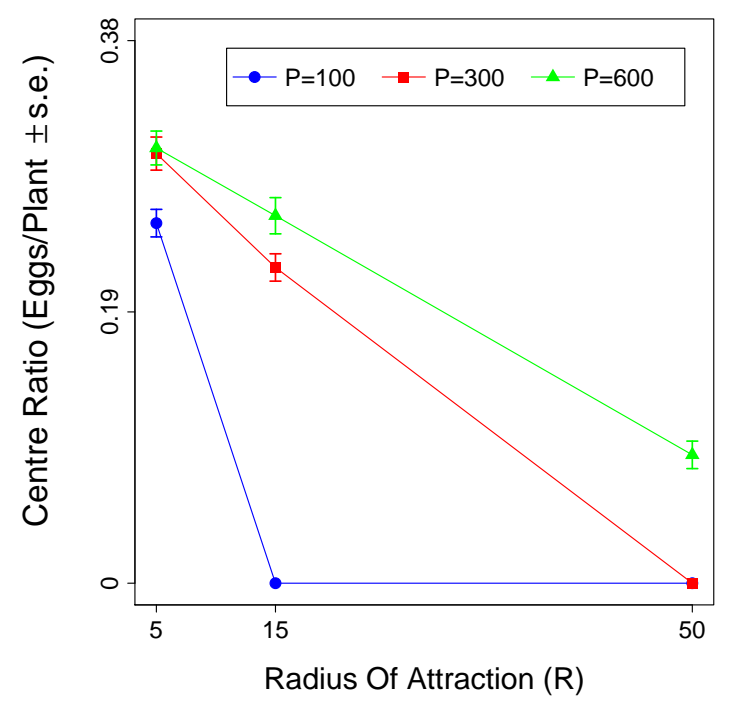

b)

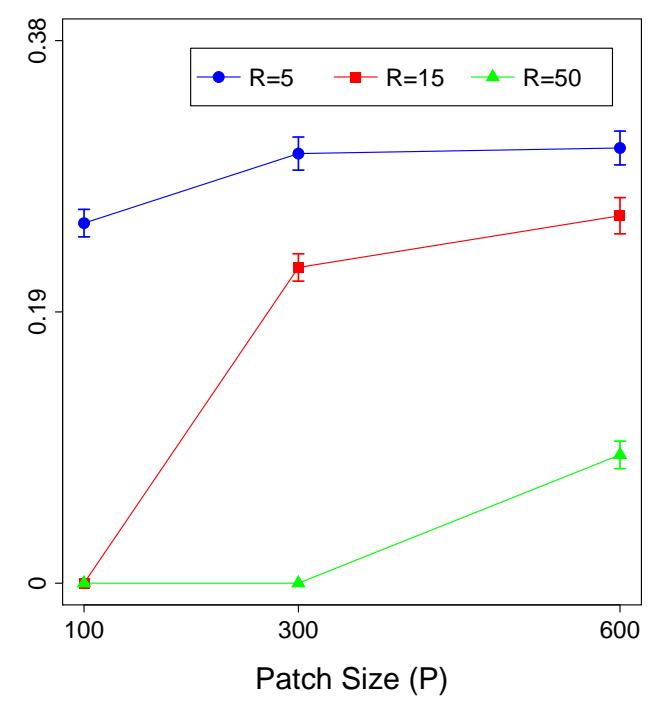

Figure A.1-2: Centre ratio vs. $R$ and $P$ - Trial B (CRW), Single Egg. Proportion of eggs per plant on centre resources (y-axis) vs. a) radius of attraction $(R)$ and $b)$ patch size $(P)$. Results are averaged over all combinations of $L$ and $k$. 


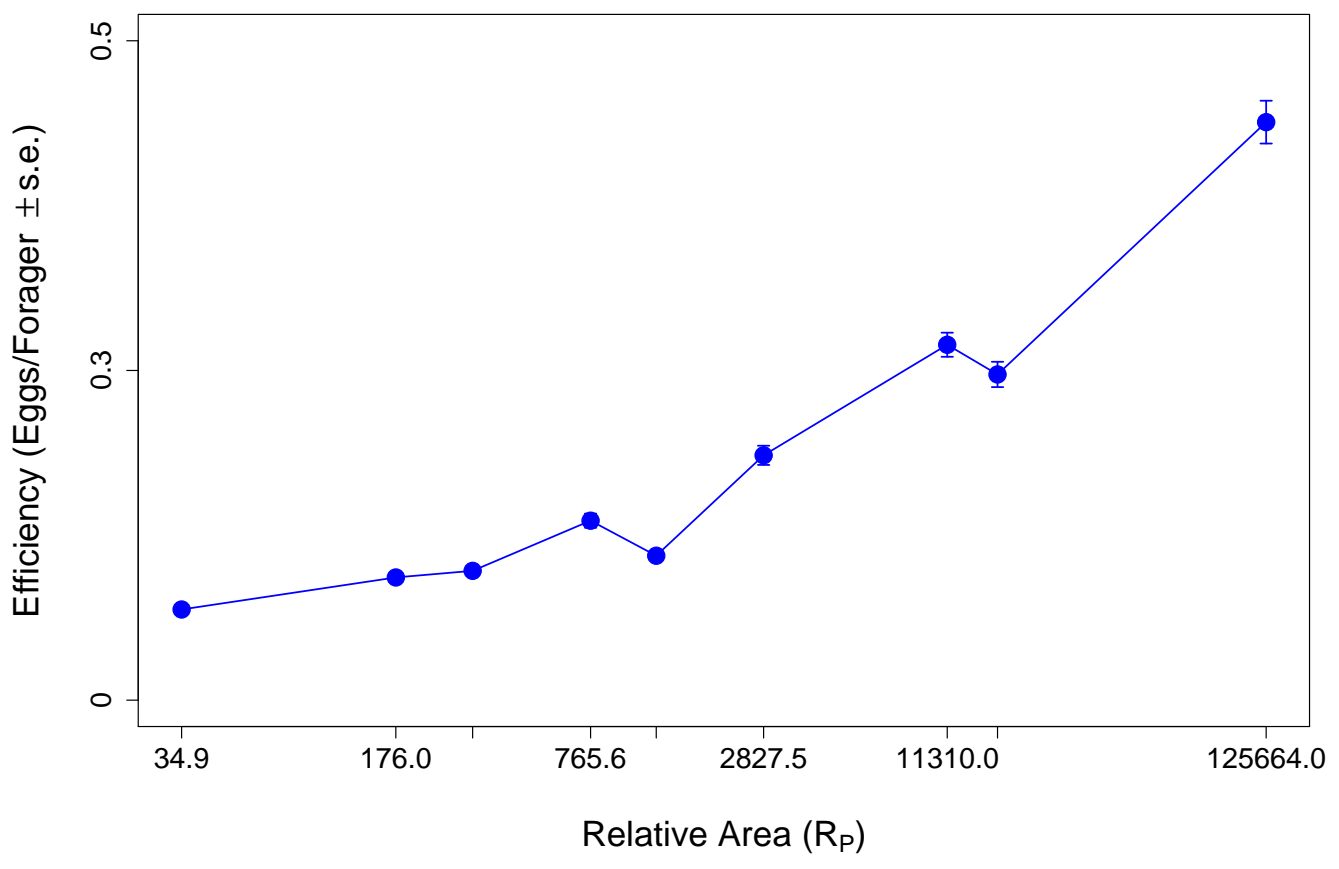

Figure A.1-3: Search Efficiency Summary - Trial B (CRW), Single Egg. Proportion of eggs per Forager released (y-axis) vs. Relative Area $\left(R_{P}\right)$. See Chapter 3 details of this calculation.

a)

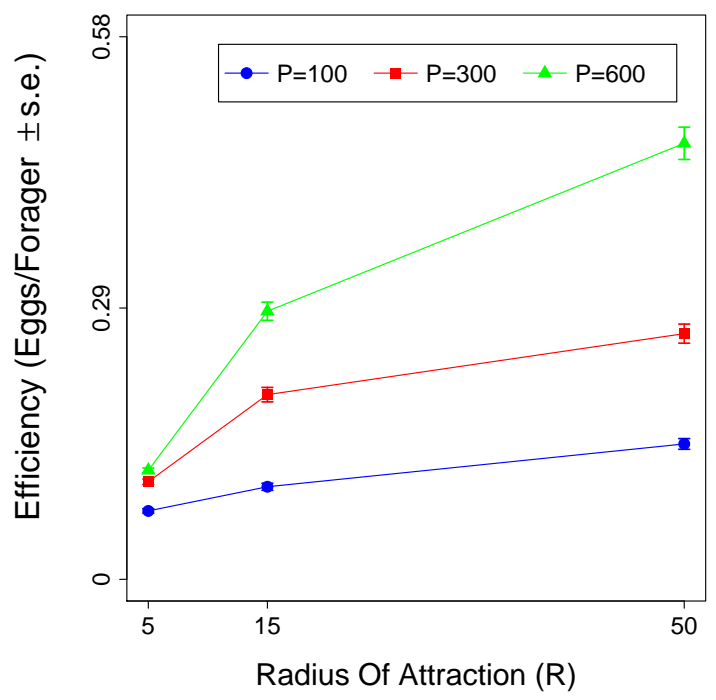

b)

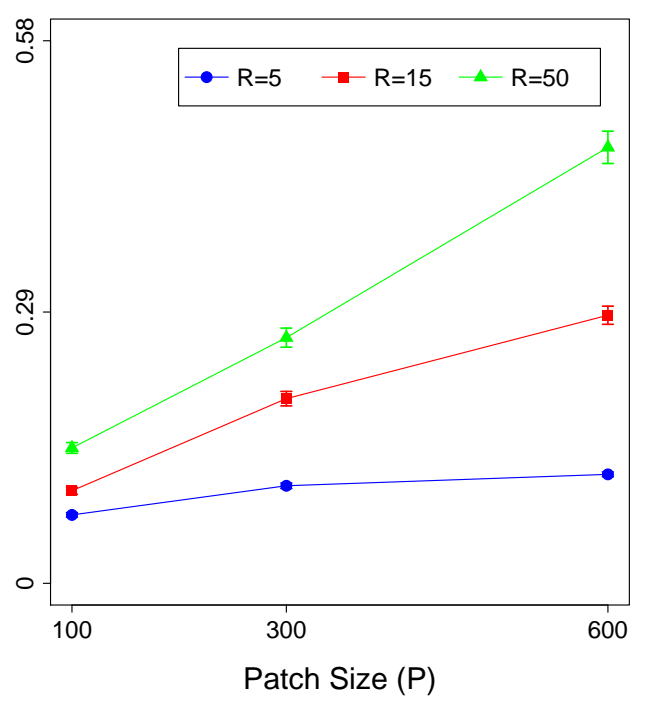

Figure A.1-4: Search efficiency vs. R and P - Trial B (CRW), Single Egg. Proportion of eggs per forager released ( $y$-axis) vs. a) radius of attraction $(R)$ and $b$ ) patch size $(P)$. Results are averaged over all combinations of $L$ and $k$. 


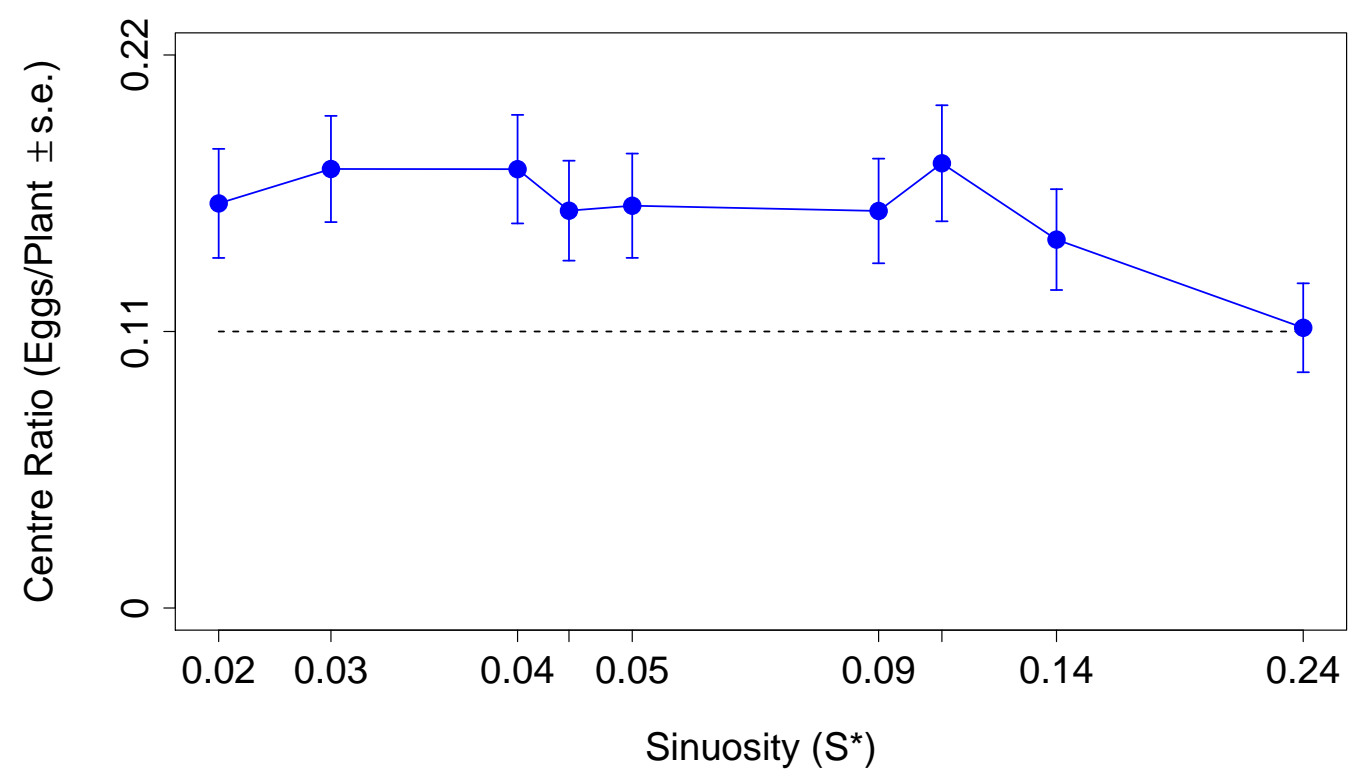

Figure A.1-5: Centre ratio vs. sinuosity - Trial B (CRW), Single Egg. Proportion of eggs per plant on centre resources (y-axis) vs. Sinuosity ( $S *)$ of forager paths. Sinuosity is a measure composed of both step length $(L)$ and turning angle concentration $(k)$

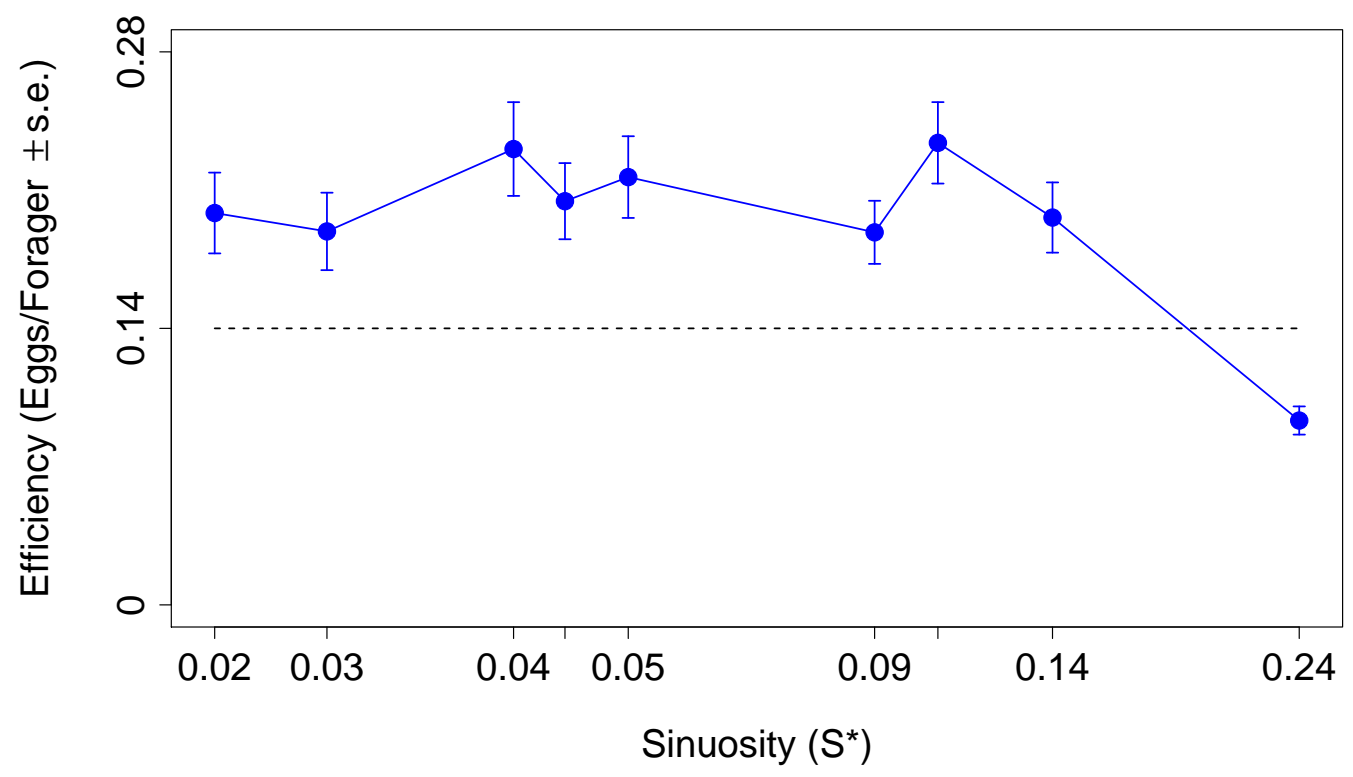

Figure A.1-6: Search efficiency vs. sinuosity - Trial B (CRW), Single Egg. Proportion of eggs per forager released ( $y$-axis) vs. a) radius of attraction $(R)$ and $b$ ) patch size $(P)$ 


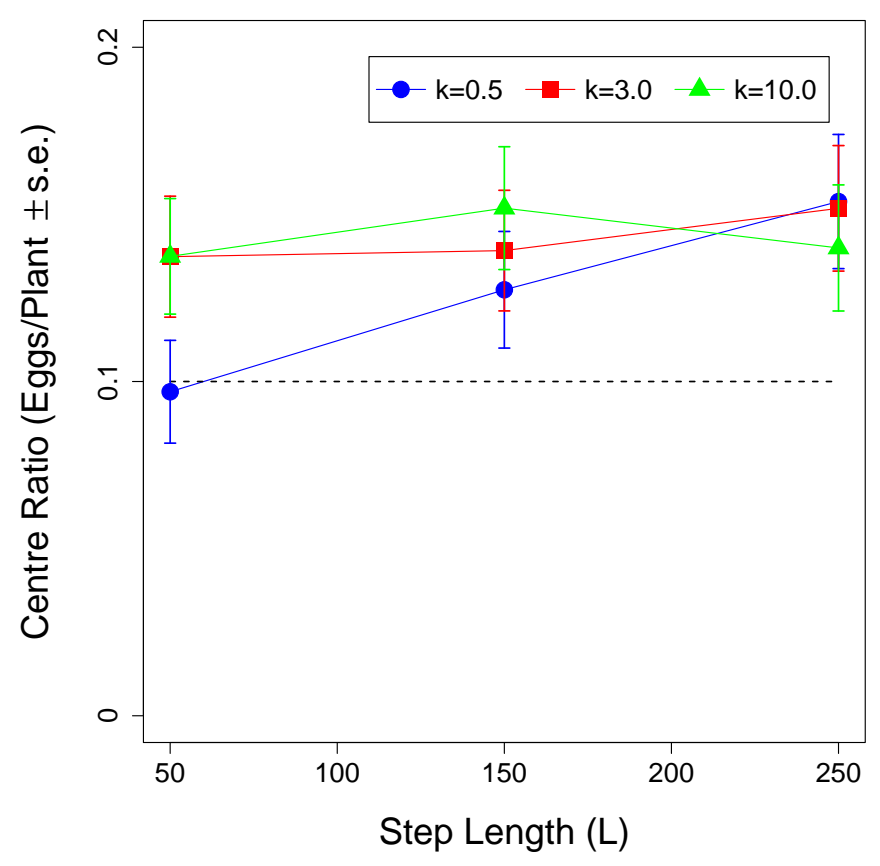

Figure A.1-7: Centre ratio vs. L and $k$ - Trial B (CRW), Single Egg. Proportion of eggs per plant on centre resources ( $y$-axis) vs. step length $(L)$ for various turning angle concentrations $(k)$. Results are averaged over all combinations of $R$ and $P$.

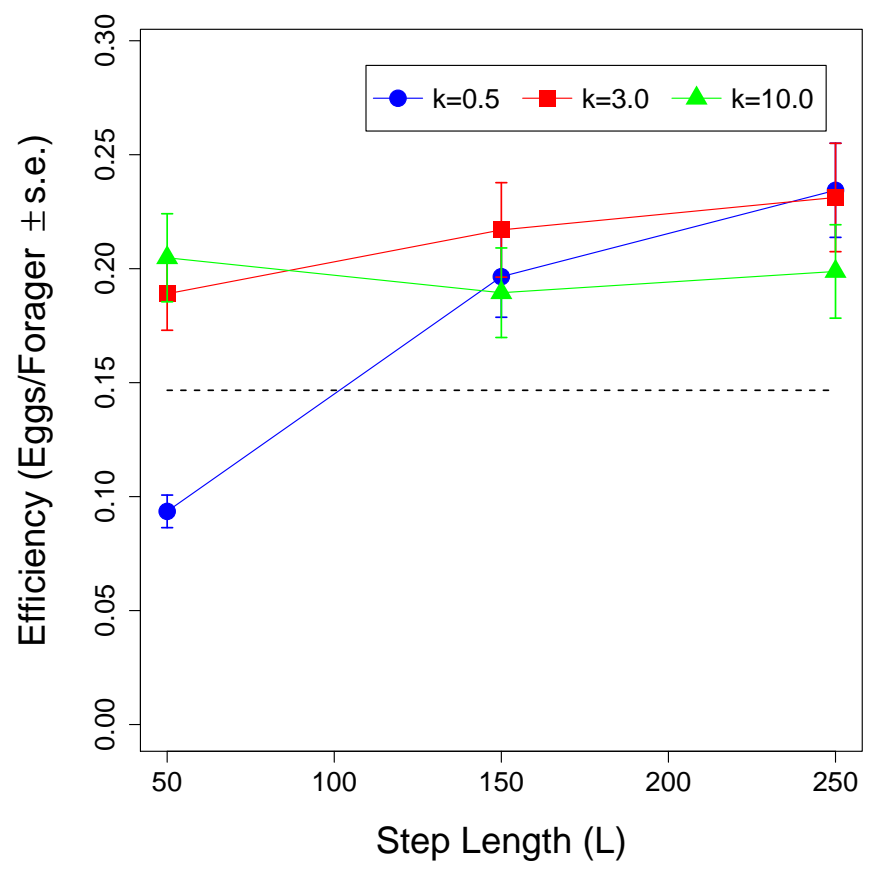

Figure A.1-8: Search efficiency vs. L and $k$ - Trial B (CRW), Single Egg. Proportion of eggs per forager released (y-axis) vs. step length $(L)$ for various turning angle concentrations $(k)$. Results are averaged over all combinations of $R$ and $P$. 

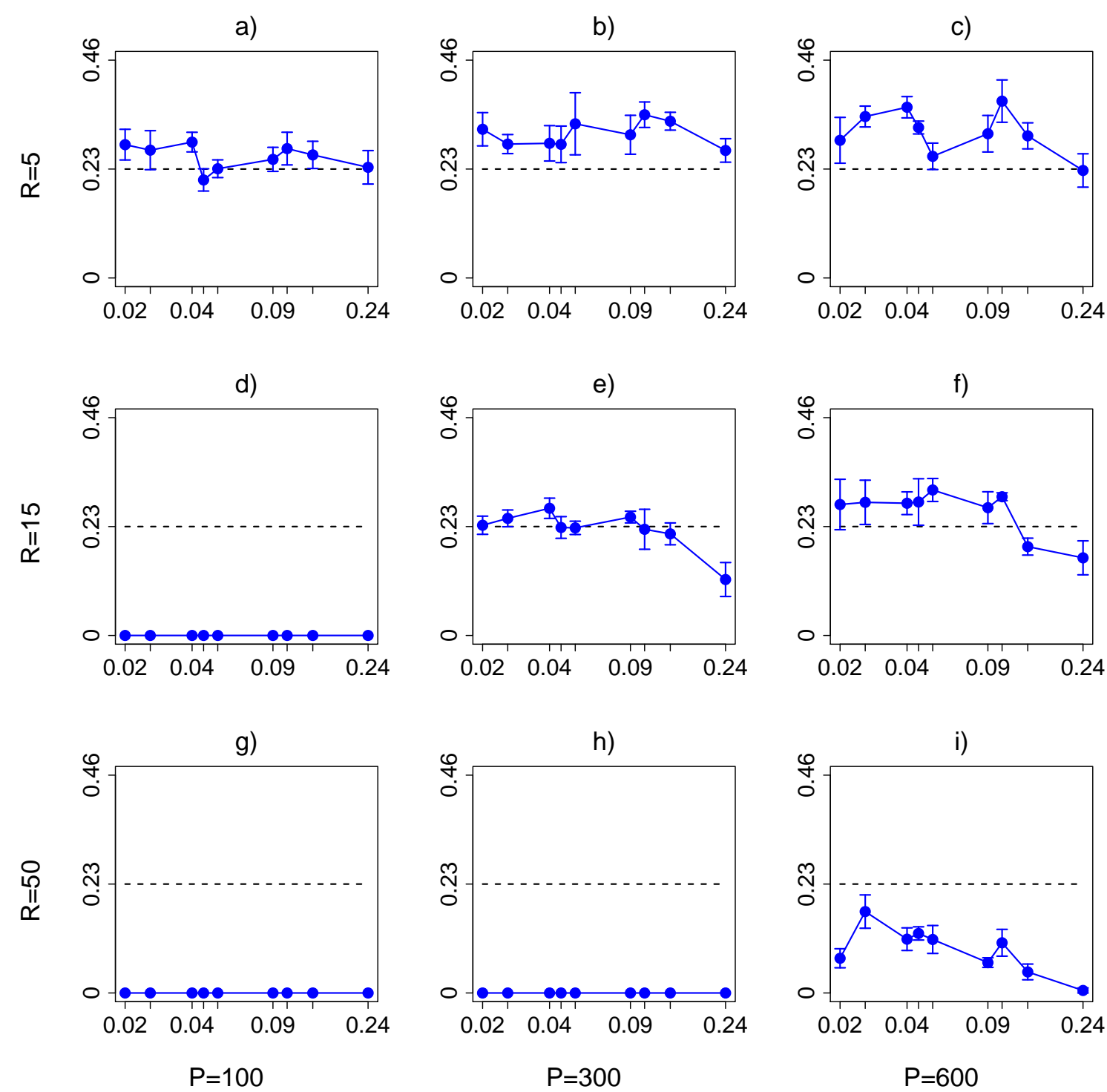

Figure A.1-9: Centre ratio vs. layout - Trial B (CRW), Single Egg. Proportion of eggs per plant on centre resources (y-axis) vs. Sinuosity is plotted for combinations of radius of attraction $(R)$ and patch size $(P)$ in each sub-plot $(a-i)$. Radius of attraction increases from top to bottom and patch size increases from left to right. The layout is such that these results may be compared with Figure 3.32 to see the patch layouts. 
a)

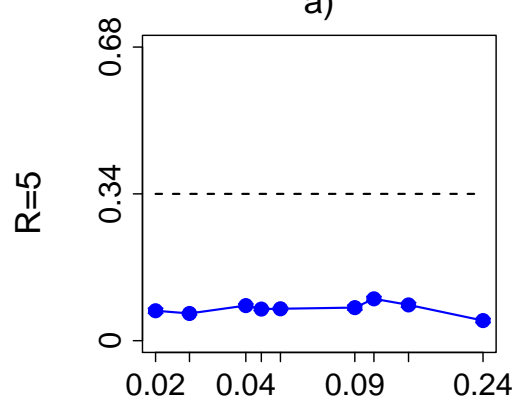

d)

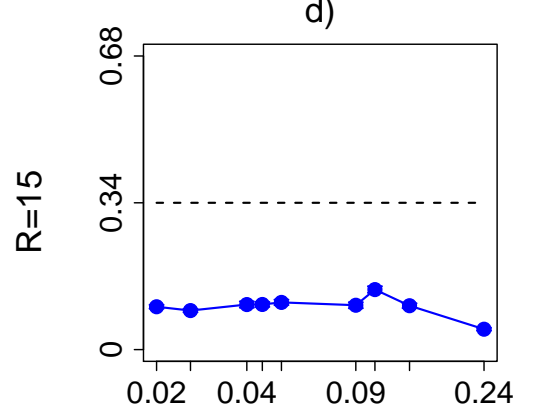

g)

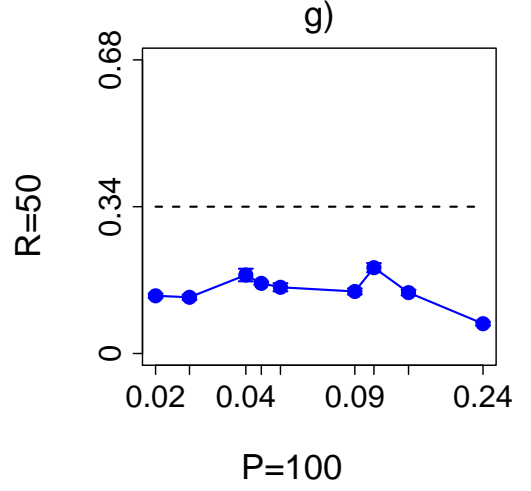

b)

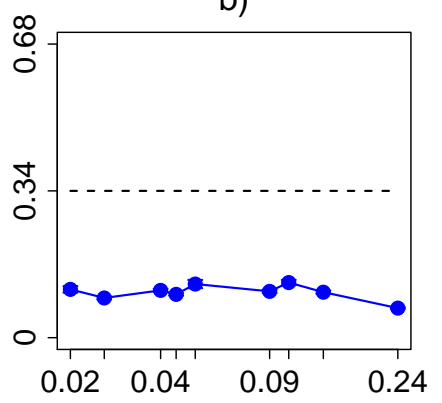

e)

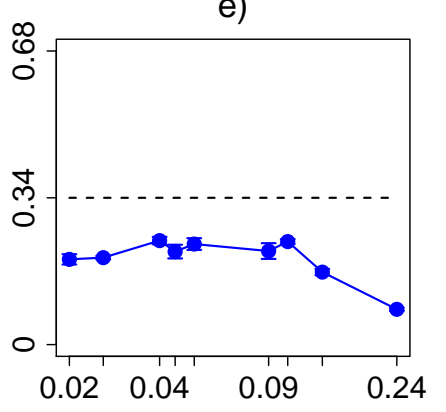

h)

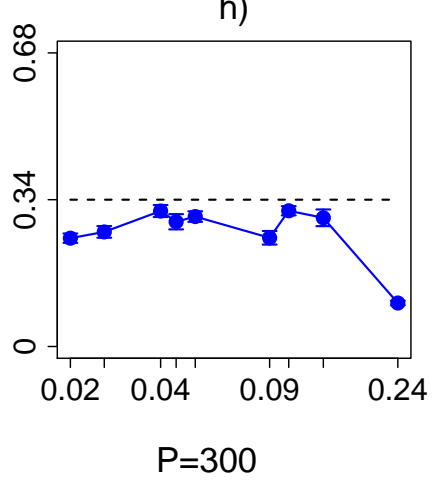

c)

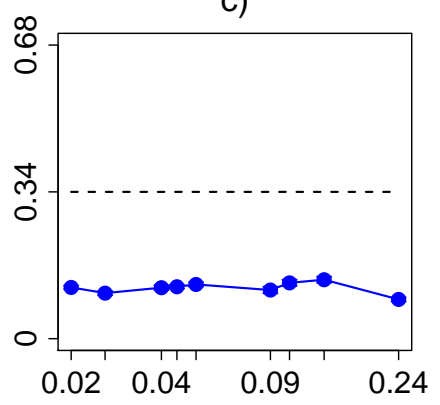

f)

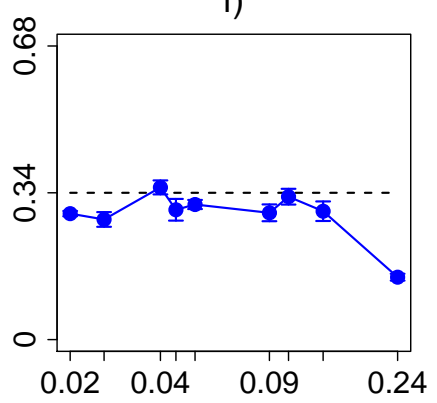

i)

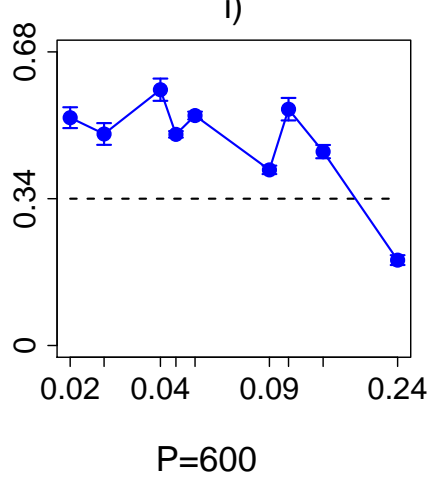

Figure A.1-10: Search efficiency vs. layout - Trial B (CRW), Single Egg. Proportion of eggs per forager released (y-axis) vs. sinuosity is plotted for combinations of radius of attraction $(R)$ and patch size $(P)$ in each sub-plot $(a-i)$. Radius of attraction increases from top to bottom and patch size increases from left to right. The layout is such that these results may be compared with Figure 3.32 to see the patch layouts. 


\section{A.2 Trial B (CRW) Multiple Eggs results}

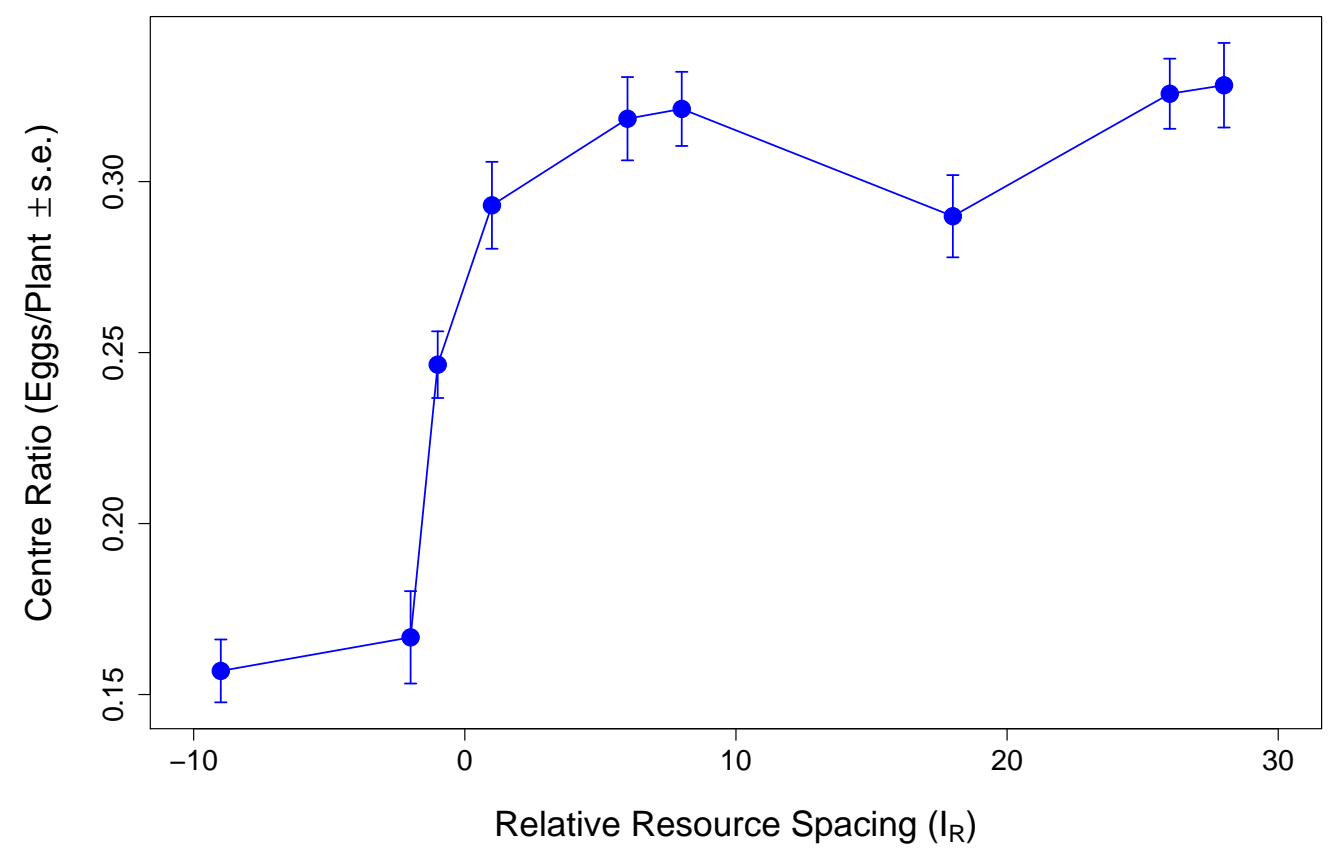

Figure A.2-1: Centre ratio summary - Trial B (CRW), Multiple Eggs. Proportion of eggs per plant on centre resources ( $y$-axis) vs. relative resource spacing, $I_{R}$ (x-axis). Negative values of $I_{R}$ indicate that the radii of attraction $(R)$ are overlapping.

a)

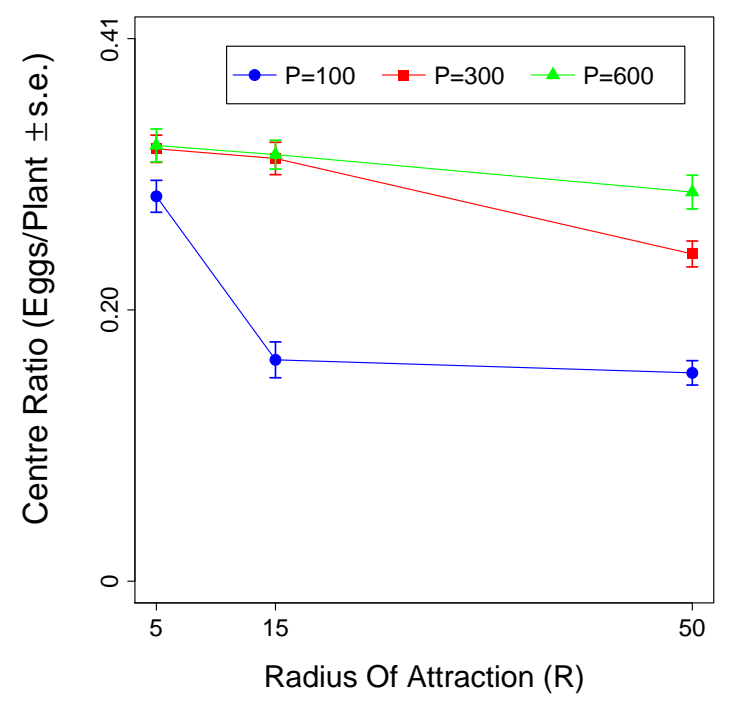

b)

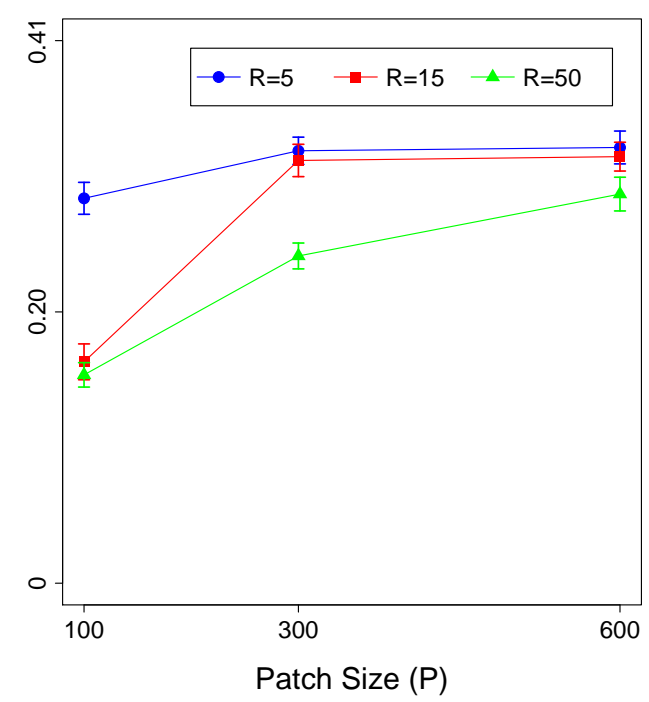

Figure A.2-2: Centre ratio vs. $R$ and P - Trial B (CRW), Multiple Eggs. Proportion of eggs per plant on centre resources ( $y$-axis) vs. a) radius of attraction $(R)$ and b) patch size $(P)$. Results are averaged over all combinations of $L$ and $k$. 


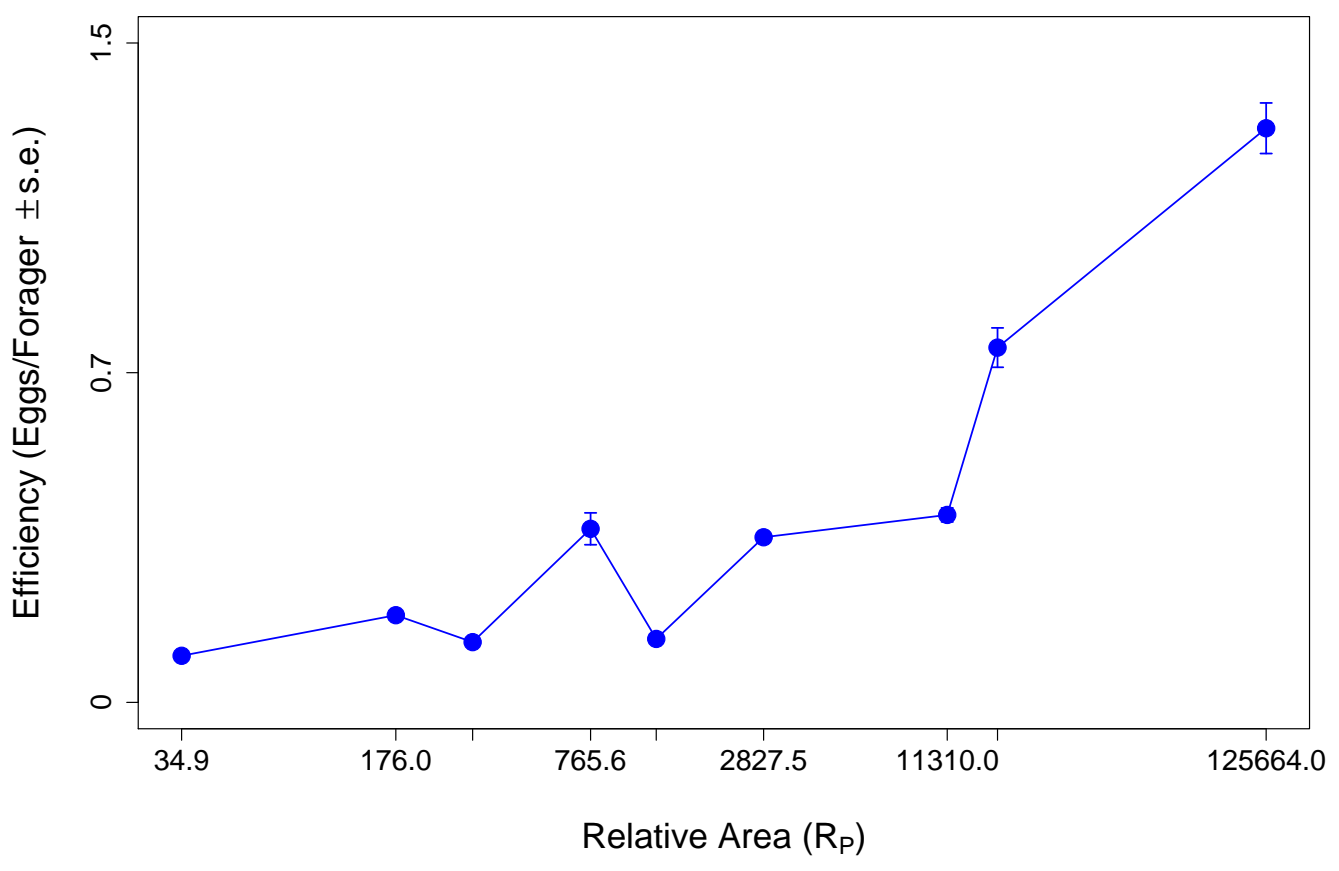

Figure A.2-3: Search Efficiency Summary - Trial B (CRW), Multiple Eggs. Proportion of eggs per Forager released (y-axis) vs. Relative Area $\left(R_{P}\right)$. See Chapter 3 details of this calculation.

a)

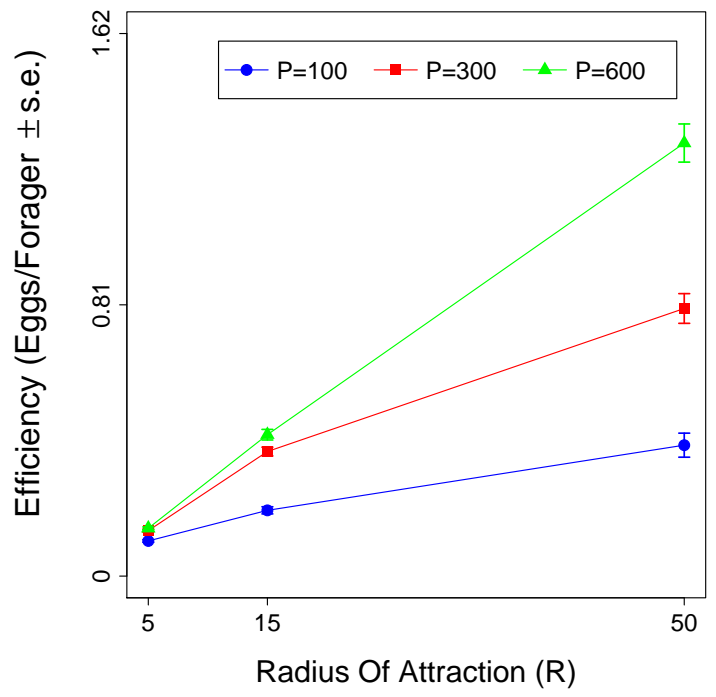

b)

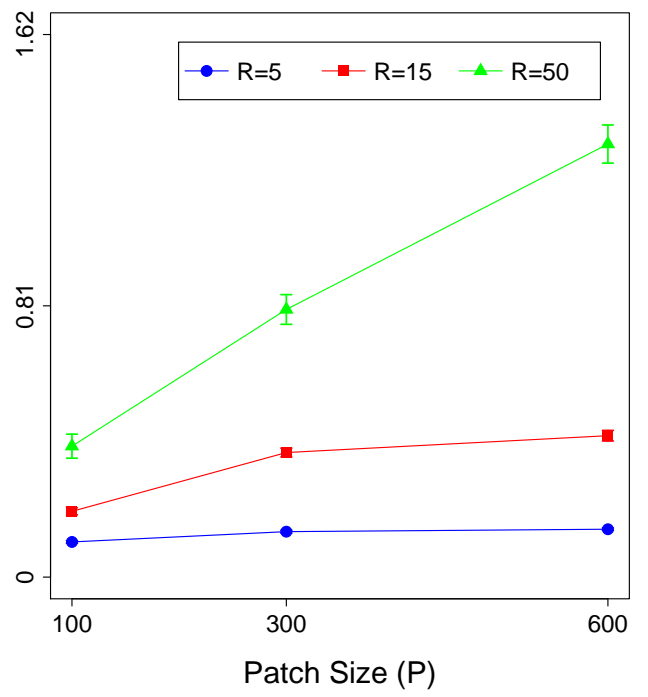

Figure A.2-4: Search efficiency vs. $R$ and P - Trial B (CRW), Multiple Eggs. Proportion of eggs per forager released (y-axis) vs. a) radius of attraction $(R)$ and $b)$ patch size $(P)$. Results are averaged over all combinations of $L$ and $k$. 


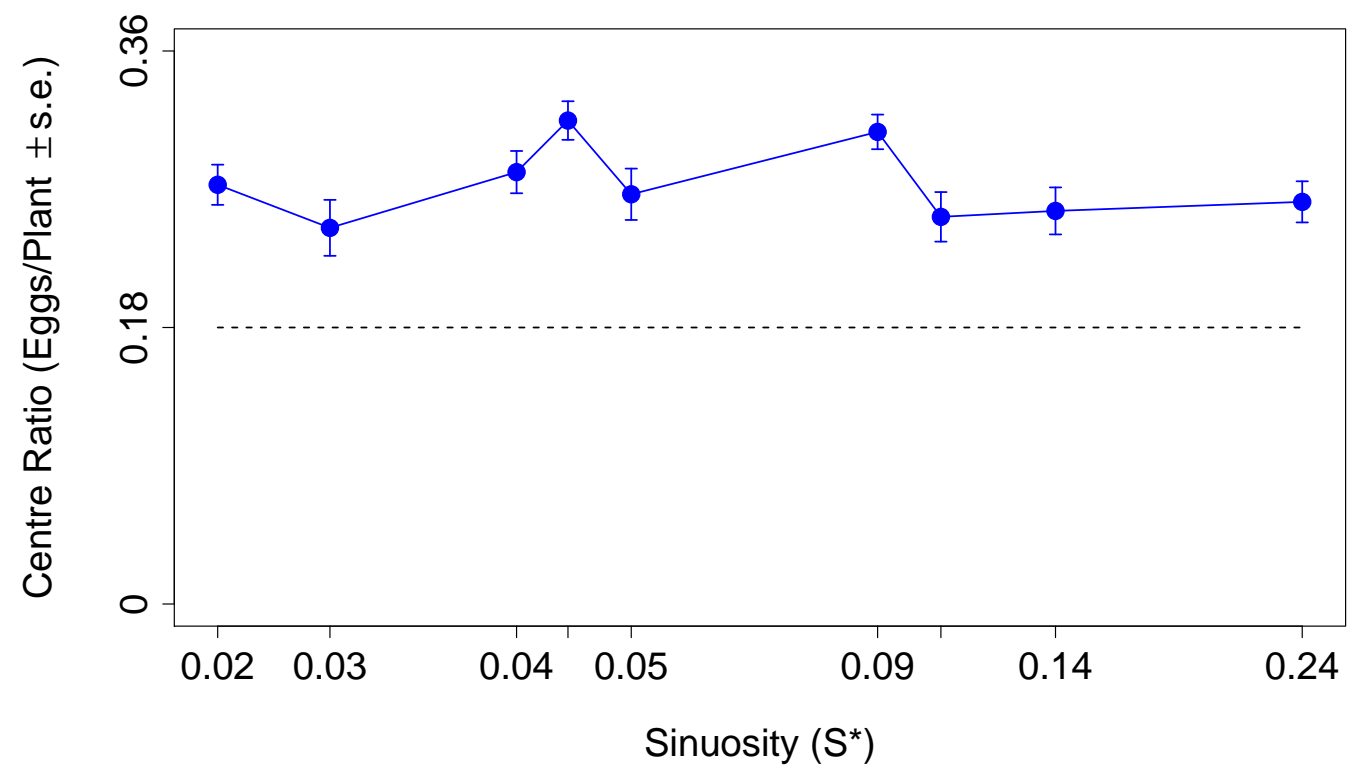

Figure A.2-5: Centre ratio vs. sinuosity - Trial B (CRW), Multiple Eggs. Proportion of eggs per plant on centre resources (y-axis) vs. Sinuosity $(S *)$ of forager paths. Sinuosity is a measure composed of both step length $(L)$ and turning angle concentration $(k)$

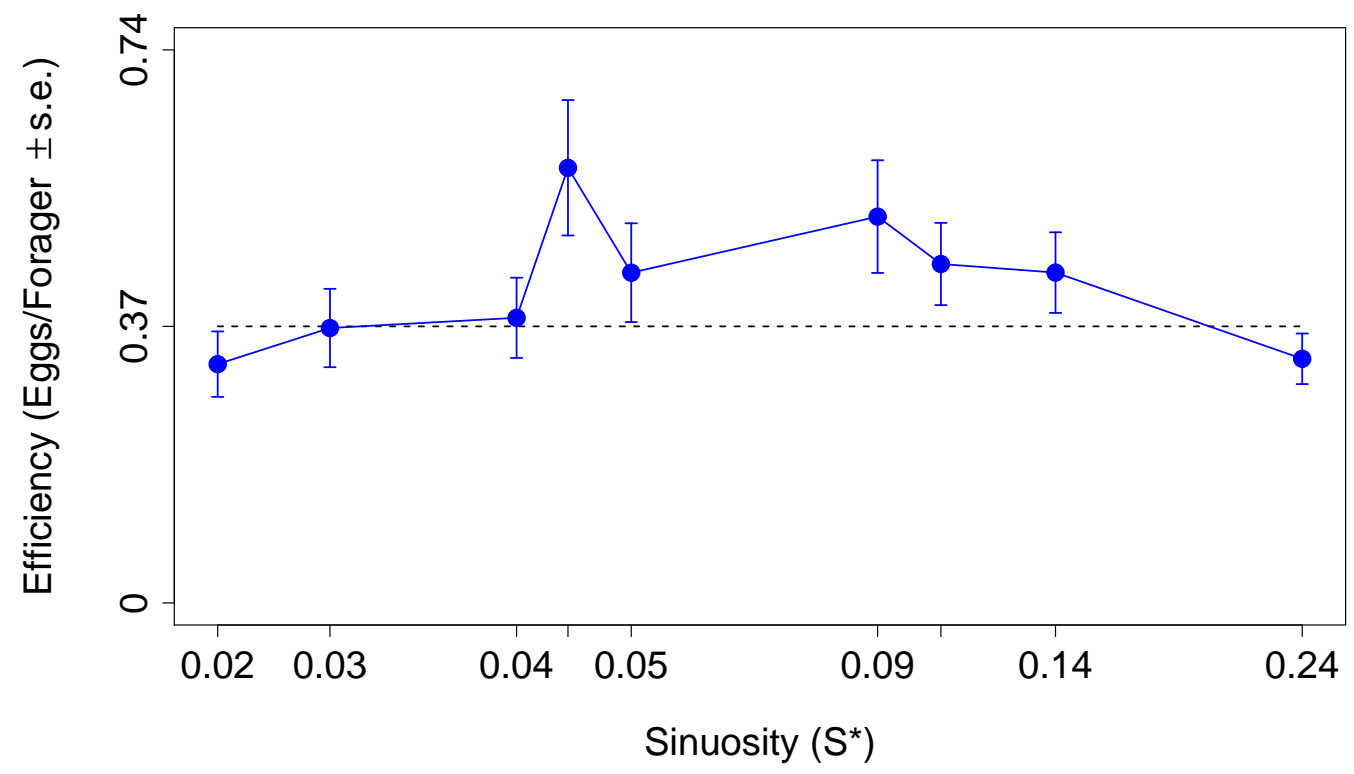

Figure A.2-6: Search efficiency vs. sinuosity - Trial B (CRW), Multiple Eggs. Proportion of eggs per forager released ( $y$-axis) vs. a) radius of attraction $(R)$ and $b)$ patch size $(P)$ 


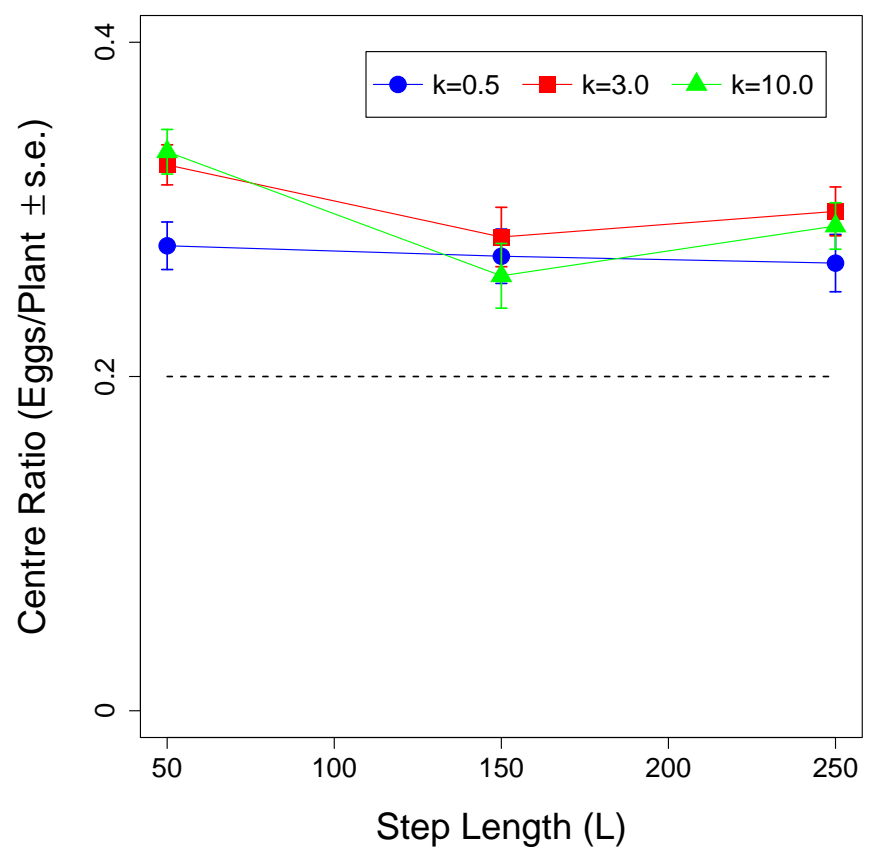

Figure A.2-7: Centre ratio vs. L and $k$ - Trial B (CRW), Multiple Eggs. Proportion of eggs per plant on centre resources (y-axis) vs. step length $(L)$ for various turning angle concentrations $(k)$. Results are averaged over all combinations of $R$ and $P$.

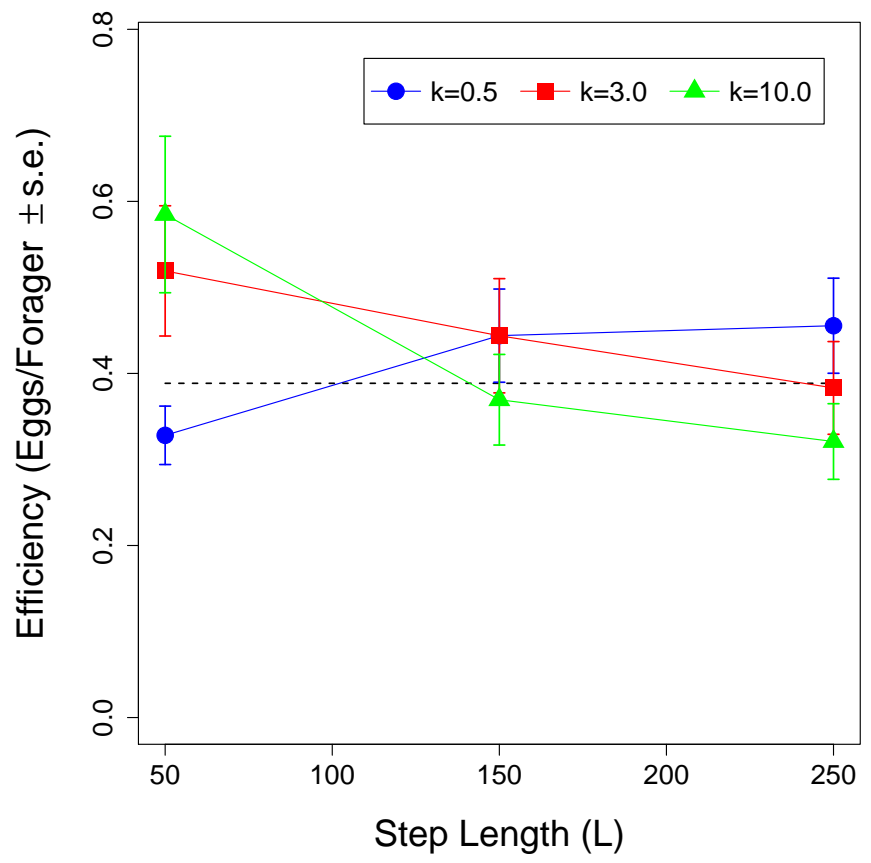

Figure A.2-8: Search efficiency vs. L and $k$ - Trial B (CRW), Multiple Eggs. Proportion of eggs per forager released (y-axis) vs. step length $(L)$ for various turning angle concentrations $(k)$. Results are averaged over all combinations of $R$ and $P$. 

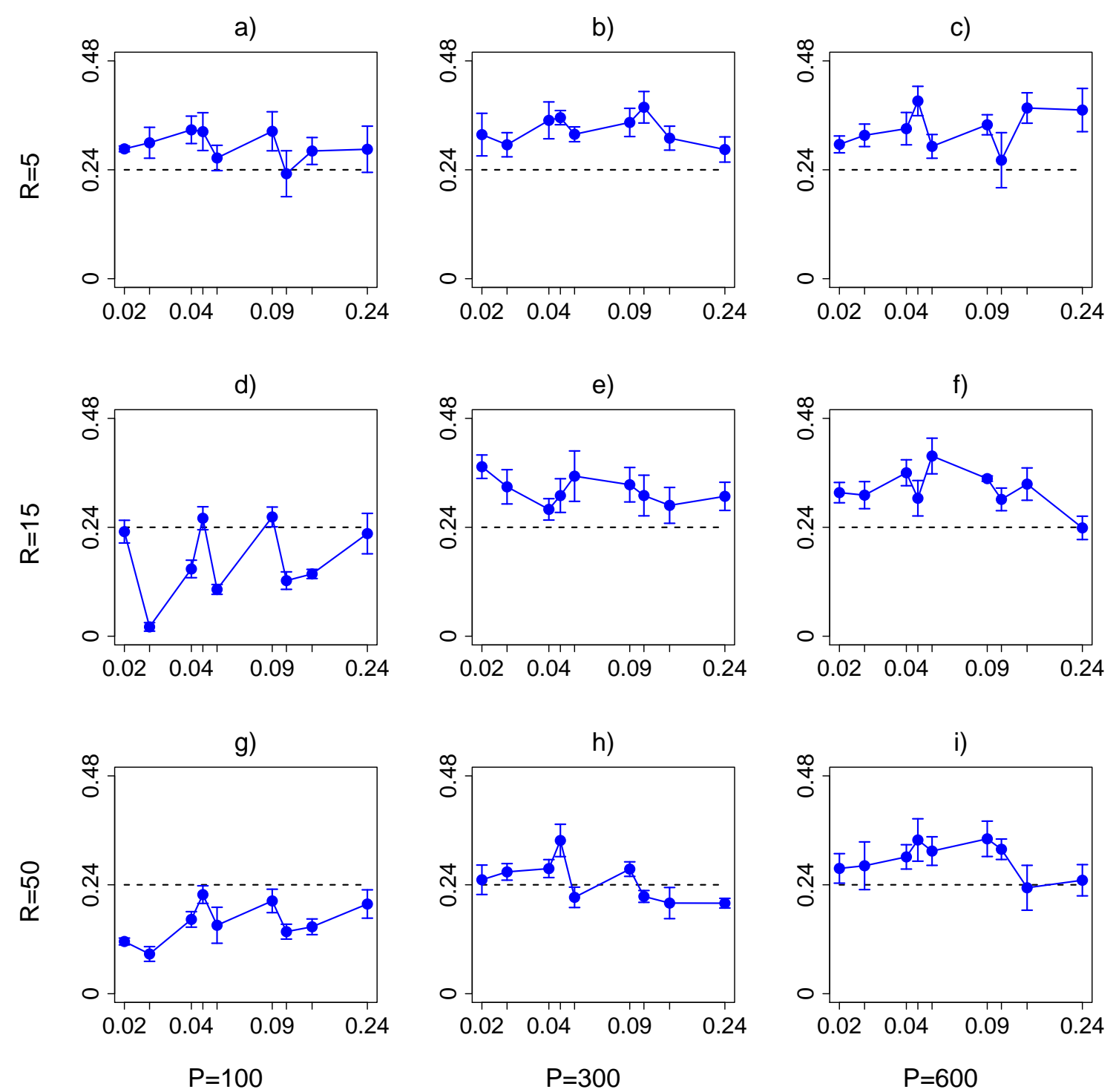

Figure A.2-9: Centre ratio vs. layout - Trial B (CRW), Multiple Eggs. Proportion of eggs per plant on centre resources (y-axis) vs. Sinuosity is plotted for combinations of radius of attraction $(R)$ and patch size $(P)$ in each sub-plot $(a-i)$. Radius of attraction increases from top to bottom and patch size increases from left to right. The layout is such that these results may be compared with Figure 3.32 to see the patch layouts. 
a)

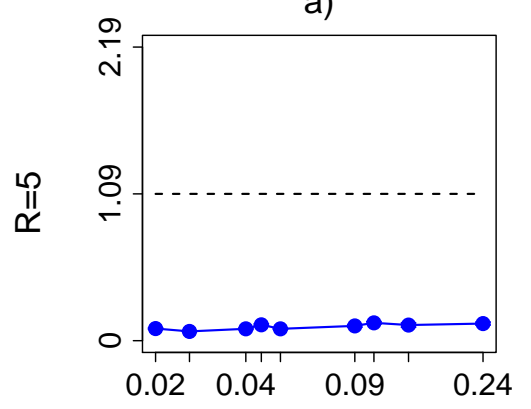

d)

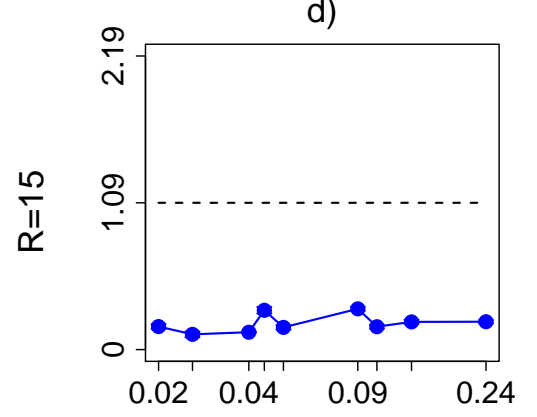

g)

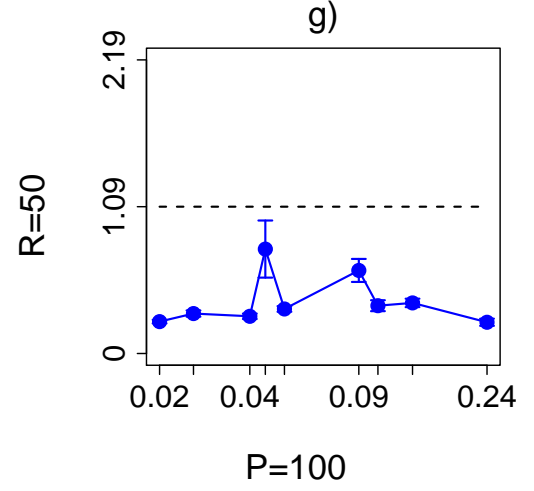

b)

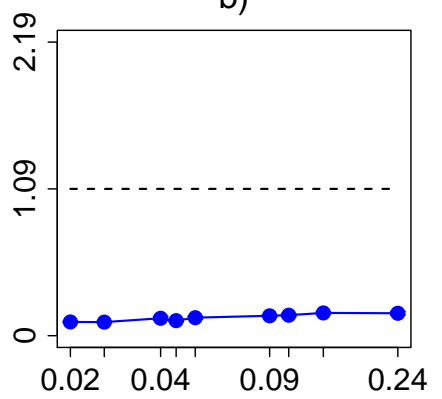

e)

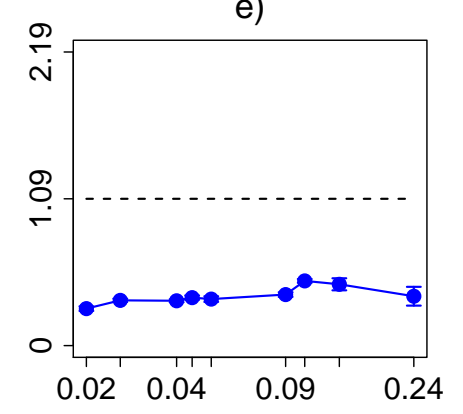

h)

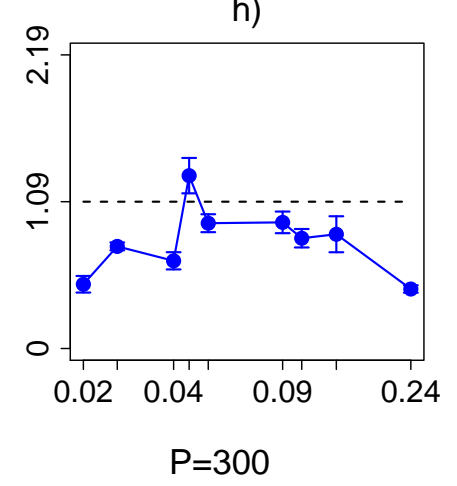

c)

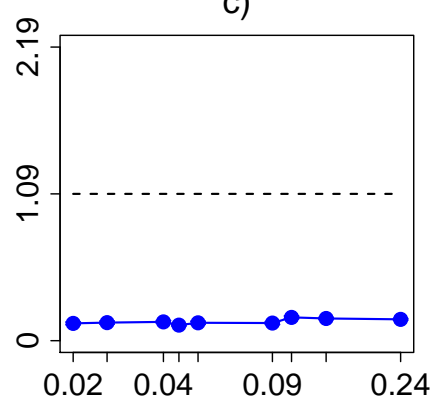

f)

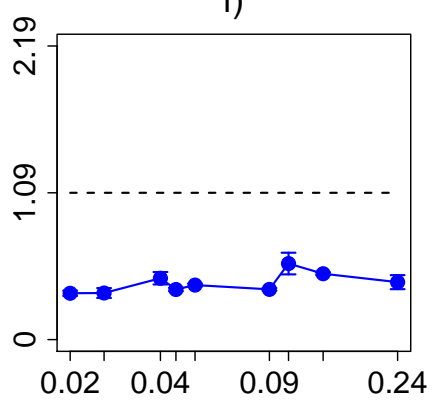

i)

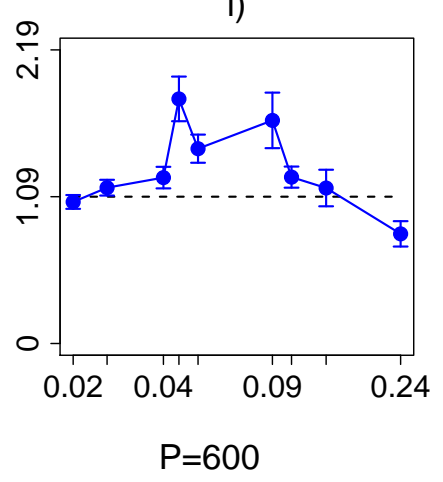

Figure A.2-10: Search efficiency vs. layout - Trial B (CRW), Multiple Eggs. Proportion of eggs per forager released (y-axis) vs. sinuosity is plotted for combinations of radius of attraction $(R)$ and patch size $(P)$ in each sub-plot $(a-i)$. Radius of attraction increases from top to bottom and patch size increases from left to right. The layout is such that these results may be compared with Figure 3.32 to see the patch layouts. 


\section{A.3 Trial C (Olfaction) Single Egg results}

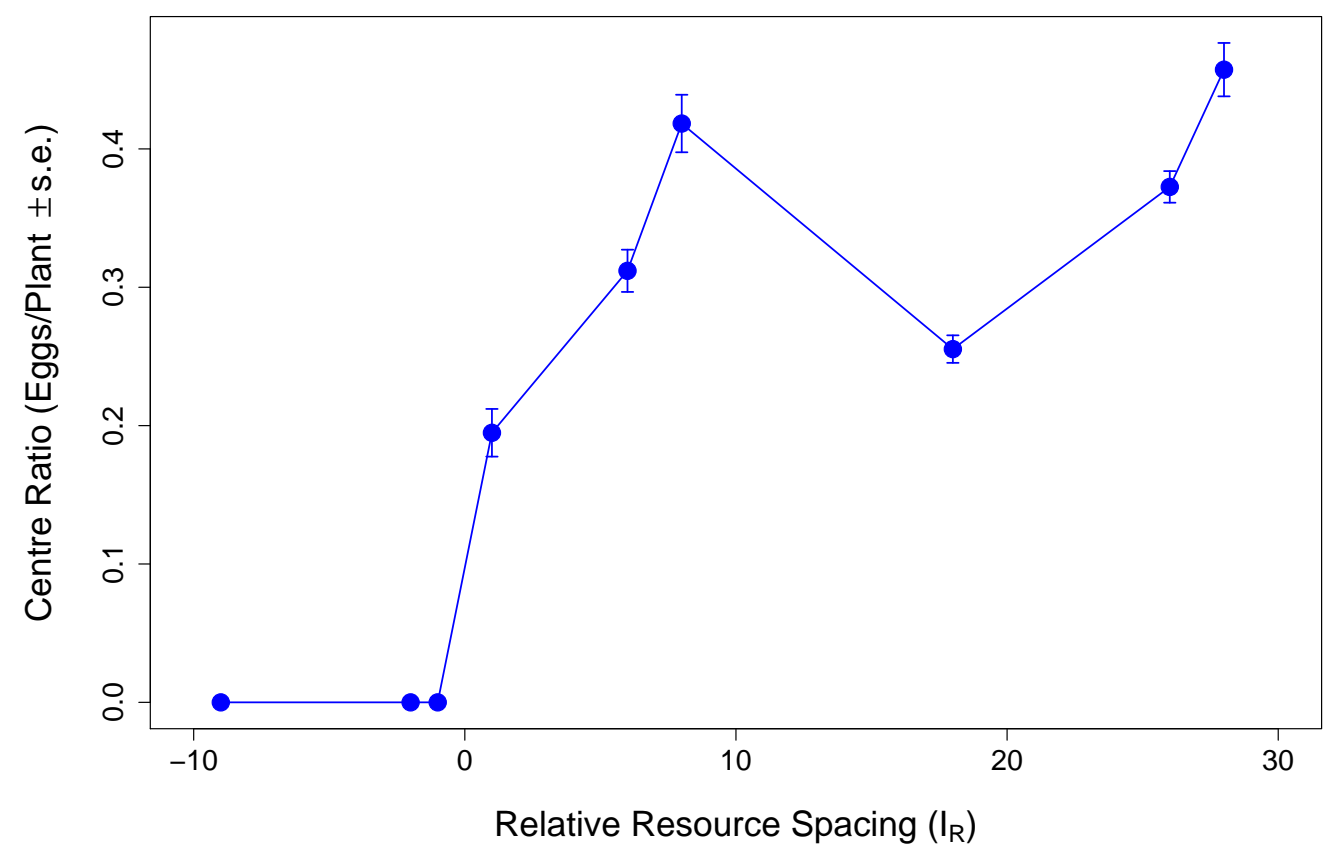

Figure A.3-1: Centre ratio summary - Trial C (Olfaction), Single Egg. Proportion of eggs per plant on centre resources ( $y$-axis) vs. relative resource spacing, $I_{R}$ (x-axis). Negative values of $I_{R}$ indicate that the radii of attraction $(R)$ are overlapping.

a)

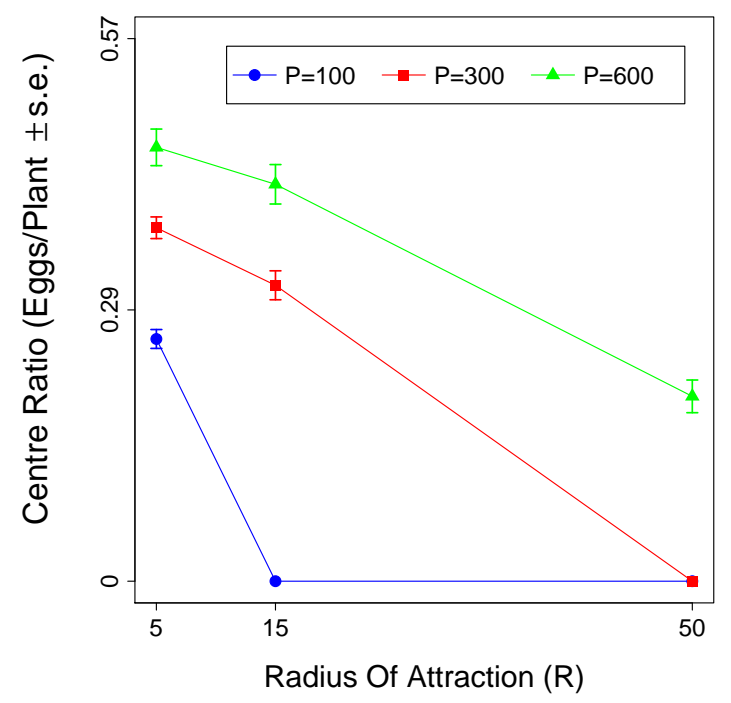

b)

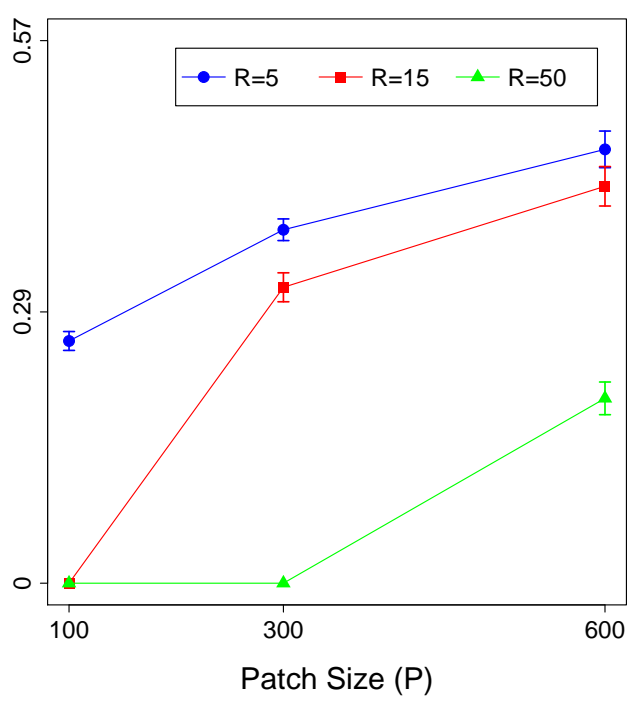

Figure A.3-2: Centre ratio vs. $R$ and $P$ - Trial C (Olfaction), Single Egg. Proportion of eggs per plant on centre resources (y-axis) vs. a) radius of attraction $(R)$ and $b)$ patch size $(P)$. Results are averaged over all combinations of $L$ and $k$. 


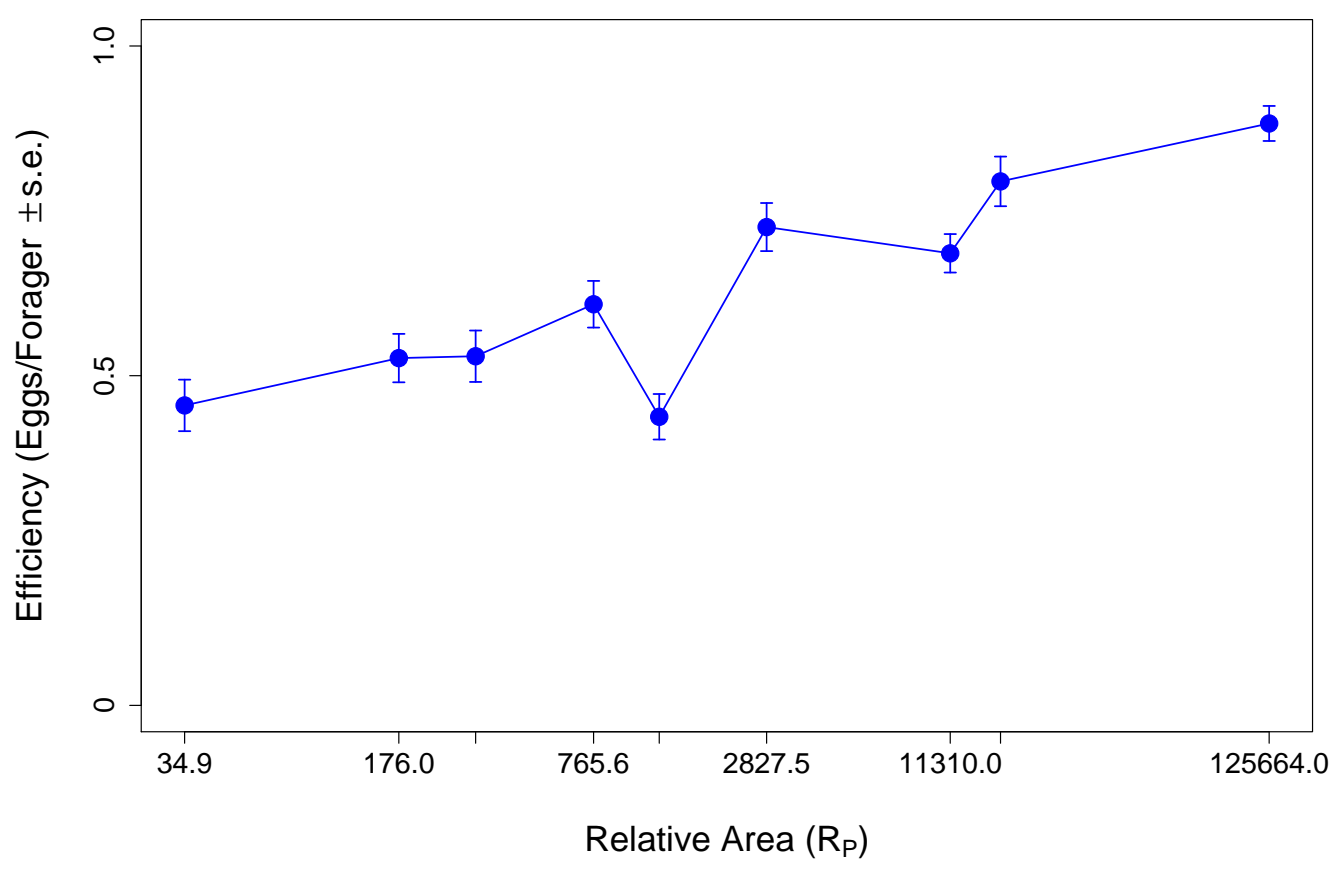

Figure A.3-3: Search Efficiency Summary - Trial C (Olfaction), Single Egg. Proportion of eggs per Forager released (y-axis) vs. Relative Area $\left(R_{P}\right)$. See Chapter 3 details of this calculation.

a)

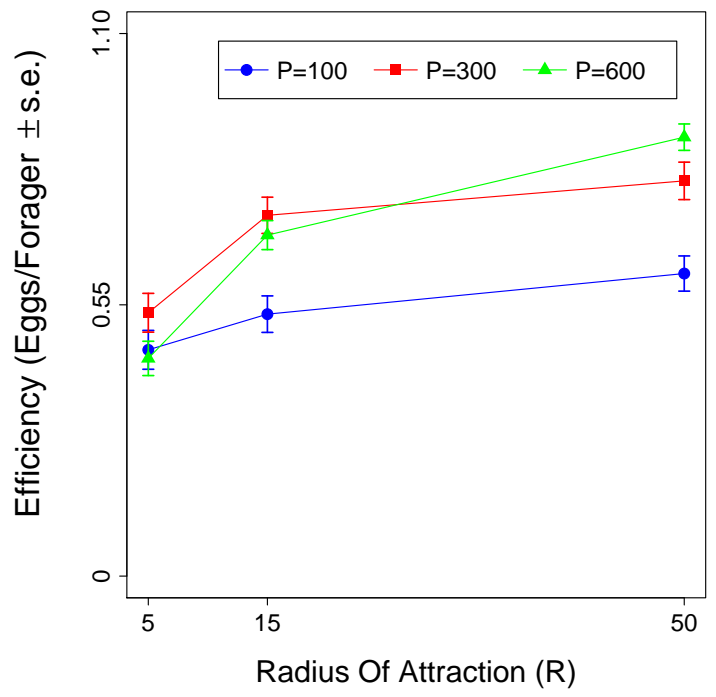

b)

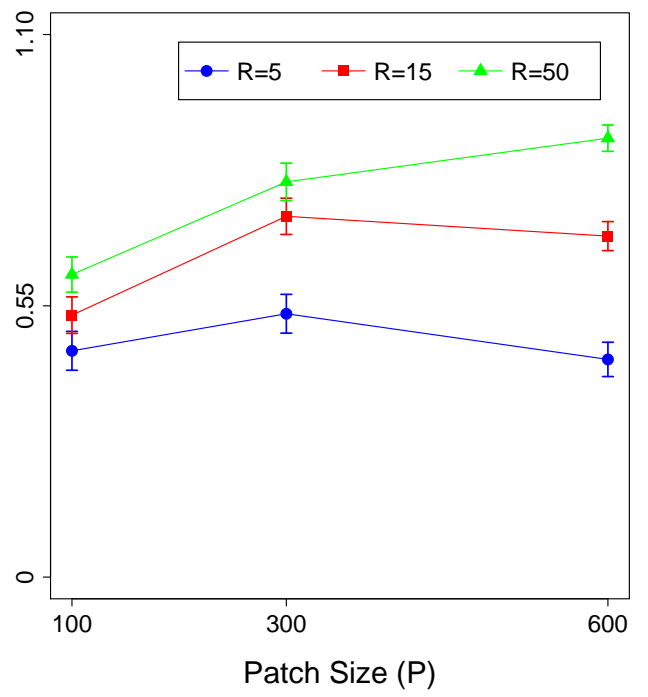

Figure A.3-4: Search efficiency vs. $R$ and P - Trial C (Olfaction), Single Egg. Proportion of eggs per forager released (y-axis) vs. a) radius of attraction $(R)$ and b) patch size $(P)$. Results are averaged over all combinations of $L$ and $k$. 


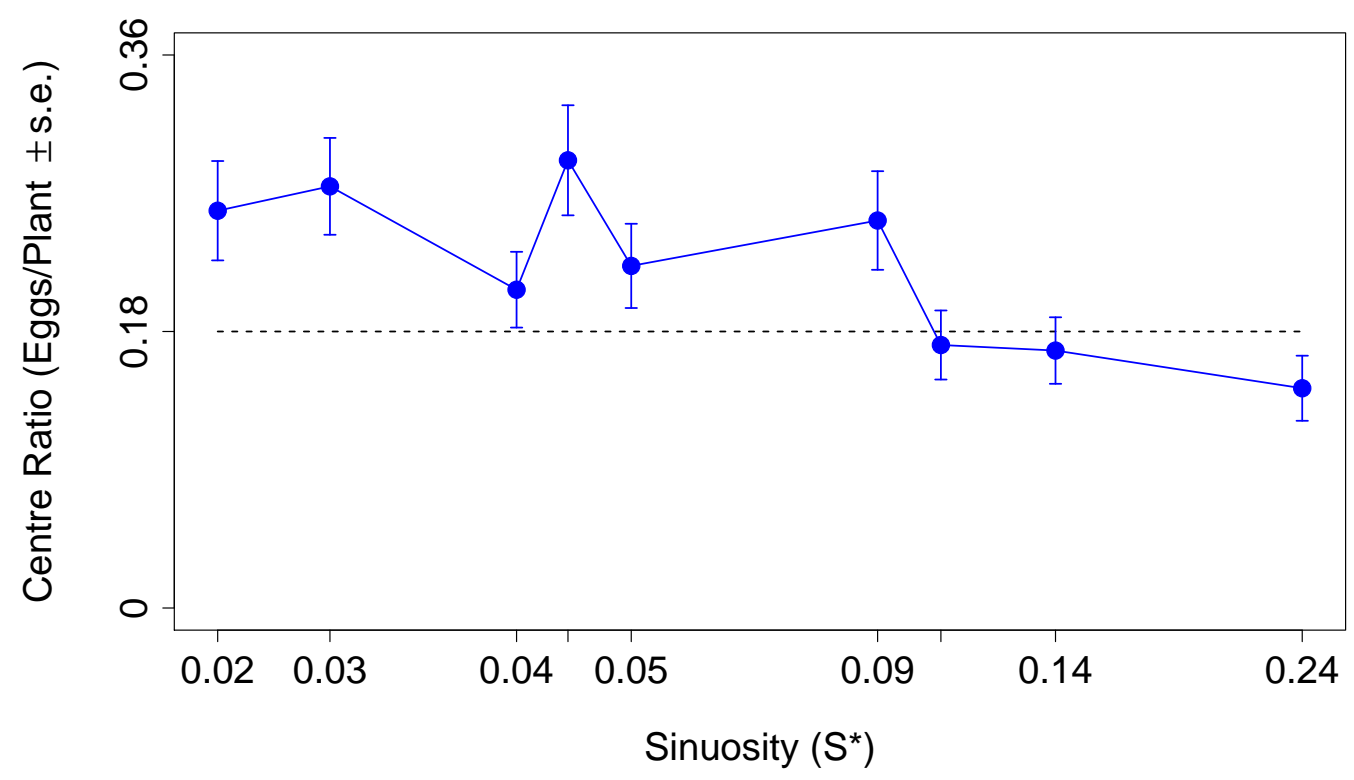

Figure A.3-5: Centre ratio vs. sinuosity - Trial C (Olfaction), Single Egg. Proportion of eggs per plant on centre resources (y-axis) vs. Sinuosity $(S *)$ of forager paths. Sinuosity is a measure composed of both step length $(L)$ and turning angle concentration $(k)$

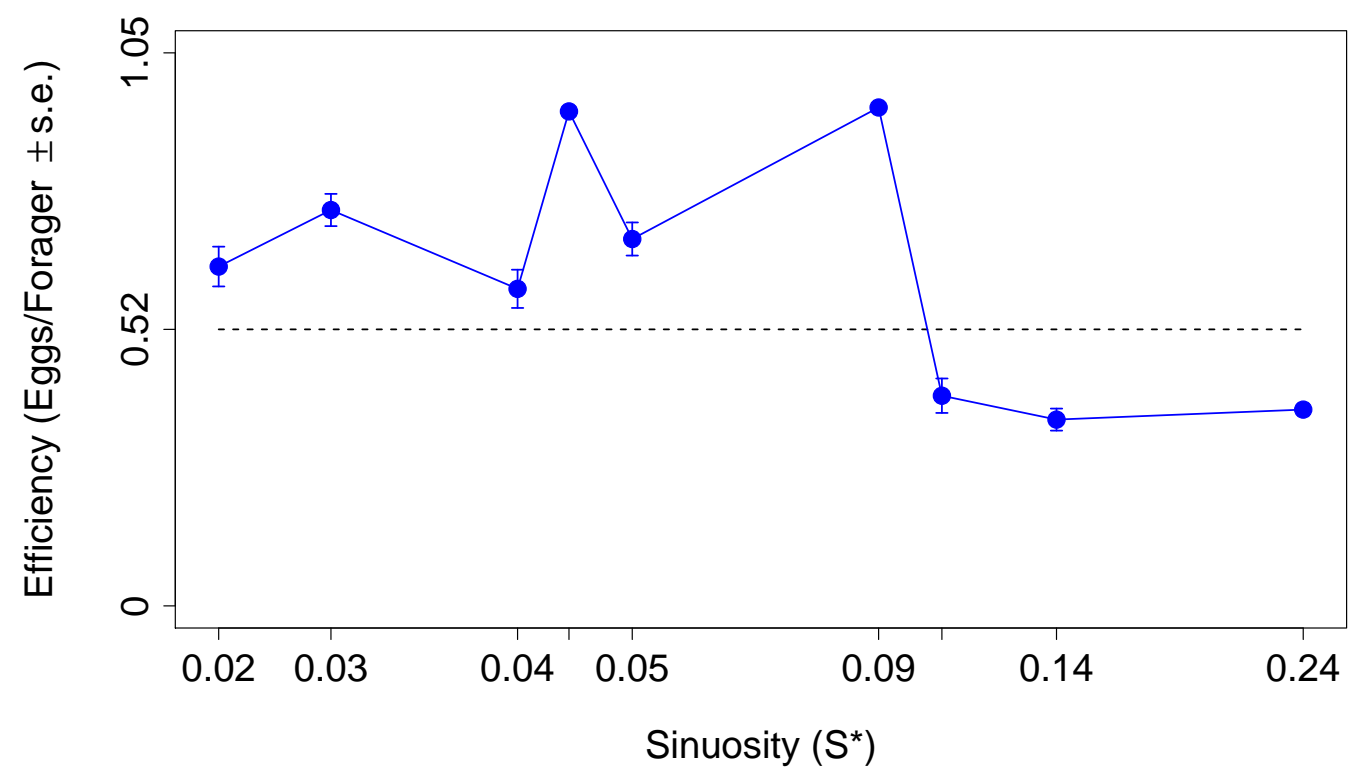

Figure A.3-6: Search efficiency vs. sinuosity - Trial C (Olfaction), Single Egg. Proportion of eggs per forager released ( $y$-axis) vs. a) radius of attraction $(R)$ and $b$ ) patch size $(P)$ 


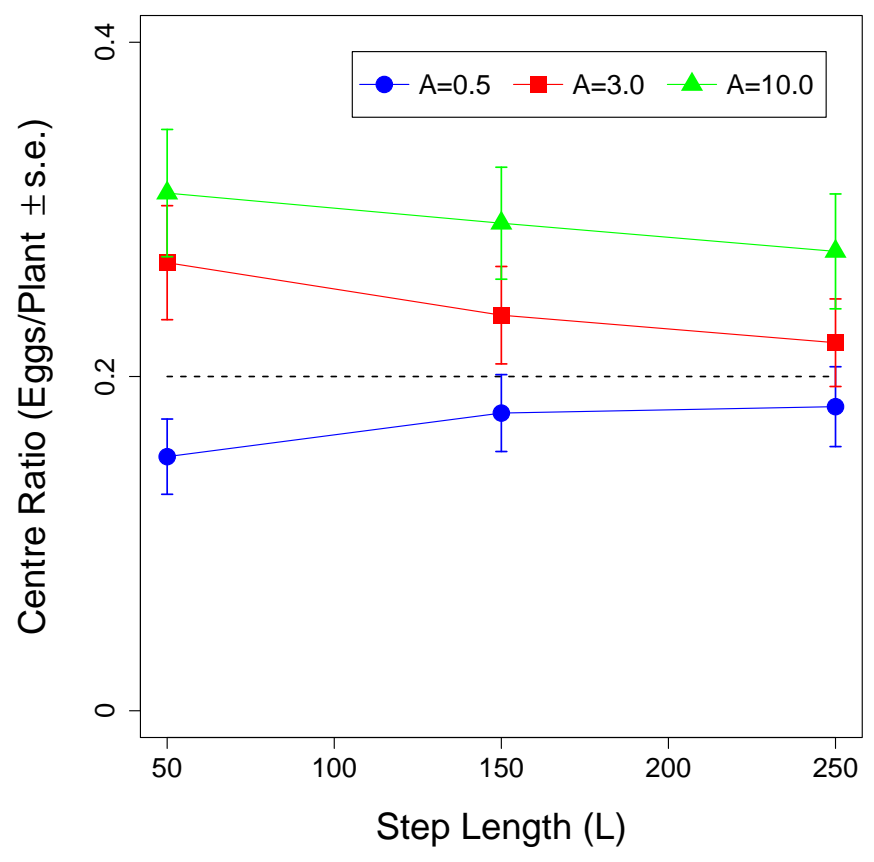

Figure A.3-7: Centre ratio ws. L and $k$ - Trial C (Olfaction), Single Egg. Proportion of eggs per plant on centre resources (y-axis) vs. step length $(L)$ for various turning angle concentrations $(k)$. Results are averaged over all combinations of $R$ and $P$.

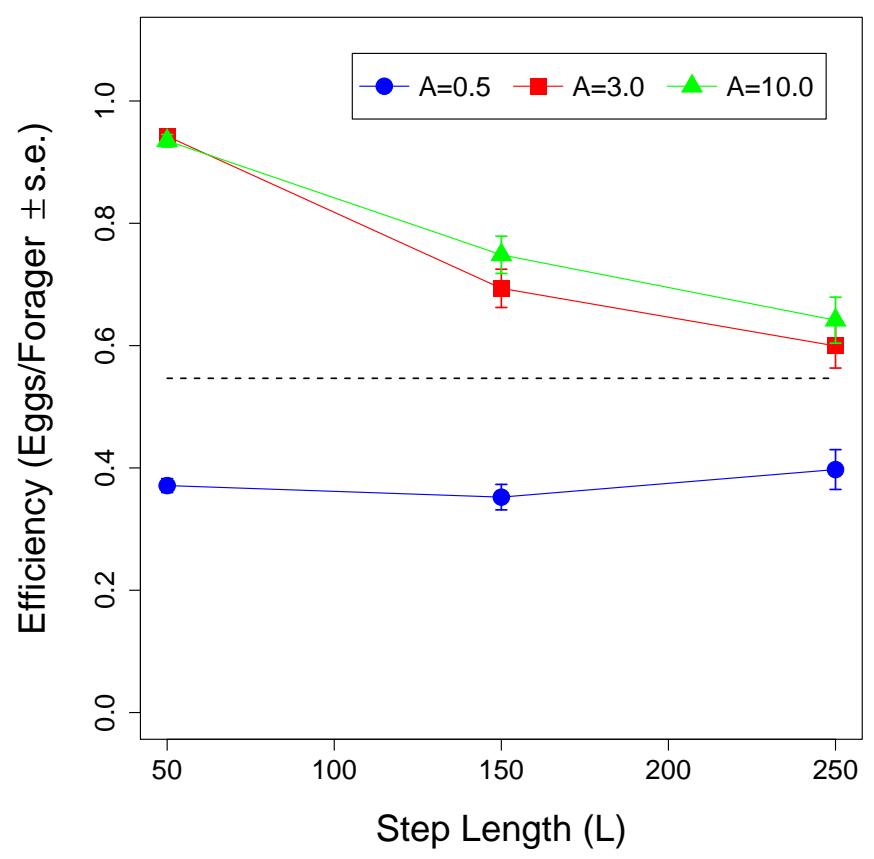

Figure A.3-8: Search efficiency vs. L and $k$ - Trial C (Olfaction), Single Egg. Proportion of eggs per forager released (y-axis) vs. step length $(L)$ for various turning angle concentrations $(k)$. Results are averaged over all combinations of $R$ and $P$. 
a)

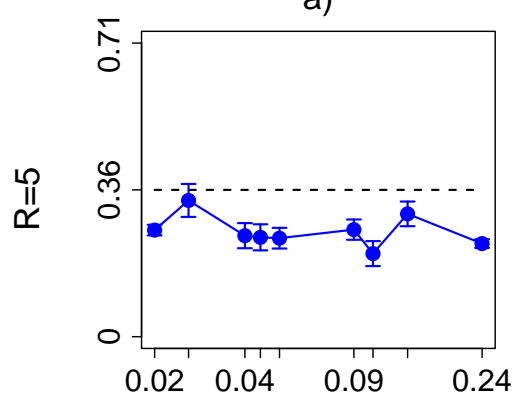

d)

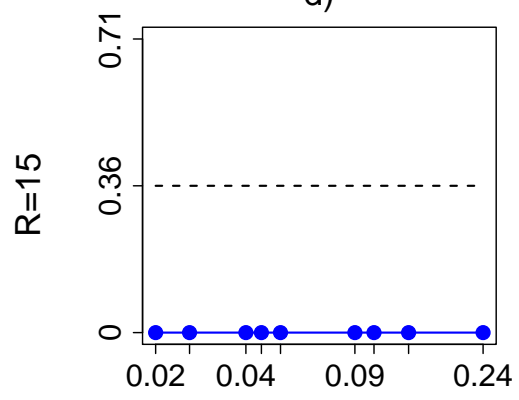

g)

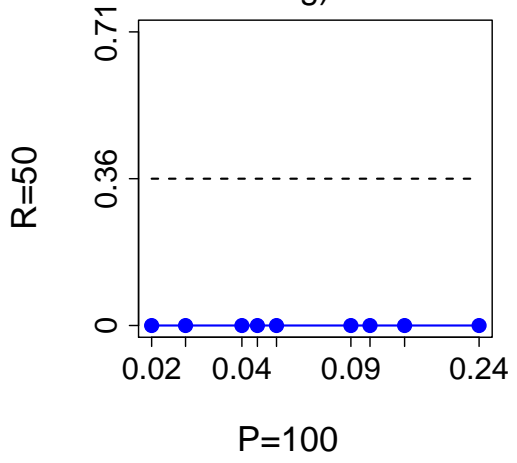

b)

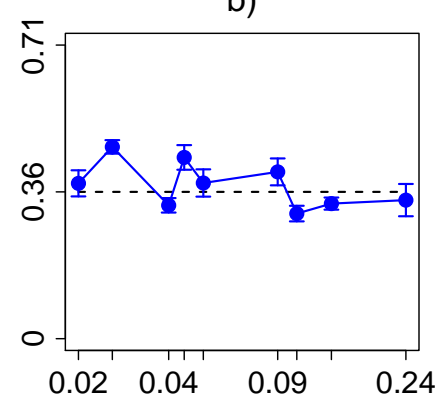

e)

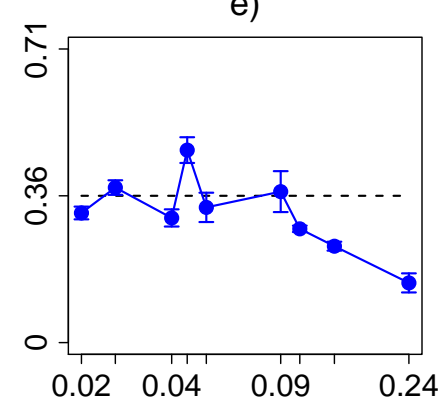

h)

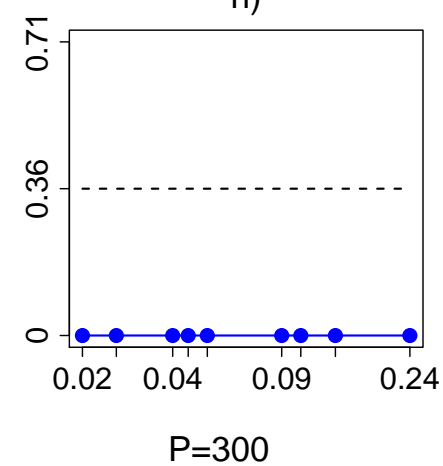

c)

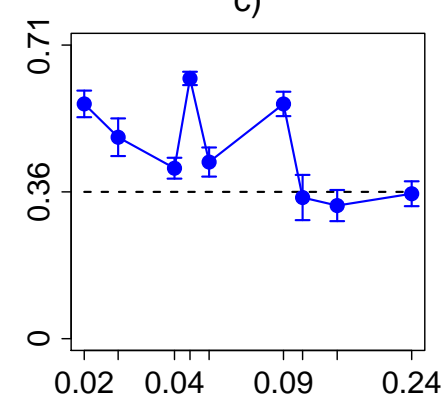

f)

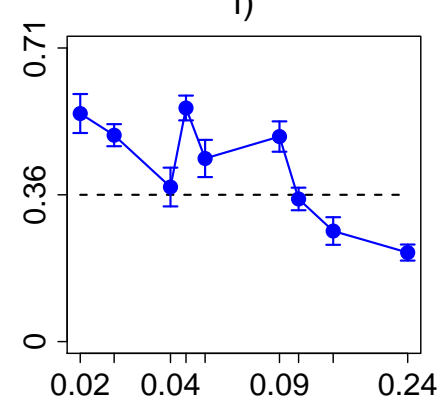

i)

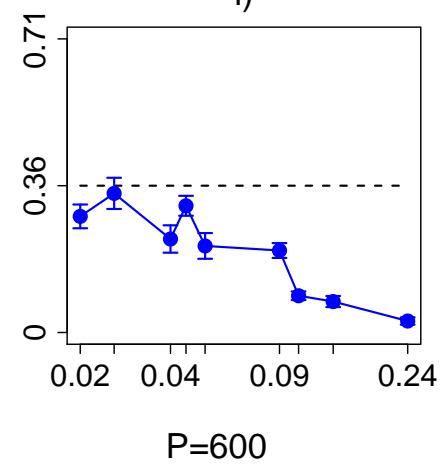

Figure A.3-9: Centre ratio vs. layout - Trial C (Olfaction), Single Egg. Proportion of eggs per plant on centre resources (y-axis) vs. Sinuosity is plotted for combinations of radius of attraction $(R)$ and patch size $(P)$ in each sub-plot $(a-i)$. Radius of attraction increases from top to bottom and patch size increases from left to right. The layout is such that these results may be compared with Figure 3.32 to see the patch layouts. 
a)

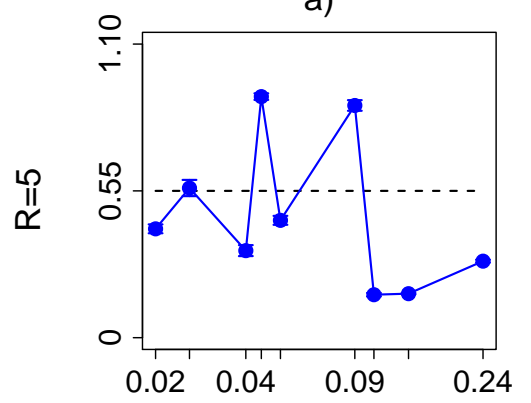

d)

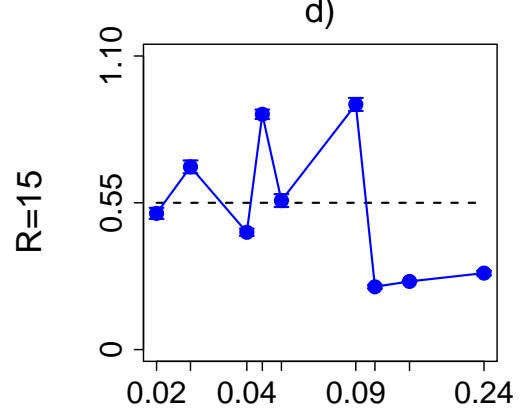

g)

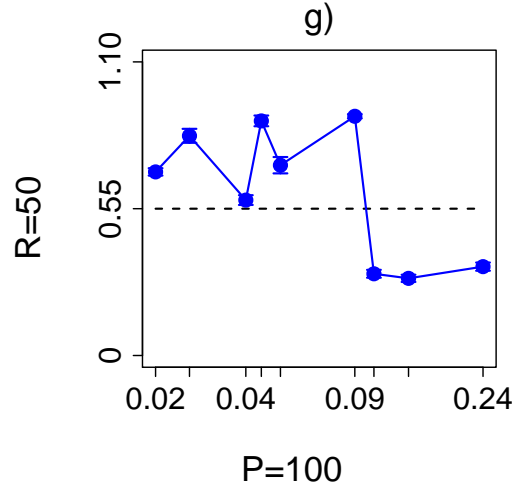

b)

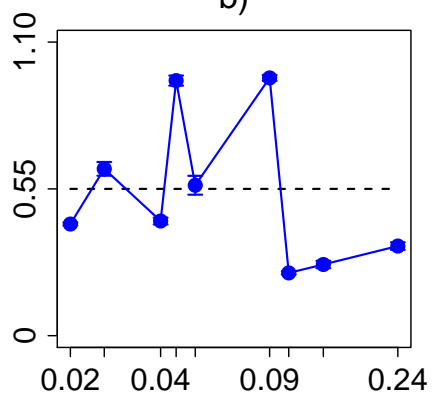

e)

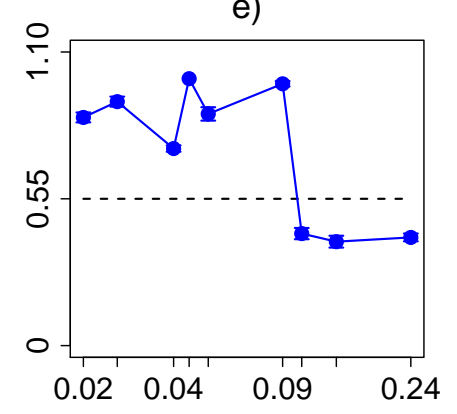

h)

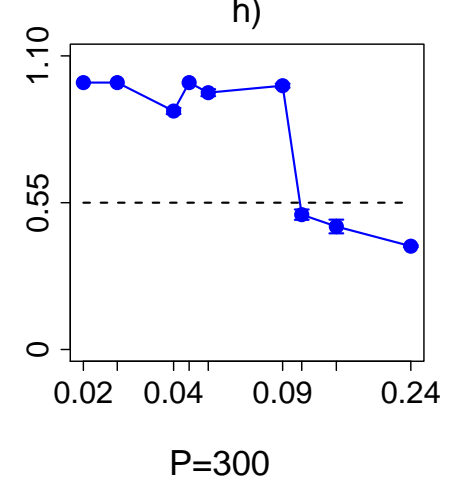

c)

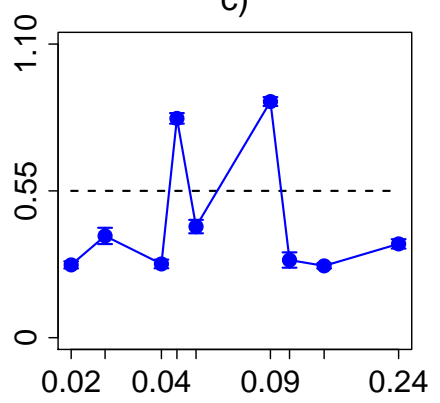

f)

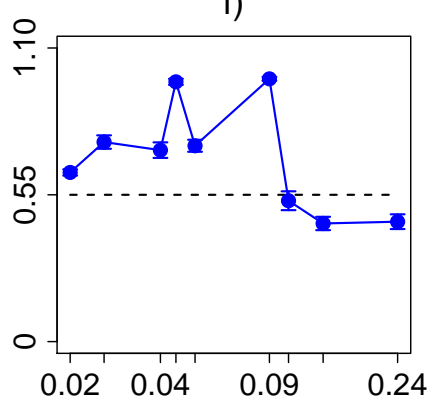

i)

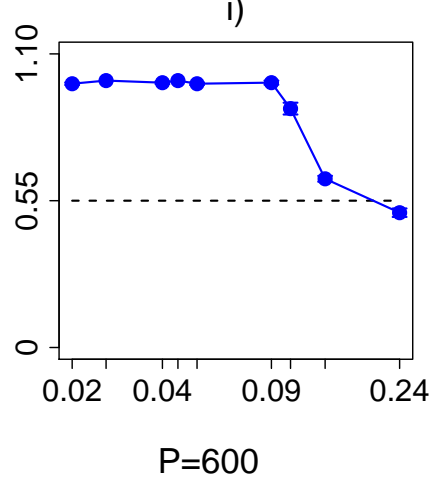

Figure A.3-10: Search efficiency vs. layout - Trial C (Olfaction), Single Egg. Proportion of eggs per forager released (y-axis) vs. sinuosity is plotted for combinations of radius of attraction $(R)$ and patch size $(P)$ in each sub-plot $(a-i)$. Radius of attraction increases from top to bottom and patch size increases from left to right. The layout is such that these results may be compared with Figure 3.32 to see the patch layouts. 


\section{A.4 Trial C (Olfaction) Multiple Eggs results}

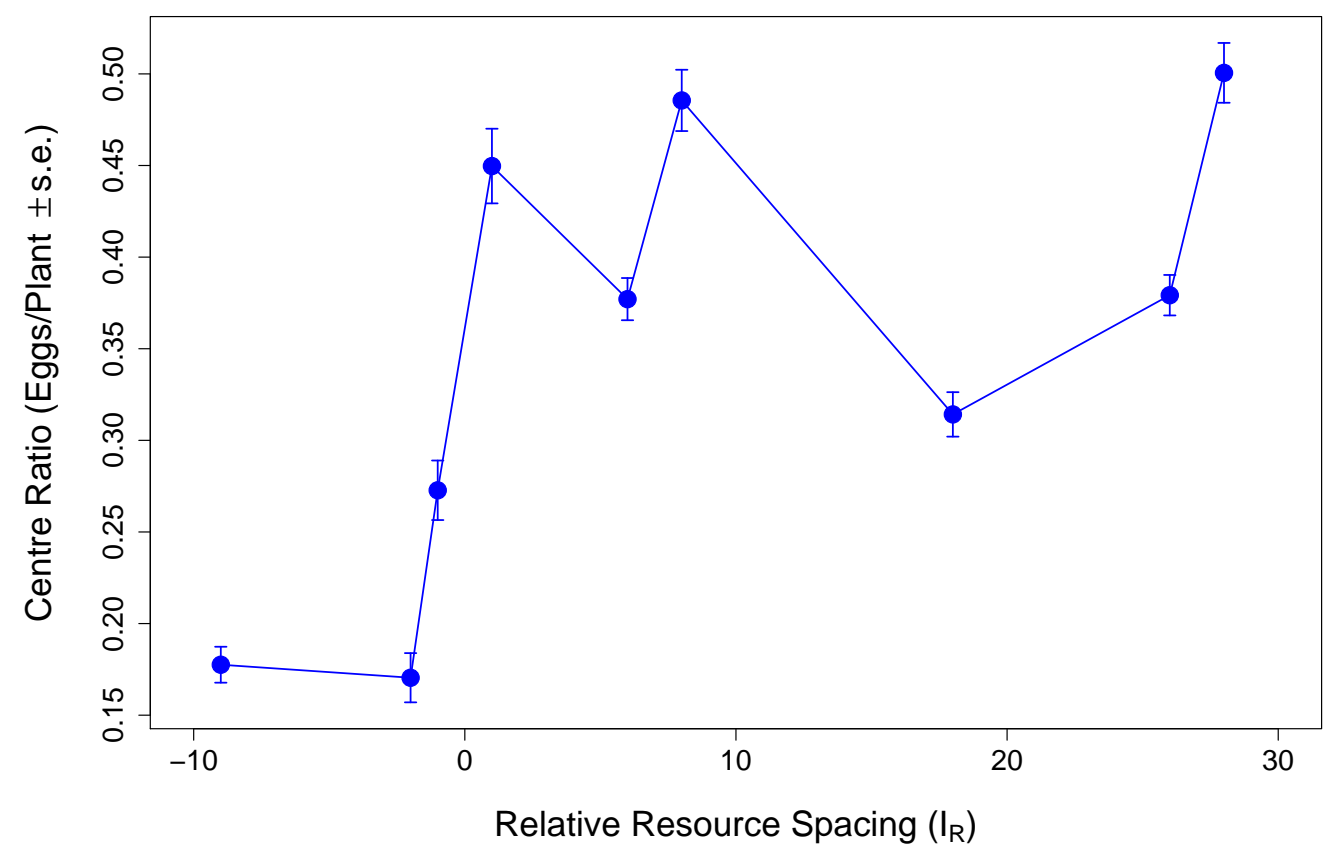

Figure A.4-1: Centre ratio summary - Trial C (Olfaction), Multiple Eggs. Proportion of eggs per plant on centre resources ( $y$-axis) vs. relative resource spacing, $I_{R}$ (x-axis). Negative values of $I_{R}$ indicate that the radii of attraction $(R)$ are overlapping.

a)

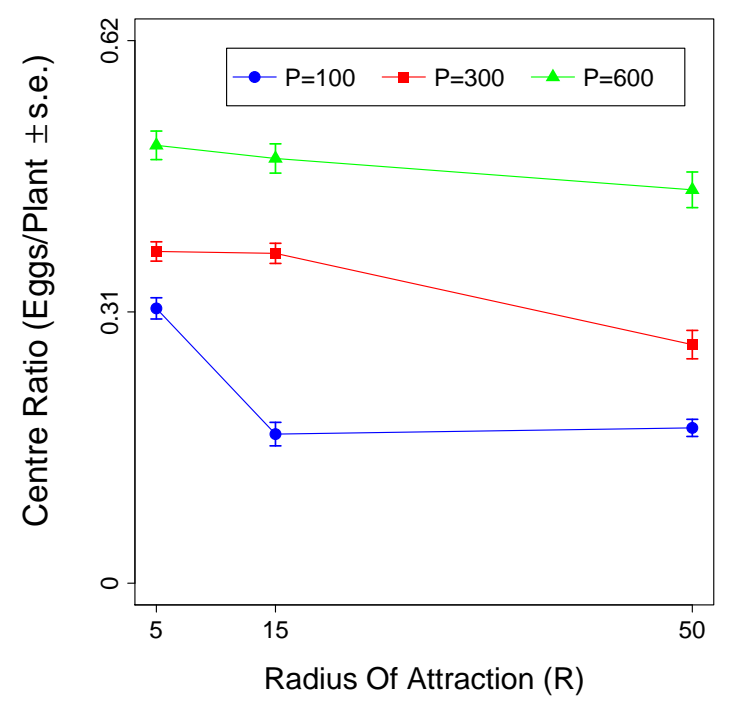

b)

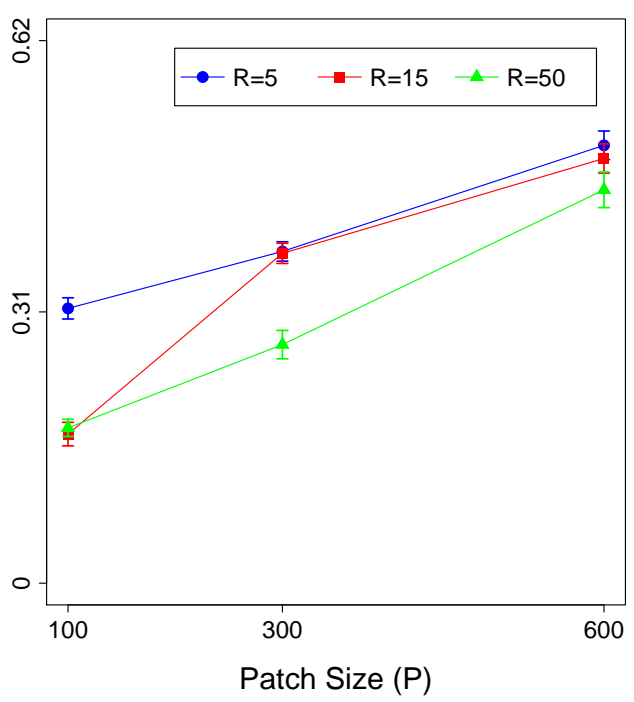

Figure A.4-2: Centre ratio vs. $R$ and P - Trial C (Olfaction), Multiple Eggs. Proportion of eggs per plant on centre resources ( $y$-axis) vs. a) radius of attraction $(R)$ and $b$ ) patch size $(P)$. Results are averaged over all combinations of $L$ and $k$. 


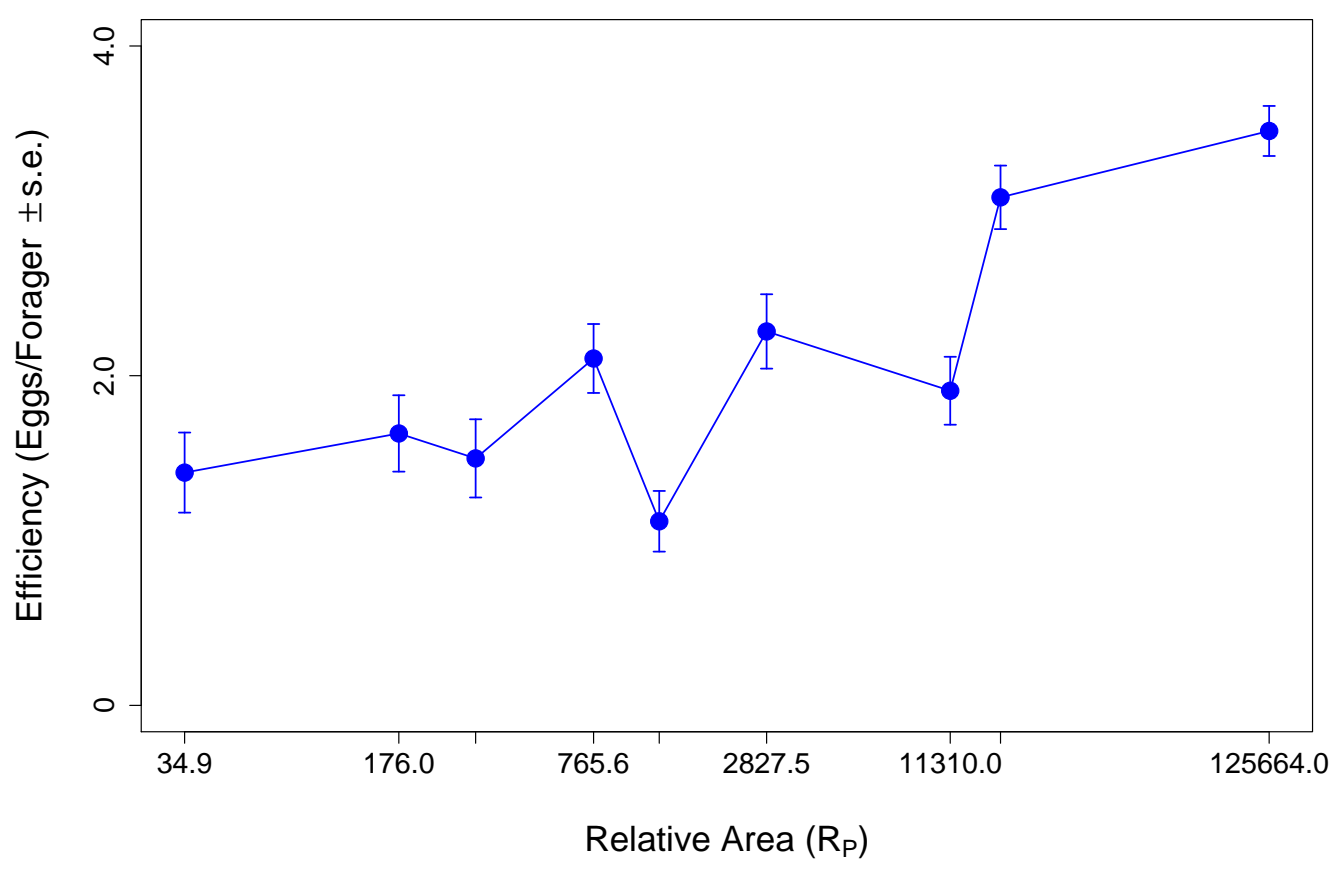

Figure A.4-3: Search Efficiency Summary - Trial C (Olfaction), Multiple Eggs. Proportion of eggs per Forager released (y-axis) vs. Relative Area $\left(R_{P}\right)$. See Chapter 3 details of this calculation.

a)

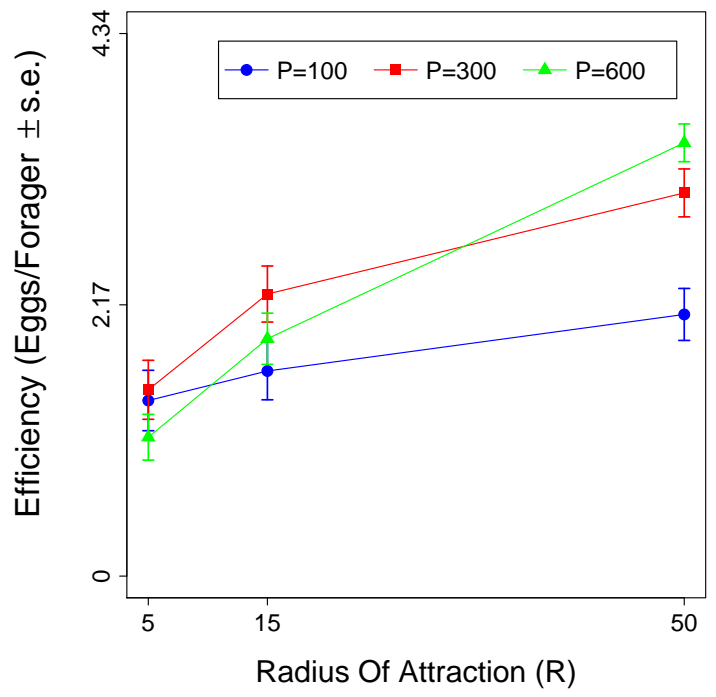

b)

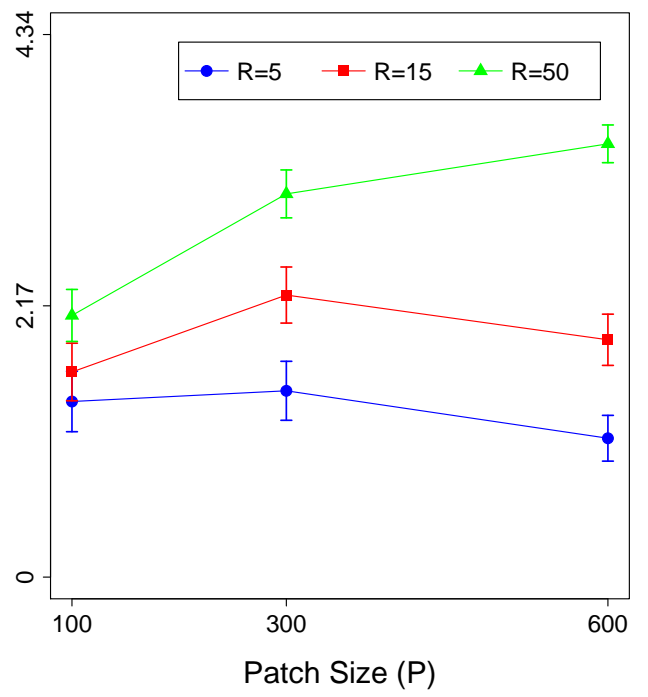

Figure A.4-4: Search efficiency vs. R and P - Trial C (Olfaction), Multiple Eggs. Proportion of eggs per forager released (y-axis) vs. a) radius of attraction $(R)$ and b) patch size $(P)$. Results are averaged over all combinations of $L$ and $k$. 


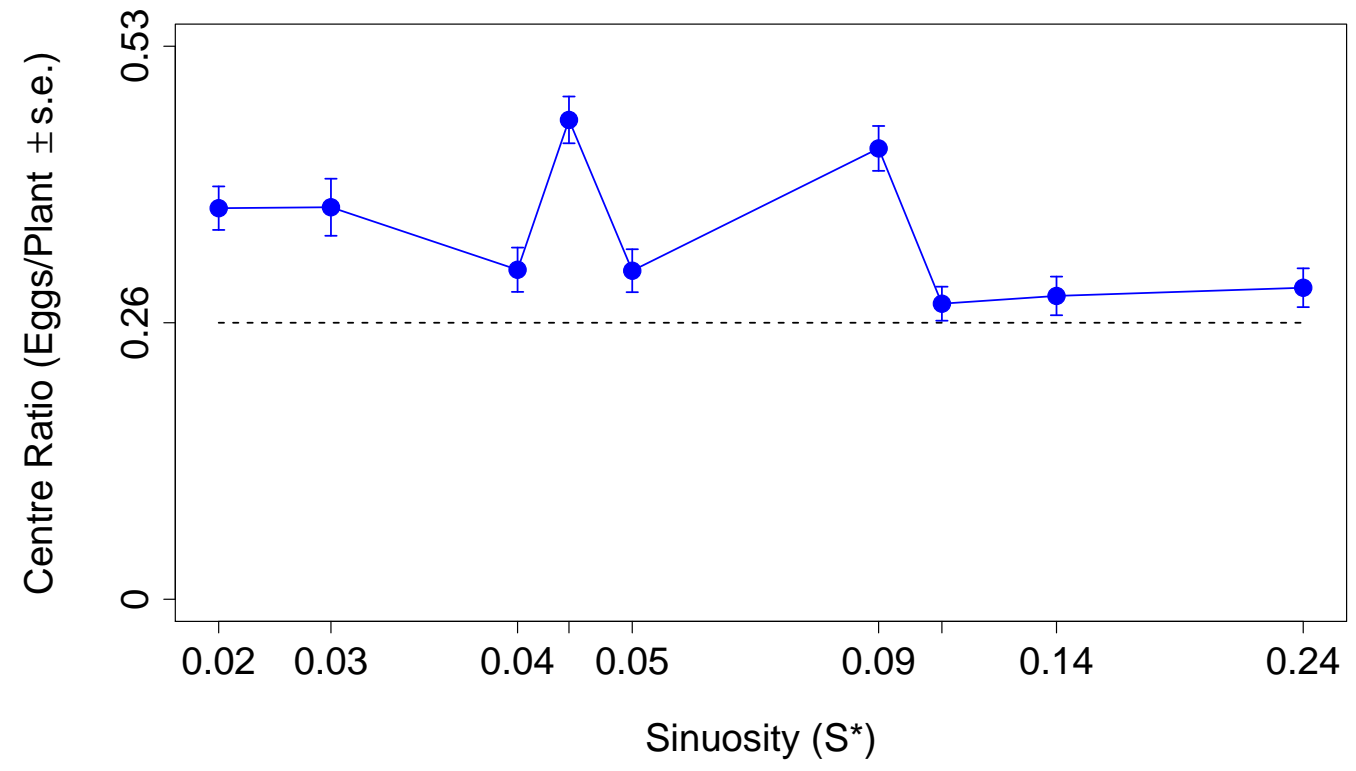

Figure A.4-5: Centre ratio vs. sinuosity - Trial C (Olfaction), Multiple Eggs. Proportion of eggs per plant on centre resources (y-axis) vs. Sinuosity $(S *)$ of forager paths. Sinuosity is a measure composed of both step length $(L)$ and turning angle concentration $(k)$

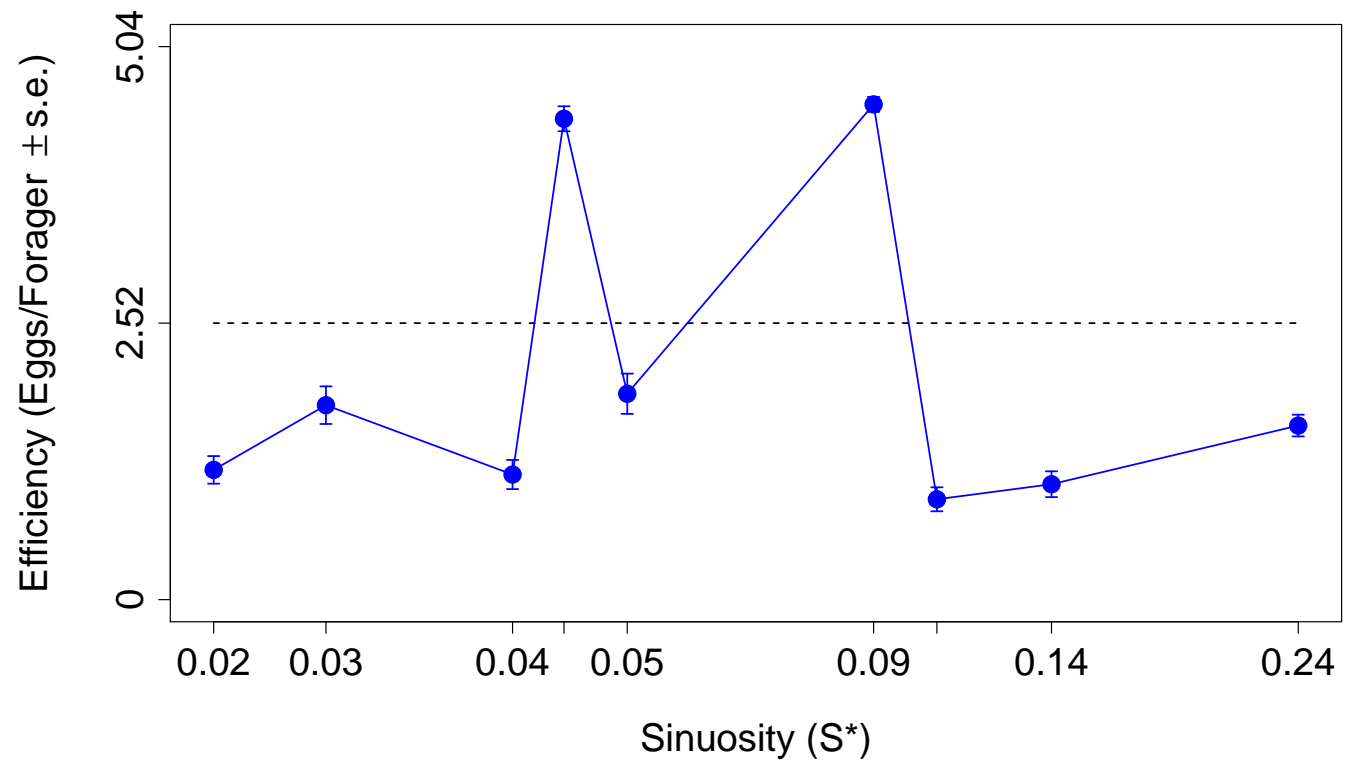

Figure A.4-6: Search efficiency vs. sinuosity - Trial C (Olfaction), Multiple Eggs. Proportion of eggs per forager released (y-axis) vs. a) radius of attraction $(R)$ and $b$ ) patch size $(P)$ 


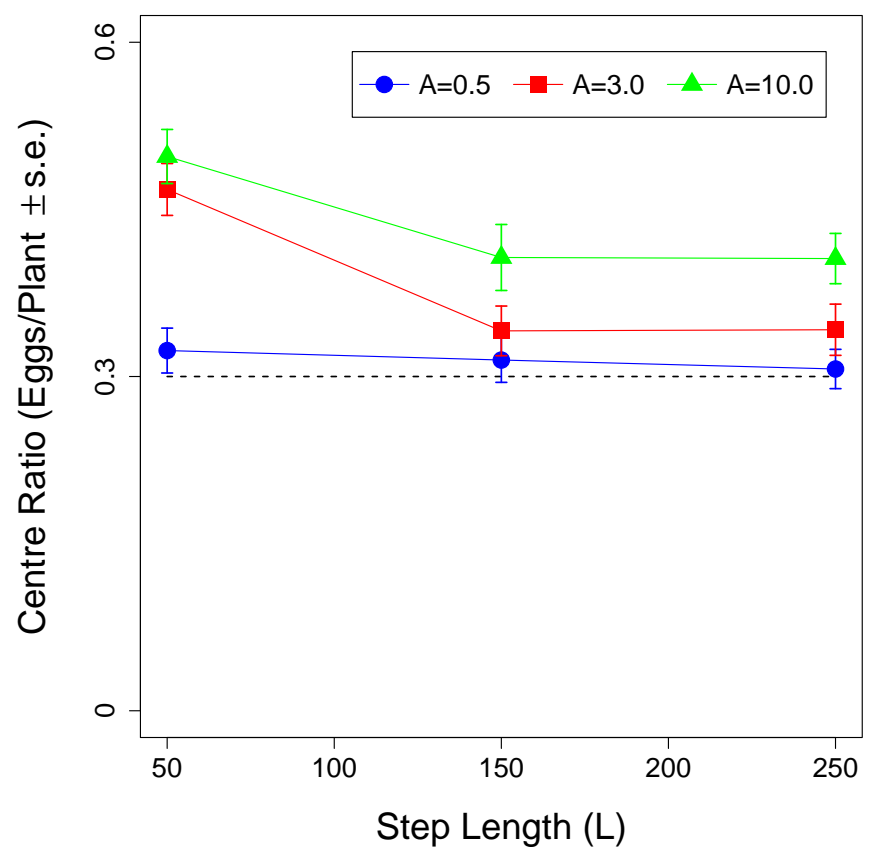

Figure A.4-7: Centre ratio vs. L and $k$ - Trial C (Olfaction), Multiple Eggs. Proportion of eggs per plant on centre resources (y-axis) vs. step length $(L)$ for various turning angle concentrations $(k)$. Results are averaged over all combinations of $R$ and $P$.

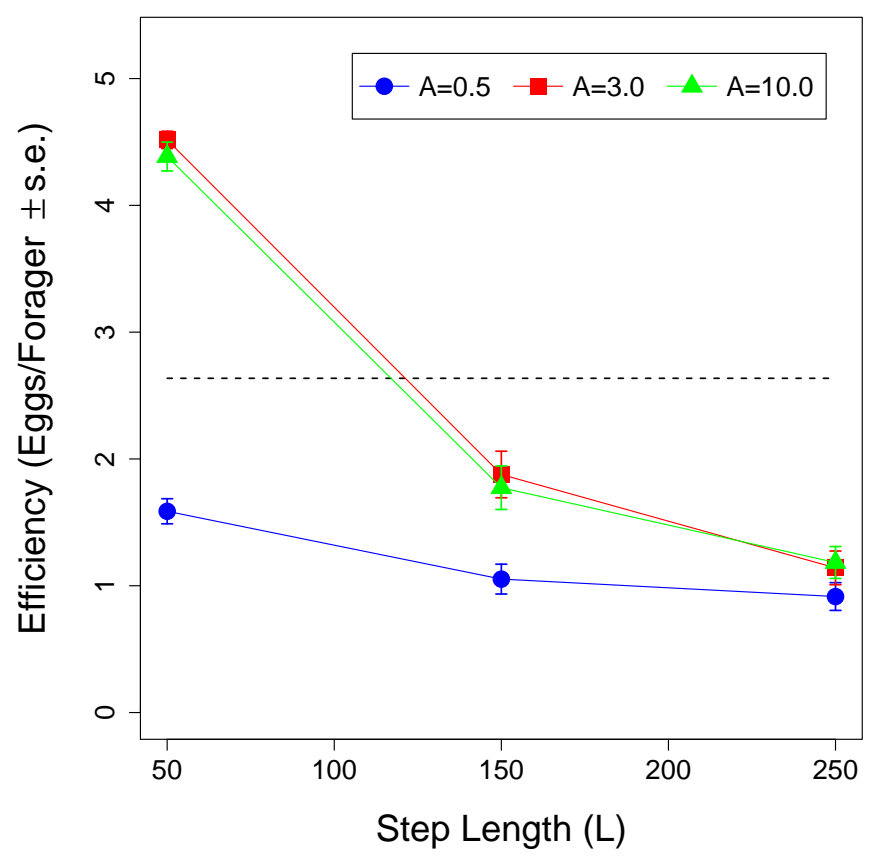

Figure A.4-8: Search efficiency vs. L and $k$ - Trial C (Olfaction), Multiple Eggs. Proportion of eggs per forager released (y-axis) ws. step length $(L)$ for various turning angle concentrations $(k)$. Results are averaged over all combinations of $R$ and $P$. 
a)

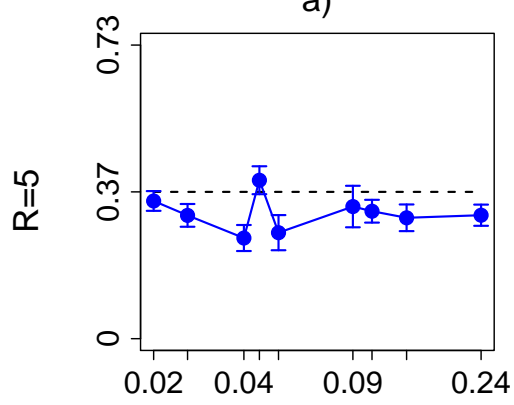

d)

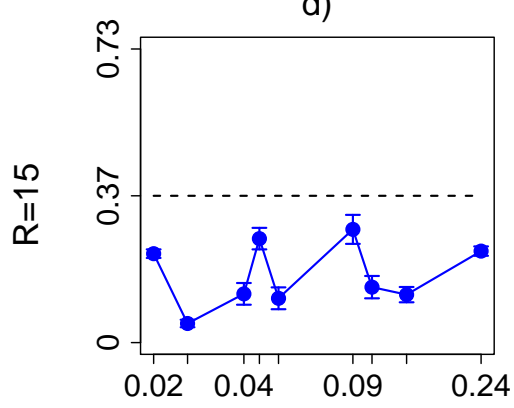

g)

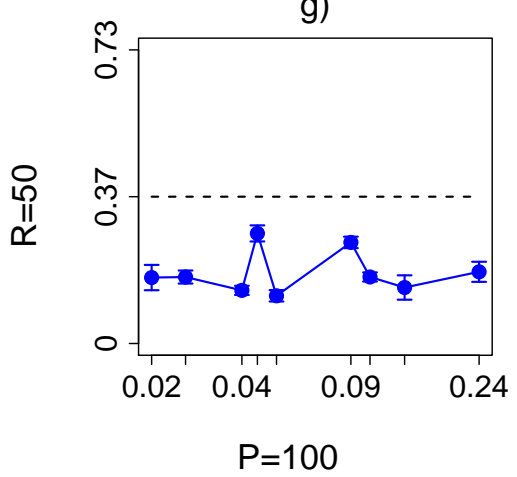

b)

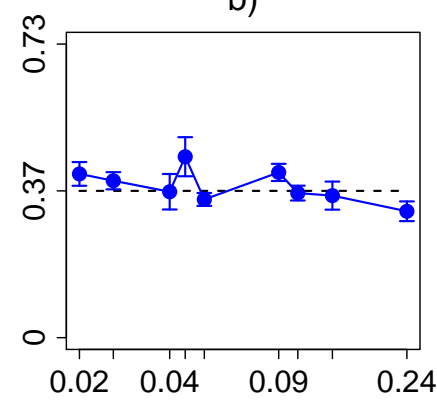

e)

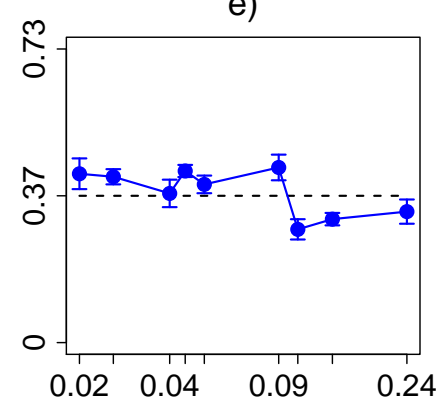

h)

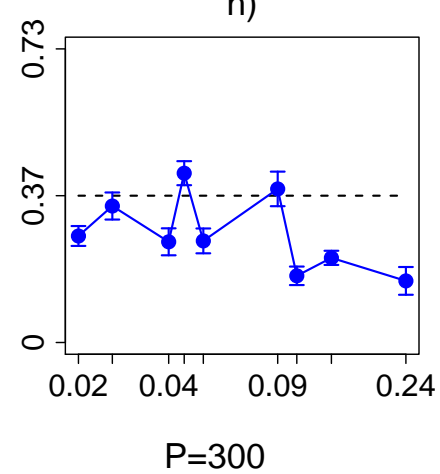

c)

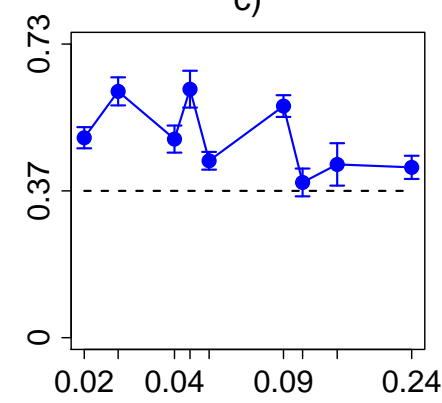

f)

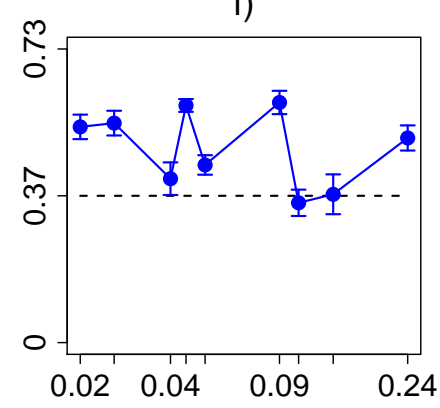

i)

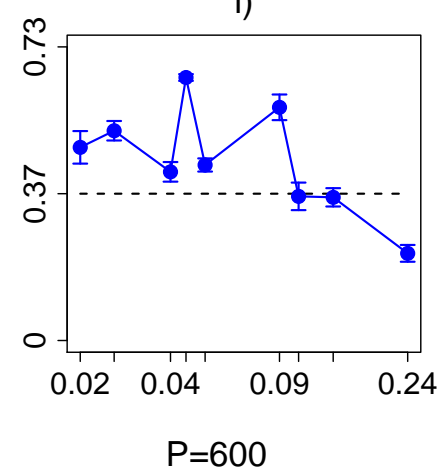

Figure A.4-9: Centre ratio vs. layout - Trial C (Olfaction), Multiple Eggs. Proportion of eggs per plant on centre resources (y-axis) vs. Sinuosity is plotted for combinations of radius of attraction $(R)$ and patch size $(P)$ in each sub-plot $(a-i)$. Radius of attraction increases from top to bottom and patch size increases from left to right. The layout is such that these results may be compared with Figure 3.32 to see the patch layouts. 
a)

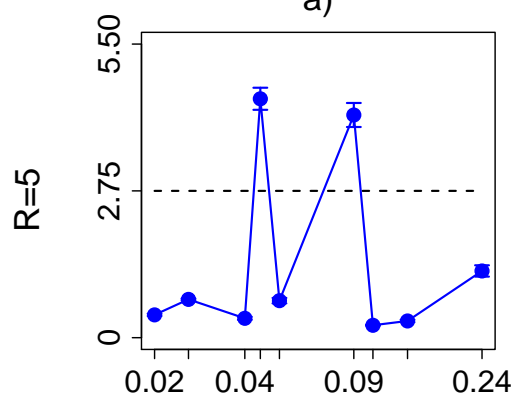

d)

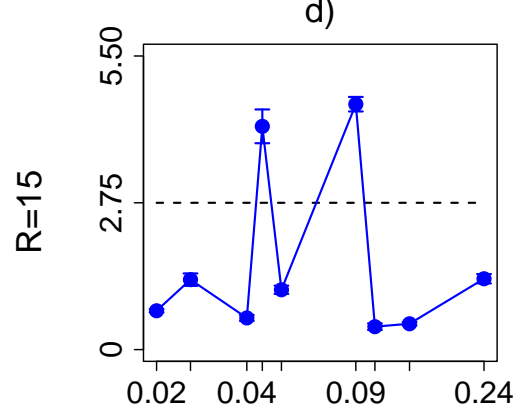

g)

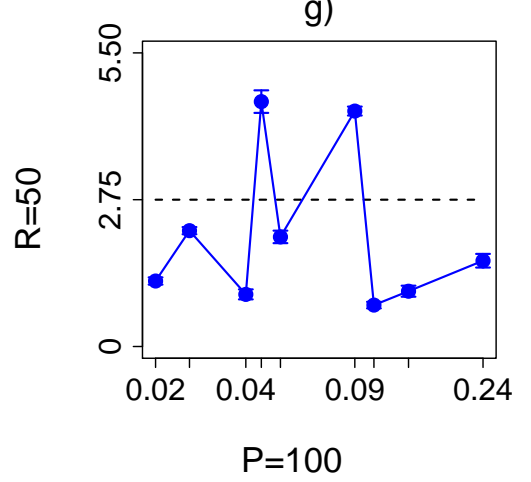

b)

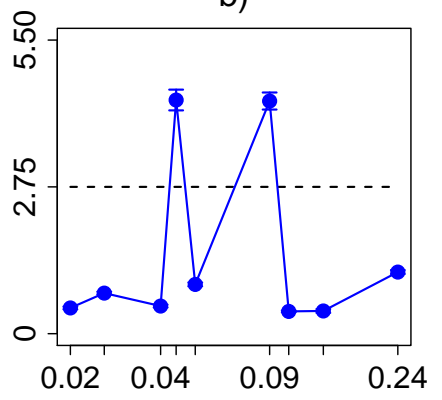

e)

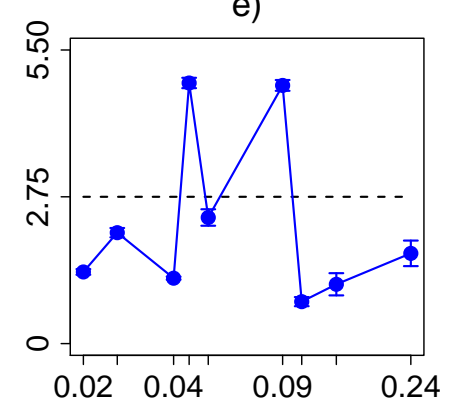

h)

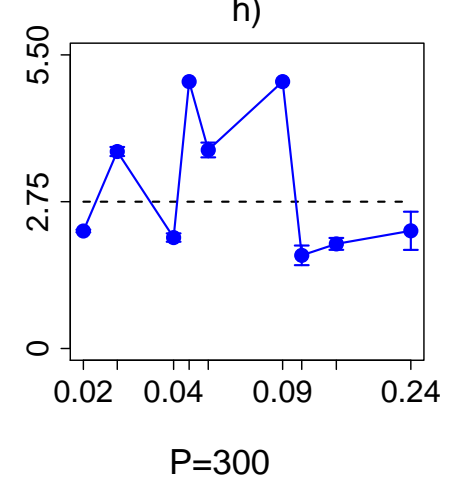

c)

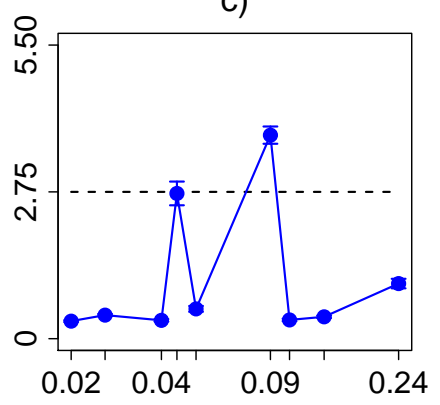

f)

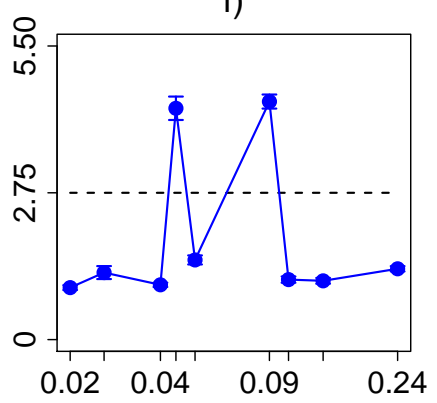

i)

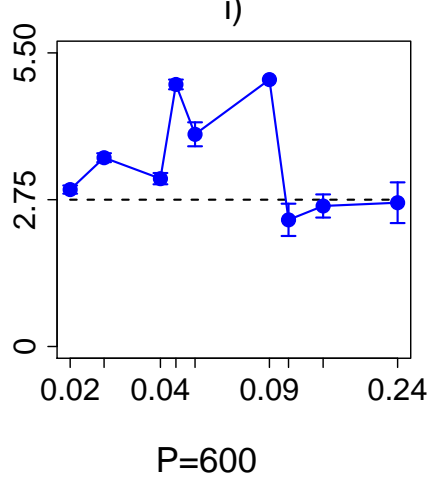

Figure A.4-10: Search efficiency vs. layout - Trial C (Olfaction), Multiple Eggs. Proportion of eggs per forager released (y-axis) vs. sinuosity is plotted for combinations of radius of attraction $(R)$ and patch size $(P)$ in each sub-plot $(a-i)$. Radius of attraction increases from top to bottom and patch size increases from left to right. The layout is such that these results may be compared with Figure 3.32 to see the patch layouts. 


\section{A.5 Trial D (Vision) Single Egg results}

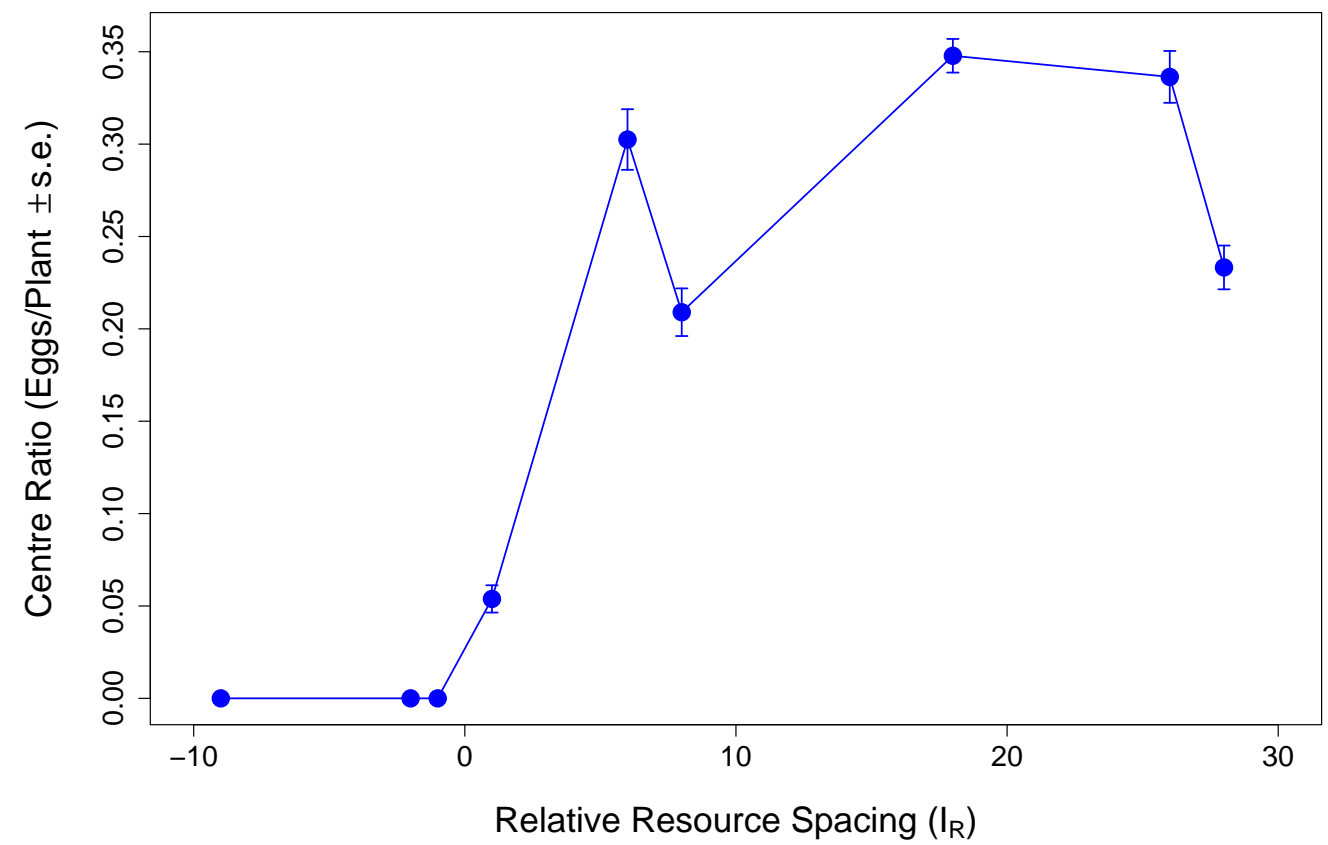

Figure A.5-1: Centre ratio summary - Trial D (Vision), Single Egg. Proportion of eggs per plant on centre resources ( $y$-axis) vs. relative resource spacing, $I_{R}$ ( $x$-axis). Negative values of $I_{R}$ indicate that the radii of attraction $(R)$ are overlapping.

a)

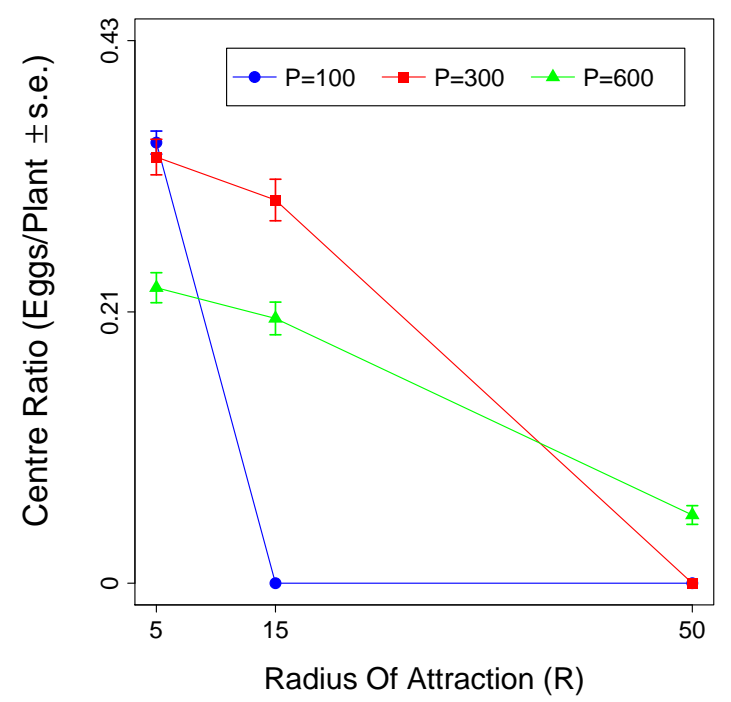

b)

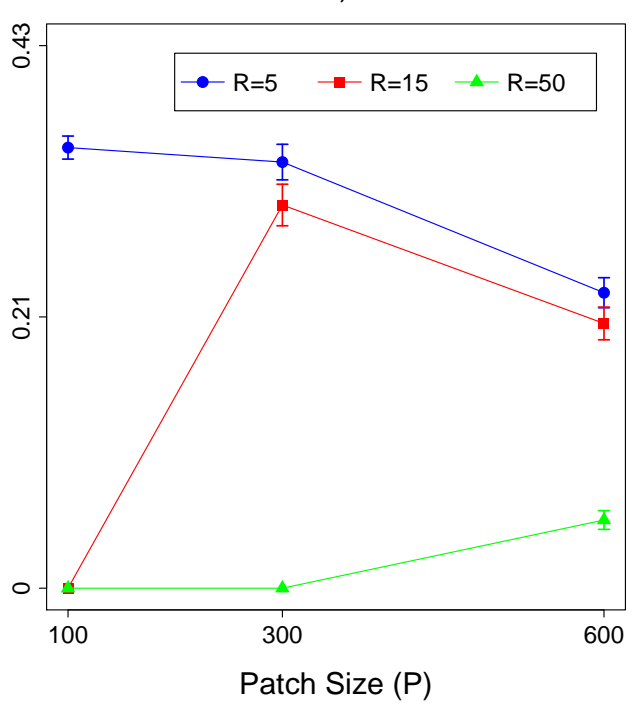

Figure A.5-2: Centre ratio vs. $R$ and $P$ - Trial D (Vision), Single Egg. Proportion of eggs per plant on centre resources (y-axis) vs. a) radius of attraction $(R)$ and $b)$ patch size $(P)$. Results are averaged over all combinations of $L$ and $k$. 


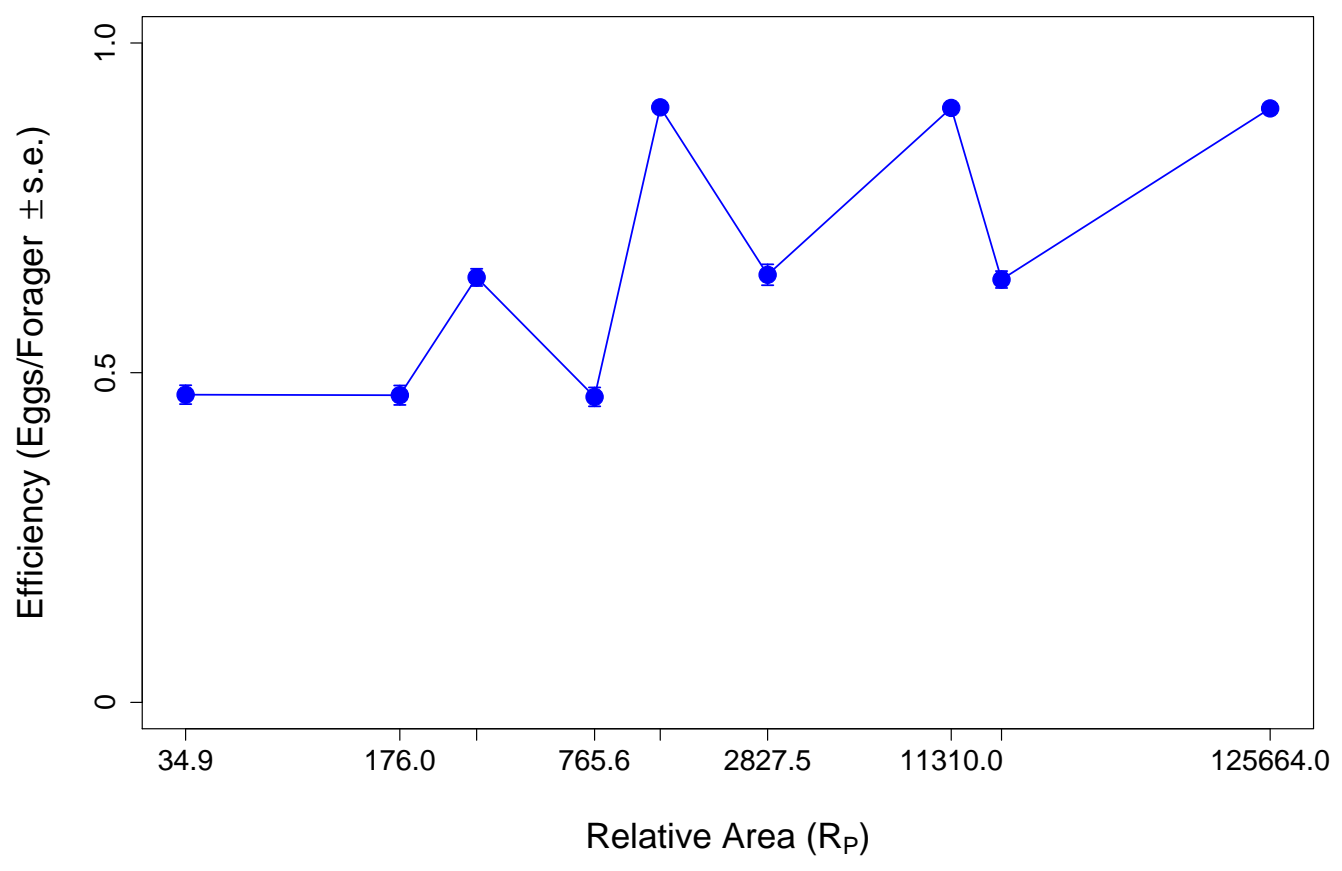

Figure A.5-3: Search Efficiency Summary - Trial D (Vision), Single Egg. Proportion of eggs per Forager released (y-axis) vs. Relative Area $\left(R_{P}\right)$. See Chapter 3 details of this calculation.

a)

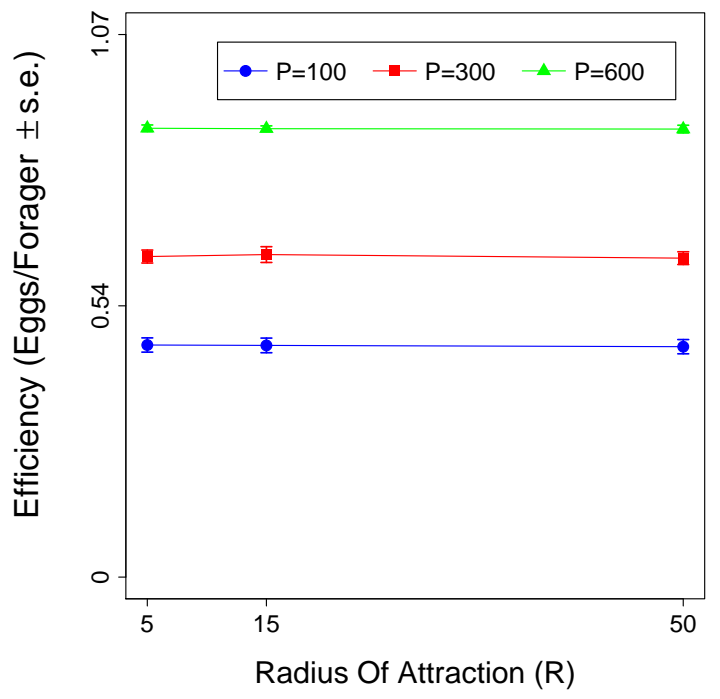

b)

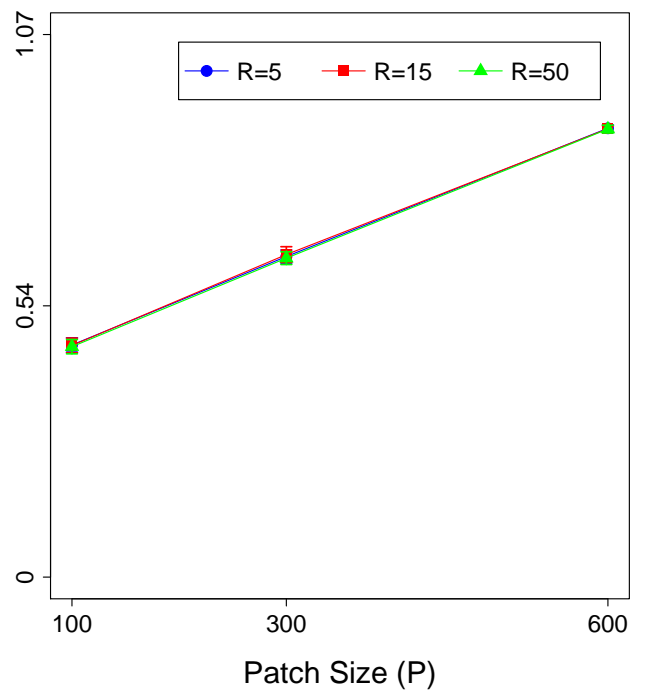

Figure A.5-4: Search efficiency vs. $R$ and P - Trial D (Vision), Single Egg. Proportion of eggs per forager released (y-axis) vs. a) radius of attraction $(R)$ and $b$ ) patch size $(P)$. Results are averaged over all combinations of $L$ and $k$. 


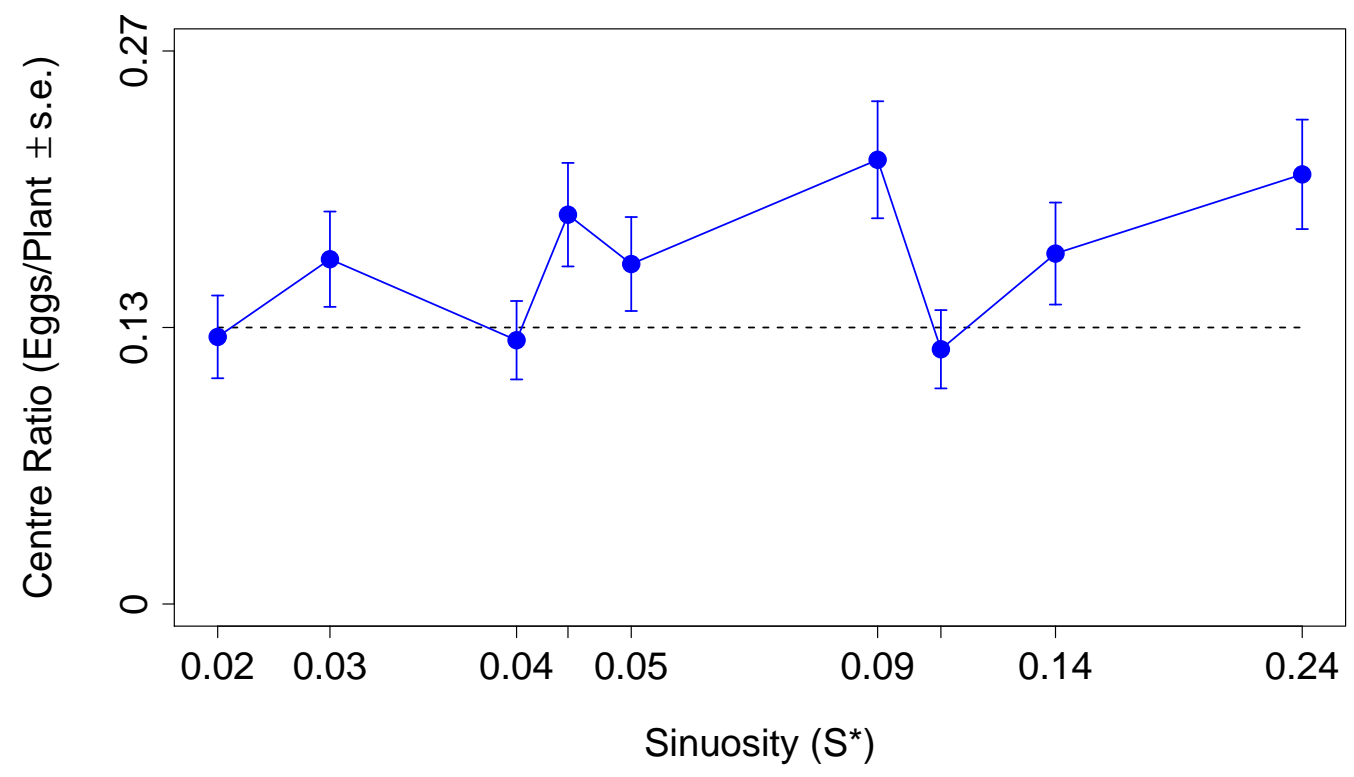

Figure A.5-5: Centre ratio vs. sinuosity - Trial D (Vision), Single Egg. Proportion of eggs per plant on centre resources (y-axis) vs. Sinuosity ( $S *)$ of forager paths. Sinuosity is a measure composed of both step length $(L)$ and turning angle concentration $(k)$

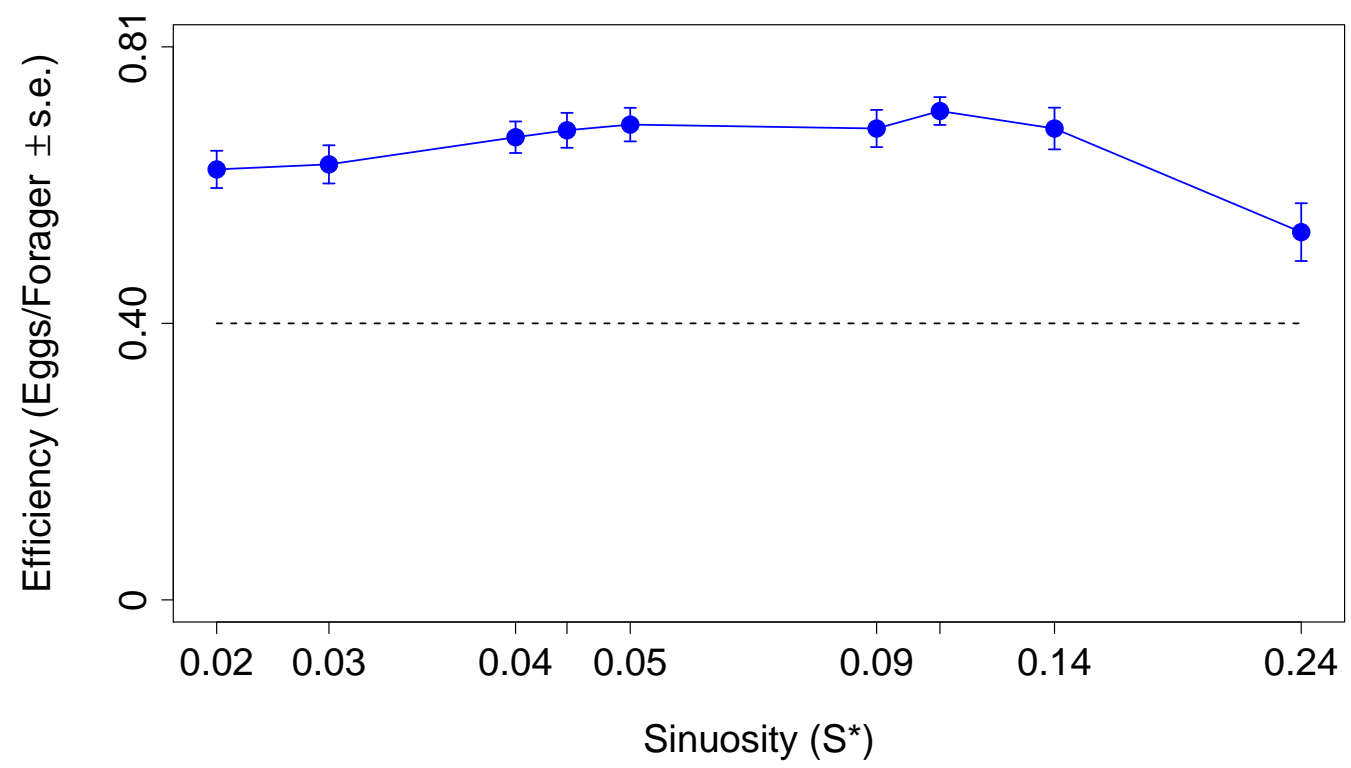

Figure A.5-6: Search efficiency vs. sinuosity - Trial D (Vision), Single Egg. Proportion of eggs per forager released ( $y$-axis) vs. a) radius of attraction $(R)$ and $b$ ) patch size $(P)$ 


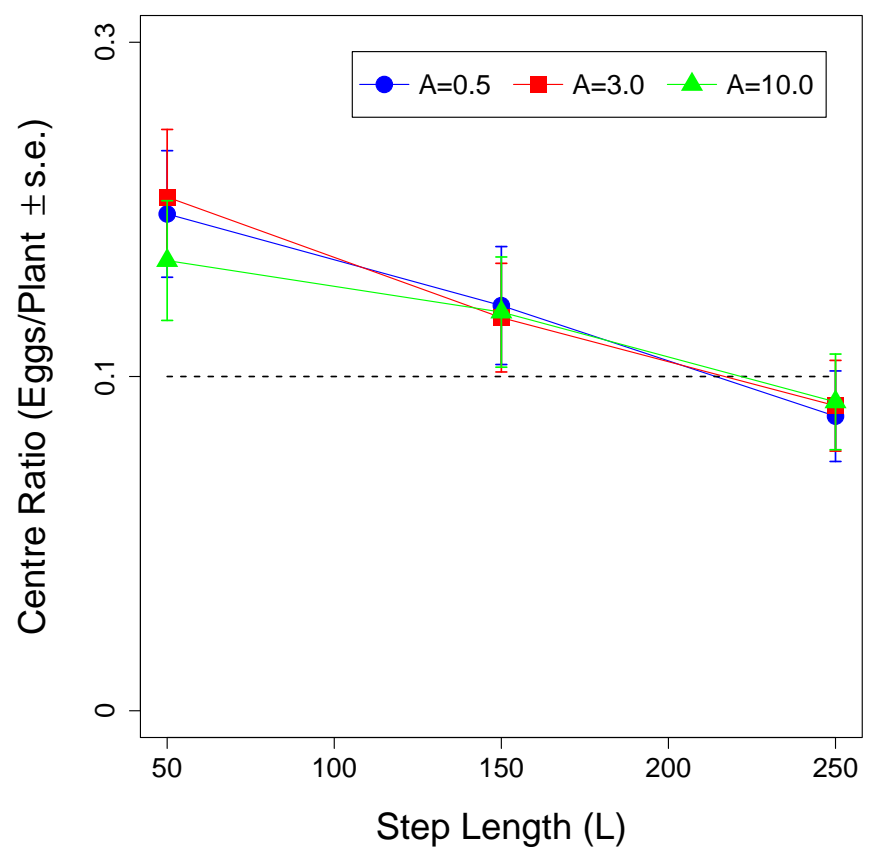

Figure A.5-7: Centre ratio vs. L and $k$ - Trial D (Vision), Single Egg. Proportion of eggs per plant on centre resources (y-axis) vs. step length $(L)$ for various turning angle concentrations $(k)$. Results are averaged over all combinations of $R$ and $P$.

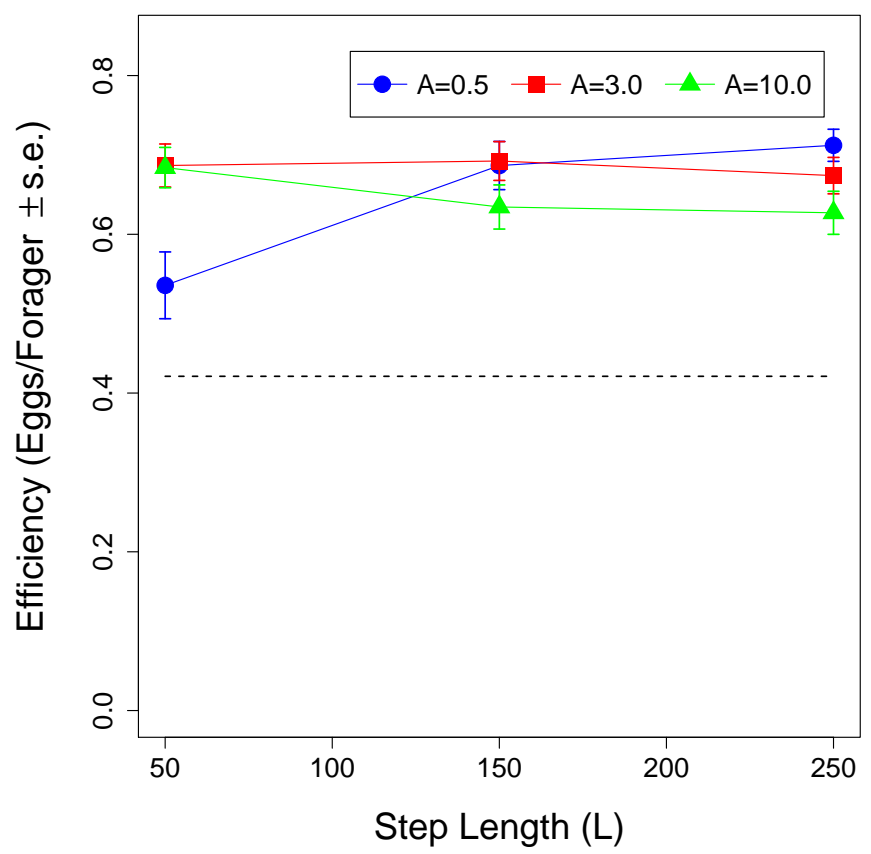

Figure A.5-8: Search efficiency vs. L and k- Trial D (Vision), Single Egg. Proportion of eggs per forager released (y-axis) vs. step length $(L)$ for various turning angle concentrations $(k)$. Results are averaged over all combinations of $R$ and $P$. 

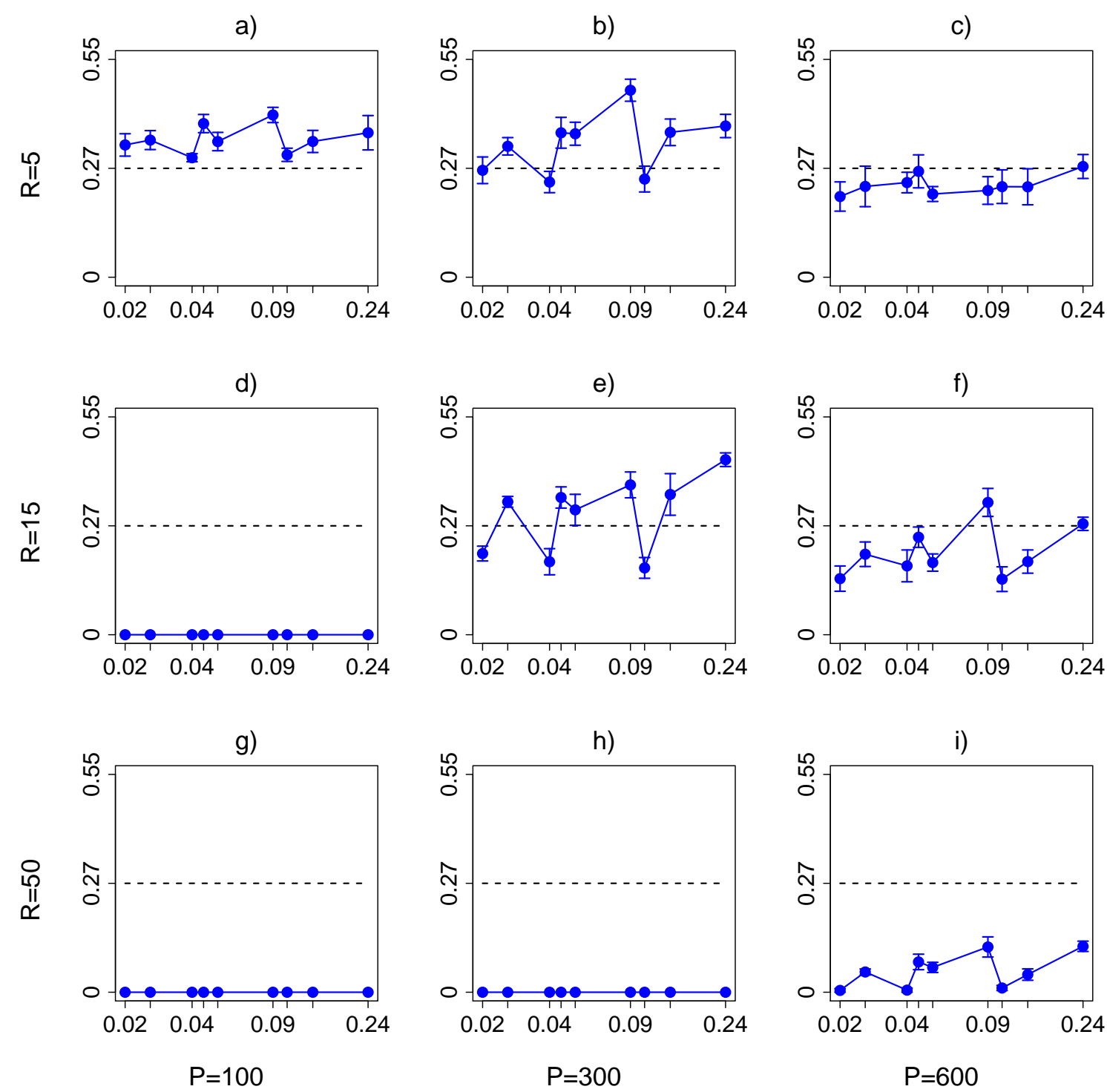

Figure A.5-9: Centre ratio vs. layout - Trial D (Vision), Single Egg. Proportion of eggs per plant on centre resources (y-axis) vs. Sinuosity is plotted for combinations of radius of attraction $(R)$ and patch size $(P)$ in each sub-plot $(a-i)$. Radius of attraction increases from top to bottom and patch size increases from left to right. The layout is such that these results may be compared with Figure 3.32 to see the patch layouts. 
a)

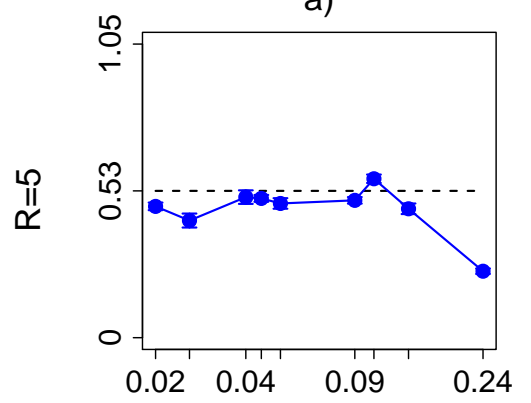

d)

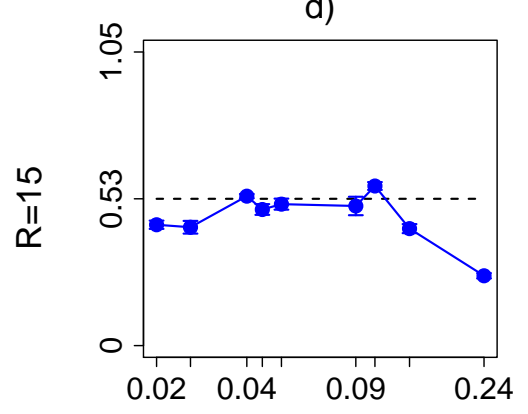

g)

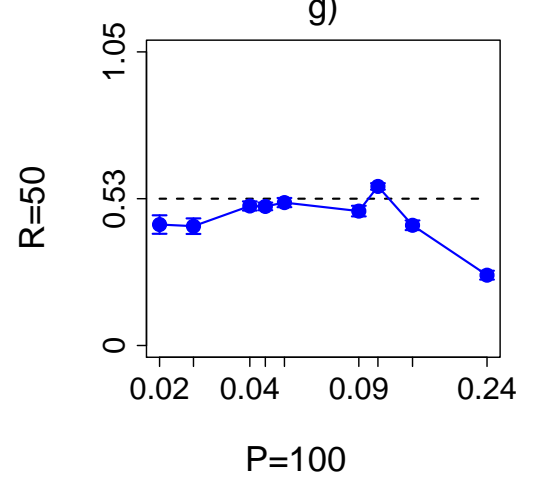

b)

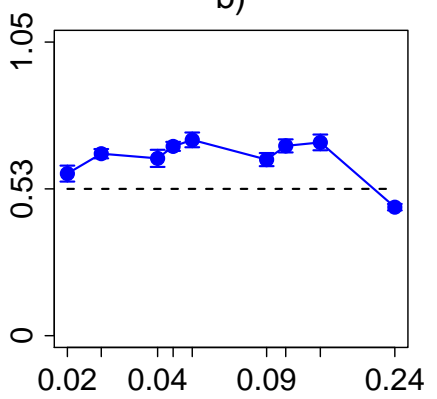

e)

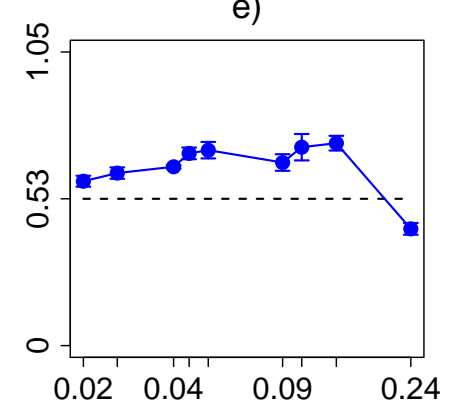

h)

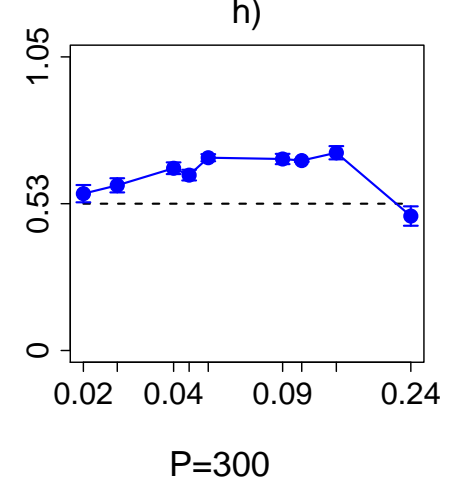

c)

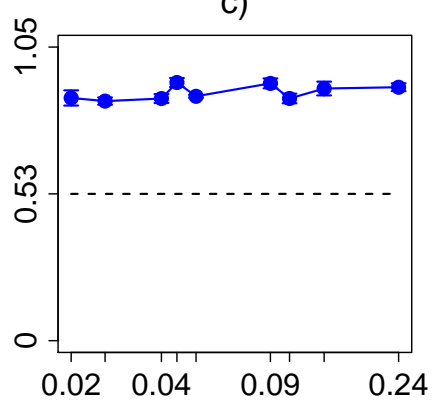

f)

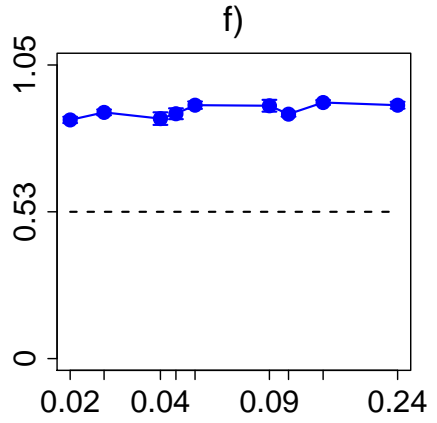

i)

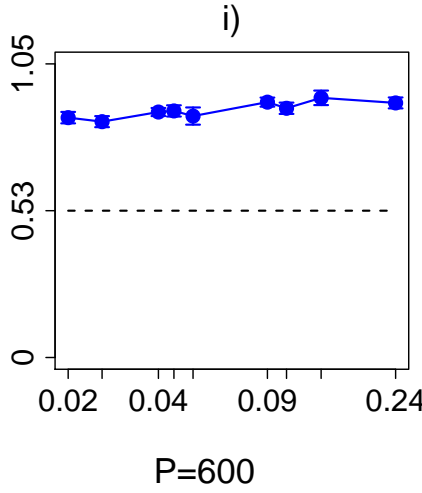

Figure A.5-10: Search efficiency vs. layout - Trial D (Vision), Single Egg. Proportion of eggs per forager released (y-axis) vs. sinuosity is plotted for combinations of radius of attraction $(R)$ and patch size $(P)$ in each sub-plot $(a-i)$. Radius of attraction increases from top to bottom and patch size increases from left to right. The layout is such that these results may be compared with Figure 3.32 to see the patch layouts. 


\section{A.6 Trial D (Vision) Multiple Eggs results}

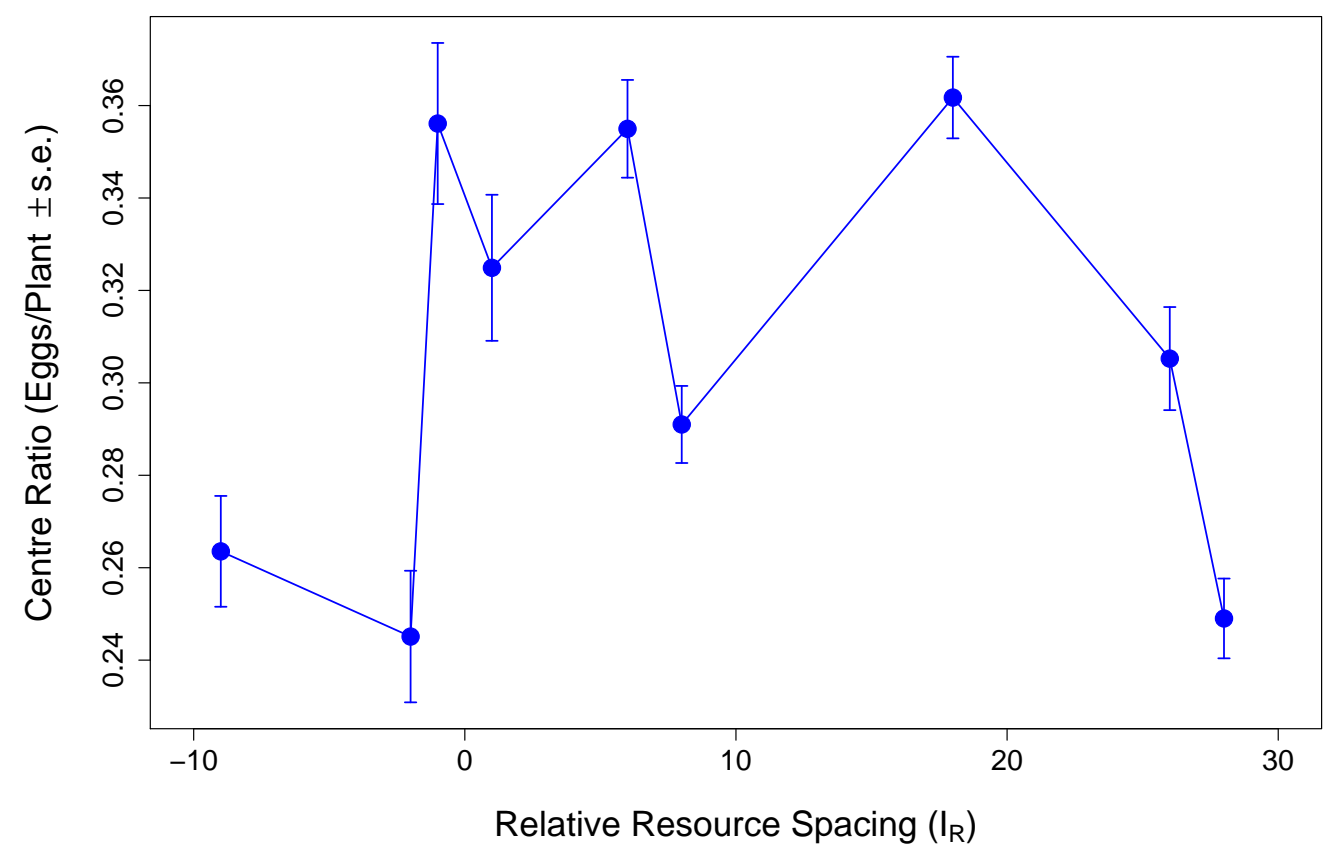

Figure A.6-1: Centre ratio summary - Trial D (Vision), Multiple Eggs. Proportion of eggs per plant on centre resources ( $y$-axis) vs. relative resource spacing, $I_{R}$ (x-axis). Negative values of $I_{R}$ indicate that the radii of attraction $(R)$ are overlapping.

a)

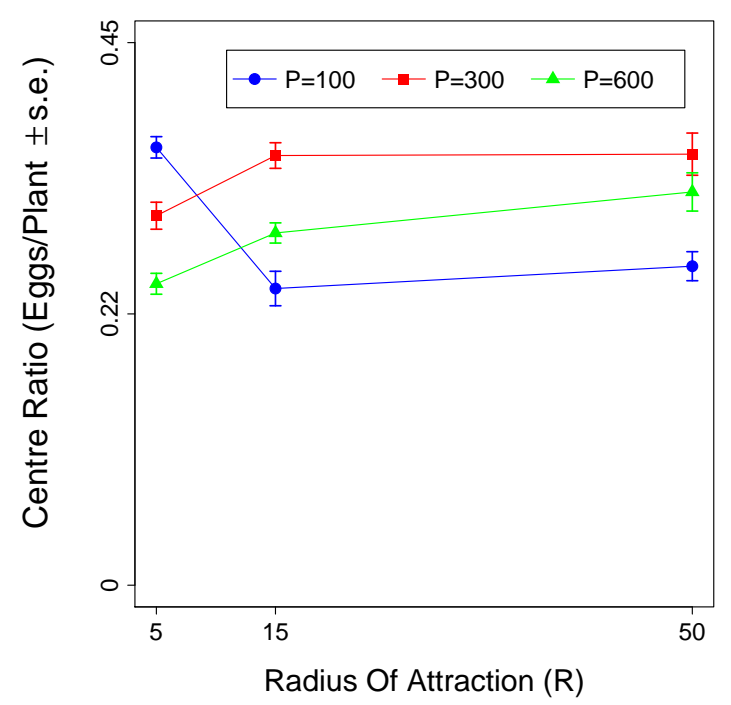

b)

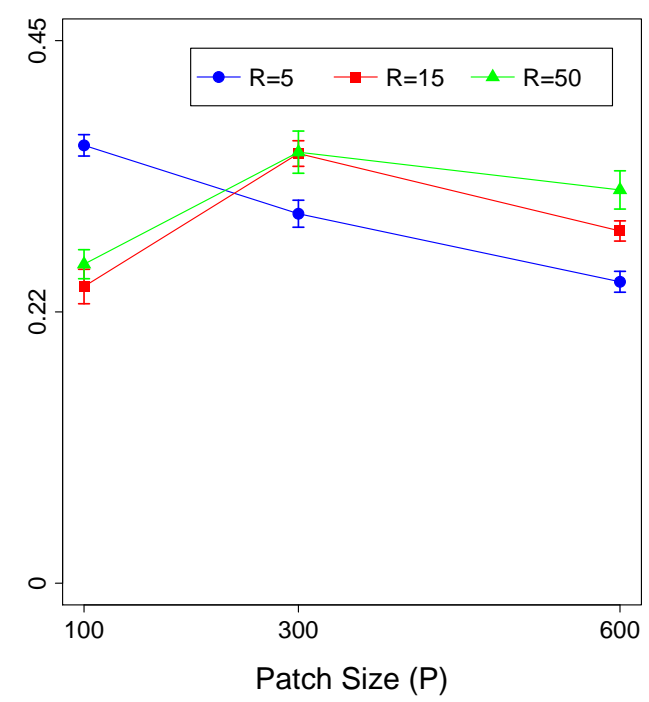

Figure A.6-2: Centre ratio vs. $R$ and P - Trial D (Vision), Multiple Eggs. Proportion of eggs per plant on centre resources ( $y$-axis) vs. a) radius of attraction $(R)$ and $b$ ) patch size $(P)$. Results are averaged over all combinations of $L$ and $k$. 


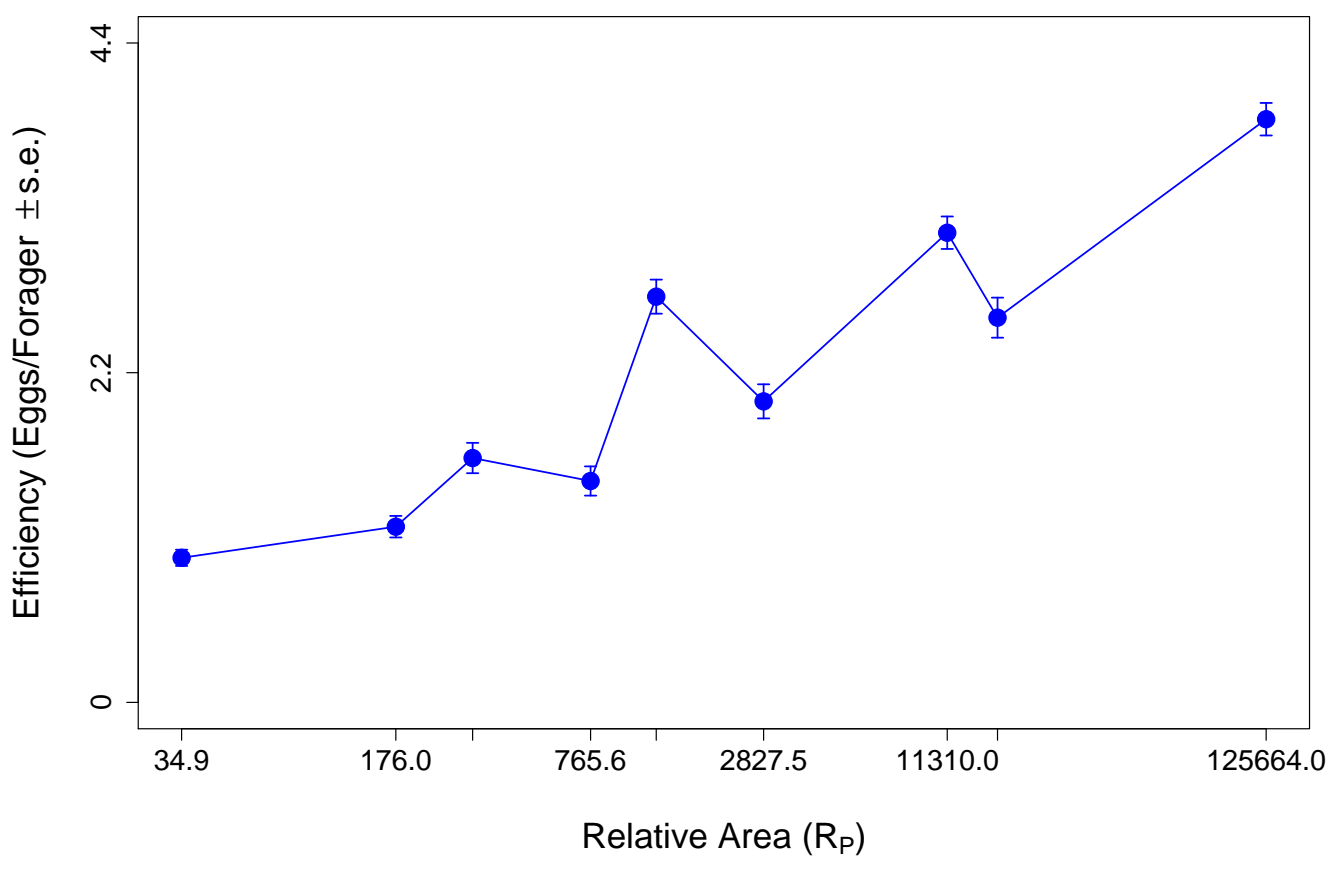

Figure A.6-3: Search Efficiency Summary - Trial D (Vision), Multiple Eggs. Proportion of eggs per Forager released (y-axis) vs. Relative Area $\left(R_{P}\right)$. See Chapter 3 details of this calculation.

a)

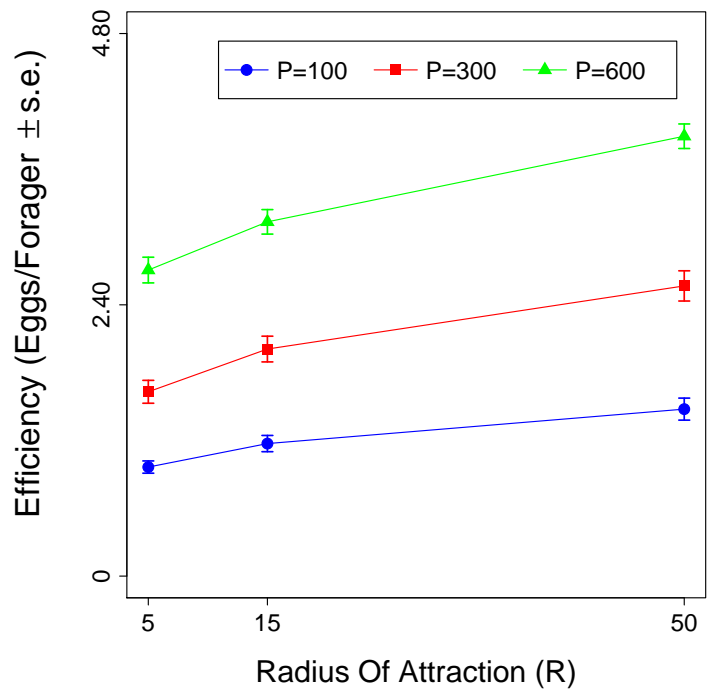

b)

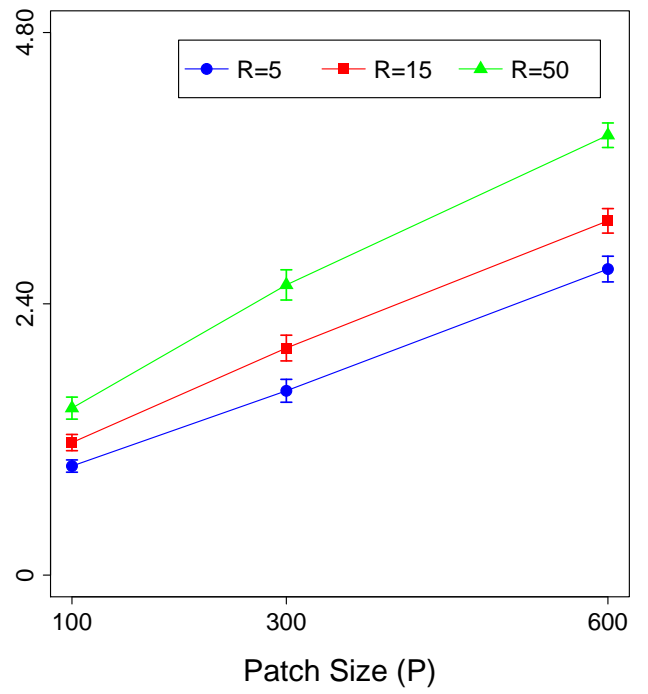

Figure A.6-4: Search efficiency vs. $R$ and P - Trial D (Vision), Multiple Eggs. Proportion of eggs per forager released (y-axis) vs. a) radius of attraction $(R)$ and $b$ ) patch size $(P)$. Results are averaged over all combinations of $L$ and $k$. 


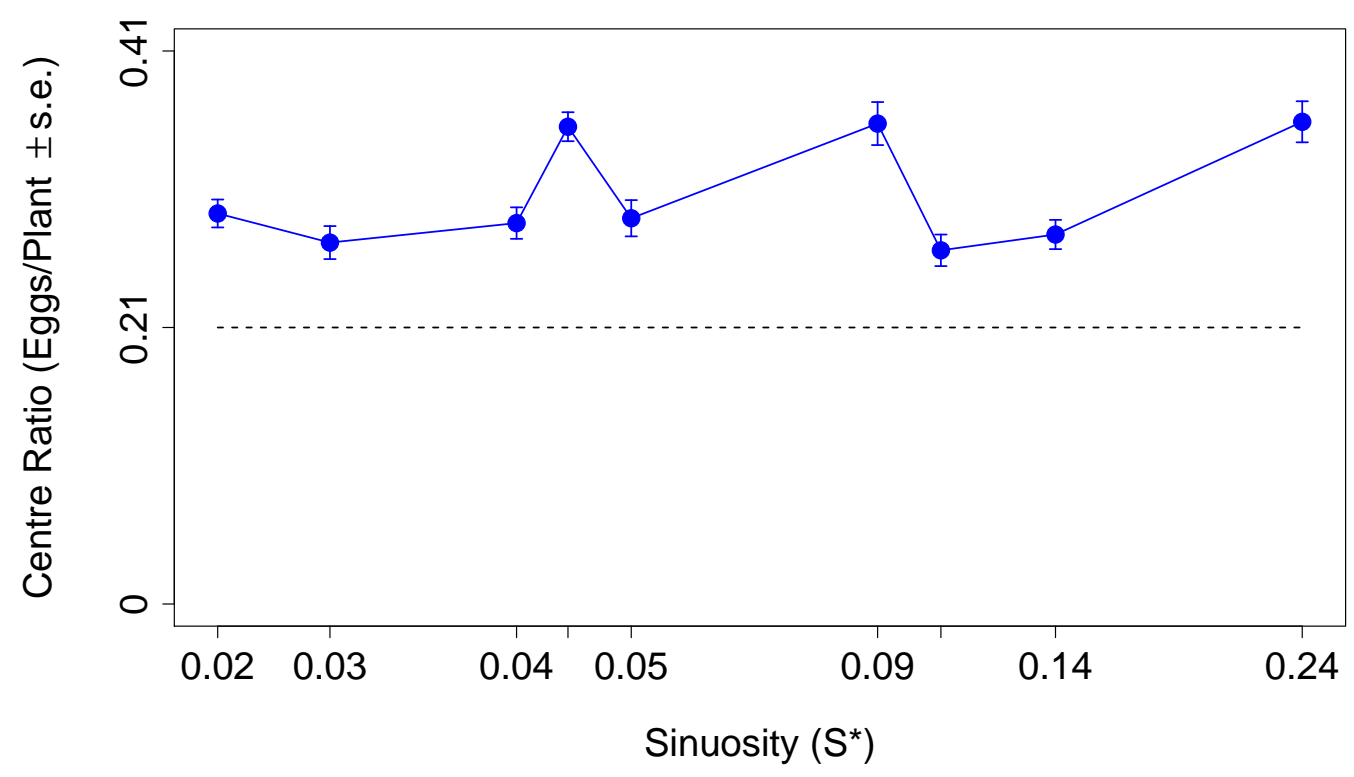

Figure A.6-5: Centre ratio vs. sinuosity - Trial D (Vision), Multiple Eggs. Proportion of eggs per plant on centre resources (y-axis) vs. Sinuosity $(S *)$ of forager paths. Sinuosity is a measure composed of both step length $(L)$ and turning angle concentration $(k)$

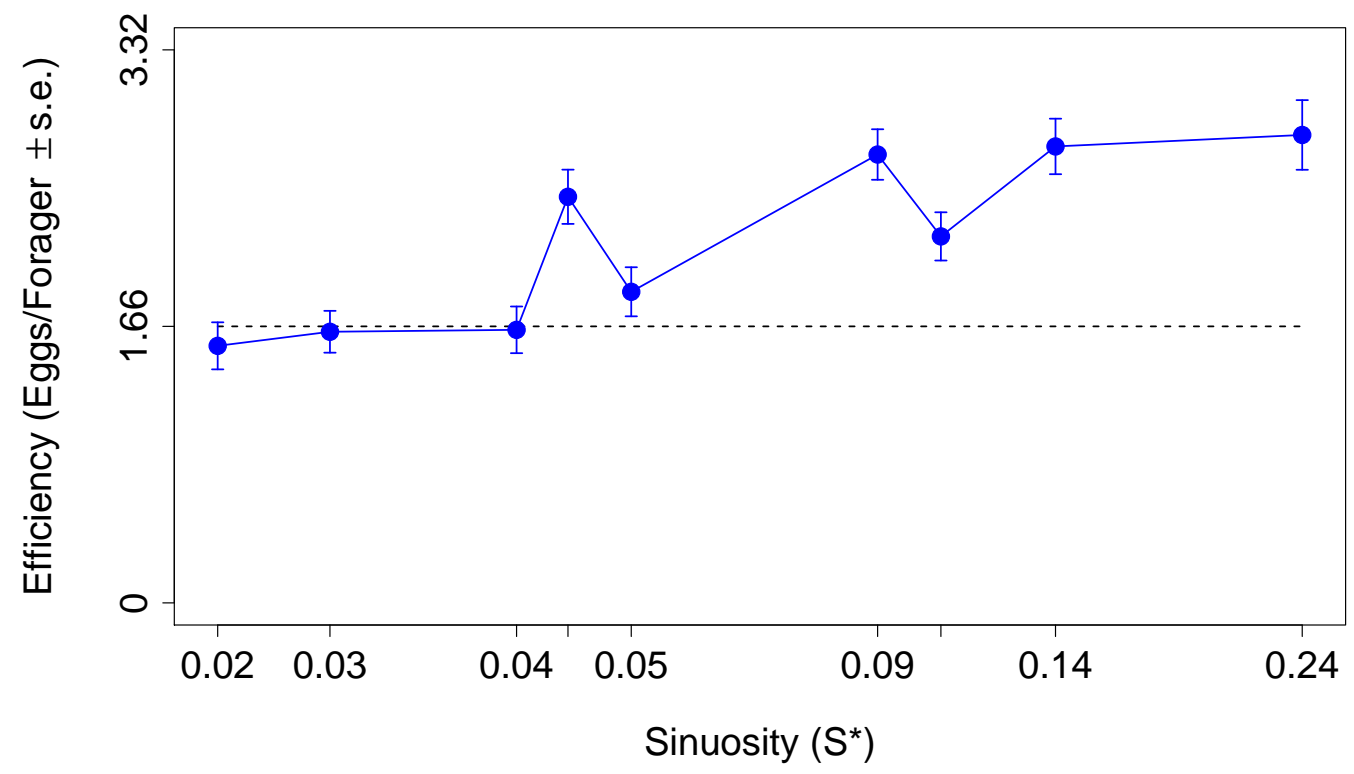

Figure A.6-6: Search efficiency vs. sinuosity - Trial D (Vision), Multiple Eggs. Proportion of eggs per forager released ( $y$-axis) vs. a) radius of attraction $(R)$ and $b$ ) patch size $(P)$ 


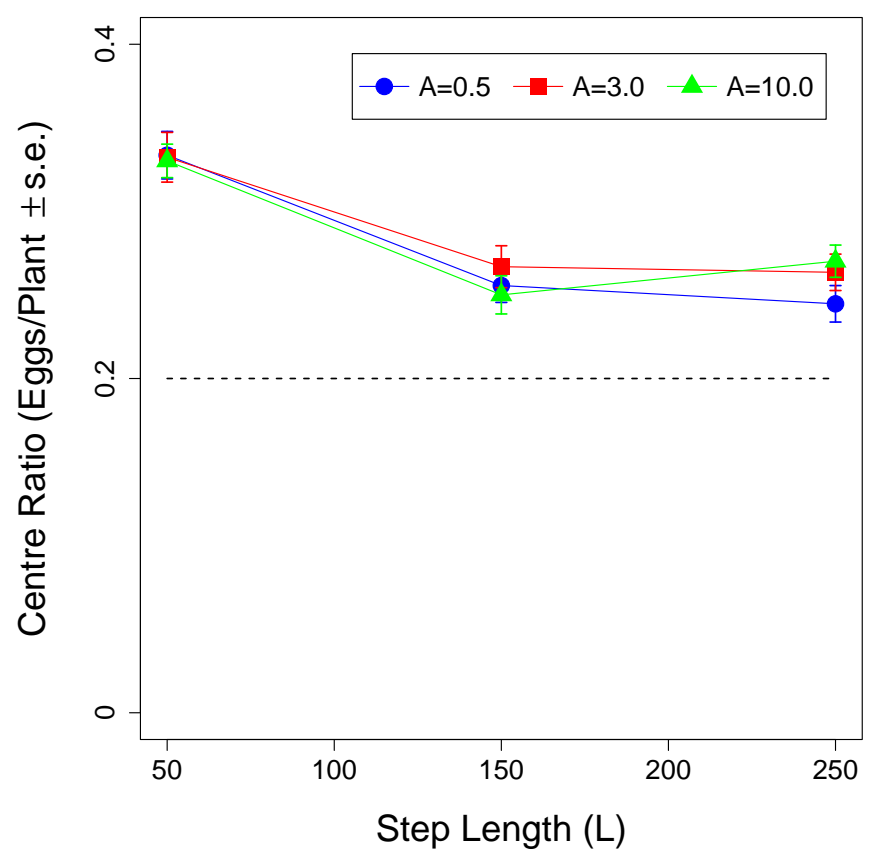

Figure A.6-7: Centre ratio vs. L and $k$ - Trial D (Vision), Multiple Eggs. Proportion of eggs per plant on centre resources (y-axis) vs. step length $(L)$ for various turning angle concentrations $(k)$. Results are averaged over all combinations of $R$ and $P$.

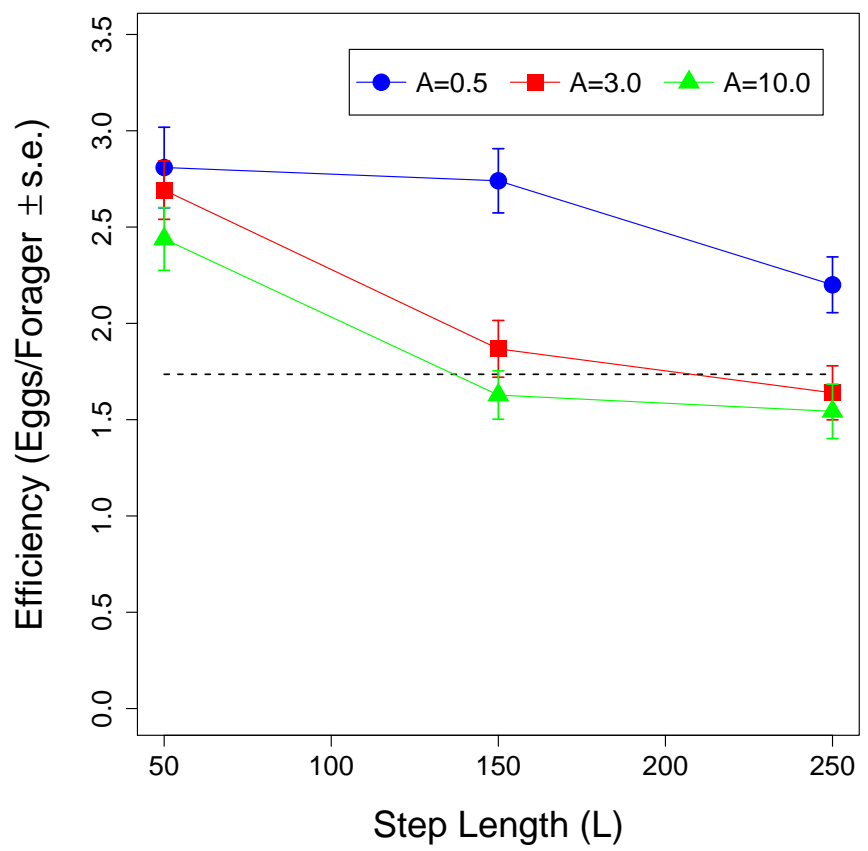

Figure A.6-8: Search efficiency vs. L and k-Trial D (Vision), Multiple Eggs. Proportion of eggs per forager released (y-axis) vs. step length $(L)$ for various turning angle concentrations $(k)$. Results are averaged over all combinations of $R$ and $P$. 

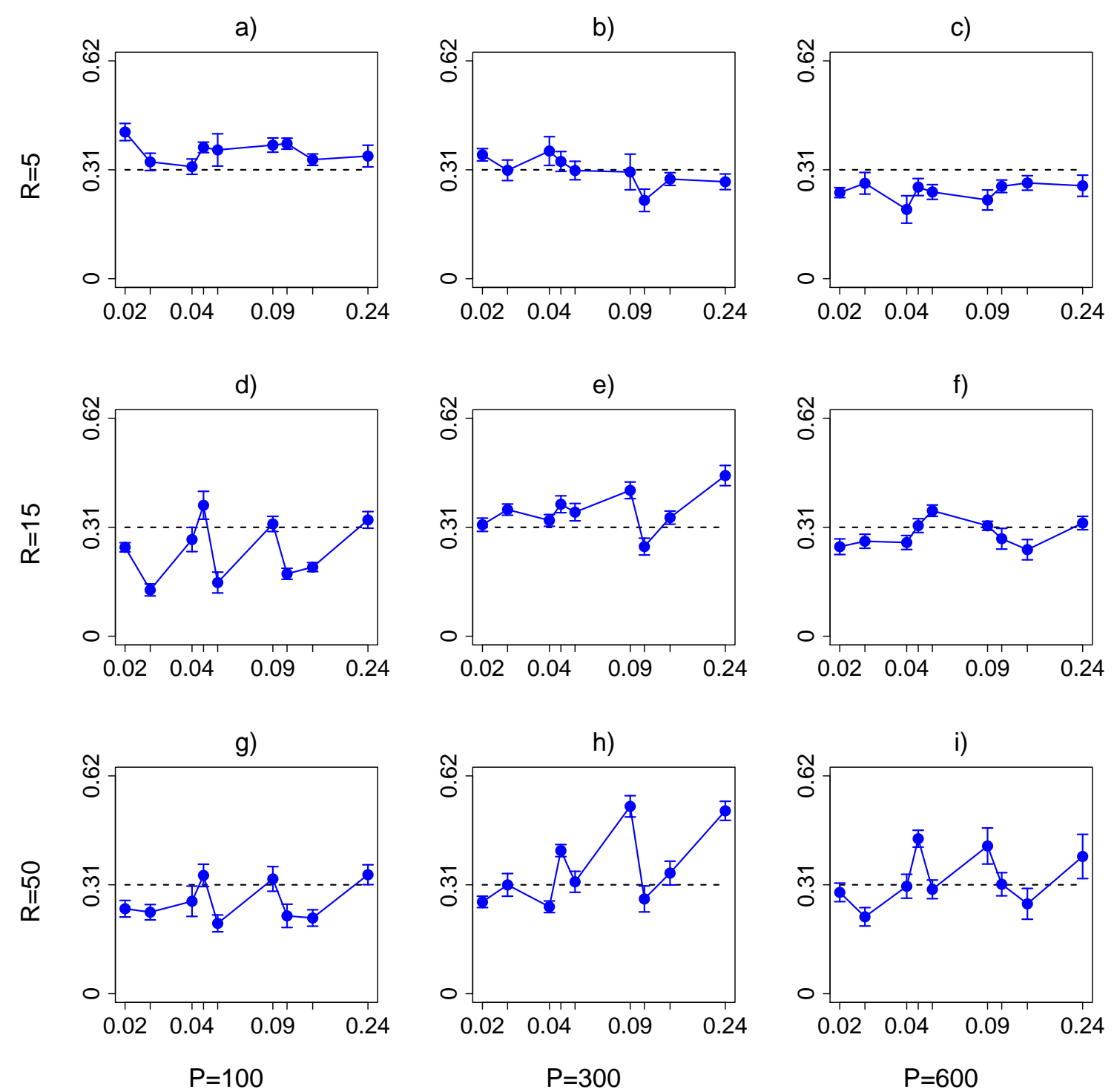

Figure A.6-9: Centre ratio vs. layout - Trial D (Vision), Multiple Eggs. Proportion of eggs per plant on centre resources (y-axis) vs. Sinuosity is plotted for combinations of radius of attraction $(R)$ and patch size $(P)$ in each sub-plot $(a-i)$. Radius of attraction increases from top to bottom and patch size increases from left to right. The layout is such that these results may be compared with Figure 3.32 to see the patch layouts. 
a)

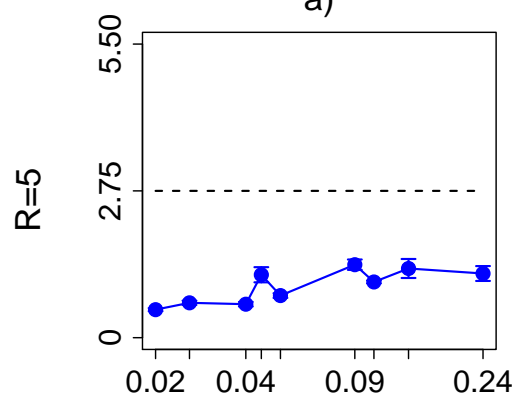

d)

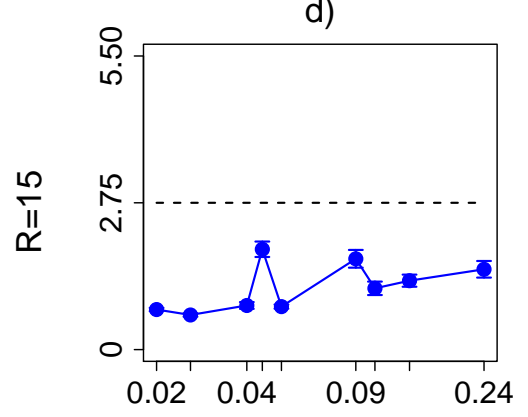

g)

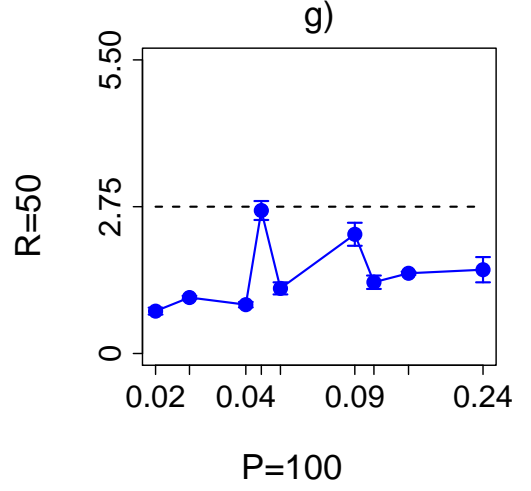

b)

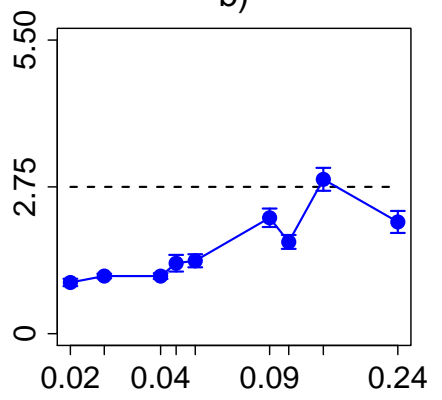

e)

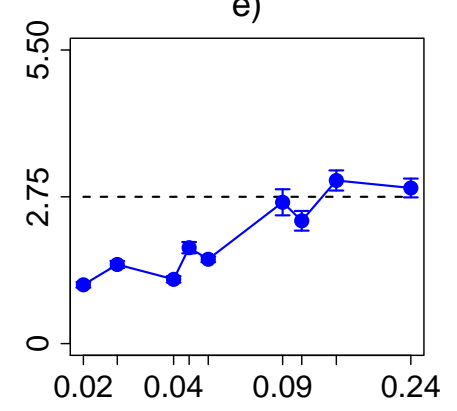

h)

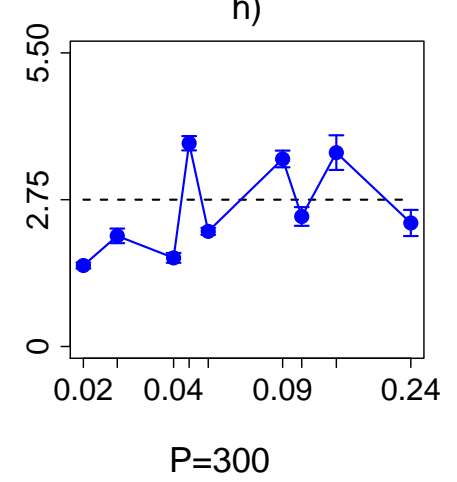

c)

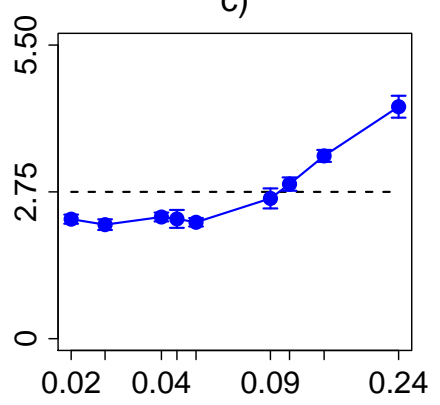

f)

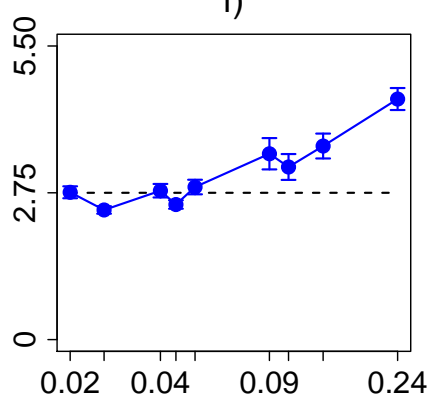

i)

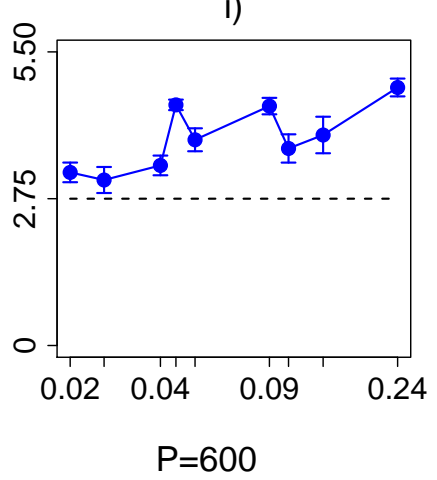

Figure A.6-10: Search efficiency vs. layout - Trial D (Vision), Multiple Eggs. Proportion of eggs per forager released (y-axis) vs. sinuosity is plotted for combinations of radius of attraction $(R)$ and patch size $(P)$ in each sub-plot $(a-i)$. Radius of attraction increases from top to bottom and patch size increases from left to right. The layout is such that these results may be compared with Figure 3.32 to see the patch layouts. 


\section{A.7 Trial E (Olfaction and Vision) Single Egg results}

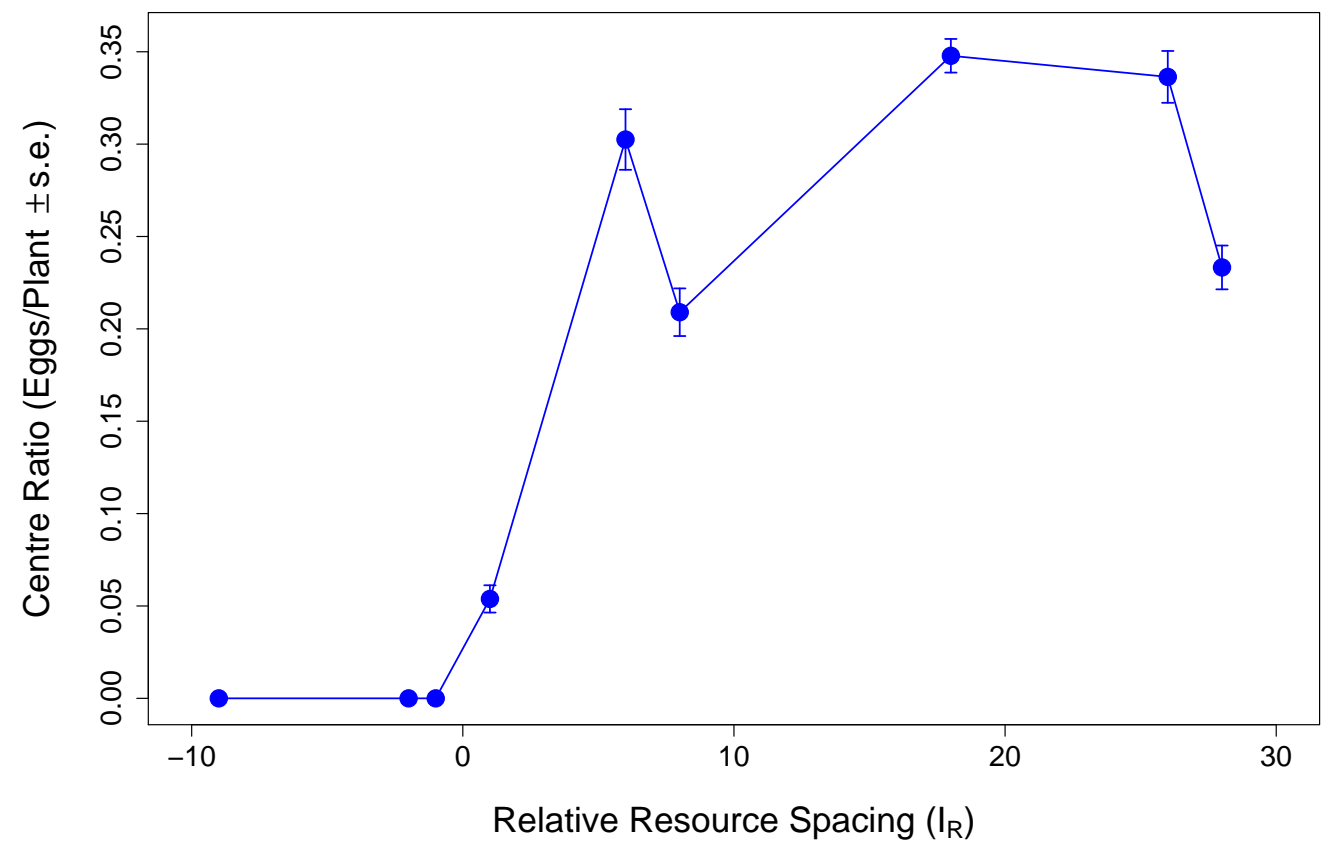

Figure A.7-1: Centre ratio summary - Trial E (Olfaction and Vision), Single Egg. Proportion of eggs per plant on centre resources ( $y$-axis) vs. relative resource spacing, $I_{R}$ ( $x$-axis). Negative values of $I_{R}$ indicate that the radii of attraction $(R)$ are overlapping.

a)

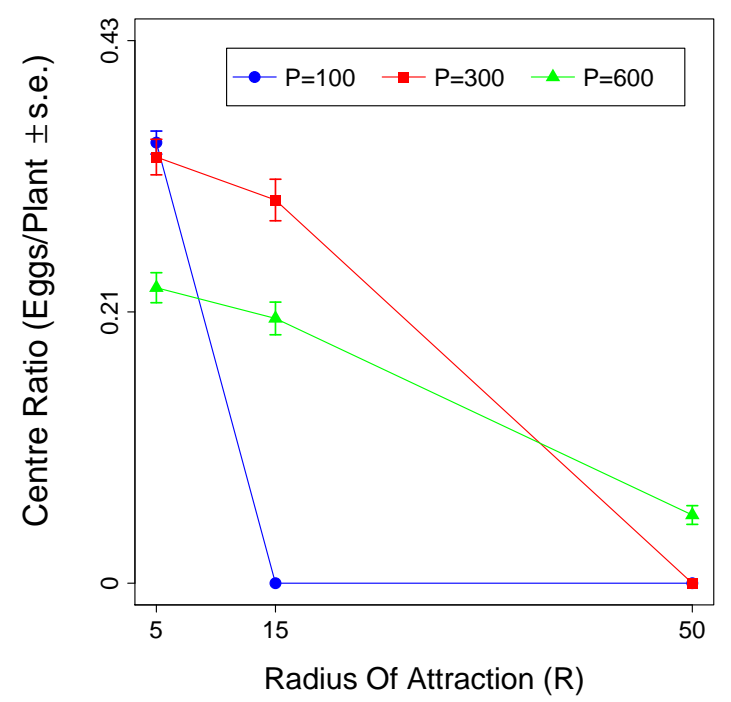

b)

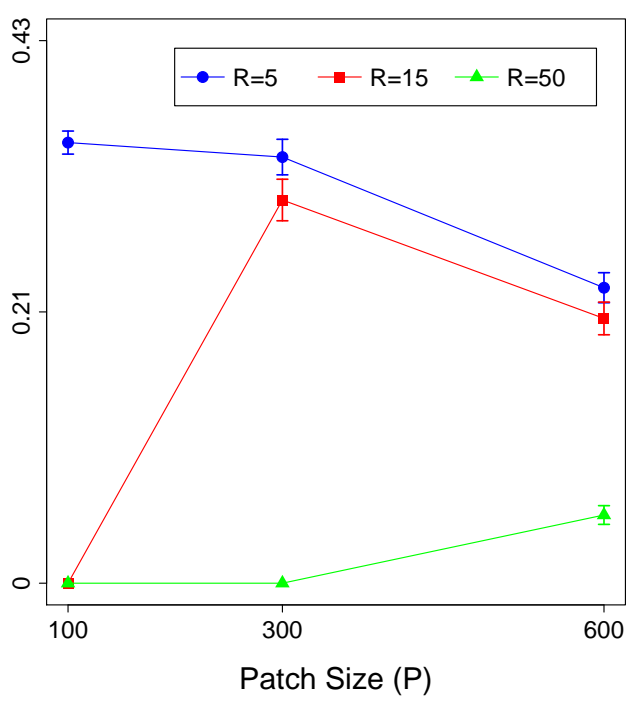

Figure A.7-2: Centre ratio vs. $R$ and $P$ - Trial E (Olfaction and Vision), Single Egg. Proportion of eggs per plant on centre resources ( $y$-axis) vs. a) radius of attraction $(R)$ and $b$ ) patch size $(P)$. Results are averaged over all combinations of $L$ and $k$. 


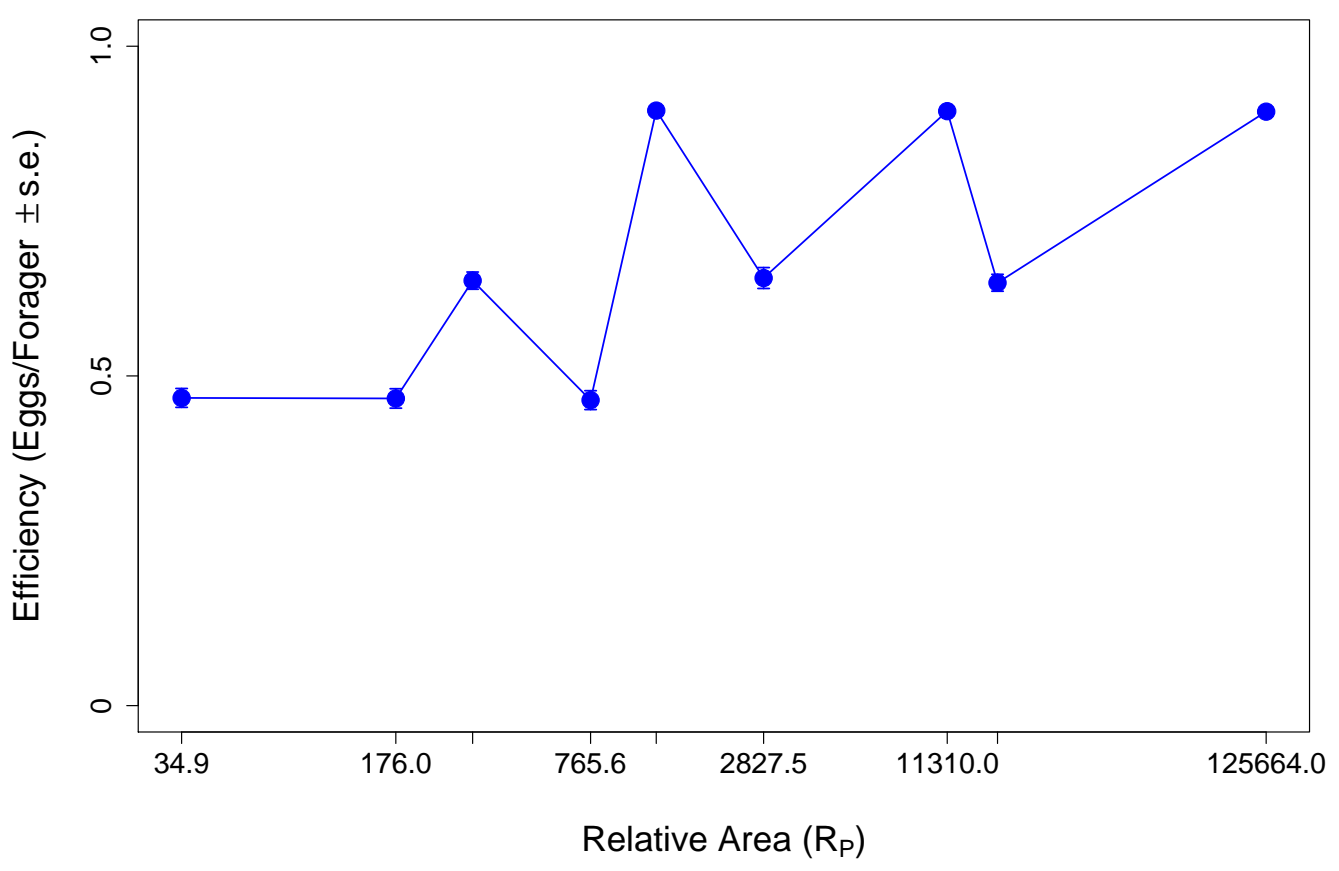

Figure A.7-3: Search Efficiency Summary - Trial E (Olfaction and Vision), Single Egg. Proportion of eggs per Forager released (y-axis) vs. Relative Area $\left(R_{P}\right)$. See Chapter 3 details of this calculation.

a)

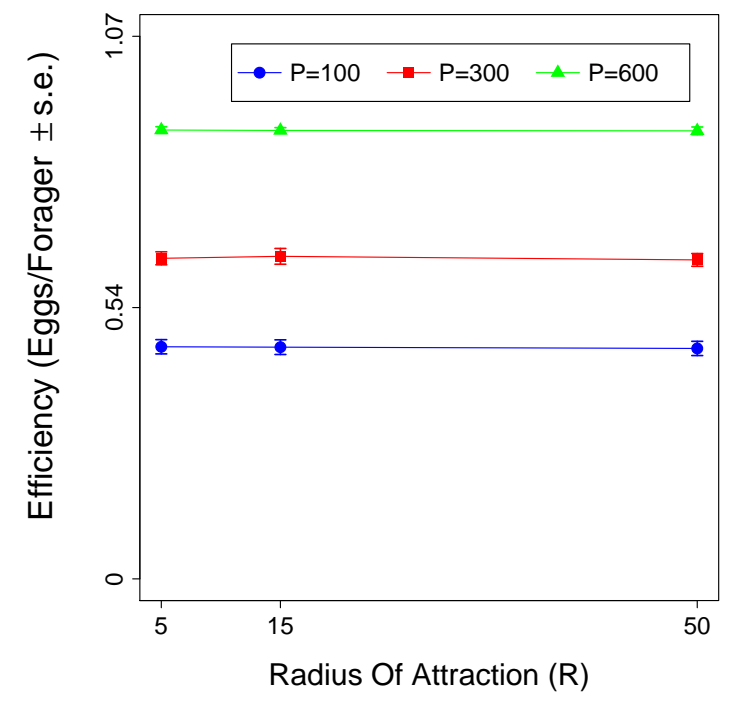

b)

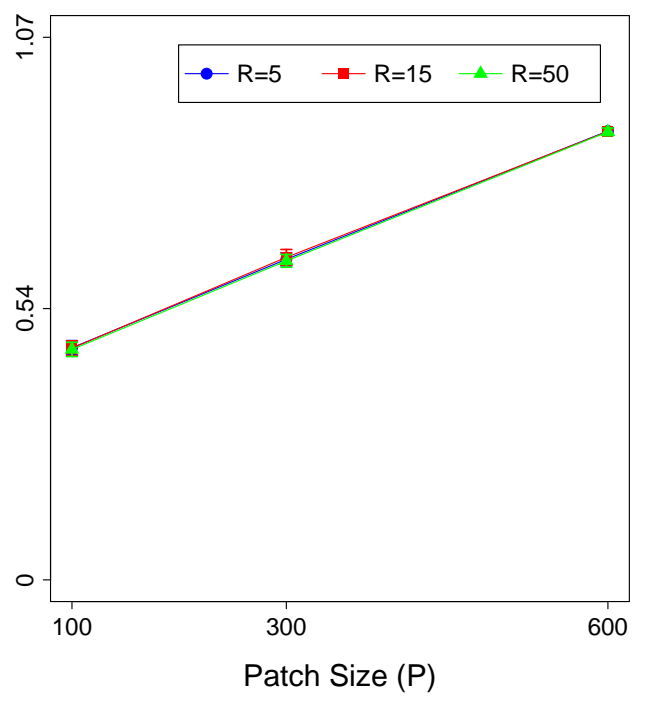

Figure A.7-4: Search efficiency vs. $R$ and P - Trial E (Olfaction and Vision), Single Egg. Proportion of eggs per forager released ( $y$-axis) vs. a) radius of attraction $(R)$ and $b$ ) patch size $(P)$. Results are averaged over all combinations of $L$ and $k$. 


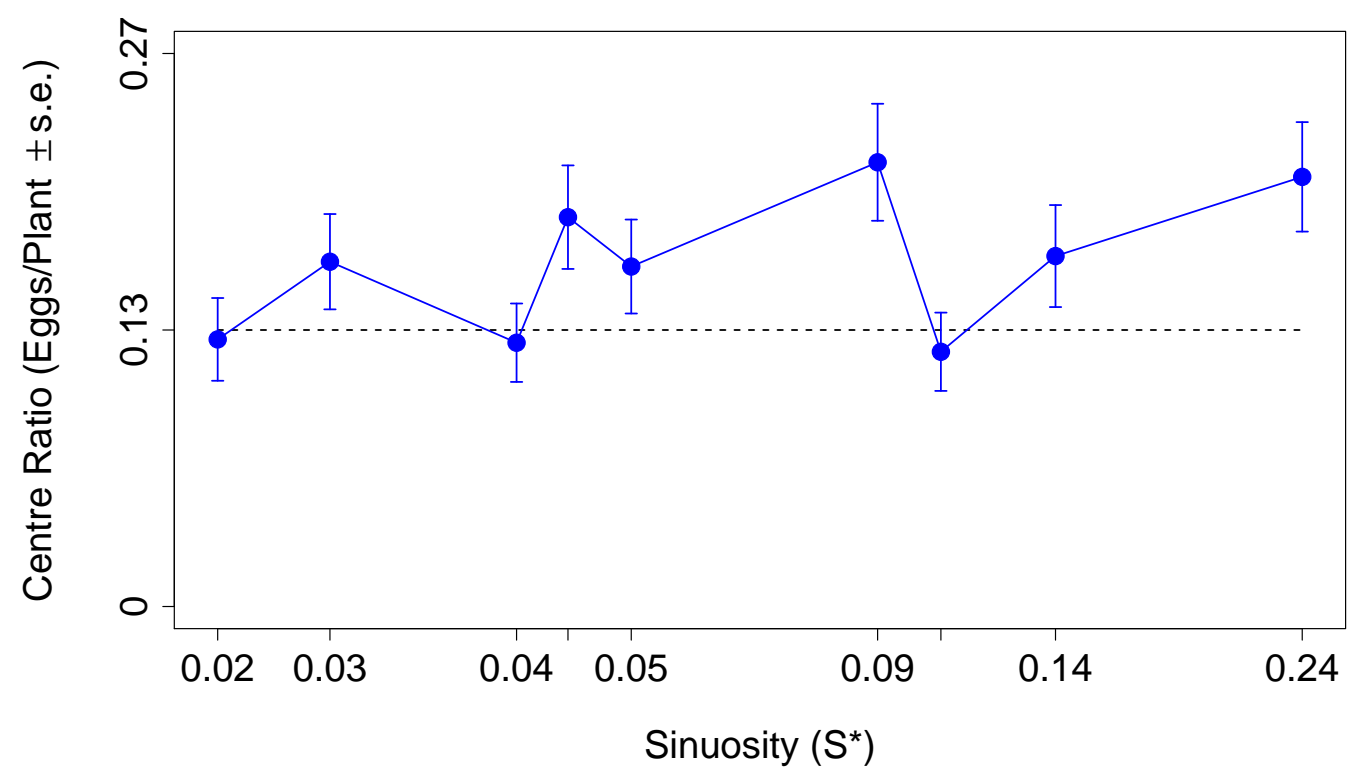

Figure A.7-5: Centre ratio vs. sinuosity - Trial E (Olfaction and Vision), Single Egg. Proportion of eggs per plant on centre resources (y-axis) vs. Sinuosity ( $S *$ ) of forager paths. Sinuosity is a measure composed of both step length $(L)$ and turning angle concentration $(k)$

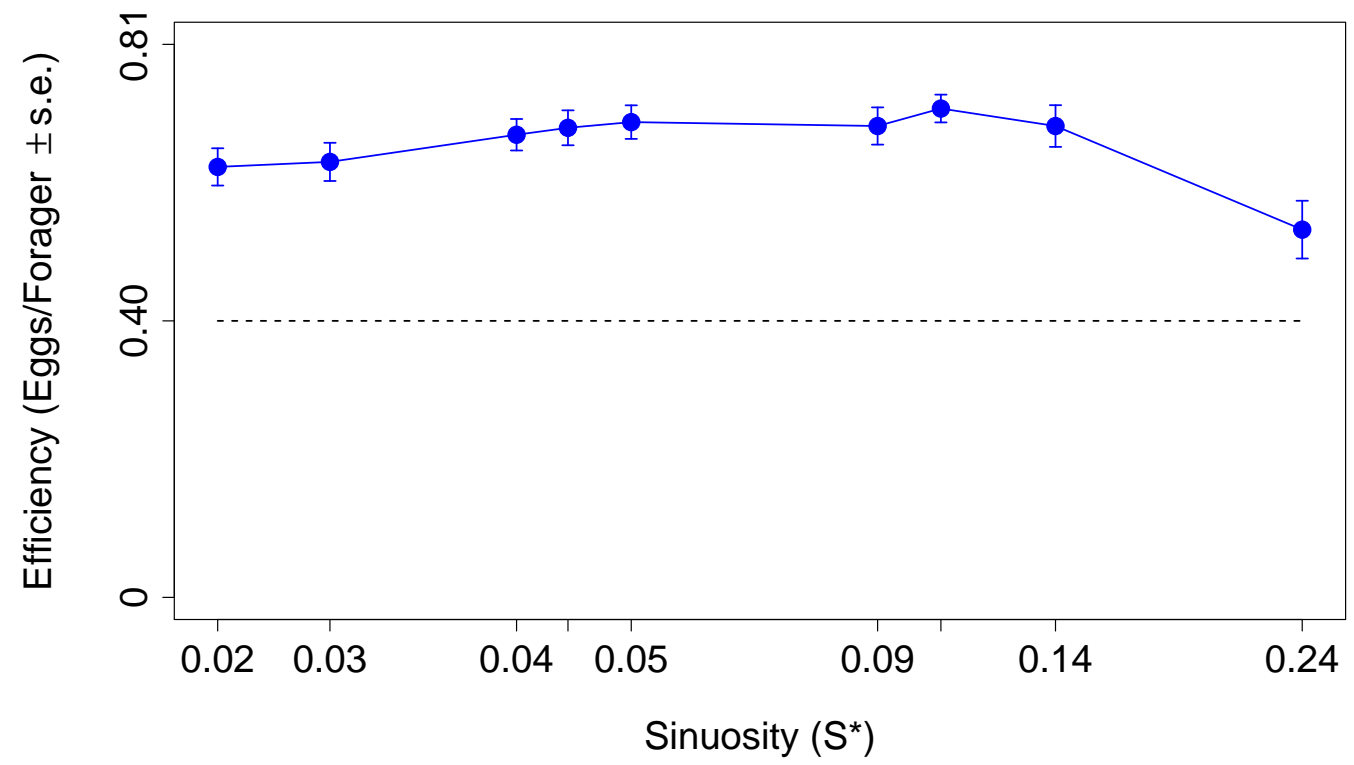

Figure A.7-6: Search efficiency vs. sinuosity - Trial E (Olfaction and Vision), Single Egg. Proportion of eggs per forager released ( $y$-axis) vs. a) radius of attraction $(R)$ and $b$ ) patch size $(P)$ 


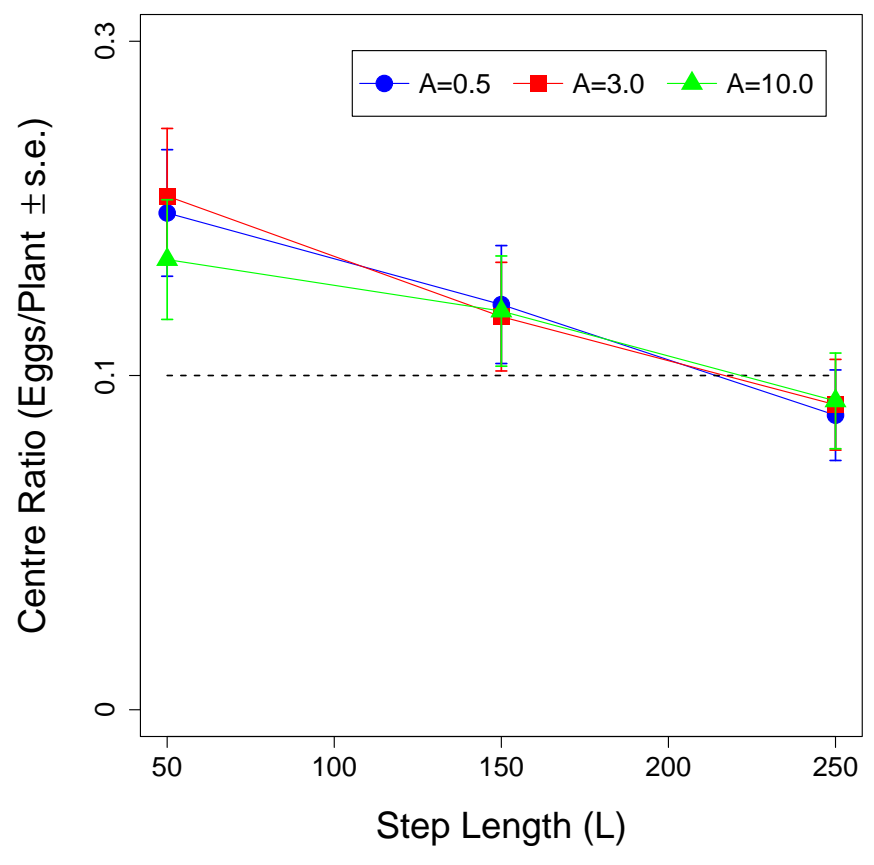

Figure A.7-7: Centre ratio vs. L and $k$ - Trial E (Olfaction and Vision), Single Egg. Proportion of eggs per plant on centre resources (y-axis) vs. step length $(L)$ for various turning angle concentrations $(k)$. Results are averaged over all combinations of $R$ and $P$.

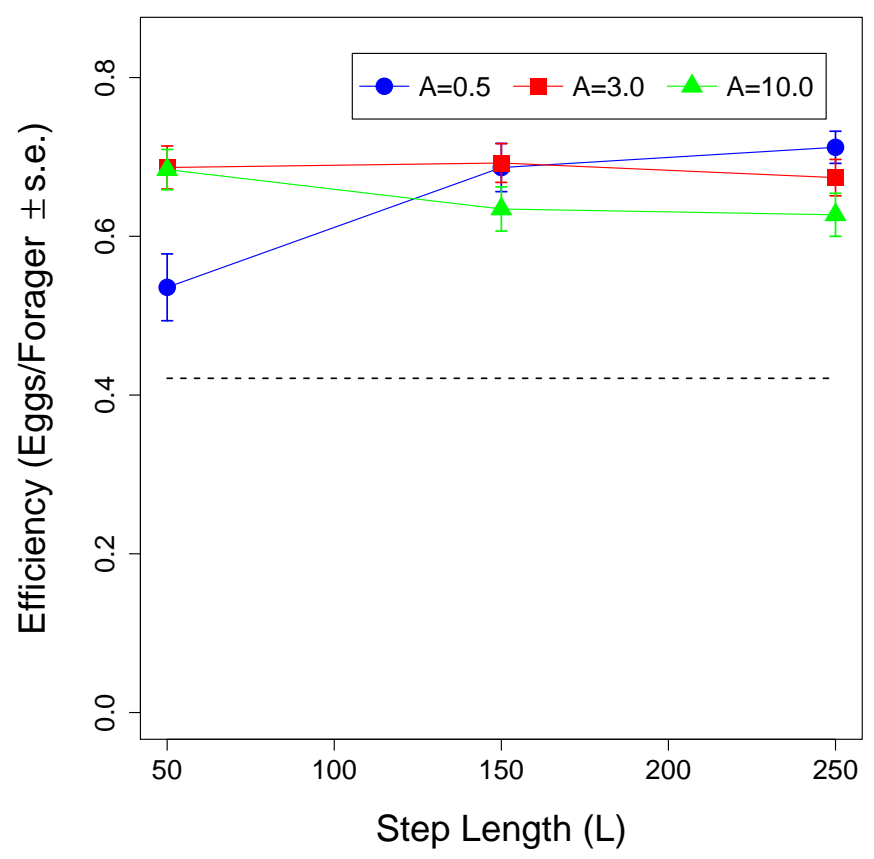

Figure A.7-8: Search efficiency vs. L and $k$ - Trial E (Olfaction and Vision), Single Egg. Proportion of eggs per forager released (y-axis) vs. step length $(L)$ for various turning angle concentrations $(k)$. Results are averaged over all combinations of $R$ and $P$. 

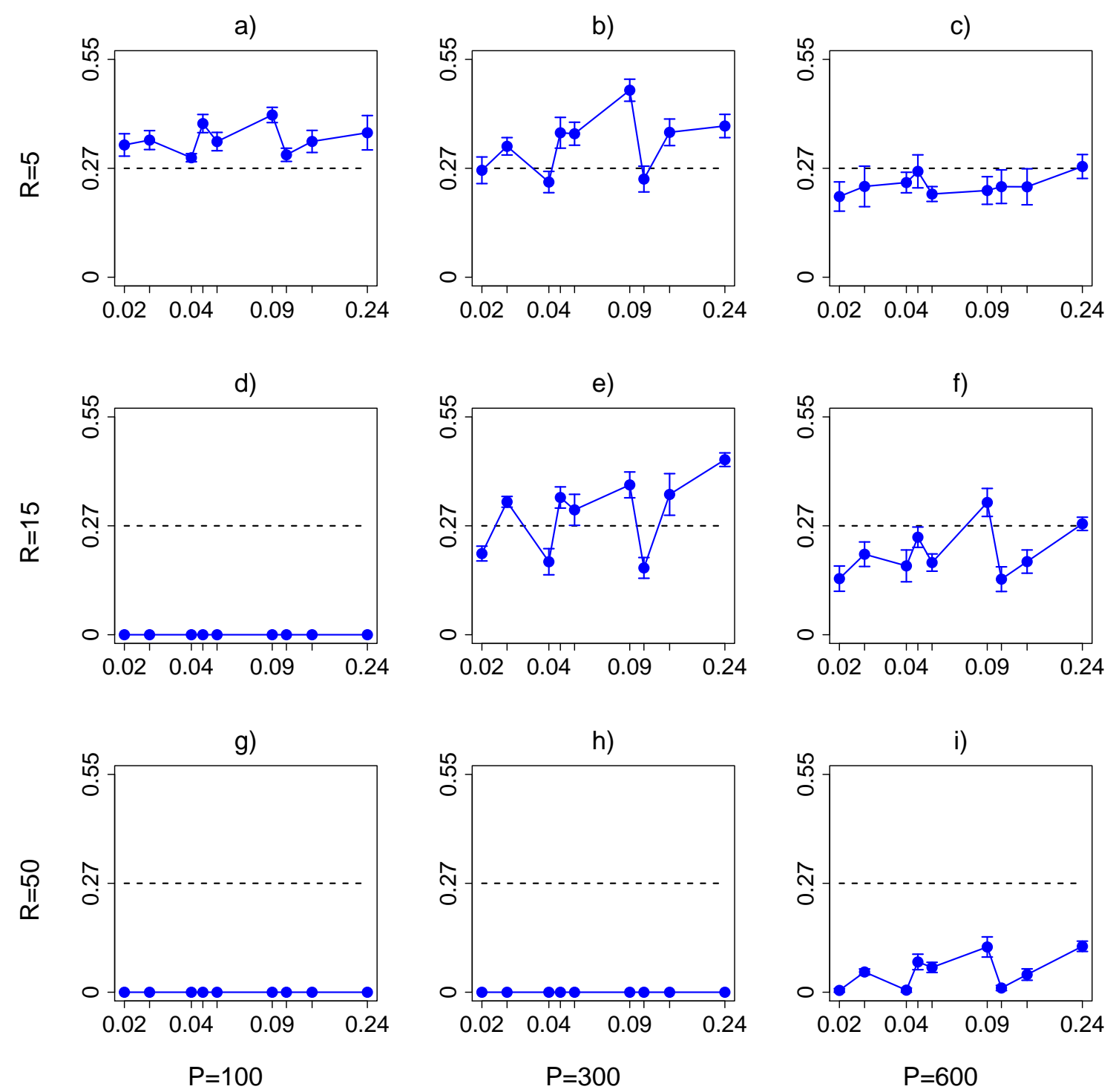

Figure A.7-9: Centre ratio vs. layout - Trial E (Olfaction and Vision), Single Egg. Proportion of eggs per plant on centre resources (y-axis) vs. Sinuosity is plotted for combinations of radius of attraction $(R)$ and patch size $(P)$ in each sub-plot $(a-i)$. Radius of attraction increases from top to bottom and patch size increases from left to right. The layout is such that these results may be compared with Figure 3.32 to see the patch layouts. 
a)

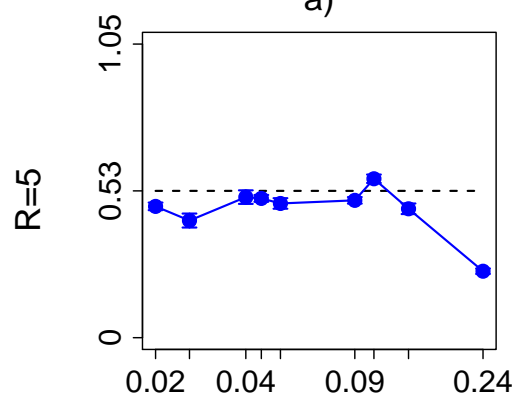

d)

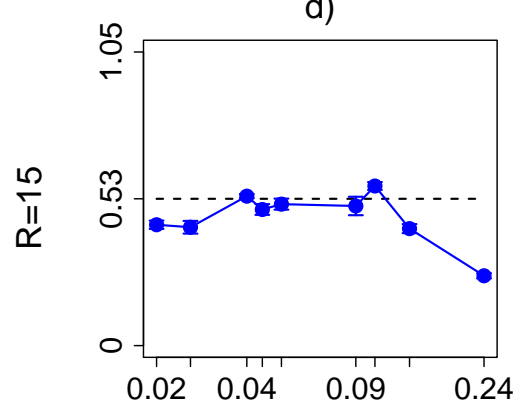

g)

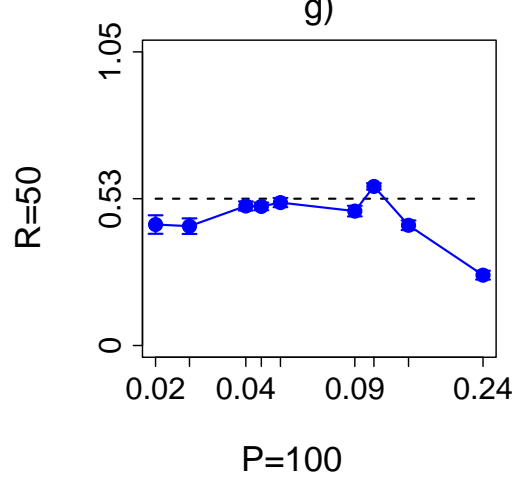

b)

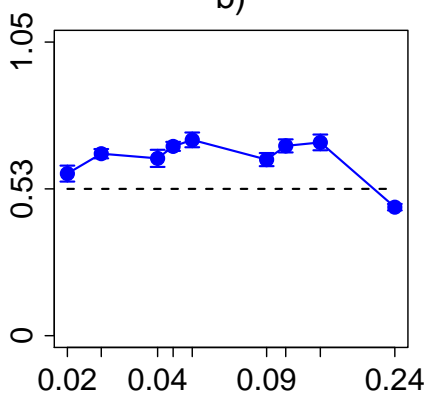

e)

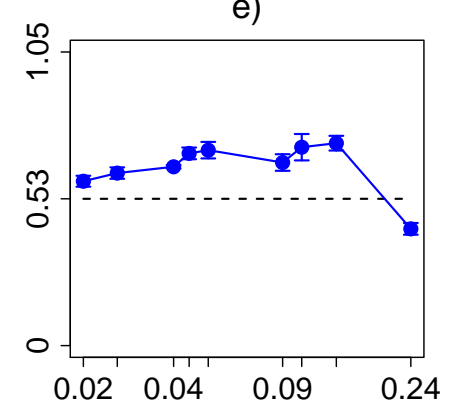

h)

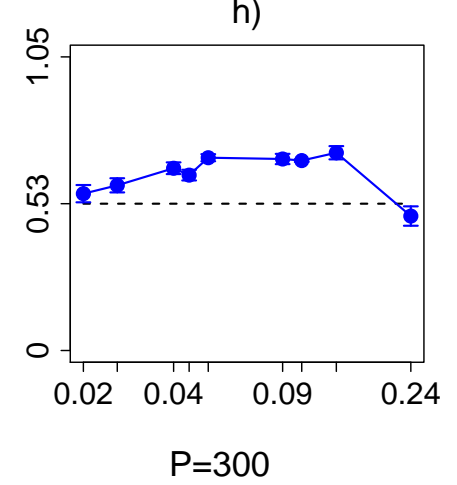

c)

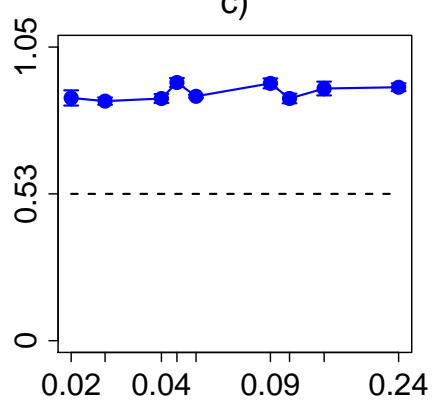

f)

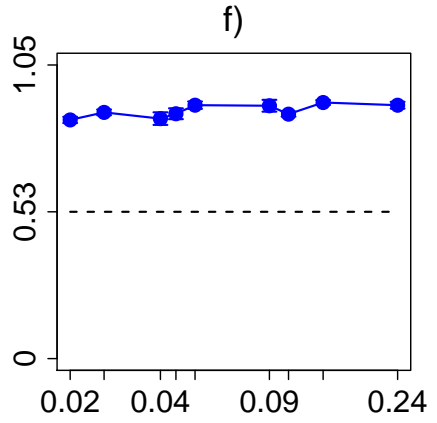

i)

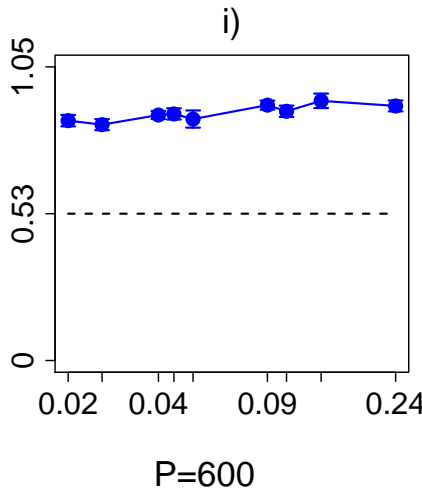

Figure A.7-10: Search efficiency vs. layout - Trial E (Olfaction and Vision), Single Egg. Proportion of eggs per forager released (y-axis) vs. sinuosity is plotted for combinations of radius of attraction $(R)$ and patch size $(P)$ in each sub-plot (a-i). Radius of attraction increases from top to bottom and patch size increases from left to right. The layout is such that these results may be compared with Figure 3.32 to see the patch layouts. 


\section{A.8 Trial E (Olfaction and Vision) Multiple Eggs results}

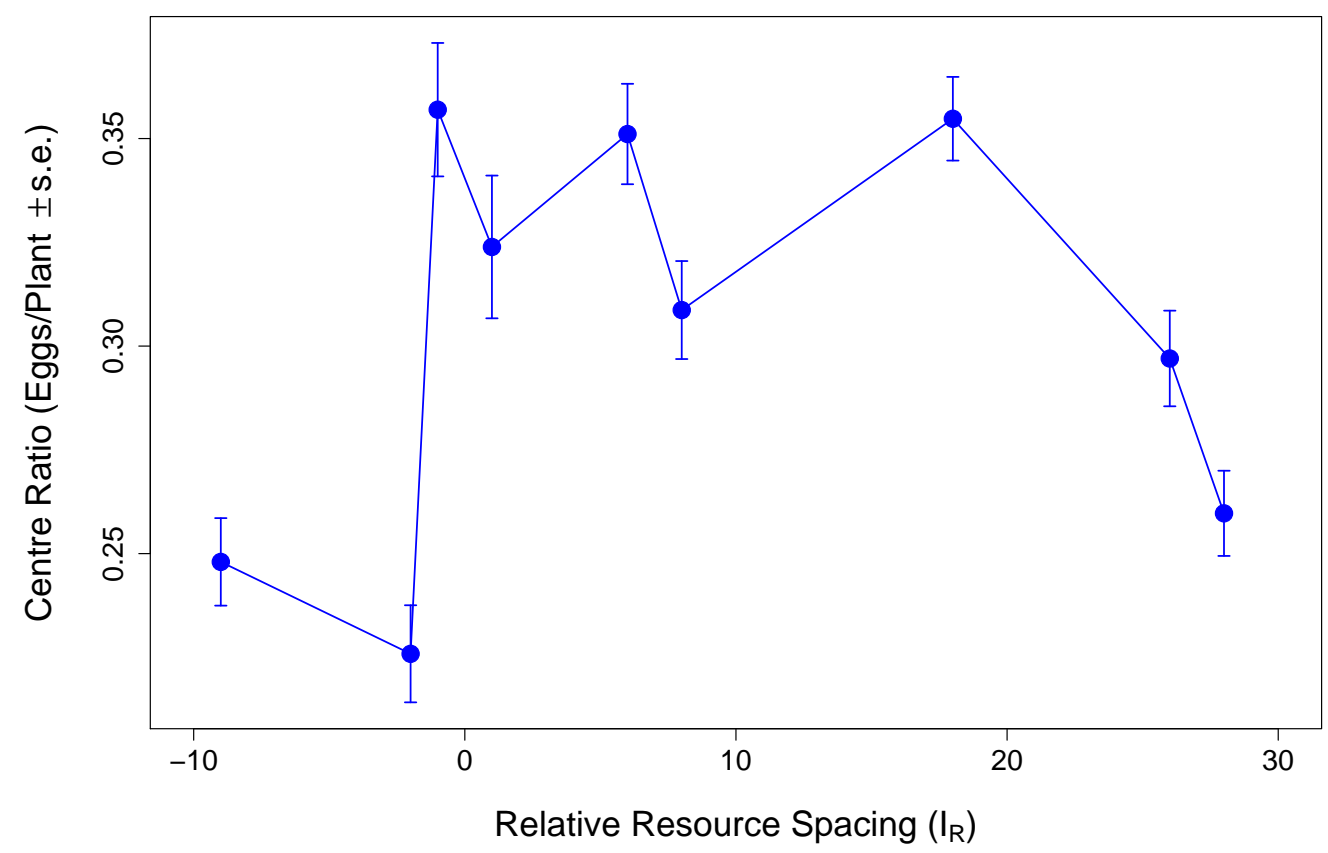

Figure A.8-1: Centre ratio summary - Trial E (Olfaction and Vision), Multiple Eggs. Proportion of eggs per plant on centre resources (y-axis) vs. relative resource spacing, $I_{R}(x-$ axis). Negative values of $I_{R}$ indicate that the radii of attraction $(R)$ are overlapping.

a)

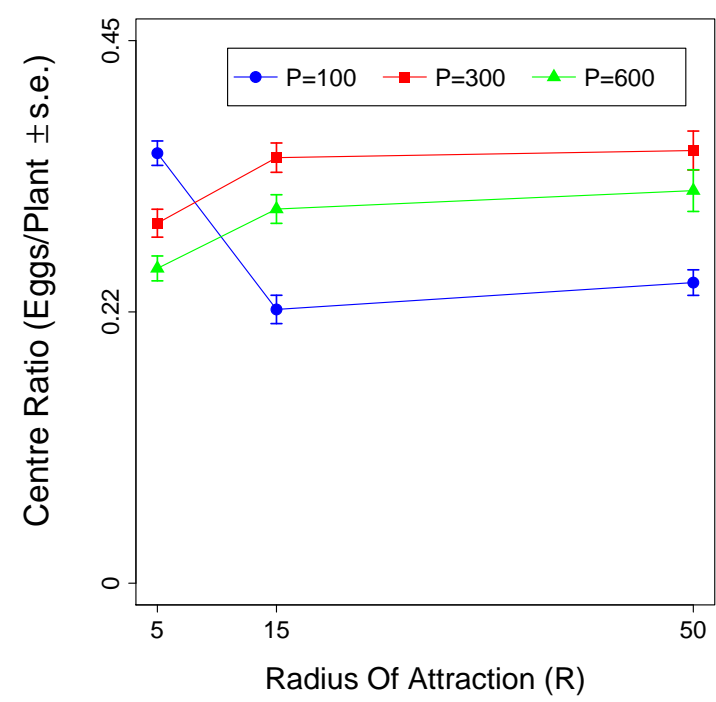

b)

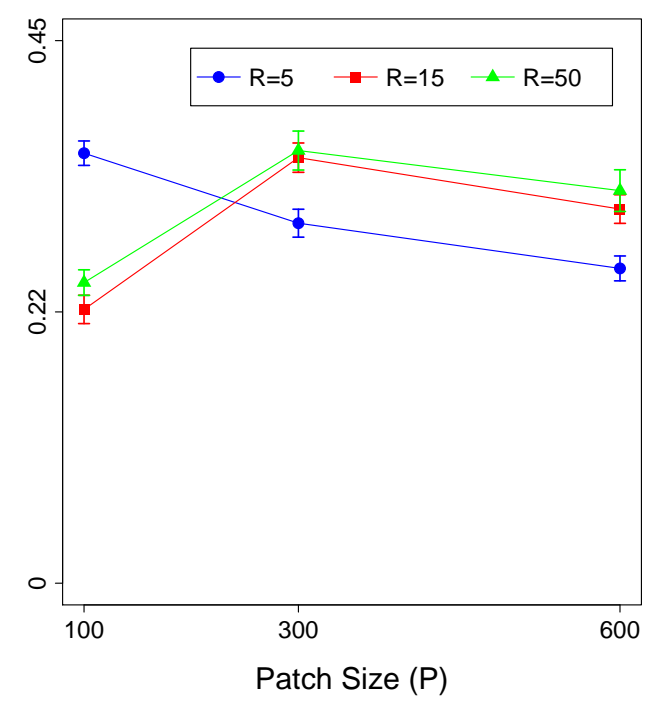

Figure A.8-2: Centre ratio vs. $R$ and $P$ - Trial E (Olfaction and Vision), Multiple Eggs. Proportion of eggs per plant on centre resources (y-axis) vs. a) radius of attraction $(R)$ and b) patch size $(P)$. Results are averaged over all combinations of $L$ and $k$. 


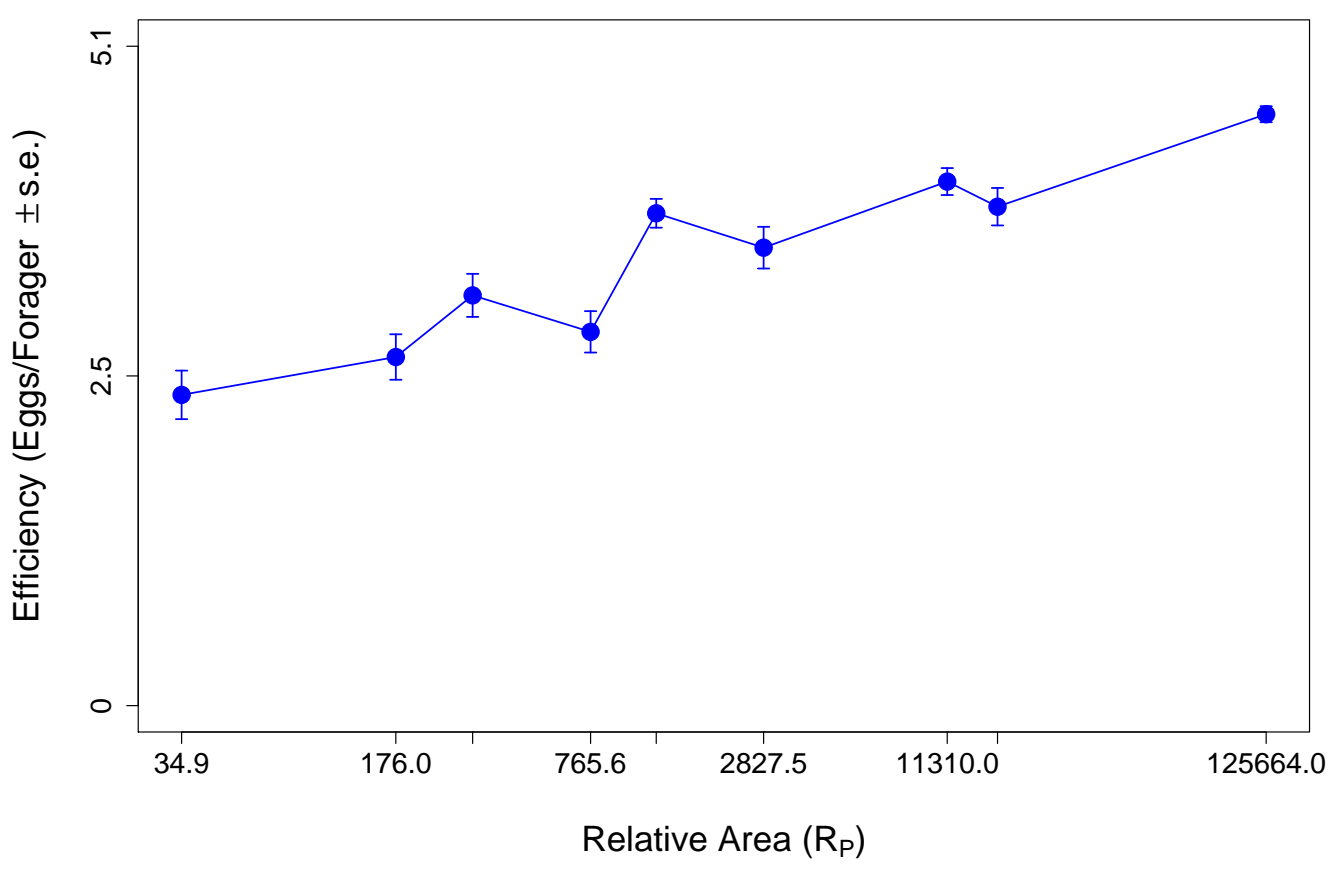

Figure A.8-3: Search Efficiency Summary - Trial E (Olfaction and Vision), Multiple Eggs. Proportion of eggs per Forager released (y-axis) vs. Relative Area $\left(R_{P}\right)$. See Chapter 3 details of this calculation.

a)

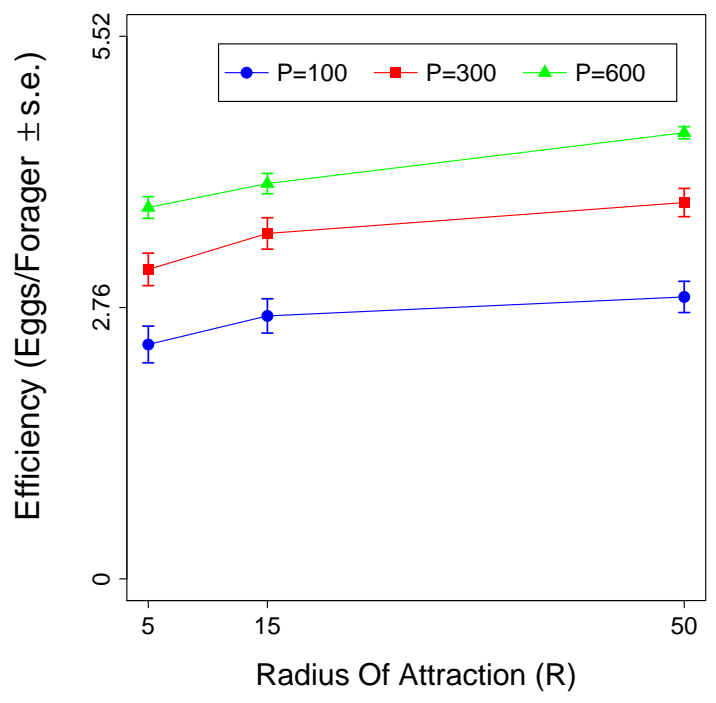

b)

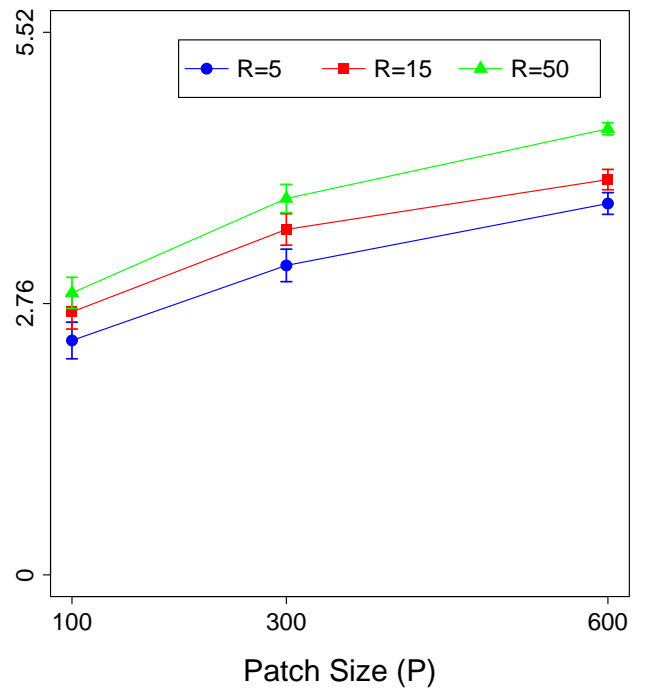

Figure A.8-4: Search efficiency vs. R and P - Trial E (Olfaction and Vision), Multiple Eggs. Proportion of eggs per forager released ( $y$-axis) vs. a) radius of attraction $(R)$ and $b$ ) patch size $(P)$. Results are averaged over all combinations of $L$ and $k$. 


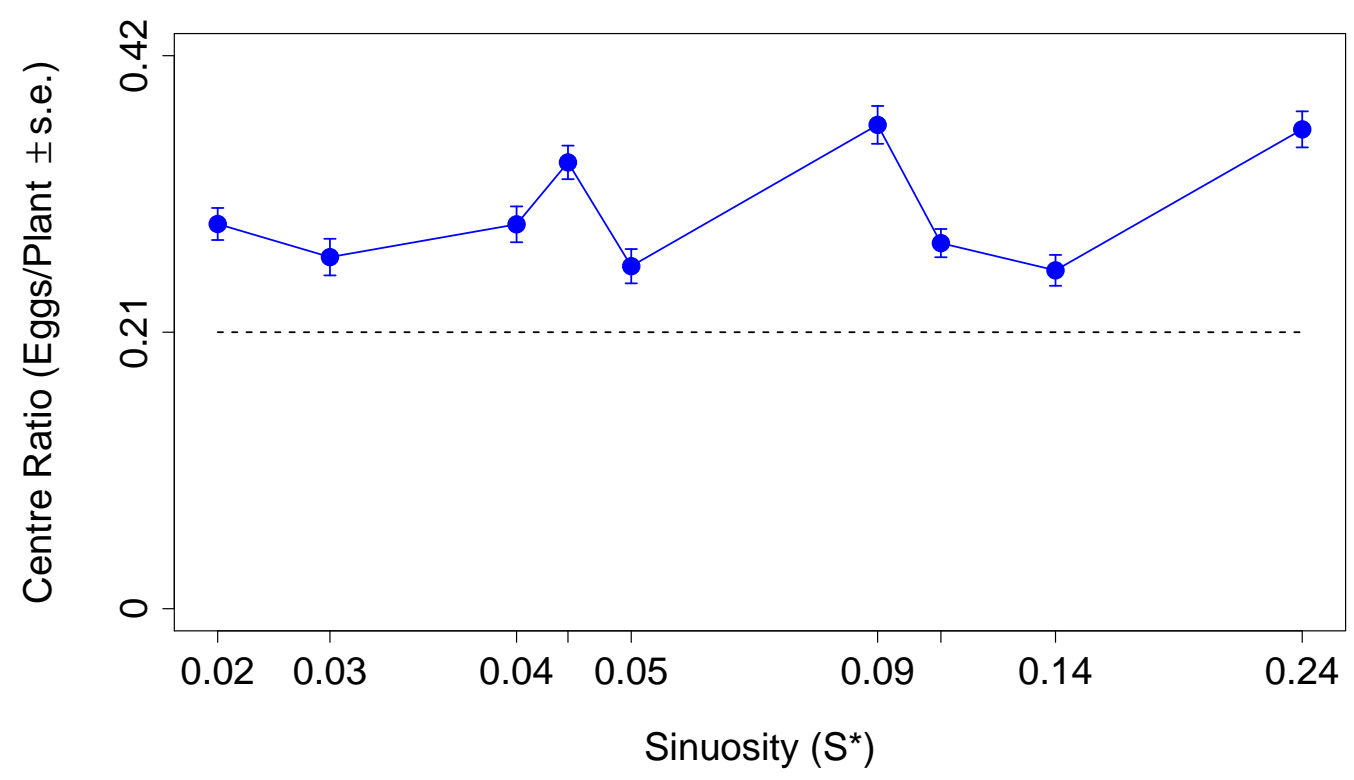

Figure A.8-5: Centre ratio vs. sinuosity - Trial E (Olfaction and Vision), Multiple Eggs. Proportion of eggs per plant on centre resources (y-axis) vs. Sinuosity $(S *)$ of forager paths. Sinuosity is a measure composed of both step length $(L)$ and turning angle concentration $(k)$

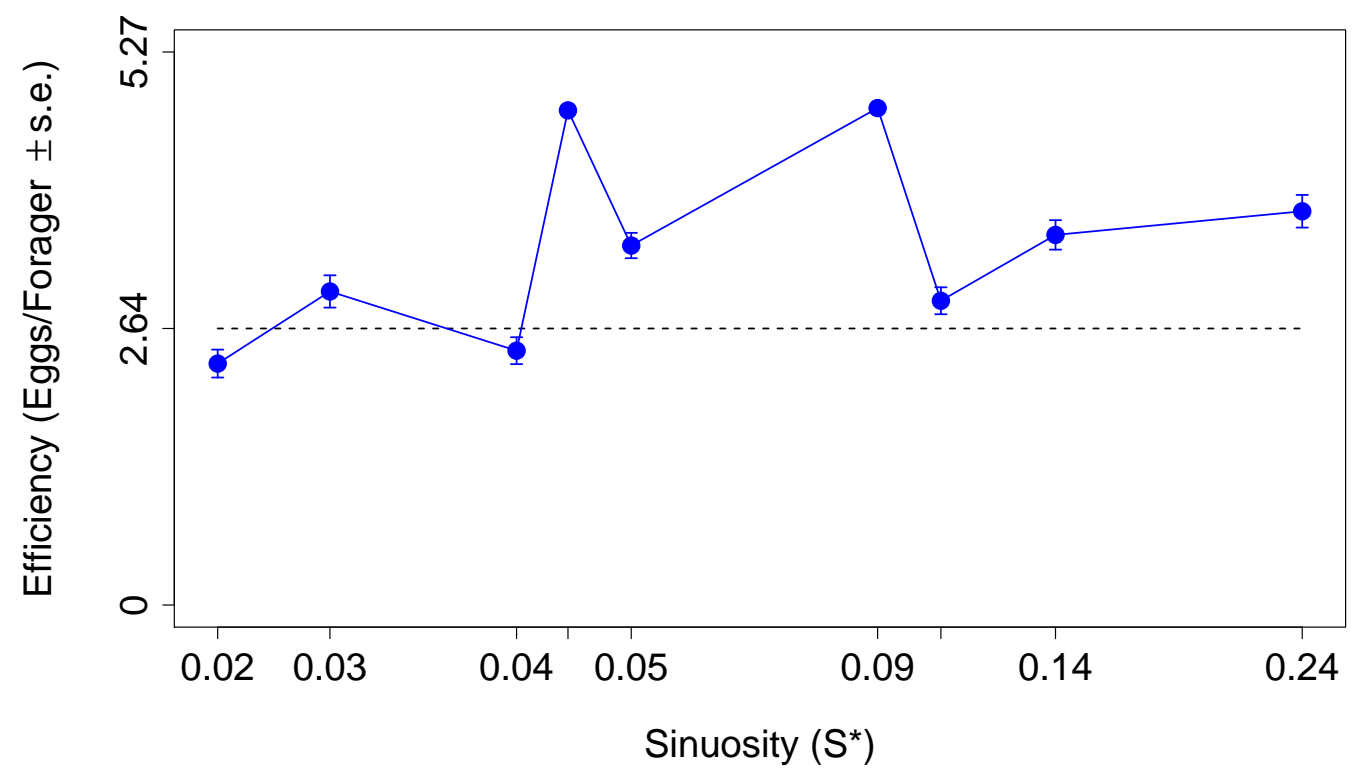

Figure A.8-6: Search efficiency vs. sinuosity - Trial E (Olfaction and Vision), Multiple Eggs. Proportion of eggs per forager released (y-axis) vs. a) radius of attraction $(R)$ and $b$ ) patch size $(P)$ 


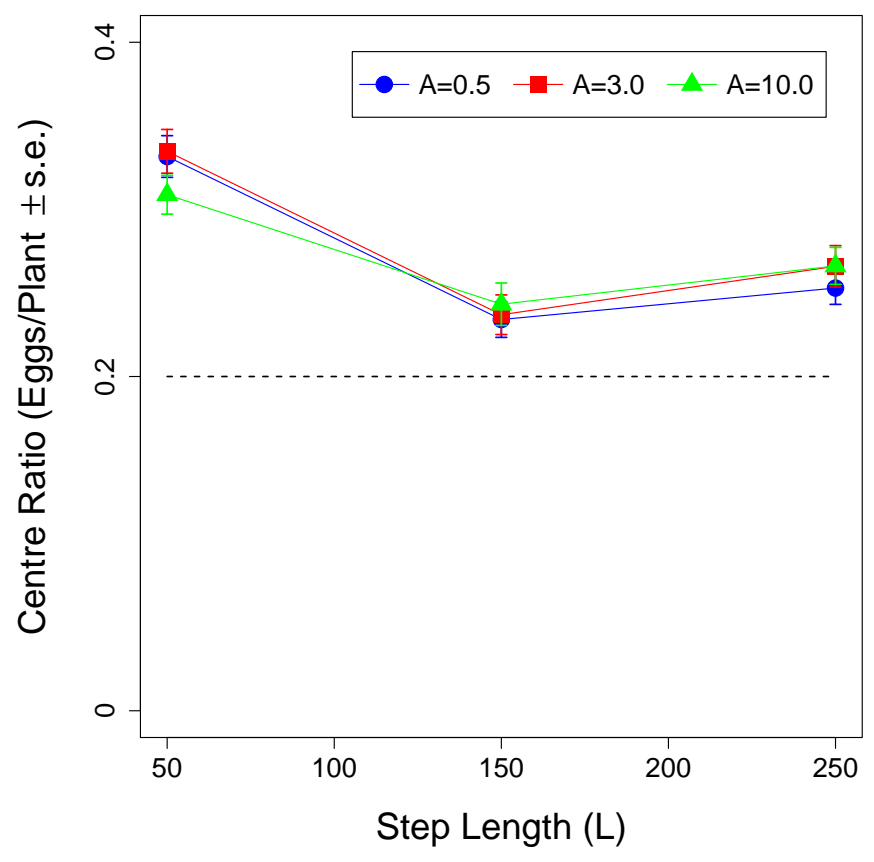

Figure A.8-7: Centre ratio vs. L and $k$ - Trial E (Olfaction and Vision), Multiple Eggs. Proportion of eggs per plant on centre resources (y-axis) vs. step length $(L)$ for various turning angle concentrations $(k)$. Results are averaged over all combinations of $R$ and $P$.

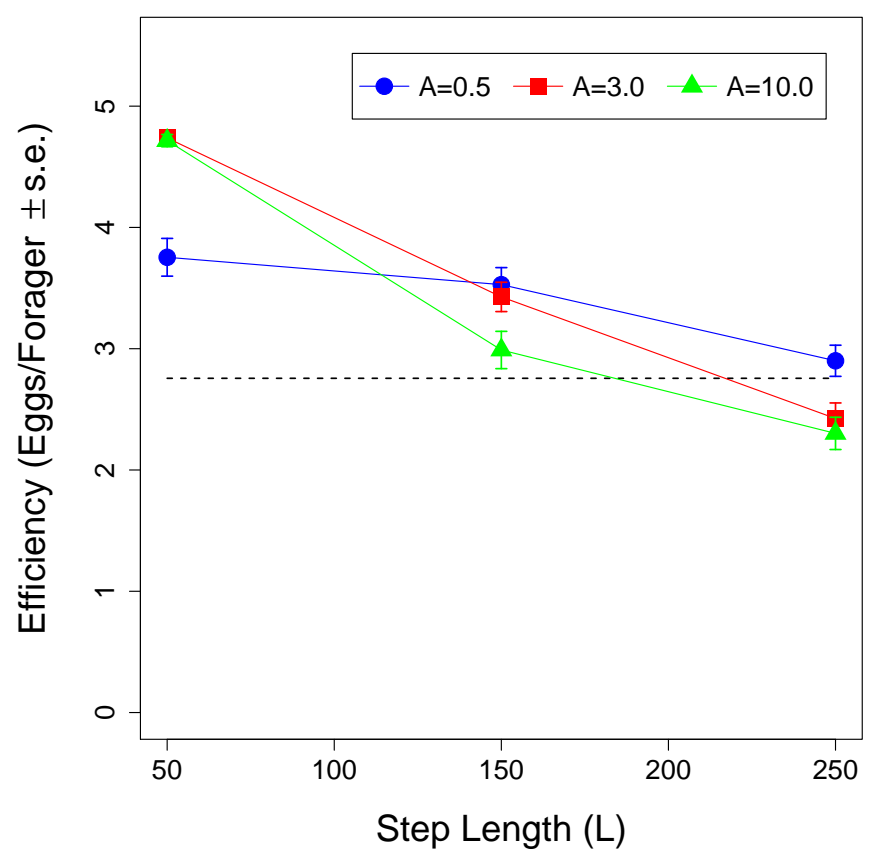

Figure A.8-8: Search efficiency vs. L and k- Trial E (Olfaction and Vision), Multiple Eggs. Proportion of eggs per forager released ( $y$-axis) vs. step length $(L)$ for various turning angle concentrations $(k)$. Results are averaged over all combinations of $R$ and $P$. 
a)

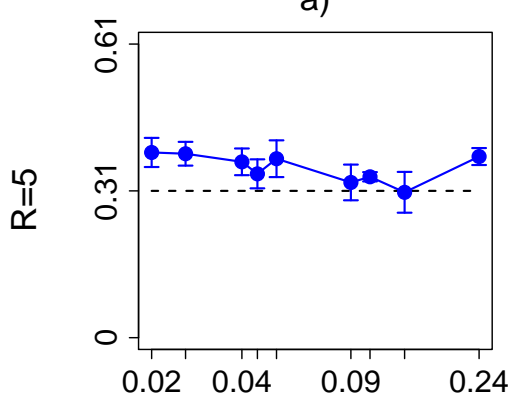

d)

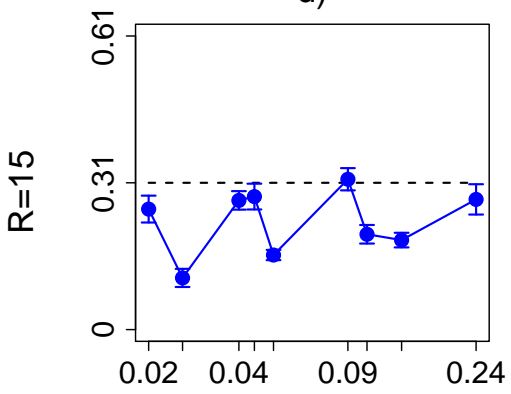

g)

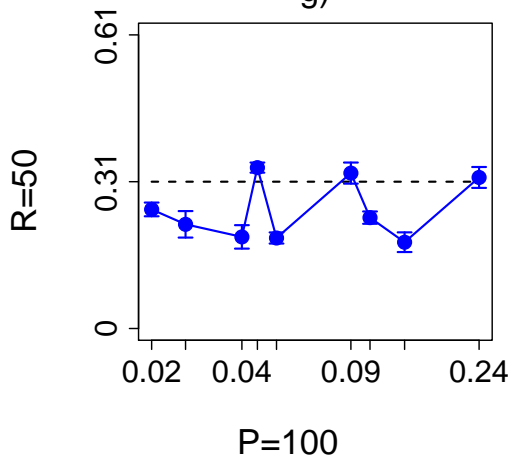

b)

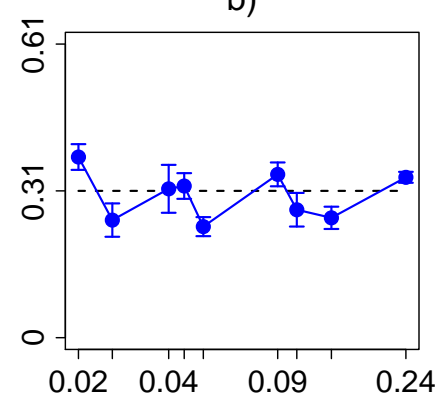

e)

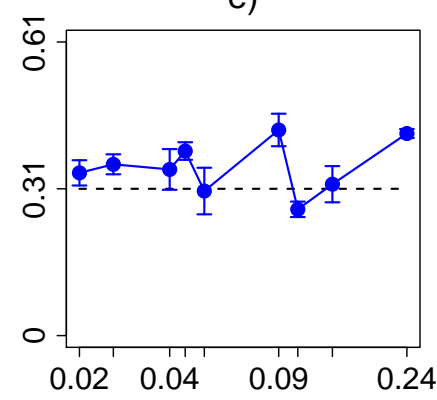

h)

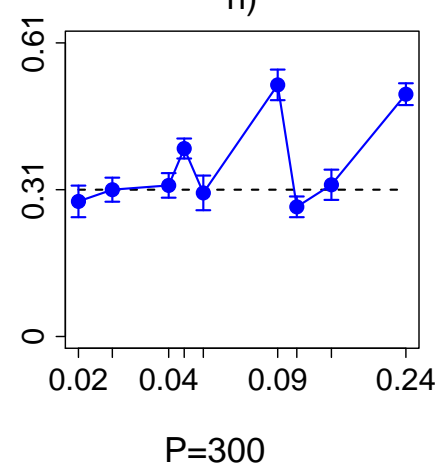

c)

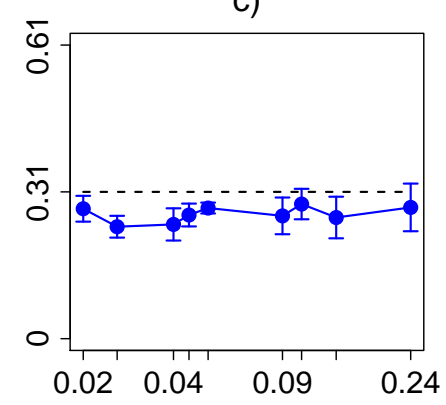

f)

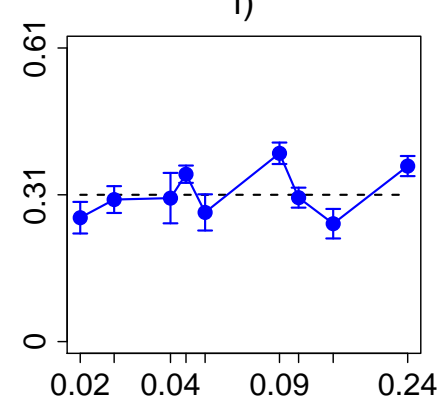

i)

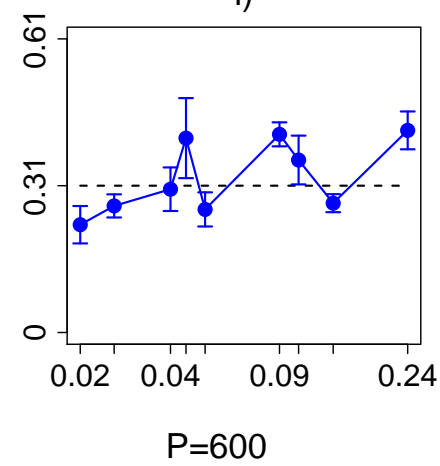

Figure A.8-9: Centre ratio vs. layout - Trial E (Olfaction and Vision), Multiple Eggs. Proportion of eggs per plant on centre resources (y-axis) vs. Sinuosity is plotted for combinations of radius of attraction $(R)$ and patch size $(P)$ in each sub-plot $(a-i)$. Radius of attraction increases from top to bottom and patch size increases from left to right. The layout is such that these results may be compared with Figure 3.32 to see the patch layouts. 
a)

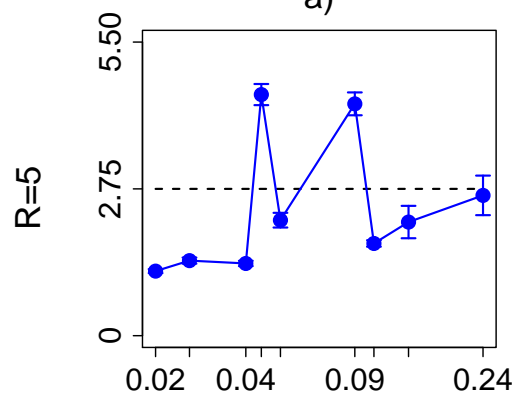

d)

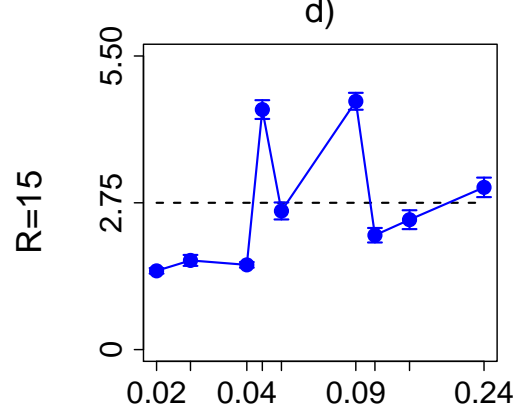

g)

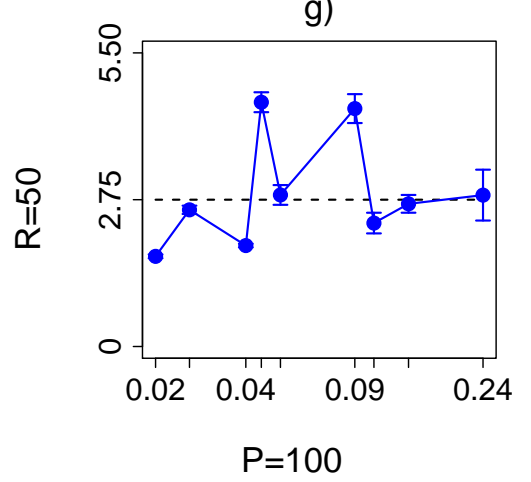

b)

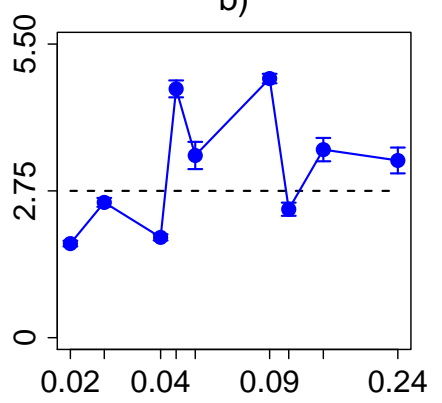

e)

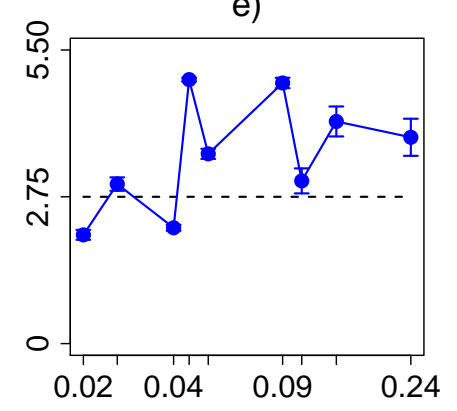

h)

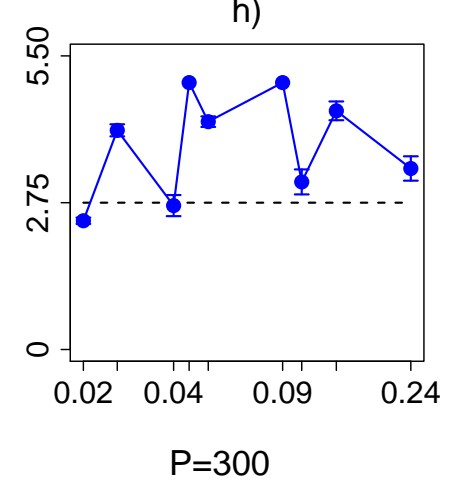

c)

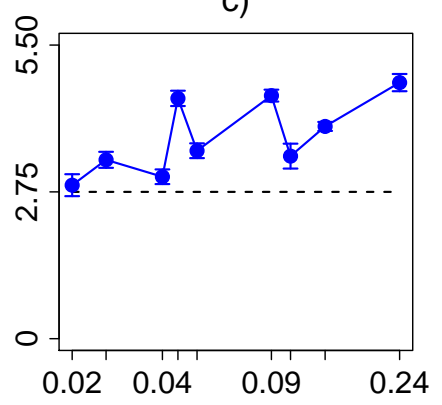

f)

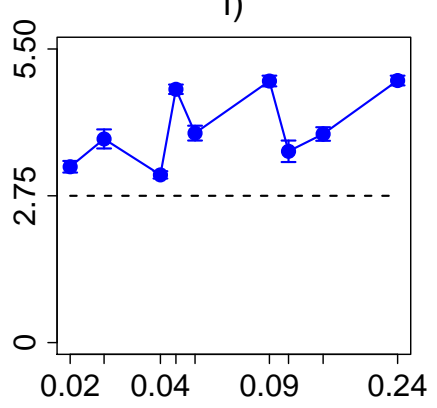

i)

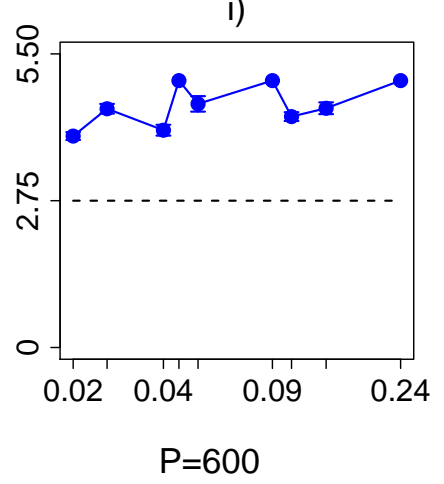

Figure A.8-10: Search efficiency vs. layout - Trial E (Olfaction and Vision), Multiple Eggs. Proportion of eggs per forager released (y-axis) vs. sinuosity is plotted for combinations of radius of attraction $(R)$ and patch size $(P)$ in each sub-plot (a-i). Radius of attraction increases from top to bottom and patch size increases from left to right. The layout is such that these results may be compared with Figure 3.32 to see the patch layouts. 
Appendix B

Sense and scale detailed simulation results 


\section{B.1 Bray-Curtis Optimisation Statistics}

\begin{tabular}{|c|c|c|c|c|c|c|c|c|c|c|c|c|c|c|c|}
\hline \multirow[b]{2}{*}{$\mathbf{R}$} & \multicolumn{3}{|c|}{ Parameters } & \multicolumn{3}{|c|}{ 1x1m (2 d.f.) } & \multicolumn{3}{|c|}{$6 \times 6 \mathrm{~m} 3$ d.f. } & \multicolumn{3}{|c|}{$48 \times 48 m$ ( 2 d.f.) } & \multicolumn{3}{|c|}{ ScaleCode (5/6 d.f.) } \\
\hline & $\mathbf{L}$ & A & BC & $\chi^{2}$ & (p) & BC & 2 & (p) & BC & $\gamma^{2}$ & (p) & BC & $\gamma^{2}$ & (p) & mean $B C$ \\
\hline 5 & 150 & 0.5 & 0.040 & 5.72 & 0.057 & 0.157 & 74.89 & $<0.001$ & - & - & - & 0.143 & 80.04 & $<0.001$ & 0.1133 \\
\hline 5 & 150 & 3.0 & 0.062 & 18.15 & $<0.001$ & 0.237 & 65.28 & $<0.001$ & 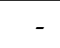 & - & - & 0.211 & 89.35 & $<0.001$ & 1699 \\
\hline 5 & 150 & 10.0 & 0.058 & 17.30 & $<0.001$ & 0.238 & 59.84 & $<0.001$ & - & - & - & 0.208 & 81.97 & $<0.001$ & 1680 \\
\hline 5 & 250 & 0.5 & 0.039 & 5.62 & 0.060 & 0.196 & 78.68 & $<0.001$ & . & - & - & 0.177 & 92.52 & $<0.001$ & 0.1372 \\
\hline 5 & 250 & 3.0 & 0.056 & 15.92 & $<0.001$ & 0.242 & 65.91 & $<0.001$ & . & - & - & 0.213 & 88.99 & $<0.001$ & 0.1706 \\
\hline 5 & 250 & 10.0 & 0.052 & 13.64 & 0.001 & 0.237 & 64.98 & $<0.001$ & - & - & - & 0.208 & 85.34 & $<0.0 c$ & 0.1659 \\
\hline 5 & 350 & 0.5 & 0.046 & 9.39 & 0.009 & 0.185 & 56.34 & $<0.001$ & - & - & - & 0.168 & 70.77 & $<0.001$ & 0.1334 \\
\hline 5 & 350 & 3.0 & 0.054 & 13.78 & 0.001 & 0.241 & 63.38 & $<0.001$ & 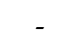 & - & - & 0.209 & 80.68 & $<0.001$ & 0.1679 \\
\hline 5 & 350 & 10.0 & 0.049 & 12.7 & 0.002 & 0 . & 61 & $<0.001$ & - & - & - & 0.207 & 80.84 & $<0.001$ & 1647 \\
\hline 10 & 150 & 0.5 & 0.124 & 61.27 & $<0.001$ & 0.145 & 120.59 & $<0.001$ & - & - & - & 0.144 & 127.92 & $<0.001$ & 0.1378 \\
\hline 10 & 150 & 3.0 & 0.080 & 22.24 & $<0.001$ & 0.192 & 87.89 & $<0.001$ & - & - & - & 0.168 & 89.75 & $<0.001$ & 0.1469 \\
\hline 10 & 150 & 10.0 & 0.068 & 15.94 & $<0.001$ & 0.195 & 82.97 & $<0.001$ & - & - & - & 0.168 & 84.05 & $<0.001$ & 0.1438 \\
\hline 10 & 250 & 0.5 & 0.119 & 51.39 & $<0.001$ & 0.170 & 106.87 & $<0.001$ & - & - & - & 0.159 & 113.15 & $<0.001$ & 0.1494 \\
\hline 10 & 250 & 3.0 & 0.082 & 23.04 & $<0$. & 0.198 & 88.01 & - & 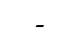 & - & - & 71 & 91.54 & $<0$ & 15 \\
\hline 10 & 250 & 10.0 & 0.072 & 18.48 & $<0.001$ & 0.203 & 81.55 & $<0.001$ & - & - & - & 71 & 84.25 & $<0.001$ & 1487 \\
\hline 10 & 350 & 0.5 & 0.105 & 37.72 & $<0.001$ & 0.158 & 88.07 & $<0.001$ & - & - & - & 0.146 & 92.57 & $<0.001$ & 0.1361 \\
\hline 10 & 350 & 3.0 & 0.074 & 18.91 & $<0.001$ & 0 . & 88.96 & $<0.001$ & - & - & - & 8 & 91.58 & $<0.00$ & 0 \\
\hline 10 & 350 & 10.0 & 0.073 & 18.31 & $<0.001$ & 0. & 90.73 & $<0.00$ & - & - & - & 34 & 92.77 & $<0.0$ & 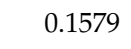 \\
\hline 20 & 150 & 0.5 & 0.211 & 190.25 & $<0.001$ & 0.169 & 187.48 & $<0.001$ & - & - & - & 0.172 & 247.47 & $<0.001$ & 0.1842 \\
\hline 20 & 150 & 3.0 & 0.172 & 109.33 & $<0.001$ & 0.176 & 137.14 & $<0.001$ & - & - & - & 0.168 & 170.55 & $<0.001$ & 0.1721 \\
\hline 20 & 150 & 10.0 & 0.174 & 114.49 & $<0.001$ & 0.180 & 131.92 & $<0.001$ & - & - & - & 0.169 & 173.74 & $<0.001$ & 0.1744 \\
\hline 20 & 250 & 0.5 & 0.192 & 147.60 & $<0.001$ & 0.111 & 154.10 & $<0.001$ & - & - & - & 0.121 & 197.61 & $<0.0$ & 0.1415 \\
\hline 20 & 250 & 3.0 & 0.176 & 117.72 & $<0.001$ & 0.201 & 135.46 & $<0.001$ & - & - & - & 0.184 & 180.62 & $<0.001$ & 0.1871 \\
\hline 20 & 250 & 10.0 & 0.168 & 107.88 & $<0.001$ & 0.181 & 130.25 & $<0.001$ & - & - & - & 0.170 & 166.02 & $<0.001$ & 0.1729 \\
\hline 20 & 350 & 0.5 & 0.195 & 147.02 & $<0.001$ & 0.139 & 163.27 & $<0.001$ & - & - & - & 0.144 & 204.53 & $<0.001$ & 0.1596 \\
\hline 20 & 350 & 3.0 & 0.170 & 108.24 & $<0.001$ & 0.174 & 125.92 & $<0.001$ & - & - & - & 0.164 & 168.90 & $<0.001$ & 0.1693 \\
\hline 20 & 350 & 10.0 & 0.178 & 121.14 & $<0.001$ & 0.189 & 133.68 & $<0.001$ & - & - & - & 0.176 & 181.27 & $<0.001$ & 0.1811 \\
\hline
\end{tabular}

Table B.1: Trial C1 (CRW). Using LEVIN-06-II layout, with Single Egg. Shows results from the bray-Curtis comparisons across various measures with the mean in the right hand column. Numbers highlighted by horizontal lines show the lowest value (best match) for each Radius Of Attraction (R). 


\begin{tabular}{|c|c|c|c|c|c|c|c|c|c|c|c|c|c|c|c|}
\hline \multirow[b]{2}{*}{ R/FD } & \multicolumn{3}{|c|}{ arameters } & \multicolumn{3}{|c|}{ 1x1m (2 d.f.) } & \multicolumn{3}{|c|}{$6 \times 6 \mathrm{~m} 3$ d.f. } & \multicolumn{3}{|c|}{$48 \times 48 m$ ( 2 d.f.) } & \multicolumn{3}{|c|}{ ScaleCode (5/6 d.f.) } \\
\hline & L & A & BC & $\chi$ & (p) & BC & $X$ & ) & BC & $\chi^{2}$ & (p) & C & & (p) & BC \\
\hline 5 & 2 & 0.5 & 0 & 45.21 & 0.001 & 0 & 23.63 & $<0.0$ & 4 & 2.15 & 0.342 & 0 & 56.88 & $<0.0$ & \\
\hline 5 & & 3.0 & 0 & 54.75 & 0.001 & 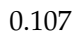 & 61.37 & $<0.001$ & 0.128 & 17.25 & $<0.001$ & 0 & 8.45 & $<$ & 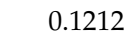 \\
\hline 5 & & 1 & & 8 & 1 & & 6 & 1 & & 15 & $<$ & & 17 & & \\
\hline 5 & 1000 & 0.5 & 0 & 53.07 & $<0.001$ & 06 & 50.33 & 0.001 & 8 & 12.89 & 0.002 & 0.127 & 78.36 & $<$ & 144 \\
\hline 5 & 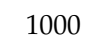 & 3.0 & 0 & 44.79 & $<0.001$ & C & 61.27 & $<0.001$ & 0.133 & 19.97 & $<0.001$ & 0.127 & 86.98 & $<$ & 169 \\
\hline 5 & & 10.0 & 0 & 3.88 & 1 & & .25 & $<0.001$ & 3 & 2 & $<0.001$ & 7 & 9 & $<$ & 1357 \\
\hline 5 & 10000 & 0.5 & 0 & 4 & $<0.001$ & 110 & 72.98 & $<0.001$ & 8 & 2 & $<0.001$ & 44 & 102 & & \\
\hline 5 & 10000 & 3.0 & 0.114 & 50.77 & $<0.001$ & 0.101 & 58.97 & $<0.001$ & 0.134 & 19.65 & $<0.001$ & 0.131 & 88.04 & $<$ & 199 \\
\hline 5 & 0 & 10.0 & 0 & 52.97 & $<0$ & 0 & 75.99 & $<0$. & 1 & 16.40 & $<0$ & 33 & 100.73 & 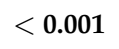 & 12 \\
\hline 1 & & 0.5 & & & & & & & & & & & & & \\
\hline 1 & 250 & 3.0 & 0 & 42.86 & $<0.001$ & 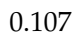 & 4 & $<0.001$ & 2 & 18.48 & $<0$ & 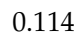 & 65.87 & & 74 \\
\hline 10 & 0 & 10.0 & 0.090 & 41.10 & $<0.001$ & 0.102 & 53.50 & $<0.001$ & 0.127 & 23.79 & $<0.001$ & 0.117 & 69.24 & & 1089 \\
\hline 10 & 1 & 0.5 & 0 & 44.81 & $<0.0$ & 0 & 42.17 & $<0.0$ & 0. & 7 & 4 & 0 & 55 & $<$ & 46 \\
\hline 1 & & ) & & T & 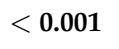 & & 4 & 1 & 0 & 2 & & & 8 & & 41 \\
\hline 10 & 00 & 10.0 & 0.101 & 50.09 & $<0.001$ & 0.108 & 56.59 & $<0.001$ & 0.114 & 19.56 & $<0.001$ & 0.118 & 74.09 & & 100 \\
\hline 10 & 000 & 0.5 & 0.100 & 46.54 & $<0.001$ & 0.110 & 53.11 & $<0.001$ & 0.095 & 16.28 & $<0.001$ & 0.112 & 67.34 & $<$ & 044 \\
\hline 10 & 000 & 3.0 & 0 & 47.34 & $<0.001$ & 0.114 & 56.90 & $<0.001$ & 0. & 22.54 & $<0.001$ & 0 & 73 & & 93 \\
\hline 1 & of & 10.0 & & & $<0.001$ & & 8.21 & & & & & & & & \\
\hline 20 & 2 & 0.5 & 0.1 & 120. & $<0.001$ & 67 & 12 & 1 & 1 & 31 & $<0.001$ & 0.173 & 16 & & 48 \\
\hline 20 & 250 & 3.0 & 0.115 & 97.13 & $<0.001$ & 0.101 & 81.04 & $<0$. & 0.072 & 26.44 & $<0.001$ & 0.108 & 101.45 & $<0$ & .0989 \\
\hline 20 & 0 & 10.0 & 0.119 & 104.46 & $<0.001$ & 0.100 & 90.76 & $<0.001$ & 0.068 & 36.65 & $<0.001$ & 0.111 & 112.33 & $<$ & 995 \\
\hline 2 & 1000 & 0.5 & 0.112 & 90.03 & $<0.001$ & 1 & 8.52 & $<0.001$ & 0. & 26.29 & $<0.001$ & 0.1 & 0 & & 4 \\
\hline 20 & 1000 & 3.0 & 0.108 & 85.99 & $<0.001$ & 0.091 & 69.33 & $<\mathbf{0}$. & 0.0 & 26.81 & $<0$ & 0.105 & 90.09 & $<$ & 931 \\
\hline 20 & 5 & 10.0 & 0.110 & 9 & $<0.001$ & 0 & 4 & $<0.0$ & 0. & 25.35 & $<\mathbf{0}$ & 0.102 & 20 & 1 & 01 \\
\hline 20 & 10000 & 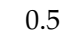 & 117 & 101.66 & $<0.001$ & 0.097 & 79.19 & $<0.001$ & 0.072 & 32.42 & $<0.001$ & 0.113 & 107.18 & $<0.001$ & 7 \\
\hline 20 & 10000 & 3.0 & 0.113 & 94.02 & $<0.001$ & 0.094 & 76.01 & $<0.001$ & 0.054 & 27.07 & $<0.001$ & 0.101 & 99.14 & $<0.001$ & 0.0907 \\
\hline 20 & 10000 & 10.0 & 0.115 & 6.13 & $<0.001$ & 0.098 & 74.02 & $<0.001$ & 0.061 & 30.78 & $<0.001$ & 0.107 & 99.65 & $<0.001$ & 0.0955 \\
\hline
\end{tabular}

Table B.2: Trial C2 (CRW). Using KAITOKE-04 layout, with Single Egg. Shows results from the bray-Curtis comparisons across various measures with the mean in the right hand column. Numbers highlighted by horizontal lines show the lowest value (best match) for each Radius Of Attraction (R). 


\begin{tabular}{|c|c|c|c|c|c|c|c|c|c|c|c|c|c|c|c|}
\hline \multirow[b]{2}{*}{$\mathbf{R}$} & \multicolumn{3}{|c|}{ Parameters } & \multicolumn{3}{|c|}{ 1x1m (2 d.f.) } & \multicolumn{3}{|c|}{$6 \times 6 \mathrm{~m} 3$ d.f. } & \multicolumn{3}{|c|}{$48 \times 48 m$ ( 2 d.f. $)$} & \multicolumn{3}{|c|}{ ScaleCode (5/6 d.f.) } \\
\hline & $\mathbf{L}$ & A & BC & $\chi^{2}$ & (p) & BC & $\chi^{2}$ & (p) & BC & $\chi^{2}$ & (p) & BC & $\chi^{2}$ & (p) & mean $B C$ \\
\hline 5 & 150 & 0.5 & 0.088 & 43.12 & $<0.001$ & 0.276 & 67.93 & $<0.001$ & - & - & - & 0.243 & 112.67 & $<0.001$ & 0.2022 \\
\hline 5 & 150 & 3.0 & 0.090 & 43.37 & $<0.001$ & 0.302 & 79.48 & $<0.001$ & - & - & - & 0.259 & 121.74 & $<0.001$ & 0.2170 \\
\hline 5 & 150 & 10.0 & 0.088 & 44.05 & $<0.001$ & 0.286 & 73.84 & $<0.001$ & - & - & - & 0.248 & 117.28 & $<0.001$ & 0.2070 \\
\hline 5 & 250 & 0.5 & 0.067 & 25.35 & $<0.001$ & 0.295 & 85.78 & $<0.001$ & - & - & - & 0.255 & 117.81 & $<0.001$ & 0.2057 \\
\hline 5 & 250 & 3.0 & 0.063 & 21.90 & $<0.001$ & 0.269 & 70.81 & $<0.001$ & - & - & - & 0.233 & 96.93 & $<0.001$ & 0.1885 \\
\hline 5 & 250 & 10.0 & 0.074 & 32.40 & $<0.001$ & 0.274 & 67.83 & $<0.001$ & - & - & - & 0.235 & 100.39 & $<0.001$ & 0.1942 \\
\hline 5 & 350 & 0.5 & 0.074 & 28.78 & $<0.001$ & 0.262 & 68.91 & $<0.001$ & - & - & - & 0.231 & 101.83 & $<0.001$ & 0.1890 \\
\hline 5 & 350 & 3.0 & 0.066 & 26.22 & $<0.001$ & 0.276 & 72.25 & $<0.001$ & - & - & - & 0.240 & 103.57 & $<0.001$ & 0.1937 \\
\hline 5 & 350 & 10.0 & 0.071 & 25.13 & $<0.001$ & 0.256 & 65.72 & $<0.001$ & - & - & - & 0.226 & 94.86 & $<0.001$ & 0.1842 \\
\hline 10 & 150 & 0.5 & 0.050 & 11.14 & 0.004 & 0.266 & 79.26 & $<0.001$ & - & - & - & 0.222 & 91.18 & $<0.001$ & 0.1796 \\
\hline 10 & 150 & 3.0 & 0.039 & 7.70 & 0.021 & 0.256 & 68.46 & $<0.001$ & - & - & - & 0.211 & 77.22 & $<0.001$ & 0.1688 \\
\hline 10 & 150 & 10.0 & 0.053 & 12.96 & 0.002 & 0.250 & 67.79 & $<0.001$ & - & - & - & 0.210 & 81.26 & $<0.001$ & 0.1708 \\
\hline 10 & 250 & 0.5 & 0.048 & 8.28 & 0.016 & 0.240 & 74.74 & $<0.001$ & - & - & . & 0.198 & 80.37 & $<0.001$ & 0.1619 \\
\hline 10 & 250 & 3.0 & 0.055 & 11.13 & 0.004 & 0.273 & 82.13 & $<0.001$ & - & - & - & 0.220 & 90.81 & $<0.001$ & 0.1826 \\
\hline 10 & 250 & 10.0 & 0.040 & 5.92 & 0.052 & 0.242 & 68.48 & $<0.001$ & - & - & - & 0.199 & 73.67 & $<0.001$ & 0.1602 \\
\hline 10 & 350 & 0.5 & 0.051 & 9.83 & 0.007 & 0.246 & 78.30 & $<0.001$ & - & - & - & 0.201 & 84.23 & $<0.001$ & 0.1659 \\
\hline 10 & 350 & 3.0 & 0.052 & 11.92 & 0.003 & 0.236 & 80.40 & $<0.001$ & - & - & - & 0.193 & 84.91 & $<0.001$ & 0.1603 \\
\hline 10 & 350 & 10.0 & 0.040 & 6.23 & 0.044 & 0.239 & 80.41 & $<0.001$ & - & - & - & 0.197 & 83.77 & $<0.001$ & 0.1590 \\
\hline 20 & 150 & 0.5 & 0.099 & 34.59 & $<0.001$ & 0.230 & 100.33 & $<0.001$ & - & - & - & 0.193 & 112.49 & $<0.001$ & 0.1743 \\
\hline 20 & 150 & 3.0 & 0.094 & 31.10 & $<0.001$ & 0.228 & 90.98 & $<0.001$ & - & - & - & 0.192 & 103.54 & $<0.001$ & 0.1714 \\
\hline 20 & 150 & 10.0 & 0.071 & 20.51 & $<0.001$ & 0.273 & 95.70 & $<0.001$ & - & - & - & 0.223 & 106.89 & $<0.001$ & 0.1891 \\
\hline 20 & 250 & 0.5 & 0.122 & 51.69 & $<0.001$ & 0.242 & 107.65 & $<0.001$ & - & - & - & 0.211 & 131.14 & $<0.001$ & 0.1917 \\
\hline 20 & 250 & 3.0 & 0.124 & 52.46 & $<0.001$ & 0.228 & 101.93 & $<0.001$ & - & - & - & 0.202 & 126.22 & $<0.001$ & 0.1846 \\
\hline 20 & 250 & 10.0 & 0.113 & 43.50 & $<0.001$ & 0.222 & 99.76 & $<0.001$ & - & - & - & 0.191 & 116.01 & $<0.001$ & 0.1753 \\
\hline 20 & 350 & 0.5 & 0.132 & 60.58 & $<0.001$ & 0.205 & 111.72 & $<0.001$ & - & - & - & 0.181 & 133.02 & $<0.001$ & 0.1728 \\
\hline 20 & 350 & 3.0 & 0.138 & 66.75 & $<0.001$ & 0.210 & 102.51 & $<0.001$ & - & - & - & 0.191 & 133.99 & $<0.001$ & 0.1798 \\
\hline 20 & 350 & 10.0 & 0.117 & 47.02 & $<0.001$ & 0.207 & 101.31 & $<0.001$ & - & - & - & 0.180 & 117.09 & $<0.001$ & 0.1679 \\
\hline
\end{tabular}

Table B.3: Trial C3 (CRW). Using LEVIN-06-II layout, with Multiple Egg. Shows results from the bray-Curtis comparisons across various measures with the mean in the right hand column. Numbers highlighted by horizontal lines show the lowest value (best match) for each Radius Of Attraction (R). 


\begin{tabular}{|c|c|c|c|c|c|c|c|c|c|c|c|c|c|c|c|}
\hline \multirow[b]{2}{*}{$\mathbf{R}$} & \multicolumn{3}{|c|}{ Parameters } & \multicolumn{3}{|c|}{ 1x1m (2 d.f.) } & \multicolumn{3}{|c|}{$6 \times 6 \mathrm{~m} 3$ d.f. } & \multicolumn{3}{|c|}{$48 \times 48 m$ ( 2 d.f.) } & \multicolumn{3}{|c|}{ ScaleCode (5/6 d.f.) } \\
\hline & $\mathbf{L}$ & $\mathbf{A}$ & BC & $\chi^{2}$ & (p) & BC & $\chi^{2}$ & (p) & BC & $\chi^{2}($ & (p) & BC & $\chi^{2}$ & (p) & \\
\hline 5 & 150 & 0.5 & 25 & 2.47 & 91 & 5 & 99 & $<$ & - & - & - & 76 & 74.80 & 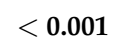 & 1354 \\
\hline 5 & 150 & 3.0 & 0.120 & 86.67 & $<0.001$ & 0.452 & 198.29 & $<0.001$ & - & - & - & 0.340 & 232.65 & $<0$ & 0.3041 \\
\hline 5 & 150 & 10.0 & 0.165 & 133.39 & $<0.001$ & 0.562 & 322.39 & $<0.001$ & - & - & - & .423 & 349.12 & -00 & 3833 \\
\hline 5 & 250 & 0.5 & 0.039 & 6.91 & 0.032 & 5 & 63.49 & $<0.001$ & 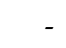 & - & - & 0.181 & 75.86 & $<0.0$ & 15 \\
\hline 5 & 250 & 3.0 & 0.086 & 51.54 & $<0.001$ & 0.380 & 132.81 & $<0.001$ & - & - & - & 0.289 & 160.51 & $<0.001$ & 0.2516 \\
\hline 5 & 250 & 10.0 & 0.132 & 94.88 & $<0.001$ & 0.471 & 221.01 & $<0.001$ & - & - & - & 0.358 & 248.37 & $<0.001$ & 3205 \\
\hline 5 & 350 & 0.5 & 0.043 & 9.04 & 0.011 & 0.219 & 68.46 & $<0.001$ & 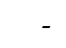 & - & - & 0.193 & 83.83 & $<0.001$ & 0.1516 \\
\hline 5 & 350 & 3.0 & 80 & 48.81 & $<0.001$ & 70 & 119.56 & $<0$. & - & - & - & 35 & 74 & $<$ & 0.2451 \\
\hline 5 & 350 & 10.0 & 0.105 & 62.22 & $<0.001$ & 0.413 & 162.44 & $<0.001$ & - & - & - & 0.313 & 185.27 & $<0.001$ & 0.2772 \\
\hline 10 & 150 & 0.5 & 0.106 & 45.75 & $<0.001$ & 0.155 & 93.61 & $<0.001$ & - & - & - & 0.145 & 101.59 & $<0$ & 01353 \\
\hline 10 & 150 & 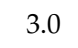 & & 4.11 & 0.128 & & 6 & $<0.001$ & - & - & - & 0.298 & 145.40 & $<0.00$ & 2 \\
\hline 10 & 150 & 10.0 & 0.086 & 32.82 & $<0.001$ & 0.498 & 254.89 & $<0.001$ & - & - & - & 0.381 & 260.09 & $<0.001$ & 3217 \\
\hline 10 & 250 & 0.5 & 0.091 & 31.21 & $<0.001$ & 0.180 & 86.60 & $<0.001$ & - & - & - & 0.161 & 90.29 & $<0.001$ & 0.1441 \\
\hline 10 & 250 & 3.0 & 0.015 & 1.11 & 0.574 & 0.347 & 107.40 & $<0.001$ & - & - & - & 0.264 & 109.60 & $<0.001$ & 0.2088 \\
\hline 10 & 250 & 10.0 & 0. & 0 & 10 & 0 & 1 & $<0.001$ & . & - & - & 26 & 17 & $<$ & 0.2658 \\
\hline 10 & 350 & 0.5 & 0 & 31.15 & $<0.001$ & 0 & 65 & $<0.001$ & - & - & - & 56 & 93.97 & $<0$ & 2 \\
\hline 10 & 350 & 3.0 & 0.044 & 7.22 & 0.027 & 0.305 & 93.88 & $<0.001$ & - & - & - & 0.246 & 102.05 & $<0.001$ & 0.1984 \\
\hline 10 & 350 & 10.0 & 0.019 & 1.97 & 0.374 & 0.384 & 131.86 & $<0.001$ & - & - & - & 0.291 & 135.46 & $<0.001$ & 0.2315 \\
\hline 20 & 150 & 0.5 & 0.196 & 165.43 & $<0.001$ & 0.115 & 151.91 & $<0.001$ & - & - & - & 0.123 & 210.49 & $<0.0$ & 01 \\
\hline 20 & 150 & 3.0 & 0.082 & 27.64 & $<0.001$ & 0.319 & 99.93 & $<0.001$ & - & - & - & 0.281 & 142.15 & $<0.001$ & 0.2276 \\
\hline 20 & 150 & 10.0 & 0.023 & 4.80 & 0.091 & 0.437 & 183.50 & $<0.001$ & - & - & - & 0.349 & 231.85 & $<0.001$ & 0.2697 \\
\hline 20 & 250 & 0.5 & 0.184 & 133.99 & $<0.001$ & 0.141 & 142.15 & $<0.001$ & - & - & - & 0.143 & 183.52 & $<0.0$ & 0.1558 \\
\hline 20 & 250 & 3.0 & 0.112 & 53.19 & $<0.001$ & 0.295 & 100.71 & $<0.001$ & - & - & - & 0.264 & 150.35 & $<0.001$ & 0.22 \\
\hline 20 & 250 & 10.0 & 0.057 & 17.48 & $<0.001$ & 0.375 & 121.92 & $<0.001$ & - & - & - & 0.315 & 167.46 & $<0.001$ & 0.2489 \\
\hline 20 & 350 & 0.5 & 0.184 & 139.44 & $<0.001$ & 0.140 & 130.76 & $<0.001$ & - & - & - & 0.140 & 182.48 & $<0.001$ & 0.1546 \\
\hline 20 & 350 & 3.0 & 0.123 & 58.29 & $<0.001$ & 0.2 & 81.70 & $<0.001$ & 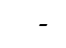 & - & - & 0.222 & 129.66 & $<0.001$ & 0.1943 \\
\hline 20 & 350 & 10.0 & 0.081 & 8.52 & $<0.001$ & 0.311 & 89.55 & $<0.001$ & - & - & - & 0.276 & 136.90 & $<\mathbf{0}$ & 0.2 \\
\hline
\end{tabular}

Table B.4: Trial D1 (Olfaction). Using LEVIN-06-II layout, with Single Egg. Shows results from the bray-Curtis comparisons across various measures with the mean in the right hand column. Numbers highlighted by horizontal lines show the lowest value (best match) for each Radius Of Attraction (R). 


\begin{tabular}{|c|c|c|c|c|c|c|c|c|c|c|c|c|c|c|c|}
\hline \multirow[b]{2}{*}{ FD } & \multicolumn{3}{|c|}{ Parameters } & \multicolumn{3}{|c|}{ 1x1m (2 d.f.) } & \multicolumn{3}{|c|}{$6 \times 6 \mathrm{~m} 3$ d.f. } & \multicolumn{3}{|c|}{$48 \times 48 m$ ( 2 d.f. $)$} & \multicolumn{3}{|c|}{ caleCode (5/6 d.f.) } \\
\hline & L & A & C & $\chi^{2}$ & (p) & B & $\chi^{2}$ & (p) & BC & $\chi$ & & C & & (p) & 1 \\
\hline 100 & 50 & 0.5 & & 4.38 & 0.001 & 1 & 186.22 & $<0$. & - & - & - & 96 & 266.76 & $<0.001$ & \\
\hline & 150 & 3.0 & & 5 & .001 & 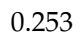 & 8 & 0 & - & - & - & 0.235 & 149.11 & $<0.001$ & 1977 \\
\hline & 0 & .0 & & 0 & 1 & & 13 & $-e_{0}$ & - & - & - & & 100 & & \\
\hline 1 & 50 & 0.5 & & 99 & 1 & & 1 & $<\mathbf{0}$ & - & - & - & & & 0.001 & \\
\hline 100 & 250 & 3.0 & 127 & 33 & 1 & 0 & 11 & $<$ & & - & - & 0.284 & 4 & 0.001 & \\
\hline & 250 & 0 & 081 & 26.73 & 001 & 5 & 4 & -4 & - & - & - & 8 & & $<0000$ & \\
\hline & (50 & .5 & & ) & & & 1 & & - & - & - & & 140.7 & & \\
\hline 100 & 50 & 3.0 & 7 & 48.17 & $<0.001$ & 0. & 76.47 & $<0$ & - & - & - & & 108.70 & & \\
\hline 100 & 350 & .0 & 068 & 17.07 & 1 & 0 & 9 & $<0.001$ & 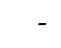 & - & - & & 9958 & & \\
\hline 600 & ( & 5 & & 7 & 1 & & 5 & & 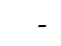 & 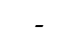 & - & & & & \\
\hline 600 & 50 & .0 & 138 & 20 & 0.001 & 0 & 23 & & - & - & - & 8 & 3 & & \\
\hline 600 & 150 & 0 & 137 & ; & 1 & 0 & 4 & $<$ & - & - & - & 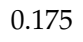 & & & \\
\hline & & 0.5 & & 1 & & & 4 & & & - & - & & & & \\
\hline 000 & 50 & 3.0 & 148 & 90.49 & 1 & 0.336 & 258.21 & $<0$ & - & - & - & 0.337 & ל & & 37 \\
\hline 00 & 250 & 10.0 & 9 & .10 & $<0.001$ & 0.194 & 83.07 & $<0 . c$ & - & - & - & 13 & 188.00 & & 752 \\
\hline 600 & 350 & 0.5 & 8 & 6.58 & 1 & 0. & 42 & $<$ & 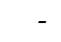 & - & - & 4 & 49 & & 15 \\
\hline 600 & 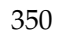 & 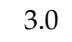 & & & & & & & 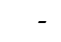 & 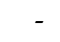 & - & & & & \\
\hline 600 & 350 & 10.0 & & & & & & & - & - & - & & & & \\
\hline 10 & 0 & 0.5 & 3 & 0.01 & 1 & 0.472 & 5 & & - & 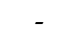 & - & 0.465 & 63 & & 68 \\
\hline 180 & 0 & 3.0 & 7 & 5.31 & $<0$ & 0.455 & 486.14 & $<0$ & - & - & - & 0 & 6 & $<0.001$ & 07 \\
\hline & & 0 & & 33.70 & & & & & - & - & - & & & & \\
\hline 1800 & 0 & 0.5 & 2 & 86 & & 0. & 5 & & - & - & - & & & & \\
\hline 1800 & 250 & 3.0 & 72 & 9.08 & $<0$ & 0.432 & 43 & $<$ & - & - & - & 0.424 & 51 & & 425 \\
\hline 1800 & 0 & .0 & 4 & 7 & 1 & 3 & 4 & $<$ & - & - & - & 0.425 & 4 & $<0.001$ & 41 \\
\hline 1800 & 50 & 0.5 & 0.189 & 50.44 & $<0.001$ & 0.450 & 493.24 & $<0.001$ & - & - & - & & $550 \mathrm{c}$ & & 0360 \\
\hline 1800 & 0 & 3.0 & 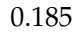 & 13 & 01 & 0.448 & 471.23 & $<0$ & - & - & - & 40 & 547.10 & $<$ & 577 \\
\hline 1800 & 50 & 0.0 & 74 & 7.03 & 0.001 & 0.444 & 450.83 & $<0.001$ & - & - & - & 0.435 & 523.25 & $<0.001$ & 0.3512 \\
\hline
\end{tabular}

Table B.5: Trial D2 (Olfaction and Vision). Using LEVIN-06-II layout, with Single Egg. Shows results from the bray-Curtis comparisons across various measures with the mean in the right hand column. Numbers highlighted by horizontal lines show the lowest value (best match) for each Visual Field Depth (FD. 


\begin{tabular}{|c|c|c|c|c|c|c|c|c|c|c|c|c|c|c|c|}
\hline \multirow[b]{2}{*}{ FD } & \multicolumn{3}{|c|}{ arameters } & \multicolumn{3}{|c|}{ 1x1m (2 d.f.) } & \multicolumn{3}{|c|}{$6 \times 6 \mathrm{~m} 3$ d.f. } & \multicolumn{3}{|c|}{$48 \times 48 m$ ( 2 d.f. $)$} & \multicolumn{3}{|c|}{ caleCode (5/6 d.f.) } \\
\hline & L & A & $C$ & $\chi^{2}$ & (p) & B & & (p) & BC & & & BC & & (p) & BC \\
\hline & 50 & 0.5 & 2 & 5.77 & 0.001 & 0 & 247.24 & $<0.00$ & - & & - & & 34 & $<00$ & \\
\hline & & 3.0 & & 7.90 & 1 & & 16 & 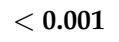 & - & & - & ( & 220.0 & & \\
\hline & 50 & 0 & & 24 & & & & & - & & - & & 202.49 & & \\
\hline 100 & 250 & 0.5 & 1 & 5.18 & 1 & 0 . & 17 & $<$ & - & & - & & & & \\
\hline 100 & 50 & 0.0 & 0 & 8.10 & 1 & 9 & 1 & $<$ & - & - & - & - & 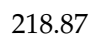 & & \\
\hline 100 & 0 & 0 & & 3.98 & 0.001 & 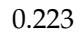 & 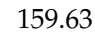 & 000 & - & & - & & 20778 & & \\
\hline 100 & 350 & 5 & & 6 & .001 & & 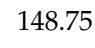 & $<$ & - & & - & & 180.92 & & \\
\hline & 50 & 3.0 & 55 & 97.56 & 1 & 0. & 117 & $<0$ & - & & - & & 14 & & \\
\hline & 350 & .0 & 161 & 8 & 1 & 0 & 1 & $<$ & - & & - & & 137.79 & & \\
\hline 600 & 50 & 5 & 3 & 2 & 1 & 1 & 5 & $<$ & - & & - & & 618.22 & & \\
\hline & 50 & 0 & 180 & 3.42 & 0.001 & 0. & 494 & & - & - & - & 49 & 58 & & \\
\hline 600 & 50 & .0 & 6 & 54 & $<0$ & 0. & 43 & $<0$ & - & - & - & 5 & 53 & $<0.001$ & \\
\hline ( & 200 & 0.5 & & & 1 & & 517 & & - & 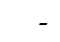 & - & & 587.97 & & \\
\hline 600 & 50 & 3.0 & 8 & 17 & $<0.001$ & 0.437 & 473.51 & $<0$ & - & - & - & 31 & 532.26 & & 517 \\
\hline 00 & 250 & 0.0 & 81 & 4.39 & $<0.001$ & 0.428 & 459.95 & $<0$ & - & - & - & 0.422 & 519.16 & & 439 \\
\hline 600 & 350 & 0.5 & 6 & 9.66 & 1 & 0 & 5 & $<\mathbf{0}$. & - & - & - & 0 & 55 & & 36 \\
\hline 600 & 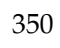 & 3.0 & & P & 1 & & & $<$ & 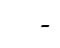 & 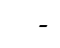 & - & & & & \\
\hline 600 & 350 & 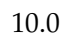 & & 1 & 1 & & 4 & & - & ( & - & & 482.93 & & \\
\hline 1000 & 50 & 0.5 & & & $<0$. & & & $<0$ & - & - & - & 0.454 & & 1 & 3637 \\
\hline 1800 & 0 & 0 & 176 & 9.74 & 1 & 0.452 & 488.31 & $<0$ & - & - & - & 0. & 58 & 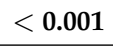 & 80 \\
\hline 1800 & 0 & .0 & 0.180 & 4 & 1 & 0 & 4 & & 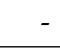 & - & - & & & & \\
\hline 1800 & 250 & 0.5 & 3 & .95 & 1 & 0. & 4 & $<$ & - & 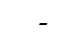 & - & 50 & 57 & & 338 \\
\hline 1800 & 0 & 3 & & & 1 & & & $<0$ & - & - & - & & 6 & & 78 \\
\hline 1800 & 0 & .0 & 0 & 2 & 0.001 & 0 & 5 & $<\mathbf{0 . 0}$ & - & - & - & 8 & 6 & 0 & 0.3714 \\
\hline 1800 & 50 & 0.5 & & 56.11 & $<0.001$ & 0.456 & 490.86 & $<\mathbf{0 . 0}$ & - & - & - & 0.448 & 566.93 & 5000 & 0.3635 \\
\hline 00 & 50 & 3.0 & 0.187 & 8.01 & $<0.001$ & 0.460 & 492.13 & $<0$ & - & - & - & 0.452 & 568.16 & $<0$ & 0.3662 \\
\hline 1800 & 350 & 10.0 & 88 & 6.40 & $<0.001$ & 0.451 & 490.62 & $<0.001$ & - & 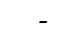 & - & 0.444 & 565.15 & $<0.001$ & 0.3608 \\
\hline
\end{tabular}

Table B.6: Trial D3 (Vision). Using LEVIN-06-II layout, with Single Egg. Shows results from the bray-Curtis comparisons across various measures with the mean in the right hand column. Numbers highlighted by horizontal lines show the lowest value (best match) for each Visual Field Depth (FD). 


\begin{tabular}{|c|c|c|c|c|c|c|c|c|c|c|c|c|c|c|c|}
\hline \multirow[b]{2}{*}{$\mathbf{R}$} & \multicolumn{3}{|c|}{ Parameters } & \multicolumn{3}{|c|}{ 1x1m (2 d.f.) } & \multicolumn{3}{|c|}{$6 \times 6 \mathrm{~m} 3$ d.f. } & \multicolumn{3}{|c|}{$48 \times 48 m$ ( 2 d.f. $)$} & \multicolumn{3}{|c|}{ ScaleCode (5/6 d.f.) } \\
\hline & L & A & ВС & $\chi^{2}$ & (p) & BC & $\chi^{2}$ & (p) & BC & $\chi^{2}$ & (p) & BC & $\chi^{2}$ & (p) & mean $B C$ \\
\hline 5 & 150 & 0.5 & 0.095 & 66.39 & $<0.001$ & 0.344 & 105.84 & $<0.001$ & 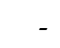 & - & - & 0.279 & 155.57 & $<0.001$ & 0.2395 \\
\hline 5 & 150 & 3.0 & 0.153 & 144.32 & $<0.001$ & 0.510 & 259.08 & $<0.001$ & - & - & - & 0.384 & 312.92 & $<0.001$ & 0.3489 \\
\hline 5 & 150 & 10.0 & 0.188 & 185.47 & $<0.001$ & 0.576 & 360.90 & $<0.001$ & - & - & - & 0.433 & 406.29 & $<0.001$ & 3989 \\
\hline 5 & 250 & 0.5 & 0.078 & 39.48 & $<0.001$ & 0.316 & 86.84 & $<0.001$ & - & - & - & 0.259 & 120.03 & $<0.001$ & 0.2177 \\
\hline 5 & 250 & 3.0 & 0.114 & 84.06 & $<0.001$ & 0.422 & 169.61 & $<0.001$ & - & - & - & 0.319 & 210.31 & $<0.001$ & 0.2850 \\
\hline 5 & 250 & 10.0 & 0.140 & 110.78 & $<0.001$ & 0.479 & 230.08 & $<0.001$ & - & - & - & 0.364 & 264.01 & $<0.001$ & 0.3275 \\
\hline 5 & 350 & 0.5 & 0.085 & 40.51 & $<0.001$ & 0 & 81.75 & $<0.001$ & - & - & - & 0.254 & 118.62 & $<0.00$ & 0.2136 \\
\hline 5 & 350 & 3.0 & 0.105 & 66.59 & $<0.001$ & 0.389 & 134.89 & $<0.001$ & - & - & - & 0.300 & 168.53 & $<0.001$ & 0.2645 \\
\hline 5 & 350 & 10.0 & 0.137 & 103.21 & $<0.001$ & 0.440 & 187.47 & $<0.001$ & - & - & - & 0.334 & 225.77 & $<0.001$ & 0.3039 \\
\hline 10 & 150 & 0.5 & 0.048 & 12.58 & 0.002 & 0.321 & 94.97 & $<0.001$ & - & - & - & 0.253 & 106.67 & $<$ & 76 \\
\hline 10 & 150 & 3.0 & 0.095 & 67.65 & $<0.001$ & 0.481 & 224.63 & $<0.001$ & - & - & - & 0.363 & 252.00 & $<0.001$ & 3128 \\
\hline 10 & 150 & 10.0 & 0.136 & 97.07 & $<0.001$ & 0.568 & 338.86 & $<0.001$ & - & - & - & 0.428 & 357.58 & $<0.001$ & 0.3775 \\
\hline 10 & 250 & 0.5 & 0.045 & 7.45 & 0.024 & 0.305 & 100.24 & $<0.001$ & - & - & - & 0.242 & 106.70 & $<0.001$ & 0.1972 \\
\hline 10 & 250 & 3.0 & 0.051 & 19.60 & $<0.001$ & 0.408 & 150.67 & $<0.001$ & - & - & - & 0.309 & 161.21 & $<0.001$ & 0.2558 \\
\hline 10 & 250 & 10.0 & 0.070 & 30.92 & $<0.001$ & 0 & 201.35 & 0. & - & - & - & 47 & 211.40 & $<0$ & 0.2919 \\
\hline 10 & 350 & 0.5 & 0.040 & 5.68 & 0.059 & 0.274 & 83.65 & $<0.001$ & - & - & - & 0.219 & 88.00 & $<0.001$ & 0.1774 \\
\hline 10 & 350 & 3.0 & 0.030 & 6.19 & 0.045 & 0.361 & 116.43 & $<0.001$ & - & - & - & 0.275 & 120.72 & $<0.001$ & 0.2222 \\
\hline 10 & 350 & 10.0 & 0.051 & 17.73 & $<0.001$ & 0 & 145.45 & $<0.001$ & - & - & - & 0.304 & 152.07 & $<0.001$ & (2) \\
\hline 20 & 150 & 0.5 & 0.065 & 17.58 & $<0.001$ & 0.279 & 96.83 & $<0.001$ & - & - & - & 0.228 & 108.00 & $<0.001$ & 0.1907 \\
\hline 20 & 150 & 3.0 & 0.041 & 9.14 & 0.010 & 0.456 & 200.28 & $<0.001$ & - & - & - & 0.355 & 219.52 & $<0.001$ & 0.2840 \\
\hline 20 & 150 & 10.0 & 0.051 & 17.23 & $<0.001$ & 0.549 & 306.34 & $<0.001$ & - & - & - & 0.415 & 328.63 & $<0.001$ & 0.3383 \\
\hline 20 & 250 & 0.5 & 0.109 & 41.00 & $<0.001$ & 0.270 & 109.04 & $<0.001$ & - & - & - & 0.232 & 130.27 & $<0.001$ & 0.2036 \\
\hline 20 & 250 & 3.0 & 0.051 & 9.33 & 0.009 & 0.368 & 126.85 & $<0.001$ & - & - & - & 0.301 & 146.79 & $<0.001$ & 0.2396 \\
\hline 20 & 250 & 10.0 & 0.022 & 2.12 & 0.347 & 0.435 & 169.57 & $<0.001$ & - & - & - & 0.341 & 192.96 & $<0.001$ & 0.2662 \\
\hline 20 & 350 & 0.5 & 0.104 & 37.63 & $<0.001$ & 0.234 & 95.71 & $<0.001$ & - & - & - & 0.202 & 112.42 & $<0.001$ & 0.1799 \\
\hline 20 & 350 & 3.0 & 0.073 & 18.24 & $<0.001$ & 0.337 & 121.38 & $<0.001$ & - & - & - & 0.287 & 145.87 & $<0.001$ & 0.2323 \\
\hline 20 & 350 & 10.0 & 0.045 & 6.92 & 0.031 & 0.357 & 114.07 & $<0.001$ & - & - & - & 0.293 & 138.26 & $<0.001$ & 0.2317 \\
\hline
\end{tabular}

Table B.7: Trial D4 (Olfaction). Using LEVIN-06-II layout, with Multiple Egg. Shows results from the bray-Curtis comparisons across various measures with the mean in the right hand column. Numbers highlighted by horizontal lines show the lowest value (best match) for each Radius Of Attraction ( $R$ ). 


\begin{tabular}{|c|c|c|c|c|c|c|c|c|c|c|c|c|c|c|c|}
\hline \multirow[b]{2}{*}{ FD } & \multicolumn{3}{|c|}{ arameters } & \multicolumn{3}{|c|}{ 1x1m (2 d.f.) } & \multicolumn{3}{|c|}{$6 \times 6 \mathrm{~m} 3$ d.f. } & \multicolumn{3}{|c|}{$48 \times 48 m$ ( 2 d.f. $)$} & \multicolumn{3}{|c|}{ caleCode (5/6 d.f.) } \\
\hline & L & A & $3 \mathrm{C}$ & $\chi^{2}$ & (p) & B & & (p) & BC & & & BC & & (p) & $n$ BC \\
\hline & 50 & 0.5 & 8 & 1.52 & 0.001 & 0 & 134.03 & $<0.0$ & - & & - & 0.195 & 194.61 & $<00$ & \\
\hline & & 3.0 & & 3.10 & 1 & 0.244 & 140.50 & -5 & - & & - & ontes & 17. & < 0001 & \\
\hline & 50 & 0 & & 98 & & & 1 & $<0$ & - & & - & & & & \\
\hline 100 & 250 & 0.5 & & .50 & 1 & 0 . & 12 & $<0$ & - & & - & & & & \\
\hline 100 & 50 & 0.0 & 2 & 9.34 & 1 & 8 & 123.36 & $<$ & 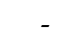 & - & - & -0 & - & - & \\
\hline & 0 & 0 & & 5 & 0.001 & 3 & 8 & 000 & - & & - & 0.235 & 17330 & & \\
\hline 100 & 350 & 0.5 & & 70.08 & .001 & 0.205 & 107.94 & $<0.00$ & - & & - & 0.14 & 141. & & \\
\hline 100 & 50 & 3.0 & 141 & 68.16 & 0.0 & 0.184 & 100.33 & $<0$ & - & & - & 0. & 12 & & \\
\hline & 350 & 1.0 & & & 1 & 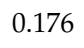 & 1 & $<$ & - & 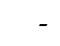 & - & & & & \\
\hline & 50 & 5 & & 8 & 1 & 6 & 2 & $<$ & - & 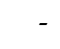 & - & & & & \\
\hline & 50 & 0 & & 0.92 & 0.001 & 0. & 137.54 & & - & 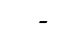 & - & 08 & 174.69 & & 75 \\
\hline & 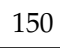 & .0 & 100 & 4 & (0. & 0. & 17 & $<$ & - & - & - & 0 & 2 & & \\
\hline & 250 & 0.5 & & 29.02 & & & & & - & & - & & & & \\
\hline 600 & 50 & 0 & 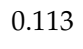 & 2.46 & $<0$ & 0. & 1 & $<0$ & - & 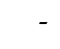 & - & ou & 214.80 & & \\
\hline 00 & 250 & .0 & 101 & 40.77 & 0.001 & 0.269 & 186.34 & $<0$ & - & - & - & 0.262 & 214.32 & & 108 \\
\hline 600 & 350 & 0.5 & $103 \quad 2$ & 7.58 & 0.001 & 0 & 185 & $<\mathbf{0}$. & 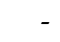 & 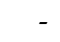 & - & 0 . & 2 & & 21 \\
\hline & 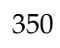 & 3.0 & & 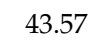 & 1 & & & $<$ & 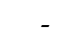 & 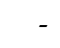 & - & & & & \\
\hline 600 & 350 & 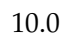 & & & 1 & & & & - & - & - & & & & \\
\hline 10 & & 0.5 & & & & & & & - & - & - & 0.219 & & & \\
\hline 1800 & 50 & 3.0 & 106 & 6.90 & 0.001 & 0.243 & 162.67 & $<0$ & - & - & - & 02 & 20 & $<$ & 968 \\
\hline & & 0 & & & & $c$ & 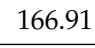 & & - & 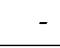 & - & & & & \\
\hline & 250 & $?$ & 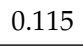 & 2 & 1 & 0 & 2 & $<$ & - & 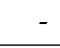 & - & 0.164 & 224.57 & & 30 \\
\hline 1800 & 0 & 3 & c & & & 2 & & & - & - & - & J & & & \\
\hline 1800 & 0 & .0 & c & 8 & 0.001 & 0 & 2 & $<0.0$ & - & - & - & 8 & 274.15 & 0 & 0.2586 \\
\hline 1800 & 50 & 0.5 & 10 & 56.16 & 0.001 & 0.212 & 200.05 & $<0.00$ & - & - & - & 0.216 & 56 & $<\mathbf{0 . 0 0}$ & 0.1840 \\
\hline 1800 & 50 & 3.0 & 143 & 78.39 & $<0.001$ & 0.349 & 291.88 & $<0$ & - & 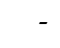 & - & 0.340 & 320.03 & $<0$ & 0.2776 \\
\hline 1800 & 350 & 10.0 & 52 & 3.04 & $<0.001$ & 0.352 & 298.10 & $<0.001$ & - & 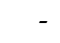 & - & 0.343 & 322.42 & $<0.001$ & 0.2827 \\
\hline
\end{tabular}

Table B.8: Trial D5 (Vision). Using LEVIN-06-II layout, with Multiple Egg. Shows results from the bray-Curtis comparisons across various measures with the mean in the right hand column. Numbers highlighted by horizontal lines show the lowest value (best match) for each Visual Field Depth (FD). 


\begin{tabular}{|c|c|c|c|c|c|c|c|c|c|c|c|c|c|c|c|}
\hline \multirow[b]{2}{*}{ FD } & \multicolumn{3}{|c|}{ Parameters } & \multicolumn{3}{|c|}{ 1x1m (2 d.f.) } & \multicolumn{3}{|c|}{$6 \times 6 \mathrm{~m} 3$ d.f. } & \multicolumn{3}{|c|}{$48 \times 48 m$ ( 2 d.f. $)$} & \multicolumn{3}{|c|}{ ScaleCode (5/6 d.f.) } \\
\hline & L & A & C & 2 & ) & B & & (p) & BC & $\chi$ & & BC & & (p) & 1 \\
\hline 100 & 50 & 0.5 & 5 & 4.72 & 0.001 & 7 & 135.61 & $<0$. & - & - & - & 0 & 205.73 & $<0.001$ & \\
\hline & 150 & 3.0 & & 91 & 0.001 & 1 & 1 & 0 & - & - & - & 1 & 2 & $<0.001$ & \\
\hline & 0 & 0 & & & 8 & & 27 & $<0$ & - & - & - & & 300. & & \\
\hline 1 & 50 & 0.5 & & 10 & 1 & & & $<$ & - & - & - & & 181. & & \\
\hline 100 & ) & 3.0 & 6 & .75 & 1 & 0 . & 13 & $<0<x$ & ( & - & - & 6 & 1 & 001 & \\
\hline 100 & 250 & .0 & - & 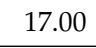 & 1 & 7 & 9 & $<0.001$ & - & - & - & 9 & 2 & $<0000$ & \\
\hline & 50 & 5 & & 68.28 & 1 & & 109.27 & & - & - & - & & & & \\
\hline 100 & 50 & 3.0 & 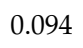 & 31.36 & $<0.001$ & 0.3 & 10 & $<0$ & - & - & - & & 147.6 & & \\
\hline 100 & 350 & .0 & 076 & 8 & 1 & 0 . & 1 & - & 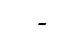 & - & - & 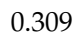 & 工 & & \\
\hline 600 & 150 & 5 & & 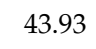 & 1 & & 2 & $<$ & - & - & - & & 20 & & \\
\hline 600 & 50 & 3.0 & (50 & 58 & 0.001 & 0. & & 20 & - & - & - & & 60 & & \\
\hline 600 & 150 & .0 & 0 & 64.36 & 0.001 & 0. & 44.00 & & - & - & - & & 89. & & \\
\hline 600 & 50 & 0.5 & 083 & 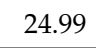 & 1 & 0 & 13 & 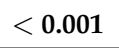 & - & - & - & 0.166 & 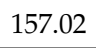 & & 1373 \\
\hline & 250 & 3.0 & & & & 0 & & & 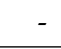 & - & - & & & & \\
\hline 00 & 250 & 10.0 & 50 & 17.27 & $<0.001$ & 0.059 & 12. & 00 & - & - & - & & 45 & & \\
\hline 600 & 350 & 0.5 & 083 & 25.14 & 1 & 0.149 & 124.98 & $<$ & 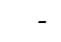 & - & - & 0 . & 13 & & \\
\hline 600 & 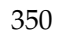 & 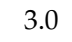 & & 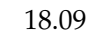 & 1 & & & & 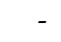 & 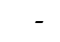 & - & & & & \\
\hline 600 & 350 & 10.0 & & & & & & & - & - & - & & & & \\
\hline 1800 & 50 & 0.5 & 966 & 38.32 & 1 & 0.214 & 184.98 & $<$ & - & - & - & & 220 & & \\
\hline 1800 & 0 & 3.0 & 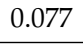 & 21.90 & 1 & 0.14 & 0.98 & $<0$ & - & - & - & 8 & 112 & $<$ & 0.1281 \\
\hline 1800 & & 10.0 & & & & & & & 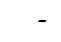 & - & - & & & & \\
\hline 1800 & 0 & 0.5 & & 37 & $<$ & 0 . & 14 & & - & - & - & & 16 & & \\
\hline 800 & 250 & 3.0 & & 47 & & 0.243 & 157.71 & & - & - & - & 0.2 & 184 & & \\
\hline 100 & 0 & 10.0 & 0 & 3 & 1 & 0 . & 1 & $<$ & - & - & 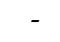 & 0.245 & 167.02 & 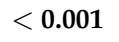 & 0 \\
\hline 180 & 50 & 0.5 & 0103 & 40.57 & $<0.001$ & 0.198 & 158.26 & $<0.001$ & - & - & - & 0.199 & 1760 & & 167 \\
\hline 1800 & U & 3.0 & 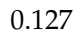 & 66 & 0.001 & 0.308 & 233.67 & $<0$ & - & - & - & 0 & 266.98 & $<0$ & 0.2 \\
\hline 1800 & 50 & 10.0 & 24 & 4.67 & 0.001 & 0.297 & 216.57 & $<0.001$ & - & - & - & 0.290 & 247.06 & $<0.001$ & 0.2370 \\
\hline
\end{tabular}

Table B.9: Trial D6 (Olfaction and Vision). Using LEVIN-06-II layout, with Multiple Egg. Shows results from the bray-Curtis comparisons across various measures with the mean in the right hand column. Numbers highlighted by horizontal lines show the lowest value (best match) for each Visual Field Depth (FD). 


\section{B.2 Egg Distributions by $\mathrm{L}$ and $\mathrm{k}$}
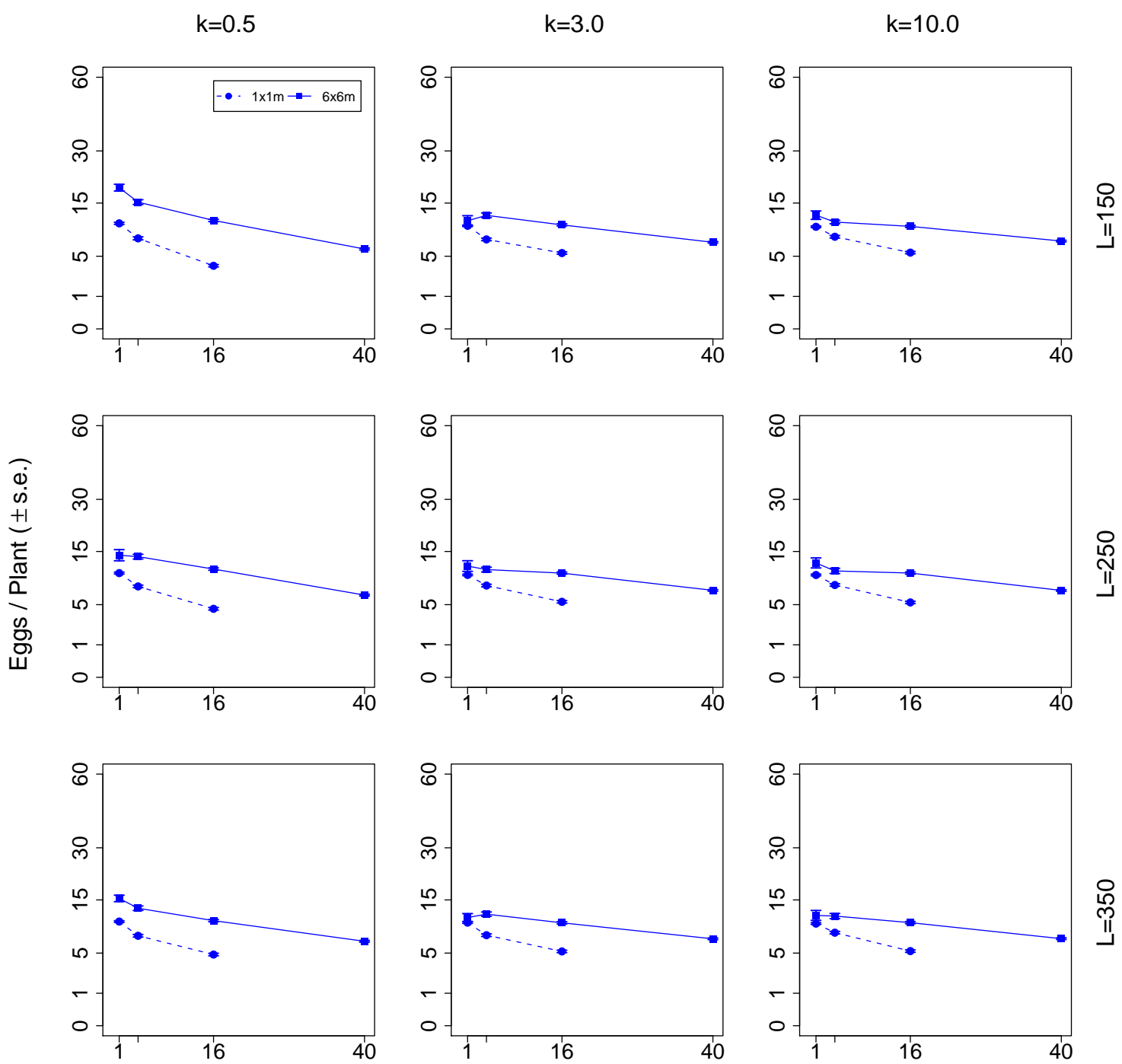

Plant Density

Figure B.1: Trial C1 (CRW - LEVIN-06-II), Single Egg, Response Summary $(R=5)$ 

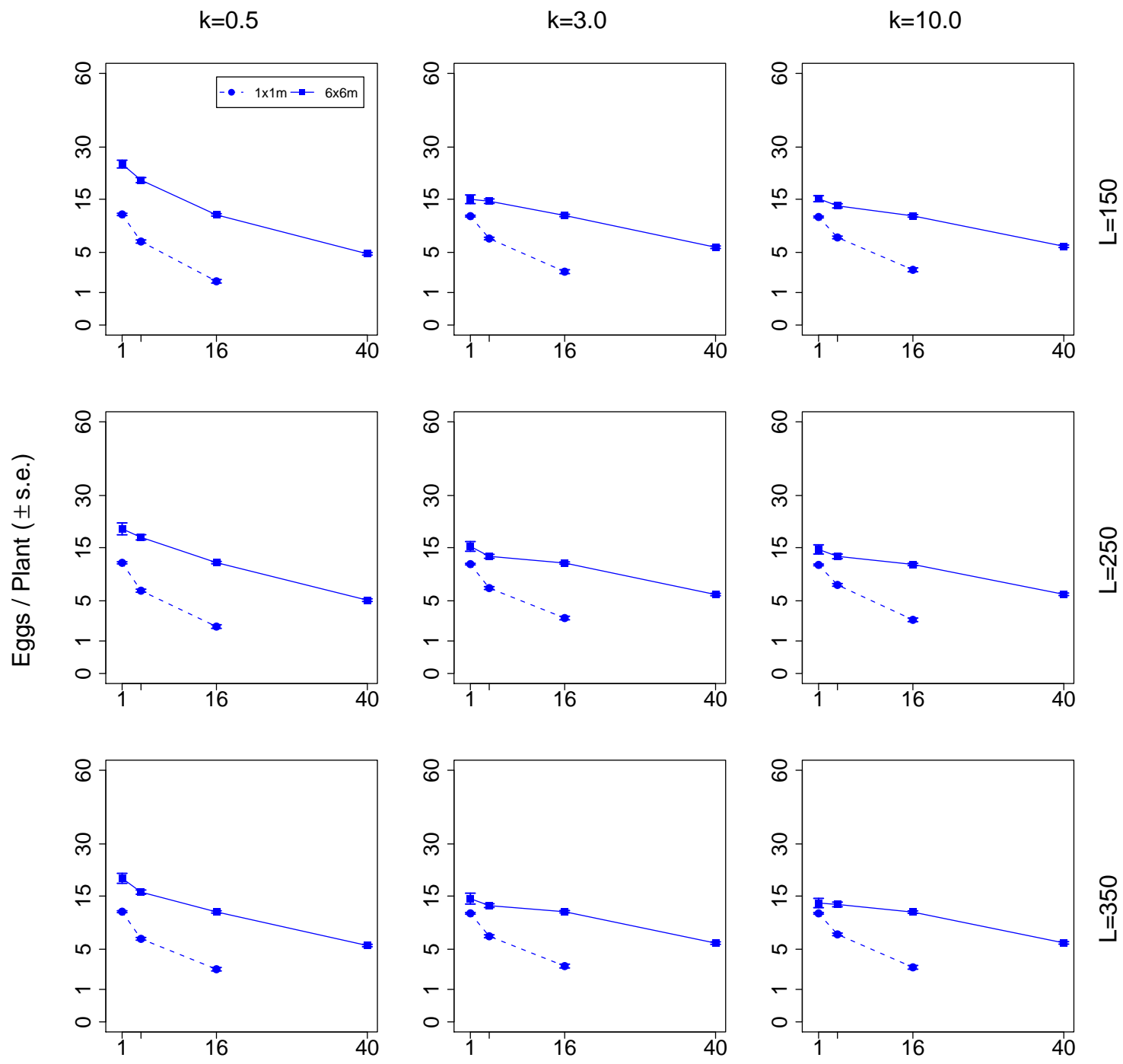

Plant Density

Figure B.2: Trial C1 (CRW - LEVIN-06-II), Single Egg, Response Summary $(R=10)$ 

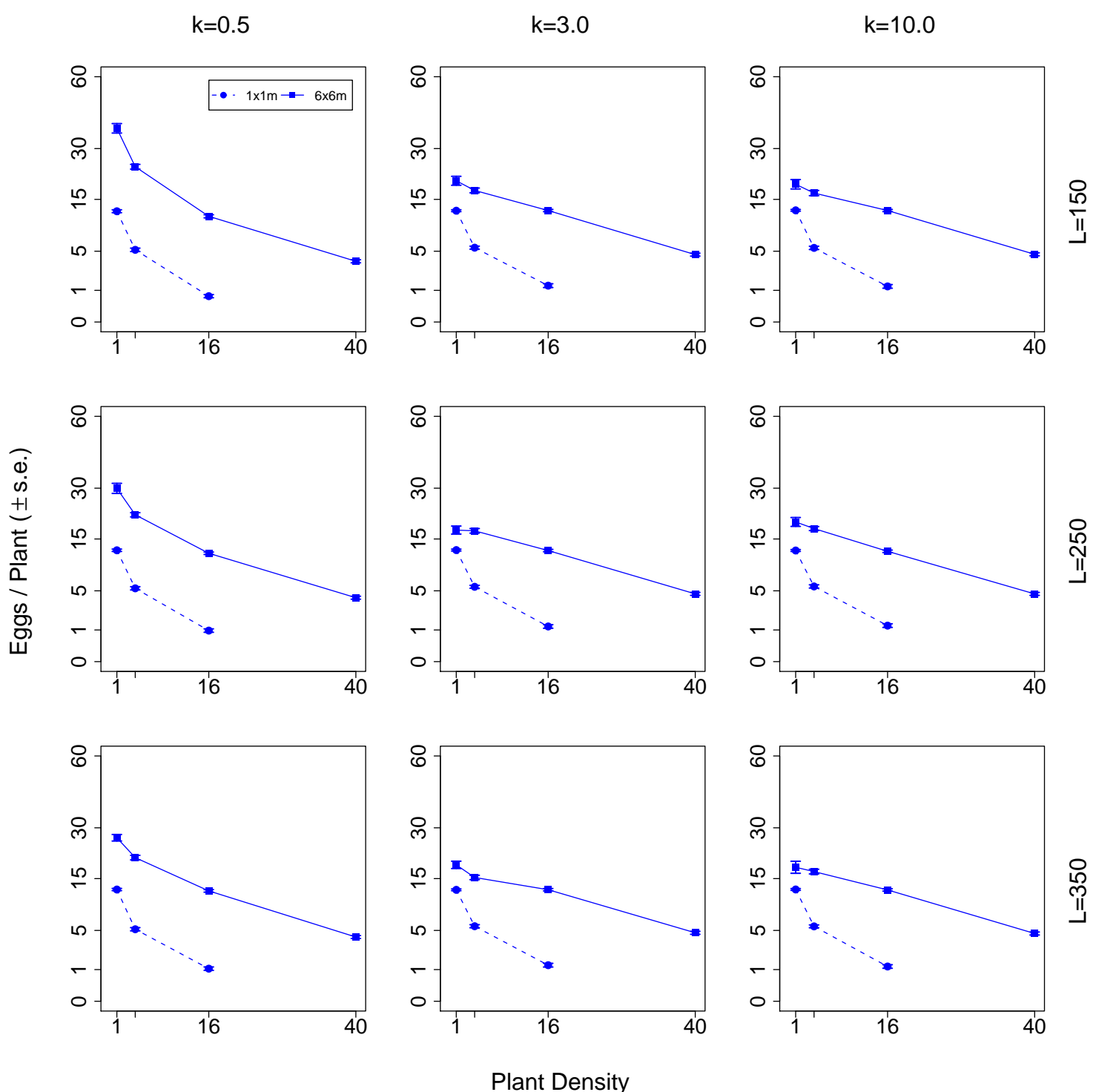

Plant Density

Figure B.3: Trial C1 (CRW - LEVIN-06-II), Single Egg, Response Summary $(R=20)$ 

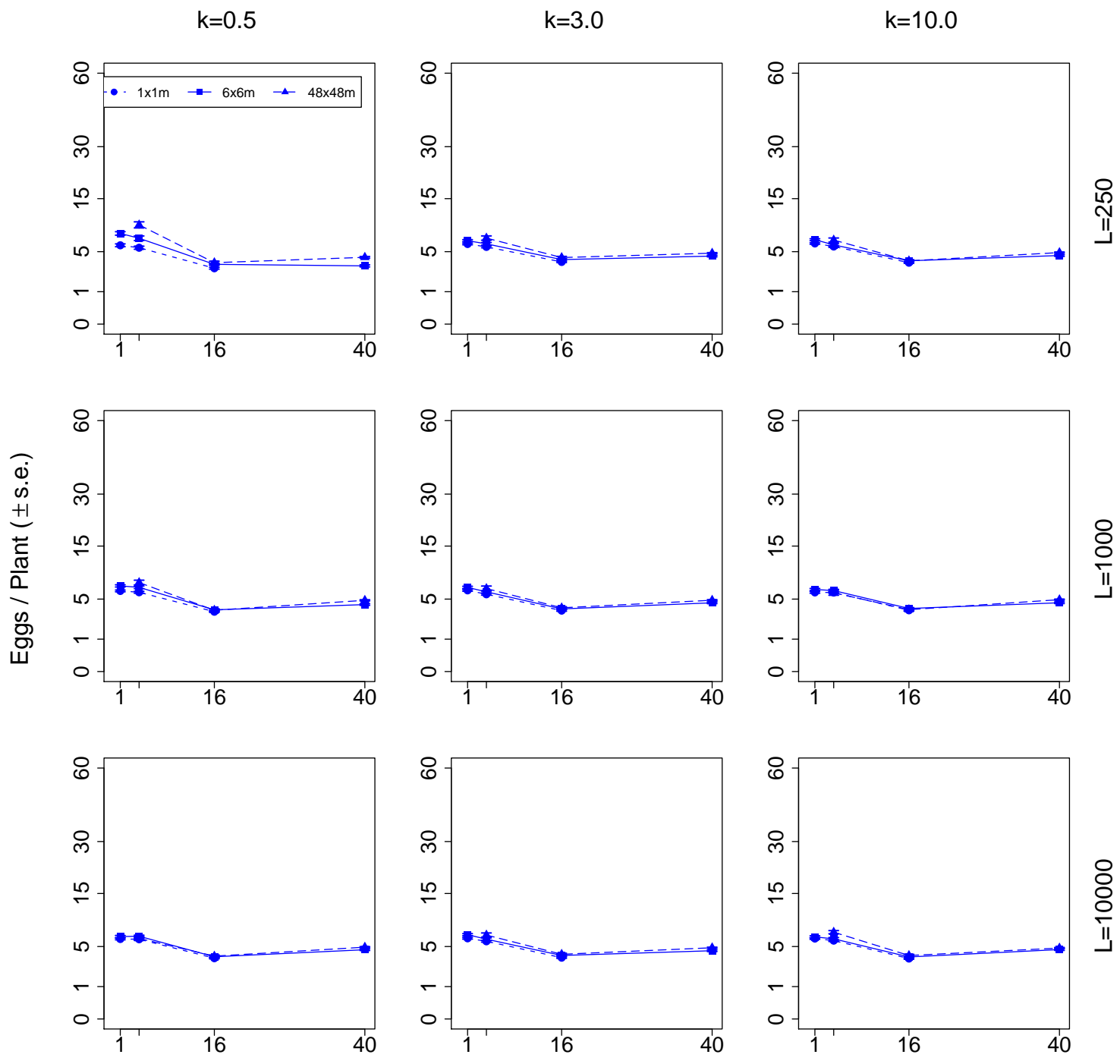

Plant Density

Figure B.4: Trial C2 (CRW - KAITOKE-04), Single Egg, Response Summary ( $R=5)$ 

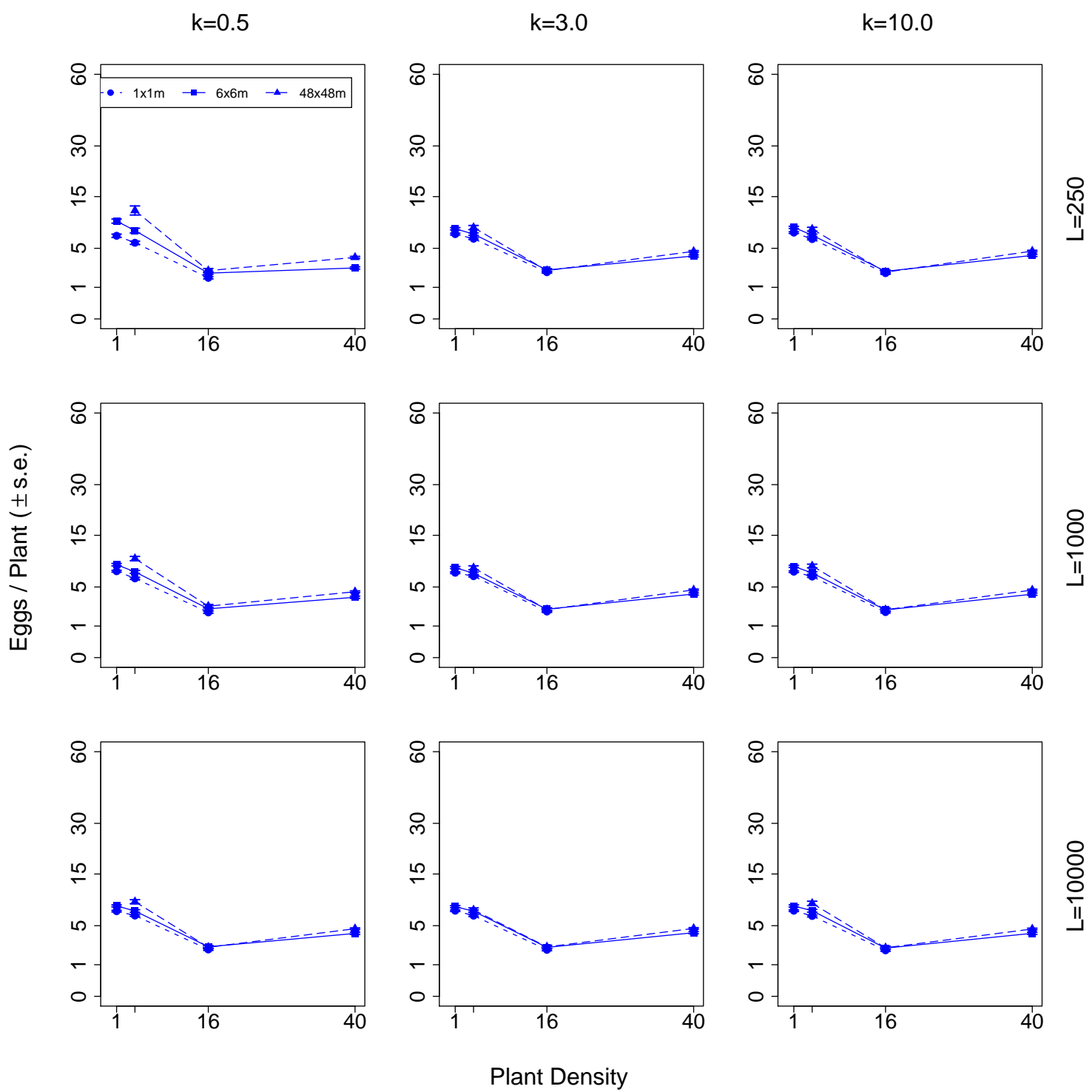

Figure B.5: Trial C2 (CRW - KAITOKE-04), Single Egg, Response Summary $(R=10)$ 

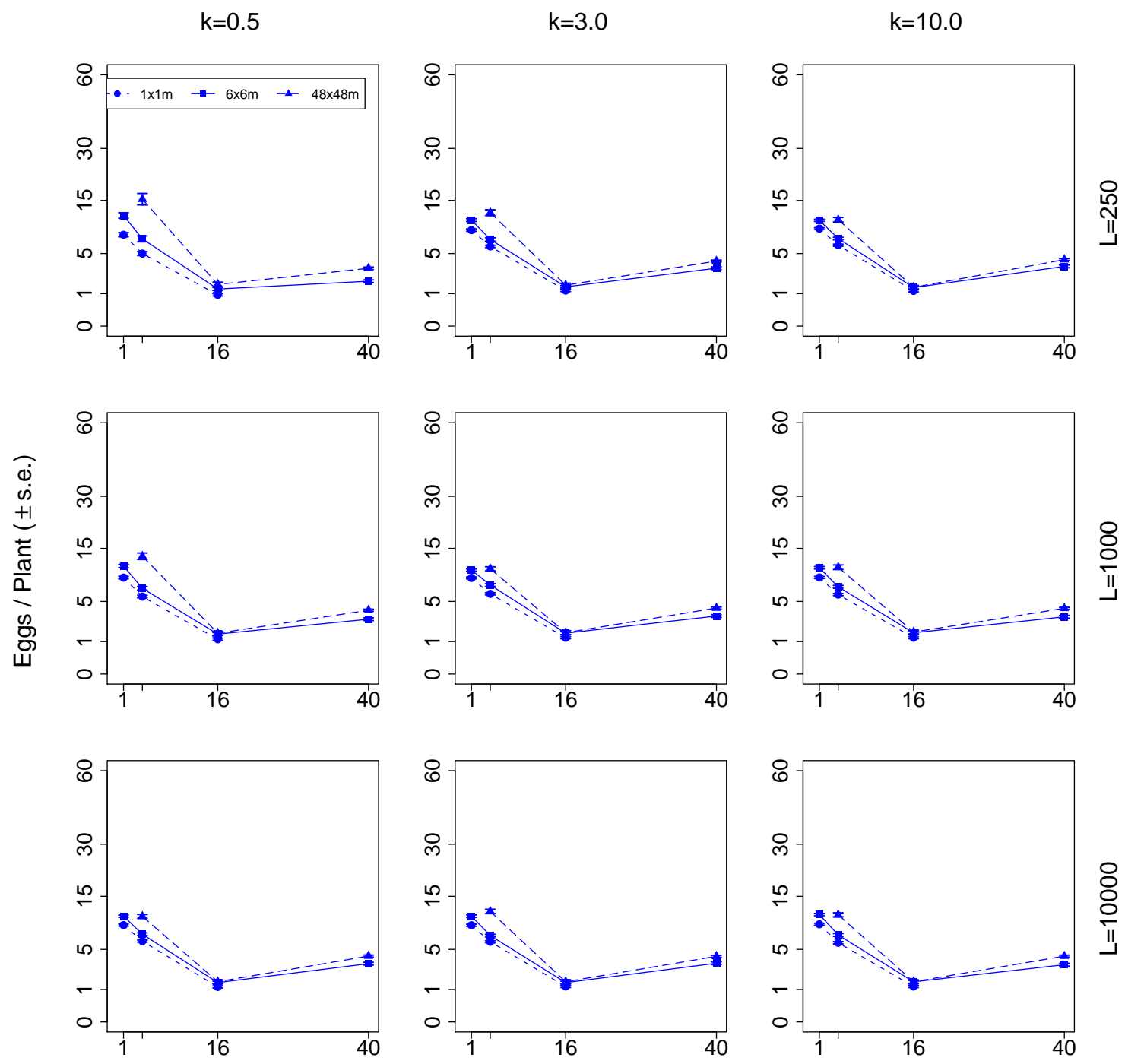

Plant Density

Figure B.6: Trial C2 (CRW - KAITOKE-04), Single Egg, Response Summary (R=20) 

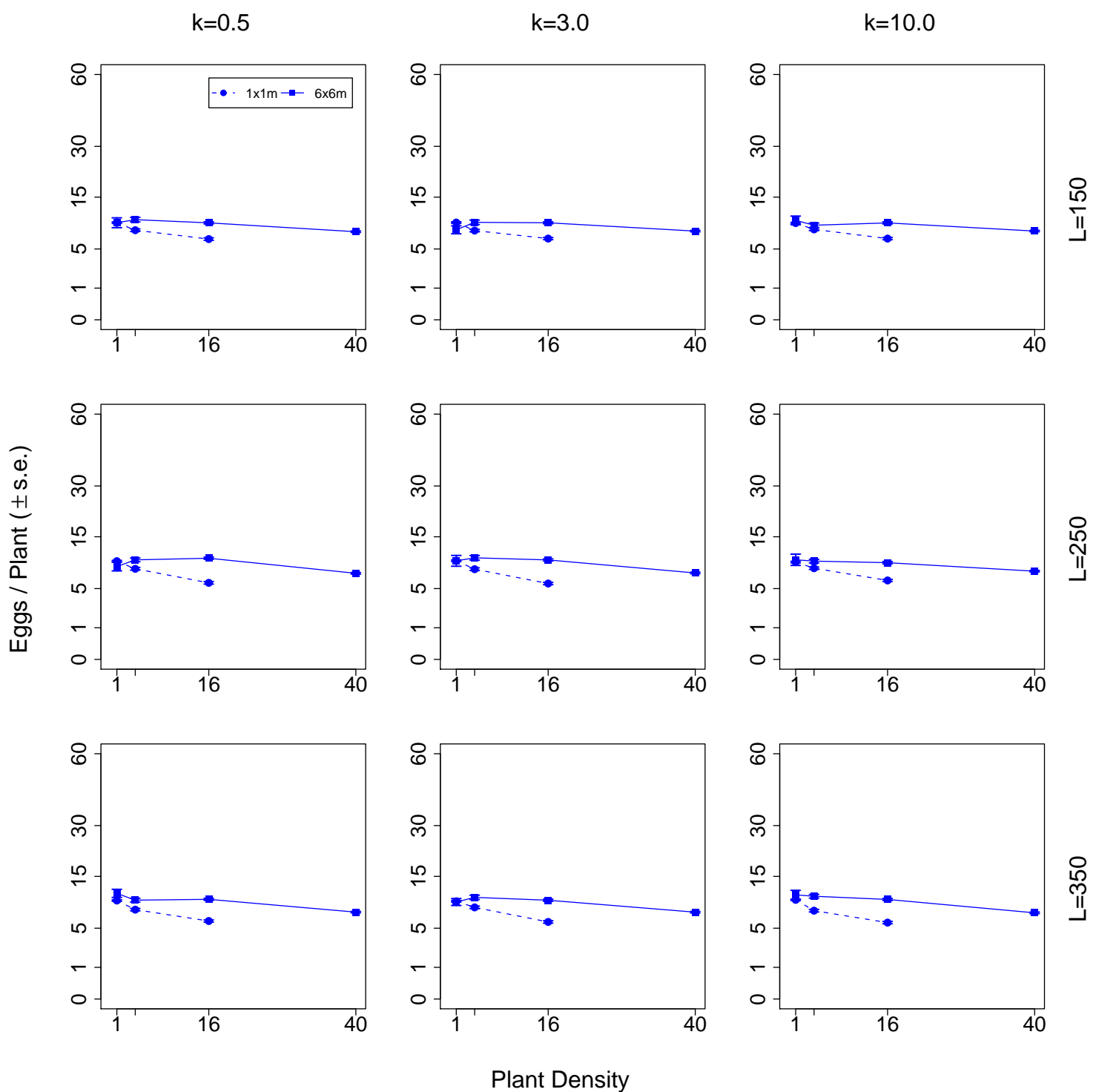

Plant Density

Figure B.7: Trial C3 (CRW - LEVIN-06-II), Multiple Eggs, Response Summary (R=5) 

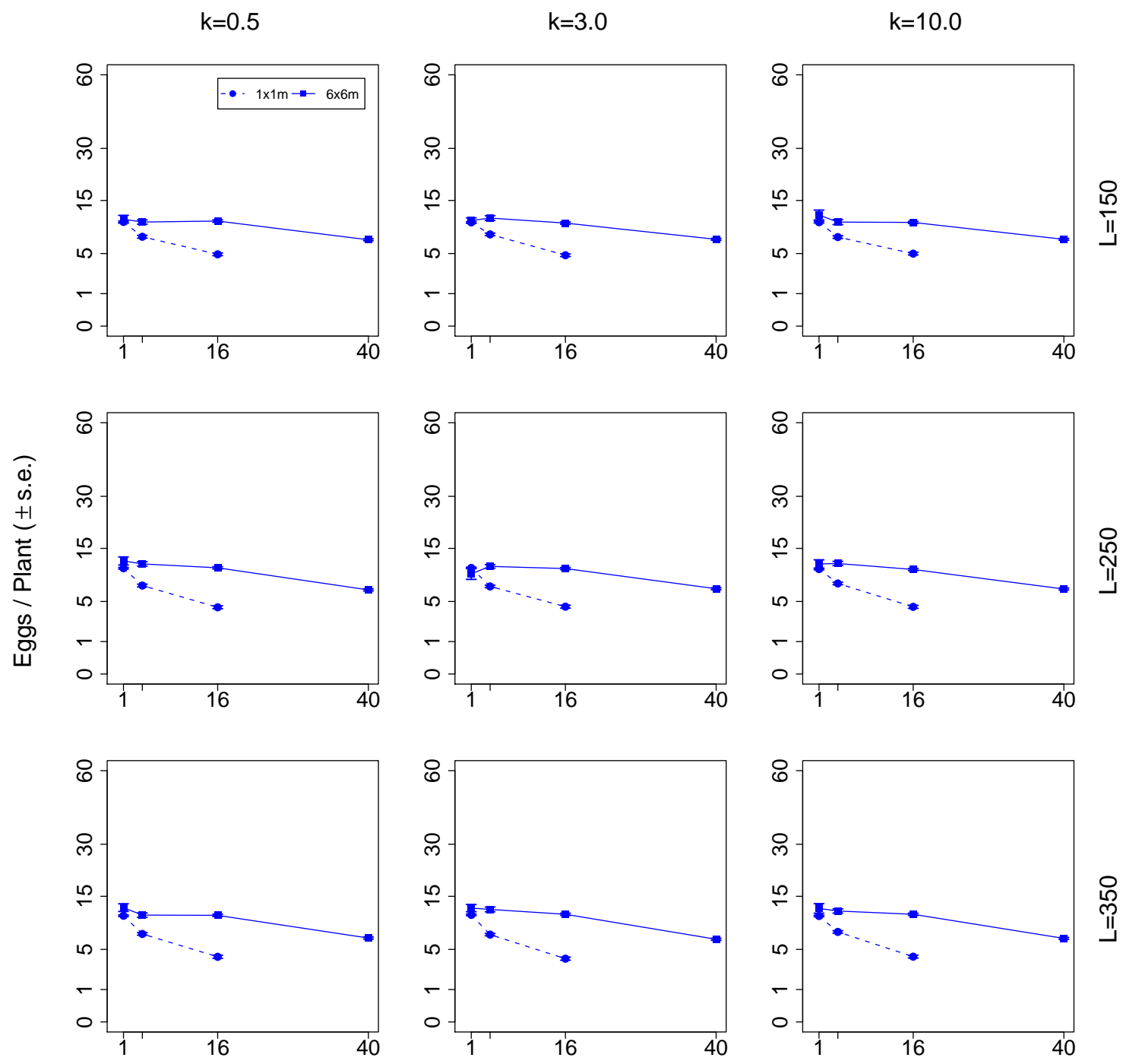

Plant Density

Figure B.8: Trial C3 (CRW - LEVIN-06-II), Multiple Eggs, Response Summary (R=20) 

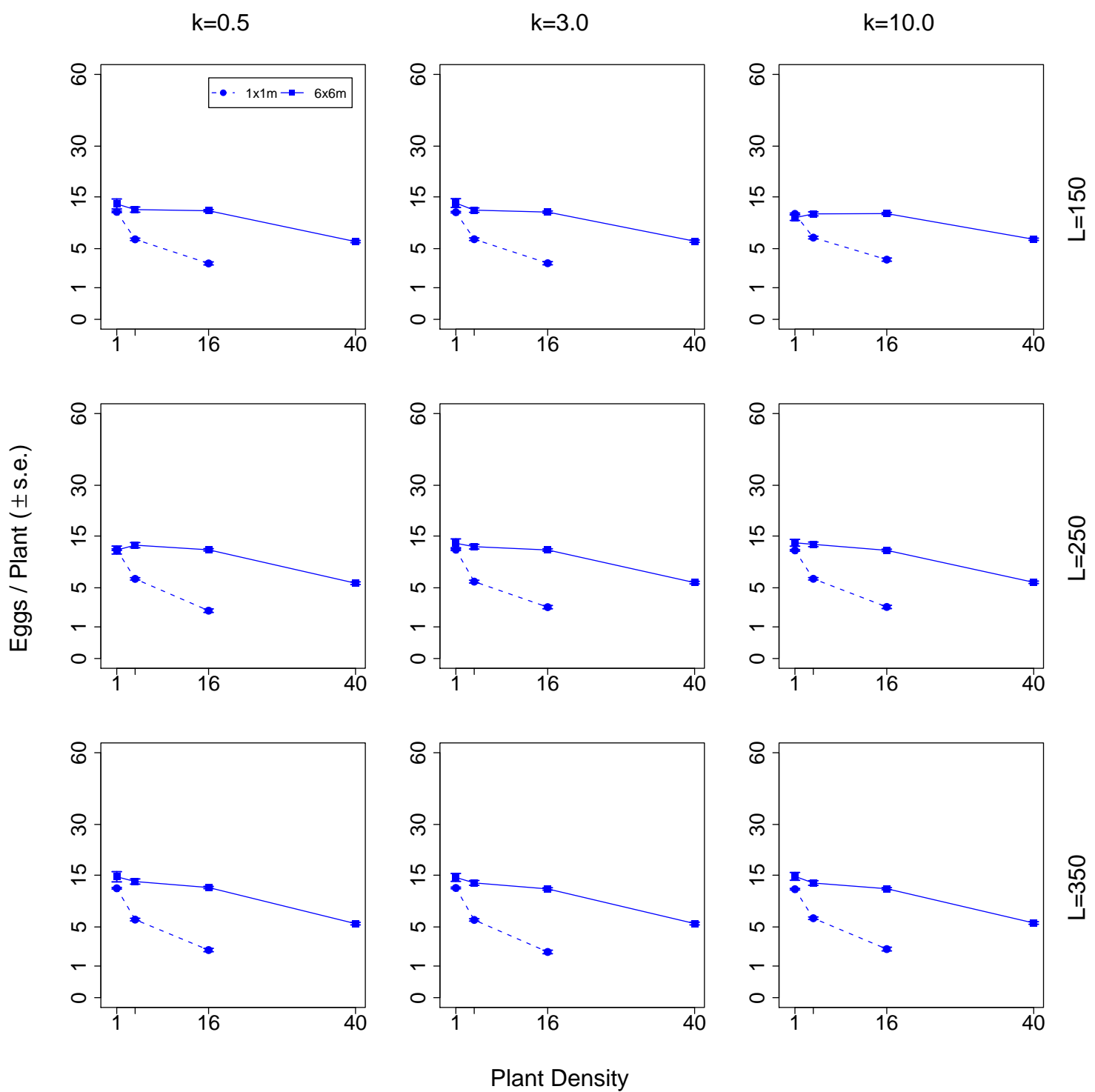

Figure B.9: Trial C3 (CRW - LEVIN-06-II), Multiple Eggs, Response Summary $(R=20)$ 

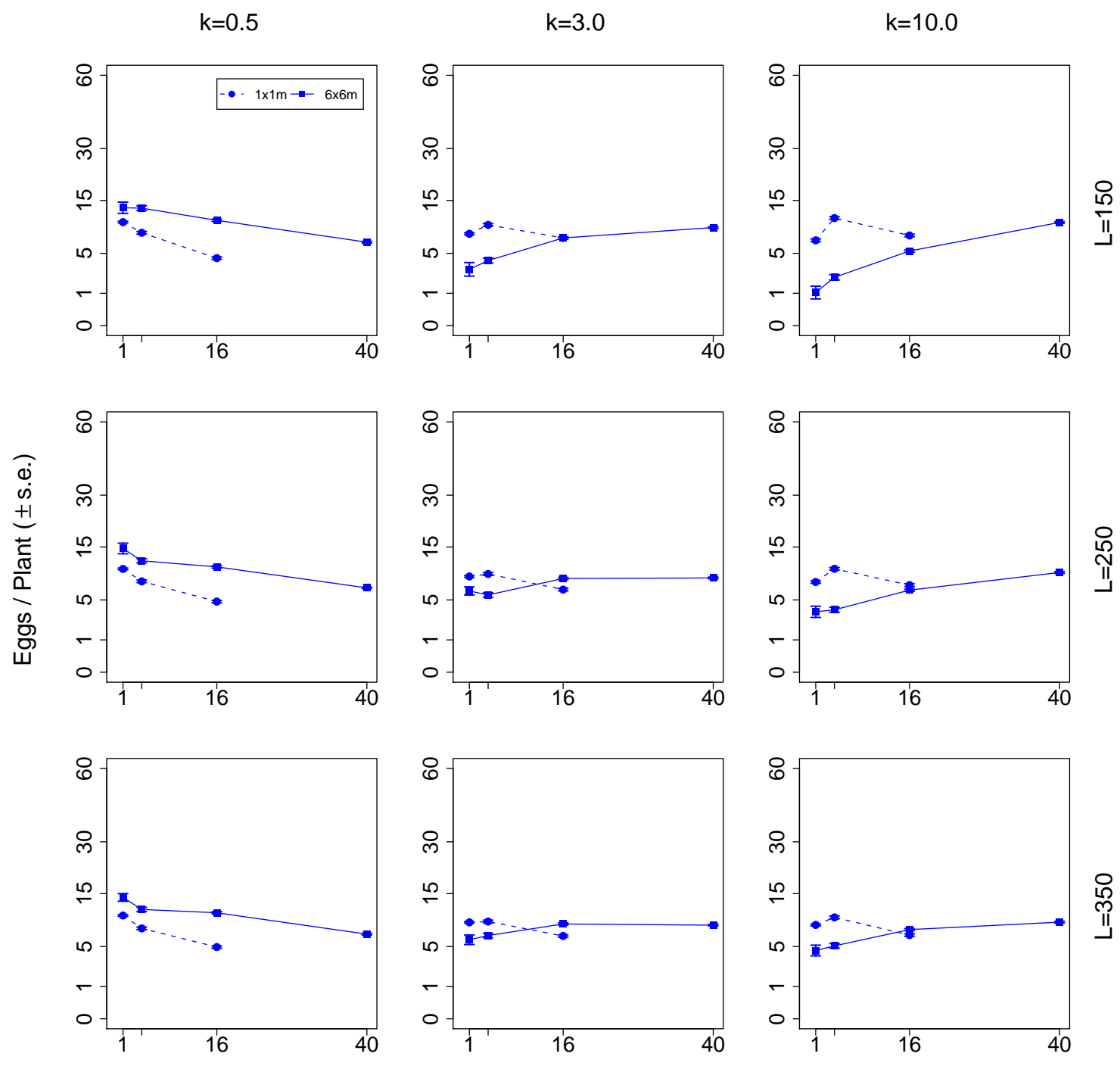

Plant Density

Figure B.10: Trial D1 (Olfaction - LEVIN-06-II), Single Egg, Response Summary $(R=5)$ 

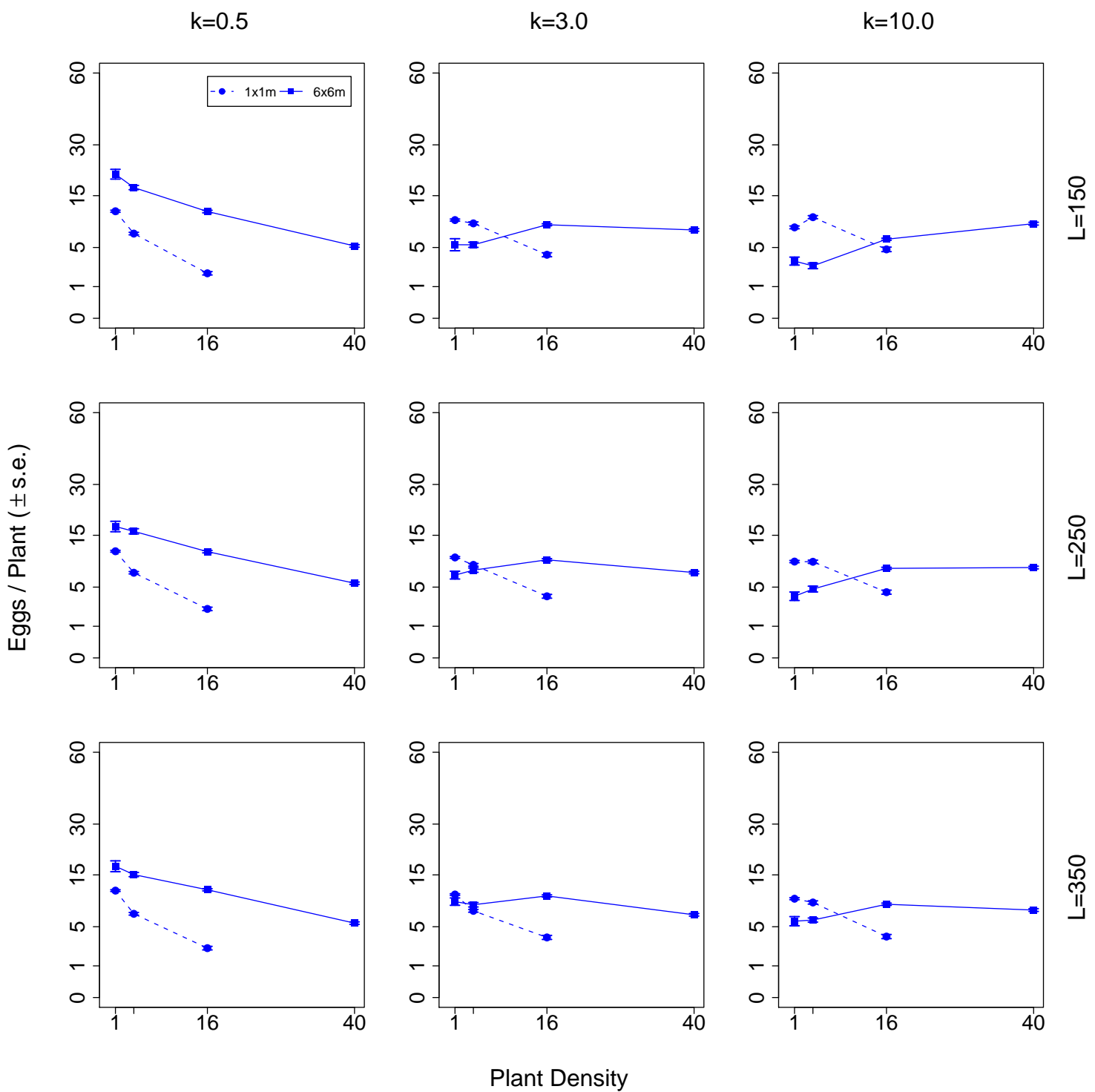

Plant Density

Figure B.11: Trial D1 (Olfaction - LEVIN-06-II), Single Egg, Response Summary $(R=10)$ 

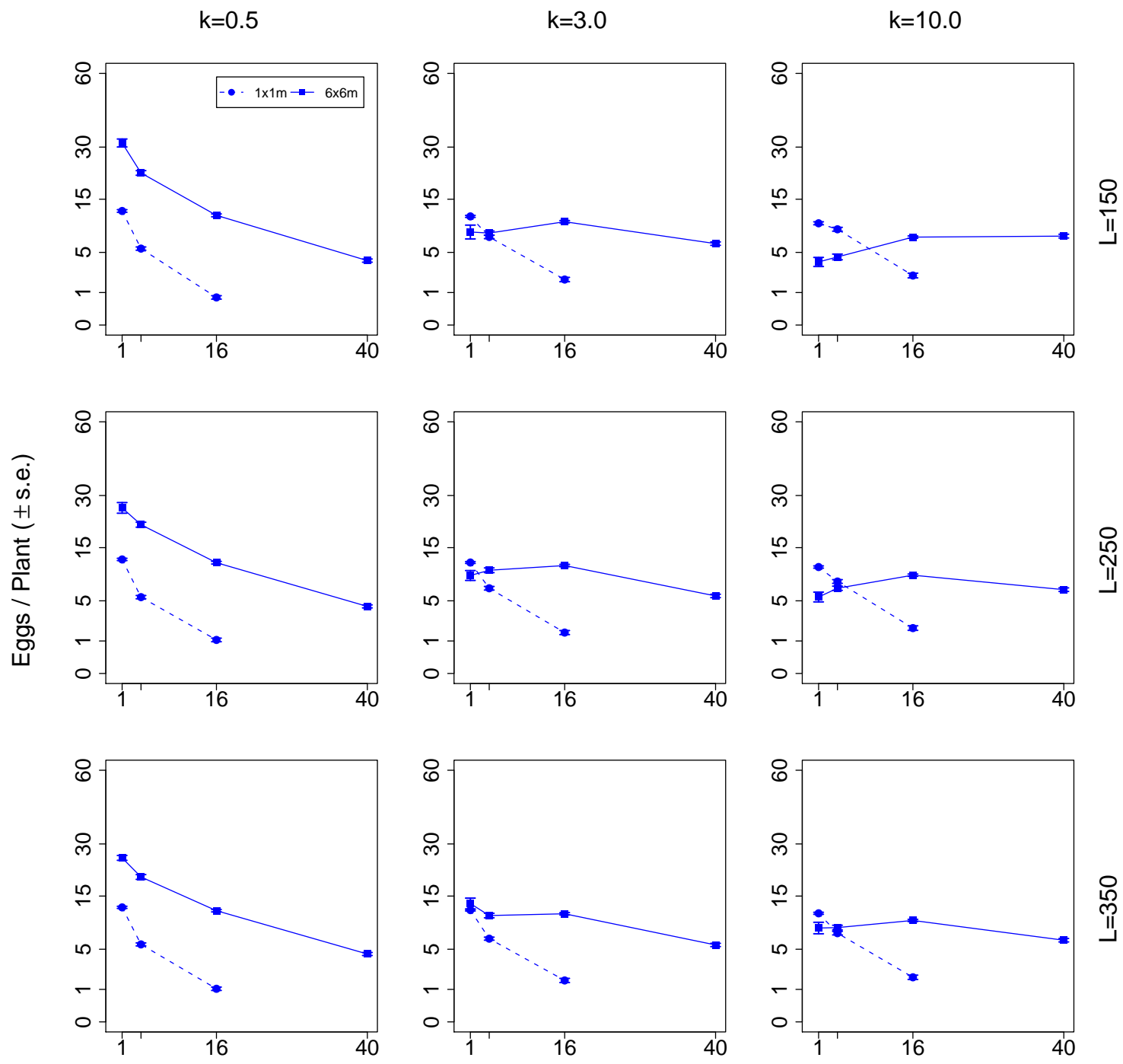

Plant Density

Figure B.12: Trial D1 (Olfaction - LEVIN-06-II), Single Egg, Response Summary ( $R=20)$ 

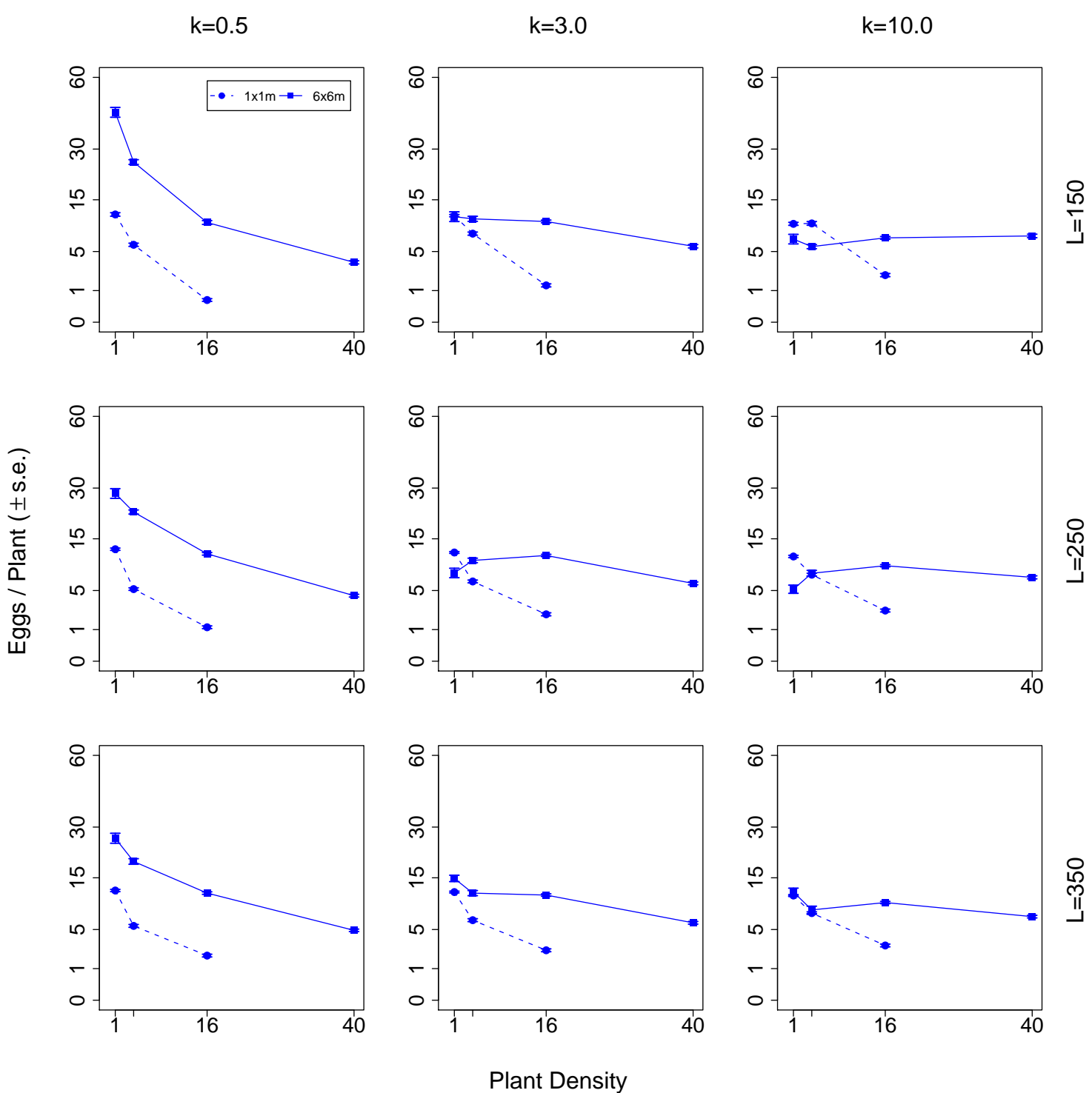

Figure B.13: Trial D2 (Olfaction and Vision - LEVIN-06-II), Single Egg, Response Summary $(F D=100)$ 

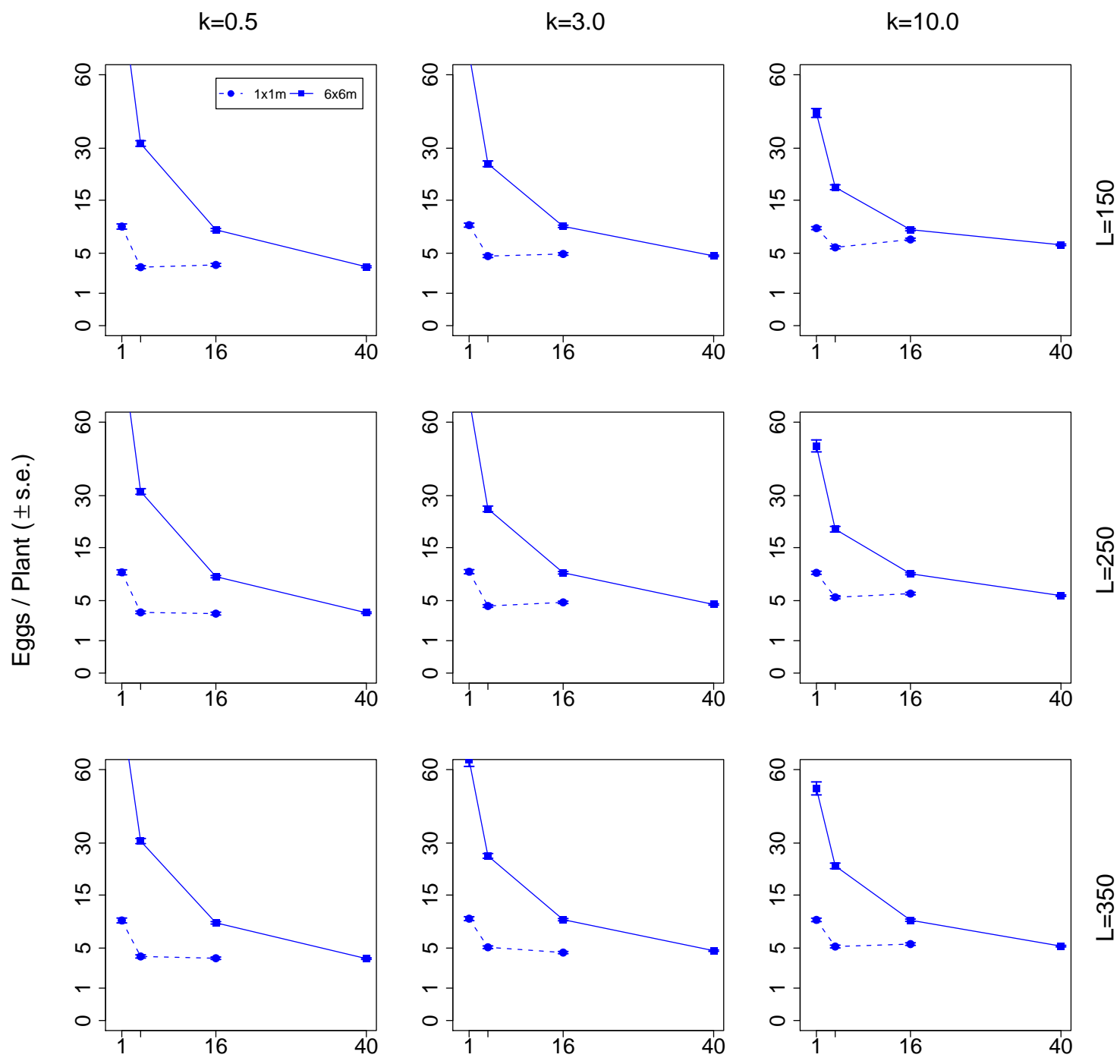

Plant Density

Figure B.14: Trial D2 (Olfaction and Vision - LEVIN-06-II), Single Egg, Response Summary $(F D=600)$ 

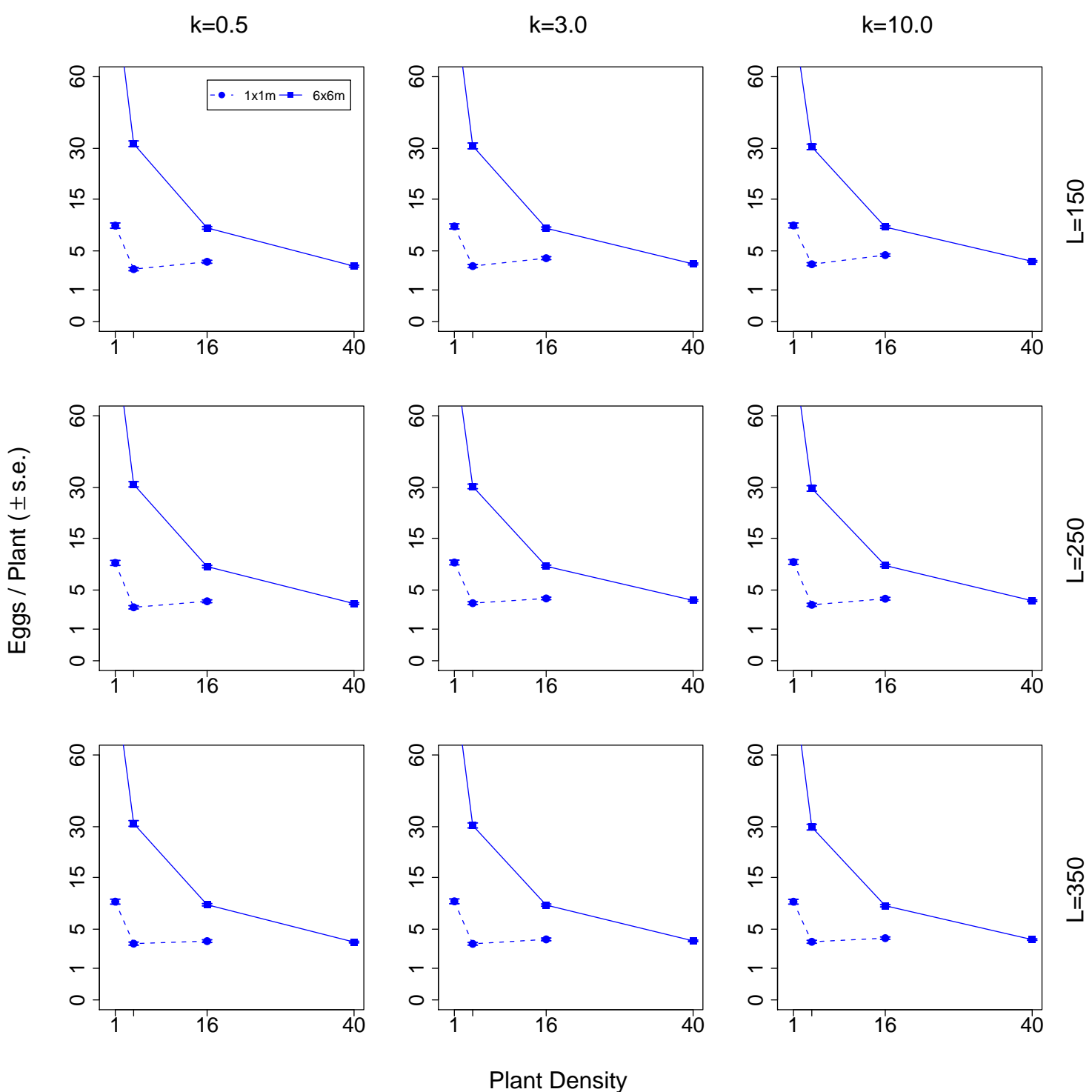

Figure B.15: Trial D2 (Olfaction and Vision - LEVIN-06-II), Single Egg, Response Summary (FD-1800) 

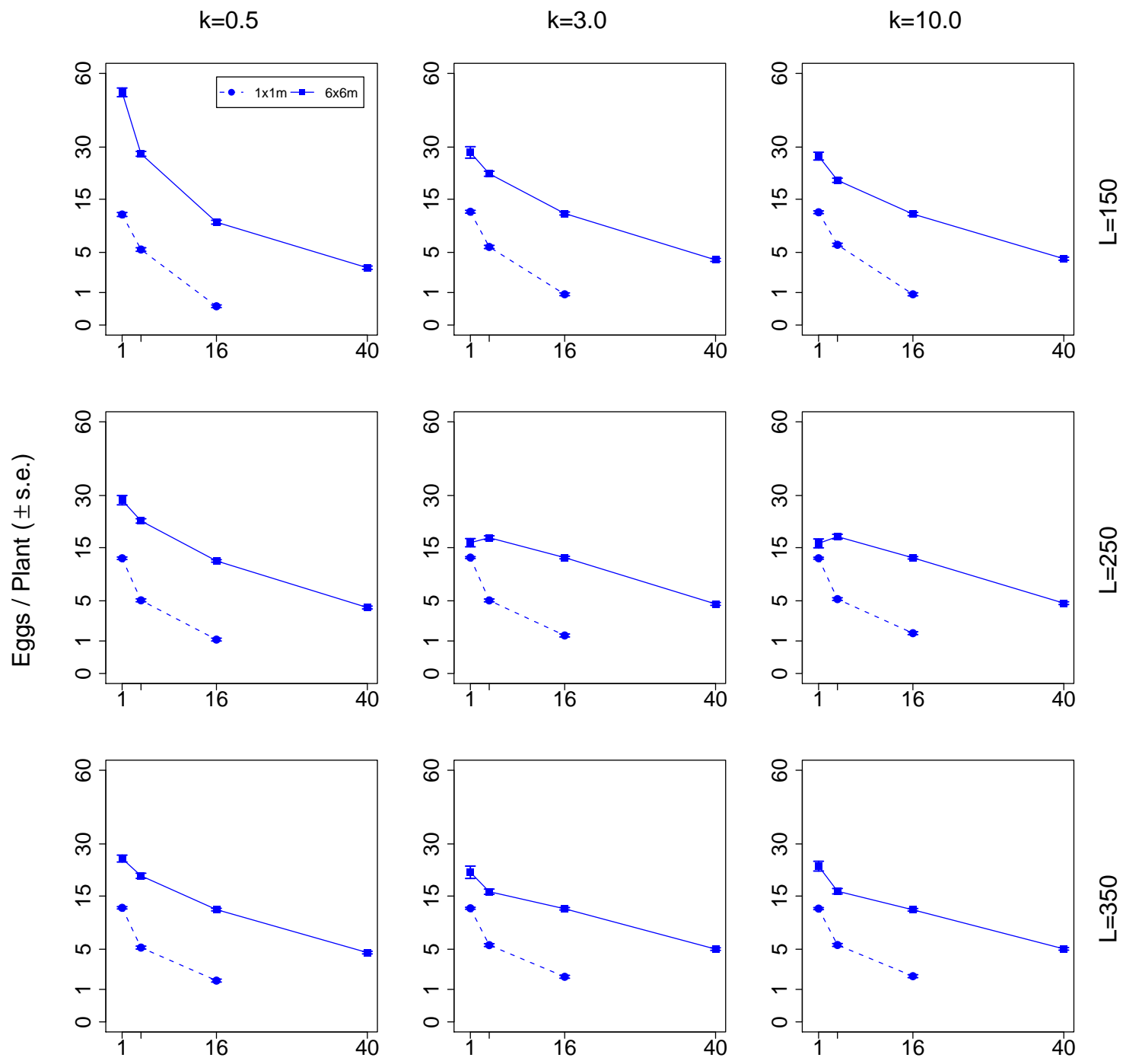

Plant Density

Figure B.16: Trial D3 (Vision - LEVIN-06-II), Single Egg, Response Summary (FD=100) 

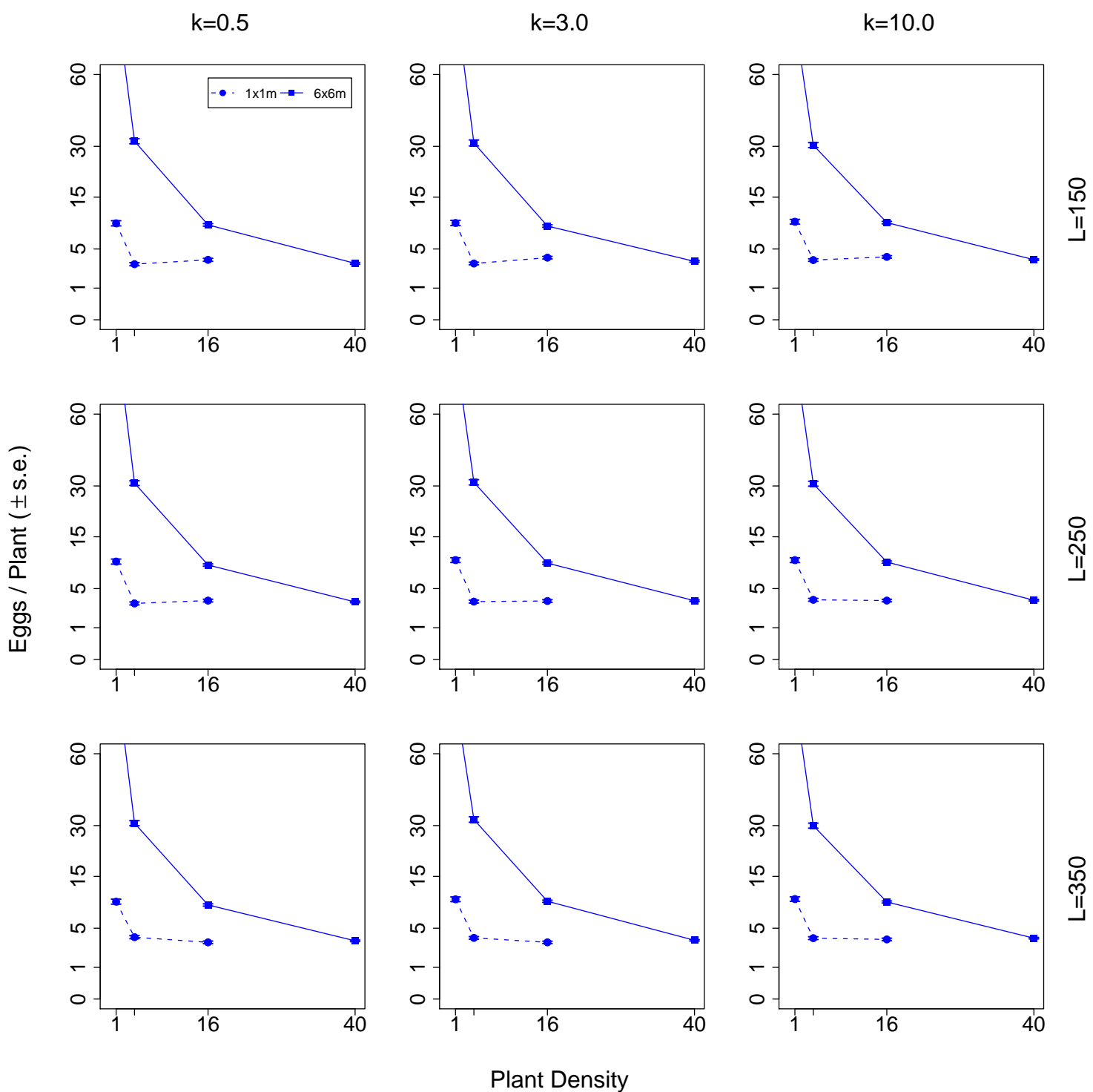

Figure B.17: Trial D3 (Vision - LEVIN-06-II), Single Egg, Response Summary (FD=600) 

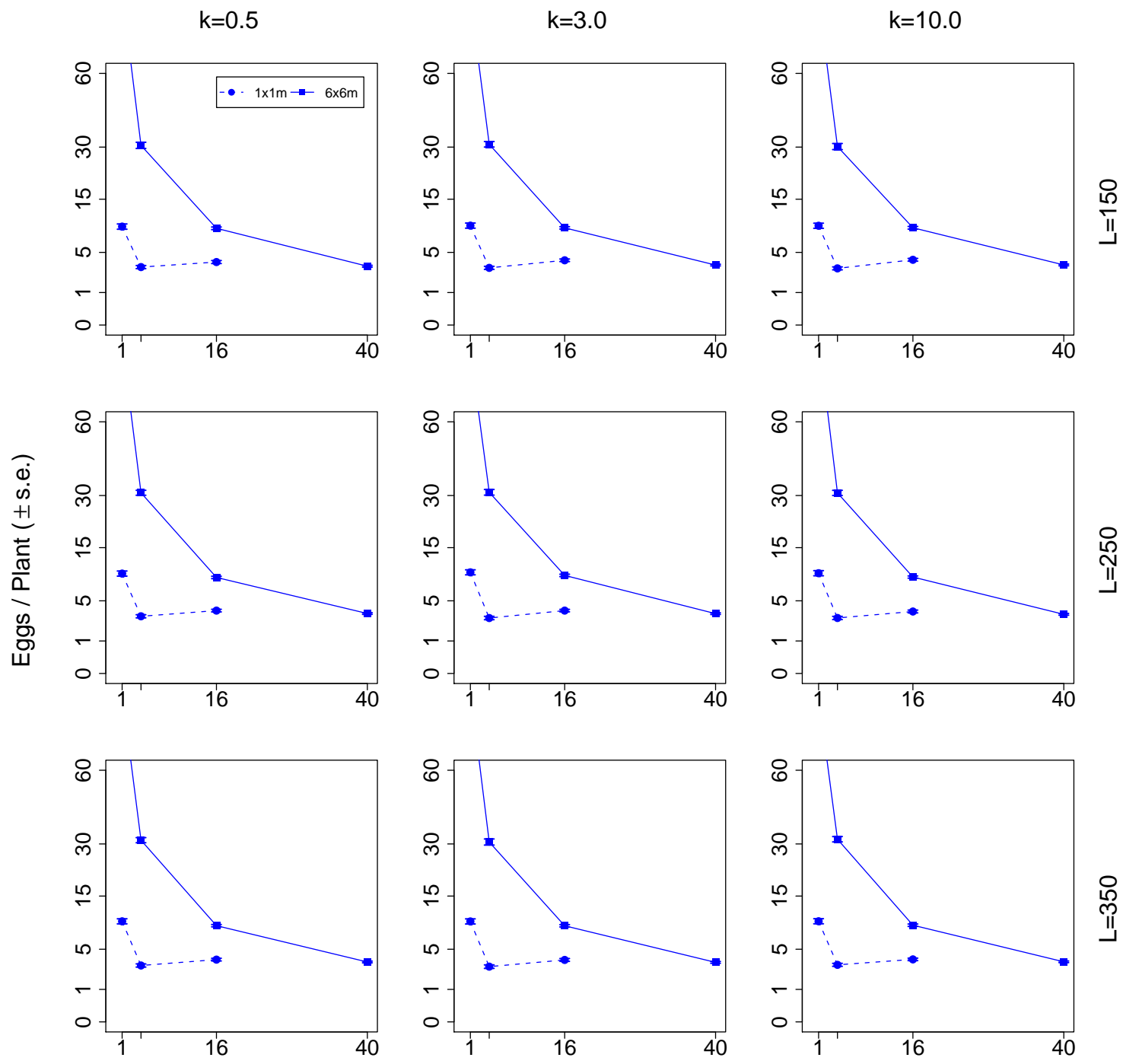

Plant Density

Figure B.18: Trial D3 (Vision - LEVIN-06-II), Single Egg, Response Summary (FD=1800) 

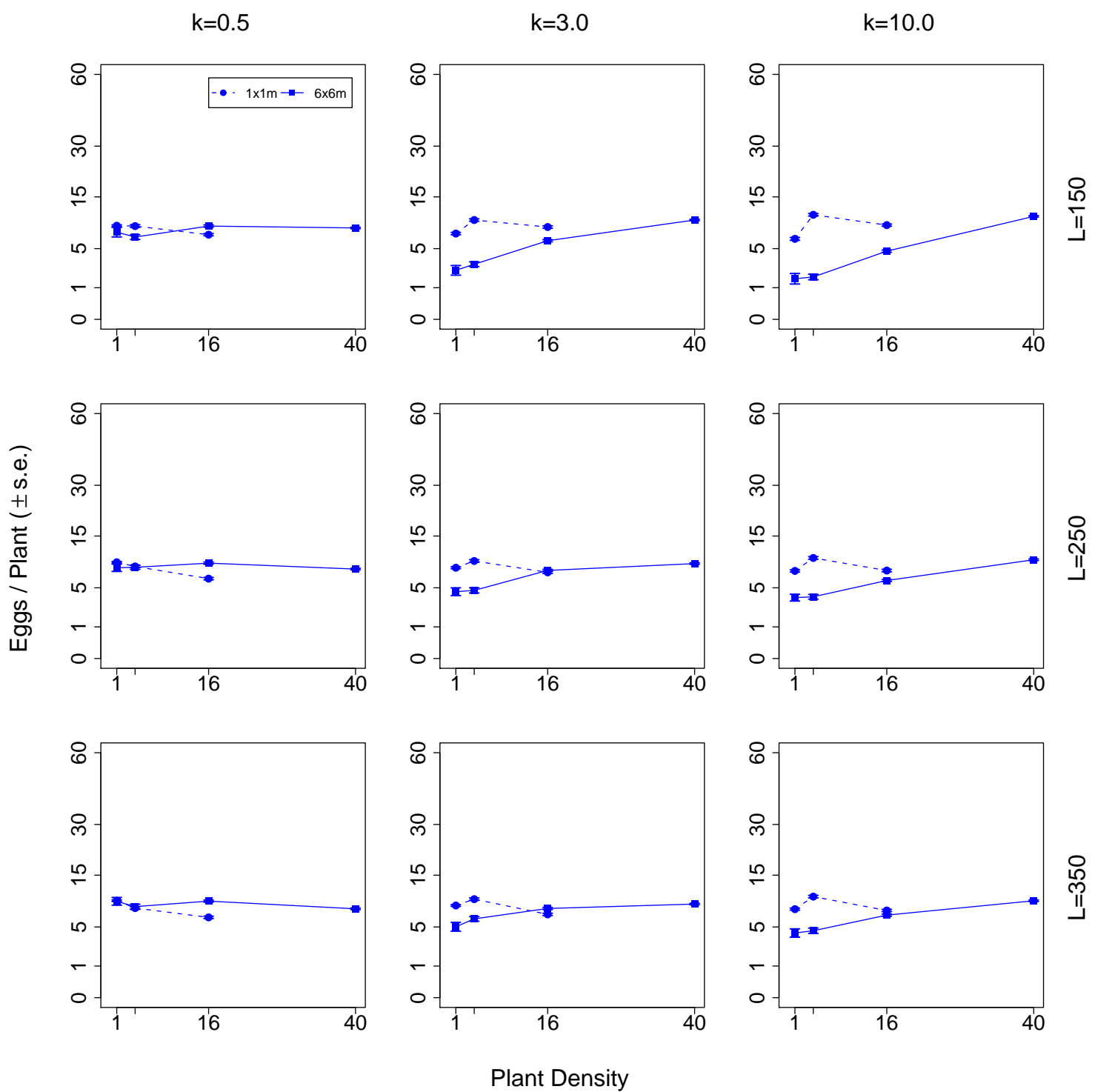

Figure B.19: Trial D4 (Olfaction - LEVIN-06-II), Multiple Eggs, Response Summary ( $R=5)$ 

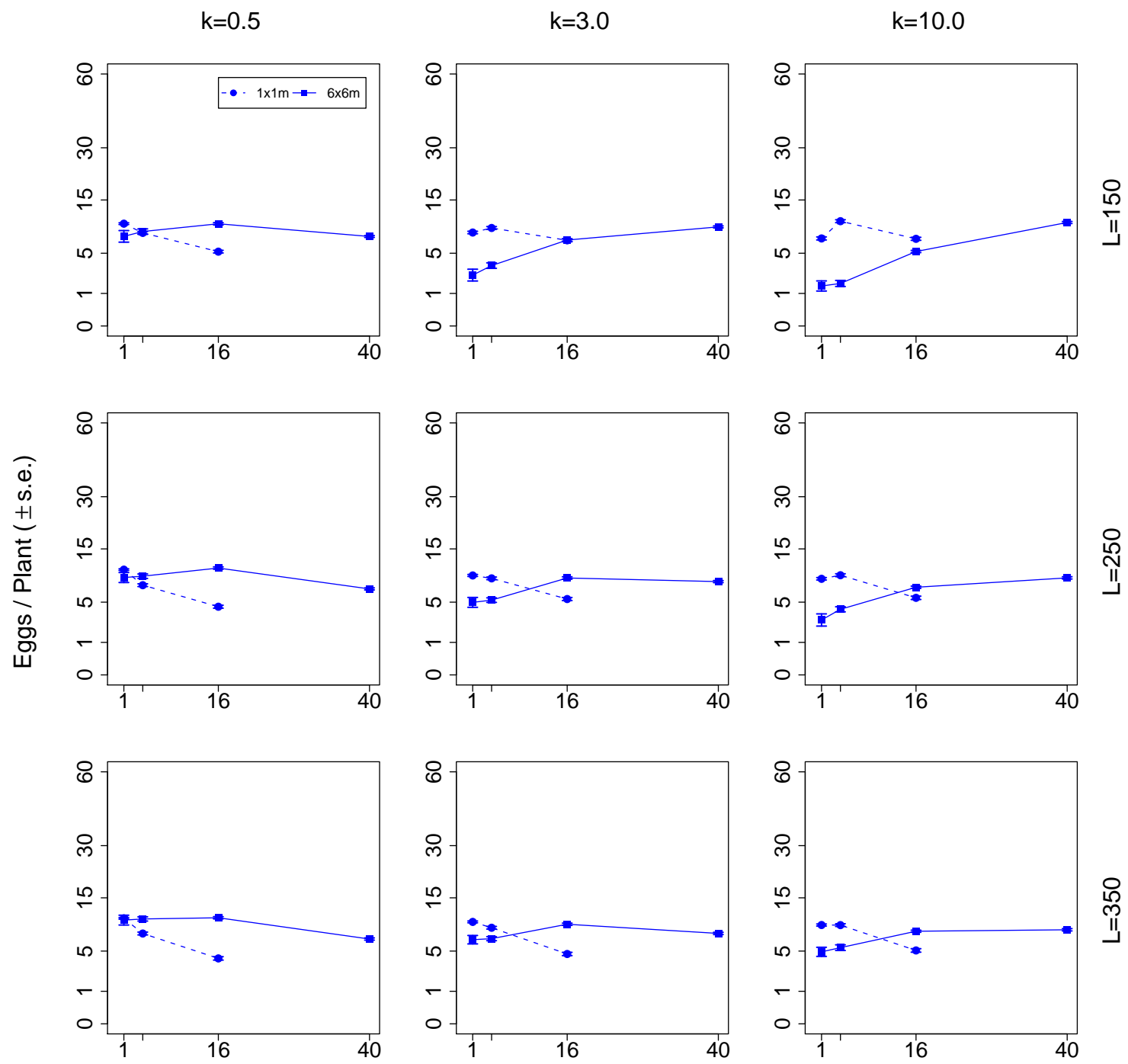

Plant Density

Figure B.20: Trial D4 (Olfaction - LEVIN-06-II), Multiple Eggs, Response Summary $(R=10)$ 

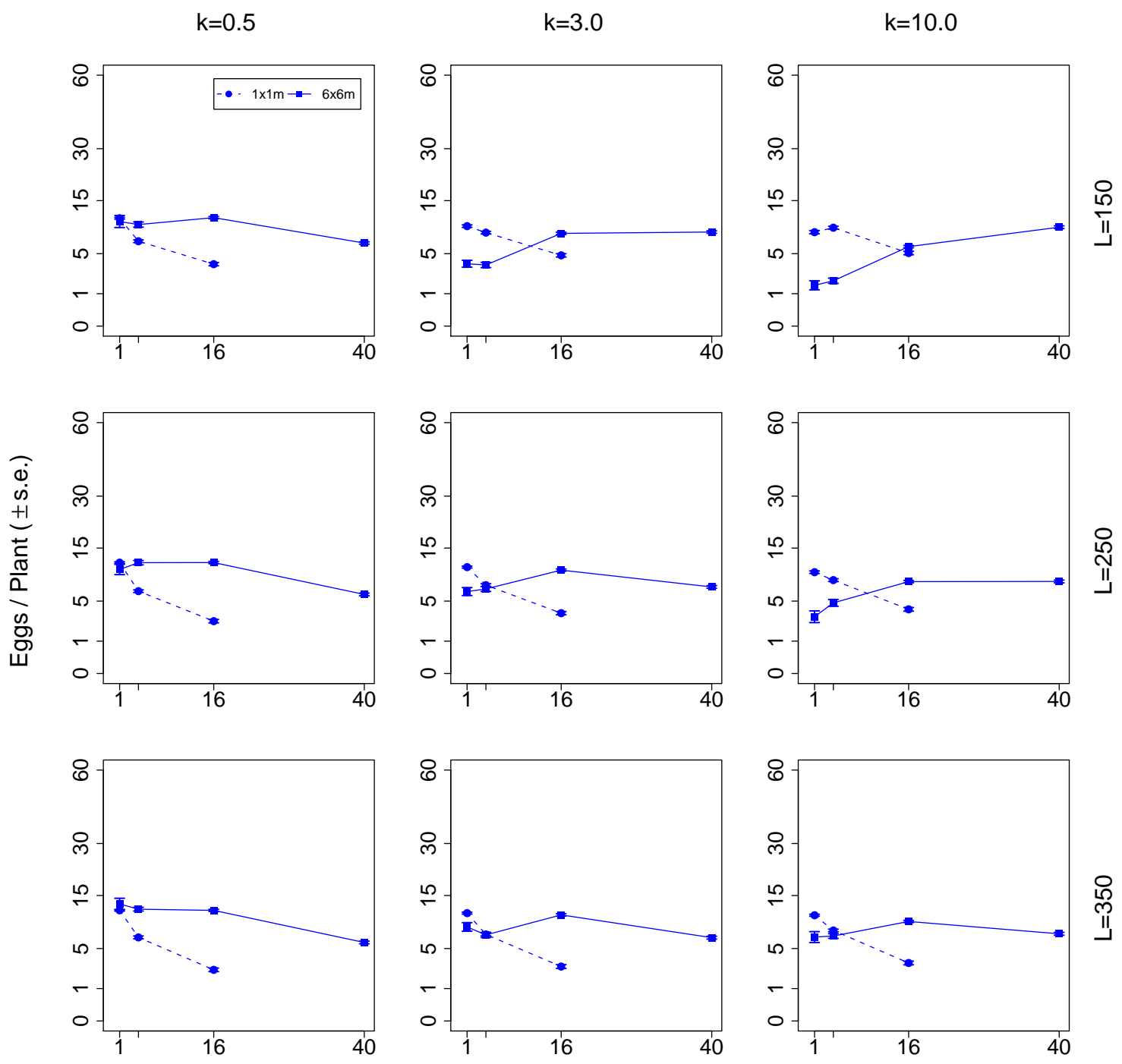

Plant Density

Figure B.21: Trial D4 (Olfaction - LEVIN-06-II), Multiple Eggs, Response Summary $(R=20)$ 

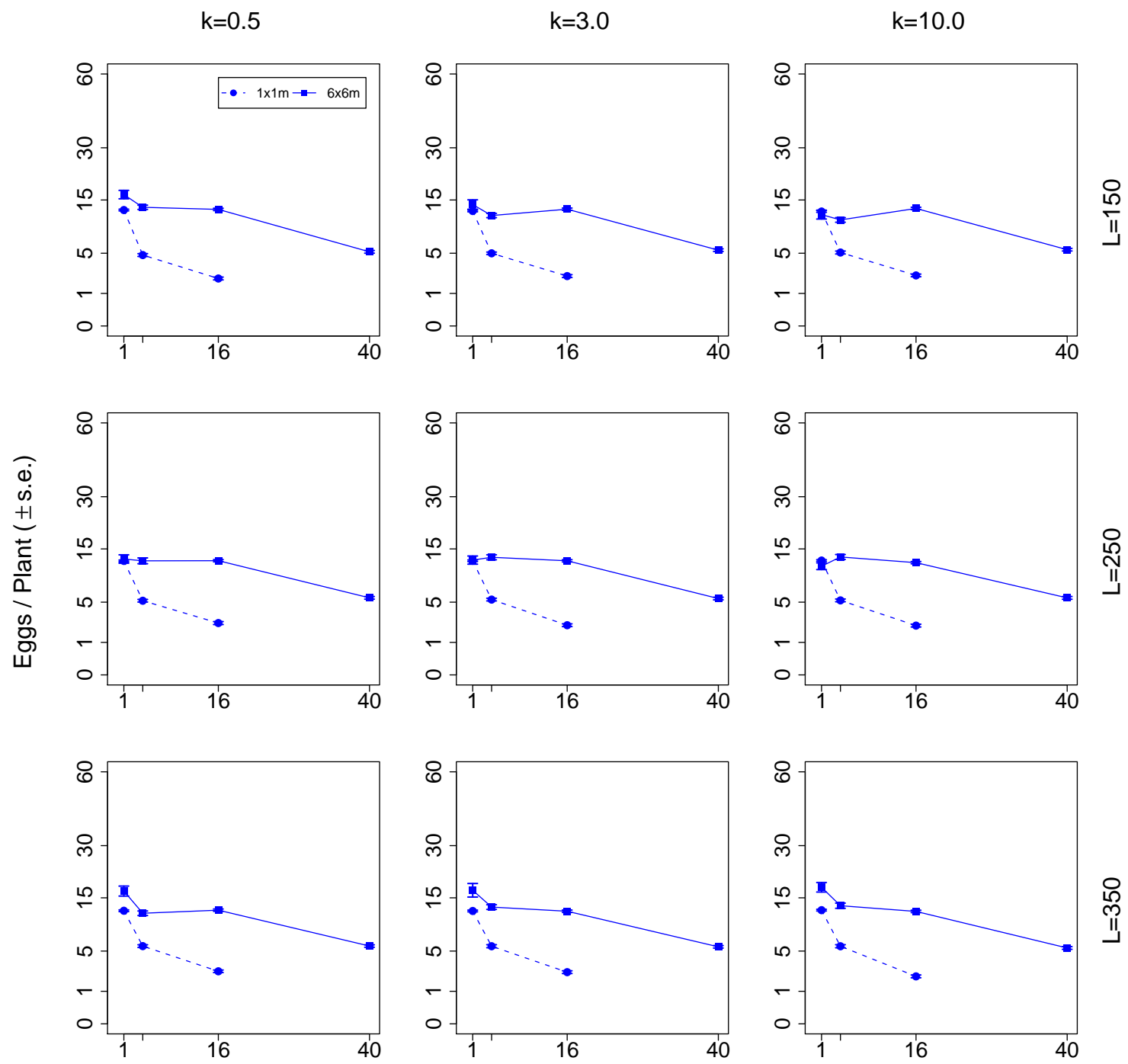

Plant Density

Figure B.22: Trial D5 (Vision - LEVIN-06-II), Multiple Eggs, Response Summary $(F D=100)$ 

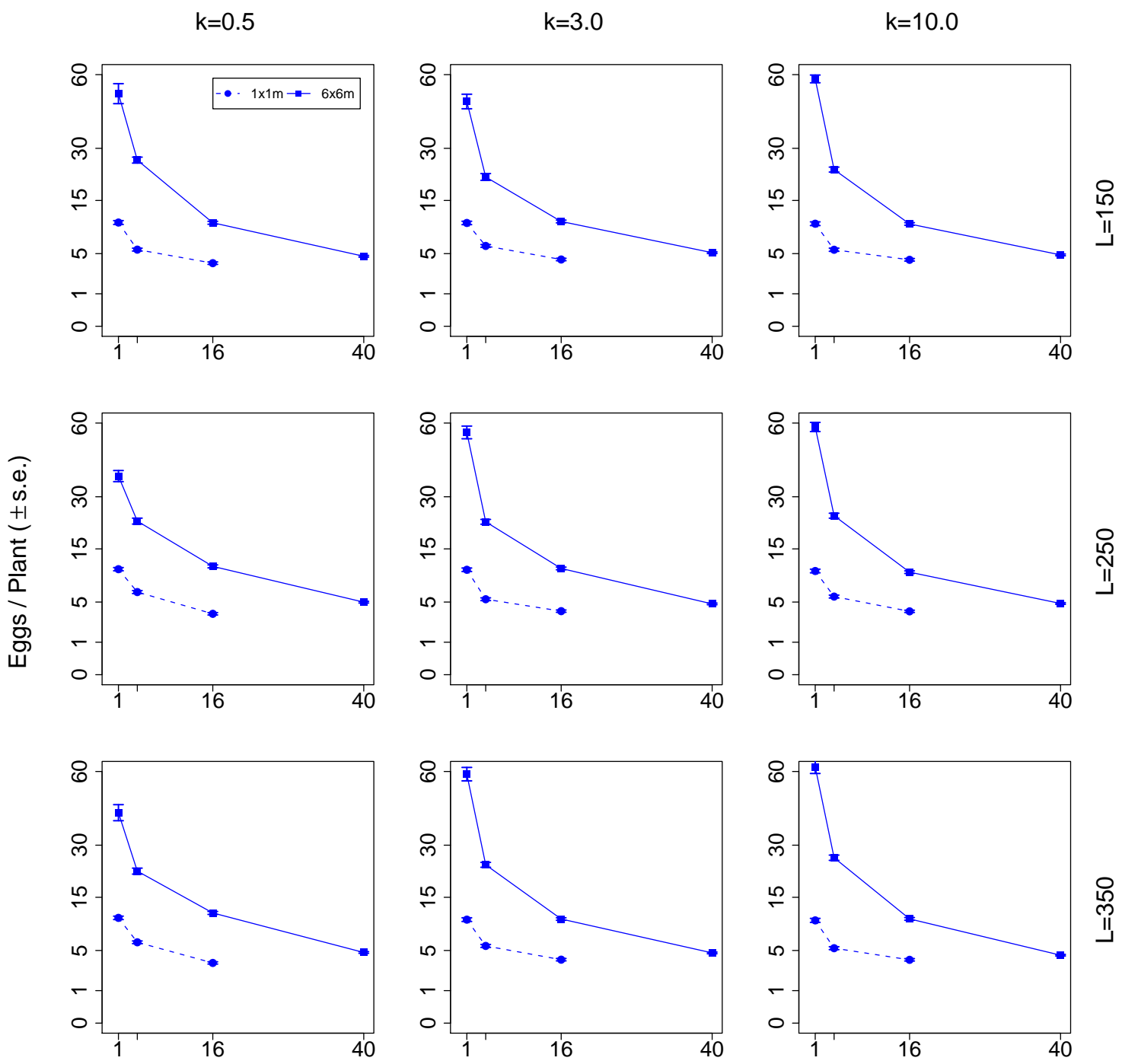

Plant Density

Figure B.23: Trial D5 (Vision - LEVIN-06-II), Multiple Eggs, Response Summary $(F D=600)$ 

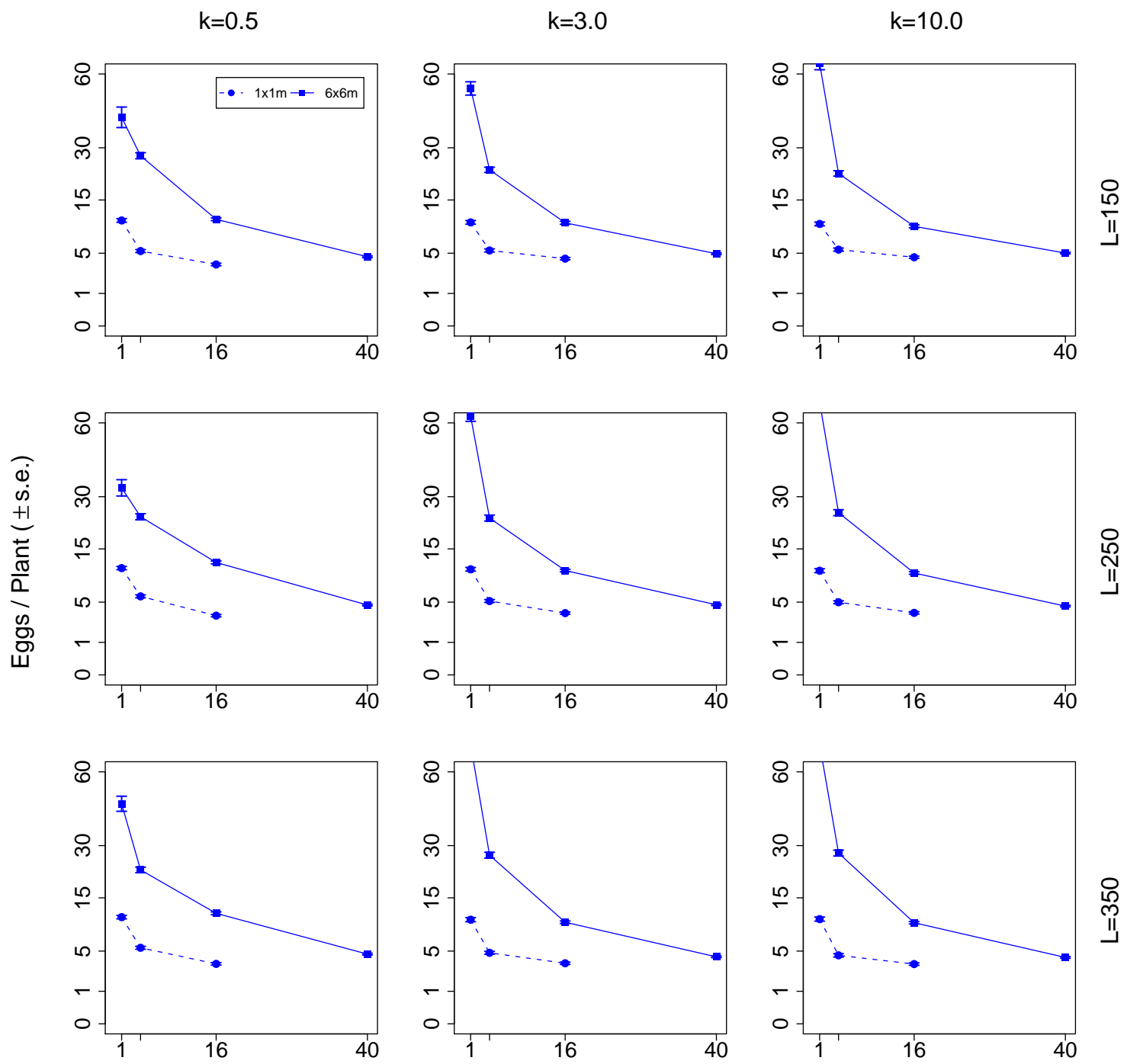

Plant Density

Figure B.24: Trial D5 (Vision - LEVIN-06-II), Multiple Eggs, Response Summary $(F D=1800)$ 

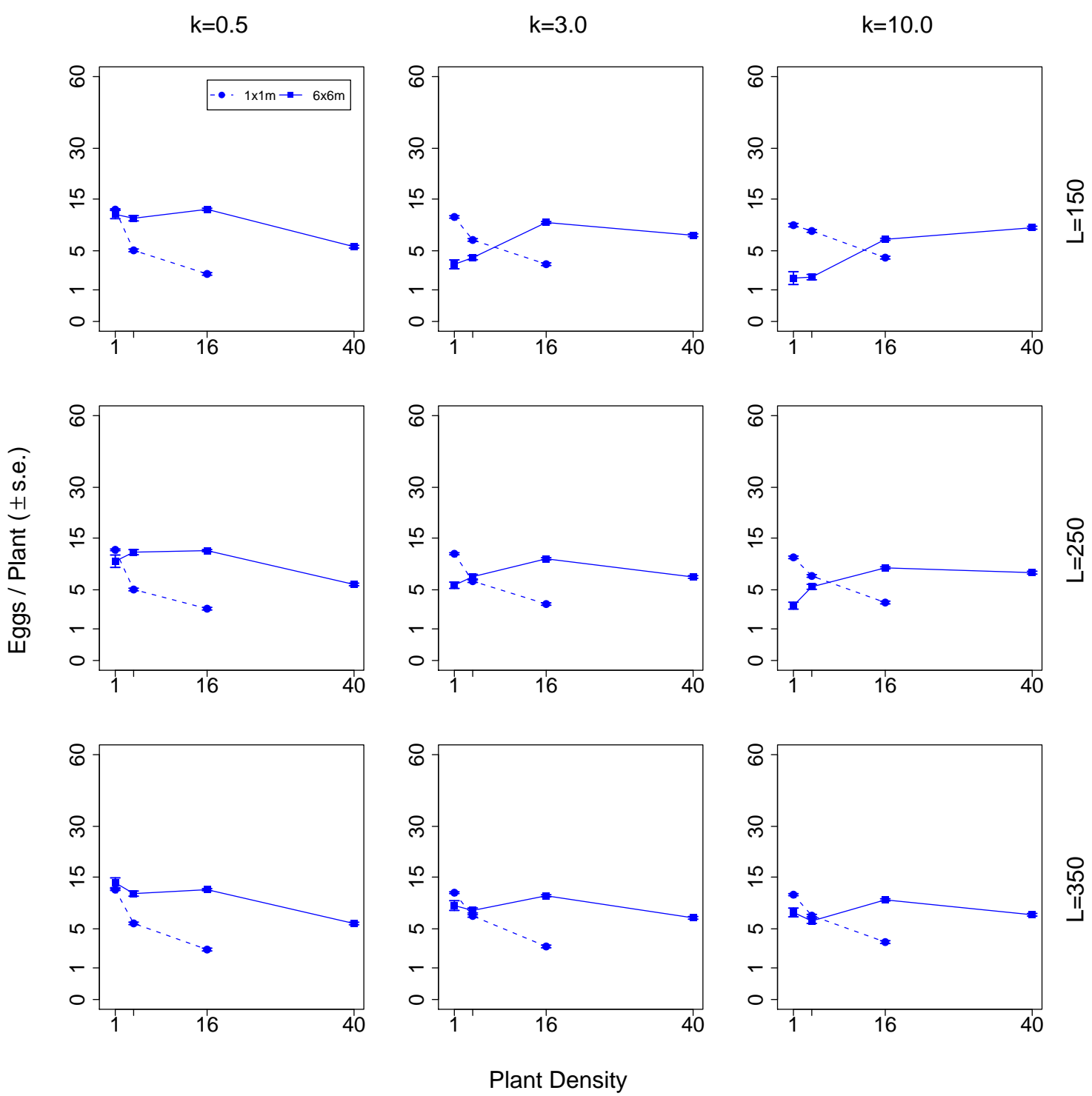

Figure B.25: Trial D6 (Olfaction and Vision - LEVIN-06-II), Multiple Eggs, Response Summary $(F D=100)$ 

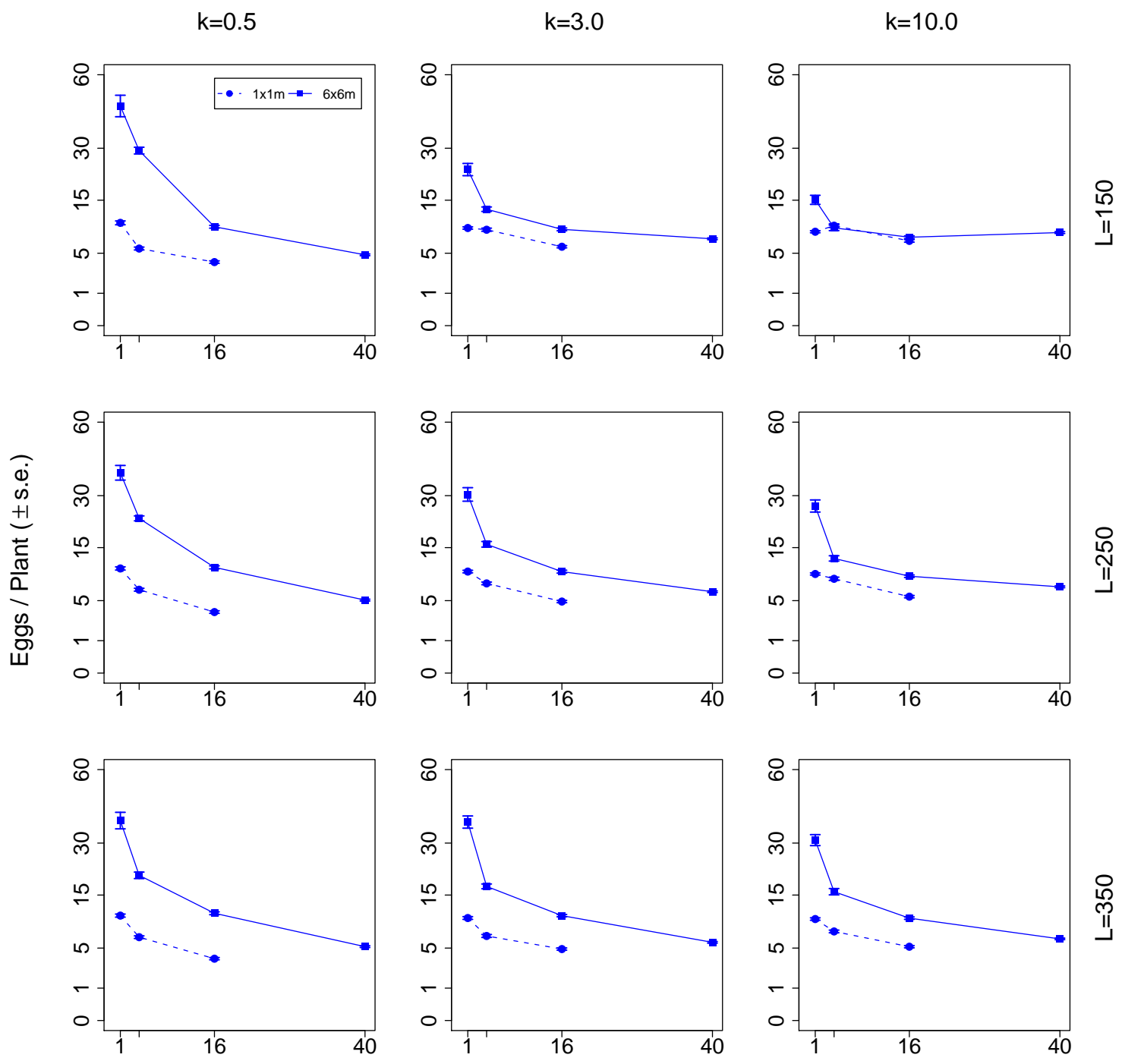

Plant Density

Figure B.26: Trial D6 (Olfaction and Vision - LEVIN-06-II), Multiple Eggs, Response Summary $(F D=600)$ 

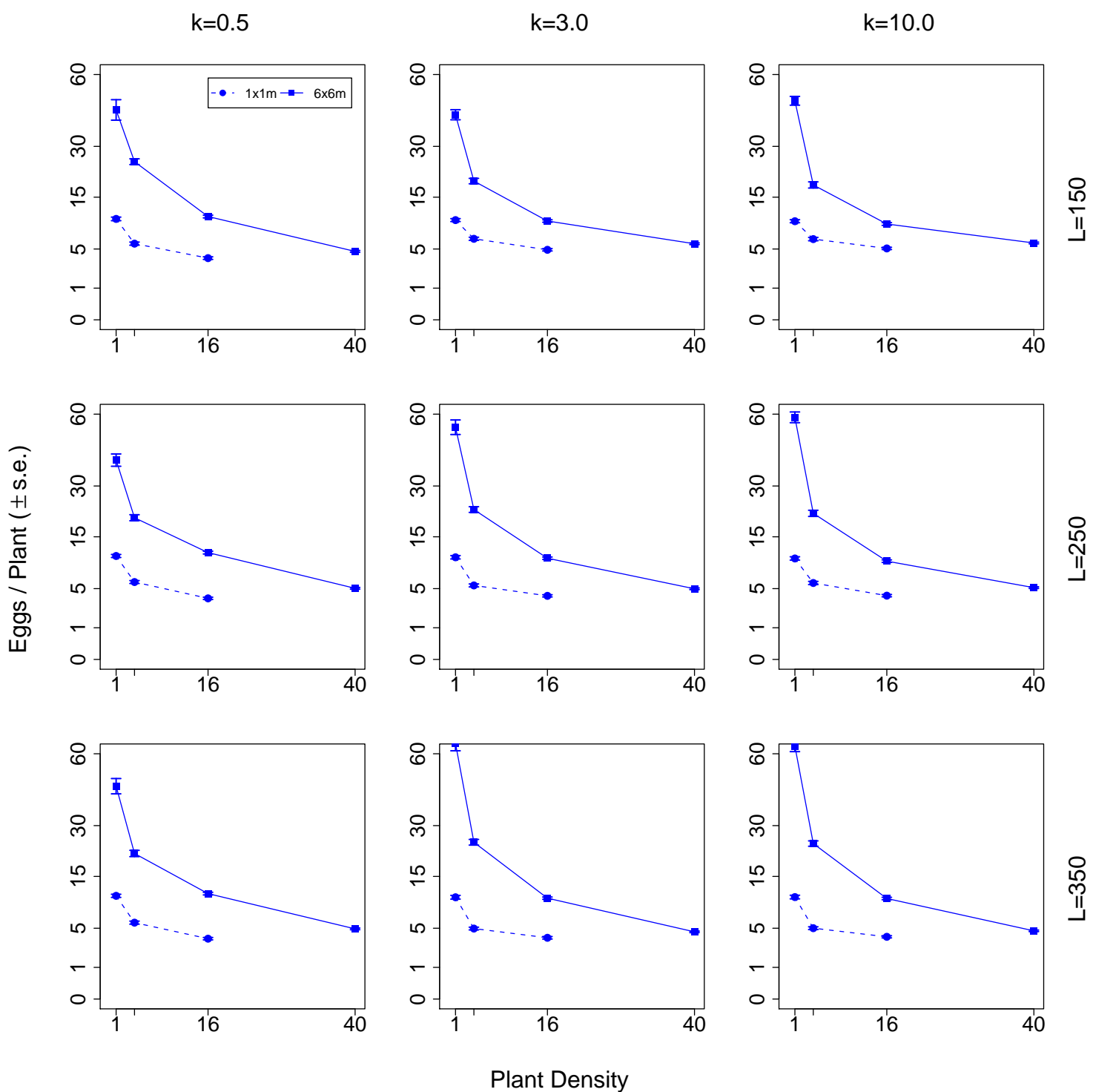

Figure B.27: Trial D6 (Olfaction and Vision - LEVIN-06-II), Multiple Eggs, Response Summary $(F D=1800)$ 


\section{B.3 Regression Statistics}

\begin{tabular}{lrrrrrrr}
\hline Scale & d.f. & slope & $\mathbf{R}^{\mathbf{2}}$ & $\mathbf{R}^{\mathbf{2}}$ (Adj.) & Std. Error & t-value & p-value \\
\hline $\mathbf{1 x 1 m}$ & 398.000 & -0.277 & 0.142 & 0.139 & 0.034 & -8.103 & $<\mathbf{0 . 0 0 1}$ \\
$\mathbf{6 x 6 m}$ & 398.000 & -0.258 & 0.155 & 0.152 & 0.030 & -8.532 & $<\mathbf{0 . 0 0 1}$ \\
$\mathbf{4 8 x 4 8 m}$ & 398.000 & -0.297 & 0.034 & 0.032 & 0.079 & -3.755 & $<\mathbf{0 . 0 0 1}$ \\
\hline
\end{tabular}

Table B.10: Table of regression statistics for KAITOKE-04 field experiment

\begin{tabular}{lrrrrrrr}
\hline Scale & d.f. & slope & $\mathbf{R}^{\mathbf{2}}$ & $\mathbf{R}^{\mathbf{2}}$ (Adj.) & Std. Error & t-value & p-value \\
\hline $\mathbf{1 x 1 m}$ & 242.000 & -0.185 & 0.052 & 0.048 & 0.051 & -3.652 & $<\mathbf{0 . 0 0 1}$ \\
6x6m & 242.000 & -0.172 & 0.019 & 0.015 & 0.079 & -2.172 & $\mathbf{0 . 0 3 1}$ \\
\hline
\end{tabular}

Table B.11: Table of regression statistics for LEVIN-06-II field experiment.

\begin{tabular}{lrrrrrrr}
\hline Scale & d.f. & slope & $\mathbf{R}^{\mathbf{2}}$ & $\mathbf{R}^{\mathbf{2}}$ (Adj.) & Std. Error & t-value & p-value \\
\hline $\mathbf{1 x 1 m}$ & 974.000 & -0.307 & 0.405 & 0.405 & 0.012 & -25.759 & $<\mathbf{0 . 0 0 1}$ \\
$\mathbf{6 x 6 m}$ & 974.000 & -0.397 & 0.285 & 0.284 & 0.020 & -19.696 & $<\mathbf{0 . 0 0 1}$ \\
\hline
\end{tabular}

Table B.12: Table of regression statistics for LEVIN-06-II simulation experiment, Trial C1 (LEVIN-06-II, CRW, Single Egg). Best fit, $R=5, L=150, k=0.5$

\begin{tabular}{lrrrrrrr}
\hline Scale & d.f. & slope & $\mathbf{R}^{\mathbf{2}}$ & $\mathbf{R}^{\mathbf{2}}$ (Adj.) & Std. Error & t-value & p-value \\
\hline $\mathbf{1 x 1 m}$ & 1598.000 & -0.195 & 0.113 & 0.113 & 0.014 & -14.286 & $<\mathbf{0 . 0 0 1}$ \\
$\mathbf{6 x 6 m}$ & 1598.000 & -0.211 & 0.166 & 0.165 & 0.012 & -17.835 & $<\mathbf{0 . 0 0 1}$ \\
$\mathbf{4 8 x 4 8 m}$ & 1598.000 & -0.159 & 0.016 & 0.015 & 0.031 & -5.062 & $<\mathbf{0 . 0 0 1}$ \\
\hline
\end{tabular}

Table B.13: Table of regression statistics for KAITOKE-04 simulation experiment, Trial C2 (KAITOKE-04, CRW, Single Egg). Best fit, $R=5, L=250, k=0.5$

\begin{tabular}{lrrrrrrr}
\hline Scale & d.f. & slope & $\mathbf{R}^{\mathbf{2}}$ & $\mathbf{R}^{\mathbf{2}}$ (Adj.) & Std. Error & t-value & p-value \\
\hline $\mathbf{1 x 1 m}$ & 974.000 & -0.302 & 0.450 & 0.450 & 0.011 & -28.253 & $<\mathbf{0 . 0 0 1}$ \\
$\mathbf{6 x 6 m}$ & 974.000 & -0.266 & 0.147 & 0.146 & 0.021 & -12.954 & $<\mathbf{0 . 0 0 1}$ \\
\hline
\end{tabular}

Table B.14: Table of regression statistics for LEVIN-06-II simulation experiment, Trial C3 (LEVIN-06-II, CRW, Multiple Eggs). Best fit, $R=10, L=350, k=10$

\begin{tabular}{lrrrrrrr}
\hline Scale & d.f. & slope & $\mathbf{R}^{\mathbf{2}}$ & $\mathbf{R}^{\mathbf{2}}$ (Adj.) & Std. Error & t-value & p-value \\
\hline $\mathbf{1 x 1 m}$ & 974.000 & -0.479 & 0.551 & 0.550 & 0.014 & -34.553 & $<\mathbf{0 . 0 0 1}$ \\
$\mathbf{6 x 6 m}$ & 974.000 & -0.503 & 0.256 & 0.255 & 0.027 & -18.306 & $<\mathbf{0 . 0 0 1}$ \\
\hline
\end{tabular}

Table B.15: Table of regression statistics for LEVIN-06-II simulation experiment, Trial D1 (LEVIN-06-II, Olfaction, Single Egg). Best fit, $R=10, L=150, k=0.5$ 


\begin{tabular}{lrrrrrrr}
\hline Scale & d.f. & slope & $\mathbf{R}^{\mathbf{2}}$ & $\mathbf{R}^{\mathbf{2}}$ (Adj.) & Std. Error & t-value & p-value \\
\hline $\mathbf{1 x 1 m}$ & 974.000 & -0.377 & 0.289 & 0.288 & 0.019 & -19.905 & $<\mathbf{0 . 0 0 1}$ \\
$\mathbf{6 x 6 m}$ & 974.000 & 0.098 & 0.008 & 0.007 & 0.034 & 2.844 & $\mathbf{0 . 0 0 5}$ \\
\hline
\end{tabular}

Table B.16: Table of regression statistics for LEVIN-06-II simulation experiment, Trial D1 (LEVIN-06-II, Olfaction, Single Egg). Multiple response, $R=20, L=150, k=10$

\begin{tabular}{lrrrrrrr}
\hline Scale & d.f. & slope & $\mathbf{R}^{\mathbf{2}}$ & $\mathbf{R}^{\mathbf{2}}$ (Adj.) & Std. Error & $\mathbf{t}$-value & p-value \\
\hline $\mathbf{1 x 1 m}$ & 974.000 & -0.515 & 0.624 & 0.624 & 0.013 & -40.202 & $<\mathbf{0 . 0 0 1}$ \\
6x6m & 974.000 & -0.578 & 0.331 & 0.331 & 0.026 & -21.970 & $<\mathbf{0 . 0 0 1}$ \\
\hline
\end{tabular}

Table B.17: Table of regression statistics for LEVIN-06-II simulation experiment, Trial D2 (LEVIN-06-II, Vision and Olfaction, Single Egg). Best fit, $F D=100, L=350, k=0.5$

\begin{tabular}{rrrrrrrr}
\hline Scale & d.f. & slope & $\mathbf{R}^{\mathbf{2}}$ & $\mathbf{R}^{\mathbf{2}}$ (Adj.) & Std. Error & t-value & p-value \\
\hline $\mathbf{1 x 1 m}$ & 974.000 & -0.524 & 0.648 & 0.648 & 0.012 & -42.354 & $<\mathbf{0 . 0 0 1}$ \\
$\mathbf{6 x 6 m}$ & 974.000 & -0.529 & 0.278 & 0.277 & 0.027 & -19.374 & $<\mathbf{0 . 0 0 1}$ \\
\hline
\end{tabular}

Table B.18: Table of regression statistics for LEVIN-06-II simulation experiment, Trial D3 (LEVIN-06-II, Vision, Single Egg). Best fit, FD =100, $L=350, k=10$

\begin{tabular}{lrrrrrrr}
\hline Scale & d.f. & slope & $\mathbf{R}^{\mathbf{2}}$ & $\mathbf{R}^{\mathbf{2}}$ (Adj.) & Std. Error & t-value & p-value \\
\hline $\mathbf{1 x 1 m}$ & 974.000 & -0.299 & 0.379 & 0.378 & 0.012 & -24.375 & $<\mathbf{0 . 0 0 1}$ \\
$\mathbf{6 x 6 m}$ & 974.000 & -0.214 & 0.082 & 0.081 & 0.023 & -9.307 & $<\mathbf{0 . 0 0 1}$ \\
\hline
\end{tabular}

Table B.19: Table of regression statistics for LEVIN-06-II simulation experiment, Trial D4 (LEVIN-06-II, Olfaction, Multiple Eggs). Best fit, $R=10, L=350, k=0.5$

\begin{tabular}{lrrrrrrr}
\hline Scale & d.f. & slope & $\mathbf{R}^{\mathbf{2}}$ & $\mathbf{R}^{\mathbf{2}}$ (Adj.) & Std. Error & t-value & p-value \\
\hline $\mathbf{1 x 1 m}$ & 974.000 & -0.316 & 0.313 & 0.313 & 0.015 & -21.086 & $<\mathbf{0 . 0 0 1}$ \\
6x6m & 974.000 & -0.606 & 0.485 & 0.484 & 0.020 & -30.280 & $<\mathbf{0 . 0 0 1}$ \\
\hline
\end{tabular}

Table B.20: Table of regression statistics for LEVIN-06-II simulation experiment, Trial D5 (LEVIN-06-II, Vision, Multiple Eggs). Best fit, FD $=600, L=250, k=0.5$

\begin{tabular}{lrrrrrrr}
\hline Scale & d.f. & slope & $\mathbf{R}^{\mathbf{2}}$ & $\mathbf{R}^{\mathbf{2}}$ (Adj.) & Std. Error & t-value & p-value \\
\hline $\mathbf{1 x 1 m}$ & 974.000 & -0.205 & 0.208 & 0.207 & 0.013 & -15.987 & $<\mathbf{0 . 0 0 1}$ \\
$\mathbf{6 x 6 m}$ & 974.000 & -0.391 & 0.319 & 0.319 & 0.018 & -21.380 & $<\mathbf{0 . 0 0 1}$ \\
\hline
\end{tabular}

Table B.21: Table of regression statistics for LEVIN-06-II simulation experiment, Trial D6 (LEVIN-06-II, Vision and Olfaction, Multiple Eggs). Best fit, $F D=600, L=250, k=3$ 


\begin{tabular}{lrrrrrrr}
\hline Scale & d.f. & slope & $\mathbf{R}^{\mathbf{2}}$ & $\mathbf{R}^{\mathbf{2}}$ (Adj.) & Std. Error & t-value & p-value \\
\hline $\mathbf{1 x 1 m}$ & 974.000 & -0.335 & 0.367 & 0.366 & 0.014 & -23.769 & $<\mathbf{0 . 0 0 1}$ \\
$\mathbf{6 x 6 m}$ & 974.000 & 0.077 & 0.008 & 0.007 & 0.027 & 2.839 & $\mathbf{0 . 0 0 5}$ \\
\hline
\end{tabular}

Table B.22: Table of regression statistics for LEVIN-06-II simulation experiment, Trial D6 (LEVIN-06-II, Vision and Olfaction, Multiple Eggs). Multiple response, FD =100, L= $250, k=10$ 


\section{B.4 Field Breakdowns}
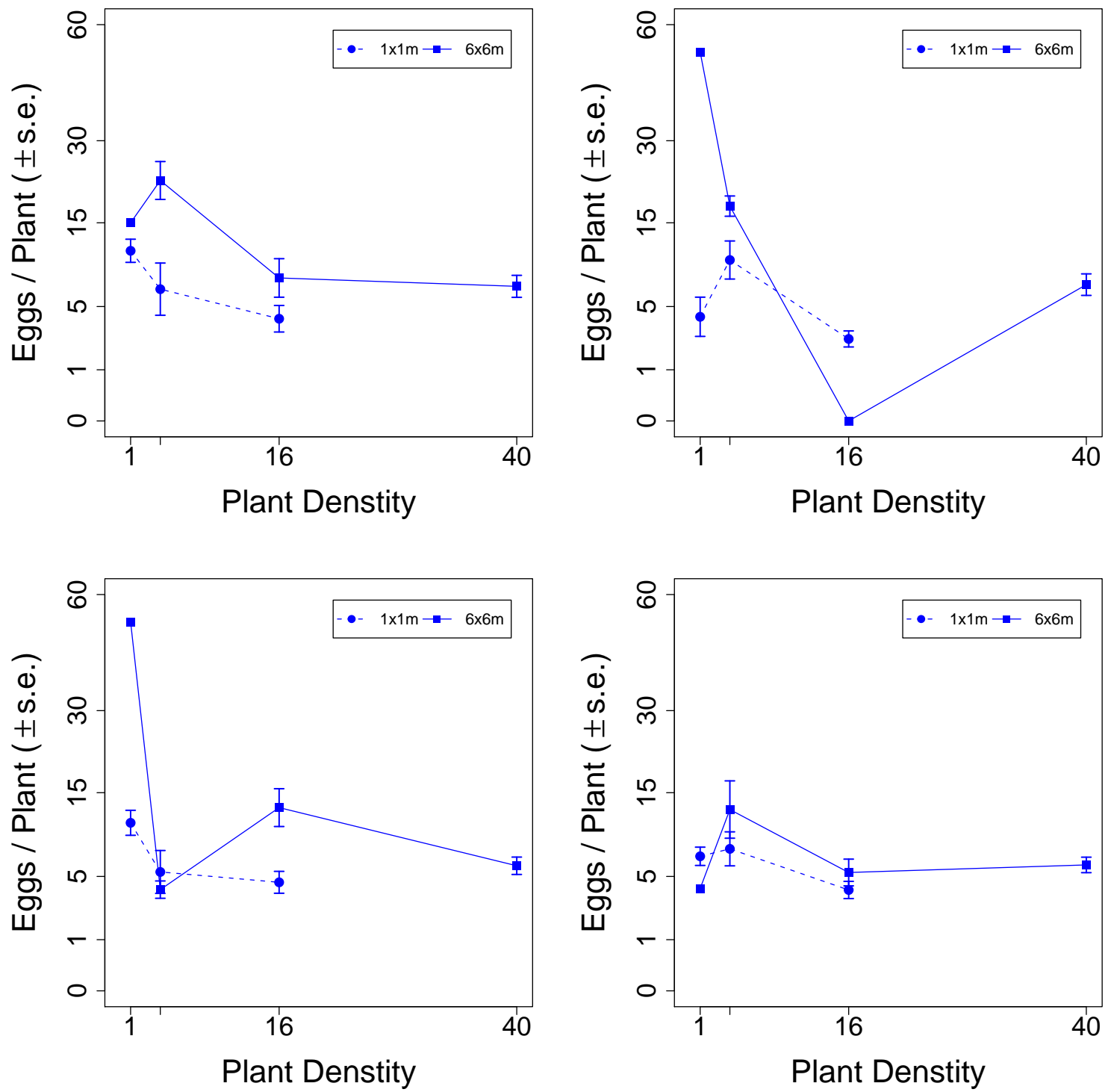

Figure B.28: Levin 2006 Field breakdown of Egg Distribution 

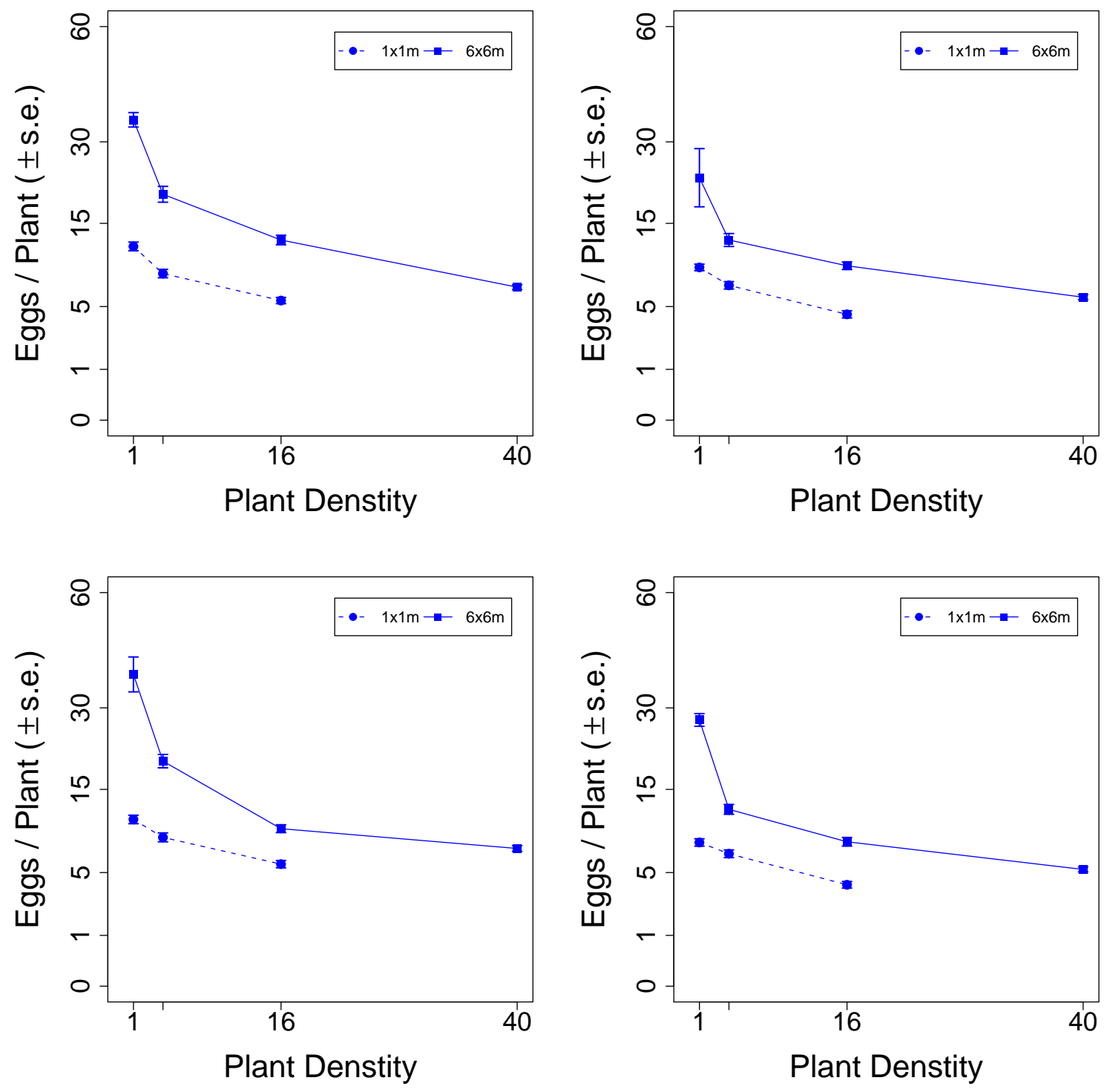

Figure B.29: Trial D6 - Olfaction Vision - Field Breakdown. FD=600, L=250, A=3.0, Multiple Eggs 

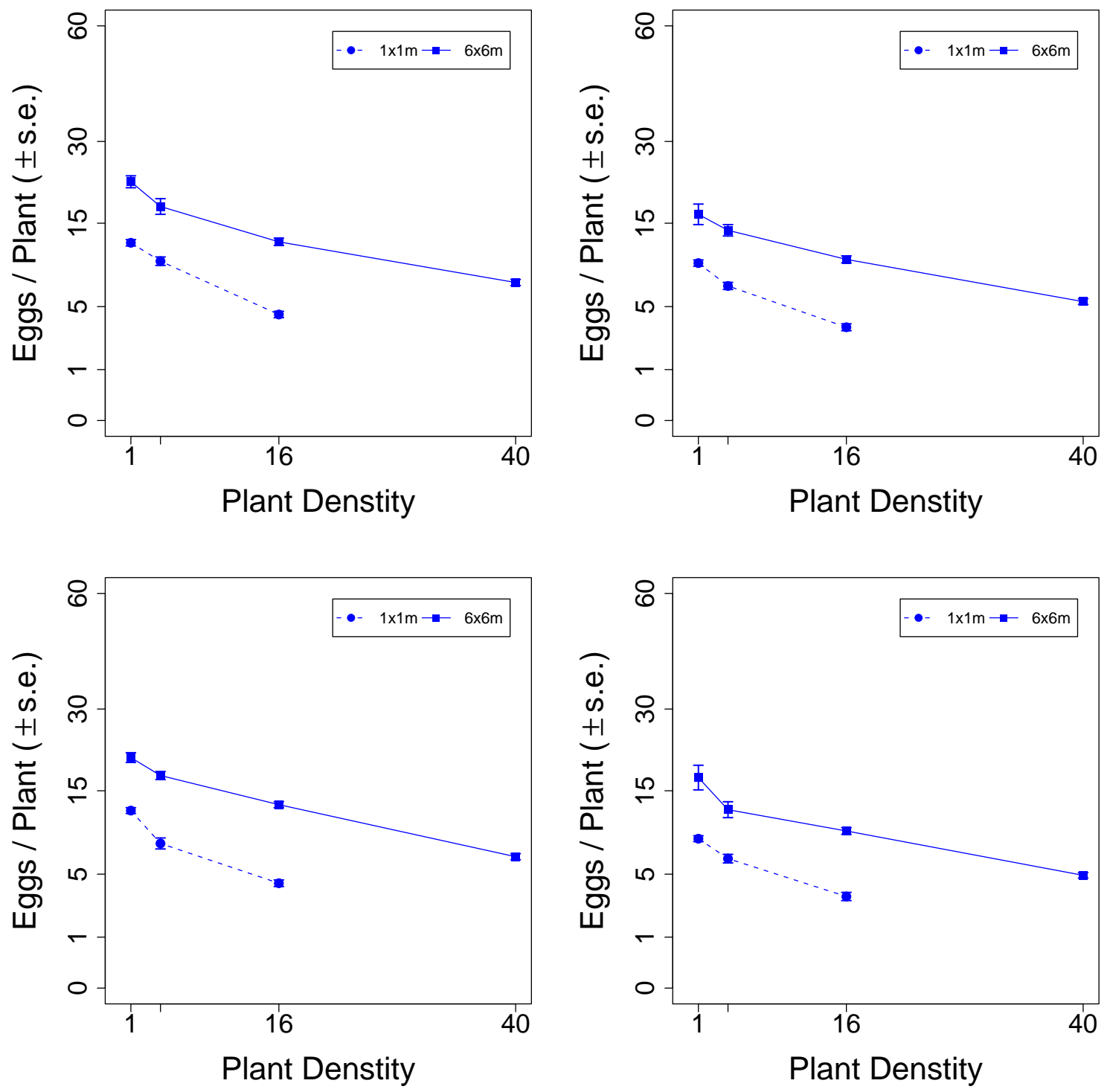

Figure B.30: Trial C1 - CRW - Single Egg - Field Breakdown. Best-fit $R=5, L=150, A=0.5$ 


\section{Appendix C}

\section{Method for generating Lévy dust or flight}

Lévy dust is a point pattern which is left after a Brownian like random walk is taken in space. Although it can be generated for 3D here it is constructed in 2D.

The main source for this method is Ogata and Katsura (1991). Reynolds (2006) also provided some assistance, as well as the obligatory Mandelbrot (1983). Kenkel and Walker $(1993,1996)$ also provide useful information about the general application of fractals to ecology.

Ogata and Katsura (1991) propose a simple method for generating a Lévy dust. The idea is that each step, the path is defined by polar coordinates $(R, \Theta)$ where $R$ is the radius (or step length), and $\Theta$ is the azimuth (degrees from north - defined in glossary of thesis). The step length is drawn at random from a distribution which is basically an inverse power function (Hastings and Peacock, 1974). This is similar to a negative exponential function except that it has a "fatter" tail. The power function distribution is defined by (C.1).

$$
\operatorname{pr}\left(R>r \mid R>r_{0}\right)= \begin{cases}1 & r \leqslant r_{0} \\ \left(r_{0} / r\right)^{D} & r>r_{0}\end{cases}
$$

$R$ is a step size chosen at random (random variable), $r$ is a particular step length, $r_{0}$ is the minimum step length and $D$ is the fractal dimension. This equation is a conditional probability equation. Secondly, defining the probability of $R$ being greater than $r$, given that it is greater than $r_{0}$.

There are two parts to understand. First, there is the concept of the minimum length, so that any $r$ below this length is excluded from the function. This means that any $R$ drawn at random must be greater than the minimum length. Hence for $r \leqslant r_{0}$, $\operatorname{pr}(R>r)=1$. $R$ will always be greater than $r_{0}$. 


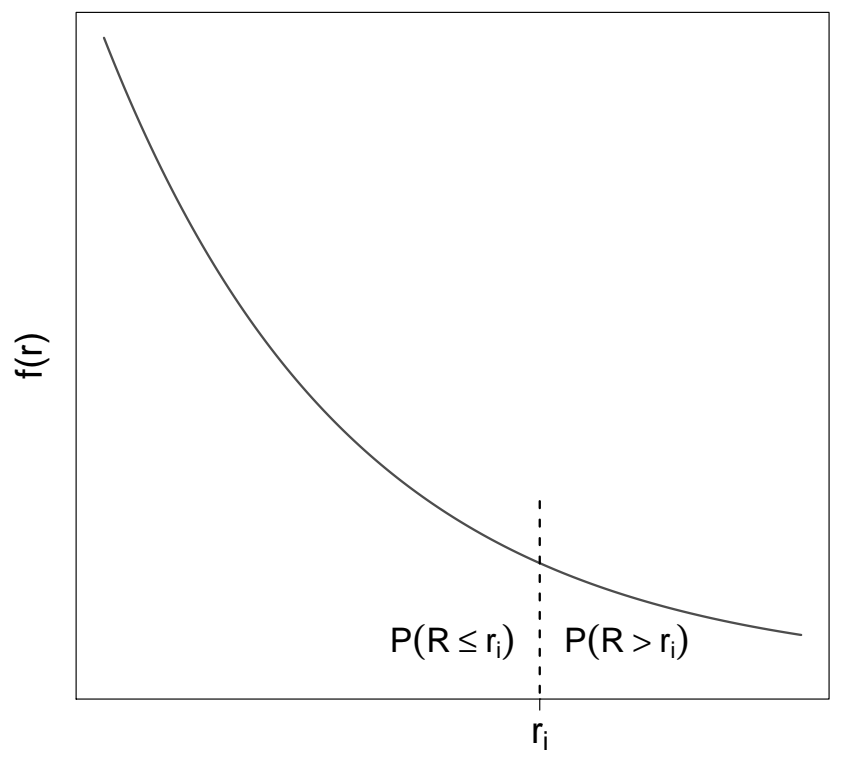

Step Length ( $r$ )

Figure C.1: Difference between expressing probability as $>$ or $<=$. The dotted line represents the point $r_{i}$ being considered. The probability $P\left(R \leqslant r_{i}\right)$ is then the area beneath the curve on the left.

For values of $r>r_{0}$, the probability that the random length $(R)$ has a value $R>r$ is defined by the expression $\left(r_{0} / r\right)^{D}$. This could be called an "upper tailed" probability function because it is expressed in terms of $R>r$ (Figure C.1). In other words, what is the probability of $R$ being greater than $r$ ? It is more usual to express it in terms of $P(X \leqslant x)=f(x)$.

If the probability of $R$ being greater than $r$ is given by $P(R>r)=x$, then the probability of $R$ being less than or equal to $r$ is given by $P(R \leqslant r)=1-x$ so the second condition from (C.1) can be re-written as (C.2):

$$
F(r)=1-\left(\frac{r_{0}}{r}\right)^{D}
$$

Which would be the equivalent writing (C.3). Figure C.2 demonstrates the difference between Equations C.1 and C.3.

$$
\operatorname{pr}\left(R \leqslant r \mid R>r_{0}\right)= \begin{cases}0 & r \leqslant r_{0} \\ 1-\left(r_{0} / r\right)^{D} & r>r_{0}\end{cases}
$$

Equation (C.3) now gives a cumulative probability distribution which can be used to generate random numbers from a uniform distribution. This is achieved by generating a uniform random number $(U)$ between 0 and 1 (essentially selecting a point at random on the y-axis of Figure C.2). By finding the inverse function $\left(F^{-} 1\right)$, i.e. rearranging (C.2) to make $r$ the subject, we can describe a function that will produce 
a step length $(R)$ given a uniform random number $(U)$ drawn from $U n i f[0,1]$. This is represented by Equation C.4.

$$
R=F^{-1}(U)
$$

If the value of the uniform random numbers is denoted by $u$, we can say write (C.5).

$$
u=F(r)=1-{\frac{r_{0}}{r}}^{D}
$$

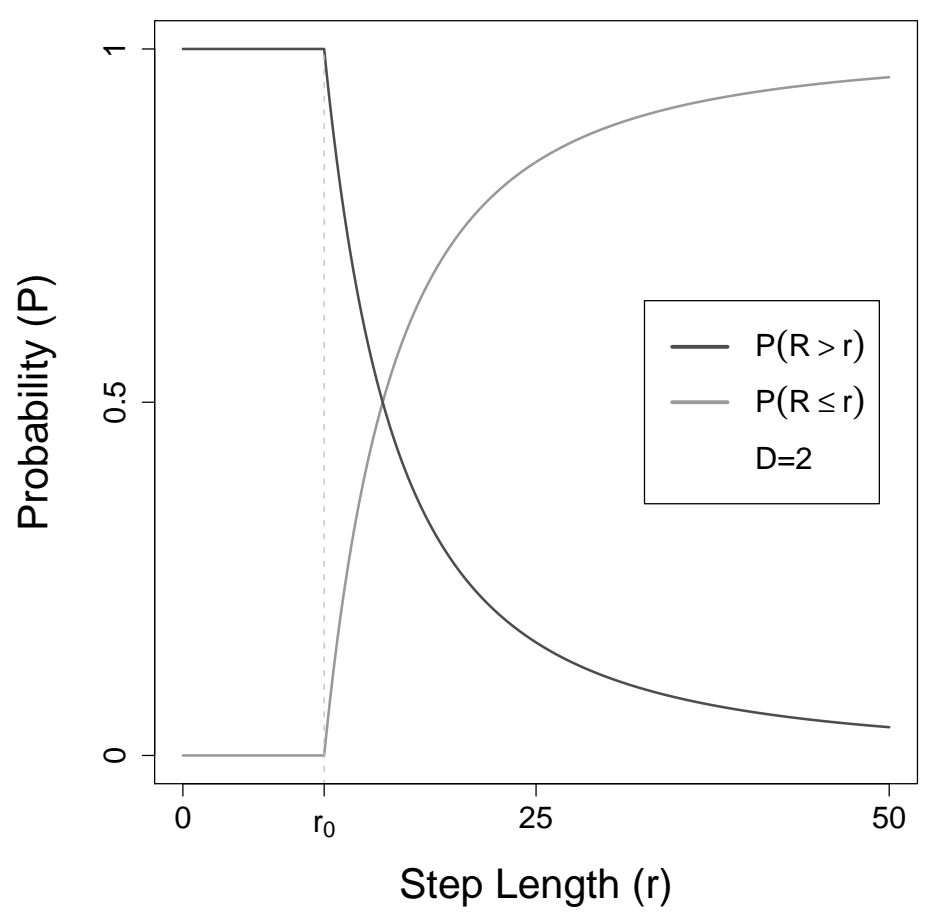

Figure C.2: Demonstration of Upper vs Lower tailed probability functions. The cumulative probability begins at 0 and tends towards 1 for the upper tailed $(P(R \leqslant r))$. The opposite is true for the lower tailed $(P(R \leqslant r))$. $r_{0}$ is shown which is the minimum step length.

When generating the random number, the value of $u$ is known, the value of $r$ which gives this probability is required. Rearranging (C.5) to make $r$ the subject leads to (C.6 and C.6).

$$
\begin{aligned}
& \left(\frac{r_{0}}{r}\right)^{D}=1-u \\
& \frac{r_{0}}{r}=(1-u)^{1 / D}
\end{aligned}
$$

Note that $\sqrt[n]{x} \equiv x^{1 / n}$, finally leading to (C.8).

$$
r=\frac{r_{0}}{(1-u)^{1 / D}}
$$


Thus for a specific random number $(U)$ generated from $U n i f[0,1]$, we can therefore generate a specific step length $(R)$ by using Equation C.8.

$$
R=\frac{r_{0}}{(1-U)^{1 / D}}
$$

Equation C.9 gives a simple transformation between a uniform random number $(U)$ and a power law probability density, with potentially infinite step size. The fractal dimension of the resulting path generated by this distribution of $R$ will be given by $D$. In this way a path or a set of points (given by the "footprints" of the path) can be generated with a specific fractal dimension (Figure C.3) There may be some issues with scaling here; sooner or later it will generate a massive step, but this will be very unlikely. A modification would be for the implementing code to simply truncate these results. Note that if $v=1-u$ and $u$ is Unif $[0,1], \mathrm{v}$ is also Unif $[0,1]$ and so we can implement the algorithm by replacing $1-u$ with simply $u$.

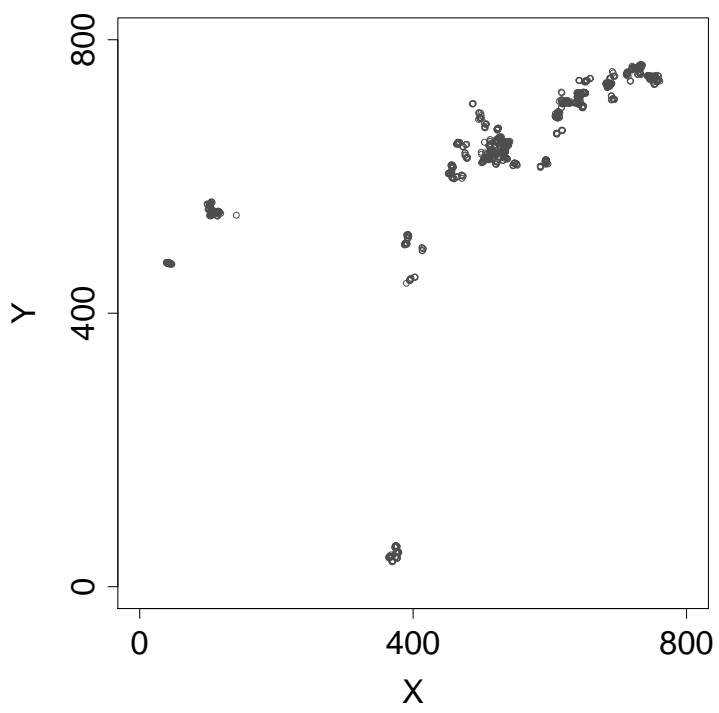

Figure C.3: Example of a Léry Dust pattern generated with a Léry walk. The fractal dimension $(D)=1$, minimum step length $\left(r_{0}\right)=2$, maximum step length (to prevent infinite step length $)=1000$, number of points $=1000$. The dimensions are arbitrary, but in the same units as the step length. This figure shows a larger area than Figures C.4 and C.5 because the pattern covers a larger area. 


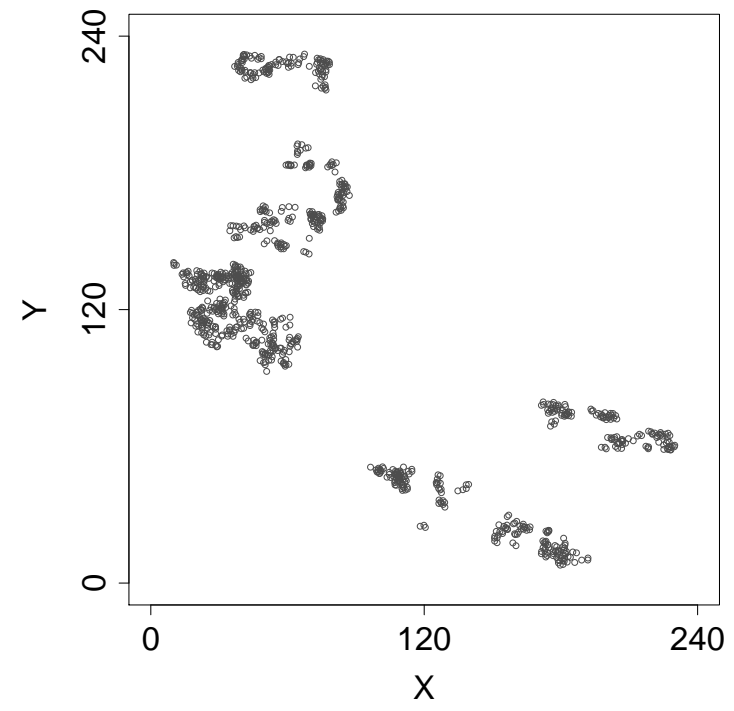

Figure C.4: Example of a Léry Dust pattern generated with a Lévy walk. The fractal dimension $(D)=1.5$, minimum step length $\left(r_{0}\right)=2$, maximum step length (to prevent infinite step length $)=1000$, number of points $=1000$. The dimensions are arbitrary, but in the same units as the step length.

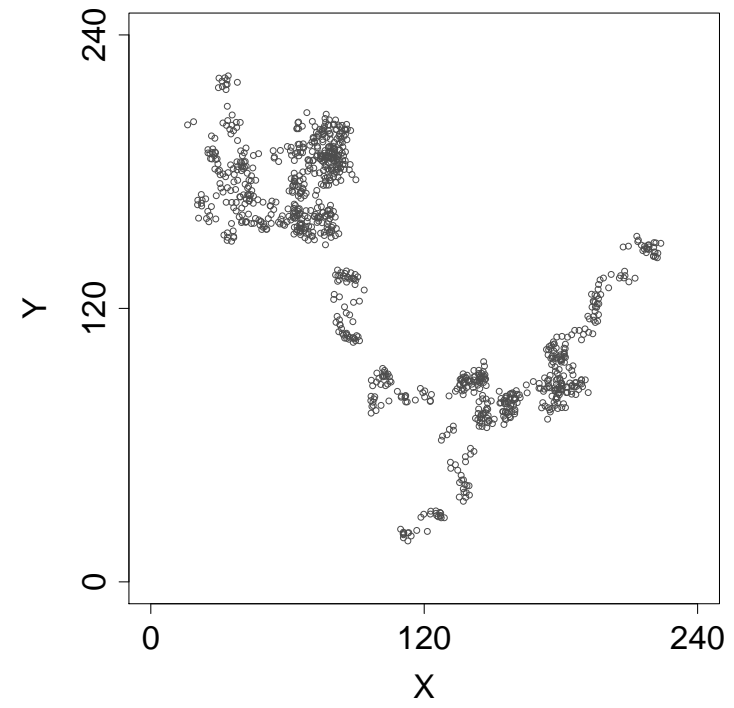

Figure C.5: Example of a Lévy Dust pattern generated with a Lévy walk. The fractal dimension $(D)=2$, minimum step length $\left(r_{0}\right)=2$, maximum step length (to prevent infinite step length $=1000$, number of points $=1000$. The dimensions are arbitrary, but in the same units as the step length. 


\section{References}

Hastings, N. and J. Peacock, 1974: Statistical Distributions. John Wiley and Sons.

Kenkel, N. and D. Walker, 1993: Fractals and ecology. Abstracta Botanica, 17 (1-2), 53-70.

Kenkel, N. and D. Walker, 1996: Fractals in the biological sciences. Coenoses, 11, 77-100.

Mandelbrot, B., 1983: The Fractal Geometry Of Nature. W.H. Freeman and Co, New York, USA.

Ogata, Y. and K. Katsura, 1991: Maximum-likelihood-estimates of the fractal dimension for random spatial patterns. Biometrika, 78(3), 463-474.

Reynolds, A. M., 2006: On the intermittent behaviour of foraging animals. Europhysics Letters, 75(4), 517-520. 